THIS BOOK IS DUE ON THE LAST DATE STAMPED BELOW

RENEWED BOOKS ARE SUBJECT TO IMMEDIATE RECALL

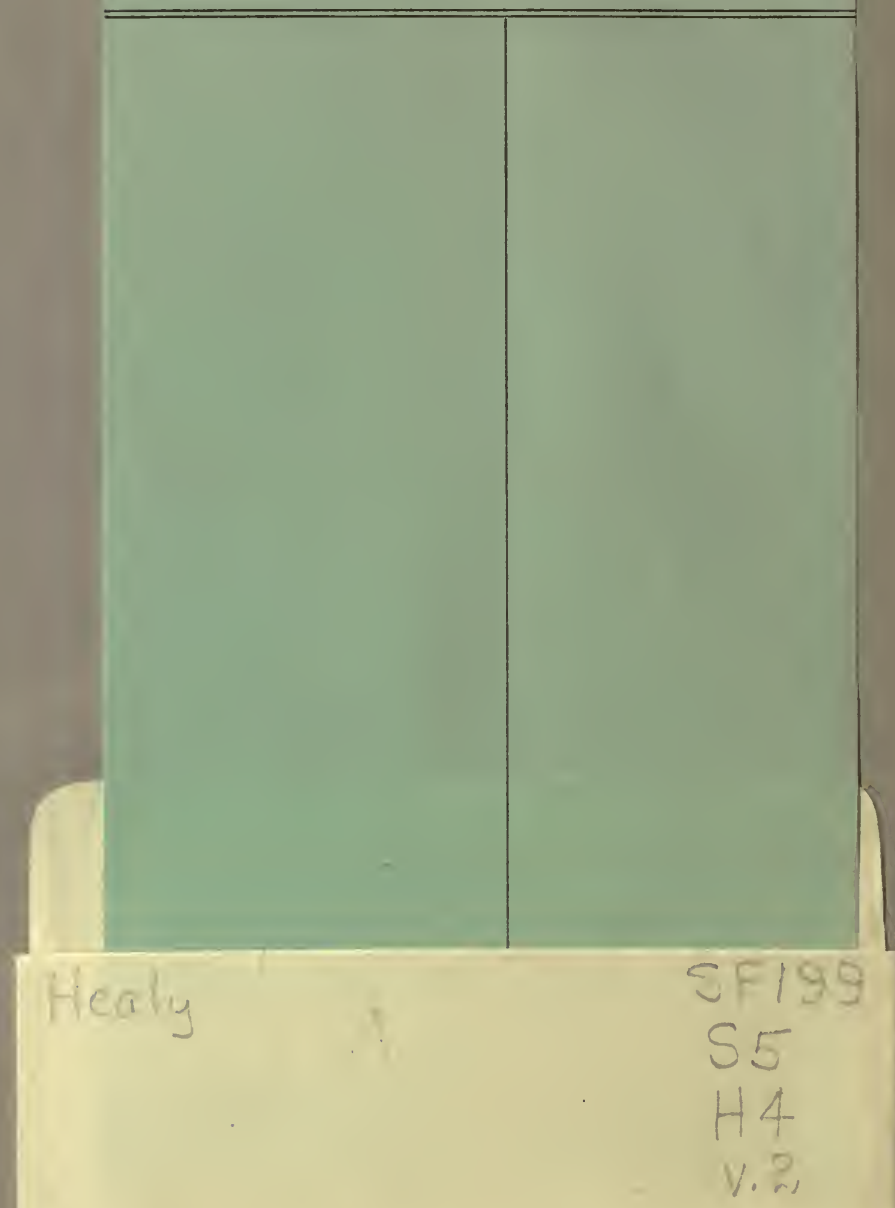




\title{
SHORT-HORN CATTLE
}

\author{
BY O. M. HEA \\ VOL. II.
}

With a Revised List of about should have been inclu 
Digitized by the Internet Archive in 2007 with funding from Microsoft Corporation 


\section{A RECORD}

OF

\section{UNFASHIONABLE \\ CRosses}

IN

\section{SHORT-HORN CATTLE PEDIGREES.}

BY O. M. HEALY.

\section{VOL. II.}

With a Revised List of about 3,000 crosses that should have been included in Vol. I.

BEDFORD, IOWA :

PUBLISHED BY O. M. HEALY.

1887. 
Entered according to Act of Congres8, in the year 1883, BY F. P. AND O. M. HEALY,

in the office of the Librarian of Congress, at Washington.

PRINTED BY PORTER PRINTING CO., Moline, ILLINoIS. 


\section{PREFACE.}

In issuing this, our second volume of Unfashionable Crosses in Short-Horn Pedigrees, we do so believing it will meet the approval and receive the patronage of Short-Horn Breeders generally. We have taken great pains to make it as near correct and complete as possible laying all prejudice aside.

This volume contains a revised list that should have been included in our first volume. The descendants of Imported Animals that were imported without pedigrees, and those whose sires have no recorded pedigrees are included in the revised list, and to avoid much confusion we have classed them as $L R$, Lost Record pedigrees, or tracing to the same, with the exception of a few families that are more numerous and better known, and whose descendants have been crossed on many of the fashionable families, are classed separate and given a name or character signifying the family they descend from.

They are as follows :

Britannia, (without pedigree) Imported in 1837, by Henry Clay Jr., of Kentucky.

Beauty, (Vol. 15, A. H. B.) Imported in or about $1834,{ }_{,}^{2} b y$ Thos. Weddle, of N. Y., got by Favorite - (no number), dam by Comus (161), by Patriot (486), etc.

Multiflora, Imported in 1833, by Walter Dun, of Kentucky ; got by Walter 11118, A. H. B. (recorded under certificate); dam White Face by Roland (563), etc. See remarks to Dun Importation, Volume 1 , of our work.

Milk Spring, (Vol. 22, A. H. B.) calved in 1835 ; believed to have been imported the same year; got by Leander (4199); dam Young Blossom by Brigade Major (817); Blossom by Planet (1325), etc. The breeding of Imported Milk Spring is considered objectionable, and is evidently erroneous as her given sire Leander (4199) was not calved until 1838 , three years after the date given of her birth. 
Rose, Imported in 1833, by Wm. Jackson, of New York ; got by Skipton, 978 ; dam Red Rose by Young Cleveland (3401) ; Carnation by Apollo (36), etc. The pedigree of Imported Rose is also believed to be incorrect.

The bull Rolla (4991), and his descendants are also included. His pedigree as recorded reads thus: Bred by Col. Van Renssaller the property of Mr. Rotch, Butternuts, Otsego Co., N. Y.; got by Admiral .(1608) ; dam Romp by Admiral (1608); Moss Rose by Young Denton (693); Rosa by Young Denton (963); Ruby by Young Denton (963). Thus it will be observed that his pedigree as recorded does not descend on the dam's side to an imported cow, but is believed by some of the best authorities to be a direct descendant of Imported Tuberose by North Star (460), (a cow of most excellent breeding) and that his pedigree should read: Got by Admiral (1608): dam Romp by Admiral (1608): Moss Rose by Young Denton (963); Rosa by Young Denton (963): Imported Tuherose by North Star (460): Tuberose by Cripple (173); Tulip by Comet.(115); Cherry by Ladrone (353): by Danby (190); by Son of Favorite (252). By comparing his pedigree with others recorded in the early volumes of the American Herd Book, and leaving off the last cross (Ruby by Young Denton), it seems very reasonable to believe that he is a descendant of Imported Tuberose, and that his pedigree as recorded is wrong. But at this late date (more than one-half a century since he was calved), no one is able to state (only by belief) whether his pedigree is right or wrong.

This cross occurs in some of the most fashionable families, and we believe is objected to only by breeders of high class pedigrees. For the benefit of those that do not object to the cross, we have endeavored to mark all pedigrees that have no other objectionable cross than Rolla as tracing (-) to Rolla.

BEDFord, IowA, Jan. 1, 1887.

O. M. HEALY. 


\section{EXPLANATION.}

P S, means the Patton Stock; M, Mrs. Motte; T, the Teeswater Cow ; D, the Durbam Cow (of the Seventeen Importation); KY I, Kentucky Importation, many pedigrees tracing to or ending in "Tracing to the Kentucky Importation," meaning the importation of 1817; COX, the Cox Importation; DAISY, Daisy by Wild, 11134; C, Caroline by Dashwood, 9731 ; R R, Red Rose by Ernesty, 10017; MUL'TIFLORA, Multiflora by Walter, 11118 (of the Dun Importation). For remarks to the above families, see first volume of this work. ROLLA, Rolla (4991) pedigrees marked as tracing to Rolla are supposed to contain no other objectionable cross. BEA UTY, Beauty by Favorite — Imported by Thos. Weddle; ROSE, Rose by Skipton, Imported by Wm. Jackson; B, Britannia, Imported by H. Clay ; MILK SPRING, Imported Milk Spring; L R, Lost Record; numerous families are included in this list, descendants of Imported Animals without pedigrees, or whose sires are without record, also pedigrees ending in "From the Imported Stock of Mr. —— " without showing direct descent from the imported Animals and those of unknown Importations. WOODS, animals supposed to not trace to any Importation. Animals with after the name means that there is a cross of such blood in their pedigree, although it may be a distant one. Numbers in parenthesis ( ), refer. to the English Herd Book, open to the American Herd Book. 



\title{
A List of Unfashionable Crosses
}

\author{
IN
}

SHORT-HORN CATTLE PEDIGREES.

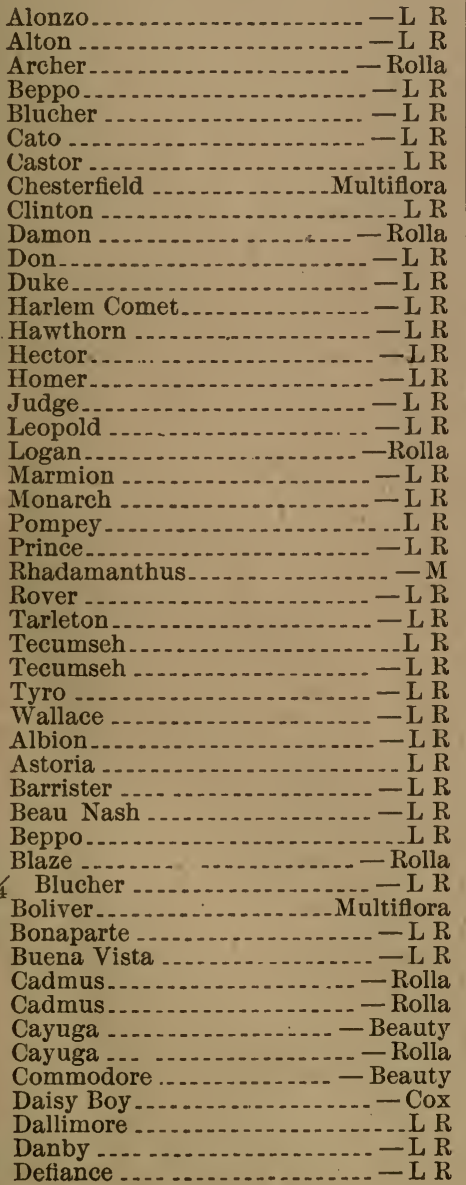

Alonzo

417

440

460

474

475

483

484

488

502

508

516

549

559

560

565

566

568

569

570

575

596

5981

618

623

625

630

635

644

652

$66^{\circ} 1$

683

685

694

698

$(721)$

724

733

739

750

751

754

767

768

785

791

808

813

815
Diamond.

-Beauty

Deke....................

Earl of Elles more ..........

Emperor, 2d.................... R

Empire................. Beauty

Exchange ................... Rose

Exchange, Jr.,............... R

Fairfax_................. $R$

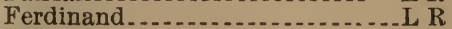

Fop ........

Fremont ............

Granite.....

Hampton ........ L R

Hampton

Hector ................

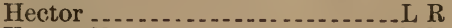

Henry Clay

Henry Clay

Henry Clay, $2 \mathrm{~d} . . . . . . . . . . . .-\mathrm{L} R$

Hero......................... Rolla

Jeffery

$1 / 2$ John Bull .................... R

Kossuth ........... R

La Fayette ....... Rolla

Lambro ............. L R

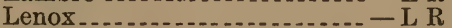

Leopard.......................... R

Little John ................... L R

Logan, 2d................... Rolla

Lord Byron .......... R

March _............... Beauty

Marius, $2 \mathrm{~d}$

Matchless ................. $-L ~ R$

Memnon............. Rolla

Adrain ................ R

Morgan .................. R

Albert .......................

Napoleon .................... $R$

Nebraska ............. Rolla

Nicholas ............. R

Nicholas Biddle............

Noble ............

Old Splendor-...........

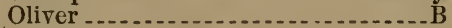

Orman

Ossian .......... Rolla

Paul Jones ................ L R

Petrarch .................. $\mathrm{R}$

Pilot .............................. 


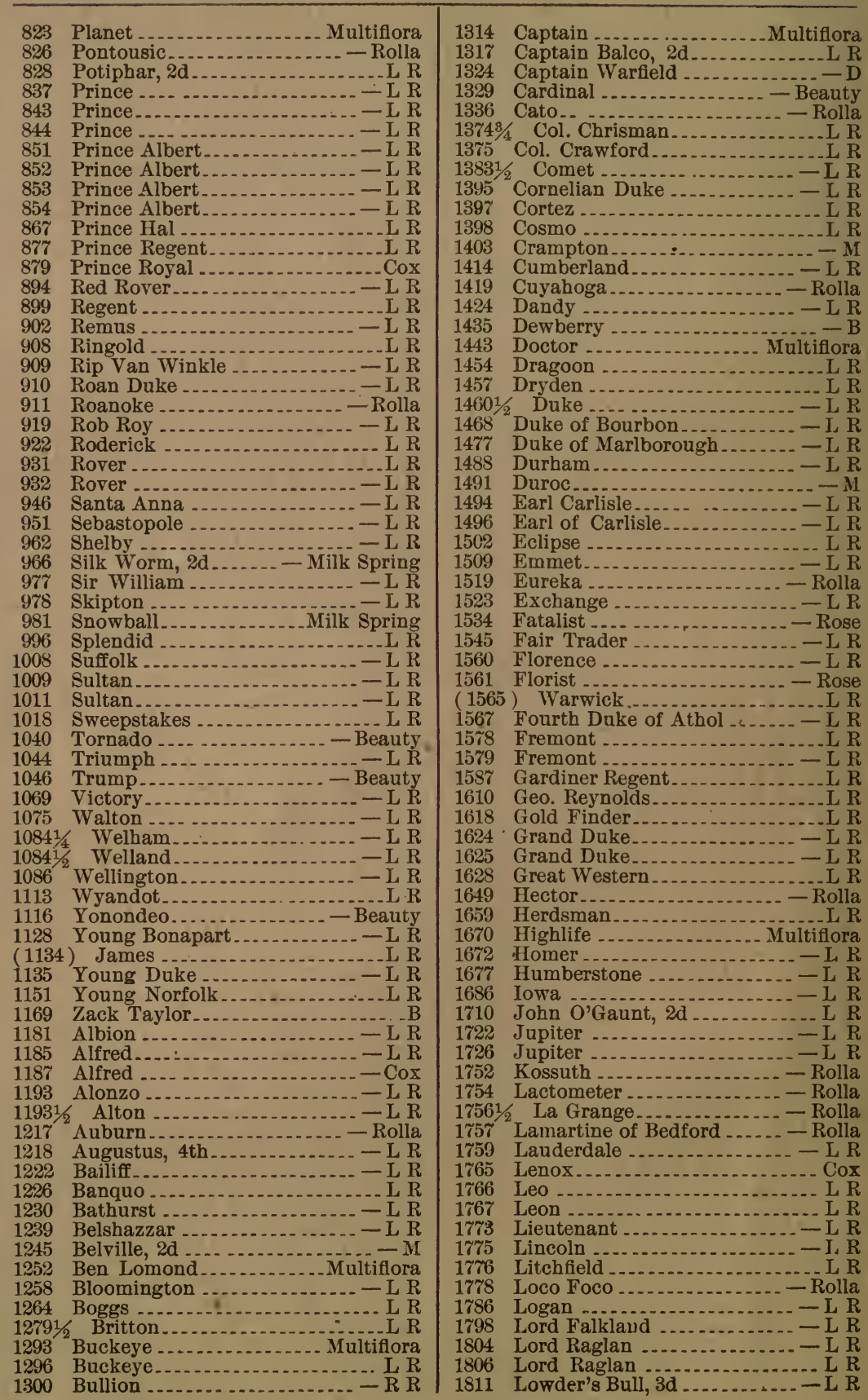




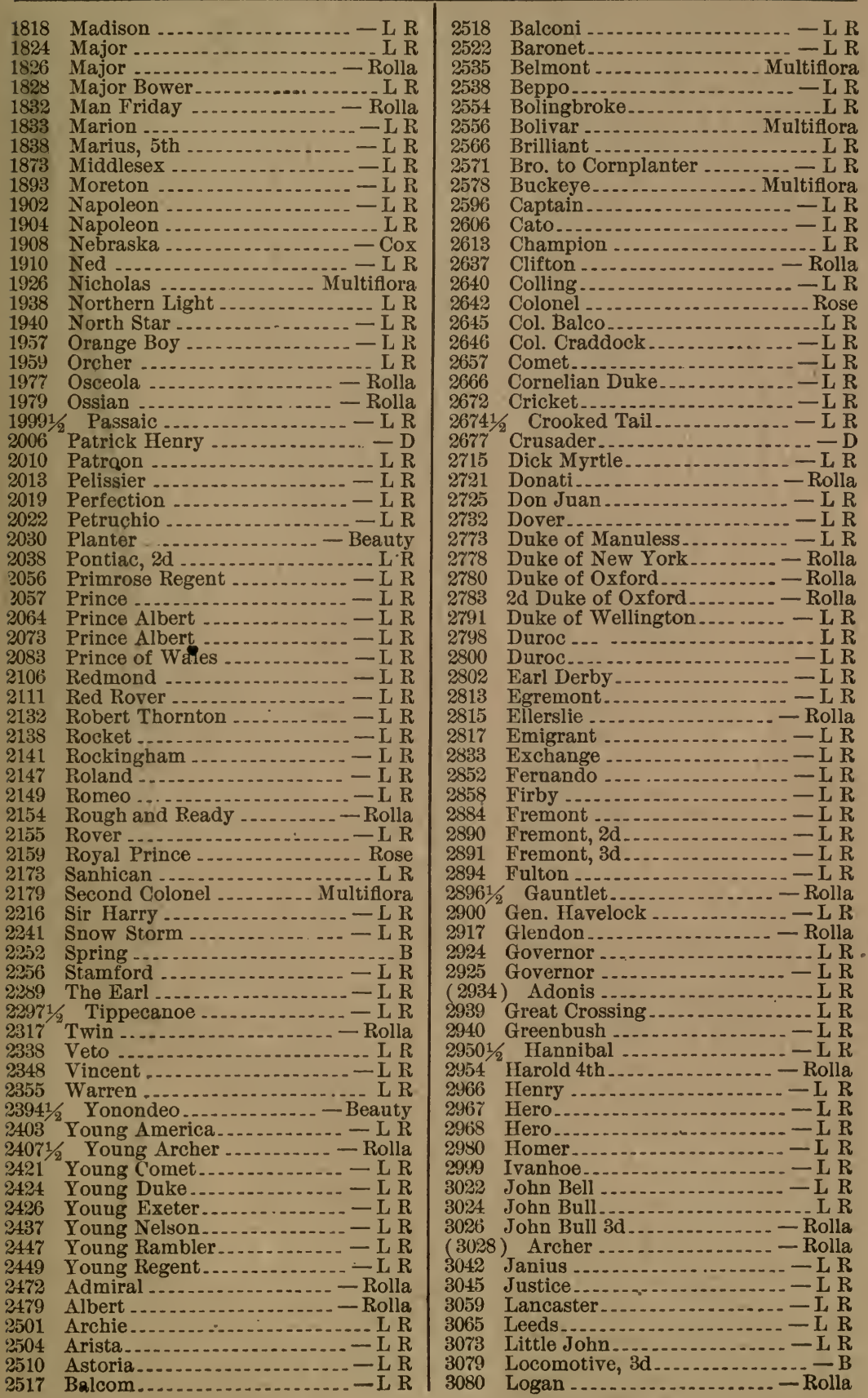


3092 Lord Raglan.... 3099 Louis Philippe............ $R$ 3106 Lowder's Bull, 11th......... - - R R 3112 Majesty ........................ R $31181 / 2$ Mark Antony 3121 Marquis, 3d .................... Rose 3138 Master Clem............. R

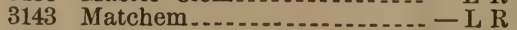
3154 Merritt .........

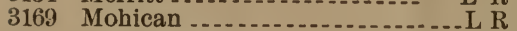
$31741 / 2$ Montezuma 3185 Napoleon ................ - L R (3185) Young Bolivar.......... - L R 3204 Norfolk ....................

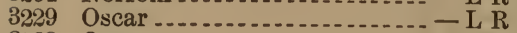
3233 Osseo........................ Rolla 3239 Oxford ...................... Rolla 3240 Oxford Lad.............. Rolla 3241 Oxford Lad, $2 \mathrm{~d} . . . . . . . . .-$ Rolla 3242 Oxford Lad, 3d ............ - Rolla 3248 Paul Morphy ................ R 3249 Paul Morphy............. L R 3269 Pompey .......... Rolla 3277 President ................. R 3285 Prince Albert ..... 3288 Prince Albert............... - L R 3291 Prince Albert............... - L R 3297 Prince Charles............ - Rolla 3298 Prince Consort............ - L R 3303 Prince Halton..................... R 3331 Red Jacket............... R 3335 Red Rover...................... 3337 Redtop...................... T 3347 Richmond.................... 3354 Robin Hood 3355 Robin Hood........ (3364) Chesterfield............ Multiflora 3368 Rolfe ................... - Rolla 3371 Rolla ...... 3374 Romeo.................. $\mathrm{L}$ 3379 Romeo........ 3381 Romeus....................... $33841 / 2$ Rover......................... R 3394 Royalton..................... 3395 Rubicon .................... Rose 3399 Sachem .......... 3401 St. George ................. L R 3419 Sebastopole ........... R (3422) Colling . ........... 3435 Sir Chas. Knightly......... - L R 3442 Sir Walter............... R 3445 Snowball ....................... 3446 Snowball 3447 Snowball................................... 3448 Snowball $-\ldots \ldots \ldots \ldots \ldots \ldots$ 3453 Snowdon's Knight.......... - L R

3454 Snowstorm................. L R

3458 Soyer ...........................

3463 Spottess .................

3464 Spotted Andes ........... L R

3472 Sthreshley, 2d........ - Long Horn

$34731 / 2$ Sumner .................. - R R

3499 Timoleon ......................... R

3513 Tyhee

3553 White Comet...................

$35 \% 0$ Yorkshire Maynard......... - L R

3583 Young Cheltenham......... - L R

3588 Young Duke................. $\mathrm{L}$

3598 Young Jupiter ................. R

3599 Young King .................
3603 Young Marquis ............. - L R

3604 Young Matchem ............. L R

3611 Young splendor..............

3613 Young Washington.........

3614 Young Welland...........

3615 Young Wellington ......... $-\mathrm{L} \mathrm{R}$

3621 Zouave Le Blane............. - L R

3622 Zouave Le Range............ - L R

3649 Archduke................... Rolla

3660 Baron of Oxford......... - Rolla

3664 Bedford .............

3677. Billy Plover.......... Beauty

3685 Bolingbroke.............

3693 Boxer........... L R

3696 Brant ...............

3704 Brilliant ...........

3705 Britton .........

3707 Bro. Jonathan

3720 Bulbo .............

3743 Charlemagne........................ R

3744 Charles 1st............... - Beauty

3745 Charles $2 d$.................. - Beauty

3746 Charley P........................

3750 Cherry Duke................ R

3779 Comus........................

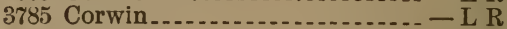

3790 Cripple.......... R

3798 Cumberland.................

3802 Dairyman....................

3805 Dandy $2 \mathrm{~d} . . . . . .$.

3825 Dootor......................... $\mathrm{R}$

3839 Duke.........................

3855 Duke of Cumberland.......- Rolla

3856 Duke of Exeter.............. - L R

3871 th Duke of Northumberland .. - L R

3872 6th Duke of Northumberland - Rolla

3830 2d Duke of Oxford................ R

3882 Duke of Page................

3884 Duke of Richmond........... R

3885 Duke of Richmond.......... $-\mathrm{L} R$

3894 Duke of Wellington

3908 Elkeso ....................... Rolla

39171/2 Eugene................. L R

3930 Fen wick ........ Rolla

3935 Firby

3942 Frank

3952 Garibaldi.................... R

3957 Gauntlet $-\ldots \ldots \ldots \ldots . . . . . . . . .-$ Rolla

(3962) Haggin's Miranda........... L R

3963 Gen. Snowball ..............

3967 Gen. Wyndham

3970 Gloster.........................

3989 2d Grand D. of Oxford...... - Rolla

3991 Grantham...............

4004 Harrold 5th................. - Rolla

4005 Harrold 6th

4006 Harrold 7th................ Rolla

4020 Hiawatha.......................

4021 Hiawatha Jr.............. Rolla

4031 Hubback ................... - L R

4037 Independence................

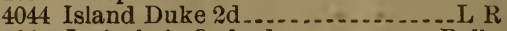

4047 Jacintha's Oxford........... - Rolla

(4053) Hotspur.................. - Rolla

4056 Jasper

4062 John Bell ..................... $R$

4076 Julius........................ $\mathrm{L} R$

$4089 \mathrm{Kwasind}$

4092 Lawrence 4 th................... - B

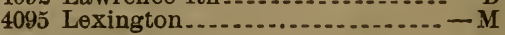


4105 Little Giant

41124 th Lord of Eryholme........ - Rolla

4115 Lord Lincoln ................... - L R 4136 Majesty ............................

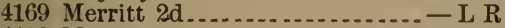

4170 Meteor

4178 Mohawk.....................

4183 Napoleon......

4186 Neptune

4198 October....... L R

4205 Oneida....................... Rolla

4215 Oskaloosa.................... - P

4217 Oxford Boy........................ R

4219 Oxford, Duke of Fordham...-Rolla

4221 Oxford of Geneva........... - Rolla

4225 Paul Morphy............... $\mathrm{L} R$

4229 Perfection

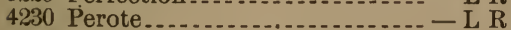

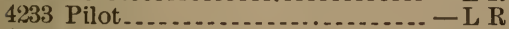

4242 Pluto...............................

4262 Prince George $2 \mathrm{~d} . . . . . . . . .-$ Rolla

4273 Prince of Oxford

4293 Red Chief.............. Multiflora

4301 Red Rover $2 \mathrm{~d}$

4313 Rip Van Winkle.............. - L R

$43331 / 2$ Royal Corsair ..............

4364 Shenandoah $2 \mathrm{~d} . . . . . . . . . . .$. Rolla

4365 Shepherd's White Bull......... - L R

4367 Shingebis................. - Rolla

4374 Sirius 4th........................

4381 Snowball .................... Rolla

4388 Somerset.....................

4389 Somerset. ......................... R

4401 Stephen A. Douglas.......... - L R

4108 Sumter......................... $R$

4413 Tecumseh.......................... R

4421 The Professor

4443 Union Boy.................... R

4455 Waburn...........................

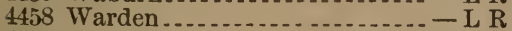

4482 Wide Awake........... - Beanty

4494 Yanadizze...............

4504 Young Comet................ - L R

4517 Young Kirk............. R

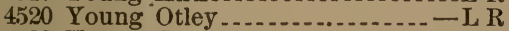

4522 Young Prince.................. L R

4525 Young Rockingham .......... $-\mathrm{L} R$

4530 Young Wellington........... L R

4535 Zed........................... Rolla

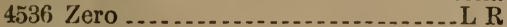

4546 Alfred .............

4552 Archer $3 \mathrm{~d}$

4557 Banbury ..................... - L R

4568 Beau of Oxford ............

4579 Berkshire Boy ............... Rolla

4584 Billy Seward .......................

4591 Boswell or Buzzy ............ L R

4610 Buena Vista.................. - L R

4617 Burnside...................... Rolla

4620 Butterfly ................... L R

4628 Cardinal...................... R

4629 Caroga

4634 Champion........................

4646 Clarendon 4th

4660 Col. Mulligan................ - L R

4665 Commodore.................. $\mathrm{R}$

4669 Commodore Foote.

(4684) Petrarch................. - Rolla

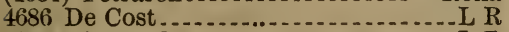

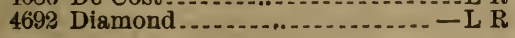

4694 Dixie.................... $\mathrm{R}$

4721 Duke of Cumberland...... - Rolla 4725 2d Duke of Exeter.......... - L R 4742 Duke of Mt. Pleasant......... $-\mathrm{L} R$ 4749 Duke of Richmond 2d........- L R 4757 Duke of Wellington........-Rolla 4763 Duke of York............. 4773 Earl of Gloster......... Multiflora 4778 Eclipse................. Beauty 4780 Elsworth ................. - Rolla 4787 Engineer..... Rolla 4791 Express ................... Rolla 4797 Financier................ Beauty 4800 Frank .......................

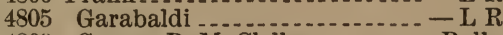
4809 George B. McClellan ...... - Rolla 4817 Gen. Butler ............... - Beauty 4826 Gen. Grant ......................... R 4829 Gen. Hooker 4840 Gen. Rosecrans.......... - Rolla 4847 Governor .................... R 4856 Grand Duke ............... $\mathrm{L}$ R 4857 3d Grand Duke............ - Rolla 4860 3d Grand Duke of Oxford - - - Rolla 4884 Hazelwood, 4th............ - L R 4898 Highlander.................... R 4910 Island Duke 3d............ L R 4922 John Gilpin ............. Rolla 4927 Junior..................... - Rolla

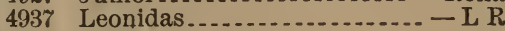
4952 Lord Lovel.................. $\mathrm{L}$ 4954 Lord Mayor of Oxford..... - Rolla 4957 Lord of Lune $2 \mathrm{~d} \ldots \ldots \ldots \ldots \ldots-\mathrm{L} R$ 4961 Lord Raglan .................... R R 4964 Lord Renfrew...... 4975 Lucifer .................... 4978 McClelland ........ R (4991) Rolla ...................... R 4997 Marquis.................. 5009 Mikado............... Rolla 5021 Monitor ................. Rolla 5033 Nero ........................ 5045 Optimus .................. (5049) St. Andrew 5050 Orion .................. - Beauty 5051 Orpheus 5053 Otsego Lad............. Rolla 5057 Oxford Laird....................... R

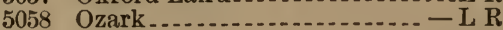
5062 Patriot ..................... Rolla 5063 Perfection ................ R 5086 Prince A!bert....................... R 5089 Prince Charles ............. R 5090 Prince Jerome ............... - L R 5096 Prince of Wales............ - L R 5098 Prince of Wales............ - Rolla 5099 Prince of Wales............. - Rolla 5105 Puritan .................... - Rolla 5110 Recruit................ Rolla 5119 Red Rover............ - Beauty 5121 Regent......... 5122 Reginald..........

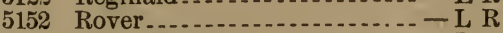
5163 San Antonia....... 5172 Seward ................................... 5175 Sigel ......................... Rolla 5189 Shamrock.... R 5191 Sheridan's Bruce......... 5195 Slasher ..................... Rolla

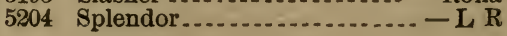




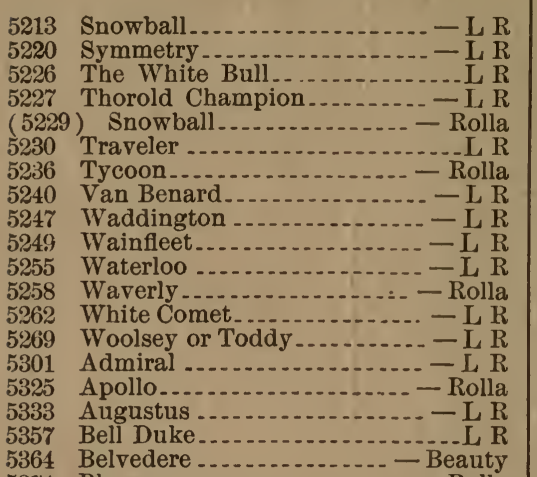

5374 Blanc.................... - Rolla

5429 Capt. Languish........... - Rolla

5432 Carnival ................... - L R

5454 Clifton Duke .............. - Rolla

5456 Col. Balco ....................... R

5472 Commodore........................

5515 Dover $\ldots \ldots \ldots \ldots \ldots \ldots \ldots$

5527 Duke Lionel

5528 Duke Reginald............. - L R

5529 Duke Roderick.............

5537 2d Duke of A mherst.........- - L R

5561 Duke of Garrard ............- - L R

5564 Duke of Grafton ........... - Rolla

5598 Duke of Oxford ................. R R

5605 Duke of Somers............. R

(5647) White Jacket............. - L R

5656 Garibaldi ....................

5663 Gen. Butler........ R

5680 Gen. Grant...............

5711 Gen. Smith

5719 George .......................... R

5720 Gladiator .........

57\%2 Glenroy ......................... R

5727 Governor ................... - L R

5731 1st Grand Duke of Kansas - Rolla

5735 Grant....................... - L R

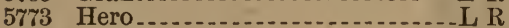

5778 Highland Duke............ - Rolla

5793 Hilpa's Oxford ........... Rolla

5801 Idaho....................

5805 Inkerman

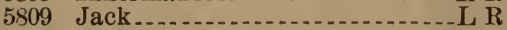

5813 Jack Hardy ................. - L R

5825 Jerome ....................... Rolla

5833 John Bright.................. - L R

5845 Juarez

5846 Judge .................... Cox

5863 Knight of Virginia......

5867 Laird of Oxford ........... Rolla

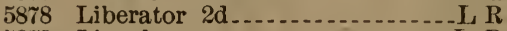

5879 Lincoln............................

(5888) Cornelius..........................

(5913) Democrat .............. Rolla

5931 Mariner......... R

5949 Mazeppa -.......... Rolla

5962 Mogul ...................... R

5989 Nero ................. Rolla

6005 Oakland Oxford.........

6013 Olympus.................. Rolla

6016 Oscar

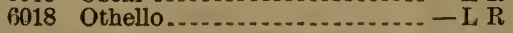

6020

6035

6036

6041

6043

6044

6047

6049

6050

6064

6077

$(6078)$

6081

6135

6146

6204

6214

6233

6244

6250 Tickler

6253 Tom Bishop ...............

6258 Triton .........................

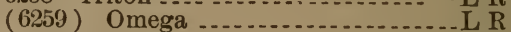

(6278) Patroon

6289 Waukegan .................. - L R

6321 Young Comet.............. Rolla

6333 Young Rappahannock....... - L R

6347 Duke of Stanstead ......... - Rolla

6351 Flirt's Airdrie............. - L R

(6357) Quaker......................

6358 John Stephenson .........-Bcauty

6367 Prince William............. - L R

6371 Rockville................... $-\mathrm{L}$ R

6395 Albert .......................

6408 American Comet......... Rolla

6427 Balbo ...........

6463 Billy, $2 \mathrm{~d} . . . . . . . .2 \mathrm{R}$

6469 Bismark.................. $-\mathrm{L}_{\mathrm{R}} \mathrm{R}$

6481 Bourbon .........

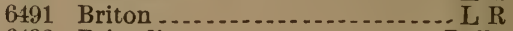

6492 Brigadier ........

6543 Clifton..................... - Rolla

6552 Col. Frank Wolford ...... L R

6555 Columbus......................

6557 Comet ...........

6573 Count Bismark

6588 Dalton .........

6615 Duke, 3d

6630 2d Duke of Amherst........ - L R

6642 1st Duke of Dexter........ -Rolla

6649 3d Duke of Exeter.......... - L R

6656 Duke of Hampden....... Beauty

6666 Duke of Maine........... Rolla

6673 Duke of Mercer ..........

6697 Duke of Virginia

6709 Earl of Rutland ......... Beauty

6751 General .................... Rolla

$67631 / 2$ Gen. Grant.....

6767 Gen. Grant................. - L R

6779 Gen. Scott...................

6782 Gen. Sheridan............

6789 Gen. Thomas..................

6790 Gen. Thomas.........................

6793 Gen. Warren........ Rolla

6795 George Starlight..........

6796 George W. Williams...... - L R

6831 Green Mountain Boy ........ - Rolla

6841 Harper ........................ L R 
6850 Hector................... $\mathrm{R}$

6870 Highlander.......... $\mathrm{R}$

6878 Hotspur.................... R

6897 Jashion ............................

6903 Jefferson...................-Rolla

6907 Jerome, $3 \mathrm{~d} \ldots . . . . . . . . . . . .-\mathrm{L}$ R

6920 John Halifax............. R

6960 Logan .......................

6967 Lord Lyon

6987 Major........................

6997 March, 1st ......................

7011 Matadore Jr., ............ Beauty

7013 Maximillian............... $\mathrm{L} R$

7031 Miffin.........................

7047 Monitor.................... R

7068 Noble................... R

7078 Orlo.............................

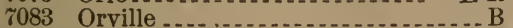

7085 Osceola

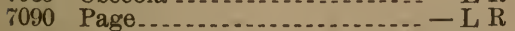

7117 Prairie Lad.............. Multiflora

¡120 Priam................. R

\%130 Prince Alfred ............. Beauty

138 Prince Napoleon .......... L R

7144 Prince of Hector ............ Rolla

7147 Prince of Shelburne ........ - Rolla

7151 Prince Tom ............... Rose

7159 Rambler

7172 Red Knight .............. $\mathrm{L}$

7181 Reginald .........

7182 Regulåtor ................. $\mathrm{R}$

7221 Root...................... Rolla

7229 Royal.....1............ Rolla

7232 Royal Duke............... Rolla

7235 Royal George

7236 Royal George............... L R

7237 Royal Prince.............. Rolla

7449 Sampson ................ R

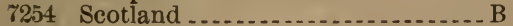

7258 Shamrock............... Beauty

7270 Sir Charles.................. - Rolla

7274 Sir John ................. Rolla

7355 Victor ..................... Rolla

7358 Victor

7380 Wellington............ R

\%383 Western Duke............ R R

7396 Windsor, 3d ............. Rolla

7407 Young Bonaparte ......... - L R

7411 Young Coronet............

7421 Young Splendor.......... L R

7454 Airdrie ................... Rolla

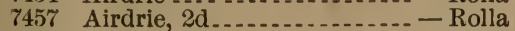

7486 Alexis, 1st ................. L R

7489 Algernon Sidney.......... Rolla

7490 Alhambra................ L R

7504 Apollo, 3d ........ Rolla

7512 Ascutney .................... Rolla

7522 Auvergne Duke ............... R

7533 Baron Bolton ..... Rolla

7534 Baron Booth ............... Rolla

7540 Baron of Uxford ........... Rolla

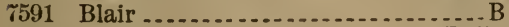

7593 Blossom's Duke.......... Rolla

7597 Bolingbroke.............. R

7620 Bruno ..................... - Rolla

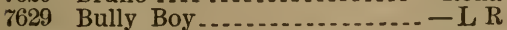

7642 Cairn Gorm ................ Rolla
7675

7692

$7 \% 03$

7708

7\%31.

760

7806

7833

7867

7885

7893

7894

7899

7907

7914

7915

7916

7928

7953

7956

7957

7961

‘963

7965

7968

7993

7994

7997

8021

8039

8042

8061

8063

8080

8081

8082

8112

8129

8132

8139

8144

8146

8150

8211

8228

8246

8273

8275

83041

8305
8308

8358

8359

8361

8366

8389

8397

8398

8399

8404

8457

8461

8462

8500

8528

8536

8541

8566

8.579

8588
Champion .............. $R$

Claflin, 2d........ Rolla

Clifton ........... R

Clifton ............... R

Colonel ................ Beauty

Corporal .................. R

Dexter ...................

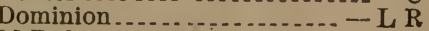

2d Duke of Adams. ........ - Cox

Duke of Athol............... $-\mathrm{L}$ R

Duke of Callaway ......... $\mathrm{L}$

Duke of Cambridge........ $-\mathrm{L} R$

Duke of Clarence.......... Rolla

Duke of Cloe............... L R

2d Duke of Dexter......... Rolla

3d Duke of Dexter......... - Rolla

4 th Duke of Dexter......... - Rolla

Duke of Florence......... Rolla

2d Duke of Kansas............ R

5 th Duke of Kansas....... Rolla

6th Duke of Kansas............ R

10th Duke of Kansas........... $\mathrm{L}$

Duke of Keene.............. $\mathrm{L}$

Duke of Kent ............. $\mathrm{L}$ R

Duke of La Colle............ $\mathrm{L} R$

Duke of New Boston ....... L R

2d Duke of New Boston..... L R

Duke of Northfield ......... $\mathrm{L} R$

Duke of Sharon........... $\mathrm{L} R$

Duke of Wellington.......

Duke of Weybridge.......... - Rose

Earl Darnley ............. Rolla

Earl Leicester .............. - L R

Earl Randor............... Rolla

Earl Spencer ............. Rolla

Eastern Duke............ - Rolla

Excelsior ............... Rolla

Felix Moss .............. R

Field Marshall ............ Rolla

Fitz Oxford.............. Rolla

Fortune . . ............... Rose

Forney ........ Rose

Frank......... Rose

Gen. Sigel

Gold Drop................... Rose

Grand Duke....... L R

Green Mountain.......... Rose

Green Mountain Boy ...... - L R

Hacienda ............... $\mathrm{L}$ R

Hagaman ..................... R

Hamilcar ........... $\mathrm{R}$

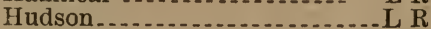

Humbolt ............ $\mathrm{R}$

Hydra .............. $\mathrm{R}$

Imperial ................. $\mathrm{L} R$

Jack ................... R

Jennie's Carlisle......... Rolla

Jerome 2d............... R

Jerome 4th............ R

Jim ............... Rose

Ketton........ Beauty

King John..... Rolla

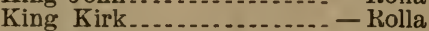

Lieutenant................. - L R

Lord Mayor of Maine...... - Rolla

Lord Valentine............ - Rolla

Loudon ..................... R

Magnet................. Rolla

Manfred .................... L R

Marmion ............... Rolla 


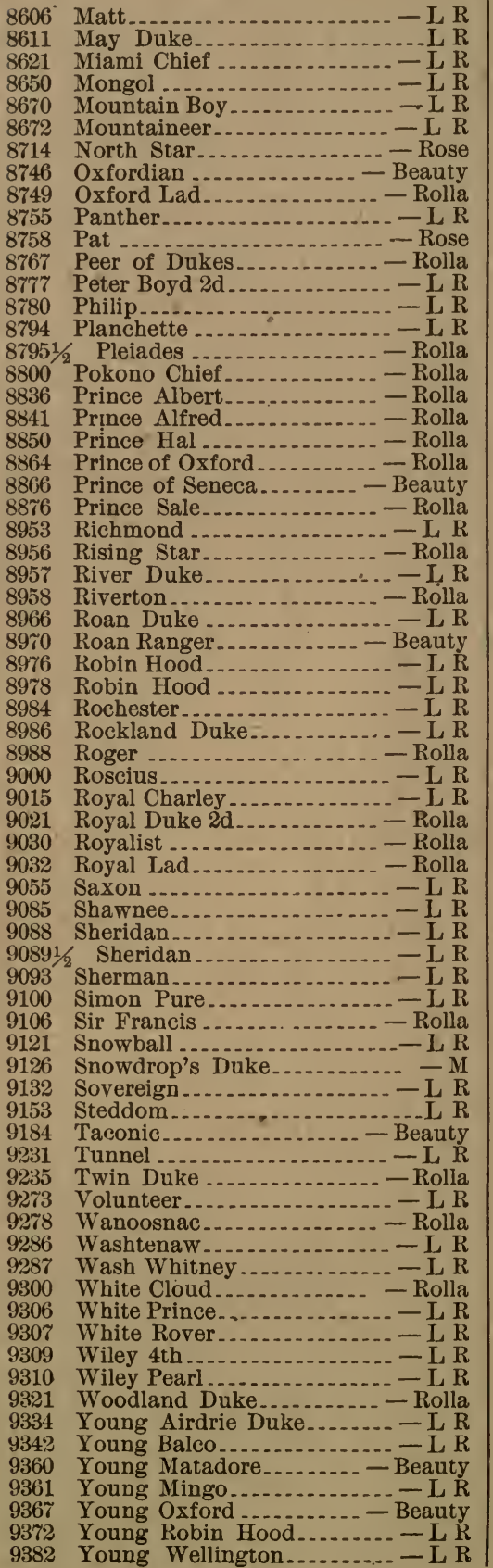

9386 Zerubabel........... $\mathrm{R}$

9388 Zouave Le Rouge 1st....... - L R

9389 Zouave Le Rouge $2 \mathrm{~d} . . . . .-\mathrm{L}$ R

9419 Airdrie of Rainbow......... $\mathrm{L}$ R

9436 Althorp Jr.................. R

9466 Balmoral........................

9467 Bamford..................

9468 Banter..................... Spring

9474 Baron.................. - Beauty

9503 Beacon ................... Rose

9525 Billy O'Gaunt........... - L R

9530 Bismark ................. L R

9536 Bloomfield Lad ............

9553 Boutwell................ - L R

9579 Burnside .................. Rolla

9604 Captain Ladd. ............. L R

9606 Carl ........................

9616 Caspedes .................. Rolla

9630 Cheops................... R

9635 Chieftian .................... $\mathrm{L}$

9638 Christmas King............. - L R

9670 Comet ....................

$96 \pi 3$ Comet ...................

9681 Comrade............... Beauty

9735 David........................ R

9756 Dixie..................... - Rulla

9772 Dover ........................ R

9775 Driver.................... - Rolla

9777 Droxton ....................

9803 Duke of Arundel............. $\mathrm{R}$

9878 14th Duke of Kansas ....... Rolla

9882 18th Duke of Kansas ....... - Rolla

9888 1st Duke of Liberty ......... - L R

9895 Duke of Mapleton ........ - Rose

9899 Duke of Mason............ - Cox

9917 Duke of North Bend......... - L R

9931 Duke of Paris............. $-\mathrm{L} R$

9936 Duke of Princeton

9948 3d Duke of Stanstead.....- - Rolla

9969 Duke of Walworth ....... - L R

9979 Dundee.................... Rolla

9994 Ed Davison................... R

10006 Emperor

10023 Exile ...................... Rolla

10029 Eureka ................... - L R

10057 Fidget's Oxford 4th ...... - Rolla

10064 Flirt's Airdrie............... - L R

10076 Furioso.................... Rolla

10100 Gen. Hill ...............

10115 Gen. Prim

10124 Gen. Thomas........... Rolla

10141 Golden Crown.......... - Rolla

10166 Grant......................

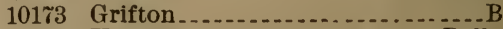

10181 Hamlet......................

10183 Handsome Jack........... - L R

10195 Hart of Montrose........ $\mathrm{R} R$

10238 Hotspur................... Rose

10300 John Bell.................... L R

10313 Kankakee Duke............ - L R

10323 King William ............ - Rolla

10326 Kirk's Oxford............ - Rolla

10327 Kirk's Oxford $2 \mathrm{~d} . . . . . . . .$. - Rolla

10337 Lawrence...............

10350 Lifford ..................... Rolla

10357 Little John

10369 Lord Derby

10375 Lord Roden................ $-\mathrm{L}$ R

10384 Lord Roden................

10390 Lothair................... Rolla 
10413

10418

10426

10440

10531

10550

10567

10572

10578

10598

10608

10640

10667

10671

10676

10678

10683

10690

10746

10758

10768

10774

10877

10901

10921

10923

10932

10946

109481/2 Sarcoxie Chief....... L R

10953 Sebastipole ............... - Rolla

10968 Shylock ................. Rolla

10975 Sir Charles............... - L R

10982 Sir Herbert ..............

10992 Snowball

10995 Snowdrop's Duke........

10999 Sovereign

11001 Splendor

11005 Spotted Bolton..... - Milk Spring

11031 St. George............... - L R

11076 Tonganoxie............... L R

11086 Tulip's D of Hamilton ........... R R

11088 Twin Balco .................... R R

11090 Uncle Sam......................

11118 Walter........................ R

11125 Wellington

(11261) Cato .................... - Rolla

11334 Baron Erskine,Clydesdale - Beauty

11344 Beacon Boy ................. - L R

11346 Beau of Oxford ........... - Rolla

11354 Beecher....................... R

11363 Belmont 3d ............... - Cox

11364 Belvedere ................ Beauty

11373 Ben Butler................. - L R

11399 Bismark ........... L R

11405 Blapco.................... $\mathrm{L}$ R

11426 Boutwell................... - L R

11438 Bringham....................

11443 Bro. to Sweepstakes............. L R

11447 Brutus...................... D

11465 Bute.................... Rolla

11466 Buttercup......................

11509 Chieftian

11512 Cincinnatus................... R

11514 Clarendon 2d ..... R

11554 Conqueror ............... - Rolla

11556 Corporal ........... R

11577 Crown Prince........... - Rolla

11621 Derby

11652 Dudley Bowers.....- Milk Spring

(11662) Lamartine ............. - Rolla
(11715) Lord Eryholme 2d...... - Rolla 11724 3d Duke of Exeter.......... - L R 11765 Duke of Jackson........... - L R 11767 21st Duke of Kansas....... - Rolla 11769 23d Duke of Kansas........ - Rolla 11772 26th Duke of Kansas .......... L R 11775 29th Duke of Kansas......... R R 11782 2d Duke of Markesville..... - L R 11783 3d Duke of Markesville..... - L R 11784 4th Duke of Markesville....- - L R 11898 Earl of Lyndale............. - Rolla 11917 Emperor Maximillian ...... - L R 119:26 Ernest ...................... B 11930 Eugene 6th 11933 Excelsior $2 \mathrm{~d} . . . . . . . . . . . .-$ Rolla 11953 Fidget's Oxford 5th ....... - Rolla 11979 Frank …........ L R (11998) Roan Oak 12004 Gaylord ................... Rose 12008 Gen. Butler .............. L R 12019 Gen. Grant.................. 12022 Gen. Hallock 12024 Gen. Howard 12025 Gen. Jackson ........... - L R 12037 Gen. Scott.............. 12055 Gibelin ............ Rolla 12073 Goodspeed...................... 12084 Gracy Pearl's Duke....... - L R 12086 Grand Duke .............. 12088 4th Grand Duke........ - Rolla 12101 Grantz Brown.................. 12108 Gunter .............. 12127 Harry Belmont ........... 12135 Hector Jr.,...................... 12136 Hendrick Hudson .......... - Rolla 12232 Kaiser ................... - Rolla 12261 Korak 12286 Leopard .................. 12288 Le Roy Chief............... Multiflora 12296 Lincoln .................... - L R 12300 Lisgar ............................ 12336 Lord Warwiok........... 12379 Magic ................... Rolla 12382 Marble $B$. of Rutland 12383 Marble Duke of Rutland ... - L R 12401 Maroon.................. R R 12418 Maximillian........ 12424 May Duke .............. R (12427) Balconi....... 12429 Mazurkum .......... 12451 Mohawk................... Rolla 12464 Montana................ R 12508 North Star............ Rolla 12527 Oneida ............ L R 12579 Perfection 12609 Pope -....... 12664 Prince Norn 12676 3d Prince of Oxford 12677 Prince of the River.....- Beauty 12680 Prince Oxford ............ - Rolla 12681 Prince Oxford . 12706 Red Bank 12731 Red Duke....... 12732 Red Duke .............. - Rolla 12733 Red Duke............... L R 12740 Red Duke............... Rolla $12 \pi 41$ Red Duke............... L R 12821 Romeo............................... 12885 Sankya ..................... Beauty 12911 Sheridan................ 
12920

12953

13023

13040

13053

13057

13060

13062

13111

13113

13114

13115

13118

13136

13137

13139

13166

13171

13182

13202

13207

13208

$(13209)$

13212

13236

13263

13267

$132 \pi 2$

13284

13292

13356

13357

13373

13379

13395

13403

(13443)

13446

13449

13462

13467

13475

13495

13511

13516

13519

13528

13529

13543

13554

13611

13615

13619

13620

13630

13640

13657

13680

13713

13745

13755

13816

13861

13912

13926

13955

13964

14012

14030

14075
Simeon Cameron..........-L R

Stantou .................. The Knight............ Rolla

Toledo

Trojan ...... Rolla

Turk

Twemlow.........

Tyeoon

Waterloo ............. Beauty

Water Prince $2 \mathrm{~d} . . . . . . . . . . \mathrm{L}$ R

Water Prince 2d.......... Rolla

Water Prince 3d............. R R

Wellington............. $\mathrm{R}$

Wiley 5th...........

Wiley Duke...................

William $3 d$.................

Young Crimsom........ R

Young Harold .................

Young Solway.........

Bellflower Prince........-Beauty

Bismark ........... Rolla

Booth Boy

4th Lord of Erybolme.. - Rolla Bert Harte .............. - Rolla Duke of Fishkill...... - Rolla King Lear.............. - Rolla Lord Chesterfield ......... - Rolla Lycurgus .............. Pioneer.............. Sir Fenwick............. $\mathrm{L}$ Alhambra...... Allen Duke ................. Appleton ............. Archon................ Rolla Aurora's Major...... Rolla Baron Airdrie........ Rolla ) Oxford Lad ........... - Rolla Bates ................. Beau of Oxford Belmont 4th ............... - Cox Belvedere ............... Beauty Ben Grant...... Rolla Bishop............ R Blaze .......... Beauty Blucher........ L Bob Block .................. Bolingbroke $2 \mathrm{~d}$ Bolingbroke $3 \mathrm{~d}$ Bowman's Oxford ........ - Rolla Bright Boy -............ - Rolla Capt. Pbillips....... Rolla Cardinal ................. - Rolla Carl 2d ....................... Rolla Carlotte's Oxford....... - Rolla Champion ............... Rolla Cherry Duke $2 \mathrm{~d}$ Cincinnatus............. $\mathrm{R}$ Col. Gracy....... Copenhagen - . Rolla Cypress Airdrie... Rolla Dandy .............. Rolla Dr. Franklin Duke Cadenza............ Rolla Duke of Clinchdale - - Milk Spring Duke of Erie.......... Rolla 4th Duke of Grandville..... $-R$ 11th Duke of Greenbush....- $-\mathrm{L} R$ 5 th Duke of Liberty 1st Duke of Marksville.....-L R 4th Duke of Richland - Milk Spring
14099

14125

14141

14172

14182

14198

14201

14204

14207

14234

14244

14245

14246

14249

14250

14255

14280

14281

14331

14360

14426

14428

14431

14440

14443

14449

14473

14523

14566

14632

14659

14665

14716

14719

14722

14726

14766

14767

14814

14821

14822

14541

14849

14854

14857

14967

14971

14973

14980

14981

14995

15017

15029

15040

15069

15076

15083

15096

15102

15112

15120

15186

15199

15223

15247

15264

15266

15285

15310

15334
Duke of the Cottage - Milk Spring

Duke of Waverly ..........- $-\mathrm{L} R$

Duncan Duke...................

Earl of Stony Brook

Edgar Jr., ............... R

Emperor ........................

Enchanter .............. R $\mathrm{R}$

Envoy

Ernly ................

Fashion

Fidget's Oxford 5th

Fidget's Oxford 6th ....... - Rolla

Fidget's Oxford 7th

Fillingree's Corporal......... - L R

Fillingree's Lad .......... $-\mathrm{L}$ R

Fizgig - .

Friar John 6th ......... - R

Frosty ..........

Gen. Grant ............. - L R

Glanford ......... $\mathrm{L}$

Gunsaule

Guy ................. Rolla

Hampshire Oxford...... - Rolla

Harker -...................... L R

Harold 8th

Harry Bassett.............. - C

Hero of Athelstane........ Rolla

Hotspur ................-Beauty

Jason ..........

Jupiter ................. $R$

King of the West.....

Kimball .........................

Liberator . . ............ Rolla

Lightning .......... Milk Spring

Lincoln ................. Rolla

Little Mac............ R

Lord Elgin............

Lord Elgin............

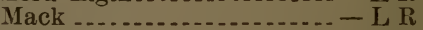

Magnet

Mainstay

Major Napier ........... Rolla

Manlius.................. Rolla

Mariner 2d............ - Rolla

Mark ......... Rolla

Mount Joy ........... Multiflora

Murat ........ R

Muscatoon

Napoleon -... Rolla

Napoleon $3 \mathrm{~d}$

New Years Gift ......... - Rose

Norwood ............ R

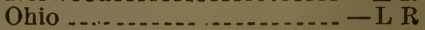

Ontario ........

Oxford Boy

Oxford Duke 5th.... Rolla

Oxford Lad ......... Rolla

Pandion.............. L R

Patchin....... Rolla

Peer ......... Rolla

Perham ............. Rolla

Prince Albert .......... R

Prince Ernest .......... Beauty

Prince of Oxford $2 \mathrm{~d}$

Rambler ..................... $R$

Red Airdrie ............... $\mathrm{L}$

Redbird ..................

Red Duke.............. Rolla

Red Muscatoon ........... R

Roan Chief.............. 
$1537 \%$

15401

15410

15446

15468

15477

15494

15521

15554

15580

15595

15598

15652

15655

15688

15689

15691

15694

15697

15702

15707

$15 \% 20$

15721

15725

15734

15755

15768

15769

15816

15818

15824

15826

15830

15858

15870

15919

15946

15958

15959

15960

15.977

15978

15985

15491

15994

16006

16010

16013

16051

16053

16060

16061

16063

16075

16077

16122

16212

16218

16224

16225

16237

16240

16317

16321

16353

16363

16395

16398

16405

16435
Romeo

Royal George

- L R

Sebastopole

Sheridan Boy.......... R

Sir Charles .........

Skirmisher -........

Star Duke.............. $R$

Suffolk........................

The Beau of Athelstane..... - L R

Thunderbolt......... - Milk Spring

Tim ........................ Cox

Vulcan .......

Waif ............. $R$

Weehawken Jr., .......... L R

Weehawken, $2 \mathrm{~d}$

Welland .................. - L R

Wellington................. R

Western Duke............ $\mathrm{R}$

West Fork _...........

White Cloud.......... Rolla

Wiley -.................... L R

Wiley, 1st......................

Wiley, 6th_...............

Winter.................... R

Young Briton .......

Young Honest Tom ........ $-\mathrm{L} R$

Young Lincoln ............ L R

Appelton _................ R Arnold's Marquis....... Bill Northrop.............

Bob Watson ............... - L R

Butterfly Lad.............. - Rolla

Duke of Jefferson .......... - L R

Excequer ................. Rolla

Marksman ................ - L R

Petruchio .........

Prince of the River, $2 \mathrm{~d} . . .-$ - Beauty

Prince of the River, $3 d \ldots \ldots$ - Beauty

Reconstruction.......... - Rolla

Shawnee Lad.............. - Rolla

Shawnee Ranger ........ Rolla

St. Patrick ............... - L R

Victor _.............. $R$

Wallace_.................

Airdrie, 4th ........... R R

Bellerus ................... Rolla

Billy Deans .............. - L R

Jemmy Deans ......... $R$

Johnny Deans

Lord of San Mateo ........ $L$

Lucellius................... L R

Marshall ..........

Rufus.......................

Stephanus

Airdrie Lad ..............

Baron Booth of Bellville ....-L L R

Baronet................. Beauty

Baron Hope.....

Baron Languish .......... Rolla

Baron Oxford............. Rolla

Baron Oxford Gwynne, 3d_- Rolla

Bob Lee............... . L R

Bob Napier ................

Bright Eyes Oxford ...... Rolla

Bud........... R

Capt. Clay . Fry

Capt. Jack

Carl, 3d ................. - Rolla
16449

16450

16462

16466

16467

16475

16479

16482

16490

16495

16507

16512

16530

16545

16580

16648

16678

16686

16692

16783

16803

16804

16832

16841

16899

16946

16999

17003

17042

17056

17085

17086

17087

17095

17097

17100

17143

17174

17182

17201

17253

17258

17264

$172 \% 0$

17299

17309

17353

17375

17376

17391

17399

17400

17416

17423

17447

17463

17475

17478

17497

17552

17569

17620

17629

17634

17635

17642

17674

17677

17766

17776
Challenger ............... $\mathrm{L} R$

Champion

Charlie Tabb ...........

Chief of Little Lehigh .....-Rolla

Cherry Duke, 4th......... - Rolla

Chieftain -.............. - Rolla

Christmas King .......... L R

Clarence

Clifton, $2 \mathrm{~d}$

Climax

Colin, $2 \mathrm{~d}$

Colonel .............. R

Colossus -

Compound ............ Rose

Crown Prince ..........

Dr. Livingston ........ Rolla

Duke ................ L R

Duke Alexis .....

Duke Jeff ................. - L R

Duke O'Gaunt.............. $\mathrm{R}$

11th Duke of Greenbush ....- - L R

14th Duke of Greenbush....- - L R

Duke of Hinkston .......... $-\mathrm{L} R$

Duke of Jefferson............. $R$

Duke of Oxford ..........

Duke of the Lake..........

Dundus .................... - L R

Earl ............... Beauty

Elm Duke .... . . .

Ethan, $2 \mathrm{~d}$

Fidget's Oxford, 8th ...... - Rolla

Fidget's Oxford, 9th ...... - Rolla

Fidget's Oxford, 10th .......-Rolla

First Chance ............... - L R 5th Fisherman's Pride ....... - L R Fitz Stanley ................. - L R Gen. Butler ................ Rolla Geneva's Duke .............. - L R Gloster's Budegroom......- - Rolla Good Samaritan ............. R R Greeley -.................. Green Mountain Prince....-L R Grove Prince ............ Beauty Halton ........... Hector Henry Wilson .... Hotspur, 1st........... R Iowa Airdrie .......... Rolla Iowa Duke........... Beauty James.................... R Jeffery Jehosephat............. $R$ Joe Hooker........................ B John Brown . . Rolla Judge Avery ......... King Henry, 8th...... L R Kirk's Oxford............ - Rolla Knatchbull................ L R Leo Lord Bemis ........... - L R Lord Languish........... - Rolla Lusty Lad............. L R MeSheridan .......... Magician ..... Magnet . . . . Major ................ Rolla Manhan .......... R Marco Modoc Chief........ Montauk................. Rolla 
17789 Mountain Boy ............. - Rolla

17800 Napoleon, 3d........... - Rolla

17803 Nast ....................... R

17856 Oneida Chief ..............

17864 Osage ......................

17866 Oscar ......... R

17868 Osceola........................

17871 Otsego Oxford..........

17875 Oxford Airdrie............. - Rolla

17911 Patroona's Oxford ......... - Rolla

17921 Penamite Duke............. - L R

17924 Perham,2d ................. Rolla

17946 Pio Nono......

17969 Pompey

18015 Prince Edward ........... $-\mathrm{L} R$

18017 Prince Geneva.............

18037 Prince of Grass Hill ..... - Beauty

18078 Puritan .................. - Rolla

18111 Red Duke, 2d

18132 Red Prince.............. - Rolla

18143 Red Star .........

18180 Roan Eari.............

182:21 Rosabella's Baron......... - Rolla

18270 Royal Prince ............... L R

18282 St. Elmo

18307 Septimus

18319 Sherman

18339 Sir Ross Winans.........

18372 Stanley

18373 Stanley

18398 Stentor

18399 Stokes_.............

18405 Stonewall 18433 The Earl .................

18436 Theopkile........ Rolla

18469 Trump ................. Beauty

18482 Van.....................

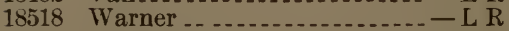

18530 2d Waterloo

18535 Wentworth

18544 West Wind

18548 White Cloud.............. - L R

18588 Young America............ Rolla

18600 Young Lincoln ........... - L R

18604 Young Noble.................. R

18613 Young Volunteer....... L R

18631 Baron of Oxford

18632 Baron of Oxford $2 \mathrm{~d}$

18644 Breadalbane............... - L R

18645 Briton ....................... R

18650 Captain ..................

18658 Cheney ..........................

18704 Farmer

18705 Fidget's Oxford, 5th ..... Rolla

18792 Glenlyon.................

18723 Gracchus........................

18725 Green Mountain Boy $3 \mathrm{~d}^{-\ldots . .-L} \mathrm{~L}$ R

18736 John Johnson ............. - L R

18767 Nelson

18772 Ontario Chief........... R

18788 Ready ...............

18790 Red Cap........

18793 Roan Carl

18802 Sir Colin........ R

18805 Sky Rocket ........... Rolla

18817 Wellington

18826 Young Volunteer ..........

18843 Airdrie....................

18850 Airdrie Lad ..............

18868
18882

18887

18892

18949

18958

18979

19015

19049

19075

19093

19124

19134

19145

19147

19173

19187

19194

19211

19270

19292

19295

19318

19323

19348

19358

19372

19374

19397

19439

19455

19512

19544

19599

19600

19622

19706

19710

19714

19809

19829

19920

19942

19995

19998

20000

20075

20097

20098

20104

20110

20111

20118

20130

20140

20148

20186

20290

20295

20298

20307

20335

20349

20354

20373

20381

20441

20459

20461

20475

20487
Altonia ................. $-\mathrm{L} \mathrm{R}$

Anita's Oxford...... Rolla

Argyle Joe .............

Baron Marion ............ - R R

Baron Oxford Gwynne, 4th-Rolla

Bashaw .................. - Rolla

Belvedere

Blucher .............

Bourbon Duke........ $R$

Brett....................

Buster.

Capt. Dick

Capt. Sayers ......... Rolla

Capt. Wiley

Champion.......

Charles Dickens.......... L R

Chautauqua.............

Christmas Day

Count Bismark

Cumberland Chief ........- - Rolla

Cyrus.................... - Rolla

Dean of Glenora

December Lad ........ L R

Dictator ............ Rolla

Dodge ......... L R

Duanes Duke, 3d.....

Duchess Duke of Airdrie.. - Rolla

Duke of Arkell _.......... - L R

Duke of Elba............

Duke of Fraser

Duke of Locust Lawn.......- - L R

3d Dnke of New Boston....-Rolla

Duke of Shelburne .......-Rolla

2d Duke of Shelburne .....- Rolla

Duke of Troy _........... $-\mathrm{L} R$

2d Earl of the Pines ....... - L R

Echo

Eclipse

Gem's Rosy Duke....... Rolla

Gen'l Marion ............

Granite Prince........

Halton

Highflyer ............... Rolla

Highland Chief....... - Beauty

Highland Duke, 2d .......... - R R

Joel................... Beauty

Jonnie......... Rolla

Jonnie, $2 \mathrm{~d}$............ Rolla

Jonathan............. Rolla

Jubilo .................. Rolla

Jubilum ................ - Rolla

Jupiter

Ketton Redux ........ Rolla

6th King of Dexter...... L R

King Philip............

Leon $2 d$.......... Rolla

Loudon Prince 1st...... - Rolla

Lucifer ................... $-\mathrm{L}$

Lumberman ............ Rolla

Magician .......... B

Mariner 2d.............. Rolla

Marquis of Solway

Martin Duke 1st............... R R

Master Sharon......... R

Max Adeler ..............

Monarch ................ $\mathrm{R}$

Moscow Duke 4th....... $R$

Mount Clare..............- Rolla

Nasby ........ $R$

New Year.................... 
20538

2054

20550

20558

20555

20599

20607

20608

20645

20646

20690

20691

20692

20696

20702

20717

20728

20733

20750

æ078:

20799

20853

20876

20392

20963

21123

2.124

21184

21197

21200

21201

2123:

(21254) Beau of Oxford......... - Rolla

21256 Vesper 2d's Duke........ Rolla

21267 Victor 2d................ L R

21280 Warrior .............. R

21291 Weehawken Lad ....... L R

21323 Wild Cap............. - Beauty

21323 Wiley Duke.......... R

213:29 Wiley Lad............... L R

21362 Young Climax.......... Rolla

21370 Young Eclipse ........ R

21374 Young Leopard........... R R

21388 Young Starlight.......... $\mathrm{L}$ R

21404 Androscoggin Lad....... Rolla

21437 Canada ................ R

21452 Colborne................. R R

21471 Daisy's Scotsman

$214962 d \mathrm{D}$ of Clinchdale. - Milk Spring

21514 Duke of Madison

21545 Ernest........................

21547 Farmer ................... R

21550 Fidget's Oxford 10th ...... - Rolla

21554 4th Fordham D of Oxford - - Rolla

21562 Fulton Duke....... - Milk Spring

21564 Gaddesby ............... Rolla

21582 Goodspeed Duke.......... L R

21584 Gov. Leslie.............. R

21594 Harry .................... R

21598 Hester's Duke .......... Rolla

21629 Lehicton ............... Rolla

21669 Major Hoyle ............. - L R

21673 Marksman ...................

21686 Messenger's Oxford

21695 Morning Star.......... Beauty

21731 Prince Albert ............. - L R

21749 Prince of Wales $2 \mathrm{~d} . . . . . . .-\mathrm{L} R$

21779 Roan Chief............. Rolla

21793 Royal George........... Rolla

21799

St. Valentine

Rolla
21807

21852

21865

21874

21900

21935

21947

21979

21984

21992

$2: 2047$

22054

$2: 2087$

2:2095

22151

22156

22158

2:202

$2 \% 03$

22:22:3

22:249

2.2267

22320

$2: 3236$

2:35̃4

22363

22427

22174

22501

2. 506

2:3507

22560

22568

22573

22575

2:35ิ 6

22613

22614

2:3647

22680

22698

22707

$2 \% 273$

22736

2:755

22756

22765

22829

2283:

22853

22863

22864

22882

2:2858

$2: 2892$

2:2901

$2: 2905$

22916

23097

23116

23131

23135

23143

23151

23154

23200

23249

23250

23281

Silence ................ Rolla

Young Comet............ $-\mathrm{L} \mathrm{R}$ Adam -.................. $\mathrm{L}$ Admiral Lord Nelson..... - L R Airdrie Gem ................. - L R Althorp ............. R Anthon .... Rolla Augustus............ Rolla Avon Beau ........... - Beauty Baltic ... . . . . . . . . . . - Rose Baron of Enterprise...... - Rolla 8th Baron of Oakwood...... - L R Barton Duke............. L R Beaumont . . . . . Rolla Bert _... Big Bonanza........... $\mathrm{C}$ Bill Burris ................... $\mathrm{L} R$ Bonar.................... Bonnie Lad...... Breadelbane................... R Brome D. of Bedford... Burnside.................. - L R Capt. Jack ........ Capt. Napier......... R Charles ....................... Charlie Hook.......... - Rolla Collegian ............... - Rolla Coma's Airdrie......... - Rose Crown Prince............ - Rolla Cupid Cupid _... _. _ _. Rolla Diamond -. - . Rolla Dick Johnson ............ - L R Dictator ................. Rolla Dillon's Duke............ - L R Dirigo . Duke _.......... Rolla Duke.................. - Rolla Duke of Alden_......... L R Duke of Belknap.......... Duke of Burlington....... $L R$ Duke of Cheshire......... - L R Duke of Danbury......... - L R Duke of EI Paso........... $-\mathrm{L} \mathrm{R}$ Duke of Freeville......... - Rolla Duke of Gablan........ - L R Duke of Goffstown....... $-\mathrm{L} R$ Duke of Labett............ R Duke of Lebanon..... Rolla Duke of Meredith ...........- L R 4th Duke of New Boston.... - L R 5th Duke of New Boston....- - L R Duke of Oxford.......... - Rolla 1st Duke Peosta ............ - L R Duke of Podunk........ $\mathrm{L} R$ Duke of Riverbank......._- L R Duke of Salem .......... - L R Duke of Springdale........ $-\mathrm{R} R$ Emperor................. - Rolla Eureka _.............. Rolla Fanny's Airdrie..... - Milk Spring Farmer ................. L R Favorite ........ Fidget's Oxford 11th ....- Rislla Fidget's Duke of Oxford.. - Rolla Freeville _................ - Rolla Gen. Webster 5th ....... - Rolla Gen. Whitefoot......... L R Giant $2 d$................ - Rolla 23282 Giaour.......................... Rolla 
23309 Goliah ..................... L R

23322 Gov. Shannon ......... - Rolla

23367 Grassmere Duke............. - L R

23372 Greenland .................. Rolla

23431 Henry Wyttenbach.... .... - L R

23444 Highflyer Turk ........... - L R

23466 Horace

23514 Jackson

23528 Jeff Davis..................

23555 Joe Merriman

23606 Kavanagh ............... - L R

23619 King Henry 8th........... - L R

23624 King of the West.......... L R

23629 Kirks Oxford 3d..........- Rolla

23647 Knight of the Roses........ - Rolla

23667 Lawrence Duke ............ - L R

23705 Lincoln Chief .............

23713 Little Duke .................

23745 Lord Byron

23761 Lord Fairfielda........ Rolla

23770 Lord Henry .........

23781 Lord Nelson

23862 Ludwig ................. Rolla

23882 Major Anthony

23906 Manager

23938 Marshall Winfield.......

239.52 Master Geneva 2d........ $\mathrm{D}$

23971 Matchless ................. L R

23972 May Boy ..................... R

23986 Mazurka's Baron .......... - L R

24033 Miss Allies' Oxford...... - Rolla

2405i Monarch............... L R

24064 Monitor......

24065 Monitor

24111 Nelson

24112 Nelson........... R R

24164 Old Splendor.

24175 Oneida Chief............. L R

24216 Oxford Boy

24:41 Oxford Echo............ L R

24255 Pat ..............................

$24: 62$ Patrick Hotspur...... L R

24263 Patrick Hotspur $2 \mathrm{~d}$

24:74 Peace Maker.............. L R

24390 Perfection's Airdrie ...... - L R

24:94 Peri's Oxford........ Rolla

24295 Philander ......... L R

24379 Prince Arthur.

24407 Prince of Avon

24425 Prince of Montgomery .... - Rolla

24428 Prince of Oxford........ $-\mathrm{L} \mathrm{R}$

24451 Princeton

24514 Red Cloud

24522 Red Coat...... R

24540 Red Duke $2 \mathrm{~d}$

24559 Red Knight........... R

24574 Red Rose Duke....... Rolla

24579 Red Rover......... R R

24603 Rinaldo.................. Rolla

24604 Rinaldo.... R R

24620 Roan Duke....... R

24623 Roan Duke........ R

$246 * 26$ Roan Lad..... R

24644 Rockdale

24678 Rose Duke of Springdale... $-R R$

24694 Rover............ Rolla

24712 Royal Crown...... L R

24769 Sanganash

24812 Sharon Duke...... Milk Spring

24848
24919

24941

24947

24952

24974

25022

25040

25096

25103

25126

25139

25159

25167

25212

25245

25250

25265

25312

$2535 \%$

25376

25537

25540

25กั44

25545

25546

25550

25551

25564

25570

25598

25605

25638

25646

25648

$2565 \mathrm{~s}$

$256 \% 6$

25690

$25 \% 98$

25807

25815

25843

25858

25856

25893

25905

25915

25934

25964

25967

26013

26031

26040

26044

26053

26077

26081

26096

26123

26124

26197

26201

26221

$262: 22$

26256

26273

26299

26302

26313

26331

26352
Star of Hillsdale .......... - Rolla

Sucker Boy ........

Sultan ................... Rolla

Summit Boy ..... $-\mathrm{L}$

Tangwotten ........... Rulla

Tipton ........ L R

Tornado ......... Beauty

Victor .................. Multiflora

Viscount $2 \mathrm{~d}$

Washtenaw Duke..........

Wellington $2 \mathrm{~d} . . . . . . . . . . . .-$ Rolla

White Prince ........... Rolla

Wild Eyes............... Rolla

Young America......... Rolla

Young Rodney .....- Milk Spring

Young Warwick....... - Rolla

Zerlina's Victor........... - Rolla

Dillon's Duke............. L R

5th Duke of Trenton.......... L K

Gloster ........................

Alva Rosuler................. R

Anak

Antaeus ............

Antinous ........... Rolla

Arago -............... Rolla

Arnold

Arrow ............... Rulla

Autocrat_...........

Ball_..........................

Baron Douglas

Baron Hubback $3 \mathrm{~d}$

Baron Rose .............. - Rilla

Baron Valentine

Baron of Woodhill, 4th.....- $\mathrm{L} R$

Becky Sharp's Duke ....... - Ruse

Bellerophon

Ben Butler

Brutus ........ D

Buncombe

Butter Ball ........

Captain Jack .......... Rolla

Cardinal …....... Rulla

Centennial ..... Beauty

Centennial, 7th ........... $\mathrm{L} R$

Champernowne ....... Rolla

Chancellor -........... R

Cherry Oxford........

Col. Grier . .......

Col. Holloway

Count Clareville........... R

Cumberland Lad......... Rolla

Dairyman . . . .

Daisy's Lad ........ Rolla

Darwin ...............

Dick Coke...... Milk Spring

Dietator ...... Rulla

Dom Pedro......... Rulla

Duane's Duke, 10th ...... - Rolla

Duane's Duke, 11th ....... - Rolla

Duke of Dexter........ $\mathrm{R}$

2d Duke of Elba.......... 5th Duke of Gabilan......... $\mathrm{L} R$ 6th Duke of Gabilan...... - L R Duke of Hendon, 2d......-Rulla Duke of Kendallville....... Ky I Duke of Madison .......... Duke of Maple Grove...... - L R Duke of Meadowside .......- L R Duke of Mt. Zion .......-Beauty 2d Duke of Peosta.......... $-L$ R 


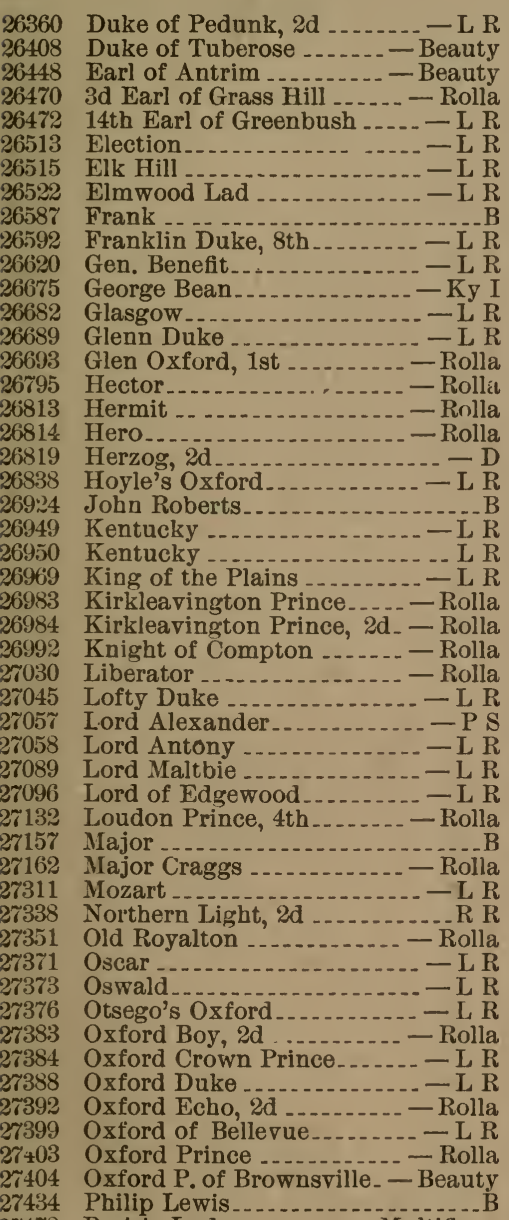

27478 Prairie Lad .............

27489 Prince _.

27501 Prince Albert, $2 \mathrm{~d}$

275\%1 Prince Hal, 2d .......... Rolla

27548 2d Prince of Muirkirk..... - Rolla

27552 9th Prince of Oxford .... - Beauty

27553 10th Prince of Oxford.... - Beauty

27558 Prince of Speedvale........ - L R

27560 Pride of the Fairy Bells ... - Rolla

27571 Prince of Wales, 2d....... L R

27578 Princeton Duke ............. R R

27615 Redbird

27633 Red Duke................ Rolla

27664 Red Rose Oxford ........... - Rolla

27674 Reformer..... R

27682 Rhodope ................. R

27716 Roan Oxford........... Rolla

27763 Rosy Duke................ Rolla

27779 Royal Booth.............. R

27793 Royal D. of Burlington .... - L R
27824 St. George $\ldots \ldots-\mathrm{L}$ R

27868 Shabonee ........ L R

27874 Sharon Airdrie, $2 \mathrm{~d} . .-$ Milk Spring

27893 Sidney Green ............. - Rolla

27980 Sunflower................ Rolla

28026 Tilden

28117 Warfield ................... R

28160 Wiley Oxford, $2 \mathrm{~d}$

28166 Winooski ................ R

2\$178 Wyoming's Champion .... - Rolla

28182 York Duke of Airdrie ....... - D

28183 Yorkshire John ............. - L R

28215 Young Sir Dimple........ Rulla

28216 Young Sir Dimple, 2d ...... - R R

2\$223 Zebulon ................ Rolla

28282 Earl of Pleasant Ridge .... - Rolla

28310 Little John........... L R

28314 Lord Clyde....................

28335 Pioneer, 2d............. $\mathrm{R}$

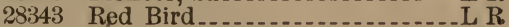

28384 Young Carlisle............ Rolla

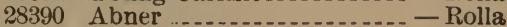

28436 Albor Chief ...... L R

28446 Alexis ........ Rolla

28458 Alstead

28160 Ambler ................... Rolla

28461 American..... Rolla

28463 Americus.................... L R

28464 Anchises ......... Rolla

28475 Armadale ................ L R

28488 Bankhead

28520 Baron of Glenfern

28547 Baron of Oxford .......... Rolla

28573 Beauty Boy ............ L R

28618 Ben Butler.............. Rolla

28624 Ben Franklin ......... R

$2 \$ 665$ Bloomfield Duke, 3d....... - L R

28670 Blossom's Briton . . . . . . Rolla

28683 Bob Price ......... R R

28684 Bonaparte.........

28687 Bonbright ...

28726 Brutus ............. R

28762 Cameo

28835 Chesterford Chief....... Rolla

28859 Clifton ......... Rolla

28860 Clifton

28867 College Hero ...........

28894 Coma's Airdrie.......... Rose

28915 Conrad ........ R

28951 Crocus Duke

28960 Cumberland Beau ...... - Rolla

28961 Cumberland Chief, 2d..... - Rolla

28967 Cypress Duke........... Rolla

29010 Dexter, 3d .............. Rose

29051 Dom Pedro

29149 Duke of Chestnut Hills.... - L R

29201 11th Duke of Gabilan

29248 Duke of Haw Patch...... - L R

29267 Duke of Kelvine Grove .... - L R

29276 Duke of Lentner........ - Rolla

29302 Duke of Mt. Pleasant _.... - L R

29303 2d Duke of Mt. Zion..... - Beauty

29312 1st Duke of Northwood..... - L R

29334 Duke of Oxford Gwynne .. - Rolla

29372 2d Duke of Sunnyside..... - Rolla

29416 Duke of Windfield....... - Rolla

29418 4th Duke of Windingvale.... _ B

29440 Earl of Ashfield ......... _ Rolla

29453 15th Earl of Greenbush .... - I, R

29462 Earl of Huron.......... Rolla 
29464 Earl of Huron, 3d

29485 Earl of Solway ............ - L R

29503 Edward ......................

29512 Elgin Prince

29513 Eli.................. R

29526 Excell.........

29550 Fearnaught

29602 Garland's Duke......... - Rolla

29627 Gen. Hayes .............. L R

29666 Gertrude's Bairn........... - R

29689 Glen Napier.............. - Rose

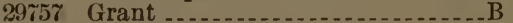

29761 Green Mountain Boy, 2d.- - Polla

29828 Hexam's Airdrie.......... - L R

29841 Hilldale Prince............ $-\mathrm{L} R$

29863 Humboldt Jr., ............ - L R

29589 Ivanhoe ................. Rolla

29903 J. C.

29942 Johnnie...........

29972 K. C. B. $2 \mathrm{~d}$

29988 King of Atha..........

30004 Kirkleavington Duke....... - Rolla

300125 th Kuight of Cambria.... - Rolla

30034 Lancer -................ - Rolla

30062 Leonidas................ L R

30068 Leopold Watson ............ - L R

30090 Lizzie's Duke ............ L R

30119 Lord Burk

30165 2d Lord Wetherby

30224 Magnum Bonum Jr.,........ _ II

30262 Malvern Oxford ......... - Beauty

30263 Manfred................... - Rolla

30294 Massina Boy ............. - Rose

30330 Mazurka, Duke of Oxford - - L R

30339 Meadow King

30359 Minna's Oxford......... Rolla

30361 Minnesinger................ - Rose

30378 Monarch of the West....... - L R

30380 Monitor

30387 Moro _.............

30392 Mountain Boy.............

30405 Myrtle's Sam...............

30410 Napoleon 3d........... Rolla

30142 Norman

30443 Northampton ............ - Beauty

30505 Oxford Brokenhorn....... - Rolla

30508 Oxford Duke.............. - L R

30515 Oxford Gywnne Baronet.. - Rolla

30527 Oxford Royal Prince....... - L R

30616 Prince ..................... Beauty

30631 Prince Albert 6 th

30632 Prince Albert 7th......... - L R

30634 Prince Antony ........... L R

30653 Prince of Harlem......... $-\mathrm{L} R$

30668 Prince of Maine............ - Rolla

30678 Prince of Oxford $2 \mathrm{~d}$

30679 10th Prince of Oxford ... - Beauty

30684 Prince Royal............. L R

30712 Prospero.................... $\mathrm{R}$

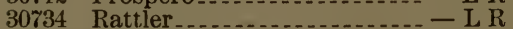

30767 Red Dandy

30780 Red Duke 3d

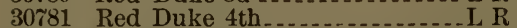

30807 Red Rose Prince........... Rolla

30810 Red Rover........... Milk Spring

30849 Roan Duke ............ - Beauty

30891 Romeo.................

(30910) Duke Frederick

30913 Rose 10th's Oxford............ R

30929
30947

30950

30970

30982

30991

$(3100$

31115

31132

31147

31171

31183

31287

31343

$313 \% 9$

31462

31495

31496

31542

31544

31562

31612

31663

31740

$3175 \%$

$3176 \%$

31780

31782

31830

31833

31850

$3185 \mathrm{~s}$

31880

31914

31925

31926

31955

31961

31998

$320: 5$

32059

$320 \div 1$

32074

32091

32095

32123

32153

32212

32224

32:25

32255

32262

32267

32282

32329

$3235 \overline{5}$

32367

$3237 \%$

32422

$324: 37$

32453

32455

32493

32497

32521

32530

32547

32555

32568

$325 \% 4$

32588
Royal Hook ............ - Rolla

Royal Lancaster $2 \mathrm{~d}$ Saginaw -............... L R Sam 2d...... $R$ Sam'l J. Tilden........ - R Duke of Oxford...... Rolla Shabonee ......... Silence - ........... Rolla Sir Walter.............. Spotswood..... Beauty Stanley Duke...... $\mathbf{R}$ Tommy Bates........... Wade Hampton............. Wellington -........ Rose Baron of Athelstane..... - L R ith D of Richland .. - Milk Spring Duke of Rosendale....... - Rolla Pontiac . . . . . 3d Prince of Grass Hill -. - Beauty Thorndale's Souv., _-_- Milk Spring Alabama . . . . . . Austin ............... Multiflora Baron Watson.......... - Rolla 2d Bates of Oak Hill....... - L R Beaumont 2d ........... - Rolla Belknap .............. Beauty Belle Duke.......... Billy Smith Bismarck Bloom's 2d Duke....... - Rolla Bob ...... Bourbon ......... Burnside 2d......... R Calm Duke of Sharon 6th_ - Rolla Cambria's Airdrie........ - Rolla Cassius ............. Beauty Cato Chief of Little Lehigh .. - Beauty Col. Cheney ........... - Beauty Constance Duke 3d........... L R Count of Wexford. - Milk Spring Courtwood ......... $R$

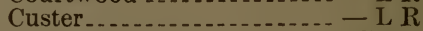
Cypress Duke 2d..... Rolla Darlington Duke $2 \mathrm{~d} . . . . .-$ - Rolla Dirigo Duke of Bedford........ $-\mathrm{L}$ Duke Cadenza......... Rolla Duke Cadenza $2 \mathrm{~d}$ Duke of Fairview .. - Milk Spring $2 d$ Duke of Florence.......... $\mathbf{R}$ Duke of Hamilton...... $R$ 9th Duke of Harlem..... - Rilla Duke of Maine........ - Rolla Duke of Oakland...... $-\mathrm{R}$ Duke of Pickering ....... - Rolla Duke of Putney $2 \mathrm{~d}$ Duke of Spring Creek...- Rulla Duke of the Valley 8th Duke of Waveland ....... - C 2d Duke of Wexford - Milk Spring Earl of Cambridge 7th.....-L $R$ Earl of Cambridge 11th...- $-\mathrm{L} R$ 4th Earl of Grass Hill..... - Rolla Earl of Jefferson ........... - R R 4th Earl of Seaham...- Beauty Earl of Winfield .......... - L $\mathrm{R}$ El Cerrito............ $R$ Emperor Excell $2 d$............ 
32618 Fidget's Oxford 5th....... - I R 32699 Genesee Prince............ L R 32721 Glen Oxford 2d .......... - Rolla 32759 Grand Duke of Airdrie.... - Rolla 32767 7th Grand Duke of Sharon - Rolla 32779 Gray Jack ............. L R 32785 Grove Prince................ L L R 32795 Hamilton Boy ............. R 32802 Harry 32306 Hayward's Roan Bull .... - Rolla 32810 Hector 2d............. Rolla 32844 Hilman ............... Rolla 32845 Hilpa Prince..... Rolla 32858 Hotspur ................ L R 32865 Hugo ..................... 32887 Ivanhoe ............... Cox 32891 Jack Prince............... - L R 32393 Jacob Faithful......... - L R 32948 Jupiter .......... L R 32969 King Sharon......_- Milk Spring 32983 Kirkleavington Oxford ... - Rolla 32984 Kirkleavington Oxford 2d_ - Rolla 32988 Knight Errant_.......... - L R 32998 Lamont ................ L R 33013 Leonidus ................. R 330225 th Liberty Duke........ - L R 33040 Longfellow ........... L R 3306:2 Lord Chestiford $2 \mathrm{~d} \ldots \ldots \ldots \ldots$ 33080 Lord Leslie ............... - M (33129) Beau of Oxford 2d ... - Rolla 33150 Lute ................... Rolla 33160 Major 33189 Manly 33194 Marquis ................ Rolla 33206 Marquis of Lorne ....... - L R 33207 Marquis of Lorne........ L R 33260 Mazurka Lad 4th 33:91 Modoc .................. L R $33: 314$ Mott Duke..... L R 333:66 Nebraska.................. $\mathrm{R}$ 33400 Oxford Beau....... R 33404 Oxford Duke........... Rolla 33485 Prairie Duke............... - L R 33491 Pride of the Fairy Bells _.. _ Rolla 33515 Prince Carl........ - Milk Spring 33522 Prince Frederick 2d...... - Rolla 33529 Prince of Hartse......... - L R 33544 Prince Royal........... Rolla 33559 Prince of Viroinia..... - Beauty 33560 2d Prince of Virginia_... - Beauty 33633 Red Duke of Mt. Zion .. _ Beauty 33635 Red Gauntlet............ - Rolla 33703 Poan Duke 2d.......... L R (33703) Duke of Oxford 33712 Robin Hood......... L R 33716 Robert Burns 33719 Rockingham ......... Beauty 33756 Rosedale John......... L R 33830 Sam Sawyer........... L R 33860 Sharon Duke.......... 3398\% Sir Allen 33936 Star Booth........ R 33962 Success.......... R 33969 Sylvester 33973 Tallyman $2 \mathrm{~d}$ 33981 Telluria Duke $2 \mathrm{~d} \ldots . . . . .-$ Rolla 34003 Tim Day 34068 Water King 34069 Water King 1st.......... Rolla 34091
34095 34106 34146 34153 34189 34914 34229 34248 34265 34302 34307 34310 34356 34358 34359 34364 34399 34393 34404 34427 34137 34440 34459 34460 34461 34491 34492 34499 34530 34575 34608 34624 34659 3466 \% 34684 34685 34686 34694 34712

34726

34756

34785

34814 34829 34834 34365 34898 34907 34908 34951 34964 34972 34979 35004 35015 35031 35037 35038

35045

35046

35076

35080

3508\%

35147

35157

35162

35166

35270

35308

35346
Whitewater Prince 2d..... - L R Wild Chieftian.......... - L R Young Denton ........ Rolla Young Honest Tom....... $\mathrm{L}$ R Capt. Kenny ..... B Duane's Duke 17th..... Rolla Earl of Dummer Park.... - Rolla Irene's Duke .......... L R 2d Mazurka Knight....... - Rolla Van Buren ........... $\mathrm{L}$ R Wild Calf ....... $\mathrm{L}$ R Aaron............ L R Airdrie Oxford Airdrie Prince......... $\mathrm{R}$ ise Airdrie Prince.... $\mathrm{L} R$ Airdrie of Summit...... L R Annabella's Prince...... - Rolla Annabella's Constant .. ... - Rolla Avon Beau 2d........... - Beauty Baron Adelaide......... L $\dot{R}$ Baronet ................ L R Baron Falconwood... $-\mathbf{L}$ R Baron of Oneida........ Rolla Baron Oxford ......... - Rolla Baron Oxford.......... - Rolla Bates Duke.............. L R Bates Duke 2d...... R R 13th Bates of Oak Hill.....- L R Belleville ............. - Beauty Bismarck .............. Rolla Bob Taylor. Rolla Bourbon Airdrie...- Milk Spring Burns ... Butterfly Boy ........... Rolla Cambridge Prince......... L R Cameron .............. Beauty Canadian ........ L $\mathbf{R}$ Capt. Dollinger ............. Multiflora Carlotta's Duke......... $-\mathrm{R}$,lla Centerville ........... Rolla Chieftian ...... L R Climax.......... Rolla Combination $2 \mathrm{~d} . . .-$ Milk Spring Compton Duke .......... - L R Constance D. of Maplelawn _...L R Dairyman 2d ........... $\mathrm{L} \mathrm{R}$ David Noble........... L R Defiance................. LR Defiant Duke........... Rolla Dom Pedro 2d..... R R Douglas............ R R Duke of Airdrie........ $-\mathrm{L} \mathrm{R}$ Duke of Alstead........ Rolla Duke Bill ........ Duke of Buffalo...... R R 4th D of Clinchdale_ - Milk Spring Uuke of Connaught........ - L R 1st Duke of Cool Spring... - R R Duke of Dover.......... Rulla 2d Duke of Dover...... - Rolla 19th Duke of Gabilan ..... - L R 25th Duke of Gabilan ..... $-\mathrm{L} R$ 28th Duke of Gabilan...... - L R Duke of Kansas.............. R Duke of Lake Park........ L R Duke of Lee............ Rolla Duke of Lincoln ..... L R Duke of Sharon $2 \bar{d} \ldots \ldots-L R$ 2d Duke of Tuberose.... - Beauty Duke of Woodstock..... 
35357 Earl 2d................. - Rolla

35369 Earl Chesterford .......... - L R

35373 Earl of Conneaut........ $\mathrm{R} R$

35376 Earl of Dexter ............ - Rolla

35380 2d Earl of Dummer Park _. - Rolla

35410 Earl of Maple Hill......... - Rolla

35434 Earl of the Tree............ - L R

35462 Empire Duke............ - Rolla

35492 Favorite of Maine Valley _..

35506 Fletcher ................. L R

35556 Gebal Tarik

35567 General Custer.......... - Rolla

35574 Gen. Fisher

35654 Gloster Prince............ - Rolla

35661 Goldfoil.................. Rolla

35688 Grand Duke ....................

35698 Grand Master Rose....... - L R

35714 Grant.................. - Rolla

35724 Guy of Warwick........ - Rolla

35\% 76 Hilpa's Duke 2d .......... - Rolla

35777 Hilpa's Hillhurst......... - Rolla

35778 Hilpa Prince 2d.......... - Rolla

35818 Iowa Chief............... - L R

35866 John

35896 Junebug -....... R R

35900 Jupiter

35937 King of the Lawn....... - Rolla

35940 King William 3d.......... - L R

35980 Leif The Lucky ............ - Rolla

36029 Lord Belvedere.......... - Rolla

36036 Lord Cornwallis........... - L R

36051 Lord Hubhack $3 \hat{d} \ldots . . . . . . . .-L R$

36058 Lord Nelson.............. - L R

36086 Lou Angeline's Duke...... - L R

36103 Loudon Prince 4th .......... - Rolla

36119 Lyonsdale Lad............. L R

36144 Major Airdrie 2d........ - Rolla

36147 Major Benson .......... - L R

36162 Major Wooster.......... Daisy

36167 Mameluke 6th........... - L R

36169 Manchester............ Beauty

36188 3d Marquis of Worcester .... _ P S

36207 Master Duke............... - L R

36224 Matterhorn

(36231) Beau of Oxford 4th.... - Rolla

36255 Milo...........

36257 Miltiades ............... - L R

36311 Ned Dimple... Rolla

36318 Nep_................ - Rolla

36330 Netherby ................ Rolla

36331 New Braintree Chief...... - L R

36371 4th Oneida's D of Mellery .... - C

36394 Oxford 7th.......... P S

36405 Oxford Boy

36410 Oxford Duke........... Rolla

36412 Oxford Lad .............. - L R

36438 Pearl .................

36468 Pickwick .................

36515 President Lincoln

36522 Prince ....................

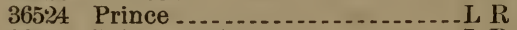

36536 Prince Arthur

36538 Prince of Barnet......... $-\mathrm{L} R$

36561 3d Prince of Grass Hill - - - Beauty

36562 4th Prince of Grass Hill. - Beauty

36564 Prince Hal 3d ............. - Rolla

36568 20th Prince of Herkimer.. - Rolla

36582 Prince of Kansas ............ - L R

36606 Prince of Paris 1st.......... - Rolla

36607
36621

36700

36752

36812

36814

36843

36851

36881

36921

36942

36961

36964

36975

36977

36983

36985

37039

37041

37049

37052

37069

37134

37197

37198

37209

37227

37236

37255

37267

37278

37289

37401

37405

37505

37517

37524

37546

37554

37578

37607

37612

37619

37638

37675

37676

$37 \% 22$

37729

$37 \% 87$

37809

37821

37857

37859

37896

37954

37961

37967

37988

37999

38033

38087

38095

38134

38142

38160

38165

38177

38196

38205

38212

38279
Prince Regent 11th......... - L R

Red Cloud..............

Red Rover............

Roan Prince............. L R

Robert Bates........... Rolla

Rosy Duke............... - Rolla

Rose's Duke of Syene..... - Rolla

Royal Dane................ L R

Sam

Saxon ................ Rolla

Seymour......

Shamrock -..........

Sheldon............... $\mathrm{R}$

Sheridan ........... Rolla

Shiloh

Short Tail ............... Rolla

Spring Valley Duke.... - L R

Squire Archie .............- - Beauty

Star Duke ............... - Rolla

Star Duke 2d.......... - Rolla

Stimpson's Duke........ L R

Thorndale Duke.......... - Rolla

Viking .................... $-\mathrm{L}$ R

Village Blacksmith_ _ Milk Spring

Wade Hampton.............. L R

Water King ................ Rolla

Wehawken Lad.......

Wildeap 2d ........... - Beauty

Wiley 5th...............

Will $O$ ' the Wisp.......... $\mathrm{L} R$

Wolverine ...................... $\mathrm{R}$

Airdrie Duke ......... Rolla

Airdrie D. of Oneida.... - Rolla

Aztec....................

Balco _............. Rolla

Banner Bearer....... R R

Baron of Bluffdale 5th

Baron Bright Eves.......- Rolla

ith Baron of Hilldale..... - - Rolla

2d Baron Rose....... ...... - Rolla

Baron Sharon............. $\mathrm{R}$

Baron Wellington 6 th......- $-\mathrm{L} \mathrm{R}$

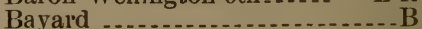

Bellerophon ....... Beauty

Bellerophon $2 \mathrm{~d} . . . . . . .-$ Beauty

Bishop .............. R

Blaine........

Boyne Water........... - Rolla

Bright Eyes Duke....... - Rolla

Brookfield Lad............. - L R

Butterfly Boy ............. - Rolla

Byram

Carlock ....... Rolla

Chief ................

Christopher ............ Rolla

Clansman -.... $\mathrm{L} R$

Colonel Dee.......

Col. Mansfield, 2d......-L R

Comstock

Cypress Duke, 3d...... - Rolla

Daisy Duke........... $\mathrm{L}$

David Noble, $2 \mathrm{~d}$

Delia's Airdrie....... $-\mathrm{L} R$

Diamond

Diamond Joe.......... $\mathrm{R}$

Dick Wiley

Doctor Haley.......

Dominion........... $\mathrm{R}$

Donn ............

6th Duke of Ashland - Milk Spring 
38282

38329

38388

38106

38417

38442

38466

38473

38520

38528

38529

38556

38565

38577

38610

38611

38623

38640

38700

38715

38724

38746

38756

38776

388119

38816

(3883

38849

38882

38909

38922

38954

38967

38983

38998

39013

39176

39192

39199

39213

39264

39267

39269

39281

39281

39293

39308

39345

39400

39404

39433

39448

39510

$3952 \gamma$

39535

39536

39539

39543

39584

39607

39622

39664

39667

39698

39749

39755

39790

39791

39820

39821
Duke of Athlone

- Rolla

Duke of Cornish........ Rolla

Duke of Glen ........... - Rolla

Duke of Grove Creek...... - Rose

Duke of Hatfield....... - Beauty Duke John ....... - Long Horn Duke of Lee Side......... Rolla Duke of Lone Jack. - Milk Spring 7th Duke of Orleans ...... - Rolla Duke of Oxford ........... - Rolla Duke of Oxford, 4th...... - Rolla Duke of Rock River....... $-\mathrm{R} \mathrm{R}$ Duke of Saline.......... Rolla 8th Duke of Skaneateles. - Beauty 3d Duke of Tuberose.... - Beauty 4th Duke of Tuberose... _ Beauty Duke of Weston.......... - Rolla Duke of Westchester....... - L R Earl of Gainer........... Rolla Earl 2d of Lake Park...... - L R Earl of New Boston..... - Beauty Eastern Star.............- L R Edmond ................ Beauty Emperor ............ R Falstaff _......... Rolla Fannie's Duke..... - Milk Spring Oxford Swell ......... - Rolla Forest Napier of Orange.... $-\mathrm{L} R$ Fusee.................. $-L ~ R$ Garfield .................. Rolla Garland's Chaplet....... - Rolla Gen. Garfield ............ L Gen. Garfield ... L R Gen. Grant .............. Gen. Hancock $\ldots$ Gen. H. Day ......... 6th Grand D. of Oak Hill Grant..................... $-\mathrm{L}_{\mathrm{H}} \mathrm{R}$ Green Mountain Prince, 3d- - L R Hamden ............... Rulla Hero Hero of Fairview . Hester's Duke ............ - Rolla Hillhurst Duke of Hilpa .. _ Rolla 3d Hilpa Prince.......... Rolla Homer............... Rolla Howard ..................... Island Chief........... Rolla

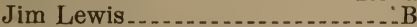
Joe Airdrie ...... Rose John R. Goodman............. B John Templeton .... - Milk Spring Kinellar's Boy _....... - Beauty Kirkleavington Lad ...... - Rolla Kirkleavington Lord ...... - Rolla Kirkleavington Lord, 2d.. - Rolla Kirkleavington Prince, 6th - Rolla Knight_._._._._._._- Milk Spring Leader.................. - Beauty Lexington .................. - Rolla Llewellyn_............. - Beauty Lord Dunsmore.......... - Rolla Lord Dunsmore, 4th....... L R Lord Sharon.............. - Rolla Lucy's Airdrie............ L R Lydia's Joe..................... - M Major................. - Rolla Major................... - Rolla Marmeluke, 4th.......... - Rolla Marmeluke, 7th......... - Rolla
39865

39889

39892

39893

39954

39994

40058

40072

40135

40141

$4014 \%$

40179

40194

40221

40233

40350

40359

$403 \% 5$

40377

40380

40389

40423

40488

40490

40494

40532

40573

40592

40652

40666

40682

40689

40690

40742

40768

40803

40809

40868

40875

40889

40906

40915

40916

40924

40932

40949

40999

41034

41039

41064

41087

( 4108

41106

41121

41129

41152

41153

41163

41165

41175

41189

4126?

( 41264$)$

41291

41429

41433

41460

41464

41518

41523
Marquils of Salem .........- $\mathrm{L} R$ Master Chilton .......... $R$ Masterman, 2d ...........-Rolla Master Porter ........... - Beauty Mendota Goldfinder ........ $-\mathrm{L} \mathbf{R}$ Monarch ................ - Rolla New Braintree Beau........ - Rolla Nippersink Boy ............. - R R Orator _... . . . . Rolla Orion, $2 \mathrm{~d}$.............. $\mathrm{R}$ Orion, 3d....... Oxford Duke ............. - L R Oxford Lad............... - Rolla Peachbud Duke ........... - L R Percy .................... Rolla Prince............... $R$ Prince Albert .............-L R Prince Belvedere _..._._- Beauty Prince of Bourbon _.....- Beauty P. Carl of Grove Creek-Milk Spring Prince Frederick, 4th ....... - Rolla Prince of Okland ......... - Rolla Rachel Duke.................. L R Ralph .................... R Ralph Van Dusen .......... B Red Duke............... Rolla Red Light.............. Red Rose Lad............. - L R Riverside Lad ...........- Beauty Roan Duke......... Milk Spring Roan Prince ........... - Beauty Robert Lee ........ Robert Lee, $2 \mathrm{~d}$ Romulus.......... - Milk Spring Rose's Oxford ...... - Milk Spring Roval D. of Sandusky _..... - L R Royal George.............. - L R Sampson................... $-L$ Santa Claus ............... L R Seaham_..._......... - Beauty Seymour................ - L R Sharon Barmpton...... - Rolla Sharon Bates............. - L R Sharon of Greentield .....- - Rolla Sidney ..................... - L R Sir James Graham ....... - Rolla Spotswood Chief......... - Rolla Sterling Marquis ............- $-\mathrm{C}$ Stonewall ........ . Milk Spring Sussex Boy ............ $R$ Telluria Duke, 4th ........ - Rolla ) Beau of Oxford ....... - Rolla Thorn ................. - Rolla Tom _............. $R$ Tom Siance .......... Tuberose Baron.........- Beauty Tuberose Prince ......... - Beauty Underedge Duke........... $\mathbf{L} \mathbf{R}$ Union ..................... -Rolla Vanderbilt ................ Rolla Vermont_............. R Wentworth Duke........ L R Conishead Duke, 3d .... - Rolla Wiley ..................... - L R Airdrie Baron Bates ...... - Rolla Airdrie D. of Oxford.....- Rolla Atticus................. - R R Babraham's D. of W'dstock_- Rolla Bill Custer .............. - R Bird Rosemount........... L R 
41544 Bourbon Bates ............- - Rose

41555 Bright Eyes Prince....... Rolla

41561 Brokenhorn ................ - L R

41596 Carleton.................. - Rolla

41598 Cedar's Earl of Sharon.... - Rolla

41642 Conewango Lad, 4th ....... - L R

41643 Conewango Lad, 6th........ - - L R

41666 Crown Prince ............ R R

41684 Darlington Duke........ - Rolla

41685 Darlington Prince.......... L R

41699 Dexter Chief .......... R

41717 Doctor Weller.............. R R

41803 Duke of Leon .............. L R

41851 Duke of Warfield ......... L R

41852 Duke of Washington............ B

41858 Duke of Woodside........ Rose

41890 Edmond, 2d............. Beauty

41894 Emigrant ............... L R

41904 Exchange................. L R

41917 Fennel D. of Lancaster .... - Rolla

41948 Gaylord's Oneida

41950 Gem's Baron............... Rolla

42058 Herman........... Milk Spring

42065 Honest John .......... L R

42070 Horatio................ R

42087 Iona's Baron ....... R

42088 Iona's Baron, 2d......... L R

42144 King Alfred.............. L R

42158 Langdon ................. - L R

42202 Maggie's Kirk ................ $-\mathrm{C}$

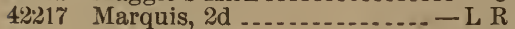

42260 Nashua King ............ L R

42302 Oxford Gwynne Baronet.. - Rolla

42304 Oxford Lad ................ _ Rolla

4232: Peri Baron ............ Rolla

42326 Peter Gentry ............ Rolla

4:3351 Prince... L R

42365 Prince of Baden

42380 Prince Leopold ............. - L R

42337 Prince Oxford Gwynne 5th - Rolla

42416 Red Chunk.............. L R

42441 Red Rose Prince......... Rolla

42543 Southard's D. of Newham - Milk S.

42575 Taylor Oxford ........... - Rolla

42589 Tiras ..................... R

42612 Valley Falls Duke....... L R

42635 Waterman.............. Rolla

42661 Young Mary's Duke....... - Rose

$426 \pi 9$ Acem ........ L R

42683 Acomb Lad........... Rolla

42715 Airdrie Sharon............ - Rose

42727 Alderman........ R

42731 Alice Duke 3d....... Rolla

42732 Aladdin ............. Beautv

42771 Babraham's D of W'dstock _ - Rolla

42790 Baron Acorn ............ - Rolla

42791 Baron of Alton ........... - L R

42792 Baron of Alton 2d....... $\mathrm{L}$

$42 \% 95$ Baron of Cambridge..... - L R

42813 Baron Rawlins............. - L R

42850 Bellfield Airdrie........... Rose

42851 Bellfield Airdrie 2d........ - Rose

42864 2d Bell D. of Sandusky ..... - L R

428675 th Bell D. of Sandusky.... - L R

42871 Bellville 2d............ - Beauty

42919 Block ... Rose

42942 Bonus .................. Rolla

42948 Boss Duke of Lakeview ... - R R

42950 Boston Duke........... Rolla

42955
42969

42979

43020

43039

43056

43058

43059

43067

43078

43113

43139

43159

43170

43185

43194

43215

43216

43217

43253

43300

43313

43337

$435+1$

43637

43641

43648

43675

43689

43699

$43 \% 15$

43761

43769

43790

43566

43882

43895

43921

43939

43947

43958

43961

43982

44034

14049

44092

44113

44155

44163

44187

44202

44207

44262

44280

44382

44394

$+4408$

44414

44418

44476

44512

44588

44623

44634

44659

44716

44737

44758

44830

44837

44886
Bright Eyes King......... - Rolla

Brookfield Boy ........... $-\mathrm{L} R$

Capt. Clover.............. - Rolla

Caroline's Loudon...... - Rolla

Champion ................ - Rolla

Charles ................ L R

Charley

Charlton Boy

Cherry Duke 6 th............ $R$

Col. Boudinot ............ Rolla

Comet ...............

Conewango Lad 3d........ - L R

Constantine ............... $-\mathrm{L}$ R

Count Newby $2 d$........ - Rolla

Croppy -................ - Beauty

Daisy's Duke........... - Rolla

Daisy's Duke 2d........... - L R

Daisy's Napier............ Rolla

De Soto ......................

Don Royal ............. - Rolla

Duke Alexander......... - Rolla

Duke Boy .................. - R R

Duke of Stone River - Milk Spring

Earl of Meansker.. _ Milk Spring

Earl of Overlake.......... - Rolla

Earl Van................ - Rolla

Emma Rue's Oxford ....... - C

Eva's Duke................. - L R

Fairy's Duke........... Rolla

Fillagree's Duke.......... $-\mathrm{L} R$

Gem of Airdrie's Earl...... - L R

Gen. Arthur ............. - L R

Gen. Grant ............... L R

Governor.............. Rolla

Grand Prince.......

Green Mountain Lad..... - Rolla

Hearne............... - Rolla

Hero . . . . . . . .

Hicks

Hilpa D. of Norwood .... - Rolla

Hobart ................... - L R

Imperial Airdrie........

Johnny .................

Joker

Kirkleavington Lord $3 \mathrm{~d}_{. . .}-$Rolla

Lancaster _................. - L R

Lone Star .......... - Milk Spring

Lord Bright Eyes 2d....... - L R

Lord Lorne............. $-\mathrm{L} R$

Lord Watton...............

Lorena's Hilpa............ Rolla

Mahone.................. - L R

Major R

Monarch $2 \mathrm{~d}$

Morton ........ $R$

Neal's Choice...........

Nero

New Boston Double D. 2d- - Rolla

Orpheus 8th............ - Rolla

Oxford Prince....... Rolla

Prairie Chief .............

Prince Bismark.......... - L R

Princess Duke $2 \mathrm{~d} . . . . . . . . .-\mathrm{L} R$

Prince Leopold

Readjuster............. Rose

Red Comet......... - Milk Spring

Red Knight............... - Rolla

Rob Roy

Roderick Dhu

Royal Prince........... Rolla 
44915

44921

44947

45025

(45086

45090

45095

4.5128

45168

45171

45198

45287

45.317

45323

45330

45331

45332

45334

45337

45349

45353

45389

45416

45472

45501

45502

45505

45521

45526
45539

45577

45.593

45594

4.5644

45657

45681

$45 \sim 33$

45785

45874

$458 \% 9$

4.5926

45939

45941

45951

45952

45967

45984

45985

45986

45987

45995

45998

46019

(.46023

$46^{\circ} 24$

46032

46033

$4605 y$

46096

46123

46132

46152

46154

46159

46177

46227

46235

$46 \% 65$

46310

46320
Sabo _... . . . 2 - Rolla St. Lawrence..... Milk Spring Senator.................. - Rolla Star of Pdunk........ R ) Lord France........... Rolla Tom Paine ......... Beauty Tonj Custer....... L R Victor ........... Rolla Wellington $3 \mathrm{~d}$ White Baron........... L R Windom Acem 2d. Alfonso .......... $\mathrm{L} R$ Alfonso ........... Annabella's Duke...... - Beauty Annabella's Duke 2d... - Beauty Annabella's Duke 3d.... - Beauty Anson Wood.......... Rolla Arabella's Duke........ - Beauty Ascot . . . . . . . . . Auburn Duke........... $-\mathrm{L}$ R Baron McKane..... $\mathrm{L}$ R Beatrizet. ........ Rose Bismarck ......... Booth's Briton........ L R

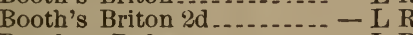
Bourbon Duke.......... L R Bridegroom ........ R Brightness Duke........ Bud O'Hope............ R Carlotta's Duke ........ Rolla Champion 2d........... Rolla Champion 3d......... Rolla Comet........... R Count Butterfly Daisy Duke 3d ........ L R Dixie ............ Duke of Bath Duke of Lexington...... Rolla Duke of Livingston ....... - L R Duke of Rockdale.. - Milk Spring Duke of Sheridan.......... $-\mathrm{L} \mathrm{R}$ Duke of Springdale......- Beauty Duke of Summit............. $-\mathrm{L}$ R 2d Duke of Swansea..... Rolla Duke of Wellington....... Rolla Dunmore 2d............. - Rolla Dunmore 3d ...........-Beauty Dunmore 4th......... - Beauty Dunmore 5th............. Rolla 6th Earl of Brookdale.....- Rolla Earl of Cedar Springs....- Beauty 3d Earl of Sharon ........ - Rolla Bushbury C. of Oxford _. - Rolla Earl of Worcester....... - Rolla Edwin Booth......... Rolla Eldorado ........ L R Eureka 2d............ Rolla Frederick 2d........... Rolla Gen. Carpenter ........... L R Gen. Garfield .......... Rolla Genesee.................. L R Geneva's G. Duke....... - Beauty Geneva Wild Eyes Duke......L R Golddust Hannibal Harkrader .................. R Hilpa Duke.............. Rolla James Allen Joker 2d................... Rolla
46342 46.349 John Templeton 2d_ - Milk Spring 46393 Kirkleavington Lad 2d .... - Rolla 46408 Lady Hester's Prince..... - Rolla 46410 Lady Sale's Kirkleavington - Rulla 46425 Leader.................. - L R 46476 Lord Duckham .......... R R 46.501 Lord Wellington 46553 Malinthas Earl 2d...... - L R 465\%6 Master Mason............. $-\mathrm{R} R$ 46590 Mayflower Prince........ - L R 46598 Merrimac River Duke..... - L R 46607 Minstrel Duke........... - Rolla 46617 Monarch................ R R 46618 Monarch $3 \mathrm{~d} \ldots . . . . . . . . . .2 R$

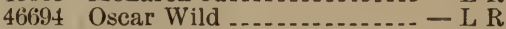
46696 Ossian ................ R (46708) Lord Turncroft Oxf'd 4th-Rolla 46711 Oxford Gwynne Baronet 2d - Rolla 46761 Pride.................... - Rolla 46762 Pride of Avon $46 \% 63$ Pride of Avou, 2d..... R 46811 Prince of Mellery ......... $-\mathrm{L} R$ 46816 Prince Robert ............. L R 46849 Rawley ....................... Rose 46861 Red Chunk, 3d ............. L R 46863 Red Cloud.................. L R 46890 Red Light.............. R 46930 Rising Star............... L R 46964 Roger Atwood...... R 46984 Rosamond's Duke...... Beauty 47000 Royal Duke.......... Rolla $470123 d$ Royal Lad ........... Rolla 47023 Royalty ........... R 47053 Sciota Lad ................. R 47057 Sempster......... R

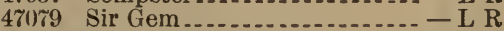
47098 Son of Jessie, 8 th 47102 South Fork Lad, 2d....... Rolla 47113 Starlight ............... 47160 47174

$471 \div 6$

47183

47193

47213

47254

47283

47308

47329

47374

47395

47407

$4 \pi 464$

47465

47478

47512

47555

$475 \% 9$

47622

4 ชิ632

47685

47752

47776

47802

47840

47862

47863

47902

47916
Tom Tuberose Duke....... Beauty Tulip's Oxford.......... - Beauty Unkamet Prince.......... L R Vanguard .................. $\mathrm{L}$ R Von Moltke............ $\mathrm{R}$ 2d Wild Eyes of Macomb _ $-\mathrm{L} R$ Young Hero ............ Rolla Acorn, 3d ............ R Airdrie McHenry Arthur ................ Rolla Banker................... Baron Birkett...... Rolla Bell Duke, 5th.......... L R Bell Duke, 6th ............ - Rolla Bell Prince.... Rolla Billy ................. Cox Bonapart ........ Bright Hopes....... Rolla Captain Hooper........ Rolla Cash Boy ............... R Col. Bright Eyes ........... - Rolla Dairy Baron.............. L R Davy Wilson............. L R

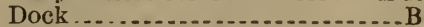
Dragon Brook Duke...... - Rolla 5th Duke of Annabella $\ldots$ - Beauty 6th Duke of Arabella _... _ Beauty Duke of Conneaut.............. R R Duke of Edgewood...... Beauty 
47963 Duke of Isanti............ - L R 48023 Duke of Penn............. - Rolla 48072 Duke of the Realm .. - Milk Spring 48079 Duke of Walnut Grove ..... . - Rose 48095 Duke of Williamstown .... - Rolla 48129 40th Earl of Greenbush ..... - L R 48131 42d Earl of Greenbush ...... - L R 48134 Earl of Lancaster............ - L R 48136 2d Earl.of Manteno ........ - Rolla 48144 Earl of Somers ............. - Rolla 48160 Emperor of the French _... - Rolla 48166 Essex Lad .................. - L R 48183 Fayette Lad .............. - Rolla 48184 Felix . . . . 48204 Frank .................. - Rolla 48314 Gold Foil, $2 \mathrm{~d} . . . . . . . . . . .-$ Rolla 48343 Guilford Champion......- - Beauty 48358 Hector................... - Rolla 48374 Hesperus ................ - Rolla 48379 Hillhurst Sharon Duke.... - Rolla 48393 Humbert................ - L R 48422 James Duke.............-Beauty 48457 John Bull, 3d .............. - Rolla 48460 John Hancock .......... - L R (484\%2) Prince Airdrie ........ - Beauty 48489 Jumbo ................... - Rolla 48532 Kirklevington, 1 of Oak Hill-Rolla 48535 Kirk's Wonder............. - Rolla 48549 Konkapot................ - Rolla 48551 Lad ...................... Rolla 48572 Leander ................. - Rolla 48588 Leopard ................. - Rolla 48589 Leopold ................... - Daisy 48619 Lord Barrington of Vermont - L R 48625 Lord France ................. - Rolla 48631 Lord Moreton.................R R

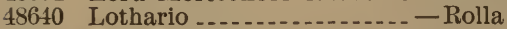
48668 Lucy's Airdrie.............. - Rose 48720 Marmaduke................ - L R 48730 Mary Duke............... - Rolla 44752 May Duke ................ - Rolla 48779 Monarch, 4th ............. - Rolla 48780 Monarch, 5th .............. - Rolla 48781 Monarch, 6th ............. - Rolla 48890 Oxford Prince, $2 \mathrm{~d} . . . . . . . . . .-$ Rolla 48892 Oxford Royal Prince ....... - L R 48893 Pansy Duke ............. R 48926 Pilot ...................... - L R 48955 Porter ...................... 48985 Prince Albert, 8 of Hemdoka - L R 48995 Prince Barrington, 2d .......- - L R 48996 Prince of Berkshire ......... - Rolla 49039 Prince William, 2d ......... - L R 49082 Red Cloud ................ - Rolla 49083 Red Cloud ................ - Rolla 49100 Red Jacket.............. - L R 49104 Red Jammy 49130 Red Vermillion................ $-\mathrm{L} R$ 49173 Rob Roy 49248 Royal Wild Eyes .......... - L R 49304 Sennett Lad........ - Multiflora 49332 Sir Charles .......... - Milk Spring 49372 Springvale Comet ........... - Rolla 49374 Squabble Hollow Duke ..... _ - L R 49387 Stirling Prince ............. - Rolla 49415 The Squire 49422 Thorndale Prince........... - L R 49425 Tillamook................... - L R 49448 2d Tuberose Duke....... - Beauty 49463 Vanquish Airdrie, $2 \mathrm{~d} . . . . . .-$ Rolla
49469

49503

49540

49584

49610

49629

49631

49635

49636

49637

49638

49643

49646

$4964 \pi$

49648

49649

49650

49651

49652

49655

49656

49657

49658

49660

49661

49663

49664

49667

49669

49672

$496 \% 4$

49675

49676

49678

49679

49683

49684

49685

49686

49694

49695

49701

49703

49706

49707

49708

49709

49710

49712

49713

49714

49715

49716

49721

49724

49725

49726

49727

49729

49731

49732

49733

49734

49735

49736

49737

49738

49739

49741

49742
Vernon Duke ................... L R

Washington ............... Rolla

Wrangle

Beau of Oxford $2 \mathrm{~d}$

Oxford Swell ............. - Rolla

Aaron ...................... - D

Aberdeen

Abingdon Duke.........

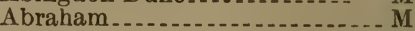

Accident .............

Achilles

Acme Duke ................. Ky I

Adam ..................

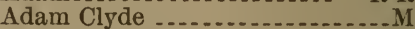

Adelaide Duke ............ $\mathrm{L}$

Adella's Duke............. - Rolla

Addie's Duke ............... Ky I

Add Walker ....................... S

Admiral

Admiral, $3 \mathrm{~d}$

Adniral of Sonora.........

Adolphos Duke........... $\mathrm{R} R$

Adonis ...................

Aesop, $2 \mathrm{~d}$

Afton Boy .................

Airdrie...................

Airdrie, 13th ...........

Airdrie of Audrain ..........

Airdrie of Bayview......... - Rolla

Airdrie Boy ................. B

Airdrie Constance Duke....- $-\mathrm{L} R$

Airdrie Duke.....................

Airdrie Duke............... $\mathrm{R}$

Airdrie Duke, 2d.......... - Rolla

Airdrie Duke, $2 d . . . . . . . . . . . . M$

Airdrie D. of Elmwood........ B

Airdrie D. of 1st Creek Run - $-R R$ 2d Airdrie D. of 1 Creek Run _- - D 4th Airdrie D. of 1st Creek Run - D Airdrie, 3d of Hickory Grave - R R Airdrie's Hook - ............ - R R Airdrie's Lad .............. $R$ Airdrie Mason ............... $\mathrm{R} R$ Airdrie of Pleasant Hill.....- $-\mathrm{R} R$ Airdrie of Pl'nt Hill, 2- Long Horn Airdrie of Pleasant Hill, 3d- - R R Airdrie of Pleasant Hill, 4th - R R Airdrie of Pleasant Hill, 5th - R R Airdrie Rose of Sharon....- - Daisy 2d Airdrie of Rosewood...- - Daisy 3d Aurdrie of Rosewood...- - Daisy 5th Airdrie of Rosewood.- - Daisy Airdrie Sharon ........... $R$ R Airdrie of Sonora....... Ajax ................. $R$ A jax A jax ............................. M Ajax Alansa Albert Albert Albert ..................... Ky I

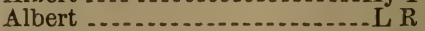

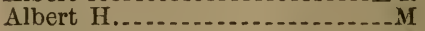

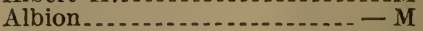
Alby's Duke ............. - Rolla Alcyon Aldevade....................... Alexander .................. $\mathrm{R}$ Alexander Hamilton.......- $\mathrm{R} R$ 
49743

49744

49745

49746

49747

49748

49749

49750

49751

49752

49753

49754

49755

49756

49757

$4975 \mathrm{~s}$

49759

49760

$49 \% 61$

49762

49763

49764

49765

49767

$497 \% 0$

49771

$497 \% 2$

49773

$497 \% 5$

49776

49777

49778

49779

49781

49783

49784

49785

49787

49788

49793

49796

49798

49799

49800

49801

49802

49803

49804

49805

49807

49808

49810

49811

49813

49814

49820

49821

49823

49826

49827

49828

49829

49830

49833

49837

49838

49841

49843

49845

49846
Alexis.................. - Cox

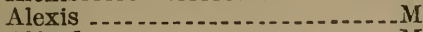

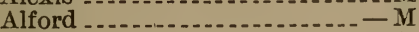

Alfred

Alfred

Alice Maud's Duke...... $-R$

Allegan Duke.............. R

Allegheny ..............

Allen County Chief......... $-\mathrm{R} R$ Allendale Loudon Duke.... $-\mathrm{R} R$ Allendale Loudon Duke $3 \mathrm{~d} . . .-\mathrm{T}$ Allendale Loudon Duke 4th Allendale Loudon Duke 8th - Ky I Allendale Loudon Duke 9th - L R Allendale Loudon Duke 10th_Daisy Allendale Loudon Duke 11th _ - T Allendale Loudon Duke 12th _ - M Allendale Loudon Duke 13th - L R Allendale Loudon Duke 14th _ - M Allendale Loudon Duke 15th .. - T Alonzo Fern ................... T Alpine...... Cox Alva ....................... P S

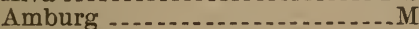
Anchorite

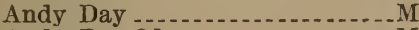
Andy Day $2 d . . . .$. Andy Johnson........... Ky I Apelles ................... $R$ Apelles $2 d$............. Apollo............... $R$ Apollo ..... Daisy Apollo ..................... Arabella Duke........... L R Araby Archduke 4th of Palmyra.....Ky I Archduke 5th of Palmyra.... - M Archer............. P S

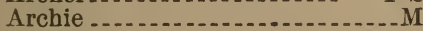
Arcola Arkakeker........ Arlington................ $\mathrm{R}$ Aroma Prince ........... - Rolla Artemus IVard Artesian Duke............ - R R Artesian Duke 2d............... S S Arthur ................... Arthur Arthur .................. Arthur .................. Arthur Arthur ................... Arthur $2 \mathrm{~d}$ Aruba's Airdrie.......... $\mathrm{R} R$ Aruba's Lancaster........ Athelstane ............... - P S Athelstane 1st ............. - R R Athlestane 3d............ - R R 2d Athol of Maple Hill..... - P S Auctioneer .................................. Audubon Duke.............. Aurelius ................. - D Aurelius Autumn Lad 4th.... Bachelor ................. Cox Bailey's Chaise................

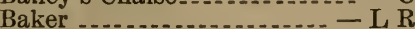
Baldy -................... Baltimore Lad............ Banker...................
49847

49849

49850

49851

49852

$4985 \overline{3}$

49855

49856

49858

49862

49863

49864

49865

49866

49867

49870

49873

49874

49875

49877

49878

49879

49881

49884

49885

49886

49891

49893

49895

49897

49898

49899

49900

49901

49902

49904

49905

49906

$4990 \%$

49914

49915

49916

49917

$49918^{\circ}$

49919

49920

49921

49922

49925

49926

49927

49928

49929

49931

49933

49935

49936

49938

49941

49942

49945

49946

49947

49948

49949

49950

49951

49953

49954

49957
Banker .................. - L R

Barmpton................. - $\mathrm{P} S$

Barmpton 2d....... P S

Barmpton Duke........... - R R

Barmpton Duke.......... - M

Barmpton I Lad ............ - R R

Barmpton's Prince......... - R R Baron................... $R$

Baron -..................... Ky I

Baron's Airdrie.......... M

Baron Bates.................. Ky I

Baron Bates........... B

Baron Bell Bates...... R R

Baron Bickerstaffe 2d..... - Rolla

Baron Beirn of Bay View ... - P S

Baron Bright Eyes 3d..... - Rolla

Baron Cecil 1st ................ Ky I

Baron Cecil 2d................. I I

Baron Cecil 3d............ $\mathbf{L} R$

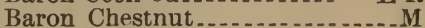

Baron of Chester............ - T

Baron Clark 2d................. Ky I

Baron Constantine 2d..... - R R

Baron Duke................. Ky I

Baron's Duke.............

Baron Dunmore............ - R R

Baron of Geneva.......... - P S

Baron of Geneva $2 \mathrm{~d} . . . . . . .-\mathrm{P} S$

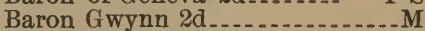

Baron Hopewell ............. - R R

Baron J 3d of Vine Hill...... - C

Baron Knightly .............

Baron's Lad............. $-R R$

Baron Leslie............... Ky I

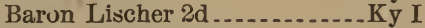

Baron Lyndale 26th........ Ky I

Baron Madison ................ - D

Baron May 3d............... - B

Baron May 4th......

Baron Oxford ..............- -

Baron of Oxford

Baron of Oxford $3 \mathrm{~d}$

Baron Oxford 5th....... - Rolla

Baron Oxford 6th ......... - Ky I

Baron Oxford 10th........- - Rolla

Baron Oxford 11th....... - P S

Baron Oxford 12th........... - M

Baron Piatt 2d............ $\mathrm{L}$

Baron Prince.......... P S

Baron of Reesville....... $R$

6th Baron of Riverside... - Cox

Baron Rose Bud............. - D

Baron of Rosedale

Baron Siddington $2 \mathrm{~d} . . . . .-$ - Daisy

Baron of Spring Brook - Long Horn

Baron of Sunny Hill ........- - R R

2d Baron of Sunny Hill...... - R R

Baron Taylor.............. - L R

Baron Warlaby ................... Rose

Baron Wild Eyes........ $R$

Baron Zoo_.................. - D

Barrett Gwynne.......... $R$

Barrett Gwynne 1st.........Daisy

Barrington .................... T

Barrington.........

Barrington 1st.........

Barrington 2d..................

Barrington 3d ...............

Barrington 4th

Barrington Airdrie $2 \mathrm{~d}$ 
49961 Barrington Duke......... - - Daisy

49962 Barrington Duke.......... - Ky

49963 Barrington Duke............ - R R

49971 Barrington Prince..................

49972 Barrington Prince........ - Beauty

49975 Barton County Duke......... - M

49976 Bashford $2 \mathrm{~d} . . . . . . . . . .2 \mathrm{R}$

49977 Bashford 3d.......... Ky I

49978 Basil Duke ... R

49979 Bassanio .......... $\mathrm{R} R$

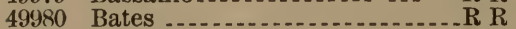

49981 Bates Prince of Brockhurst _ - Cox

49982 Battle Axe 5th............ Ky I

49983 Battlefield ................... D

49984 Baxter.................

49985 Beaconsfield ........ D

49986 Beason 49987 Beatly's Lord Sharon..... R R

49989 Beau of Richland......... - Rolla

49990 Beauty's Airdrie......... _ - L R

49991 Beauty's Baron ............ - P S

49992 Beauty's Duke........... - R R

49993 Beauty's Duke..........

50001 Bedford Lad ............ - R R

50002 Bedrock ................... D

50003 Beecher .................... P S

50004 Beecher............................

50005 Beecher

50006 Beecher of Luray ........... - C

50007 Belina Gwynne.............. M

50008 Bellaire ....................... Ky I

50012 Belle's Beauty................. $\mathrm{T}$

50014 Bell Duke............

50015 Bell Duke........... D

50016 Belle's Duke............ Ky I

50019 Bell Duke................ - L R

50020 Bell Duke......................

50021 Bell Duke............... Rose

50024 Bell Duke Jr.,...........

500255 th Bell Duke of Elkhart......... D

50026 Belle Duke of Flat Rock ...... L R

50027 3d Belle Duke of Maple Hill - R R

50030 Belle Duke of Sugar Grove _ - R R

50031 Belle Duke of Vine Hill ...... - D

50032 Bell Duke of Walnut Hills .. - R R

50034 Bellefield Airdrie 3d......... - R R

50035 Bellefield of Eagle Dale....... - D

50039 Belle's Kirk .................... T

50040 Belle's Kirk $2 \mathrm{~d}$

50041 Bell's Louan .............. - R R

90042 Belmont .................. R R

50043 Relmont .............. R

50044 Belmont Duke 6th

50045 Bell Prince..............

50046 Bell Prince............

50047 Bellerophon................ R R

500515 th Belvedere of Rosemound .- - C

50055 Ben ..................... L R

50056 Ben . ........... R R

50057 Ben

50059 Benny ........

50060 Ben Battle........................

50061 Ben Bolt .............

50062 Ben Butler.......... Cox

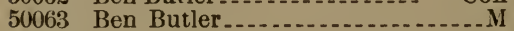

50064 Ben Butier........ B

50065 Ben Butler ..... W

50067 Ben Butler..................

50068 Ben Butler.............. L R

50069
50070

50071

50072

50073

50074

50075

50076

50077

50078

50079

50080

50082

50083

50085

50089

50090

50091

50092

50093

50094

50095

50096

50101

50102

50103

50104

50106

50107

50108

50109

50110

50111

50112

50113

50114

50115

50116

50118

50119

50120

50121

50122

50123

50125

50126

50127

50128

50129

50130

50131

50132

50133

50134

50135

50136

50137

50138

50140

50141

50142

50143

50144

50145

50146

50148

50151

50152

50154

50156

50157
Ben Crofton -............- R R

Ben Franklin

Benjamin of Hazelhurst.....

Ben Hill . . . . .

Ben Hill 1st................. Ky I

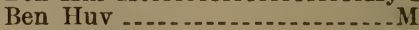

Ben McClure.....

Benonia ..........................

Ben of Pleasant Home.....-P S

Ben Thorndale............ $\mathrm{R} R$

Ben Trimble..............

Benedictine.............. R R

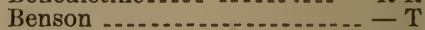

Benton

6th Berthold

9th Berthold

10th Berthold............ - M

11th Berthold .........

Berryman .........

Bertram 7th.......... $R$

Bessie's Lancaster............ Ky I

Bessie's Oenanthe.......... $-R$

Bethlehem...............

Bethlehem ....... R R

Better Times.......

Bettie's Duke... $\mathrm{R} R$

Beulah's Duke 5th...... $R$

Billy .............. P S

Bill Bayless....................... M

Billy Boy ............

Billy Boy ..........

Bill Burriss............ $\mathrm{R}$

Billy Craggs.......... Cox

Billy Crofton ........ Cox

Billy Cruickshank........ - R R

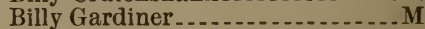

Billy Hanna...........

Billy Harrison.............. - D

Billy Harrison................. Ky I

Bill of Hillside......... $\mathrm{L}$

Bill Hodgkin............ $R$

Billy Malone.............. $-R R$

Bills Muscatoon

Bill Nye ................ P S

Bill Rowe.......... - Long Horn

Billy Watson ........

Bird's Duke.............. Ky I

Bird Wilson ................

Birthday Duke ................. P S

Bishop......................

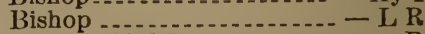

Bishop Airdrie...

Bismarck............

Bismarck ...........

Bismarck........ Long Horn

Bismarck........

Bismarck .......

Bismarck $2 \mathrm{~d}$

Bismarck 2d..............

Bismarck Junior.......... - M

Black Bird Chief............. R

Black Nose ................ $-\mathrm{L} R$

Blaine

Blocky Tom.......................

Bloom's Baron .........

Bloom's Duke......... M

Blossom's Duke....... Cox

Blucher ............. - Long Horn

Blucher Boy............ P 
50158

50159

50160

50161

50162

50163

50164

50169

50170

50171

50172

50173

50176

50177

$501 \% 8$

50180

50181

50182

50183

50184

50190

50191

50192

50193

50195

50196

50198

50199

50200

50201

50202

50203

50205

50206

50208

50210

50214

50215

50216

50221

50222

50223

50225

50227

50228

50230

502233

50234

50235

50236

50237

50238

50240

50242

50243

50244

$50: 46$

50247

50248

50249

50251

50253

50254

50260

50261

50265

50266
Blue Beard

.

Blue Mason _............... - R R

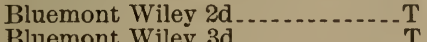

Bluemont Wiley 4th........

Blue Prince............ $\mathrm{R}$ R

Bluff Duke........................

Blythedale Champion...... - L R

Blythedale Champion 6 th $_{-.}-\mathrm{L} \mathrm{R}$

Blythedale Champion 7th .. - L R

Blythedale Champion 8th..- $-\mathrm{L} R$

Blythedale Champion 9th .. - L R

Blythedale Sharon $2 d . . . . .-R ~ R$

Bob .................. R R

Bob ....................

Bob Dixon $2 \mathrm{~d}$

Bob Hunter ...............

Bob Ingersoll

Bob Ingersoll......... Cox

Bob Ingersoll.................

Bob Ridley 2d........ D

Bobs .......................... M

Bobtail............

Bob Walker............ $\mathrm{R}$

Bolivar................. Rolla

Bolivar............... P S

Bonaparte ............. $-R$ R

Bondholder .............. - L R

Bonny Boy ................ L R

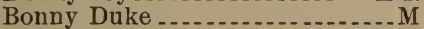

Bonny Lad............. $\mathrm{R} R$

Bonny Lad......................

Bonny Lad................. P S

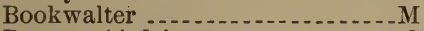

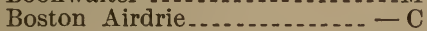

Boston Duke 2d........ Rolla

Boulder Duke.................... P S

Bouncer ............... P S

Bourbon ................... B

Bourbon of Highland...... - Ky I

Boxer .......................... M

Boxwood S............ $R$

Bracelet Duke............ - M

Bracelet's Duke............... - M

Bracelet's Duke............. $R$ R

Bracelet's Duke........... - R R

Bradford.................. $R$

Bradford Duke...........

Breadalbane 12th ............ $-\mathrm{T}$

Breadalbane 13th.............Ky I

Breadalbane 14th ............. R

Breadalbane of Raisin

Breadalbane of Raisin $2 \mathrm{~d} . . . .-\mathrm{M}$

Breastplate 4th..............- - M

Breastplate of Spring Branch - - M

Bride's Baron ............... Rose

Bride's Chevalier............ D

Bridegroom................ $\mathrm{R}$

Bridegroom's Prince...... - Rolla

Brigham Yonng-.......... - L R

Bright Eyes.............. $\mathrm{R} R$

Bright Eye 2d........ Cox

Bright-Eyed Lad........... - M

Bright Red......................

Bristow ...............

Broad Plan.....

Bross ........

Brutus ....... Woods

Brutus..................... Rose
50267

50268

50270

50272

50276

50277

50278

50279

50280

50281

50284

50287

50288

50289

50290

50293

50294

50295

50296

50297

50298

50299

50300

50301

50302

50303

50306

50307

50325

50326

50327

50328

50333

50335

50336

50337

50338

50339

50340

50341

50342

50343

50344

50345

50346

50347

50348

50349

50351

50352

50353

50354

50355

50360

50361

50362

50363

50364

50365

50366

50367

50369

50370

50371

50372

50377

50378

50380

50381

50382
Brutus.................. $\mathrm{R} R$

Brutus .......... P S

Buckalew . -............. Cox

Buckeye Chief $2 \mathrm{~d} . . .$.

Buffalo Bill ...............

Buffalo Jim.......... Ky I

Bull Aleck ................... I I

Burke_....................... Ky I

Burnside................................

Burroughs 2d.................. I

Bushranger Ben........... - R R

Butterfly 2d..................

Butterfly's Lad ......... Rolla

By Blackmarr......... $R$

Byron ............... $R \mathrm{R}$

Cadmus ............. $\mathrm{R}$

Cadmus ................ R

Cæsar ................ Cox

Cæsar............ B

Cæsar...................... R R

Calico Charlie........... $\mathrm{R} R$

Caligula.................. - M

Caligula ...................... $R$

Calvert Duke.......... $R$

Calydon …....... R R

Cambria Boy .......... R R

Cambria's Duke 5th....... - R R

Cambridge ......................

Cambyses.................. R R

Camilla's Prince......... - Cox

Campbell's Duke........... - R R

Campus Duke............. - Rolla

Capt. Berregan..................

Capt. Clem ................

Capt. Cockrill ... ... - Milk Spring

Capt. Collier ..... . . . . . . . . . L R

Capt. Fisher......... R R

Capt. Fred................

Capt. Jack.....

Capt. Jack

Capt. Jack ............ R R

Capt. Jack

Capt. Jack-_..........

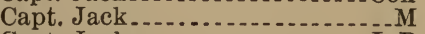

Capt. Jenks..............

Capt. Jinks ................

Capt. Lee.......... Cox

Capt. Lee .............. R R

Capt. Wilford ........

Capt. Nat..............

Capt. Pacy

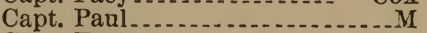

Capt. Taylor................ Rose

Carroll ............................. R

Carroll Wild Eyes.............. R R

Carter Harrison .............

Cash Clay .........

Cashier, $2 \mathrm{~d}$

Cashier, $3 d$.............. $R$

Cashier, 4th............. R

Casket's Thorndale.......... $-R R$

Cass ...................... - Cox

Cassada ...........

Cassius .................. Rolla

Cassius M. Clay.............. R

Cato Duke..........

Cato of Melleary ........ $R$

Cavalier................. $R$

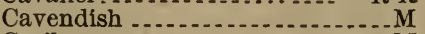

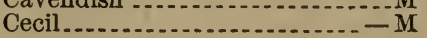




\section{3}

50384

50385

50386

50387

50390

50391

\section{3}

50395

50397

50399

50400

50401

50403

50404

50405

50406

50407

50408

50409

50410

50413

50414

50415

50417

50418

50419

50420

50422

50425

50429

50430

50431

50434

50435

50436

50437

50439

50440

50441

50442

50443

50445

50446

50447

50452

50453

50454

50455

50456

50457

50459

50460

50461

50464

50465

50466

50468

50469

50470

50471

50475

50476

50477.

50479

50480

50481

50482

50483
Cecil D.

Cecil G.

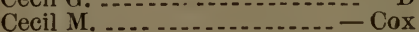

Cedar Boy ....... Cox Cedar's 2d Earl of Sharon. - Rolla Center Boy, 2d ............... - M Centredale Beauty -...... Cetywayo _............... Challenger ....... Champion... Ky. I Champion Champion of Marble Cliff -. $-R R$ Champion Q............. $\mathrm{R} R$ Chap Briant.......... Chaplet Duke Chaplet Duke, $2 \mathrm{~d}$ Charger ..... Charlie.......... Charlie of Hillside............... 1st Champion of Oakdale....- M Chariton Duke........... Charlie Manderson.......... - M Charlie Marquis .......... Charles O'Malley ............ $R$ Charles Sumner........... Cox Charm's Nectar ........... - Rolla Chartiers Duke..... D Chatham Duke Chautauqua Chief.................. Cherry Duke ............ Cherry Oxford $3 \mathrm{~d}$

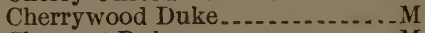
Chestnut Duke ..................... Chevalier 13th Chevalier 14th Chevalier 15th ............ Rolla Chevalier's Goodness ...... $\mathrm{R} R$ Chief ..... Chief Chief's Gem............ Chief of Greene Chief Joseph ........... Chief Ouray Chief of Salem Chieftain ............ Christmas Ranger ........ $R$ Christopher............ S S Christopher Columbus ..... - M

Chuck Chumley . Church's Duke ... Cinderella's Duke ........ $\mathrm{L} R$ Clarence .................. $R$ Clarence $2 \mathrm{~d}$ Clarendon Jr., Claude........ Claude............... Claudius ........ Ky I Claudius......... Claudius Duke........ Clifford ........... Clifton Clifton Duke of Airdrie $2 \mathrm{~d}$ Climax ....... Climax of Vine Hill Clinton $2 d . . . . . .$. Clinton Duke Clinton Duke.
50484 50485 50486

50487

50458

50489

50490

50491

50492

50493

50494

50497

50498

50499

50500

50501

50502

50503

50505

50506

50507

50508

50509

50510

50511

50513

50514

50515

50516

50517

50518

50519

50520

50521

$505 \% 2$

50523

50524

50526

50527

50529

50530

50531

5053:

50533

50535

50536

50537

50540

50542

50544

50545

50546

50547

50549

50551

50552

50553

50554

50555

50556

50557

50558

50566

50569

50570

50571

50572

50573

50576

50577
Clinton Duke .................. M

Clinton Earl

Clio

Clio of Shoal

Clipper..................

Cloud

2d Clover Duke ......... $R$

Clover Lad............... P S

Clyde.............................

Clyde Wonder

Collum ..........

Colonel .

Colonel -...

Colonel

Colonel ........ $\mathrm{D}$

Colonel

Colonel

Colonel Ällen.........................

Colonel Babb .................

Colonel Bates ..........................

Colonel Blanden

Colonel Brady ........... Ky I

Colonel Brice............. Ky I

Colonel Briton......... Cnx

Colonel Cass ......... P S

Colonel Cloyda

Colonel Crisp -........... R R

Colonel Cross

Colonel Dick

Colonel Dollinger.....- Multiflora

Colonel Don .

Colonel Elliot

Colonel Foote Jr.,

Colonel Fulkerson_............ Ky I

Colonel Glen ............

Colonel Harbinger

Colonel Higgins .........

Colonel Holloway......... P S

Colonel Lewis .................... C

Colonel Motte...........................

Colonel Musket..........

Colonel Musket 4th .......

Colonel Pocy ......... Cox

Colonel Scott .

Colonel Shaw

Colonel Smith ............... - C

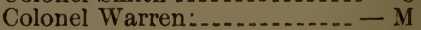

Colorado Boy

Columbus Airdrie............

Columbus Duke.............. R R

Comanche Chief.............. D

Comet

Comet

Comet

Comet .......

Comet

Comet

Comet

Comet

Comet $2 \mathrm{~d}$

Comet 4th

Comfort's Wiley 15th..... - Daisy

Commander Bly ........... R R

Commodore........

Commodore...... Milk Spring

Commodore ...................

Commodore

Commodore V.

Compromise 
50578 Compton Duke..... D 50579 Comus 3d.

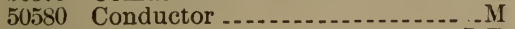

50581 Congor ...................................

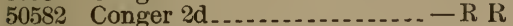

50586 Conrad.............. D

50587 Constance Airdrie 2d

50594 Coral......................................

50599 Cossack 2d............ D

50600 Cotton......... L R

50602 Count Bismarck

50605 Count De Lille

50606 Count Hurat ............. R R

50607 Count Noble............. M

50609 Count Wild Eyes 2d...... - Daisy

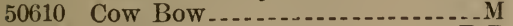

50614 Crocket

50615 Crofford's Duke

50619 Crown Prince.... Rolla

50620 Crown Prince .............. P S

50622 Crown Prince of the West ... _ L R

50623 Crusader ................... Daisy

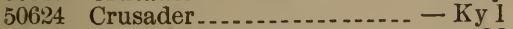

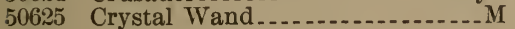

50628

50629

50630

50631

50635

50636

50637

50688

50639

50640

50642

50648

50650

50653

50654

50655

50656

50658

50659

50660

50661

50662

50663

50664

50665

506660

50667

50669

$506 \% 0$

50671

50672

50673

50674

$506 \% 5$

50676

$506 \pi 7$

50680

50681

50682

50684

50685

50687

50688

50690

50693

50696
50697

50698

50699

50700

50702

50704

50705

$50 \% 06$

50707

50709

50 r10

50711

50712

50713

50714

50715

50716

50717

50721

50723

50724

50725

50727

50728

50729

50730

50732

50734

50735

50738

50739

50740

50741

50742

50743

50744

50749

$50 \pi 50$

50751

50752

50757

50759

50760

50761

50763

50765

50766

50767

50768

50769

50771

50773

50774

50775

50776

50777

50778

50779

50781

50783

50784

50785

50786

50787

50788

50789

50790

50792

50794

50796

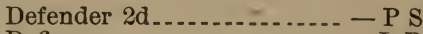

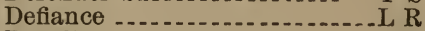

Degoli _............ $\mathrm{R}$

De La Matyr....... Ky I

Denmark...........................

Derby Duke 2d........... D

Derby of Springport.......... Ky I

Deronda ................... $\mathrm{D}$

Derrick ...............

Desmoro........

De Soto

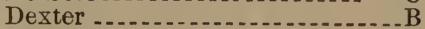

Diadem .................. Cox Diamond

Diamond.........................

Diamond.............. Rolla

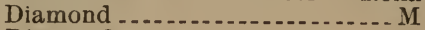

Diamond .......

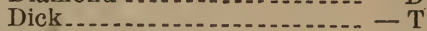

Dick

Dick

Dick Allen ............ - Cox

Dick Gentry

Dick Hnbbard

Dick Johnson ........... P S

Dick Nailer...............

Dick Ramsey ...........

Dick Swiveler ........... - Cox

Dick Taylor $2 \mathrm{~d} . . . . . . . . . . . . . R$ R

Dick Thompson........... P S

Dick Tone_..............

Dick Turpin ......... L R

Dickson's Pride.... - Milk Spring

Dictator . . . . . . . .

Dill .................. $\mathrm{D}$

Dill W. Booth.... R R

Dinah's Oxford 5th...... - Rolla

Dinah's Oxford 6th....... - Rolla

Dinah's Oxford 7th...... - Rolla

Dinah's Oxford 8th..... - Rolla

Diomed.............. $\mathrm{R}$

Dirigo _......... $\mathrm{R} R$

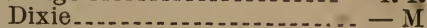

Dixie .................. Ky I

Dixie Duke 4th

Dixon Lad............... - L R

Dixon Mason......... - L R

Doc Black ........

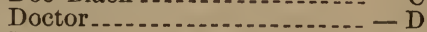

Doctor ................

Doctor $3 \mathrm{~d} . . . . . . . . . .6 y$ I

Doctor Griffith ......................

Doctor Jo .........................

Doctor's Prince.............

Doctor Sharon.......... $\mathrm{L}$

Doctor Sharon 2d........ - L R

Doctor Sharon 3d....... - L R

Doctor Tanner................

Dolly Varden Duke......

Dombey ................. $\mathrm{L} R$

Dominie Samson........... - Cox

Dominion ............... M

Dominion Jr.,

Donald .....................

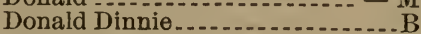

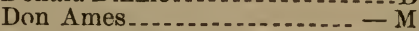

Don Cameron

Don Colson 2d............ - Cox

Don John........................

Don Juan........ 
50797

50799

50800

50801

50802

50803

50804

50805

50806

50807

50808

50809

50810

50811

50812

50813

50814

50815

50816

50817

50818

50820

50821

50822

50823

50824

50825

50828

50829

50838

50840

50841

50842

50843

50844

50845

50846

50847

$508+8$

50850

50851

50852

50853

50854

50855

50856

50859

50861

50862

5086.5

50867

50869

50870

$5087 \%$

50874

50886

50887

50888

50889

50890

50891

50893

50894

50895

50896

50897

50898

50899

50900

50901
Don Mazurka.

- D

Dom Pedro.................

Dom Pedro........ Rolla

Dom Pedro $2 \mathrm{~d}$

Don Piatt.............

Don Quixote........................

Don Thorndale.......... P S

Donald Lewis................. $R$

Dora's Duke...............

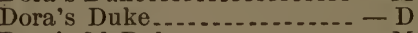

Dora's 2d Duke......................

Dora's Lad.................. - M

Double Breastplate.............. S S

Double Doctor............. II

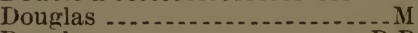

Duuglas ............

Doxander.......... L R

Draco

Duchess Lad...........

Duchess Loudon Duke..... - Cox

Duchess Loudon Duke 2d.... - D

Dude ............................

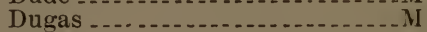

Duke

Duke

Duke

Duke........... II

Duke Adelaide....... P S

Duke Adelaide 2d......... - P S

3d Duke of Alameda........ $\mathrm{C}$

5th Duke of Alameda.......... P S

Duke of Albert Lea.......... M

1st Duke of Alderly ....... $\mathrm{R} \mathrm{R}$ 3d Duke of Alderly ........ $R$ 4th Duke of Alderly ........ - R R Duke Alexander......... - Daisy Duke of Allegheny .............. Cox 3d Duke of Allegheny............. Duke Allen ................. R K 54th Duke of Allendale....... M ith Duke of Altaham......- $R$ R 12th Duke of Altaham........ - M 13th Duke of Altaham...... - M Duke of Alton............ $\mathrm{L} R$ Duke of Altona...... P S Duke Ameleck $2 \mathrm{~d} . . . . . . .$. Duke of Argyll............ 1st Duke of Arrow............ R Duke of Ascot ................ Ky I 15th Duke of Ashland........ M 2d Duke of Astoria.......... $R$ R Duke of Athelstane............. Ky I $2 d$ Duke of Athlone......... Rolla Duke of Athol............ R R Duke of Aylmer......... Duke of Bath 11th.......... Duke of Bath 12th....... - L R Duke of Bath 13th.........- - L R Duke of Bath 14th........ - R R Duke of Beaman........ P S Duke Bedford ............ R Duke of Beloit.......... $R$ 2d Duke of Beloit........... $\mathrm{L} R$ 3d Duke of Beloit........ - L R Duke Bentley ................. 2d Duke of Benton........... Ky I Duke of Berkshire.......... P S Duke of Blackburn Duke of Bloomfield Duke of Bloomfield.
50902

50904

50907

50909

50910

50911

50912

50913

50914

50915

50920

50921

50922

50923

50924

50925

50926

50927

509:S

$509: 29$

50930

50931

50933

50934

50935

50936

50937

50938

50939

50940

50941

$5094:$

50943

50944

50945

50947

50951

50952

50953

50954

50956

$5095 \%$

50958

50963

50964

50965

50967

50970

50972

$509 \% 3$

$509 \div \%$

50978

50979

50980

$509 \times 1$

50982

50984

50985

50986

50997

50992

50993

50995

50996

50997

50998

50999

51000

51001

51003
Duke of the Blossom ............ D

1st Duke of Bluemont.......

Duke Boonsborough........... Ky I

Duke of Breadalbane........... D

Duke of Bridgewater .......... Ky I

1st Duke of Bridgewater...- Ky I

4th Duke of Broadhead........ - M

Duke of Bromley ......

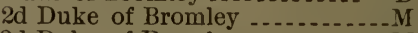

3d Duke of Bromley ........... M

9th Duke of Brownsville.....- R $\mathrm{R}$ 10th Duke of Brownsville... - R R 11th Duke of Brownsville...- $-\mathrm{R} R$ 12th Duke of Brownsville...- - R R 13th Duke of Brownsville...- - R R 7th Duke of Brownwood ........ M Duke of Buena Vista.......- Cos Duke of Buffalo ........... D Duke of Buffalo $2 \mathrm{~d}$ 2d Duke of Caledonia ........ $\mathrm{P}$ S Duke of Calypso .......... $R$ Duke of Cambria.......- Daisy Duke of Carroll ........... Duke of Cass......... Duke of Cedar Croft ...... 2d Duke of Cedar Croft Duke of Center............... Duke of Chagrin 3d Duke of Chartiers ......... - T 2d Duke of Chelmsford Duke of Cherry Creek ......- - Cox Duke of Chestnut ................ Duke of Chestnut Grove .....-D Duke Chesterford ...........- L R 4th Duke of Chieo ............... Duke of Clark.......... Daisy Duke of Clintonville ....... $\mathrm{R} R$ Duke of Cloverdale .......... - M Duke of Clover Meadows...-- L R

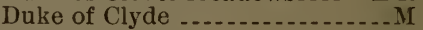
Duke of Columbia...... Duke of Columbus .........-R $R$ Duke of Connaught ........ 4th Duke of Crystal Spring ...Ky I Duke of Cumberland............M Duke of Cummington $\mathrm{Jr}_{.,-}-$Rolla Duke of Derby ....... - Long Horn Duke of Delaware.......... - D Duke of Dunreath ........... - C Duke of Durham 2d Duke of Edgewood...- - Beauty 5 th Duke of Edgearood ........... B 6th Duke of Edgewood ............. Duke of Elba............ Duke of Elden........ Duke of Elkhorn ... M Duke of Elkhorn.......... - Cox 2d Duke of Ellinwood....... - M 3d Duke of Ellinwood.......- i 5th Duke of Ellison......... D Duke of Elmwood........... 3d Duke of Elmwood......... - D Duke of Evergreen ............. P S 2d Duke of Evergreen .......... P S 3d Duke of Evergreen .......... - D 4th Duke of Evergreen ......... P S 5th Duke of Evergreen......- P S 6th Duke of Evergreen......... D Duke of Eylar.................. T Duke of Fairview ......... 
51004

51005

51006

51007

51010

51012

51013

51016

51017

51018

51019

51021

51022

51023

51024

51025

51028

51029

51030

51031

51033

51034

51036

51037

51038

51039

51040

51041

51042

51043

51045

51046

51047

51048

51049

51050

51051

51052

51053

51055

51056

51058

51059

51060

51061

51062

51063

51064

51065

51067

51068

51070

51078

$510 \varangle 1$

51083

51084

51085

51086

51087

51088

51089

51090

51091

51092

51093

51094

51096

51097

51106

51107
Duke of Fairview .......... $\mathrm{R} R$ 4th Duke of Fairview ........ $-M$ 5th Duke of Fairview 13th Duke of Fairview ........ 5th Duke of Fancy Creek ... Ky I 3d Duke of Ferndale......... $-\mathrm{D}$ Duke of Fernleigh......... L R Duke of Flat Rock Duke Fleming ......... R R

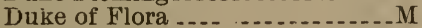
Duke of the Forest........ $-\mathrm{L} \mathrm{R}$ Duke of Forest Shade..... $-\mathrm{R} R$ 2d Duke of Forest Shade ... $-R R$ Duke of the Fountain ..... $-R R$ 1st Duke of the Fountain.... - P S Duke of Franklin .......... D Duke of Gardner ........ Duke of Garfield ............... D Duke of Geauga 3d Duke of German......... Ky I Duke of Ginger Hill......... Duke of Goodness .............. Ky I 58th Duke of Goodness..... - R R Duke of Grandview 1st Duke of Granvilie......... Ky I 2d Duke of Granville.......... Ky I 5th Duke of Granville .......... Ky I 6th Duke of Granville....... Ky I Fth Duke of Granville ......... Ky I 3d Duke of Grasmere........ - R R 1st Duke of Gratiot....... Ky I Duke of Green................ M Duke of Green 2d Duke of Green Hill ............M 2d Duke of Green Hill .......... 3d Duke of Green Hill...... Cox Duke of Greenwood ........ P S Duke of Greenwood....... $-\mathrm{L}$ R Duke of Greenwood........... 2d Duke of Greenwood...... L R Duke of Grindstone........ P S Duke of Guilford ........... Daisy

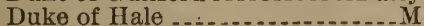
Duke of Halton....... Cox Duke of Hancock ........... P S Duke of Harper................ Duke of Hatfield Duke Hawthorn ............ - M Duke of Hazel Grove ........ Duke of Heosho 2d...... L R Duke of Hickory Grove.... $-\mathrm{L} R$ Duke of Highland......... $\mathrm{D}$ Duke of Hilliard .......... R R 2d Duke of Hinkston ........ 1st Duke of Homestead... - Ky I 2d Duke of Homestead .... - Ky I 3d Duke of Homestead .... - Ky I 4th Duke of Homestead..... - Ky I 5th Duke of Homestead.... - Ky I 6th Duke of Homestead........ Ky I Duke of Honey Creek ........... M 2d Duke of Honey Creek ..... - M 2d Duke of Honsick.......... Cox 2d Duke of Hopewell....... Cox Duke of Hornby ............ $-\mathrm{L}$ R Duke of Howard .......... L R Duke of Indian Creek......... - M Duke of Inghurst.............. Duke of Jefferson $3 \mathrm{~d} \ldots \ldots \ldots-M$ Duke of Jefferson 4 th ......... L R
51108

51109

51111

51112

51113

51114

51115

51116

51120

51121

51122

51128

51129

51130

51132

51133

51134

51135

51136

51137

51139

51140

51141

51142

51143

51144

51145

51146

51147

51148

51149

51151

51152

5 I153

51154

51155

51158

51162

51163

51164

51165

51167

51168

51169

51170

51172

51173

51174

51180

51181

$5118 \%$

51185

51186

51187

51188

51189

51190

51192

51194

51195

51196

51197

51198

51199

51200

51202

51203

51204

51205

51206
Duke of Jefferson 5th ........ - M

Duke of Jefferson $6 \mathrm{th} . \ldots . . . . \mathrm{L} \mathrm{R}$

Duke of Jessamine .......... $-\mathrm{C}$

Duke John . ................. R R

Duke John ................... Ky I

Duke John ................... M

5th Duke John

Duke Johnson ................... M

Duke of Kinnellar..........

Duke of Kinsley ............. - D

Duke of Kirklevington....... $-\mathrm{M}$

2d Duke of Lakeside......... - Cox

Duke $3 d$ of Lamartine ......... - T

Duke of Lancaster........... R R

Duke of Lankford ............

Duke of Laprairie........ - Daisy

Duke of Larchland......... $-\mathrm{R} \mathrm{R}$

Duke Latimer................. Daisy

Duke of Lebanon........... $\mathrm{R} R$

11th Duke of Lebanon..... - R R

Duke of Lexington......... - Rolla

3d Duke of Liberty ............ $-\mathbf{M}$

5th Duke of Liberty ......... - M

8th Duke of Liberty ......... L R

Duke of Lima.............. Duke of Lima $2 \mathrm{~d}$ Duke of Lima 3d............. Duke of Linn Home....... - Ky I Duke of Linnwood .......... Duke of Litehfield........... - L R Duke of Livingstone ........ L R Duke of Locust Grove-Milk Spring Duke of Locust Hill ............ Ky I Duke Logan .................. Duke London .......... R R 2d Duke of Lone Oak........... Ky I Duke of McLean Park..... - Rolla Duke of Madison.......... $\mathrm{R} \mathrm{R}$ 1st Duke of Maple Grove....- L R 2d Duke of Maple Grove.... $-\mathrm{R} R$ 3d Duke of Maple Grove.... - L R Duke of Mapleton 2d ........ - L R Duke of Marble Cliff ....... - R R 2d Duke of Marble Cliff..... Woods Duke of Marion ................ Ky I 13th Duke of Marlboro ......- K R 16th Duke of Marlboro ...... $-\mathrm{R} R$ Duke of Maroa .............. Duke of Meadows.......... $R \mathrm{R}$ Duke of Meadow Brook .... - L R Duke of Meadow Lawn .... $-\mathrm{R} R$ Duke of Mecca Duke of Mecklenburg-Milk Spring 2 D. of Mecklenburg - Milk Spring Duke of Mercer..... - Long Horn Duke of Mesopotamia..........P S Duke of Milford.......... L R Duke of the Mohican..........Ky I Duke of the Monte........ Ky I

Duke of Montrille.......... - D

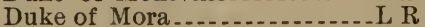

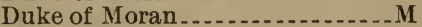

Duke Morris ................ $-\mathrm{D}$

1st Duke of Morrison...... $\mathrm{R} R$ Duke of Pontiac.......... - L R Duke of Nebraska.......... Duke of Nettlewood..........-C Duke of Newark. 3d Duke of Newark 7th Duke of Newark............ Ky I 
51208 6th Duke of New Forest... - Rolla

51211 Duke of North Liberty ....... - M

51212 2d Duke of North Liberty .... - M

51213 Duke of Northumberland......... D

51214 Duke of Norwood............... D

51215 Duke of the Glade......... - D

51217 Duke 1st of Oakdale........ - D

51220 3d Duke of Oakland........ $\mathrm{R} R$

512:1 4th Duke of Oakland...... - R R

51222 Duke of Oakland 6th....... $-\mathrm{D}$

51223 Duke of Oaklawn........ R P

51224 Duke of Oak Ridge.............. P S

51225 Duke of Oak Ridge.......... D

$5122 \tau$ 2d Duke of Oakwood......... - T

51228 3d Duke of Oakwood.........

51229 5th Duke of Oakwood..... - L R

51230 6th Duke of Oakwood...... - L R

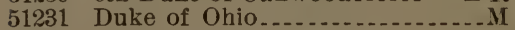

51232 2d Duke of Onondaga...... - Cox

51233 Duke of Ontario......... R R

51234 Duke of Ontario........ Cox

5123.5 Duke of Orange.......... - Cox

51236 Duke of Orange..........

51238 19th Duke of Orleans ....... - Cox

51239 8th Duke of Osage......... - P S

512409 th Duke of Osage......... - P S

51241 10th Duke of Osage...... - Cox

51242 11th Duke of Osage....... - Cox

51243 Duke of Owl Creek .............

51245 Duke of Palmer.......

51246 Duke Peabody .............. Wrong

51250 Duke of Pleasant Hill...... - R R

51251 Duke of Pleasant Ridge...... - M

51252 Duke of Pleasant Valley...... - B

51254 Duke of Ponta........... M

51255 Duke of Portage......... L R

51256 Duke of Portage................ Ky I

51257 Duke of Portage......... - L R

51258 1st Duke of Prairie Lawu... - M

51259 2d Duke of Praire Lawn..... - M

51260 3d Duke of Prairie Lawn... - R P

512615 th Duke of Prairie Lawn......R R

$512622 \mathrm{~d}$ Duke of Raisin ........ $\mathrm{R} R$

51266 Duke of Richland........

51267 2d Duke of Richland....... D

51269 20th Duke of Richland.... - Cox

51271 Duke of Richmond............ - M

$512 \gamma_{2}$ 15th Duke of Ridgevale..... - L R

51273 8th Duke of Rio........... - P S

51274 9th Duke of Rio.......... - P S

51275 10th Duke of Rio......... P S

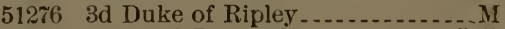

51277 Duke of Riverside.... R

51278 Duke of Riverview.........

$512 \% 9$

51280

51232

51286

51287

$5128 \mathrm{~s}$

51289

51292

51293

51295

51296

51297

51298

51299

51300

51301
51302

51303

51304

51305

51316

51317

51318

51319

51320

51321

$513: 3$

51329

51330

51333

51335

51336

51337

51339

51344

51345

51346

51350

51351

51352

$5135 \% 3$

$5135 \% 6$

51357

51358

51359

51360

51361

51362

51363

51364

51365

51366

51368

51369

$513 \% 0$

$513 \% 1$

51372

51373

$513 \% 4$

51375

$513 \% 6$

$513 \% 7$

$513 \% 9$

51380

51381

51383

51384

51385

51386

51387

51368

51389

51390

51391

51392

51397

51398

51400

51401

51402

51404

51405

51406

51407

51408

51410 1st Duke of Sharon....... - Daisy

Duke of Sharon.......... P S

Duke of Sharon............. $-\mathrm{D}$

Duke Sharon .............. R R

9th Duke of Skara Glen.... $-\mathrm{R}$ R

10th Duke of Skara Glen.......... C

11th Duke of Skara Glen... - R R 12th Duke of Skara Glen.... - R R Duke of Slater............ $-\mathrm{R} R$ 3d Duke of Smith's Grove.... - M Duke of Spottsilvania........ $-\mathrm{C}$ Duke of Spring Hill ....... - Rolla 1st Duke of Spring Valley -. - R R Duke of Stanford ............. M 1st Duke of Stock Place..... - D 13th Duke of Stock Place..- R R Duke of Stoner 2d......... $\mathrm{R}$ R Duke of Summerset....... $-\mathrm{L}$ R 7th Duke of Swansea....... - Cox Duke of Sweetwater.......... Duke of Sycamore............ Daisy Duke of Tama.............. M Duke Taylor............... L R Duke of Thayer............... I I Duke Thornpson.......... - Ky I 10th Duke of Trout Run.... - M Duke of Troy ....... - Long Horn Duke of Troy .............. - L R 1st Duke of Troy......... $\mathrm{L}$ R 2d Duke of Troy Duke of Trumbull............... M Duke of Truro............. P S Duke of the Valley ........... Ky I Duke of Vermillion...... $\mathrm{R} R$ Duke of Vermillion......... - Ky I Duke of Vernon............ Cox Duke Victor................. Duke of Vine Hill......... Cox Duke of Walnut 7 th....... $-\mathrm{L}$ P Duke of Walnut 10th....... - I, R Duke of Walnut Grove.....- R R Duke of Walnut Grove............ M Duke of Walnut Hill....... $\mathrm{L}$ R 2d Duke of Warren....... $\mathrm{R} R$ 4th Duke of Washington.... - T Duke of Washington Creek - - M 3d Duke of Watson ............ Ky I Duke of Waveland........ R R Duke of Wellington....... $-\mathrm{L} \mathrm{R}$ Duke of Wellington........ R 3d Duke of Wellington....... Ky I Duke 4th of IVheatland.......Ky I Duke 1Sth of Wheatland..... - C Duke 20th of Wheatland.... - D Duke Wiley ........ R R Duke of Willow Brook.... $-\mathrm{R} R$ Duke of Willow Park........ $-\mathrm{D}$ Duke of Willow Wood..... - M 6th Duke of Winfield..... Cox Duke of IVoodside........ - Rose Duke of York ................ I I 2d Duke of Iule Grove......... 5 th Duke of Yule Grove........ T 8th Duke of Yule Grove.... - Cox Dumpling . ................. L R

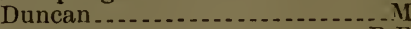
Dunmore Dunmore .............. $\mathrm{R} R$ Durock ................... Duch Wilson.... 
51411 Dynamite.............

51412 Eagle Louan Duke........ - R R

51414 Earl of Airdrie................ Cox

$514153 \mathrm{~d}$ Farl of Allen......... - Cox

514175 th Earl of Allen .......... - Cox

51418 7th Earl of Allen......... - Cox

51419 8th Earl of Allen........... - Cox

51425 Earl Cartwright............. D

51431 Earl Craggs.............. R

51435 Earl of Eagle Dale............. S S

51440 Earl of Goodness......... - Cox

51442 8th Earl of Grass Hill ...... - Cox

51443 Earl of Hampden 2d..... - Rolla

51444 2d Earl of Hanover...... - Beauty

51448 1st Earl of Knox......... D

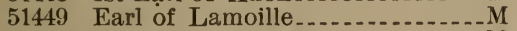

51450 Earl of Lamiolle 2d................ M

51451 Earl of Locust Grove...........

514535 th Earl of Maple Hill........ D

51454 6th Earl of Martin

51455 8th Earl of Martin ...................

51456 9th Earl of Martin ............ Cox

51458 Earl of Mineral Point...... - R R

51462 Earl of Montrose............ - D

51465 Earl of Oakdale........... R R

51467 Earl of Oak Ranche...........L R

51468 2d Earl of Osborn................

51470 Earl of Pickering

51471 12th Earl of Raisin .......... - M

5147.2 13th Earl of Raisin......... - M

$514743 d$ Earl of Richmond......... - D

51475 4th Earl of Richmond....... - D

51476 Earl Rosamond......... R R

51477 Earl of Russell .......... $\mathrm{R} R$

51479 Earl of Shalon

51483 Earl of Sussex................ Ky I

$514 \times 6$ Earl Vane 2d...............

51490 Earl 6th of Wheatland........ - C

51492 Farl of Windsor.......... M

51493 Earl of Wingate

51494 Earl of Winterset........... - M

51498 Easter Prince ............. P S

51499 Eastman ................ P S

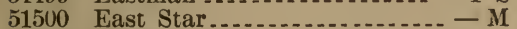

51501 Echo

51502 Eclipse...................

51503 Eclipse

51504 Eetor

51505 Ed

51507 Edgewood Chief ............ - C

51508 Edgewood Clyde.................. M

51509 Edison

51510 Ed Salmon

51511 Ed White........ Cox

51512 Edwin................. M

51513 Edwin Booth ........... R R

1515 Effingham Duke........... Daisy

51518 Eldred 2d .................... M

51519 Ella Vail's Duke........ Ky I

51521 Elegant.................... Cox

51522 Eli Perkins.............. Cox

51524 Ella's Airdrie..............

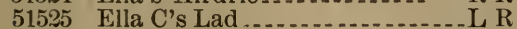

51526 Eilen's Duke................ Daisy

51527 Elm Duke.............. Kv I

51528 Elmer .................... R

51529 Elm Grove Duke...... D

51533 Eminent Commander..... - R R

51534 Emperor ...................... R

51536
51537

51538

51541

51542

51543

51545

51546

51548

51549

51550

51551

51552

51553

51554

51555

51556

51557

51558

51561

51563

51565

51567

51568

51569

$515 \% 0$

51571

$515 \% \%$

$515 \% 4$

$515 \% 6$

$515 \% 7$

$515 \% 8$

515581

$5158 \%$

51583

51584

51585

51586

51587

51588

51589

51592

51598

51599

51600

51601

51602

51608

51609

51610

51611

51613

51615

51616

51617

51618

51619

51620

51621

51622

51625

51626

51628

51629

51630

51631

51632

51634

51635

51636

51637
Emperor -.............. Cox

Emperor-............

Enchanter Clyde................

Enchanter Clyde $2 d$.

Engineer ...................... D

English Nineveh................

Enterprise . ...............

Erastus ...................

Erin's Duke................ B

Ethelbert 2d................

Etta's Duke............ $R$

Eugenie Lad....................

Eula's Muscatoon

Eureka ...................... - M

Eureka

Evelyn's Lad ............ - Daisy

Eventide's Bly _.............. - P S

Excelsior ................ Rolla

Fairfax ............... Ky I

Fairview Duke .........

Fairy Boy ...........

Falconwood ........

Falstaff 4th............... $-\mathrm{L} R$

Fancy's Airdrie............ $-R$ R

Fancy Boy ................ $-\mathbf{M}$

Fancy's Doctor.......... $-R$

Fancy's Duke............ - Ky I

Fanny's Duke ............. - D

Farmer Boy .................... Ky I

Fashion's Doctor..........

Favorite ................. C

Favorite

Favorite 4th ............. - P S

Favorite 5th

Favorite 6th............

Favorite of Sonora........... - D

Fawsley Duke............ Rolla

Fayette Boy ................. L R

Fayette Duke............... R

Fear Not 2d..............

Fencer..............

Fidget's Hillhurst........

Fidget's Hillhurst 2d......- - Cox

Fidget's Oxf'd of Sunnv Hill - R R

2d Fidget's Oxf'd of S. Hill - Rolla

Fidget of Richland....... - Rolla

Financier............. $M$

Fine Style_.......... P S

First Choice

Fitz Favorite............... - M

Fitz Oxford Gwynne....... - Cox

Flora's Duke............. C

Flora's Dnke............. L R

Florence Duke............

Forence's Oxford ......... $-L$

Florida's Duke 9th..... - L R

Forest ................

Forest Airdrie ........

Forester ...................... Ky I

Forest Lad ............... $-R$ R

Forest Lad

Forest Shade Duke......... - R R

Fortune's Duke......... - L R

Fourth Duke........... $-\mathrm{R} R$

Frank

Frank

Frank

Frank

Frank ....................... S

Frank Hunt................ 
, 51638 Frank James ............ - M

51639 Frank Moore

51640 Frank 1st of Pleasant Hill .. - R R

51641 Frank Sharon............ - R R

51642 Franklin ......... R

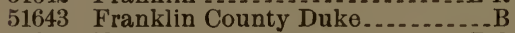

51644 Fred ..................... P S

51645 Fred $\ldots$

51646 Fred......... M

51647 Frederick

51648 Frederick.......... R

51650 Fred Buckhardt.......... D

51651 Fred Gebhardt.......... L R

51652 Freddie Gebhardt............. $-\mathrm{D}$

51653 Fred O'Neal of Sunnyside... - D

51654 Fred Red Jacket............ - R R

51655 Fremont

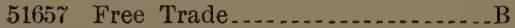

51658 Freya's Boy ..... . . . ..... - Cox

51659 Frontenac

51660 Frosty

51661 Frosty

51663 Gaily's Prince Louan ...... R R

51665 Gallant Duke............. R R

51667 Gallatin Jack.............

51668 Gallatin Roan............ - P S

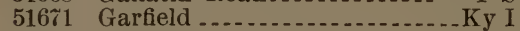

51672 Garfield ................. R R

51673 Garfield

51674 Garfield

51675 Garfield............. Ky I

51676 Garfield

51677 Garfield

51678 Garfield ....................... R

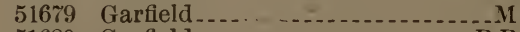

51680 Garfield

51681 Garfield

51682 Garfield

51683 Garabaldi........ R R

51685 Garrison

51686 Garryowen .............. Rolla

51687 Gaston Airdrie ........ P S

51688 Geauga Chief........ S S

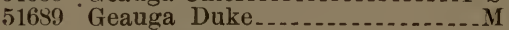

51691 Gem Duke............ L

51694 Gem's Duke 2d

51697 General ..................... R R

51698 General Arthur ......... R R

51699 General Barmpton...................

51701 General Butler.......... - R R

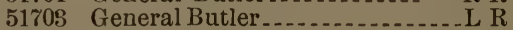

51704 General Butler......

51709 General Combs .......... R R

51710 General Custer.........

51711 General Garfield ........ L R

51712 General Garfield

51715 General Garfield ................. R

51716 General Garfield

51718 General Garfield................ I I

51719 General Gartield......... R R

51720 General Garfield.......... - P S

51721 General Garfield.......... - L R

51722 General Garfield .................

51723 General Garabaldi.................

51724 General Grant................. S

51725 General Grant........... - P S

51726 General Grant...............

51727 General Grant

51728 General Grant 3d........ P S

51730
$51 \% 32$

51733

51734

51735

51736

51737

51738

$517 \pm 0$

51741

51742

51743

51745

51746

51747

51748

51749

51750

51752

51754

51755

51756

51757

51758

51780

$517 \% 2$

$51 \% 80$

51781

51783

51784

51785

51786

51787

51790

51791

51792

51 r93

51794

51795

51796

51799

51803

51804

51805

51806

51807

51809

51810

51811

51812

51813

51814

$5181 \%$

51821

51522

$518: 25$

$518: 29$

51830

51831

51833

51834

51835

51836

51837

51839

51840

51841

51842

51843

51844
General Hancock......... $-\mathrm{R}$ R

General Hancock ............. M

General Hancock

General Jackson .............. I I

General Jackson ............. P S

Gencral Lee................. M

General Lee............... R R

General Logan.

General Logan ............... P S

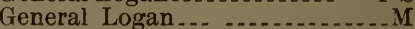

General Logan 4th

General Napier ...........

General Pierce.......................

General Price............... D

General Rose..........

General Scott...... - Long Horn

General Sheridan ........ R R

General Sherman

General Terry ........... L R

General Tyler................

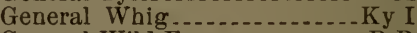

General Wild Eyes........

General Wolsey............... L R

Geneva Lad....................... R

Geneva Richmond.......... Ky I

Gentle Glen.................... Ky I

Gentle Tom

George Chase.

George Dixon

George Gaines.... M

George Gaston ........... M

George Gauger

George of Pleasant Home... P S

George W..... M

George W. McCoy ............ M

George Washington....... L R

George Washington .........

George Washington ....... R R

George Winfield

Gibraltar ............ R

Gladstone Jr.,

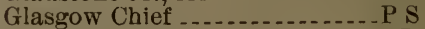

Gledhow Duke

Glendale Lad............ P S

Glen Gary $\ldots \ldots \ldots . . .1 \mathrm{~L}$

Glenwood's Duke....... R R

Glick........... M

Gloster's Hero......... Rolla

Golden Crown ....... D

Golden Crown of Liberty ..... - D

Gold Drop .................. Cox

Gold Drop of Sonora

Gold Dust .............. R

Golden Duke of Sciota

Goldfinder's Duke......... L R

Goliath .....

Governor Cleveland........

Governor Foster......... R R

Governor Gear................ Ky I

Governor Glick........

Governor Glick .......... Ky I

Governor Glick........... P S

Governor Glick ......... R R

Governor Hall ........... Cox

Governor Pattison

Governor Phelps

Governor Porter.......... Ky I

Governor Rusk.....-Long Horn

Governor St. John.............

Governor Sherman 
51845 Governor Sherman .......... - T

51846 Governor Sherman

51847 Governor Vance ....................

51848 Governor Yates............... - M

51850 Gracie's Duke of Woodside... - M

51851 Grand Acklam Boy ............... B

51853 Grand Airdrie 2d................. M

51854 Grand Airdrie $2 \mathrm{~d} . . . . . . . . \mathrm{R}$ R

51857 Grand Duke .............. - L R

51860 Grand Duke ..............

518694 Grand D. of Cherry Wood_- P S

51870 Grand D. of Fountain Farm - Ky I

51876 Grand Duke of Sharon 5th - Daisy

51877 Grand Duke of Washington _- - D

51882 Grand Loudon Duke......... - M

51883 2d Grand Loudon Duke ...... - M

51884 Grand Master............ Wrong

51885 Grand Oxford ............

51887 Granger.........................

51888 Granger

51889 Granger Duke 2d....... M

51891 Grant 2d ............... R

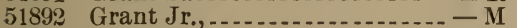

51894 Gray Boy .............. R R

51896 Greenwood

51897 Groom of Greenfield....... - R R

51898 Groton Star.............. - Cox

51899 Groveland Prince............... R

51900 Grundy

51901 Grundy King

51903 Guilford Bell Duke....... - R R

51904 Guilford Royal Lad ........ - R R

51905 Gus..................

51907 Gwynne Duke...... C

51908 Gypsy Chief ............... L R

51910 Hamlet

51913 Hamlin

51914 Hancock.................... D

51915 Hancock......... Long Horn

51916 Hancock ................... - M

51917 Hancock

51918 Handel .............

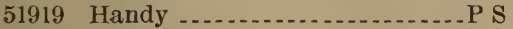

51920 Hank Baker.......... P S

51922 Hankins' Loudon Duke 6th_ - L R

51923 Hannibal ............... - R R

51924 Happy New Year ......... D

51925 Happy Thought ........ Rolla

519:26 Hard Castle...............

519:8 Harmony

51929 Harper

51930 Harrington M. A.

51931 Harrison......... P S

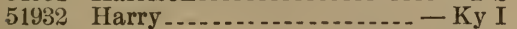

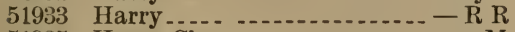

51935 Harry Clay .............

51936 Harry Grant .............. Ky I

51938 Harvey .............. Rolla

51939 Hatfield Friday ........ - Beauty

51940 Hawkeye .......... D

51941 Hawkeye Boy Whitefoot....... M

51943 Hayes ..........................

51944 Hazel Kirk......... D

51945 Hearne ................. $\mathrm{R} R$

51946 Heber........... P S

51947 Hector............. D

51949 Hector

51950 Hector

51953 Hemp Ridge Gem ....... M

51954
51957

51959

51960

51961

51962

51963

51965

51966

51967

51969

51970

$519 \gamma 1$

51972

51973

51974

51977

519 8

51979

51980

51981

51982

51983

51986

51987

51991

51993

51994

51995

51996

51997

51998

52000

52001

52002

52003

52004

52005

52007

52008

52010

52011

52012

52013

52015

52017

52019

52020

52021

52023

52024

52028

52029

52032

52034

52035

52038

52039

52041

52044

52045

52047

52048

52049

52052

52053

52054

52055

52056

52057

52058
Henry - . . . . .

Henry Clay ............. R R

Henry Clay ............ R

Henry Clay $2 \mathrm{~d} . . . . . . . . . . . .-\mathrm{T}$

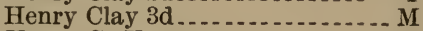

Henry C. Young............

Henry Ward Beecher......... Ky I

Herbert.................. $\mathrm{R} R$

Hercules................. $\mathrm{R} R$

Hercules ..............

Hercules ..........

Herder . . . . . . . .

Hero

Hero

Hero

Herod............. R

Hersher Duke........... Rolla

H. H. Downing ....

High Bank............. - Daisy

Highlander.....................

Highland Duke.......... - Rolla

Highland Duke ...........

Highland Lad ..............

Highland Lad ............. L R

Hilpa Duke 4th

2d Hilpa Duke of Norwood - Rolla

Hilpa Mistletoe......... Rolla

Hiram ..............

Hiram IVeeks

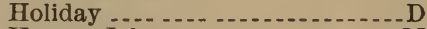

Honest John ....................

Honkey Boy ..... M

Hood

Hooker ................. Cox

Hoosier Boy ........... Ky I Hoosier B'plate of Sp'ng Branch-M

Hope..... Ky I

Hopeful $2 \mathrm{~d} \ldots \ldots . . .2 \mathrm{P} S$

Hopeful Duke..... R R

Hopewell 2d............. R R

Hope's Wild Eyes ......... $-\mathrm{M}$

Horace ......................

Horace Greeley .

Howell Hero..........

Hubbard ............ - Long Horn

Hudson's Hubback...... - Rolla

Hugo ...................... P S

Hugh Logan .........

Hulcie .............

Humbled..... Rose

Hurricane...... M

Hyperion ................ R

Ike

Illustrious 1st of Panora.... $-\mathrm{L}$ R

Imperial ...........

Imperial Duke .

Imperial Duke .......... R R

Imperial Prince........... Ky I

Independence Day ........ $-\mathrm{R} R$

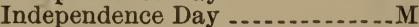

Independent...........

Independent Lad........ $\mathrm{R} R$

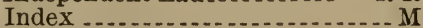

Indian Prince .............. $\mathrm{C}$

Indiana Phyllis ............. Cox

Indicator …............ $\mathrm{R} R$

Indispensable ...............

Indo _.................... T

Ing

Ingomar ........... $\mathrm{R}$ 


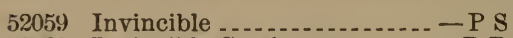
52060 Invincible Goodness ........ $-R$ R 52061 Invincible Henry ............ - Cox 52062 Iowa Boy ...................... 52064 Iowa Chief 52069 1st Irene Duke of Flat Rock - Cox 52070 Ireneus 3d................... R R 52071 Iron Duke... 52072 Iroquois........................ 52073 Isabel's Duke................ - M 52074 Israel Putnam ......... D 52075 Ivanhoe................... Ky I 52077 Jack..................... R R 52078 Jack 52079 Jack Bradley ............... - L R 52080 Jack Downing....................... 5:2081 Jack's Gift.............. 52082 Jack Goudy .............. Ky I 52083 Jack Hearne............... 52084 Jack McNeere................. S S 52085 Jack Richmond ............... - D 52086 Jackson .................. $\mathrm{R} R$ 52087 Jackson .................... 52088 Jackson ................ R R 52089 Jackson 52090 Jackson 52091 Jacob Astor............. - Rolla 52093 Jake ................... R R 52094 Jake............ 52096 James A. G.,............ R R 52097 James Forbis............... $-\mathrm{D}$ 52099 January $14 \ldots \ldots \ldots$

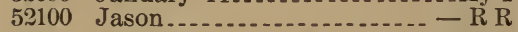
52101 Jason W.,................................. 52102 Jay Cooke......... Ky I 52106 Jay Gould 52108 Jay's Red Jacket.......... D 52110 Jean Valjean............. P S 52111 Jeff $\ldots . . . . . . .60 x$ 5211\% Jeff Davis.......... - Milk Spring 52113 Jeff Davis ................. D 52114 Jennie's Duke.............. L R 52115 Jennie's Oxford............. - R R 52116 Jericho

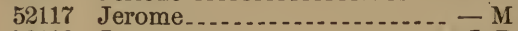
52118 Jerome.................. L R 52119 Jerome Eddy ........................ 52120 Jerry 52122 Jerry Smith....................

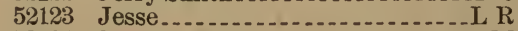
52124 Jessie's Duke.............. - M 52125 Jessie's Grant................ - M 52126 Jesse James.............. R R 52127 Jesse James.................... Ky I 52128 Jewett ........................ Ky I 52130 Jim

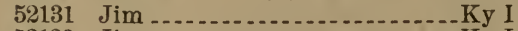
52132 Jim

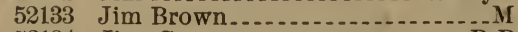
52134 Jim Crow 52135 Jim Dillard 52136 Jim Eakin ................. 52137 Jim Hopkins ................... S S 52138 Jim Keen ...................... P S 52139 Jim Taylor .......... L R 52140 Jim William's............ 52142 Joab.............................. 52143 Jockey Boy 52144 52145
52146 52147 52149 52150 52151 52153 521.4 52157 52159 52160 52162 52164 52165 52167 52169 521\% 52171 52172 52173. 52175 52176 52177 52179 52182 52183 52184 52185 52186 52187 52189 52190 52191 52192 52193 52194

52195

52196

52198

52199

52200

52201

52202

52203

52205

52206

52:208

52209

52210

52211

52212

52213

52214

5:2216

52217

52218

52219

52220

52222

522233

52224

52226

52227

52228

52:229

52231

52232

52234

52:236

52237
Joe

Joe

Joe Älen

Joe B., .......

Joe The Banker.......... - D

Joe Bowers .................. M

Joe Breckenridge.........

Jo Eglentoun .................... S

Joe Gerig ................... $\mathrm{R}$

Joe John ................. - C

Joe Mason...........

Jo Richmond...........

Joe Shelby ................. L

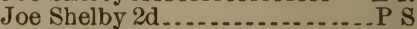

Joe Walters ............... D

John ......................... I

John A...........................

John A.

John H............

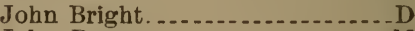

John Brown.......

John Calvin:.............. - M

John Finn .................

John Halifax...........

John Henry

John Hoover . . . . .

John Hurr

John Livar................ $R$

John Morgan..................... R

John R Page............... L R

Johnny Richardson....... - Daisy

Johnnie Sands............ - Ky I

John Sherman............ D

John Smith ........ - Milk Spring

John Stakedaub........... - P S

John Sweed ................ P S

John W ........................

Jonah ................... $-\mathrm{M}$

Jonathan ..............

Jonathan ........

Jonathan ...................... B

Joppa

Joseph................. $\mathrm{R}$

Josephus.................. -

Josephus..................

Josh ...............

Josh Brown....... R R

Joshua ..............

Joshua.................. R

Joslin ............... Cox

Joy Duke......

Jubilee Duke 5th........ R R

Judge ..........................

Judge

Judge $\mathrm{F}$

Judge Royal ..............

Judge Taylor

Julius ........................ M

Julius Lemoyne...........

Jumbo .................... L

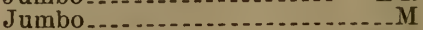

Jumbo

Jumbo

Jumbo .............

Jumbo

Juno Roan............. $\mathrm{D}$

Jupiter .............. Cox

Jupiter ................

Jupiter $2 \mathrm{~d} . . . . . . . . .$.

Jupiter 3d 
52238

52239

52240

52241

52242

52243

52244

52245

52246

52247

52248

52251

52252

52253

52256

5:2:257

52258

$52 \div 259$

52263

52264

$52: 265$

52266

$5: 2267$

52:268

52ว 0

52271

$5: 272$

52274

$522 \% 6$

52277

52278

52:79

52283

52:285

52286

52288

52292

52293

52303

52306

52313

52316

5:2317

523:1

$5: 323$

52326

52328

52329

52331

52335

523:37

52339

52340

52344

52345

52346

52347

52348

52349

52:350

52352

52353

52354

52355

52356

52357

52359

52360

52361

52362
Jupiter 4th

- D

Jupiter 5th............... P S

Jupiter Tth Jupiter Knight of Aylesby .. - R R Justice Lad ............ T Juvenile Lad.......................... J. W. Judy ............. D J. W. Spratley Kalon Kansas Bondholder........ Wrong Kansas Boy ..................... I Kansas Jo Katie's Kirklevington ......... - D Katie's Kirklevington $2 \mathrm{~d} . . . . .-\mathrm{D}$ Kentuck................ $\mathrm{R} R$

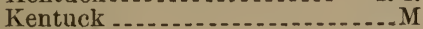
Kentucky -....... Daisy Kentucky -............... D Kentucky Duke......... L R Kentucky Prince.............. R Killpatrick ................... D Kindred............. R R King ................. King Clovis............ King David............. Daisy King Duke........... R King Duke $2 \mathrm{~d}$ King George........- Milk Spring King George................. M King James.. King Laeir................... M King Morris .......... D King Richard $3 \mathrm{~d} . . . . . .$. King of the Roses $2 \mathrm{~d} . . . . . . . \mathrm{Ky}$ I King of The Valley .......... D King of Wayne County ........ - D Kingston Lad............. $R$ R King Athol .................... $-\mathrm{D}$ Kirklevington $\mathrm{D}$ of Oakdale $-\mathrm{R} R$ Kirklevington Lad 3d....... - - R R Knight....................... I

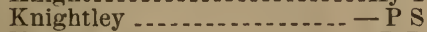
Knightley 1st ............. - R R Knightley Gem .......... Knightley Prince 1st..... - Beauty Knox -..................... Cox Kocosion Chief....... Koehler ........................ Ky I Kosciusko of Melleray ....... - R R Lad ............................... D

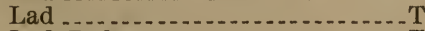
Lad Duke.... Lady's Duke................. Laird ........... Laird $2 \mathrm{~d}$.................. - P S Lake County Star........... M Lancaster.................... R R Lancaster's Junius ......... - M Landlord ........................ Cox Lang's Duke of Kirkl evington - D Langhorn's Airdrie.......... - R R Lant ...................... Larkin Boy ........... Laura's Duke................ Rose Laura's King ................... M Laura's Lad................... M Leba.............. Legal Tender....... LeGrand .............. Rolla

LeGrand
52363 Leon Airdrie......... - Milk Spring 52368 Leon Goodness..... - - Milk Spring

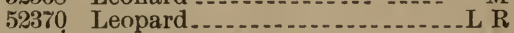
52371 Leopard of Melleray ...... P S 52372 Leroy 52378 Lewis Brentwood 4 th.......... 52379 Lewis Brentwood 5th............ M 5:382 Liberty Duke................. P S

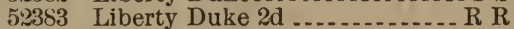
52384 Liberty Duke 3d............... M 52385 1st Lieutenant............. D 52386 Lillie 6th Duke............ 52387 Limbo............. R R

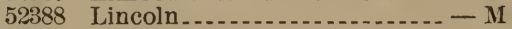
52389 Lindale Duke ............ R R 52392 Lippencott 52393 Lisbon ....... Daisy 52394 Little Ben .......... 5:395 Little Giant............... R R 52396 Little Giant................... 52397 Little John ......... Long Horn 52399 Little Vic .................. L R 52401 Llewellyn Prince 52402 Locomotive .................. D

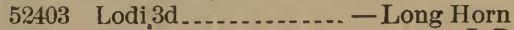

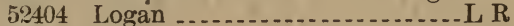
52405 Logan .................. 52406 Logan 5th 52407 Logan 6th

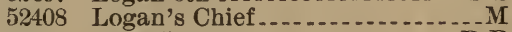
52410 Lone Jack ................ R R 52411 Lone Star ............. R R

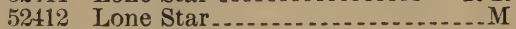
52413 Long Branch................ M 52415 Long John ............... 52416 Lorain's Duke ............ - L R 52422 Lord Albert .............. 52424 Lord Ashton 2d ......... P S 5:425 Lord Austerlitz ............ 52427 Lord Barmpton 52435 Lord Beaconsfield......... - R R 52436 Lord Bickerstaffe 2d...... Rolla 52437 Lord Boleyn............... Ky I 52438 Lord Brampton .... - Milk Spring 52439 Lord Broughton .......... - Cox 52442 I.ord Cavendish $\ldots \ldots \ldots \ldots \ldots$ L R 52446 Lord Clyde 52447 Lord Cornwallis.......... - Cox 52453 Lord Francis................... M 52454 Lord Geneva $2 \mathrm{~d} . . . . . . . . . .-$ Cox

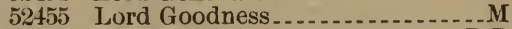
52457 Lord Graham 7th..... R R 52458 Lord Haley .............. L R วั2460 Lord Hester.............. Ky I 52463 Lord Leslie................ - T 52467 Lord Lorne 52470 Lord Mario ............. R R 52473 Lord Matchless 2d ........ Rolla 52474 Lord Mayor of B. L. .... - R K 52475 Lord Michael............... D 52477 Lord Mortimer......... R R 52480 Lord Nelson.............. L R 52481 Lord Nelson... ............. Cox

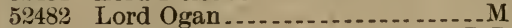
52485 Lord Ragland............. 52486 Lord Raymond ........... - Cox 52491 7th Lord Red Rose......... - M 52494 Lord Renick 
52495 Lord Richmond........... - D

52496 Lord Richmond $2 \mathrm{~d} \ldots \ldots . .$.

52497 Lord Rouald ...........

52498 Lord Roscoe............... D

52500 Lord Sale 2d........... R R

52501 Lord Sharon........ - Milk Spring

52502 2d Lord Sharon.......... - Rolla

52503 Lord Shelburne............. _ T

52504 Lord of The Sleeper

52510 Lord Winchester $3 \mathrm{~d} . . . . . .-\mathrm{L}$ R

52511 Lord York 4th

52512 Lorenzo .................. R R

52513 Lothario .............. R R

52514 Lothian

52515 Lou's Baron .....................

52516 Louan's Duke....... R R

52517 Louan's Duke ........... M

52518

52519

52520

52521

52522

52523

52524

52525

52526

52527

52528

52529

52530

52531

52533

52543

52544

52545

52546

52548

52549

52550

5255:

52553

525.54

52555

52556

52557

52558

52560

52561

52562

52563

52564

52567

52568

52569

52570

52572

52573

$525 \% 9$

52582

52583

52586

52587

52588

52589

52590

52591

52592

52593

52594

52596

Louan's Iuke ....... R R Louan's 3d Duke of Oneida_ $-R R$ Louan Lad 3d............. $\mathrm{R} R$ Louan's Orange Duke...... $-\mathrm{R} R$ Louanjo of Newark ........ Ky I Loudon Jr., ........... R Loudon Beck.............. Daisy Loudon Constance....... Rolla Loudon Constance $2 \mathrm{~d} . . . .-$ Rolla Loudon Duke............ $\mathrm{R}$ R 2d Loudon Duke ............... Loudon Duke $3 \mathrm{~d} \ldots . . . . . . . \mathrm{R} R$ Loudon Duke 17th........ - Cox Loudon Duke 32d....... $\mathrm{R} R$ Loudon Duke of Forest.... $-R R$ 2d Lnudon Duke of Marlboro-R R Louan Duke of Melleray ..... - M Loudon Duke of Willowwood-R R Loudon Lad................ - M Loudon Noxubee........ B Loudon Prince 18th....... - Rolla Loudon Prince of Sp'ng Branch-T Loudon's Star......... R R Louie.............. R R Louis Napoleon............. Loyal Duke............. R R Loyal Duke............. D Loyal Prince............... P S Lucius . Lucknow Lucretius ............... R R Lucy's Princeton $2 \mathrm{~d} . . . . . .-\mathrm{R} R$ Lucy's Princeton $3 \mathrm{~d}$ Luona Duke .............. - M Lycurgus ........ M Lynn Duke .... M Lynn Lancaster. Mack Lancaster...... T Mackinaw McDonough Duke...........-M Madison......................... Mae Glen Duke........... Magenta................ Ky I Mager .................... Ky 1 Magician

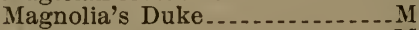
Maid's Duke... M Major Major Major Major.......... Major
52597 52598 52599 52600

52601

52602

52603

52604

52605

52606

52607

52608

52609

52610

52611

52612

52614

52615

52616

52618

$5: 2620$

$526 \% 1$

52622

52626

52627

52628

$526 \% 9$

52630

52631

52632

52634

52635

52636

52637

52638

5:639

52642

52643

52644

52646

52648

52650

52651

52652

52655

52656

5:2657

52661

52662

52666

52667

52669

526\%0

$526 \% 2$

52673

$526 \% 4$

52678

$526 \% 9$

52680

52681

$526 \mathrm{~S} 2$

52683

5:685

$5268 \pi$

52692

52693

52694

52695

52696

52700
Major................... R

Major

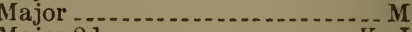

Major $2 \mathrm{~d}$

Major ..................... Cox

Major Barrington ........ $-R$ R

Major Barrington 2d......... $\mathrm{T}$

Major Barrington $3 \mathrm{~d} \ldots \ldots . . . \mathrm{L} \mathrm{R}$

Major Barrington 4th ........ $-\mathrm{T}$

Major Barrington 5th......- L R

Major Bates of Maple Grove - L R

Major Bates of Pine Run... - L R

Major Brown ........... L R

Major Casey ..........

Major Challenger ....... R R

Major Coleman

Major Downing

Major Duke ..............

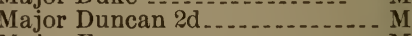

Major Evans ......... M

Major Henry

Major Hill .................. Ky I

Major Hook ........... R R

Major Leslie $2 \mathrm{~d} \ldots . . . . . . . . .-\mathrm{PS}$

Major Ryland ............... T

Major Longfellow ..... Daisy

Major Warfield ......... - Woods

Major Wellington ........ - Rose

Major Wells ............ $\mathrm{R} R$

Major Wilson ......... $\mathrm{D}$

Make Play ........ B

Malaka Duke........... P S

Malcolm............... Rolla

Malcolm Q. ............ R R

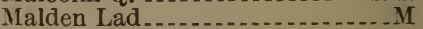

Malo

Mameluke Clyde

Mammoth Spring Duke .... - Cox

Manchester............. L R

Maple ........

Marcus

Maria's Prince........ Rose

Mariner Duke............... Daisy

Marmaduke ........... R

Marquis....... R

Marquis...... $\mathrm{R}$

Marquis $2 \mathrm{~d}$

Marquis of Floyd

2d Marquis of Gleneyre.... $\mathrm{L} R$

Marquis of Lynn

Marquis Maynard.......

Marquis of Walnut Hill.....- M

5 th Narquis of Wilderberg ... - C

th Marquis of Wilderberg. - - L R

8th Marquis of Wilderberg -- - Cox

9th Marquis of Wilderberg -. - Cox

Mars ......... P S

Mars of Melleray...... $\mathrm{R}$

Marshall 4th .......

Marshall Mazurka......... D

Marshall Ney ................ Ky I

Marshall Duke 2d............

Mary's Champion.......... B

Mary's Duke........

Mary's Prince... M

Mary's Prince........

Mary's Son...... M

Mason

Mason Prince of Highland......D 
52701

52702

52704

52705

52706

52708

52711

52712

52713

52714

52715

52717

52719

52721

52722

52724

52725

52726

52728

52729

52730

52731

52732

52733

52735

52736

52737

52738

52739

52742

52743

52744

52745

52750

52757

52760

52761

52762

52763

52764

52765

52766

52767

52772

52773

52774

52775

52776

$527 \%$

$527 \% 8$

52 81

52782

52783

52784

52787

52788

52789

52790

52791

52792

52794

52795

52797

52798

52799

52800

52802

52803

52805

52810
Master Airdrie 6th

Master Airdrie 7th

Master Bridegroom ........ - $\mathrm{R} R$

Master Clover

Master Craggs

Master De Garmo......... D

Master Gains.................. D

Master Gwynne........

Master Hopkins ............

Master Jupiter........... $\mathrm{R} R$

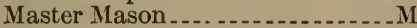

Master Oakland

Master Piatt .......... $R$

Master Rose....................

Master Sharon

Master Taylor $3 \mathrm{~d}$

Master Taylor 4th ......... - P S

Master Taylor 5th......... $-\mathrm{P}$ S

Matchless

Matchless ....................

Matilda's Duke........

Matilda Duke............. $-\mathrm{D}$

Matt Adams................. $R$

Matt Gault

Mattie's Duke.......... R R

Maxwell .................. M

May Bird's Duke......... Cox

May Boy ....................... R

May's Breastplate 2d......- M

May 4th's Duke........... D

May's Lick Duke.................. Rose

Mayflower's Grandson -.....- - L R

Maynard Lee................. - C

Mazurka Beau............. - Rose

Mazurka Duke............ P S

Mazurka King -.............

2d Maz. Lad of Elmw'd- M'k Spr'g

Mazurka's Oxford........... - P S

Mazurka's Oxford 4th...- Ky I

Mazurka's Oxford 35th....- Ky I

Mazurka's Oxford 36th....... - T

Mazurka's Oxford 37th....... - M

Mazurka Prince........... $\mathrm{R} R$

Meadow Duke of Mazomanie _ D

Meadow Lad................ - P S

Meadow Lark............

Medalist 3d

Medea's Dr. Martin

Medora's Oxford ......... $R$

Melbern Duke............. P S

Mendota Lad.............. D

Menelaus ......................

Mentor................ $R$

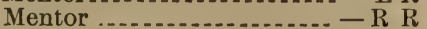

Mercer Chief ....... - Long Horn

Merlin

Merrill Duke......

Merry Monarch.........

Midnight ............ Cox

Mike_.................

Mill Creek Duke........

Miller Duke................ Rolla

Milo _................ R

Milo

Milo

Minden Duke..........

Minnesota ........................

Minnesota Duke........... $-\mathrm{R} R$

Minnie's Airdrie........... $-R$

Minotaur..................-Rose
52811

52812

52817

52818

52819

52820

52821

52822

52823

528:4

52825

52826

52827

52828

52829

52830

52831

52832

5:2833

52835

52836

52837

52838

52839

52841

52842

52843

52844

52845

52847

52848

52849

52850

52852

52853

52854

52855

528556

52858

52859

52860

52861

5:2863

52864

52866

52867

52870

52873

52874

52876

52877

52879

52880

52881

52882

52883

52884

52885

52886

52887

52888

52890

52891

52892

52893

52894

$52 \checkmark 95$

52897

52898

52899
Mion Duke

Miraculous ..........

Mississippi....................M

Model............... R R

Moderator...................

Modoc ..............

Moffitt's Bull............... - D

Mollie's Duke.........

Mollie's Duke............. R

Molly's Duke.......................

Molly's Nero....................

Molly's Oxford

Monarch

Monarch

Monarch _........................

Monarch

Monarch

Monarch

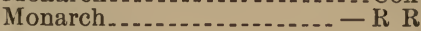

Monarch $2 \mathrm{~d}$

Monarch 7th........... - Rolla

Monarch 8th ............. - Rolla

Monarch 9th .............. L R

2d Monarch of Fountain ..... L R

Monday ................ P S

Moniteau Ravenswood...... - M

Monitor ...............

Monitor...................

Monitor ............. M

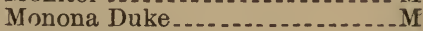

Monroe Boy .................. Ky I

Monroe Breastplate............ T

Mont ... Breastplate.......

Moonlight .........

Moore's Duke............... $\mathrm{R}$

Mordaunt ........................

Moreton Lad...................

Morgan ..............

Morning Star............... R

Morning Star................. P S

Morris Longfellow

Mortimer..................... $\mathrm{R}$

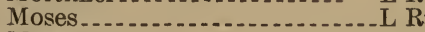

Mossy Face...... - Beauty

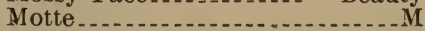

Mozart ............

Mt. Blanchard Duke........ _

Mulino Boss .........

Mulino Duke................

Mundo....................... $R$

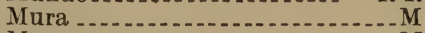

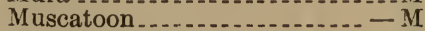

Muscatoon $3 \mathrm{~d}$

Muskegan ................

Muskingum Chlef......... D

Myron Duke 2d......... - M

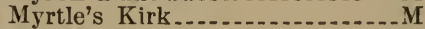

Mystery's Duke ............ Cox

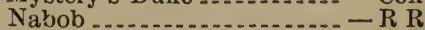

Nail Driver.........

Nannie Lee Duke........ D

Naperville Duke.............. L L R

Napoleon .............. $R$

Napoleon 1st..........

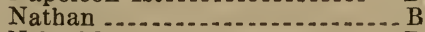

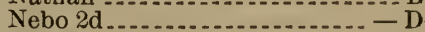

Nebraska

Nectar...........

Nectar Boy .........

Ned........................ 
52901

52902

52903

52905

52906

52907

52909

52910

52911

52913

52914

52917

52918

52920

52921

52922

52923

$529: 24$

52925

52926

52929

52930

52931

52933

52934

52935

52937

52941

52942

52943

52944

$5: 2945$

52946

52948

52949

52950

52951

52958

52959

52960

59961

52962

52963

52964

52966

52967

52968

52970

52971

52973

52974

52975

52977

52980

52982

52983

52987

52993

52996

52999

53000

53001

53002

53003

53004

53006

53007

53008

53011

53012
Nellie's Airdrie .............. - R R

Nelly Bly's Duke.......... -Rolla

Nelly Bly's Duke 2d....... - Rolla

Nellie's Lord ................. - $\mathrm{C}$

Nell's Twin Boy ...................... S

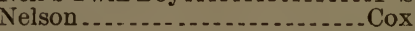

Neptune -....

Neptune ..................... KV I

Neptune

Neptune

Nero ...

Nero

Nerove

Nettie's Duke of Woodside...-M

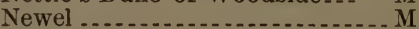

Newsboy

New Year's Star........... Woods

Nicholas .................

Nick

Nicodemus ............... Rolla

Nobby

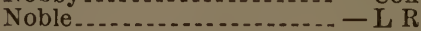

Noble....

Noble Beauty

Nobleman.......... $R$

Nobleman ..........................

Noble Richmond

North Hope

Northern Light .....- - Long Horn

North Star -

North Star ............. P S

North Star of Decorah......-L R

Northumberland ..........- Ky I

Northumberland $3 \mathrm{~d}$

Norton's 5th Baron Lindale...-Ky 1

Norton's 8th Baron Lindale...- - D

Norwich Chief $2 d$.............- D

Oakdale 2d................. $R$

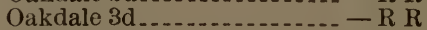

Oakland Boy

Oakland Duke ..............

Oakland Duke

Oakland Duke .......

Oakland Prince ......... R R

Obadiah ........... R

Oceanus $2 \mathrm{~d}$

Octavia Duke......

Odo Russell.................... T

Ohio Boy .... R R

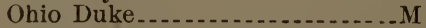

Ohio Granger...........

Old Judge .......

Olie $2 d . . . . . .12$

Oliver

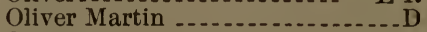

Omaha...........

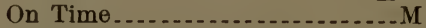

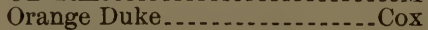

Orean's Duke of Airdrie..........

Orlando

Orpha's Duke............... Ky I

Orpha's $2 d$ Duke ...........

Orphan ................

Orphan's Airdrie ......

Orphan Boy ...............

Orphan's Geneva $2 \mathrm{~d}$

Orphan Lad ............... Ky I

Orthoceres ................... P S

Osage Giedhow

Oscar Wilde..................
53013 Oscar Wilde...............

53015 Oscar Wilde................. MI

53016 Oscar Wilde..................

53017 Oscar Wilde......................

53018 Oscar Wilde............... P S

53019 Oscar Wilde.................

53020 Osceola

$5302^{2}$ Othello .................. Rolla

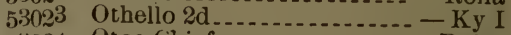

53024 Otoe Chief ............. Daisy

53025 Otoe Chief $2 \mathrm{~d}$

53026 Ottawa Chief ............. R R

53027 Otter Creek Chief ..........- L R

53028 Ouray ........................ P S

53029 Owen Torney ........

53030 Oxford

53031 Oxford

53032 Oxford Beauty .............. Daisy

53033 Oxford of Bellevue....... - L R

53034 Oxford Bly's Blossom...... - P S

53035 Oxford Brant ................... R

53036 Oxford Buckeye............ P S

53038 Oxford Carter............. - Cox

53039 Oxford Clyde .............

53041 Oxford Duke................ Rolla

53042 Oxford Duke.............. - R R

53043 Oxford's Duke.............. L R

53044 Oxford Duke .............. Rolla

5:3047 Oxford Duke ............. Rolla

53049 Oxford Duke of Airdrie.... - Cox

53050 Oxford Duke of Alexis..... - R R

53051 Oxford Duke of Kansas....... - M

53052 Oxford Goodness ...........- Rolla

53053 Oxford's Grand Duke ....... - R R

53054 Oxtord's 6th Graud Duke...- $R \mathrm{R}$

53055 Oxford Hope.............. - L R

53059 Oxford Lad ..................

53064 Oxford Major $2 \mathrm{~d} . . . . . . . . . . .-\mathrm{D}$

53065 Oxford Maynard ............... - D

53070 Oxford Netherby -......... Cox

53072 Oxford Phyllis ............ R R

53074 Oxford Prince...............

$530 \% 6$ Oxford Richmond....... L R

53078 Oxford Rose................ P S

53079 Oxford Sharon $2 \mathrm{~d}$

53080 Oxford of Vinewood ........ $\mathrm{L} R$

53081 Oxford White Rose

53083 Oxtord Young ................ L R

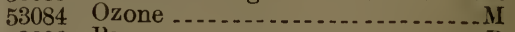

53086 Pan $\ldots . .$.

53087 Pandoro's Republic ......... R R

53088 Panic.......................

53090 Park Duke............ Ky I

53092 Parsons .......... Ky I

53094

53096

53101

53102

53104

53106

53107

53108

53110

53112

53113

53114

53118

53121

53122

53123
Partner

Patrol

Paxton Duke

Peace Maker

Pearl's Airdrie

Pearl Duke 2d.................

Pearl Duke 2d............... - D

Pearl Duke 2d

Pearl Prince $2 \mathrm{~d} . . . . . . . . . . . .$.

Pedro ..................................

Peerless................. $R$

Peleg -............... P S

Peri Duke of Norwood

Perley ................. $\mathrm{R}$

Peter

Peter Cooper 
53125

53127

53129

53131

53132

53133

53134

53135

53140

53143

53146

53147

53151

53152

53153

53154

53156

53157

53158

53159

53160

53161

53162

53163

53164

53165

53166

53168

53171

53174

53175

$531 \% 6$

53177

53180

53181

53182

53183

53187

53188

53190

53191

53192

53193

53196

53197

53198

53199

53:00

53201

53202

53203

$53: 04$

53206

53208

53210

53211

53212

53213

53214

53215

53216

53217

53218

53220

53221

53222

53224

53225

532:26

53227
Peter Cooper ..................

Pete of Pleasant Home......

Peter Right ................. - D

Phil Sheridan

Phil Sheridan ............ R

Phil Sheridan

Phil Sheridan ...

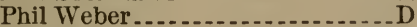

Phyllis Duke ........... Ky I

Phyllis Duke............. - L R

Phyllis Duke of Hamburg .- - L R

Phyllis Duke of Tuberose - Beauty

Pilgrim ........ Ky I

Pilot_...

Pilot_..................... M

Pilot.............. Ky I

Pilot . ... . .

Pine Hill Lad B........

Pine Hill Lad H............. - D

Pine Hill Lad N............ D

Pine Hill Lad Q

Pine Hill Lad R. ............. - D

Pine Hill Lad S. ......... D

Pine Hill Lad T. ........... $\mathrm{R}$ R

Pine Hill Lad V.

Pioneer................. R R

Pioneer . . . .

Pioneer 3d............. Rolla

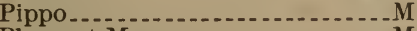

Pleasant Mason

Plumcreek Duke..........

Pluto ...................... $-M$

Polonius .

Pompadour ............. L R

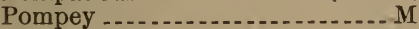

Pompey of Melleray ....... $-\mathrm{R} R$

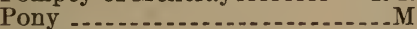

Porsena

Portland Boy ............. $-\mathrm{L} R$

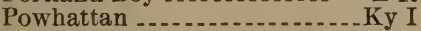

Prairie Duke........ - Multiflora

Prairie Duke.................. D

Prairie Duke........ $R$

Prairie Lad .............. P S

Prairie Prince................ $-\mathrm{C}$

Prairie Thorndale......... $\mathrm{R} R$

Pre-Emption Duke......... - PS

President Clyde.................. M

President Garfield........

Press ...................... R

Press $2 \mathrm{~d}$

Preston Lad.......

Pride's Duke.......

Pride of The Ranch....... $\mathrm{R}$ R

Prince ........................ $\mathrm{T}$

Prince....... Rolla

Prince............

Prince

Prince...... P S

Prince................ Rolla

Prince .......

Prince.................... Cox

Prince.............................

Prince....... L R

Prince..................

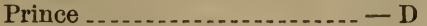

Prince Airdrie............ - Daisy

Prince Airdrie......

Prince Albert ................ - D

Prince Albert
53228

53229

53230

53231

53232

53233

53235

53241

53244

53245

53246

53248

53250

53251

53252

53253

53254

53257

53258

53259

53263

53264

53265

53267

53268

53271

53272

53273

53277

53278

53279

53280

53282

53284

53285

53286

53288

53289

53290

53291

53292

53293

53295

53296

53297

53298

53299

53300

53302

53304

53305

53307

53308

53309

53310

53311

53312

53313

53314

53315

53317

53318

53321

53322

53323

53326

53327

53330

53331

53332
Prince Albert ............ $-\mathrm{L} R$

Prince Albert................. Ky I

Prince Albert................- R R

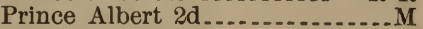

Prince Albert 3d...............M

Prince of Altoona....... Cox

Prince of Arlington....... Ky I

Prince Augustus................. Ky I

Prince Bismarck...........-Cox

Prince Bismarck............ - R R

Prince of Bourbon........ - L R

Prince of Boyle............. - L R

Prince of Bridgeport........ - D

Prince Cadenza......... Rolla

Prince Charlie.............. - L R

Prince Charlie............. - Cox

Prince of Cherry Creek..... - Cox

Prince Comet ....

Prince of Concord......... $\mathrm{R} R$

Prince Creon ............ Cox

Prince Edward ............... Ky I

Prince Edward ................ T

Prince of Eryholme............ Ky I

Prince of Fairmount..._._. - Cox

Prince Filbert.............. - Cox

Prince of Glen Eyre.......... - M

5th Prince of Grass Hill _- - Beauty

6th Prince of Grass Hill_ - Beauty

Prince of Hanover 3d..........Ky I

Prince of Hanover 4th........Ky I

Prince of Hanover 5th......... Ky I

Prince of Hanover 6th........ Ky I

Prince Imperial $2 \mathrm{~d}$

Prince of Jackson 2d.............M

Prince John .............

Prince of Laclede........... - P S

Prince of Lamoille.......................

Prince Leonard 6th...........

Prince Leonard 8th

Prince Leopold................Ky I

Prince of Locust Grove_._Multiflora

Prince of Logan............ - L R

Prince Louanjo.............. R R

Prince of Lune............ - Cox

Prince of Luray.............. Ky I

Prince Malone 4th .......... - Cox

Prince Malone 5th

Prince Malone 6th ........... - Cox

Prince of The Marys ....... - Rolla

Prince Mazurka Rose 2d.......Ky I

Prince Napier ................ - M

Prince Napier 6th.............

Prince Napier 9th....... $R$

Prince of Newton

Prince of Oakland $3 \mathrm{~d} . . . . . . .-\mathrm{P} S$

Prince of Oakland 4th.....- - P S

Prince of Oakland 5th...... - P S

Prince of Orange....... M

Prince Orange................... S S

Prince of Orange.......... - R R

Prince Oxford

Prince Oxford 4th ...........

Prince Regent

Prince of Rio ..............

3d Prince of Riverdale...... $R$ R

Prince Royal 5th........... - Rolla

Prince Rupert............. - R R

Prince Sharon............. $\mathrm{R}$

Prince of Shortman ........ $R$ R

Prince of Spring Hill ........ Cox 
53333 Prince of Sycamore ........ - P S

53334 Princeton

53335 Princeton ............... M

53336 Princeton

53337 Princeton Duke............

53338 Prince of Tupelo........ R

53340 Prince Valentine......... $\mathrm{R} R$

53342 Prince of Wales....................

53343 Prince of Wales...... D

53345 Prince William ..............

53346 Prince of Willowdale....... P S

53350 Princetta's Son .......... Rolla

53351 Printer............... R R

53354 Professor ........... R R

53355 Prohibition........... R R

533.56 Promise.......... R R

53357 Prospect Duke............

53358 Proud Duke................... I

53360 Proud Duke of Bladensburg - P S

53361 Proud D of The Highlands.--Ky I

53363 Proud Gem ................

53366 Pythias

53367 Queen's Counsel

53368

53369

$533 \pi 0$

$533 \% 1$

53373

53374

53375

$533 \pi 6$

53377

53378

53379

53380

53381

53382

53383

53384

53385

53386

53387

53388

53389

53390

53391

53393

53394

53395

53398

53399

53400

53401

53403

53405

53407

53408

53409

53410

58411

53413

53415

53416

53417

53418

53420

52421

53423

53424

53426
Queen's Duke

Quickstep 2d............... - Cox Ragnarok .........

Ralph

Randolph ......

Randolph

Randolph ......... Cox

Ranger ........... $R$ R

Rattler .........

Ray mond

Red - . Rolla

Red Airdrie.........

Red Airdrie Duke.... R R

Red Albert................ Ky I

Red Beauty's Airdrie............. MI

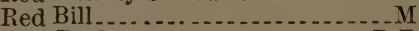
Red Bird............... R

Red Bird .

Red Boy

Red Boy ............

Red Branch.........

Red Buck........

Redbud .......... Cox

Redbud

Red Bull

Red Champion.

Red Charlie................

Red Cheney....... Milk Spring

Red Cloud.................

Red Cloud.................

Red Cloud ............ R R

Red Cloud.........

Red Cloud

Red Cloud......... Cox

Red Comet........ Daisy

Red Diamond...... P S

Red Duke........ R R

Red Duke.............

Red Duke...... R

Red Duke........

Red Duke..............

Red Duke. Duke..... Rolla

Red Duke...........................
Ranger.......

Red Duke.
53428

53429

53430

53432

53434

53435

53436

53437

53438

53439

53440

53441

53442

53443

53444

53445

53446

53447

53448

53449

53450

53451

$5: 3452$

53453

53454

53455

53456

53457

53458

53459

53460

53461

53462

53463

53464

53465

53466

53468

$534 \% 0$

53474

$534 \% 5$

53476

$534 \% 7$

53478

53479

53480

53482

$5: 3483$

$5348 \%$

53488

53489

53490

53491

53492

53493

53495

53496

53499

53500

53501

53502

53503

53504

53505

53506

53509

53510

53511

53512

53513
Red Duke.-................... M

Red Duke.....

Red Duke...... I

Red Duke...............

Red Duke...........

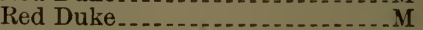

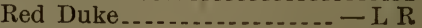

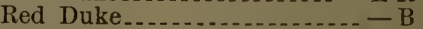

Red Duke................... Ky I

Red Duke ......... Milk Spring

Red Duke...........

Red Duke............ Woods

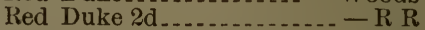

Red Duke of Anoka 3d.......-Cox

Red Duke of Anoka 4th...- $-\mathrm{R} R$

Red Duke of Monticello........Cox

4th Red Duke of Vine Hill...- - M

Red Dunmore............ $R$ R

Red Eagle............. $R$

Red Earl

Redemption

Redfield

Red Gauntlet....................

Red Ranger............ Ky I

Red Harry

Red Jack

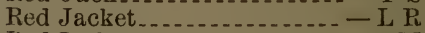

Red Jacket.......................

Red Jacket.................

Red Jacket...........

Red Jacket............................

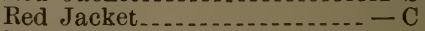

Red Jacket.........

Red Jacket................... Ky I

Red Jacket.................. Ky I

Red Jacket....................

Red Jim ....................

Red Joe

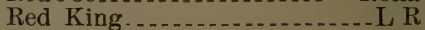

Red Knight of Edgewood..- - Ky I

Red Lad 3d.............. R

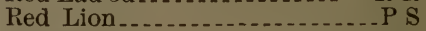

Red Mack

Red IIajor

Red Marquis Mazurka

Red Monarch

Red Oak ............

Red Oxford......

Red Oxford......

Red Pepper......... I

Red Phyllis......... $R$

Red Prince..... P S

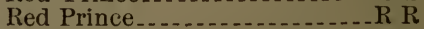

Red Prince.... P S

Red Robin

Red Rose Breastplate....... Woods

Red Rover............... L R

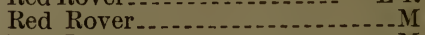

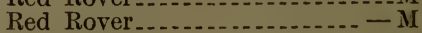

Red Rover.................. Ky I

Red Rover

Red Royal

Red Sam of Bell Creek.... Ky I

Red Sharon ................ Daisy

Red Star

Red Tom

Red Warlaby

Red Wood

Reformer ....................... 
53514 Reformer ............ Cox

53516 Regulator

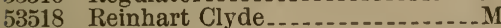

53519 Relda's Duke............ Ky I

53520 Remus ............. Milk Spring

53524 Renick Airdrie...................

53525 Renick Duke............... T

53526 Renick Duke... L R

53533 Resumption............... Cox

53534 Reuben

53535 Reuben.Lowder........... $\mathrm{R} R$

53536 Revenue

53537 Revival

53538 Rex .........................

53539 Rhoda's 2 d Duke.......... - Cox

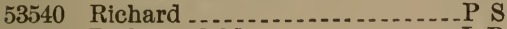

53542 Richmond $2 \mathrm{~d}$

53543 Richard 16th........... R R

53544 Richard 17th............ R R

53545 Richard Sharon

53547 Richland Duke .......... P S

53548 Richmond Lad......... R R

53549 Ridge Farm Prince....... D

53550 Rising Sun......................

53554 Roan Billy

53555 Roan Billy

53556 Roan Chief.... P S

53557 Roan Chief.................

53558

53559

53560

53561

53562

53564

53565

53566

53568

53569

53576

53577

Roan Chief.........

Roan Duke..........

Roan Duke..... M

Roan Duke....... R R

Roan Duke............... R R

Roan Duke.......

Roan Duke........

Roan Duke......................

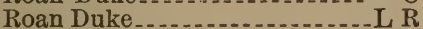

Roan Duke................

Roan Duke of Lynn ........ L R

Roan Duke of Sharon.........Daisy

53580 Roan Henry .............. R R

53581 Roan Henry ................

53583

53584

53585

53586

53587

53588

53589

53590

53591

53592

53593

53594

53595

53596

53597

53598

53599

53600

53602

53603

53604

53605

53606

$5360 \%$

53608

53609

53610

Roan Oxford..............

Roan Prince..........

Roan Prince.....

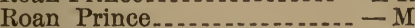

Roan Prince........

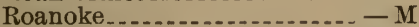

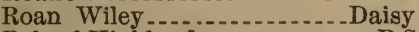

Rob of Highland...P S

Rob Roy

Rob Roy Roy

Rob Roy ..............

Rob Roy .........

Rob Roy

Robert................ R R

Robert Brewer.... R

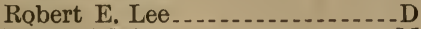

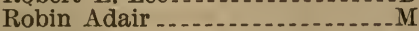

Rocket _..........

Rocket's Chief............ R

Rocket of Marble Cliff ..... R R

Rockingham .....

Roderick............. R

Rock River Lad 60th..... - Cox

Rock River Lad 61st........ - Cox

Rock River Lad 62d........ - Cox

Rock River Lad 63d........ - Cox

53611
53612 Rock River Lad 65th....... - Cox

53613 Rock River Lad 66th....... - Cox

53614 Rock River Lad 67th.....-Cox

53615 Rock River Lad 68th.......... T

53616 Rock River Lad 69th

53617 Rock River Lad r0th....... - Cox

53618 Rock River Lad 71st..............M

53619 Rock River Lad 72d....... - Cox

53620 Rock River Lad 73d........ - Cox

53621 Rock River Lad 74th...... - Cox

53622 Rock River Lad 75th.......-Cox

53624 Rock River Lad 77th....... - Cox

53625 Rock River Lad 78th ...... - Cox

53626 Roderick................ L R

53629 Rolet............

53630 Rolla

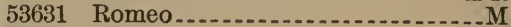

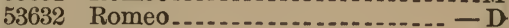

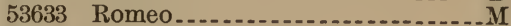

53634 Romeo.........

53635 Romeo................. R R

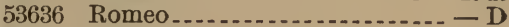

53637 Romeo................... R

53638 Romeo........................... R

53639 Romeo 2d................ L R

53640 Romeo Duke................

53611 Roscoe

53642 Roscoe.................... Ky I

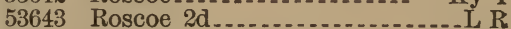

53644 Roscoe C.,................... R

53645 Roscoe Conkling

53646 Roscoe Conkling

53648 Rosa's Baron .................

53649 Rosabella's Duke........ Cox

53650 Rosebud Duke................. C

53652 Rosebud's Oxford........ - Rolla

53653 Rosebud's Rob............ L R

53655 Rosa Cypress Duke........... M

53656 Rosedale Airdrie 2d........... Ky I

53658 Rose Duke................ P S

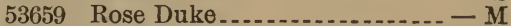

53660 Rose Duke................. P S

53661 Rose Duke................ Daisy

53662 Rose's Duke............... Cox

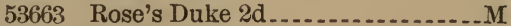

53665 Rosy Duke 2d............ Rolla

53666 Rose Duke 3d............... D

53669 Rosy F. Lee.......

53671 Rose's Kirk ........

53673 Rosy Lad...................... Ky I

53674 Rosy Lee.........................

53675 Rosalie's Prince.......... R R

53678 Rosemary's Constance Duke - Cox

53681 Rosamond's Duke............... Iy I

53682 Rosamond Duke.......... R R

53683 Rose Prince...............

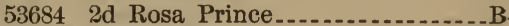

53686 Rosenbaum ...........

53688 Rosicrucian ................ L R

53690 Rover..........................

53691 Rover

53692 Rover

53693 Rover

53696 Rover Duke Jr.,...............

53700 Roy-Clyde

53701 Royal Airdrie............ Rose

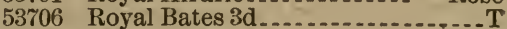

53709 Royal Blossom ............ Rose

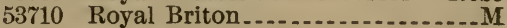

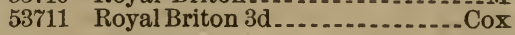


53712 Royal Briton 4th............... Cox 53713 Royal of Cabery .............. Ky I

53717 Royal Commander........... - D

53719 Royal Dave.................

53720 Royal Duke.............. - Daisy

53721 Royal Duke.........................

53722 Royal Duke................. - D

53724 Royal Duke.................. - D

53725 Royal Duke ...................

53726 Royal Duke.............. Ky I

53730 Royal D. of Bloomfield Jr., .. - R K

53731 Royal Duke John 3d......... - L R

53734 Royal Exchange................. D

53736 Royal George

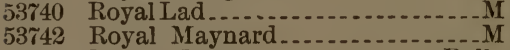

53743 Royal Nectar............. - Rolla

53745 Royal Oxford............ Ky I

53747 Royal Prince...............

53749 2d Royal Red Rose........ - L R

$53 \% 58$ Royal Wiley 2d............. $-\mathrm{R} R$

53759 Ruby's Chief...............

53760 Ruby's Duke 3d............. R

53761 Ruby's Duke of Fairview 2d....P S

$53 \% 62$ Ruby Prince................. - R R

$53 \pi 63$ Ruby's Sharou.............

53764 Rufus ...............................

$53 \% 65$ Rupert

53766 Rural Prince.....

53767 Rustic

53768 Ruthsburg Laddie............ M

53769 Sabinus ............

53770 Sachem $2 \mathrm{~d}$

$537 \% 1$ St. Clair ................... - P S

53773 St. Elmo 6th ...........................

53\%74 St. James.................. Cox

53775 St. John

537\%6 St. John

53778 St. Luke........

53779 St. Patrick.

53780 St. Patrick ..................

53781 St. Valentine

53782 Sallie's Lad.................. - P S

53783 Sam ..........................

53784 Sam

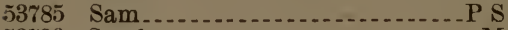

53786 Sambo

53787 Sambo

53788 Sam Cox......................

53789 Sam Gwynne.........

53790 Sam Hunter............... P S

53792 Sam Knightley

53793 Sam Marshall ............... - Rose

53794 Sam Patch ................. R

53795 Sam Patch............

53796 Sam Patch

53797 Samson

53798 Sam Tate.................

53800 Sam Tilden................ Daisy

53801 Sam Weller............... $\mathrm{L}_{\mathrm{R}}^{\mathrm{R}}$

53803 Sanford.....................

53805 San Martin..........

53807 Santa Anna....... T

53808 Santa Claus.........

53809 Saucy Boy 6th.........

53810 Scarlet Duke................ - R R

53812 Scipio _.................... M

53813 Scott

53815 Scottswood Duke......... R R

53816
53817

53818

53819

53820

53821

53823

53824

53825

53826

53828

$538: 29$

53830

53831

53832

53833

53836

$5383 \pi$

53839

53842

53843

53845

53851

53855

53858 .

53866

53868

53869

53874

53876

53877

53879

53880

53882

53886

53887

53888

53889

53890

53893

53894

53895

53896

53897

53898

53899

53900

53901

53902

53904

53905

53906

53907

53910

53911

53912

53913

53914

53915

53918

53919

53920

53922

53923

53924

53926

53927

$5392 \mathrm{~s}$

53929

53930

53931
Scrabbler $\ldots \ldots \ldots \ldots$

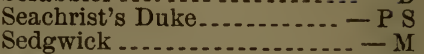

Selah's Duke.............

Selden Dake..............

Senator..................

Senator .................

Senator

Senator ............

Senator

Senator

Senator A.,

Senator Clark ............ Cox

Seraph's Duke $2 \mathrm{~d}$............. - Cox

Seraph's Duke 3d...........-Cox

Seward Corporal .................. R R

Seymour .................

Seymour

Shakespeare............

Shakspeare.........

Sharon .......................

Sharon Boy

Sharon Chips

Sharon Duke $2 \mathrm{~d} . . . . . . . . . . .$.

Sharon Duke of Kansas...-- R R

4th Sharon Duke of Oakdale.. - II

Sharon Duke of Oakwood..... - B

Sharon of Greenfield.......-Rolla

Sharon Knightley ... - Milk Spring Sharon Lad................. - D Sharon of Maple Hill........ - D Sharon of Marble Cliff......-R R Sharon of Riverside ........ $R$ R Sharonius..................

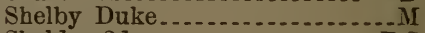
Sheldon 2d . Sheldon $2 \mathrm{~d}$ Sheldon's Chief............. $R$ R Sheridan ................ Ky I Sheriff . . Sheriff................. Sheriff Cooper...... Sherman ............... R R Sherman ...... Sherman

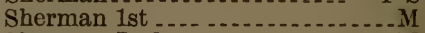

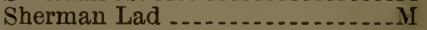
Shiner

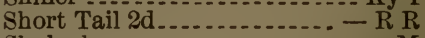
Shylock.

Shylock ..................... M

Sidney P...... Milk Spring

Silver Drip

Silver Duke.........

Silver Horn ........ Milk Spring

Silver Lake Duke ............. - M

Silver Star. .

Simpson.......... P S

Sir Alfred... Rose

Sir Arthur. Daisy

Sir Charles ..............

Sir Charles ....... R R

Sir Claudius....... Daisy

Sir Edward ................... D

Sir Edward .......

Sir Edwin

Sir Garnet Wolsley ....... Cox

Sir George 2d.....................

Sir Henry ....................... 
53932 Sir Henry of Empire 3d _....._ - D

53933 Sir Herman ..............- $\mathrm{L}$ R 53938 Sir Manti....................- R R

53940 Sir Masher ....................... B

53941 Sir Piercie ........................ D

53942 Sir Renick

53944 Sir Thomas..................-L R

53945 Sir Thomas ................... D

53947 Sir Weatherspoon

53948 Sir Williams

539491/2 Smith's Nobleman............. M

53950 Snowball

5395 I Snowbank

53952 Snowflake.................. - P S

53953 Sodom Duke...............- L R

53954 Soggy

53955 Sol_....................... P S

53956 Solicitor

5395̃7 Sonora Duke................ - D

53959 Sooy

53961 Sparswood Duke............ - R R

53962 Spartacus.................... - L R

53963 Speculation.................... Cox

53966 Splendor...................... Ky I

53967 Spotted Boy

53968 Spotted Duke ................. - D

53969 Spotted Jim ......................

53970 Spotted Jim ....................- - D

53971 Spotted King -................... P S

53972 Springdale Airdrie $3 \mathrm{~d} . . . . . .-\mathrm{R} R$

53973 Springdale Boy .................. M

53974 Springdale Duke ............- $\mathrm{R} R$

53975 Springfield $2 \mathrm{~d}$...............- $-\mathrm{R} \mathrm{R}$

$539 \pi 6$ Springfield Ward $2 \mathrm{~d} . . . . . . . . .-\mathrm{C}$

53977 Spring Hill Duke

53978 Spring Hill Oneida ..........- $\mathrm{R} \mathrm{R}$

53980 Springwood................. - L R

53981 Squire Boon

53982 Standard

53984 Stapleton Duke .............-_ - M

53985 Star........................... T

53986 Star

53987 Stark .....................-Cox

53989 Star City Duke $2 \mathrm{~d} . . . . . . . . . .-$ M

53991 Star Duke ..................... M

53993 Star Duke

53994 Starlight_...................- - R R

53995 Starlight....................... Ky I

53996 Starlight Duke............. - - L R

53997 Starling ...................... Ky I

53998 Star's Oxford.-.......... R R

53999 Star of Rock Creek............ - M

54001 Star of the West................ R R

54002 Star of the West............- Cox

54003 Statesman .................... Ky I

54005 Statesman $2 \mathrm{~d}-. . . . . . . . . . . . . . .-M$

54006 Staver -...................-Cox

54007 Stem Winder................. Ky I

54008 Stentor -...................-Rolla

54009 Sterling Duke.............. L R

54010 Stockdale Duke....................

54011 Stockholm ...................- T

54012 4th Stockland Prince-....-- R R

54013 Stockton's Glory ............... - M

54014 Stonewall ...................- Ky I

54015 Stonewall Jackson .......... - R R

54016 Straight Horn .............- Ky I

54017 Strathmore - ............... - R R

54020 Sua's Oxford -................. - D

54021 Sucker Boy
54022

54024

54028

54029

54030

54031

54032

54033

54034

54036

54037

54038

54040

54041

54043

54045

54046

54047

54048

54050

54051

54052

54053

54057

54058

54059

54060

54061

54062

54065

54066

54067

54070

54071

54074

54075

54076

54077

54078

54080

54081

54083

54086

54088

54089

54090

54092

54093

54094

54096

54097

54098

54099

54100

54101

54103

54104

54105

54106

54108

54109

54110

54111

54112

54113

54114

54115

54116

54120

54121
Suda's Duke............... $-R$ R

Sultan ............. P S

Superior ...........

Superior Briton .......... $\mathrm{R} R$

Surprise........................

Sutherland Prince.......... - D

Sutherland Prince $2 \mathrm{~d}$

Swandown ....................

Swansea Premium

Sweepstakes................ D

Sweepstakes Louanjo ........ Ky I

Sycamore Prince ............ - T

Syracuse.................

Tally Duke................ - M

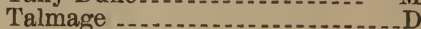

Tam O'Shanter

Tam O'Shanter .............

Tama Jim..................

Tamerlane..................... Cox

Tarquin.....................

Tarquin

Tarquinius ............. Cox

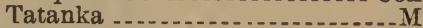

Teaser.........

Tabo .................... T

Teeswater ........ T

Tempest..........

Tepin

Terre Haute Prince

Thanksgiving Day

The Cardinal ........... $\mathrm{R} R$

The Colonel .......... $\mathrm{R}$

The Earl _.............

The Judge ........... $R$

The Scotchman

Thermometer .......... Cox

Theseus ..........

Theseus $2 \mathrm{~d}$.................

Thomas Walker............. - D

Thorndale D. of Goodness..- $-R R$

Thorndale D. of Mason....... Rose

Thorndale Newton ......... $R$ R

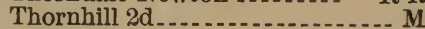

Thrasher .......................

Tilden ...................... Ky I

Tilden.........

Titus_.............. P S

Titus ................... $\mathrm{L}$

Tobeck..........................

Tom

Tommy

Tommie B. .......

Tom Barnes............. - L R

Tommy Bates............

Tomboy ................. P S

Tom Barns $2 \mathrm{~d} . . . . . . . .8 \mathrm{~S}$

Tom Boston ........... D

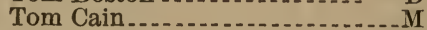

Tom Corwin .............. R R

Tom Crowder

Tom Hendricks ......... - R R

Tom Miller ..........

Tom Moore

Tom Scott .........

Tom Smith

Tom Smith 2d ........... - Rolla

Tom Taylor.............

Tom Wallace............ P S

Trimble Duke............ Ky I

Tristram Shandy 2d......- Rose 
54122

54123

54124

54125

54126

54127

54128

54130

54131

54134

54135

54136

54137

54139

54140

54141

5ั4142

54144

54146

54147

54148

54149

54150

54151

54152

54155

54157

54162

54163

54164

54165

54167

54168

54171

54173

$5417 \%$

54178

54179

54180

54181

54182

54185

54192

54194

54195

54197

54198

54199

54200

54201

54203

54206

$5420 \%$

54208

54209

54210

54212

54215

54216

54217

54218

54219

54220

54221

54222

54223

54224

54225

54226

54228
Troilus Trojan Trubadour .......... P S Troy's Boy True Blue ............. Trumbull .................. $\mathrm{R} R$ Trustee ............. R R Tup ..................... $\mathrm{S}$ Turck ................ Twin Brother. Twin D. of Locust Grove...... C Tyndall ..................... R Tyrone $2 \mathrm{~d}$ Umpire, Duke of Airdrie..... $-\mathrm{B}$ Uncle Dan........................ I Uncle Rufus...................... S Underedge Duke........... University .................. P S Uric ........... R

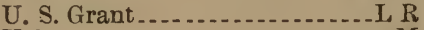
Val .......... Valentine................... Valley Duke................ Valley Duke ................ Valley King................. Van Vanderbilt.................... P S Vellum Oxford's Prince.. - Beauty Vendee....................... Ky I Verbena Duke........... L R Vermillion Boy............... Ky I Vesper Lad................... $-\mathrm{C}$ Vesper Star................ Vic . . . Victor Victor ...... L R Victor $2 \mathrm{~d}$ Victor Haley..................... I Victor Hugo............... P S Victoria's Duke.................. Ky I Victoria Duke................... Victoria's Poppy Vincent............ R R Vinton Violante's Duke........ $\mathrm{L}$ Virgie's Duke............. R R Virgie's Duke $2 \mathrm{~d} . \ldots . . . . .-R ~ R$ Virgil _... R R Virginia Duke.......... $R$ Virginius................. L R

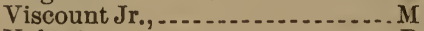
Volunteer................ D Vulcan ...................... S S Vulcan Vulcan 2d..... R R Wabash $2 \mathrm{~d} \ldots \ldots-\mathrm{L} R$ Waddle .................... Cox Wade Hampton Jr., Waid's Nectar. Wakeman Clyde ................... Waldo......... Walker Walker................ Ky I Wallace..................... Wallace Walnut Lad... R R Walter Scott. Warder Warfield .......... R R Warlaby ............ R R
54230

54231

54332

54233

54239

54241

54242

54245

54250

54255

54256

54257

54261

54263

54268

54270

54271

54272

54273

54275

54279

5ั4280

54281

54283

54284

54285

54286

54287

54288

54289

54290

54291

$5429 ?$

54294

54295

54296

54297

54298

54299

54301

54303

54307

54311

54314

54316

54317

54318

54321

54322

54323

54330

54331

54332

54333

54335

54336

54340

54341

54342

54345

54346

54347

54349

54350

54351

54354

54355

54356

54360

54361
Wash Henderson ............. - MI

Washington

Washington $2 \mathrm{~d}$

Washington $3 \mathrm{~d}$

Waterford ...................... Cox

Waterloo $3 \mathrm{~d}$

Waterloo 4th

Waterloo Bell Duke $3 \mathrm{~d}$

Waterloo Geneva........... - R R

Waterloo of Racoon Valley _ - $R R$ 4th Waterloo of Racoon Valley - Ky I 9th Waterloo of Racoon Valley_Ky I Wauseon Waveland Lad......... Webster ................... $M$ Wellington Wellington................ - L R Wellington.............. Cox Wellington Boy................... S Western Boy .............. - D Western Duke.............. - M West Grove Lad.............. Westriew Colonel............. Ky I Whalebone White Cloud White Cloud....... Milk Spring White Creek White Oak Chief White Prince ................. - I White Stockings.......... White Stockings............... C White Stockings Bull.........- $\mathbf{M}$ White Stockings........... Ky I Whitewater Chief............. - C Whitewater Duke............ L R Wide West_.................. $-\mathrm{D}$ Wiggins ............ T Wilber Wild Australian...... Cox Wild Boy ........................ S Wild Eyes................. - M Wild Eyes Duke......... Ky I 4th Wild Eyes of Armada.. - Cox Wild Eyes Prince.......... - Rolla Wild Eyes Prince........... — Rose Wild Eyes Victor.......... - L R

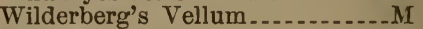

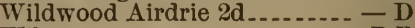
Wiley ...................... $\mathrm{R} R$ Wiley Wiley Duke of Hustondale $2 \mathrm{~d}-\mathrm{M}$ Wiley Duke of Hustondale $3 d-M$ Wiley Duke of Hustondale 4th .- D Wiley Favorite .............. - M Wiley Oxford $3 \mathrm{~d} . . .$. Wiley Oxford 5th William Chestnut......... William Duke................ - M William $\mathrm{H}$. Neece.......... - P S Willowwood Favorite........... C

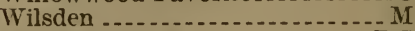
Winfield's Duke..... Windsor Lad ................. - D Winnebago Chief

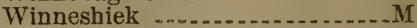
Wisconsin Duke........ Wonderful Lad................... M Woodburn .............. R R Woodhill Prince........... - Rolla Wood Jack................ — Ky I 
54363 Woodland Duke......... W W. P.,....... L R Wrangler ............... - Cox Wye Comet................ - R R Wyoming Wyoming Excelsides ............. Yankee Boy ..................... Ky I Yorkshire Lad............ - R R Young America............... - M Young Bismarck.......... C Young Byron............. $\mathrm{R} R$ Young Cæesar .................Ky I Young Challenger..................M Young Chatham ............. - D Young Councillor...............M Young Doctor ................ R R Young Duke............ D Young Duke............................. Young Englishman Young Fannie's Duke........ - M Young Governor............. - M Young Hazlewood.............. L R Young Hero . . ............... $-R$ Young Henry ............. - P S Young Hero Young Irishman............. - M Young Loudon Duke...... - R R Young Lyndale .............. $-\mathrm{C}$ Young Major......... R R Young Marquis........... - Ky I Young Mary's 3d Baron .... - - R R Young Mazurka Oxford........... Young Mentor ............ R R Young Oxford Young Oxford $2 \mathrm{~d} . . . . . . . . . . \mathrm{D}$ Young Phenom .................. Young Prince Young Richard ............ - R R Young Rocket ............ - R R Young Sharon............. Young Thorndale.......... - Cox Zack Taylor ........................ Zagony Zaphrentis.............. Zenas.......................... Zerlina's Knight.......... - Rolla Zimlich ........................ T Zoar .......................... D

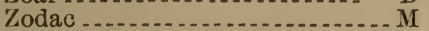
Zoe's Duke of Winfield....... R Zora's Airdrie ................ Rose Zora's Mazurka Duke.........Rose Abe .......................... M Abe Abe Abelino Gonzalez .......... - M Aberdeen 2d ................. Abe Renick ............... $\mathrm{R} R$ Abigail's Duke .......... Abigail Duke 4th ......... $R$ Accident ............. Acco of Turkey Run ........ - C Aclam Boy ................... D Acme Barmpton 1st ...... - Daisy Acme Barmpton 2d....... - Daisy Acme Barmpton 3d....... - Daisy Acme Barmpton 4th ........ - L R Acme Barmpton 5th.......- Daisy Adah's Duke.............. - Ky I
54455

54456

54457

54458

54459

54461

54462

54467

54469

54474

54477

54478

54480

5448 ?

54483

54484

54490

54491

54493

54496

54497

54500

54501

54502

54507

54508

54512

54513

54516

54517

54518

54519

54520

54521

54522

54523

54524

54525

54527

54528

54529

54530

54531

54535

54537

54538

54533

54540

54541.

54542

54543

54544

54545

54546

54548

54560

54561

54562

54563

54564

54565

54566

54567

54568

54569

54572

54573

$545 \% 4$

54576

54577
Addie's Pride.................... Ky I Adjutant............ Ky I Admiral ......... R Admiral ............... P S Admiral............... Daisy Agate's Duke ............. - R R Agitator ............. Beauty Airdrie ............. R R Airdrie of Alder Creek ..... - Cox Airdrie Boy .................. - M 2d Airdrie Duke .......... - R R Airdrie D. of Brownsville .. - R R Airdrie D. of Elmwood 2d ..... - B Airdrie Duke of Oxford ...- - Rolla 6th Airdrie D. of. Sandusky .... L R Airảrie Duke of Wayne... - Rolla Airdrie Hillhurst ............. - R R Airdrie Lad............... - R R Airdrie of Marsh Spring ......Airdrie Oxford ............ - Rolla Airdrie Peabody 2d.............. M Airdrie Royal .............. R R Airdrie of Saline $2 \mathrm{~d} . . . . . . . .-\mathrm{P} S$ Airdrie Sharon.............. $-R$ R Airdrie 2d of Springdale ...... - M Airdrie Sutherland ........... - M A jax.............. Albert 4th...........

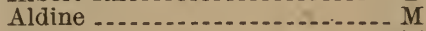

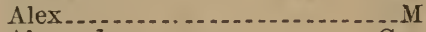
Alexander-................... Cox Alexander Kendall.......... P S Alfonso $2 d$............... $R$ Alfred ................ Alice Duke 1st ............ Alla's Bull .................

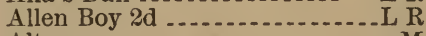
Altum ............................ Alvin G................... L Alwood ............... T Ambros............ Ky I Amelia's Lad.............. America .............. Ancona .................... $R$ Andy Day ................. Ky I Andy Johnson ............. D Angie's Duke.............. Daisy Annabella's Duke......... - Rolla Annabella's 3d Duke..... - Rolla Annas Prince............. L R Annetta's Duke............ Annie's Cherub........... Ky I Anteros .................... Anthony....................... Ky I Appanoose Chieftain ...... - Ky I April Duke.................. - Cox April Fool ...................... D Arabella's $2 \mathrm{~d}$ Booth........ Arad........................ Arbiter..................... $R$

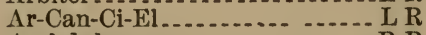
Archduke................. $\mathrm{R}$ Arch Duke of Ivy .......... $R$ Arch Duke of Oakdale........ M Arlington................. R R Arm of Gideon.......... Arnold ................... P S Arrow ................ Rolla Arthur.................... Ky I Arthur Woods............... Ky I 
54578

54579

54580

54581

54583

54584

54585

54586

54587

54588

54589

54590

54591

54592

54593

54594

54595

54596

54597

54599

54603

54605

54607

54608

54610

54612

54614

54615

54617

54618

54619

54620

54622

54623

54624

54627

54628

54630

54632

54633

54635

54636

54637

54642

54643

54644

54645

54646

54647

54648

54649

54650

54654

54658

วั4659

54661

54662

54663

54664

วั466\%

54669

$546 \% 0$

54671

$546 \% 4$

54675

54676

54677

54683

54684

54688
Asaph ..................... - P S

Ashby

Ashland

Ashland Duke...... $\mathrm{T}$

A. T. Stewart ......... M

Audrain ............

Audermon

Audubon Breastplate....... Ky I

Auglaize Baron ............ Ky I

August Duke............... M

Augustus.................... M

Augustus......

Augustus ........... D

Augustus $2 \mathrm{~d}$

Aurora Duke................

Autocrat ................... $R$

Auxvasse A,........... K y I

Babbitt Gwynne..........

Badger ............... P S

2d Badger Boy ....... M

Barclay ......... $R$

Barnabas ...........

Barney

Barney ..........

Barnum..................... R

2d Baron Adelaide ........ M

Baron Antrim .............. $R$

Baron of Appanoose

Baron Bickerstaffe 2d .....- - Rolla

Baron Boone ............... Ky I

Baron Breastplate.......... Cox

Baron Bright Eyes .........-Cox

Baron Chief ............ P S

Baron Cloud................ Cox

B. Constance 1 of Woodlawn - R R Baron Duke 9th .............. - D Baron of Elmwood............. Ky I Baronet ................. II 4th Baron of Gloster..... - Cox Baron Gwynne 3d...... - Cox Baron Gwynne 5th ......... - Cox Baron Gwynne 2 of Mapledale - D 3 Baron Gwynne of Mapledale - D Baron Jupiter of Pleasant Hill_R R Baron Leonard 12th .......... - . II Baron Lind $2 \mathrm{~d}$ Baron Lischer 3d ...... M Baron McGregor Baron of Maine Valley Baron of Maple Hill _............. M 2d Baron of Maple Hill......... D Baron Monroe.......... Baron's Prince............... Baron of Ridgeland... - Multiflora Baron of Rio ............. - Ky I Baron Rosamond .........-Rolla Baron sharon -............-D Baron of Sp'ng Br'k 2 - Long Horn Baron Steuben ............ Baron Trimble ............. $R$ Baron Wabash Baron Wadsworth 5th........ Baron Wadsworth 6th...... - Ky I Baron Woodhill 21st......... - L R Baron Yeomans.............. - Cox Barrington .................. D Barrington 6th Barrington of Pleasant Home - R R Barrington of Sonora.......- $R \mathrm{R}$ Barrister....................
54690

54691

54692

54693

54694

54696

54697

54698

54699

54700

54704

54705

54706

54709

54710

54711

54712

54713

54714

54715

54716

54717

54718

54719

54720

54721

54722

54723

54725

54726

54727

54730

54731

54733

54734

54735

54737

54738

54739

54741

5474

54745

54746

54747

54748

54749

54750

54752

54753

54754

54757

5475

54759

54761

54762

54763

54764

54766

$5476 \%$

54769

54771

54 7\%

54774

54775

54777

54778

54779

54780

54781

54782
Barrister-.............. Milk Spring

Barton Bates............... D

Bashford 4th .............. Ky I

Bashford 5th...............

Bashford 6th

Bates Oxford $2 \mathrm{~d}$

Bates Oxford 3d.......... R R

2d Bates of Richland....... P S

Batson Duke................. Ky I

Battery Boy ................ - L R

B. Carlton .............. Ky I

B. C. Jackman ........ Cox

Beaconsfield 5th ........... Cox

Beau of Clover Hill

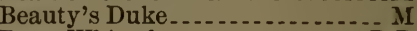

Beau Whittaker .......... $\mathrm{R} R$

Bedford.............

Bedford

Bedford's Duke... D

Beecher............ P S

Beecher....... P S

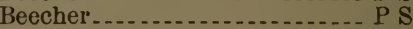

Beech Hill Duke......... $R$

Beethoom ............ Cox

Bekerans ............ D

Belden ......................

Bell's Airdrie ........ Cox

Bell Airdrie 4th......... II

Bell Boy ............ L R

Belle's Dandy ........

Bell Duke............. Ky I

Bell Duke.................. L

Belle's Duke 3d................. Daisy

6th Bell Duke of Elkhart......... D

Bell Duke of Fairholme $3 \mathrm{~d} .--\mathrm{R} R$

Bell Duke of Ferndale......... - D

4th Bell Duke of Maple Hill _ - R R

2d Bell Duke of Milford.... - $\mathrm{R} R$

3d Bell Duke of Milford....- $-R R$

Bell Duke R.,............ - R R

Bell Duke Rover........ R R

Bell Duke S.,............ R R 6th Bell Duke of Sandusky ..... L R 7th Bell Duke of Sandusky.... - D

Bell Gwynne.............. - Cox

Bellville ........................ Daisy

Belleville Duke .......

Belmont.................... Ky I

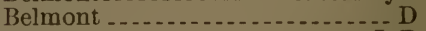

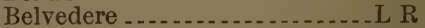

Ben

Ben ...........

Ben-................. L

Ben Barmpton ....... Daisy

Ben Bigstaff .........

Benbo Duke............... D

Ben Bolt ......................

Ben Brewster ..............

Ben Brown

Ben Butler........................

Ben Butler.........

Ben Butler-......

Ben Butler................ Ky I

Ben Butler................ Ky I

Ben Butler.........

Ben Butler 2d

Benedictus .............................

Ben Harrison .

Benjamin .............. P S

Ben of Maine Vailey ...... - Cox 


\begin{tabular}{|c|c|}
\hline 54783 & Bennie R. \\
\hline 4784 & Ben Royal \\
\hline 85 & Ben Russe \\
\hline 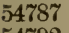 & Ben Tyler... \\
\hline 792 & Bertram 8th \\
\hline 793 & Bertram of Elk \\
\hline & $\begin{array}{l}\text { Bertram of Gra } \\
\text { Bessie's Oenan }\end{array}$ \\
\hline 796 & Bicker Lad 2d. \\
\hline & Big Bob - \\
\hline 0 & Bill Watso \\
\hline 1 & Billie... \\
\hline 2 & Billy Andre \\
\hline & Billy Bass \\
\hline 4 & Billy Beardsle. \\
\hline & Billy Bly. \\
\hline 6 & Billy Boy \\
\hline & Billy Boy \\
\hline & Billy Bra \\
\hline & Billie D., \\
\hline & Billy Duke \\
\hline & Billy of Emp \\
\hline 2 & Billy K., - \\
\hline 4 & Billy The Pri \\
\hline & Billy Wel \\
\hline 6 & Birthday- \\
\hline & 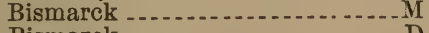 \\
\hline 9 & Bismarck \\
\hline & $\begin{array}{l}\text { Bismarck } \\
\text { Bismarck }\end{array}$ \\
\hline 324 & Bismarck. \\
\hline & Bismarck \\
\hline 27 & Bismarck Chie \\
\hline & Blaine ... \\
\hline 29 & Blaine.. \\
\hline & Blaine.. \\
\hline 1 & Blaize .... \\
\hline & Bliss \\
\hline 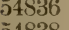 & $\begin{array}{l}\text { Bloom's Duke } \\
\text { Blucher }\end{array}$ \\
\hline & $\begin{array}{l}\text { Blucher-. } \\
\text { Blucher .. }\end{array}$ \\
\hline 40 & $\begin{array}{l}\text { Blucher -- } \\
\text { Blucher .- }\end{array}$ \\
\hline & Blue Valley C \\
\hline & - R R \\
\hline 4 & Bob..... \\
\hline & Bob Barmptol \\
\hline & Bob Bates. \\
\hline 7 & Bob Ingersoll \\
\hline 9 & Bob Lincoln - \\
\hline & $\begin{array}{l}\text { Bob Lincoln..- } \\
\text { Bob Price... }\end{array}$ \\
\hline & $\begin{array}{l}\text { Bob Price } \\
\text { Bozaris.. }\end{array}$ \\
\hline & Bold Boy \\
\hline & Bolingbr \\
\hline & - Rolla \\
\hline 7 & Bolivar - \\
\hline & Bolivar .. \\
\hline & Bolivar :- \\
\hline & Bolton Duke 5th. \\
\hline & Bonaparte \\
\hline & Bonnie Borgia Dul \\
\hline & Bonnie Duke..... \\
\hline & $\begin{array}{l}\text { Bonnie of Merino Va } \\
\text { Bonnie Prince }\end{array}$ \\
\hline & $\begin{array}{l}\text { Bonnie Prince...... } \\
\text { Boom 2d }\end{array}$ \\
\hline & $\begin{array}{l}\text { Boom } 20 \\
\text { Boon } \mathrm{Cl}\end{array}$ \\
\hline & Boreon. \\
\hline & Boston \\
\hline & Boston Duke.. \\
\hline & $\ldots B$ \\
\hline
\end{tabular}

54876

54877

54878

54879

54880

54881

54882

54883

54884

54885

54886

54888

54891

54893

54894

54895

54896

54897

54902

54904

54905

54906

54907

54908

54909

54910

54911

54912

54913

54915

54916

54917

54918

54919

54920

54922

54923

54924

54925

54926

54927

54929

54930

54981

54932

54933

54935

54936

54937

54938

54939

54940

54941

54942

54943

54914

54945

54946

$5494 i$

54949

54950

54951

54953

54954

54955

54956

54957

54958

54959

54960
Bourbon Duke........... - R R

Bourbon Duke $2 \bar{d}$

Bourbon Duke........

Bowman's Duke......... L R

Boyles' King

Bradford Lad 5th ............. Ky I

Bradford Lad 6th........ R R

Bradford Lad 7th............. Ky I

Bradford Lad 8th............. Ky I

Bradner ........................ I

Brady .................... Cox

Brandywine................ - L R

Brazen Nose.................

Breadlebane $3 \mathrm{~d}$

Breadlebane 3d......... $-R ~ R$

Breastplate...................... R

Breastplate of Pleasant Hill._._._.

Breckenridge .............. - M

Bridgegroom .................... S

Bride's Warlaby ............... Rose

Brigadier ...................

Brigadier Barmpton............

Brigham …...................

Brigham Young........

Brigham Young

Bright......................

Bright Eyes Duke............ - D

Bright Eyes Duke........ Cox

Bright Eyes Duke......... R R

Briliant.................. Ky I

Brimstone .................. - D

Bristow ................

Briton ...............

Briton ......... R

Briton $2 \mathrm{~d}$

Broken Leg............ $R$

Brooke 4th .............. - Cox

Brookfield .............. Ky I

Brooks_..................

Brookside Favorite......... - R R

Brookside Lad 4th ............ - D

Bruce . Rolla Bruce

Bruno ................. Ky I

Brush Creek Lad......... - Ky I

Brutus ................... - Cox

Brutus -......

Brutus ...............

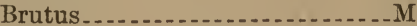

Bub

Buckeye -................... - D

Buckeye Traveler......... $R$

Buckner Lad............. - M

Budd ..........................

Bud Turner..............

Bud Renick....................

Buffalo Bill ........................

Buffalo Bill............... - L R

Buffalo Bill...........................

Bullion .......... P S

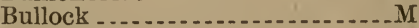

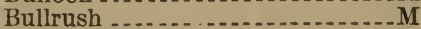

Burlington $\mathrm{Boy}$

Burnside Bill ...............

Burris Boy ................... Ky I

Bussy

Buster $2 \mathrm{~d}$

Butler .............. R R

Butter Boy

Butterfly's Champion.....- $\mathrm{R} \mathrm{R}$ 


\begin{tabular}{|c|c|}
\hline 1964 & Cadiz Ant \\
\hline & $\begin{array}{l}\text { Cadiz Arthu } \\
\text { Cadiz Clint }\end{array}$ \\
\hline 967 & Cadiz Duke \\
\hline 968 & Cadiz Earl. \\
\hline & Cadiz Leslie \\
\hline & Cadiz Milum \\
\hline & Cadiz Wild $\mathrm{H}$ \\
\hline & Cæsar -...- \\
\hline $\begin{array}{l}974 \\
977\end{array}$ & $\begin{array}{l}\text { Caldwell }- \text { - } \\
\text { Calvert } 2 \mathrm{~d}\end{array}$ \\
\hline & Cambria Boy \\
\hline & Cambria's D. \\
\hline & Cambria Fav \\
\hline & Cambria Prir \\
\hline & Cambria Pri \\
\hline & Cambridge \\
\hline & - - R R \\
\hline & Cameron Bea \\
\hline & Campachi...... \\
\hline & Campbell's \\
\hline & Canada La \\
\hline & $\ldots-M$ \\
\hline 00 & $\ldots-T$ \\
\hline & M \\
\hline & $-\mathrm{LR}$ \\
\hline & Captain of \\
\hline & $-\mathrm{R} R$ \\
\hline & $\ldots P S$ \\
\hline & $-\mathrm{PS}$ \\
\hline & - Woods \\
\hline & Captain $\mathrm{Kr}$ \\
\hline & $-\mathrm{PS}$ \\
\hline 2 & Captain M \\
\hline & $\begin{array}{l}--M \\
-D D\end{array}$ \\
\hline & D \\
\hline & Cox \\
\hline & - Woods \\
\hline & $\cdots-\mathrm{C}$ \\
\hline & Carduchi - \\
\hline & $\ldots-M$ \\
\hline & 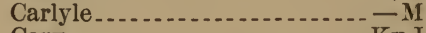 \\
\hline & - Ky I \\
\hline & Casabianca \\
\hline & $\begin{array}{l}\text { Cash } \\
\text { Cash Conrad }\end{array}$ \\
\hline & - R R \\
\hline & Cox \\
\hline & - Beauty \\
\hline & $--\mathrm{R} \mathrm{R}$ \\
\hline & $-\mathrm{PS}$ \\
\hline & Ky I \\
\hline & - Woods \\
\hline & Cavalier ..... \\
\hline & Cedar Farm \\
\hline & $\begin{array}{l}\text { Centennial. } \\
\text { Center }\end{array}$ \\
\hline & - T \\
\hline & $-\overline{K y ~ I}$ \\
\hline & Champion... \\
\hline & $--\mathrm{L} R$ \\
\hline & $\mathrm{L}$ \\
\hline & Champion Star \\
\hline & Champion of $t$ \\
\hline & Chat \\
\hline & \\
\hline
\end{tabular}

55062 Charles $2 \mathrm{~d}$.................... - L R

55064 Charles Dickens............. P S

55066 Charles D'Orleans .......... - P S

55067 Charley ................... R

55068 Charlie ...... R R

55069 Charlie C..........

55070 Charlie C.

55071 Charley henney.............

55072 Charley Lang

55074 Charlie Scott................

55075 Charley Stewart............. P S

55076 Charlie Wallace ..........

55077 Charmer........................

55078 Charm's Cherry .......... - Rolla

55079 Chautauqua Boy.............. L R

55081 Cherry

55082 Cherry Booth..............

55083 Cherry Duke............ $\mathrm{R}$

55085 Cherry Duke.............. Cox

55086 Cherry Grove Duke ........ - R R

55087 Cherry Lad 11th........... - Cox

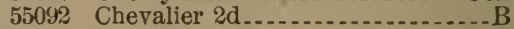

55093 Chil Hazard .................. L R

55096 Chippewa.................. R

55097 Chorister................

55100 Christopher Columbus........ P S

55102 Cicero....................

55104 Cincinnatus................. - D

55105 Circumference.................

55106 Clan Charlie Lid.........................

55107 Clarendon

55108 Clarendon $2 \mathrm{~d} . . . \ldots . . . . . . .60 x$

55109 Clarence

55110 Clarence $3 \mathrm{~d}$

55111 Claybourn ............. Ky I

55112 Clayton......................

55114 Clie............................

55116 Climax .................

55117 Climax ........................... S S

55118 Climax of Ashbrook ............ D

55119 Climax Blossom............

55120 Climax of Elmwood.......... Cox

55121 Climax Lad..................... P S

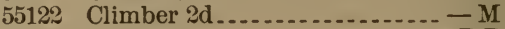

55123 Clinton

55124 Clinton Knight ........... K y I

55125 Clio .......................... R

55126 C. L. Morgan

55127 Clover Duke ................ - R R

55128 Clydus......................

55129 Climer...................

55130 Coin.........................

55131 Cold Spring .................

55133 Colonel................ L R

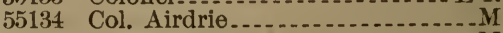

55136 Col. Cantrill ................... MI

55137 Col. Casterline................. Ky I

55139 Col. Crisp...................

55142 6th Col. Gloster........ - Cox

55143 8th Col. Gloster ........... Cox

55144 Col. Gregg ......................... M

55146 Col. Harding ................

55147 Col. Heckor

55148 Col. Hibbarda... M

55151 Col. Hodgson

55152 Col. Holmes........... Daisy

55153 Col. Horton -......... D

55154 Col. Loudon

55155 Col. Moore........................

55156 Col. Morgan................. - D 
55157 Col, Rice

55158 Col. Sawyer ................. Cox

55159 Col. Selsor

55160 Col. Tredennis ............. - R R

55162 Colorado....................

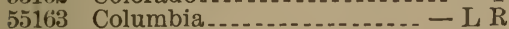

55164 Columbus................ - L R

55165 Columbus................

55166 Columbus 4th

55167 Columbus Alpha

55168 Comet.........................

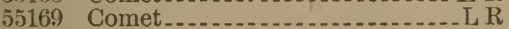

55170 Comet......... R

55171 Comet........................ R

55172 Comer

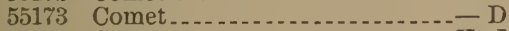

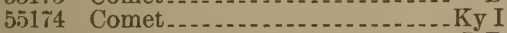

55175 Comet............

55176 Comet ................. P S

55177 Comet...........................

55178 Comet .................. - L R

55180 Comet of Maine Valley ........ L R

55181 Commander.................

55182 Commander....... D

55184 Commodore

55185 Commodore $2 \mathrm{~d}$

55186 Commodore Bun ............. L R

55187 Commodore Nutt............ - R R

55190 Conqueror Chief $2 \mathrm{~d} . . . . . . . . . . . \mathrm{L} \mathrm{R}$

55191 Conqueror of Brush Creek. - Ky I

55193 Constance Carlisle........... - P S

55196 Constant ...............

55197 Constantine of Locust Grove

55198 Constantine 2 of Locust Grove_- C

55199 Continental.................- Cox

55200 Convolous Major ............ Cox

55201 Convoy Romp............. - R R

55203 Cook ................. Beauty

55204 Cook ........... R

55205 Cook's Duke................. - C

55206 Coolspring Manley......... - Ky I

55207 Cooper .................. - I, R

55208 Cora's Duke............. R R

55209 Cora's Marquis ............ - R R

55211 Cornelius.................... L R

55212 Cornelius..........................

55215 Cotton Tail

55216 Cottonwood Matchless......- L R

55218 Count Cabarrus..........

55219 Count Cloquet.......... - R R

55220 Count of Fayette.......

55221 Count of Freedom .......... - Cox

55222 Count of Freehold......... - L R

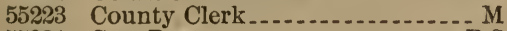

55224 Cow Boy

55225 Crawford ......... L R

55227 Cropys Duke......... Ky I

55228 Crown Prince............

55229 Crown Prince .............. - L R

55230 Crown Prince...............

55231 Crown of Sonnenberg ......- L R

55234 Cullen Rex................

55235 Cullen's Sharon ............

55236 Cumberland.............. P S

55238 Curtis...................... R

55239 Custer -........ Beauty

55240 Cyclone.......................... R

55\%42 Cyclone........................ D

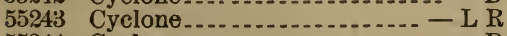

55244

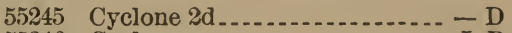

55246 Cyclops

55247 Cypress Duke $2 \mathrm{~d} . . . . . . . .-$ Rolla

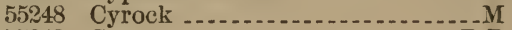

55249 Czar........................ R

55250 Dado ........................

55251 Dahlia Duke............. D

55252 Daisy's Duke.............. - L R

55254 Daisy's Duke............. - R R

55255 Daisy Duke

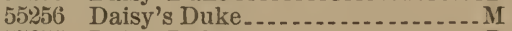

55257 Daisy Duke........... D

55258 Daisy Duke 4th.......... L R

55259 Daisy Duke 5th

55260 Daisy Duke of Elmwood...... Ky I

55261 Daisy Duke of Utoe........... - C

55263 Daisy's Prince.................... R

55264 Dakota $\ldots \ldots \ldots \ldots$

55265 Dakota Duke....................

55266 Dan.........................

55267 Dan............................. S

55268 Dan ................

55269 Dan Able...................... P S

55:\%0 Danby ..................... -

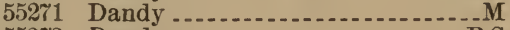

55272 Dandy

55273 Dandy ............................

55274 Dandy

55275. Dandy Duke.... $\mathrm{M}$

552\%6 Dandy Jim .............. L R

55278 Daniel Webster.......

55279 Dannet ............... Cox

55280 Dan Rice........

55281 Dan Rice..........

55282 Danver ........................... Daisy

55285 Darby Duke 19th....... - S S

55286 Darby Duke 20th

55287 Darby Duke 21st............ - P S

55288 Darby Duke 22d............ R R

55289 Darby Duke 23d........ P S

55290 Darby Louan Duke.......... - L R

55291 Dartford Duke ............... - P S

55292 Dave ...................... - Cox

55293 Dave Perrine

55294 Dave Sherman............ - D

55295 David ........................

55296 David Crockett.......... Ky I

55297 David Gordon.............. - M

55298 Davis .......................

55299 Davis Duke........

55301 Davy Crockett............. P S

55302 Davy Crockett............... R

55303 Dawson Duke...............Rose

55304 Deacon .................... - M

55305 Deacon

55306 Deacon Dean

55307 Deccan ............

55308 Deer Creek Lad............ - M

55309 Delavan Springwood........ - M

55310 Delong ................... P S

55311 Delos

55312 Demonstration...............

55313 Dennie ................... $\mathrm{R}$

55314 Denton

55315 Denver.............................

55316 Derby ..........

55317 Derby

55318 Desmoro................. - Cox

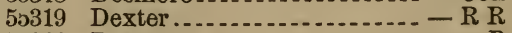

55320 Dexter 
55321

55322

55323

55324

55325

55326

55327

55328

55330

55331

55332

55333

55334

55335

55336

55337

55338

55339

55341

55342

55343

55344

55345

55346

55347

55349

55351

55353

55354

55355

55356

55357

55358

55359

55360

55361

55362

55363

55364

55365

55366

55367

55368

55369

55370

55371

55372

55373

55374

55376

55377

55378

55381

55382

55383

55384

55389

55390

55391

55392

55393

55394

55395

55396

55397

55398

55399

55400

55401

55402
Dexter.

Dexter

Dexter

酯

Diamond...................... Ky I

Diamond

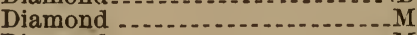

Diamond

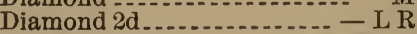

Diamond Duke................... S

Dick ..................... Multiflora

Dick .........

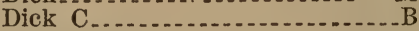

Dick Dallis........... Rolla

Dick Duncan

Dick Johnson .............. Ky I

Dick Johnson..........

Dick Keen

Dick Leeside.............. - Cox

Dick Leslie................ _ P S

Duke Romulus..........

Dick Sharon................ $D$

Dick of Sonora........

Dick Taylor.................. $-L$

Dick Taylor................ Ky I

Dick Taylor $3 \mathrm{~d} . . . . . . .$.

Dick Tinto.....................

Dick Watson........... P S

Dick Williams..............

Did Duke................. - T

Dimple ....................

Doctor ................

Doctor $2 \mathrm{~d}$

Doctor Berzilius ............... Cox

Doctor Dix...................

Doctor Gangannelli............... Cox

Doctor H.,......... R

Doctor Hinsey

Doctor Lind ............... R R

Doctor of Maine Valley........ $\mathrm{L}$

Doctor Martin 7th

Doctor Merrill ............... - R R

Doctor Montgomery....... - C

Doctor Moore Jr., ................

Doctor Napier ................. R

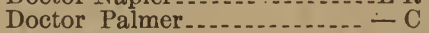

Doctor Shaftoe........... D

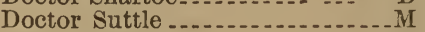

Doctor Sweeny

Doctor Young .........

Dollie Fragrant................ S S

Dom Pedro

Don Acme..... P S

Donald Duke 15th

Donald Duke 16th............ R R

Donald Duke 17th......... R R

Don Allilly ........... S

Don Carlos........ R R

Don Duke. .................

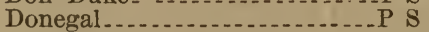

Don Eureka..... P S

Don John..........................

Don Juan .................. P S

Don Ladiesman

Don Linwood....... M

Don Louanjo......

Donnell ........................

Don Thonrose.......

Don Quixote.......................

Door Village................. Daisy

Dor....................... Cox
55403 Fourth Dora's Duke......... - M

55407 Double Star Duke.......... - L R

55408 Douglas.................... $\mathrm{P}$ S

55409 Douglas ................. $\mathrm{R} R$

55410 Dowdall

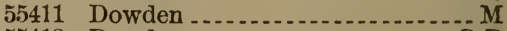

55412 Dresden

55414 Duane's Duke 24th...... - Rolla

55415 Duchess Duke ........... R R

55417 Dude ..........................

55418 Dude................... Cox

55420 Duke

55421 Duke............

55422 Duke...............................

55424 Duke....... Cox

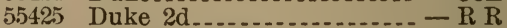

55427 Duke of Airdrie............ - Cox

55428 2d Duke of Albert Lea

55429 Duke of Albion................ S

55430 Duke Alexis.............. $\mathrm{R}$ R

554315 th Duke of Allen......... - Cox

55432 47th Duke of Allendale....... - T

55433 2d Duke of Almont............. R R

55434 5th Duke of Alstead...... - Rolla

55435 ith Duke of Alstead...... - Rolla

55436 8th Duke of Altaham....... - M

55437 9th Duke of Altaham

55438 Duke Amelia.............. R R

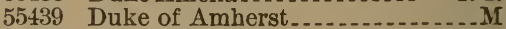

55441 Duke of Anoka........... R R

55442 Duke of Arcola...................

55443 Duke of Argentville............

55446 Duke of Armstrong Prairie $-\mathrm{R} R$

55450 Duke of Ashwood........... Cox

55451 Duke of Assyria............ - Cox

55452 2d Duke of Atwater........ - L R

55453 Duke of Auburn.......... $\mathrm{R} R$

55456 Duke of Austin 3d........... Ky I

55457 Duke of Austin 4th............ Ky I

55458 Duke of Auxvasse.......... - R R

55459 Duke of Aron............. - M

55460 4th Duke of Avondale........ - M

55463 1st Duke of Barryville..... . $\mathrm{R} R$

55464 Duke of Bates................. R

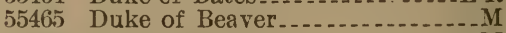

55466 Duke of Beechland.......... - M

55467 Duke Bell .................. R R

55468 2d Duke of Benton .......... Ky I

55469 1st Duke of Beriman....... $\mathrm{T}$

55470 Duke Blane................. $\mathrm{PS}$

55471 2d Duke of Blendon............ B

55472 Duke of Blooming Grove........

55473 Duke of Boke's Creek ......... R R

55474 Duke of Boone.............. - L R

55475 3d Duke of Boston

55477 Duke of Bradford......... Cox

55481 Duke of Brooklin ............. Ky I

5548 ; 14th Duke of Brownsville ... - R R

55484 Duke of Bulgar................. Ky I

55486 2d Duke of Cambridge...... - R R

55488 Duke of Carrolton............ R

55489 Duke of Casawago.......... Ky I

55490 Duke of Cass............. $\mathrm{L}$

55492 Duke of Cecil .................... R

55495 Duke of Cedar Hill........ - Cox

55496 Duke of Chatfield............ L R

55497 Duke of Cherry Grove ........ - C

55498 2d Duke of Cherry Grove..... - M

55499 3d Duke of Cherry Grove..... - M

55500 Duke of Cherry Hill............ Cox 
55501

55502

55503

55504

55505

55506

55507

ก๊5508

55509

55510

55511

55512

55513

55514

55515

55516

55517

55520

ร็ธ521

55522

55523

55524

55526

55527

55528

55529

55530

55531

555032

55533

55540

55541

55542

55543

55544

55546

55547

55548

55552

55553

55554

55555

55556

55557

55560

55561

55565

55566

55569

55570

55571

55572

55573

55574

$555 \%$

อัธธธ์ 6

55577

55578

55579

55580

55581

55582

55583

55584

55585

55586

55588

55590

55591

55592 9th Duke of Chico - Ky I

10th Duke of Chico.......... Ky I 18th Duke of Chico......... P S 19th Duke of Chico ........... Ky I 20th Duke of Chico........... Ky I 22d Duke of Chico............ L R 24th Duke of Chico .......... L R 25th Duke of Chico ........... P S 27th Duke of Chico ............ - P S 31st Duke of Chico ..................... 32d Duke of Chico ............ L R 33d Duke of Chico 34th Duke of Chico........... P S 35th Duke of Chico........... Ky I 37th Duke of Chico Duke of Clague................ $\mathrm{R}$ 1st Duke of Clarks ...... Ky I Duke of Clay Ridge ......... - L R Duke of Clermont ........... Duke Clifton of Weston....- $\mathrm{R} R$ 7 Duke of Clinchdale - Milk Spring Duke of Clinton........... Cox Duke of Coal Creek ....... - Cox Duke of Columbia ........ - Cox Duke Columbia 2d ......... - Cox 3d Duke of Columbia...... - Cox Duke of Conneaut........... D 2d Duke of Conneaut....... D Duke Constant 4th Duke of Crawfish .........- - L R Duke of Cumberland......... - M Duke of Cumberland ........ Duke of Cuyahoga Duke of Cypress...........- Rolla Duke of Cypress........... - Rolla Duke of Doyle 3d............. Ky I Duke of Duncanson ............ L R Duke of Edinburgh 3d Duke of Edgewood......... - M Duke of Edinburgh ............ Cox 3d Duke of Elder Park ............ 4th Duke of Elder Park Duke of Elizabeth ....... Duke of Elk................. Duke of Elmwood.............. Cox Duke of Elmwood.......... M Duke of Endfield .......... $R$ 4th Duke of Enterprise ..... - R R 8th Duke of Evergreen.......... P S 9th Duke of Evergreen ......... P S 10th Duke of Evergreen..... - P S

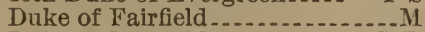
Duke of Fairfield Duke of Fairview . ........ - D Duke of Fairview ................. Duke of Fairview............... Duke of Fairview 1st Duke of Fairview ........ - R R Duke of Fairview ........... - M 3d Duke of Fairview .......... - C 4th Duke of Fairview........ 14th Duke of Fairview .......... M Duke of Farley -.... Duke of Farmdale......... P S 2d Duke of Fayette 3d Duke of Federal Hill ......... M 4th Duke of Ferndale....... $\mathrm{L} R$ 12th Duke of Forest Hill ...... L R 13th Duke of Forest Hill.....- - L R 14th Duke of Forest Hill .... - L R
55593

55594

55595

55596

55597

55600

55601

55602

55603

55604

55605

55606

55608

55611

55612

55613

55614

55615

55016

55617

55618

55620

55623

55624

55625

55627

55628

55629

55630

55631

55632

55633

55635

55636

55637

55638

55640

55641

55642

55644

55645

55646

55648

55649

55651

55652

55653

55654

55655

55656

55657

55659

55661

55662

55663

55664

55665

55666

55667

55668

55669

55671

$556 \% 2$

55673

55674

55675

55676

$556 \%$

55678

55679 15th Duke of Forest Hill ...._- L R

16th Duke of Forest Hill....- $-L ~ R$

6th Duke of Forest Home........ M

Duke of Franklin .............. D

Duke of Fulton ............- L R

Duke of Glen........Pedigree False

Duke of Glenerie..................M

Duke of Gloster........... Rolla

Duke of Gloster.............

Duke of Goodness........ R R

Duke of Goodness........... - R R

2d Duke of Goodness........ - R R

Duke of Grafton ............

Duke of Grand Prairie......... - D

Duke of Granite ................ B

8th Duke of Granville ....... Ky I

9th Duke of Granville ......... Ky I

10th Duke of Granville........ Ky I

11th Duke of Granville........ Ky I

Duke of Gratiot.............. - L R

Duke of Green $2 \mathrm{~d} . . . . . . . . . . \mathrm{Ky} \mathrm{I}$

Duke of Greenwood ....... Ky I

6th Duke of Grovedale ..... - Cox

Duke of Hanover............. R

Duke of Harrison ......... - Cox

Duke of Helena............... Rose

D. Helena of Prickly Pear.... - M

Duke of Highland .............. T

12th Duke of Highland.... - Cox

Duke of Hillier........... D

3d Duke of Hillside............ T

4th Duke of Hillside......... T

Duke of Hopkinsville

Duke of Howell............ Ky I

Duke of Humber............ - L R

Duke of Illinois ..............

Duke of Imperial ....

Duke of Independence..... $-\mathrm{L} R$

2d Duke of Indian Hill..... - Cox

2d Duize of Inverness ...... - Cox

3d Duke of Inverness ........ - Cox

4th Duke of Inverness ....... - L R

$2 \mathrm{~d}$ Duke of Irwin............... Ky I

Duke of Iveness .............

Duke of Jasper -............ - Cox

Duke of Jasper.............. P S

6th Duke John of London ..- - L R

Duke of Julien ............... B

Duke of Juliette..............

Duke of Kent ............... R R

Duke of Kennickinnic...... - L R

Duke of Lake......................

Duke of Lakin..................

Duke of Lancaster............ S S Duke of Lansing ........... - L R Duke of Lawnsdale .......... M 1 Duke of Leavenw'th-Milk Spring Duke of Lee $2 \mathrm{~d}$............. - Rolla Duke of Leesburgh ........ - Rose Duke of Lewis ....... Woods Duke of Liberty .......... - R R Duke of Lincoln Duke of Linwood........ $\mathrm{R}$ 3d Duke of Linwood........ $R$ Duke of Littleton ............ Ky I 14th Duke of Locust Grove.... - C 15th Duke of Locust Grove...- - M 16th Duke of Locust Grove...- - M 17th Duke of Locust Grove... - - M 18th Duke of Locust Grove... - M 
55680 Duke of Lorne 55681 Duke of Louan $2 \mathrm{~d}$ 55682 Duke of Lydia ............. D 55683 Duke of McLean .................. M 55684 4th Duke of McLean Park - - Rolla 55687 8th Duke of McLean Park _ - Rolla 55689 3d Duke of Madison ......... - R R 55690 Duke of Malta .............. L R 55693 Duke of Maple Grove ..... - D 55694 Duke of Maple Grove...... - R R 55695 2d Duke of Maple Grove.... - R R 55697 1st Duke of Maple Park ...... - M 55698 Duke of Maquoketa........ - Ky I 55699 2d Duke of Maquoketa........ - M 55700 Duke of Mason ............. - P S 55702 4th Duke of Mayslick ..........Rose $55 \% 04$ Duke of Mazurka ........... - M 55705 Duke of Meadow Lawn ... - Daisy 55706 1st Duke of Medina ........... - D 55707 Duke of Miami.................... 55708 Duke of Mifflin 55709 1st Duke of Milgrove....... - R R 55710 Duke of Monroe .......... $\mathrm{R} R$ 55711 Duke of Montclam....... $\mathrm{R} R$ 55713 Duke of Mount Clemens.... - Cox 55716 2d Duke of Oakdale .......... - D 55717 4th Duke of Oakdale......... - M 55718 Duke of Oakgrove......... 55719 Duke of Oakgrove......... - Ky I 55720 Duke of Oak Hill ........... - R R 55721 Duke of Oakland 55722 Duke of Oakland 55723 Duke of Oakland............ P S 55725 Duke of Oak Park......... - R R 55726 2d Duke of Orchard Hill........ Cox 55727 Duke of Osceolä......... - Daisy 55728 Duke of Otoe............ 55729 Duke of Otsego........... - Cox 55730 Duke of Oxford...................... 55735 2d Duke of Pataskala 55736 3d Duke of Pauline............. Ky I 55737 4th Duke of Pauline........... Ky I 55\%38 Duke of Pinewood............ 55739 Duke of Platte............. $\mathrm{R} R$ 55740 Duke of Pleasant Grove...... $-\mathrm{D}$ 55741 Duke of Pleasant Home.... $-\mathrm{R} R$ 55742 Duke of Pleasant Ridge....... - M 55743 Duke of Poe............... - Cox

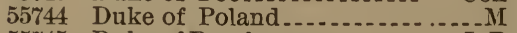
55745 Duke of Pomfret................... R 55746 Duke of Portage $2 \mathrm{~d} . . . . . . . .2 \mathrm{P} \mathrm{S}$ 55747 Duke of Portland........... - L R 55748 Duke of Pulaski........... $-\mathrm{R} R$ 55749 Duke of Randboro $2 \mathrm{~d} . . . . . . . . . . \mathrm{L} R$ 55752 Duke of Richfield................. 55753 Duke of Richland

55754 Duke of Richland............. - D

55755 2d Duke of Richland - Loug Horn 55756 3d Duke of Richland ............ M 55757 10th Duke of Richland ..... - Ky I 55758 11th D. of Richland - Milk Spring 55760 Duke of Ridgely .............. Daisy 55761 14th Duke of Ridgevale ......... Cox 55763 Duke of Riverview .......... - Cox 55764 Duke Robert.................. - M 55765 4th Duke of Rock River Valley _PS 55766 Duke Rose 3d................. Ky I 55767 Duke of Rosedale 2d........... M 55768 2d Duke of Rose Lawn....... - B 55769 Duke of Roseneath......... - L R
557\%0 Duke of Russell ................. 55771 Duke of Rutledge 55772 1st Duke of Sandstone..... $-R R$ 55773 1st Duke of Scales Mound.. - R R 55774 Duke of Sedgwick ............ $\mathrm{D}$ $55 \% 5$ Duke of Shanghai 55776 Duke of Sharon........... 55777 Duke of Sharon............. $-\mathrm{L} R$ $557 \% 9$ 3d Duke of Sheldon.......... 55780 4th Duke of Sheldon......... - P S 5.7852 Duke of South Fork ........ - Cox 55784 Duke of Springdale 1st.......... S S 5578.5 11th Duke of Springdale....... - M 55787 Duke of Spring Grove ........ - D 55788 1st Duke of Spring Hill .....- R R 55789 Duke of Spring Hill......... - $\mathrm{D}$ 55792 Duke Sterling $2 \mathrm{~d}$ 555 93 Duke Sterling 3d........... - M 55794 Duke of Stillwater........... $-\mathrm{L} R$ 55796 Duke of Stoner........... 55797 Duke of Stonewall.............. R $557982 \mathrm{~d}$ Duke of Stonewall .......... R 55799 Duke of Stringtown. - Long Horn 55801 Duke of Suez ............ D 55802 Duke of Sugar Grove.......... 55805 Duke of Taylor .............. 55808 Duke of the Realm 55809 Duke of the Rhine.......... - D 55810 Duke of the Rivers....... - Cox 55811 Duke of the Roses......... - P S 55812 Duke of the Valley ........... - C

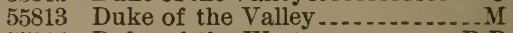
55814 Duke of the West....... R R 55815 Duke of Thorme 5th........ - D 55816 Duke of Thorne 6th........ - D 55817 Duke of Tipton............... Ky I 55818 4th Duke of Troy 55819 Duke of Underedge......... $-\mathrm{R} R$ 55821 Duke of Van Buren....... - M 55822 Duke of Vandalia............ 55823 1st Duke of Venedocia.....- R R 55824 1st Duke of Vermillion....... - M $558252 \mathrm{~d}$ Duke of Vermillion...... $\mathrm{R} \mathrm{R}$ 55826 3d Duke of Vermillion.... - Ky I 55827 3d Duke of Vermillion..... - Ky I 55828 4th Duke of Vermillion..... - Cox 55829 Duke of Vinewood 3d...... - R R 55831 Duke of Warpole.......... $-\mathrm{R} R$ 55832 3d Duke of Warren........ $-\mathrm{R} R$ 55833 Duke of Washington ...... - Rolla 55834 2d Duke of Washington...... - D 55836 . Duke of Watertown 55837 Duke Wellington 55838 Duke of Wellington 55839 5th Duke of Wellington........ Ky I 55840 Duke of Wells.......... 55841 Duke of Weston 55842 Duke of West Point.......... Cox 55843 Duke of Wheatland......... Ky I 55844 Duke of White Oak........... M 55845 Duke Wild Eyes........... - R R

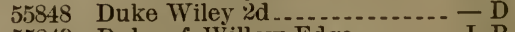
55849 Duke of Willow Edge...... - L R 55850 2d Duke of Willow Grove..... - M 55851 Duke of Windham........ - L R 55852 Duke of Winona.......... $\mathrm{P} S$ 55853 Duke of Winslow....... 55855 Duke of Wood County..... $-\mathrm{R} R$ 55858 Duke of Wood Lawn 55860 1st Duke of Yellow Creek..... - T 
55861 Duke of York............. - Ky I 55862 5th Duke of Yule Grove........ 55865 Duncan..................................... 55866 Dunlap D of Chestnut Grove _ - D 55867 Dunmore Duke............ - R R 55869 Dutch Lad................ - R R 55870 Dutchman..................

55871 Dwina.................................... 55872 Eagle ....................... Ky I 55873 Earl.................................... 55874 Earl ........... Ky I 55875 Earl

55876 Earl Second.......

55877 Earl 3d.................... M

55878 Earl 4th

55879 Earl of Alamo.... D

55880 Earl of Alamo $2 \mathrm{~d}$

55881 9th Earl of Allen........... - Cox

55885 Earl of Briar Hill............. - I

55886 Earl of Carroll

55890 Earl of Clarendon....... $\mathrm{R} R$

55892 Earl of Clifton................. R R

55894 5ch Earl of Conneaut....... - R R

55896 Earl of Crete.............. - Rolla

55898 Earl Derby 11th .................M

55899 Earl Derby 12th

55900 Earl Derby 13th

55901 Earl Derby 14th..................M

55902 Earl Duke....................

55903 Earl of Eaton............. - L R

55904 Earl of Elk Farm........... - C

55905 Earl of Elmwood........... Cox

55906 Earl of Endwood.............. Cox

55909 2d Earl of Geddes........... - Cox

55910 Earl of Genesee............... L R

55911 Earl of Glen.............. - Rolla

55917 Earl of Goodness 8th....... - L R

55918 45th Earl of Greenbush..... — L R

55919 46th Earl of Greenbush...... - L R

55921 Earl Henry............... - R R

55922 Earl Hill ....................... - D

55925 2d Earl of Lyndenwall............ M

55926 1st Earl of Marlette............. L R

55927 2d Earl of Meadow Brook ........M

55929 Earl of Newport............. . R

55930 Earl of Oak Creek

55931 Earl of Oak Hill.............. - D

55932 Earl of Oakland .......... Rolla

55933 2d Earl of Onondaga ...... - Cox

55934 Earl of Ovid........... - Cox

55935 Earl of Oxford .............. - R R

55937 Earl of Pleasant Hill

55938 Earl Prince 2d .........................

55939 Earl of Racoon Hollow -.... - P S

55942 Earl of Rochester...............

55943 Earl of Rochester.......... P S

55944 1\%th Earl of Ruralusia...... - P S

55945 20th Earl of Ruralusia.......... Ky I

55948 4th Earl of Sharon ......... - L R

55949 Earl of Sunnyside.......... - R R

5๊550 Earl of Syracuse ........... - Cox

55951 2d Earl of Syracuse ......... - Cox

55952 Earl of the Oaklands........ - Cox

55953 Earl of the Roses........... - R R

55957 Easter Boy

55958 Easter Hero..............

55959 Easton Duke...........

55960 Easton Prince............... - M

55961 Ebenezer

55962
55963

55964

55965

55966

55967

55968

55969

55970

55971

55973

55974

55977

55978

55979

55980

55982

55983

55984

55985

55987

55988

55989

55990

55991

55992

55993

55994

55995

55996

55997

55998

55999

56000

56001

56002

56003

56004

56006

56007

56008

56009

56011

56012

56013

56014

56015

56016

56017

56018

56019

56020

56021

56025

56027

56029

56031

56032

56034

56035

56036

56038

56040

56042

56043

56045

56047

56049

56050

56051

56052
Eclipse _................. $-\mathrm{R} R$

Eclipse -............. $\mathrm{R} R$

Eclipse ......... R R

Ed Davidson

Eddie Bald win................ Ky I

Eddie Hulse 5th..........- $R$ R

Edgehill Duke............ - Cox

Edinburgh Duke........... - Cox

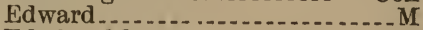

Edwin of Sonora.........

Edwinus .................... $M$

Elba Champion........... $-\mathrm{L}$

Election ...............

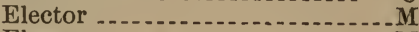

Elevator -.......................

Elkhorn Chief.........- Multiflora

Elkhorn Duke _............ - Rolla

Ellah's Barmpton .......... - R R

Ellah's Duke...............

Elmer F.................... $\mathrm{T}$

Elmhurst Lad

Elmsmere Lad................. Cox

Elmwood Lad

Elroy

Elsie's Underley..............-D

Elvina D. 1st of Locust Grove_-C Elwella's Breastplate........ - L R

Emperor................... $\mathbf{M}$

Emperor

Emperor..................

Emperor -... Cox

Emperor

Emperor Bates ........ $\mathbf{M}$

Empire .............. Cox

Empire.................. L R

Empire Star ................

Endymion-

Erasmus . .

Ernest................ Ky I

Eros_.......................

Eros

Ethan Allen.........................

Euclid............................

Eugene ............. Ky I

Excelsior .......................... Daisy

Exchange..........

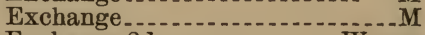

Exchange 2d......... Wrong

Fairchance Oxford...... - L R

Fair Duke............. Ky I

Fairer Eclipse ............. - L R

Fairfield Prince .............. $-\mathbf{M}$

Fairy Gift..........

Fancy Duke........ $R$

Fancy Lad ........ P S

Fancy Major 2d....... Ky I

Fancy Major $3 \mathrm{~d}$

Farmer's Glory

Farnese.......................

Fashion Duke......... D

Fausett Duke................ L R

Favorite ................ D

Favorite .............. $\mathrm{R}$

Favorite..................

Favorite of Empire..... D

Felix Grundy ........... $-\mathrm{L} R$

Furguson

Fidget's Oxford 6 th

Filigree Gem 6th..............

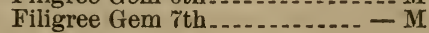




\begin{tabular}{|c|c|c|c|}
\hline 56053 & Financier Jr., - & 56141 & Gem's Duke.. \\
\hline $\begin{array}{l}6055 \\
6058\end{array}$ & $\begin{array}{l}\text { Fletcher } \\
\text { Flora's Duke }\end{array}$ & $\begin{array}{l}56142 \\
56143\end{array}$ & $\begin{array}{l}\text { Gem Duke....- } \\
\text { Gem's Duke }\end{array}$ \\
\hline 3059 & Flora's Orange Boy.- & 56144 & Gem's Duke 3d \\
\hline 060 & Florentia's & 56145 & General. \\
\hline 061 & Flotis..... & 56148 & General Bank \\
\hline 062 & Flower Lad & 56149 & Gen. Bonapa \\
\hline 3064 & Fluke..... & 56150 & Gen. Castellar \\
\hline 3065 & Fogy ... & 56151 & Gen. Crook. \\
\hline 3066 & Fonda. & 56152 & Gen. Custer. \\
\hline 067 & Fontenelle & 56153 & Gen. Deverear \\
\hline 071 & Forester- & 56154 & Gen. Garfield \\
\hline 73 & Forest King & 56155 & Gen. Garfield \\
\hline 074 & Forest Port & 56156 & Gen. Garfield \\
\hline & Forest Prin & 56157 & Gen. Garfield \\
\hline 076 & Forest Prin & 56159 & Gen. Grant \\
\hline 077 & Forest Prin & 56160 & Gen. Grant. \\
\hline 078 & Forest Rar & 56161 & Gen. Grant. \\
\hline 079 & Fortune.. & 56162 & Gen. Grant \\
\hline 3080 & Fortune.. & 56163 & Gen. Grant of \\
\hline 081 & Fortune.. & 56164 & Gen. Green ... \\
\hline 82 & Foster .... & 56165 & Gen. Hageman \\
\hline 083 & Fountain Boy & 56166 & Gen. Hanc \\
\hline 84 & Fountain Hea & 56167 & Gen. Han \\
\hline 085 & Fourth of Jul & 56168 & Gen. Harrison \\
\hline & Frank ..... & 56169 & Gen. Hill... \\
\hline 087 & Frank.- & 56170 & Gen. Hood \\
\hline & Frank Cro & 56171 & Gen. Hooker. \\
\hline 090 & Frank Cushi & $561 \% 2$ & Gen. Hopewell \\
\hline 991 & Frank Forre & 56173 & Gen. Howard \\
\hline 094 & Frank James & 56174 & Gen. Lee.. \\
\hline 095 & Franklin. & 56175 & Gen. Lee.. \\
\hline 96 & Franklin $2 \mathrm{~d}$ & 56176 & Gen. Logan. \\
\hline 97 & Franklin $\mathrm{Du}$ & 56178 & Gen. Milroy - \\
\hline & Frank L. Ma & 56180 & Gen. Oglesby \\
\hline 00 & Frank Wats & 56181 & Gen. Quinn. \\
\hline & Fred........ & 56182 & Gen. Richmond \\
\hline 4 & Frederick. & 56183 & Gen. Ricl \\
\hline 06 & Frederick W & 56184 & Gen. Richmond \\
\hline 07 & Fred Grant. & 56185 & Gen. Richmond \\
\hline 108 & Fred of Main & 56187 & Gen. Scott. \\
\hline 109 & Fred R. & 56189 & Gen. Taylor 4th \\
\hline 6110 & Fred Watson & 56190 & Gen. Thomas. \\
\hline 111 & Fremont.-... & 91 & Gen. Wils \\
\hline 11 & Fremont.. & 56193 & Geneva Bas? \\
\hline 13 & Frenchman & 56195 & Geneva Duke. \\
\hline 4 & Frenc & 02 & Geneva Lad \\
\hline & Friedla & 56207 & Geneva La Paz \\
\hline 6 & Ky I & 208 & Geneva M \\
\hline & Frontier Scot & 56209 & Geneva Wellingto \\
\hline 19 & $-D$ & 56210 & George \\
\hline 20 & Fullerton Chi & 56211 & George - \\
\hline 01 & Gabriel ... & 56212 & \\
\hline 122 & Gallant ... & 56213 & George G. Vest. \\
\hline 125 & Gambetta & 56214 & George Gaston J \\
\hline 26 & Gam & 56215 & George M. - \\
\hline 27 & Gar & 56216 & Georgetown Duke \\
\hline & & 56217 & George Vest - \\
\hline & Gar & 56218 & Geo. Washington \\
\hline & Gari & 56219 & Geo. Washington \\
\hline & Garfield.. & 56220 & Geo. Washington \\
\hline & Garfield & 56221 & Geo. of Willowed \\
\hline 56133 & Garibaldi $3 d$. & 56222 & Gervais ..... \\
\hline & Garland Duke & $562: 23$ & Gil-blas.. \\
\hline & Garland D. $2 \mathrm{~d}$ of Calumet_- $-\mathrm{R} R$ & 56224 & Gilmore... \\
\hline & Garrison Duke. & $562: 35$ & Giltedge $2 \mathrm{~d}$ \\
\hline & Gaudaloupe $2 \mathrm{~d}$ & 56226 & Gladiator.. \\
\hline & Gay Duke... & 56327 & Gladstone \\
\hline & $\begin{array}{l}\text { Gay Dul } \\
\text { Gem Du }\end{array}$ & 56228 & Gladstone- \\
\hline & $\mathrm{m} \mathrm{Di}$ & & Gladstone..- \\
\hline
\end{tabular}


56230

56232

56233

56234

56235

56236

56237

56238

56240

$5624 \mathrm{I}$

56243

56244

56245

56246

56247

56248

56249

56250

56251

5625 \%

56253

56256

56257

56258

56259

$5626 ?$

56263

56264

56265

56267

56268

56269

56270

56271

56272

56273

5627

56276

56277

56278

5627

56280

5628

5628 ?

56283

56284

56285

56286
56287

56288

56289

56290

56291

56292

56295

56301

56302

56305

56306

56314

56315

56316

56317

56318

56319

56320

56321

56324

56325
Gladstone

$-\mathrm{D}$

Glanford-............

Glen .................

Glenallan -.................

Glenvil Duke............. - L R

Gloster Du...................... D

Gloster's Duke of Richmond...L R 4th Gloster of Pine Grove.... - Cox

Gold Dust................. - P S

Golddust

Golden Duke..........

Golden Land

Golden Prince............... Ky I

Golden Prince 2d.............- -M

Golden Prince 17th..........

Golden Prince 18th........... $\mathrm{T}$

Golden Prince 19th

Golden Prince 20th .............. T

Golden Prince 21st................T

Golden Prince 22d.............. T

Golden Seal .................

Goldfinder of Ashbrook..... - P S

Goldfinder's Duke 2d............ R

Goldfinder of Melleray.......-P S

Goliah ..................-Cox

Goodness Duke............. - L R

Gortschakoff ............ - Cox

Governor -...............- Cox

Governor Foster............

Governor Anthony

Governor Butler

Governor Crittenden ............M

Governor Foster -.......... - M

Governor Glick -..........

Governor Glick -.............. - T

Governor Glick..........-- M

Governor Gwynne..........- Cox

Governor Hamilton ............ Daisy

Governor Hamilton

Governor Hoadley ............... Ky I

Governor Iles................ $R$

Governor Ireland

Governor Kirkwood $2 \mathrm{~d}$

Governor Palmer................ S

Governor St. John ................M

Governor St. John

Governor Sherman.......... Ky I

Governor Stone................ Ky I

Governor Yates................... R R

Gracie's Duke of Elmwood -..-M

Grace's Sharon ........... Wrong

Graham -.................. - M

Grand Airdrie $3 \mathrm{~d} . . . . . . . . . .$.

Grand Baron .................

Grand Duke

Grand Duke of Boardman.-...P S

2d Grand Duke of Boardman....P S

Grand Duke of Mitchell $2 d_{\text {. }}-\mathrm{L} R$

2d Grand Duke of Oakhill...... - T

Grant ......................-M

Grant .................. - P S

Grant -.............. Ky I

Grant

Grant

Grant

Grant Jackson ............. - R R

Grandville of Sonora ........ - P S

Grasmere's Barmpton....... - R R

Green Duke ................ - P S

Greenfield Lad................ M
56326

56328

56329

56330

56331

56332

56333

56334

56337

56338

56340

56341

56344

56345

56346

56347

56348

56349

56352

56353

56354

56355

56356

56357

56360

56361

56362

56363

56365

56366

56368

56369

56370

56371

56372

56373

56374

56376

56378

56380

$56 \dot{384}$

56386

56388

56391

56392

56393

56394

56395

56397

56398

56399

56400

56401

56402

56403

56404

56405

56406

56407

56408

56409

56410

56411

56412

56413

56414

56415

56416

56417

56418
Green Lawn Sharon........ - R R

Greenwood Boy ................... Cox

Greenwood Chief............... Ky I

Greer's Wild Eyes_..........- - R R

Gregory ......................... Ky I

Grinnell ....................

Grip -........................

Grubber .............

Gus Leland ................ $\mathrm{R}$

Gustavus.....................

Guthrie Duke..............

Guy

Hambledon ..............

Hamilton ................ - M

Hamlet ..............

Hamlet ..................- $\mathbf{M}$

Hamlin Lad....... Cox

Hammond ................. -

Hancock _..............

Hancock

Hannibal_................. - L R

Harbinger ................ Cox

Hardy -................. - M

Harlequin ........ T

Harold .................... Wrong

Harold ................... Ky I

Harold $2 \mathrm{~d} . . . . . . . . . . . . . . . .-D$

Harper …................. S

Harris Knight...................

Harrison ............... - Rose

Harrison's Duke.......... - R R

Harrison Chief...............-M

Harry -...................... M

Harry -...........................

Harry Carroll...............

Harry Lewis....................

Haskell .....................

Hatton Duke-.......... Ky I

Hawkeye Lad................-M

Hayes $2 \mathrm{~d}$..................... $\mathrm{PS}$

Headlight................... $-\mathrm{L} R$

Hector

Helena's Duke............ Ky I

Henry -.................

Henry Archer.............................

Henry Clay .................... R

Henry Clay -.................... S S

Henry Clay......................

Henry Louan

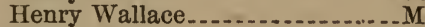

Henry Ward .................. R

Henry Ward Beecher.......- - P S

Henry Ward Beecher..............

Henry Ward Beecher..........L R

Hercules.....................

Hercules ................. - P S

Hercules ...............

Hermit_...............

Hermit .................. - P S

Hero ..................

Hero

Hero of the West........

Herchel

Heselrigge -..............

Hezekiah ...................... $R$

Hickman's Duke............. - P S

Hickory ...............-Cox

Hickory Duke............... - Cox

Hickory Duke............... - L R

Hickory Lad....................... 
56419 Highflyer ................. - R R

56420 Highland Lad ............... - Cox

$56 \pm 21$ Highland Laddie ................ Ky'I

56422 Highthorn .................... Daisy

56423 Hilda's Duke.................... M

56424 Hillhurst 2d................. P S

56428 Hillhurst Sharon D.5th-Milk Spn'g

56429 Hilpa Duke............... - Rolla

56430 Hilpa's Duke of Biggsville _. - P S

56431 Hilpa's Duke of Brookston .. - P S

56432 Hilpa's Duke of Kirkwood... - P S

56434 Hiram Bledsoe................ - M

56435 Homer....................

56436 Homer

56437 Honest John

56438 Honest John................. R

56439

56440

56441

56442

56443

56444

56445

56446

56447

56448

56449

56450

56451

56453

56454

56456

$5645 \gamma$

56458

56459

56461

56463

56464

56467

56469

56470

56477

56478

56479

56480

56481

56482

56484

56485

56486

56487

56488

56489

56490

56491

56492

56493

56495

56498

56499

56500

56501

56502

56503

56504

56505

56506

56507

56508

56509
Honest Tom

Honor Bright............... - L R

Hoosier Dick.................. Ky I

Hopeful................ R R

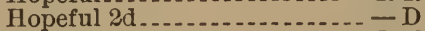

Hopewell 7 th.......... Ky I

Hopewell 8th..............

Horace.......... $\mathrm{R} R$

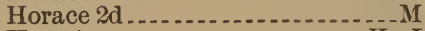

Horatio .............. Ky I

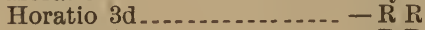
Horatio 4th ................ R Horn Buckle.............. Housatonic Major ........ - Rolla Hubback ...... Hubbard..... Hubbard ................. Hubbardton Boy ......... L R Hugo Humbolt ...... Humphrey's Airdrie Duke......M Huxley Boy ................ Ky I Ianthus .......... Ida's Marquis .......

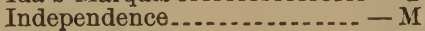
Independence ............ Indian Chief.......... P S Ingalls ..................... Ingomar ...... Cox Invincible.... Daisy Iron Duke ............. Isabel's Duke $2 \mathrm{~d}$ Ivanhoe................... Ivanhoe................... R Ixion ......... Jack ................. P S Jack Bean ........ R R

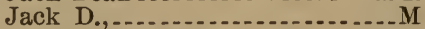
Jack of Deer Hill....... P S Jack Hancock ................... Cox Jack Lewis .............. Jack Ross.............. P S Jack Sheppard $2 \mathrm{~d} . . . . . . . . . . . M$ Jackson................ L R Jack Strauss Jack Young ....... P S Jacob .... . Jacob........ Jacob Skines James A. Garfield ............ P S James G. Blaine..... James G. Blaine......... P S James Helm ........ - Milk Spring
Honesty ................. R
56510 James Monroe.................... S S

56512 James .........................

56513 Jay Gould

56515 Jeff ..............................

56516 Jeff Davis

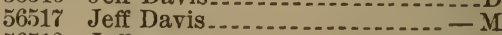

56518 Jefferson

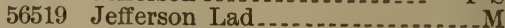

56520 Jeff Thompson.............

56521 Jennie's Star Duke............. R R

56522 Jerry ...................................

56523 Jerry Campbell...............

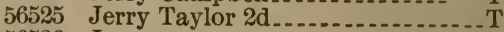

56526 Jess

56527 Jesse

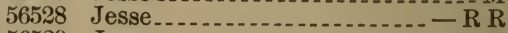

56529 Jesse

56530 Jessie's Airdrie.........

56532 Jessie's Duke................

56533 Jesse James..................... Ky I

56534 Jesse James ..................... M

56535 Jesse James........................

56 377 Jewel .........................

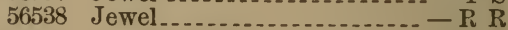

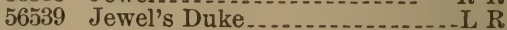

56540 Jeweler .............................

56542 J. H. Porter.........................

56543 Jim

56544 Jim Acklam........................

56545 Jim Blaine....................... Ky I

56546 Jim Davis..................

56547 Jim Fisk

56548 Jim Fisk

56549 Jim G. Carlisle...............

56551 Jim Lane...................

56552 Jimmy ............................. R

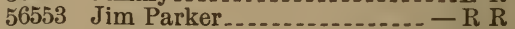

$56554 \mathrm{Jim}$ Pete........................

56555 Jim Taylor.......................

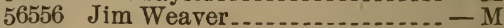

56558 Joe

56559 Joe

56560 Joe Blackburn

56501 Joe Bowers ....................... Ky I

56562 Joe Davis ..................

56564 Joe Granger................

56565 Joe Hull Jr., ........... Cox

56566 Joe Harding

56568 Joe Jefferson

56569 Joe Johnson................................

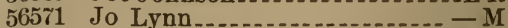

56572 Joe of Pettis.................. R

56574 Joe Shelby ............................... S

56575 Joe Smith ...................... S S

56576 Joe Taylor $2 \mathrm{~d}$

56577 Joe Witch ..................... R

56578 Joe Wright .......

56579 John.............................

56580 John

56581 John $2 \mathrm{~d}$

56582 John A. Dix ...................... S

56583 John A. Logan..................

56584 John Barker........ T

56585 John Brown ................. D

56586 John Bull ........................ Cox

56587 John Breckenridge............. Ky I

56588 John C. Breckenridge Jr.,.. - Ky I

56589 John Diamond Jr.,......... - D

56590 John E. Logan...............

56591

John Glenn .................... Rose 


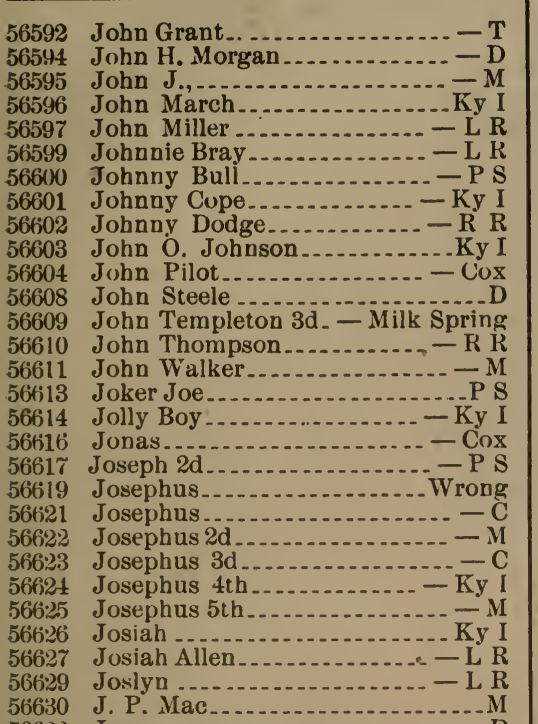

5663: Juan

56634 Jubilee Bell's Duke........ Ky I

56635 Jubilee Duke................ - T

5663 Judge

56640 Judge Kelly..................

56611 Judue Kinne............ R R

5664:) Judge Miller......................

$566+4$ Julius................ T

56645 July Duke............. Cox

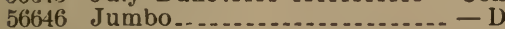

56617 Jumbo................... Cox

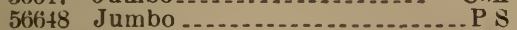

56649 Jumbo

56650 Jumbo........................ R

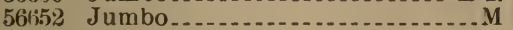

56653 Jumbo.................. . Cox

56651 Jumbo

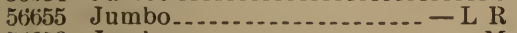

56656 Jumbo........................

56657 Jumbo

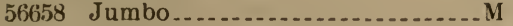

56659 Jumbo

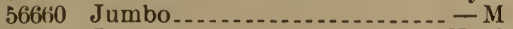

56661 Jumbo......................... Ky 1

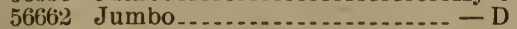

56663 Jumbo of Hornby $2 \mathrm{~d} . . . . . .-$ L R

56664 Junius of Lone Rock......... - I

56665 Jupiter _..................... M

56667 Jupiter of Coal Creek

566668 J. West Goodwin............... Ky I

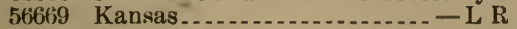

56670 Kansas Chief ...............

56672 Kansas Lad............... P S

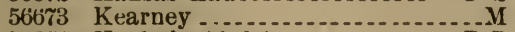

56674 Keefer's Airdrie............ $-\mathrm{R} \mathrm{R}$

56675 Kellogg ........

56676 Kent

56677 Kentucky .............

56680

56681

56682

56684

56685

56686

56687

56688

56689

56690

56691

56692

56695

56696

56697

56698

56700

56702

56703

56710

56711

56 ช6

56717

56718

56719

56720

56722

$56 \% 25$

$56 \%: 27$

56728

56731

56733

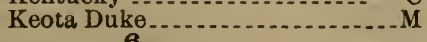

$56 \% 04$

56726

$567 \% 29$

56732

56734

56735

56736

56737

56738

56739

56740

56741

$56 \% 42$

56743

$56 \pi 44$

56745

56746

$56 \pi+7$

56748

56ช49

56751

56 เัน

56753

56755

56756

56757

56759

56760

56761

56762

56763

$56 \pi 86$

56767

$56 \% 68$

56769

56ชช0

Keystone................... D

Keystone Boy

King of Berlin

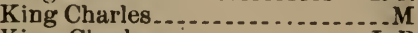

King Charles...... L R

King of Cherry Hill ....... - Rolla

King of Diamonds ......... $\mathrm{R} R$

King Duke..........................

King Humbert...............

King of Maple Grove....... D

King of Maine Valley........L R

King Rene............... R King Rose................... R

King Rover............. $\mathrm{R}$

King of Scots................ $R$

Kingston ..............................

King William King William 2d........ R R King of Woodlawn Kirklevington Duke 2d...... - P S Kirklevington Duke of Alexis_- D Kirklevington Lad 3d ..... - Rolla Kirklevington of Mt. Zion . - R R Kirklevington 2d of Oukland $-\mathrm{R} R$ Kirklevington $3 d$ of Oakland - Cox Kirklevington 4th of Oakland - Cox Kirklevington Oxford 3d.. - Rolla Kirklevington Prince....... _- Cox Kirklevington Prince....... $-\mathrm{R} \mathrm{R}$ Kirkpatrick .............. Ky I Kirkwood ...................... Cox Knight of Brant $2 \mathrm{~d} \ldots \ldots-$ Cox Knightley Sharon......... R R Knighi of the Red Cross..... L R 2d Knight of the Ridge....... Knight of the Thistle...... Knight of Viewland........ Rose

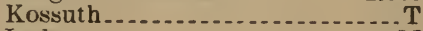

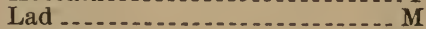
Laddie ................. Lady's Pringe........ Lia Harpe Boy ........... P S Lalla's Airdrie Duke......... - L R

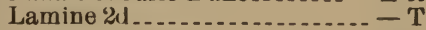
Lauark Baronet...............L R Laucaster Duke............ P S Last Rose................ L R Last Son of Sixth Earl..... - R R Laudable ..................... Cox Laudable Airdrie............. P S Laura's Councillor...............M Laura's Duke of Ashbrook ... _ D Lawnfield ..................... R Law . . . . . Lavton ........... L. B. Sims ...... L. D., ............................. I

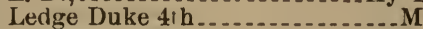
Ledge Duke 5th ........... Lee McGavoch............ Rolla Lehigh ................ L R Leland .................... P $\mathrm{S}$

Lentner Lad 3d.............. Leo................................ R Leo $\ldots \ldots$ Leo . . Leonardo ......... R 56772

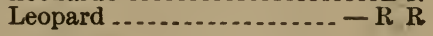


56773 Le Roy ....................

56774 Le Roy..................... Ky I

56775 Leslie .................................

56778 Leslie Duke's Lena.......... Daisy

56779 Leslie Hopewell

56781 Leslie Porter ....................

$56 \pi 82$ Lew Bennett_.............. - Daisy

56783 Lewis E. Montgomery _.......

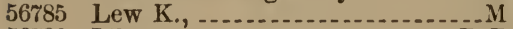

56788 Liberty

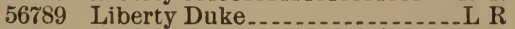

56790 Lieutenant..................

56791 Lillie's Duke............... R

56792 Lillie $\mathrm{O}$. Hero............... - M

56793 Lillypad........................... $\mathrm{R}$

56794 Lima Springwood............. - M

56795 Limerick Lad 2d........... R R

56796 Limoni ......................

56797 Lincoln

56799 Linwood Starlight ..........

56800 Little Cottonwood.......... R R

56803 Little John........................ R

56804 Little Short................... R

56806 Liven Wheeler................. Ky I

56807 Lizzie C's Duke............ - L R

56808 Lizzic's Duke............... R R

56809 Lizzie Nell's Duke.......... - M

56810 Lizzie's Perfection............. - T

56811 Lloyd ............................

56813 Locomotive

56815 Lofty ........................... Ky I

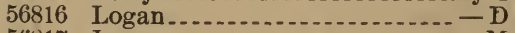

56817 Logan ....................................

56818 Logan

56819 Logan ............................

56820 Logan

$56: 321$ Logan of Dawson....... - M

56822 Lone Jack 2d....................

56823 Longfellow ................. D

56824 Longstreet ................. Ky I

56825 Longstreet..................

56\$27 Lord Alvin .............. - R R

56831 Lord Baron

56837 Lord Burk $2 \mathrm{~d}$

56839 Lord Byron .................... Ky I

56840 Lord Calliphron

56841 Lord Cavendish ................ T

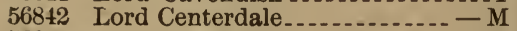

56845 Lord Clarendon

56848 Lord Duchess Airdrie $2 \mathrm{~d} . . . . . .-\mathrm{C}$

56849 Lord Duchess Airdrie Nell..... - C

56850 Lord Duke................. R R

56851 Lord Fairfield ..................

56856 Lord Hainault...............

56857 Lord Harry ...................... S S

56859 Lord Kent...........................

56861 Lord Letton.............................

5 t862 Lordley ....................... P S

56863 Lord Littleton..............

56865 Lord of Lorne................

56866 Lord of Lorne

56867 Lord Lorn

56869 Lord of Maine Valley ........ $-\mathrm{R} R$

$568 \% 02 \mathrm{~d}$ Lord Marquis of Miaini .......M

$568 \pi 1$ Lord Mayor 2d............. - R R

56875 Lord Orontes..........

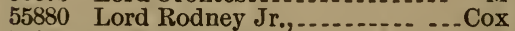

56881 Lord Roseberry .............. D

55883 Lord Shelburn.............

56884 Lord Shelburn $2 \mathrm{~d} . . . . . . . . . .-\mathrm{P} S$
56885

56886

56887

56888

56889

56890

56892

56893

56894

56896

56897

56849

56902

56903

56904

56905

56906

56907

56909

56910

56911

56912

56913

56914

56915

56916

56917

56918

56919

56923

56924

56925

56926

56927

56928

56929

56930

56931

56935

56936

56937

56938

56940

56941

56942

56943

56944

56945

56946

56947

56948

56949

56950

56951

56952

56954

56955

56958

56959

56960

56962

56963

56464

56965

56967

56968

56469

56970

56971

56972

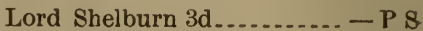

Lord Shelburn 4th........ - Ky I

Lord Shelburn 5th............ - M

Lord Shelburn $6 \mathrm{th}$

Lord Shelburn 7th.........

Lord Sbelburn 8th .......... P S

$3 \mathrm{~d}$ Lord of Spring Hill........ - T

4th Lord of Spring Hill

Lord Strawberry ..................

Lord of the Hills ...............

Lord of the Realm.................. Cox

Lord Wallflower 4th.......... - M

Loren . ......................

Lorenzo Dow

Loretus ........................... Cox

Lottie's Duke.....................

Louan Duke................. R R

Louan's Duke $2 \mathrm{~d}$

Louan Duke 2d of Calumet_ - $R$

Louan D. of Walnut Grove.. - $R$

Louan George............. $-\mathrm{R} R$

Louanjo ...................... R

Louan's Lad....... R R

Louan Lad............... R

Loudon Airdrie

Loudon Airdrie Duke 4th ..... - D

'Loudon Airdrie D. 5th ..... Woods

Loudon Boy -............

Loudon Chief.............. $\mathrm{L}$

2d Loudon D. of Oakdale

2d Loudon D. of Rush ......... L R

Loudon Duke of Sonora....- $R$ R

Loudon D. of Weston -.... - R R

Loudon D. of Woodside...- $R$ R

Loudon $D$. of Woodside $2 \mathrm{~d}_{-}-\mathrm{R} R$

Loudon D. of Woodside 3d. - R R

Loudon D. of Woolworth... - R R

Loudon Gem ....... R R

Loudon Sharon D..- Milk Spring

Louisa's Duke.................. M

Louise's Duke...............

Lowland Duke............

Lucifer

Lucille's D. of Gooduess ... - R R

Lucky Duke $2 d$............ Ky I

Lucy's Duke ..............

Lucy's Princeton 4th

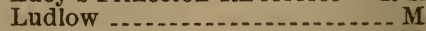

Lummix Bub...............

Lyon Duke................ Ky I

Macbeth Clydo 4th

MeCallough .................. C

MeDonald

McGregor-.........

McGregor...................

MacGregor of Elmwood...- $-\mathrm{L} R$

Mac...................... $-M$

Madriver Valley Chief..... - Ky I

Madriver Valley Chief $2 \mathrm{~d} . .-\mathrm{Ky}$ I

Magenta's Duke...............

Magician ................. $\mathrm{L} R$

Magnum Bonum....... Cox

Maid's Baron ............... $-\mathrm{R} R$

Major......................

Major ......................... T

Major

Major 3d..........................

Major 3d.................. - R R

Major Airdrie......................

Major B .................. Cox 
$569 \% 4$

56976

56977

56980

56982

56933

56984

56985

56986

56987

56988

56989

56990

56991

5699.2

56993

56994

56998

57000

$5 \% 001$

$5 \% 002$

5\%003

57004

57005

5\%006

$5 \% 007$

5т008

57009

5ช010

$5 \% 011$

57012

$5 \% 014$

$5 \% 015$

5 \%016

5\%017

$5 \% 019$

5 r020

57021

$5 \% 022$

57023

57024

57026

57027

57028

-57030

57031

57032

$5 \% 033$

57034

57035

5 r037

57043

57045

57047

57048

57049

5ช0อั0

5 \%051

57053

57054

57057

57059

57060

57061

57062
Major Bates 2d

Major Cantrill......M

Major Gwynne......

Major Logan............ R R

Major Mack...............

Major Miner..... Cox

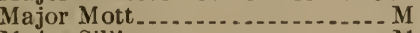

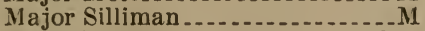

Major Vinton................... Cox

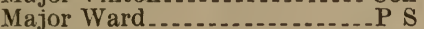

Makobo........ D

Malcolm.............. R R

Mal Quinn ............... R R

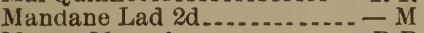

Mango Mazurka ........... $\mathrm{R} R$

Mankato.......... Ky I

Maple Earl 3d......... $R$

Mapleshade ................. R

Marcehill ................ Cox

Marcus ............ R

Marcus ....................

Marion .........

Marion County Lad ......... M

Marion Duke............ $\mathrm{L}$

Mark Antony ......... R R

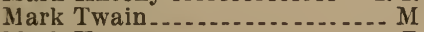

Mark Young

Marquis ........ Daisy

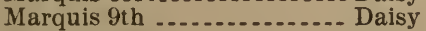

Marquis 10th............. R R

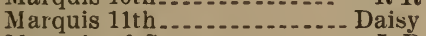

Marquis of Cottage........ $-\mathrm{L} R$

Marquis of Dundee....... S

Marquis of Edbrun

Marquis of Elmwood........ Cox

Marquis of Greenwood

Marquis of Kengrove....... $-D$

5 th Marquis of Locust Grove..... M

Marquis of Luzerne ...... $\mathrm{R} R$

2d Marquis of Pembroke.... - Cox

Marquis of Three Groves.... - L R

9th Marquis of Wilderberg - _ Cox

15 th Marquis of Wilderberg - $-\mathrm{L} R$

Marshall ................. $-\mathrm{D}$

Marshall 4th................

Marshall Victor................. Ky I

Martin Calit............

Mary's Airdrie ........... R R

Mary Ann's Duke.......... T

Mary's Constance ........ $\mathrm{P}$ S

Mary's Loudon....... R R

Master.................. $\mathrm{Cox}$

Master Ascot.............. R R

Master Harry ............

Master Miller.......

3d Master Monica......

Master Roscoe............. R

Master Sheffielder.......... D

Master Villard ........... Cox

Master Wilderberg ............ M

Matchless .......... D

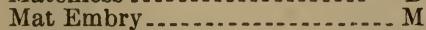

Matilda's Duke.... Daisy

Mat Offield............. Ky I

Max.............................

Maxim

Maximillian Duke............ Ky I

Maxwell....................

Maxwelton....................
57067

May 6th

57068 May Bell's Duke.............. D

57069 Mayberry ...................

57070 May Duke.................. M

57071 May Duke 4th............... R

57072 May's D. of Elmwood........ Ky I

57073 Mayflower's King ......... - K R

57075 6th Mayflower Prince ....... - M

57077 Mazoo.......... Cox

57080 Mazurka Boy ............ $-\mathrm{L}$ R

57083 Mazurka Lad ................ Ky I

57084 Mazurka Lad................. M

57085 Mazurka Lad 4th.............. Ky I

57086 6th Mazurka Lad of Elmwood-R R

57090 Melrose Lad.................... M

5rog1 Memnon

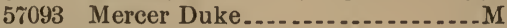

57095 Miami Duke................. M

57096 Mianui Duke.............. R R

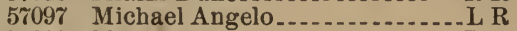

57099 Microcosm.................. R

57100 Midshipman Easy...... $-\mathrm{L} R$

5\%101 Midway ................. L R

57102 Midway Monarch...... $\mathrm{R}$ R

57103 Mike............

57104 Mike Tompson.............

57106 Millard...................... I

57107 Mill Creek Lad.............. Daisy

57108 Milford Sheriff........... - L R

57111 Milton Long.............. D

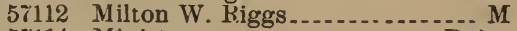

57114 Minister.................. Daisy

57115 Minnie's Airdrie.......... - Ky I

57116 Minnie's Challenger......... _ M

57118 Minnie's Duke ............. R K

57119 Minnie's Duke 2d............

57121 M. J. Durham..............

57123 Modoc.....................

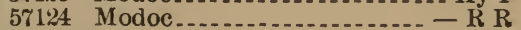

57125 Modoc Chief................. R

57127 Mogul..................... Rolla

57128 Mogul........... Ky I

57129 Mohawk.................... Ky

57130 Mollie's Duke of Athol ..... - R R

57131 Moloch Chief.............. - Daisy

57132 Moltke ................. Cox

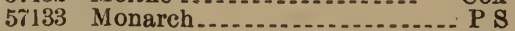

57134 Monareh

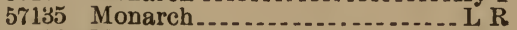

57136 Monarch

57138 Monarch of Elmwood ..... - L R

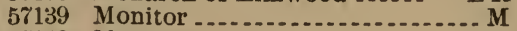

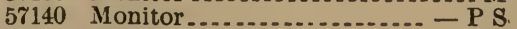

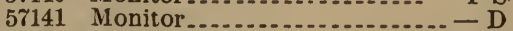

57142 Monroe Chief ..... L R

57144 Montauk Chief..........

57145 Monte Christo............ Cox

57146 Montezuma.............. M

57147 Montezuma Chief........ R R

57148 Montpelier Duke........... $\mathrm{D}$.

57149 Moody................... Cox

57151 Moore's Fidget.............. P S.

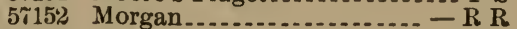

57153 Morgan

57155 Mormon

57156 Morning Star ............-Cox

$5715 \mathrm{~s}$

57159

57160

57161

Morris .

Moses

Mosley 


\begin{tabular}{|c|c|c|c|}
\hline 62 & Mountain Bo & 57258 & Oliver Opt \\
\hline 163 & Mountain Jack & 57259 & Oliver Tw \\
\hline 8164 & Mt. Sterling Duke. & 57260 & Oliver Twist \\
\hline 7165 & Mount Vernon & 57261 & O'Neal.... \\
\hline 166 & Moxom ........ & 57263 & Orange $\mathrm{B}$. \\
\hline 57167 & Mozart $2 \mathrm{~d} . .$. & 57266 & Orange Duk \\
\hline 57169 & Muscatoon Duk & 57268 & Orange Kin \\
\hline 57172 & My Boy $\ldots . . .$. & 57270 & Orange Peel \\
\hline 57173 & Myrtle Prince & 57272 & Orange Seed \\
\hline 57174 & Myrtle's Valentir & 57273 & Orcutt Duke. \\
\hline 57175 & Nabob of Maine & 57274 & Orean $9 \mathrm{th}$. \\
\hline 8177 & Napoleon..... & 57275 & Orenus $3 \mathrm{~d}$ \\
\hline 57178 & Napoleon ... & $5 \% 276$ & \\
\hline 57140 & Napoleon ... & 57277 & Orin $2 d$ \\
\hline 7181 & Napoleon Bona & 57278 & Orin $3 d$. \\
\hline 7182 & Nasby ......... & 57279 & Orion ... \\
\hline 57183 & Nathan..... & 57280 & Orion . \\
\hline 57184 & Nebeoshea Lad & 57281 & Orion 4th. \\
\hline 57186 & Nebraska Boy. & 5\%282 & Orlando .. \\
\hline 57187 & Nectar's Archo & 57283 & Orloff... \\
\hline 57189 & Neglect & 57284 & Ormshy -- \\
\hline 57190 & Nelly Bly's Dul & 57285 & Orpha's Dar \\
\hline 57191 & Nellie's Duke. & 57286 & Orphan .. \\
\hline 7192 & Nellie's Duke o & $57 \cdot 287$ & $-\operatorname{Cox}$ \\
\hline 57193 & Nelson....- & 57289 & Orphan Bu \\
\hline 57194 & Nelson ... & $57 \div 292$ & Orphan $\mathrm{L}$ \\
\hline 57195 & Nemaha Princ & 57293 & Orphan Lady \\
\hline & Nemo & 57294 & Orpheus $13 \mathrm{th}$. \\
\hline 57199 & Nero......... & 57295 & Orrmoore.. \\
\hline & Nero..... & 57296 & Orville. \\
\hline 57202 & Nettie's Duke & 57297 & - Osborn's DL \\
\hline 57203 & Nevada 5th.. & 988 & Oscar... \\
\hline 57204 & Newton ..... & 57299 & Oscar. \\
\hline 57 & Newton... & 57300 & $-\operatorname{Cox}$ \\
\hline 57207 & New Year's B & 57 & $-\mathrm{RR}$ \\
\hline 57200 & New I & 02 & Ose: \\
\hline 57210 & New Year's Diy & & Osear V \\
\hline 57: & New Year's Prid & $n 4$ & O-car Wil \\
\hline & Nicator $2 \mathrm{~d} . .$. & 57 & Oscar Wil \\
\hline 14 & Nich & 66 & Oscar Wilc \\
\hline 16 & $\mathrm{Nic}$ & & Oscar Wil \\
\hline 17 & Duke. & 8 & Oscar Wilde 20 \\
\hline & Nickel Plate. & 09 & Ossian... \\
\hline 19 & Nickel Plate. & & Othello. \\
\hline 57220 & Nicodemus.. & 57311 & Othello Harr \\
\hline 57221 & Nimrod .... & $5 ?$ & Otoe ...... \\
\hline 57222 & Nim & & $\operatorname{Cox}$ \\
\hline $5 \% 224$ & Duke.. & 14 & $\operatorname{Cox}$ \\
\hline & Nol & & \\
\hline 57 & Dick. & & $\mathrm{R} R$ \\
\hline & Oxford & & Ky I \\
\hline 57 & & & rd $32 \mathrm{~d}$ \\
\hline & $\mathrm{NC}$ & & $33 d$ \\
\hline 32 & No Gold.... & 57321 & Oxford Barm! \\
\hline & North & & $-\operatorname{Cox}$ \\
\hline 57236 & Northumberland of $\mathrm{K}$ & $5 \%$ & $\ldots P S$ \\
\hline 57237 & $2 \mathrm{~d}-\mathrm{Ky} \mathrm{I}$ & $5 i$ & $\mathrm{Ky} \mathrm{I}$ \\
\hline 57238 & berland Lad. & & Oxford Duk \\
\hline & Nor & & $-\operatorname{Cox}$ \\
\hline 57242 & Nor & & - R R $R$ \\
\hline & N. Pet & 57334 & Oxford La \\
\hline & Oakdale Prince & & \\
\hline 57247 & Oak H. S., & 57336 & $d \mathrm{~L} .4 \mathrm{~d} 3 \mathrm{~d}$ \\
\hline & Oakland Brea & & d Lad of \\
\hline & Oakley $3 \mathrm{~d}$. & 57341 & Oxford of Sprin \\
\hline 57252 & Ocean Star......... & 57344 & Oxford Wilda... \\
\hline 57253 & October Baron. & & $\ldots M$ \\
\hline & Odoacer . & & Paddy \\
\hline 5 & Oclahoma Bool & & $-\operatorname{Cox}$ \\
\hline & Old Boss. & 57351 & Punsy's Duke... \\
\hline
\end{tabular}


57352

57353

57355

57356

57357

57358

57359

57360

57361

57362

57363

$5 \pi 364$

57368

$5 \% 369$

$5 \% 3 \% 0$

57371

57372

57373

$573 \pi 4$

57375

57376

$5 \% 377$

57378

57379

57380

57381

$5738:$

57385

57386

57388

57391

57393

57394

57395

57396

573.97

57398

57349

57403

57405

57406

57407

$57+03$

57409

5 ז410

$57+11$

57412

57413

57414

57415

57419

57420

574:1

5742:2

57427

$574: 28$

5 4229

$57+30$

$57+31$

57433

57435

57436

57437

57438

57439

$57+40$

$57+41$

57442

57443

57444
Paragon Boy 3d.

Paris

Pascal

Patch .................... Ky I

Pat Donan.

Pathfinder.....................

Pathtinder.......................

Pathfinder................

Patrick .................... - M

Pat Sutherlin...........

Patsy ..............................

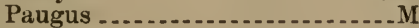

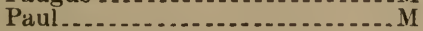

Pauline's Curtis........

Paul of Poland........... $R$

Pax................... $-D$

Paxton............

Paxton Duke......

Paxton Duke $3 \mathrm{~d}$

Pearl ................ M

Pearl Cheney

Pearletta's Airdrie Duke.... - $R$ R

Peck's Bad Boy........... - R R

Pedro Bad Boy-.....

Peeler........

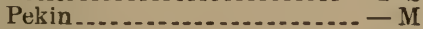

Percy

Perfection ..................... M

Perfection.........

Peri Duke 3d........... Rolla

Pete Goben ................. R

Peter........................

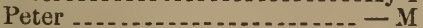

Peter Airdrie........ $R$

Peter Bee ...................... R

Peterman's Bull................ P S

Peter Perkins............. M

Petros................ Ky I

Philip Clairborne............. Ky I

Philip Phyllis............

Philip Sheridan........... $-\mathrm{L} R$

Phil L., ..................... L R Philomel ..................

Phil Sharou................. - D

Phil Sheridan.............- Cox

Phil Sheridan............ - Daisy

Phœnix ....................

Phoenix

Phounix Gem ..........

Picador ..............

Pickle................

Pierce's Marquis ............ $-k R$

Pilgrim .................. $\mathrm{R} R$

Pirate ........ Cox

Pixie's Duke ................. R

Placida's Airdrie 4th ........ - R R

Placida's Airdrie 5th ....... $\mathrm{R} R$

Pleasant ...................... $R$

Plum Grove Duke 3d....... - M

Plum Grove Duke 5th......... - M

Plum Grove Duke 6th......... - C

Plum Grove Duke 7th......... - C

Plum Grove Duke 9th......... - C

Plum Grove Duke 10th........... C

Plum Grove Duke 11th.........-C

Plum Grove Duke 12th........- C

Plum Grove Duke 13th......... .. C

Plum Grove Duke 14th.........

Plum Grove Duke 15th......... - C
57445 Plum Grove Duke 16th.......... C

5 7446 Plum Grove Duke 17th........ C

$57+52$ Plum Grove Duke $23 \mathrm{~d} . . . .-\mathrm{Ky}$ I

57453 Plum Grove Duke 24th.... - Rolla

57454 Plum Thicket Duke............ - C

57455 Plum Thicket King ...................

57456 Plumwood Jr................. R

57457 Plumwood B. J............... Daisy

57458 Plumwood B. K............ R R

57459 Plumwood B. L.......... Daisy

57460 Plumwood B. M. M...... Daisy

57461 Plumwood B. N........ R R

57462 Plumwood B. O............ R R

57463 Plumwood B. P............. R R

57464 Plumwood B. Q........... Daisy

57465 Plumwood B. R............. Daisy

57466 Plumwood B. S. ............... C

57467 Plumwood B. T.

57468 Plumwood Hero.......... R R

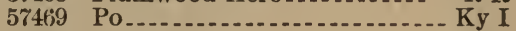

57471 Pompey

5\%472 Pony

57474 Pop Eyes.......... Ky I

57476 Portage Duke 2d............. R

57477 Portland Bates ........... Rose

57478 Potato Garden Billy............. S S

57479 Prairie Duke .......... Ky I

$57+80$ Prairie King 2d......... Multiflora

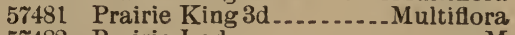

57482 Prairie Lad.................. - M

57485 Prentiss

57486

57488

57491

5749:2

57493

$5 \% 494$

57495

57493

$5 \% 497$

57498

57499

57500

57501

57502

5 รัธ03

57504

57505

57506

57507

57508

57509

57510

$5 \% 512$

57513

57514

57516

57517

$5 \pi 518$

57520

57521

57522

57523

57524

57525

$575 \div 26$

57527

57528

57529

57530

President..........................

President Arthur.

Pride of Nelson ..................

Pride of Pine Hill .............

Pride of Saginaw

Pride of Scotland .......... - L R

Pride of Sugar Grove........ - T

Pride of Winterset............ KV I

Priest....................

Prince................... P S

Prince

Prince...........

Prince............ P S

Prince........................ Ky I

Prince...............

Prince................... Cox

Prince ..........

Prince ............... $R$

Prince......................

Prince $2 \mathrm{~d}$

Prince $2 \mathrm{~d}$........................

Prince $3 \mathrm{~d}$

Prince of Airdrie ......... - Woods

Prince Airdrie............... - R R

Prince Airdrie.............. $-M$

Prince Albert.............

Prince Albert.............. R R

Prince Albert ............ Cox

Prince Albert............ _ P S

Prince Albert............ Ky I

Prince Albert ............... -

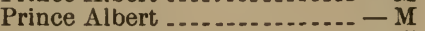

Prince Albert........

Prince Albert..............

Prince Albert ........ ........- M

Prince Alfred of Elmwood.......P S

Prince Allen ..................

Prince Allen ............... - Cox

Prince of Anoka........ Multiflora 
57531

57532

57533

57536

57537

57538

57539

57542

57543

57544

57545

57546

57547

57548

57549

57550

57551

57552

57554

57555

57559

57560

57561

57562

57563

$570 ิ 6 อ$

57566

57567

57568

57569

57570

57571

57572

57573

57574

57575

$575 \% 6$

57578

57579

57580

$5 \% 581$

57582

57583

57584

57585

57587

57588

57589

57590

57591

57595

$5 \pi 596$

57597

57599

57600

57601

57602

57606

57608

57610

57612

57613

57614

57618

57620

57621

57622

57624

57625

57627
Prince of Appanoose........ - P S

Prince Arthur................ L R

Prince Arthur............... M

Prince Arthur

ith Prince of Ashbrook .......... D

8th Prince of Ashbrook..........

Prince Ashwood ............. - R R

Prince of Balbyhaize.......... L R

Prince of Barrington 2d .....- Cox

Prince Bassanio ................ C

Prince Bismark............. P S

Prince Bismark ............... - M

2d Prince of Bourbon ............ B

Prince of Brooklyn ........ - R R

Prince Butler.............. $R$

Prince Byron ........... P S

Prince Charlie ........... T

Prince Clarendonn

Prince of Clay Ridge........... Ky I

3d Prince of Cottage Hill...... - T

Prince Ellington 2d..........- Cox

Prince of Elmira............ Cox

Prince of Erie.............

Prince Fairfield

Prince of Franklin ........ L R

Prince Geneva ................... M

Prince George .............. P S

Prince of Glenwood .....- Beauty

Prince of Golda..........

Prince of Grovedale ..... - Beauty

Prince Gwynne 3d................ T

Prince Gwynne 4th .............. T

Prince Haight .............. Cox Prince of Hanover 7 th

Prince of Hanover 8th ..........

Prince of Hanover 9th ..........

11th Prince of Hanover............

Prince Henry ................

Prince Huglett ........... Ky I

Prince John............... D

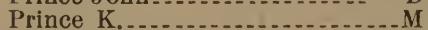

Prince of Lanark

Prince of Lenawee.......... Daisy $2 d$ Prince of Lenawee.......... Ky I Prince I.eon ................. Prince Llewellyn ............. - D Prince Llewellvn $2 \mathrm{~d} . . . . . . . .-\mathrm{M}$ 1" P.of Lumbardy Park - Multiflora $11^{\prime \prime}$ P.of Lumbardy Park-Multiflora Prince Louis ................. - D Prince Napier $4 \mathrm{th}$ Prince Napier 13th........... - M Prince of Norwich........... M Prince of Oakland .......... $\mathrm{L}$ 3d Prince of Orange......... $R$ 4th Prince of Orange............ B 6th Prince of Orange....... - Cox Prince of Plainview ........ $-\mathrm{R} R$ Prince of Riverside $3 \mathrm{~d} . . . . . .-R \mathrm{R}$ Prince Rosamond .........- $\mathrm{R} \mathrm{R}$ Prince of Silver Lake............... Princess' Duke .............. - Rolla Prince of the Luckimute ..... $-M$ Princeton of Maple Grove........ D Prince of Wayne $2 d . . . . . .-\mathrm{L} R$ Prince William -......... $-\mathrm{R} R$ Prince William $3 \mathrm{~d}^{-. . .}$ Prince W. Wiley ............ R R 22d Prince of Wyaconda........ B Proctor .................
57628

57629

$5 \% 630$

57633

57634

57635

57636

57637

57639

57640

57641

57642

$5 \pi 644$

57645

57646

57647

57649

57651

57652

57653

$5 \% 654$

57657

57658

57659

57660

$5 \% 662$

57663

$5 \% 064$

$5 \% 665$

$5 \% 667$

57668

57669

$5 \pi 670$

$5 \% 6 \% 1$

$5 \% 675$

$5 \% 676$

$5 \% 677$

$5 \% 6 \% 8$

57679

$5 \% 680$

5 7681

$5 \pi 682$

$5 \% 683$

5.684

5768.

$5 \% 687$

57688

$5 \% 690$

57691

57692

57693

$5 \% 695$

57696

$5 \% 697$

57698

57699

57700

5\%701

$5 \% 702$

$57 \% 03$

57704

57705

57706

57708

57709

57710

57711

57712

$5 \% 713$

5 ร714
Proctor Knott.

Prospect................. R

Prune Booth ................ R R

Psamtik_..................

Putnam

Pyrrbus.......................... B

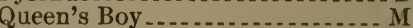

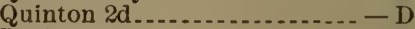

Racine.....................

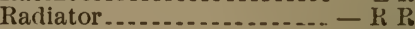

Railroader.......................

Rambler...............

Rambler........ $\mathrm{R}$

Randolph...................

Ranger ..................... Ky I

Ransom....

Rapidan................... $\mathrm{R}$

Rattler...................

Ravenswood Lad.......... $\mathrm{R}$ R

Raymond.................... Ky I

Red Alphonso....................

Red Beau ...........

Red Beauty

Red Ben............... Ky I

Red Bird .................. P $\mathrm{S}$

Red Bird .................... Ky I

Red Bird..................... L R

Red Bird .............................

Red Boy ...................

Red Boy -.............. $\mathrm{R}$

Red Boy ................ Ky I

Red Boy 2d................... M

Red Buck ...................

Redbud Prince 3d........... - M

Red Chief.........................

Red Chief

Red Cloud.................

Red Cloud

Reã Cloud....................... R

Red Cloud ................

Red Cloud.......................

Red Cloud................ - Rolla

Red Coat

Red Cross Knight............. Rose

Red Diamond............. Cox

Red Dick ...................... T

Red Duke.................

Red Duke............... - Cox

Red Duke..........

Red Duke.....................

Red Duke............. R R

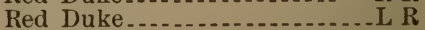

Red Duke..... - .......... - Cox

Red Duke.............. Ky I

Red Duke........ - Long Hurn

Red Duke......... P S

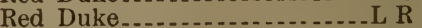

Red Duke..............

Red Duke $3 \mathrm{~d}$

Red Duke Jr., ............. - Ky I

Red Duke of Bloomfield.... - R R

Red Fox................ P S

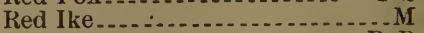

Red Jack

Red Jack..................

Red Jacket ................

Red Jacket

Red Jacket....................

Red Jacket............... R R 
57715

57716

$57 \% 17$

57719

$5 \% 720$

$577: 21$

$5772 \%$

$57 \% 23$

5\%721

57725

$5 \% 7: 27$

$5 \% 728$

57729

5 ร731

5773:

57733

$57 \% 34$

57736

57 737

57739

57740

$5 \% 741$

57742

$5 \% 744$

57745

5 746

57747

57748

57749

57750

5ั752

57753

57758

5ร760

$57 \% 61$

$57 \% 62$

57763

5\%764

5ชช65

5ั'766

$5 \% 767$

5 ร768

57769

577\%0

57771

$577 \% 2$

$577 \% 3$

$57 \% 74$

$57 \% 75$

5รส77

57780

57781

57782

57783

57784

5\%787

5 788

57789

57790

57791

5779:

5รั93

57795

57796

57797

57799

57800

57801

57803

57804
Red Jacket

Red Jacket..... . . . . . . . . . . . . . M

Red Jacket ......................

Red Jacket.................... Ky I

Red Jacket...................... L R

Red Jupiter. . . . . . . . . . . . . . . . P S

Ked King................. - L R

Red King

Red Knight.................. $R$

Red Lad

Red Lad............ R

Red Lad................ $R$ R

Red Lad ............ D

Red Major ............... D

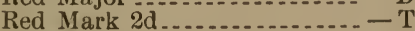

Ked Mary's Duke......... M

Redmond .............. T

Red Oak...........................

Red Oak..................... R

Red Prince................ Cox

Red Prince.................... Cox

Red Prince...................

Red Prince.................. Ky I

Red Prince...................... R R

Red Prince.................

Red Prince................... D

Red Prince of Dover........... $\mathrm{L}$

Red Prince of Maplewood... - R R

Red Ralph ................. L R

Red Ranger................ R R

Red Rock................... I

Red Rock

Red Rover............. $R$

Red Rover........

Red Rover............... Cox

Red Rover................. L R

Red Kover........................ D

Red Rover................... - D

Red Rover $2 \mathrm{~d}$

Red Rover 2d..................M

Red Royal................ Daisy

Red Sharon.

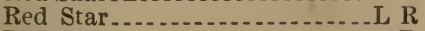

Red Star................... D

Red Star..................... R

Red Thorndale

14th Red Thorndale...............

Red Underly ....................

Red Watson.............

Referee ....................

Remus......................

Remus.....................

Remus ...........................

Remus....................

Renick................

Renown

Republic ............... L R

Rescue........................... I

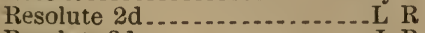

Resolute 3d...................... L R

Restituimus..............

Reuben ........

Reward.................

Rex

Rex Magnus......................

Rhomboid.................... R

Richard....................

Richard ...................

Richard S.,............. R

Richard S. $2 \mathrm{~d} . . . . . . . . . .$.
57806

57807

57808

57809

57810

57811

57812

57814

57816

$5 \% 817$

57818

57819

57820

5\%821

57822

57823

57824

57826

57827

57828

57829

$5 \% 830$

57831

57832

$5 \% 833$

57834

5783.

57836

57837

57838

$5 \% 839$

57840

57841

57812

57843

57844

57845

57846

57848

57850

57854

57855

57856

5785ว

5\%858

57860

57862

57863

57864

57865

57866

57867

57868

57869

57870

57872

$5 \pi 873$

57875

57876

57877

57878

57879

5 ז880

57881

57882

57883

57884

57885

57886

57887
Richerson

Richland Prince................. M

Richmond 2d................ D

Richmond 3d.....................

Richmond Boy ........... L R

Rifleman..................

Rival.......................... Ky I

Roan Airdrie.............. D

Roan Boy.............

Roan Boy ........................

Roan Boy................

Roan Boy ..................... $-M$

Roan Boy

Roan Chief .................. - M

Roan Duke.............. Cox

Roan Duke.............. Cox

Roan Duke.............

Roan Duke...............

Roan Duke................... R R

Roan Duke $2 \mathrm{~d}$

Roan Duke 2d................ - M

Roan Duke of Bunceton......- D

Roan Duke of Prattsville........ Cox

Roan Eagle................

Roan Frosty............... R

Roan Jim .................... S

Roan Lad................

Roan Lad ................ R

Roan Major.............. $\mathrm{R}$

Roan Monarch................ Ky I

Roan Napoleon ............. - Cox

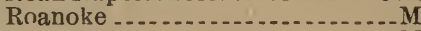

Roanoke .................... M

Roan Prince...................

Roan Prince................ $\mathrm{R} R$

Roan Prince..............................

Roan Prince......... Ky I

Roan Prince............ P 8

Roan Prince........... Rolla

Roan Springwood....... M

Roany .................... Daisy

Robt. Airdrie ........... Daisy

Robt. Colling ............ R R

Robt. Lee...............

Robt. Miller................

Robt. Sherman $2 \mathrm{~d}$

Robt. Wiley .....................

Robin Adair......

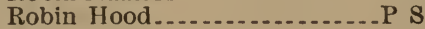

Robin Hood.................

Robin Hood ........................

Rob Roy......................

Rob Roy

Rob Rny ................

Rob Roy.........

Rocket.................. $R$

Rock Island Duke...........

Rock River Lad 79th........... P S

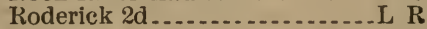

Roderick Dhu

Rodolph...............

Rodolph.......

Roland ...................

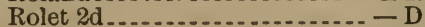

Rollo ..............

Roman Duke.......

Roma's Richmond........ - Ky I

Romeno Duke................ $-M$

Romeo Oxford

Romulus .............................. 


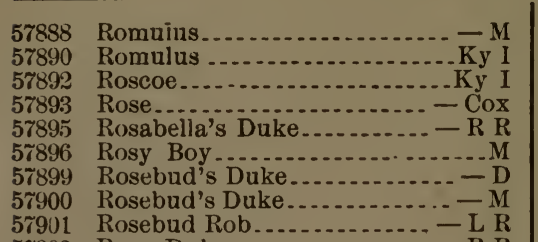

57902 Rosy Duke...................... R R

57903 Rose's Duke ................... - M

57404 Rose Duke........................ - M

57907 Rosa Duke of Maple Mound - - D

57908 Rose's Filigree Lad.............. R R

57911 Roseman

57914 Rosamond Duke

57916 Rosamond Duke 2d.........- - R R

5 7917 Rosamond Duke 2d.........- R R

57918 Rosy Prince

57919 Rose's Prince................-M

57920 Rosy Royal's Duke............. - M

5 5r9:2 Rose Thorn's Underley ...... - L R

57923 · Rosewood Duke...............Ky I

$579242_{2 d}$ Rosewood Duke.............Ky I

57925 Rover ......................... P S

57927 Rover of Grandview............ Ky I

b7929 Roxie's Lad .....................Ky I

57930 Roy ........................... R R

57931 Roy

57932 Royal -...................- Cox

57934 Royal Airdrie.................. R

$5 \pi 936$ Royal Angelica.............- - R R

$5 \tau 941$ Royal Bob..................... - D

57942 Royal Bob Ingersoll..........-Ky I

57943 Royal Bourbon .............- - Rose

57944 Royar Commander..........-- Ky I

57945 3d Royal Doctor W.,............ B

57946
$5794 \tau$

57948 Royal Duke

57949 Royal Duke................- R R

57951 Royal Favorite...............- L R

$5 \imath 953$ Royal George....................

57954 Royal George.................R R

57956 Royal George ................- Cox

$5 \pi 958$ Royal Huntingdale..........- - R R

57459 Royalist Duke ..............- - Cox

$5 \imath 962$ Royal Ned ...................... Ky I

57963 Royal Oxford Jr.,

57964 Royal Perfeetion...............-D

57965 Royal Prinee................- - L R

57966 Royal Prinee....................... M

57967 Royal Prine of Evergreen.... Ky I

57970

57971

$5 \pi 972$

$5 \pi 974$

$5 \pi 975$

57976

57977

57978

57979

57980

57981

5798:

$5 \pi 983$

57981

57985

57986

57988
57989

57990

57991

57992

57993

57994

57995

57996

57997

57998

57999

58000

58001

58002

58003

58004

58005

58006

58007

55008

58009

58010

58011

58012

58014

58015

58016

58017

58018

58019

58020

58021

58022

58023

58024

58025

58026

58028

58030

58033

58034

58035

58036

58037

58039

58040

58041

5804:2

58043

58044

58045

58047

58049

58050

58051

58052

58053

58054

58055

58056

58057

58058

58059

58060

58062

58064

58065

58066

58067

58068
Rush W. Wagner..............

Sabine .......................

Sachem $3 \mathrm{~d} . . . . . . . . . . .60$

Sailor Boy ................... - Cox

St. Ayr..................

St. Bernard..................... M

St. Charles ........................ T

St. Charles Star........... Rolla

St. Clair

St. David Bloomfield ........-Cox

St. Elmo......................... R

St. Elmo

St. Elmu ............................

St. Elmo 2ad.................

St. Elmo 3d............... - P S

St. Elmo Chief..................... R

St. James.................. - R R

St. Louis......................

St. Louis.................... R

Salamander $2 \mathrm{~d}$

Salem ....................... S

15th Salem Prince..........- $\mathrm{R} R$

Sam ...............................

Sam Houston ...................

Sammy Wright................ $R$

Sam Pack ..........................

Sam Patch

Sam Porter.......................

Sam Queen

Sam Rundall...................... M

Sam Renick............... $\mathrm{R}$

Samson .....................

Sam Steadman .................

Sam Tilden ....................... M

Sam Tilden................. M

Sanuel Blooinfield ..........-Cox

Sandusky Chief ................ D

Sangamon King ............. - Cox

Santa Fe.......................... Ky I

Santague.......................

Saxon Keith of Watertown....L R

Schenectady Chief..........-- L R

Schuyler Lad 2d............. - M

Sciota Redman................ - D

Scipio of Maine Valley .....- $-L R$

Scotland ................... - M

Scott ....................... Rolla

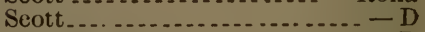

Seottish Lad ................. D

Scott Law ................... $L$

Scout ….......................

Sebastian ............... Ky I

Secretary Folger ............. M

Security ...................... Cox

Sedley

Sedgwick

Sedgwick Duke............ $\mathrm{R}$ R

Selsor Duke .................. Rose

Senator

Senator B.,.................... D

Senator Bonth $2 \mathrm{~d} \ldots \ldots . . . . . . . . . . P S$

Senator Booth 4th ................P S

Senator Booth $6 \mathrm{th}$.................... II

Senator Hoar...............- $-\mathrm{L} R$

Senator Perkins.............- $\mathrm{R} R$

Seneca....................... L R

Seramis.......................

Sergeant......................- $-\frac{L}{R}$

Sergeant ...................... 
58070 Seward Chief 4th............ D 58071 Seymour 58072 Shamrock.................. Wrong 58073 Sharon ............................. S 58074 Sharon Airdrie 2d 58077 Sharon Boy 2d............ R R 58078 Sharon Boy 3d.......... $\mathrm{R}$ K 58081 Sharon Charlie............. - P S

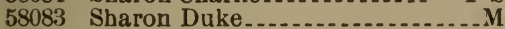
58084 Sharon Duke 2d......... R R 58089 Sharon Geneva's Duke....... R R 58093 Sharon Rose Lad................ R R 58094 Sharon of Wells................. 58095 Sharpsburg Duke.......... D 58096 Shattuck................. 58097 Sheldon 58098 Sheridan 58099 Sheridan....................... 58100 Sheriff 58101 Sherlock ............. R R

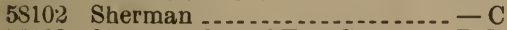
58103 Sherman Jr., of Elm Grove_ - R R 58104 Sherman's Pride ...................

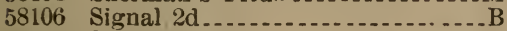
58107 Silas Gale $3 \mathrm{~d}$ 58108 Silkie's Gloster............ Cox 58109 Silver Cloud................. - Cox

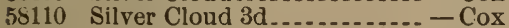
58113 Silver King ................ - Cox

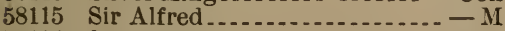
58116 Sir Alphonso 58117 Sir Arthur............... D 58118 Sir Arthur........ 58119 Sir Booth................ Rose 58121 Sir Charles................ Cox 58122 Sir Colin Campbell ............ Cox 58124 Sir Edward............. Rolla 581:26 Sir Garnet.................... D 58129 Sir George.................... I 58130 Sir George 58131 Sir Henry of Empire 4th..... - D 5813:) Sir Henry of Empire 5th..... - D 58133 Sir James of Balsam ........ - L R 58141 Sir Leonard............... $-\mathrm{L}$ R 58142 Sir Richard..................... S 58143 Sir Walter............... S S 58144 Sir William................... R 58145 Sitting Bull............... 58146 Smith's Duke................ S 58147 Smuggler.................. Ky I 58148 Snowball of Wetherfield ....- Cox 58149 Snowflake ................. P S 58150 Soapstone.........................

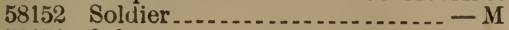
58153 Solon 58154 Son of Lady Chilton......... - M 58156 Sonsie Boy ............... Beauty 58157 Sovereign...............

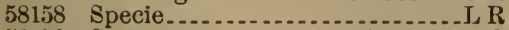
58159 Spectator......... Spencer Duke................ Spot Duke.............. R Spring Duke.................... $R$

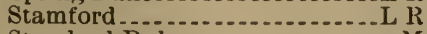
Standard Duke.............. Star Duke................ Daisy Star Duke........................

58175
58176

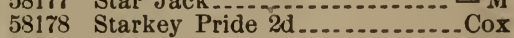

58179 Starlight..................... P S

58180 Starlight.................. R R

5818 Starlight's Marquis............ T

58183 Star Manor................ R R

58185 Star Prince................ P S

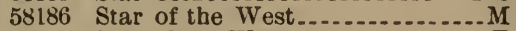

58187 Star of the West............ T

58188 Star of the West Jr.,......... Ky I

58189 Star Victor.............. R R

58190 State of Missouri's Duke....... . M

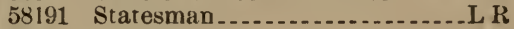

58192 Sterling Lad ...................

58194 Stibb

58196 Stonewall 2d............. P S

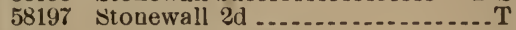

58198 Stonewall Jackson ........ S S

58199 Stonewall Jackson ........... S S

58200 Storm........................

58203 Success ..................... R

58205 Sue's Mariner............. M

58206 Sultan ...................... Ky I

58207 Sultan Dekalb............. T

58208 Sultan of Maine Valley......... Ky I

58210 Summer Cloud..... ......... - M

58212 Sumner

58213 Sunbeam's Duke.............. - D

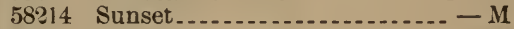

58215 Sunset.................... M

58216 Superb ..........

58:217 Surprise..................... B

58218 Surprise Edward ................ Ky I

58220 Smart's White Bull .............

58222 Sylvanus................. Cox

58:23 Sylvester.......... R R

58:24 Symmetry ................

58:25 Symmetry ................ R R

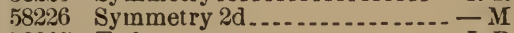

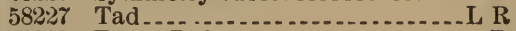

58228 Tama Duke ................. D

58:29 Tam Glenn 2d ................ S

582:30 Tarkio Chief................... S

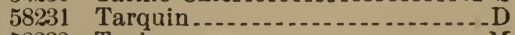

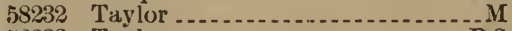

58233 Taylor

58234 Taylor Duke.............. $\mathrm{R}$ R

582:37 Tecumseh................. L R

58239 Telephone.....................

58241 Tempter

58:213 Texas .............

$5 \$ 246$ Thaddeus....

58247 Thaddeus .....................

58:248 Thaddeus Peabody ....... - R R

53251 The Clerk................

5825 Thomas.................. L R

58254 Thompson .............

58255 Thorndale Duke........... C

58256 Thorndale Noble..... Daisy

58258 Tidy's 4th Duke ............ - P S

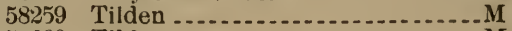

58:60 Tilden ...........................

58261 Tilden..............

58262 Tilden

58264 Timothy Tugmutton...... - L R

58265 Tip Bedford.............

58266 Tippecanoe.................. Ky I

58267 Tippecanoe

58268 Tippecanoe Ranger.............. D 
58269

$58: 20$

58271

$582 \pi 2$

58273

58275

58276

58277

$58: 78$

58:279

$58: 280$

58281

58282

58283

58284

58285

$58: 286$

$53: 287$

$58: 288$

58289

$58 \div 290$

58291

$58: 92$

58293

58:294

58:295

$58: 96$

58297

58298

$58: 99$

58300

$58: 304$

58305

58306

58307

58308

58309

58310

58311

58312

58313

58315

$5 \times 317$

58318

58319

58320

58321

58322

58324

58326

58:327

58:329

58330

58331

58332

58338

58339

58340

58342

58343

5834 วิ

58347

58348

58349

58350

58351

58352

$5835: 3$

58354

58355
Tip Top. $\mathrm{R} R$

Titus

Tobe Hinick

Tobias of Elkheart............. D

Toddy ........................... P S

Tom

Tom .......... P S

Tom Benton

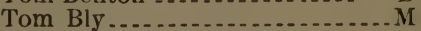

Tom Corwin

Tom Grimes

Tom Hopkins ............... - D

Tom Johnson .................. L R

Tom Johnson

Tom Kanter ............... Cox

Tom Martin . .

Tommy Bray ...........

Tom Ochiltree............. Ky I

Tom Paine............... R

Tom Power............... P S

Tom Robinson

Tom Scott ........... P S

Tom Thornapple....... Cox

Tom Thumb............... T

Tom Tyson......... P S

Tom IVard ............

Tom Young -.............. $\mathrm{C}$

Tornado _............ T

Towneley ............... $\mathrm{R}$

Townsend Duke of Sharon... - M

Tracy _.................... P S

Triumph ............. L

Trolius....................... R

Troy Baronet =............ $\mathrm{R} R$

Trump................

Tubal Cain ...... P S

Tucker.......................

Tupper........

Turco

Turkey Creek Tommy

Turuey's Duke............. - R R

Twilight .................. Daisy

Uberhale

Uchee Chief.............

Uncle Abe...................

Uncle Tom

Uncle Tom.....................

Uric of Riverview...........

U. S. Grant............... L R

Valentine Duke..........

Valley Baron................

Valley Duke...................

Valley Duke....... Milk Spring

Valiswa .............. T

Vandyke....................... M

Van Prince $2 \mathrm{~d} . . .$.

Van Snyder.............. Cox

Van Wert Jupiter........ L R

Vasco

Vassar Prince -.............. L R

Veteran .....................

Viceroy ..............

Victor

Victor

Victor ....................... $\mathrm{k}$

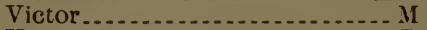

Victor....................

Victor

Victor
58356

58357

58361

58369

$583 \% 0$

58371

58372

58373

58374

58375

58382

55384

$5 \$ 385$

$5 \$ 386$

58387

58389

58390

58391

58392

58393

58395

58396

58397

$5 \$ 398$

58399

$5 \$ 400$

58401

58403

58404

58405

58406

58407

$58+10$

$58+12$

58416

$58+18$

58419

58420

5842:3

$584: 24$

58425

58426

58427

58428

55429

58131

$58+32$

58433

58434

$5843 \pi$

58438

58439

$58+40$

$58+42$

$58+43$

58444

$58+45$

$5 \mathrm{~S}+46$

58147

58448

58449

58451

58452

58453

$5 \$ 454$

58455

58456

$58+57$

58458

58459
Victor $2 \mathrm{~d}$

Victor $22 \mathrm{~d}$

Victor Hugo

Victora Duke of Mayville $2 \mathrm{~d}_{\ldots} \ldots$ - I

Victora D. of Mayville $3 d_{-.}-L R$

Victora Prince 2d..........- - Rolla

Victora Prince 3d.......... - Rolla

Victora Prince 4th ........- - Rolla

Victor 2d of Springdale......... T

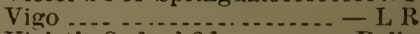

Violet's Oxford $2 \mathrm{~d}$......... Rulla

5th Viscount of Glen Leven ... - - C

Volkova Lad............. M

Vnltaire ........................... S S

Waddler's Loudon Duke ... - $\mathrm{R}$ R

Waldeck ............ D

Wallace ..............

Wallace............... Ky I

Walter........

Walter O ............. D

Walter Vance..............

Walton $2 \mathrm{~d} . . . . . \ldots \ldots$

Wamsutta..........................

Wanderer.................. D

Ward

Ward

Warden Noble 4 th....

Warren

Warren Duke 2d.............. Ky I

Warrior ..................

Warsaw Duke....... P S

Washington............. D

Waterloo 7th ............. Cox

Waterloo Bell Duke 4th ....- R R

Waterloo Prince........... Ky I

Waterville..................

Waterville.................... D

Waubansie Duke......... - L R

Waverly's Earl ..... - Milk Spring

Waxenhorns Duke............ P S

Wayne ...................

Vebster

Weehawken Prince... ....... - C

Welcome Boy .................. M

Welcome Duke 3d....... D

Well Duke 3d............... R R

Wellington.............................

Wellington

Wellington........... R

Wendling .... . .......... - Rolla

Wesley

Western Chief............ L R

Western Duke................ L R

Western Duke........ D

Western Lad......... - D

Western Star................. II

Western Star ........ D

West view Duke............. Ky I

Westville .................. M

Whicher

White D. of Fort Cribbs...... - M

White Prince................ - D

White Star...................

White Stockings........... - L R

Whitewacer Boy ............ D

Whitewater Duke $2 \mathrm{~d} . . . .$.

Wichita........... $\mathrm{R}$

Wiggins ........... Cox

Wild Calf............... - L R

Wild Clover ............. - Rolla 
58465

58467

58469

58171

58472

58473

58476

58477

58478

58480

58482

$5 \mathrm{~S} 484$

58485

58489

58491

58492

58493

58498

58499

58501

58503

58504

58505

58506

58507

58510

58511

585513

58514

58515

58516

58519

58521

585222

58523

58524

58525

58526

58527

58.528

58529

58530

58532

58533

58534

$585: 37$

58538

58539

58541

58542

58543

58544

58545

58.546

58.448

58.51

$585 \% 6$

58716

58717

58718

58721

58723

58724

58725

58726

58727

58728

58729

58730

58731
Wild Eyes Prince ........... - Cox Wild Eyes Springwood......... Cox Wild Eyes Wellington..... - Rose Wild Oscar...................... Cox Wiley Wiley Acklam............ $\mathrm{R}$ Wiley Grant .................. D Wiley Wild Eycs............. $\mathrm{R} R$ Willie ..................................

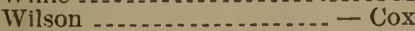
Windom ............................ Winfield Wink................ Rose Winter Grass ................. R Wolfenden ................ - Cox Woodbine's Duke........ Woodburn ........... P S Woodland Duke......... R Woodlawn Duke $2 \mathrm{~d} . . . . . . .-\mathrm{R} R$ Woodpecker Ridge......... - I, R Yates ..................... $\mathrm{L}$ R Yorkshire Duke.............. L R Yorkshire Lad ............... L R Young Arthur Young Bellvelle Lad............. R Young Brainard ............... M Young Butterfly ........... D Young Duke ................ - D Young Duke of Cedar........ - D Young Duke of Plinnwood....- - C Young Duke of Winfield ...- $R$ Young Fortune ............ L R Young Fredirick -.......... - L R Young Grant ................. - D Young Harrison ........ Daisy Young Hearne ......... $R$ Young Jonah ............. - P S Young Jugurtha.............. R R Young Juniter................ D Young Lord .................... M Young Loudon Duke........ - L R Young Major............. _ L R Young Marlow................ D Young Martin .......... Ky I Young Marvel ..................... B Young Mazurka.......... Young Minister of Hainesville.... M Young Oxford ................ L R Young Rockingham.......... L R Young Royal Briton.......... Ky I Young Ruby ................... Young Schroeder.......... Young Viscount............... Young Wellington Zach Taylor Zero Conishead Duke 3d....... - Rolla Aaron.......................... S Abar's Bull............ S Abel Sharon...................... S Accident ......... P S Adam .............. Multiflora

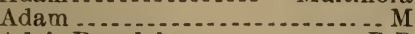
Ada's Rosedale.......... $R$ Addison Chief............... - C Addition ......................... I I Adolph ........................... Adolphus................. Advance....................... Ad Walker................... Ky I
58732 Agency Duke ................. M

58738 Airdrie's Chief .............. - D

58739 2d Airdrie Climax.............. B

58743 Airdrie of Helendale.......... - T

58745 Airdrie Lad................ - R R

58746 Airdrie Lad............... R R

58749 Airdrie Prince................ $M$

58750 Airdrie Prince $2 \mathrm{~d} . . . . . . . . .-$ Rose

58751 Airdrie Red................ Daisy

58753 Airdrie Renick of Finley - - Woods 58755 Airdrie Sharon $2 \mathrm{~d} . . . . . . . . .-\mathrm{R} R$ 58756 Albert Ross................. $\longrightarrow \mathrm{M}$ 58757 Albia...................... 58758 Alkion.......................... R

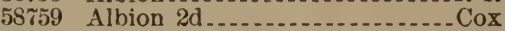
58760 Albion of Cottage 58761 Albo ............................. Ky I 58762 Alexander $\mathrm{H} . . . . . . . . . . . . . .2 \mathrm{~S}$ 58763 Alexander Hamilton .......... Daisy 58764 Alexander J..................... M 58765 Alfred .......................... $58 \pi 66$ Alfred Batès ..................... 58767 Alfred Chief ................. 58768 Allen 58770 Almont $2 \mathrm{~d}$ 58771 Almont's Duke........... M 58772 Alonzo......................... Ky I 58773 Alpha........................ M 58774 Altona's Duke .............. Cox 58 \%75 Amboy .................... P S 58776 Ambrose .......... 58777 Anes......................... 58778 Annie's 2d Duke............ - M 58780 Antonio........................... 58781 Apelles ................. R R 58784 Arabella Duke .......... - Beauty 58785 Archibald .................. L R 58787 Aristocrat ................ 58788 Arkansas Duke............. 58789 Arlington ................

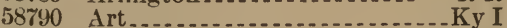
58791 Arthur.................... R

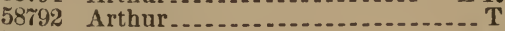

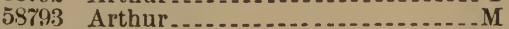
58797 Asshur................... R R 58798 Athelstane $2 \mathrm{~d}$ 58800 Atreus ............... R R 58801 Auctioneer 58802 Auditor 2d................... L R 58803 August 58804 Aurelia's Duke............ - R R 58805 Australia's Gwynne ........ - Cox 58806 Baden Duke 2d.............. - L R 58807 Baden Duke 7th........... - Rolla 58808 Baden Duke 11th.......... - Rolla 58812 Barmpton Duke........... - P S 58814 Barmpton Joe.............. - R R 58815 Barnaby Rudge............. 58816 Barney ........................... 58817 Barney ................................ 58819 Baron .................. Cox 58820 Baron Adelaide ........ 58822 Baron Bloom ............ - Cox 58823 Baron Booth.............. - Cox 58824 Baron Booth of Maplewood. - R R 58825 Baron.Bright Eyes 2d ..... - Rolla 588:26 Baron Bright Eyes 4th ..... - - Rolla 58828 Baron Cecil 5th................. Ky I 58829 Baron Cecil 6th............ 58830 Baron Cecil 7th............. R R 
58832

58833

58834

58835

58836

58838

58839

58841

58843

58345

58847

58852

588.53

58854

58855

58857

58869

58877

58878

$58 \times \div 9$

58880

58881

58884

58885

58886

58887

58888

58889

58890

58891

5889:2

58893

58894

58895

58898

58899

58900

58901

58902

58.903

58904

58905

58906

58908

58911

58912

58914

58916

58917

58918

58919

58920

58921

58922

58924

58925

58926

58927

58928

58930

58932

58933

58934

58935

58936

58937

58938

58939

58940

58941
Baron of Eaton

Baron of Elk Farm

Baron $2 d$ of Elk Farm.......

Baronet.................... $\mathrm{L}$

Baronet..................... $\mathrm{L}$

Baron Frantic ........ Rolla

Baron Frantic 4th ..........- Rolla

Baron of Geneva $3 \mathrm{~d}$

Barou Grissim .......... Rose

Baron Gwynne 3d........ - Rolla

Baron's Lad 2d.......... $\mathrm{R} \mathrm{R}$

Baron Oakland $2 \mathrm{~d}$

Baron Princeton $2 \mathrm{~d}$......... P S

Baron Renick Jr., ........... Ky I

Baron Rose ..................... D

Baron Taylor ............ - L R Barrington Lord of Webster - L R Basil Duke _.......................

Basil Duke 3d

Bates ..................

Batcs Duke...........................

Bates Dnke.....................

Beaumont's Last. .........- M

Beauty's Duke ....... - Multiflora Beauty's Grand Duke........... R R

Beaver 2d.........................

Beazly

Beckham ...........

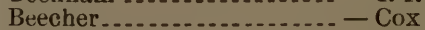

Belina Duke ........ M

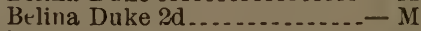

Belina Duke 3d..................

Belle Duke.................. - M

Bell's Duke.................. Ky I

Bell Duke 2d............. $\mathrm{R} R$

Bell Duke 2d................... - M

Bell Duke 3d.............. Rolla

Bell Duke 3d................. - M

Bell Duke of Clyde......... Cox

Bell Duke D................ - Cox

Bell Duke of Elm Grove....... L R

Bell Duke of Liberty ........ - Cox

Bell Duke of Manhattan.... - R R

Belle's Lad ................ P S

Belmont ............ Daisy

Belmont ............ D

Belvedere

Belvedere Tommy ............... Cox

Ben ...................... R R

Ben B. Bigstaff .....

Benbolt

Ben Bolt

Ben Butler.................

Ben Butler.............

Ben Butler................. $\mathrm{R}$

Ben Butler.......... Ky I

Ben Butler.................

Ben Butler................. $\mathrm{R}$

Ben Butler.................... Ky I

Ben Butler......................

Ben Butler..........

Ben Franklin

Ben Hill..........................

Ben Isaacs.................. L R

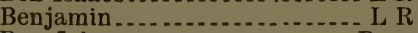

Ben Johnson .

Ben Lomond ............ Daisy

Ben Perkins................ P S

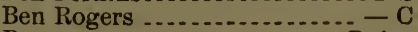

Benton ....................... Daisy
58943

58945

58946

58947

58948

58949

58950

58951

58952

58953

58954

58956

58957

58958

58961

58962

58963

58964

58965

58966

58967

$5896 \mathrm{~s}$

58971

58972

58973

58974

58975

$5 \$ 976$

58978

58979

58950

58981

58982

58983

58984

58985

$5898 \pi$

58988

58989

58990

58991

58992

58993

58994

58995

58996

58997

58998

58999

59002

59003

59004

59005

59006

59007

59008

59009

59010

59011

59012

59013

59014

59017

59018

59019

$590: 30$

59021

59022

590:23

59024
Ben Wiley _.................. Cox

Berkshire Boy ............ P S

Berkshire Grey ............... - L R

Berlin Oxford...............

Beverwyck 2d......................

Bill Nye .....................

Bill Wiley

Billy

Billy C. .

Billie Chatham............... Ky I

Billy Pierce.............. Cox

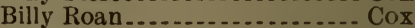

Birthday ................ R R

Bismarck

Bismarck ...... Ky I

Black Hawk

Blanche's 2d Duke..........

Blizzard..................... Ky I

Blockey ..................... Ky I

Blucher ...................

Blucher....................

Blythedale Champion 11th.. - L R

Blythedale Champion 12th..- - L R

Blythedale Champion 13th ..- - L R

Blythedale Champion 14th .. - L R

Blythedale Ernesty ............. R R

Blythedale Ernesty $2 \mathrm{~d} . . . . . . . R \mathrm{R}$

Blythedale Sharon 3d........ D

Bob..................... L R

Bob Allen ..................

Bob Baker...........

Bob Lee ................

Bob Lee.....................

Bob O'Link....... P S

Bob Ridley

Bolivar....................

Boliver Carter........ II

Bombast.................. Cox

Bonanza ............ D

Bonanza ....................

Bond's Duke of Elmhurst........ D

Bonnie Duke 2d ..............

Boo.........................

Booth of Waterford

Boss Andy .................. D

Boston .............

Boston Duke......

Bothwell Chief.......... $\mathrm{R}$

Brack ...................... Ky I

Brampton.....................

Bravo....................... D

Bravo

Breadalbane $16 \mathrm{th} . . . . . . . .5 y$ I

Breadalbane 17th

Breadalbane 19th

Breadalbane 20th............ Ky I

Brcadalbane 21st ..........

Breadalbane $22 \mathrm{~d} . . . . . . . . . . . .5$

Breadalbane 24th............

Breastplate Louanjo $3 \mathrm{~d} . . . .-\mathrm{L} R$

Bride's Airdrie................. Rose

Bridegroom .................. Co:

Bright Duke...............

Bright Eves Duke...............

Bright Lad................. Rolla

Brilliant.................... Rolla

Bristol Duke.............. Ky I

Bristol Duke ............... $-\mathrm{L}$ R

Britannia Duke ................ L R 
59026 59028

$59(129$

59030

59031

59033

59036

59037

59038

59039

59041

59043

59045

59049

59050

59052

59053

59055

59059

59064

59065

59067

59068

59069

59070

59071

59073

59074

59076

$590 \div 7$

$590 \div 8$

$590 \% 9$

59080

59081

59083

59084

59085

59086

59087

54088

59089

59091

59092

59095

$59096^{\circ}$

59097

59101

59102

59103

59107

59109

59112

59115

59117

59118

59119

59120

59121

59123

59124

59125

59129

59130

59131

5.)132

59133

59134

5913 .

59136

59137
Brock ................ $\mathrm{R} R$ Brook's Duke of Bluemounds $-R R$ Bruce........................ L R Bruno ........................ Daisy Brutus................... $\mathrm{R}$ R Buckeye Lad................ R Buena Vista .......... L R Buffalo Bill..................... M

Buffalo Bill .......................... I

Bugler........................ Ky I

Bullskin Duke.............. - Rose

Burlington............. L R

Bustamente................ $-\mathrm{D}$

Cadiz............. R

Cæsar .................. M

Calvin ............... R

Cambria Duke 2d ......... $\mathrm{R} R$

Cambria Duke 17th ....... - R R

Cambridge Duke ......... L R

Camilla Duke ............... - R R

Campagnola.................. Cox

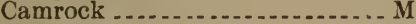

Canadian Chief

Canadian Prince of Oakland .. _ M

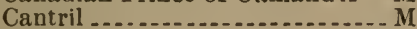

Capital

Captain.................. Ky I

Captain............... L R

Captain Carlisle.................M

Captain Claude.......... Ky I

Captain Darnall.............

Captain DeLong............... - M

Captain Dick................... Ky I

Captain Eaton................

Captain Hendricks...... Rolla

Captain Jack............ - Ky I

Captain Jack

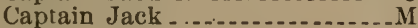

Captain Jack................. - M

Captain Jack................. M

Captain Morris............ $-\mathrm{P}$ S

Captain Wilson...................

Careful Jim................ L R

Carl........................ T

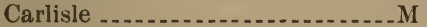

Carlisle..................... P S

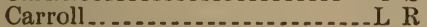

Cash Boy............... $\mathrm{R}$ R

Cassius ....................

Catchpenny

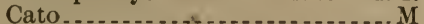

Centennial's Duke..._- Multiflora

Challenger............... R R

Champion................ R

Charles...................... P S

Charles Allen................

Charles Loudon......... L R

Charles O'Malley........... R

Chautauqua Boy ........... - Cox

Chenoweth Duke.........Multiflora

Cherry Duke........... T

Cherub .....................

Cherub of Osborn

Chester......................

Chicago Duke.......... P S

Chickasaw.................

Chief ........................

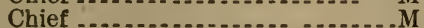

Chief of Spring Branch.......

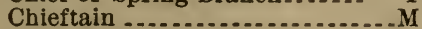

59141 Chitopa.................

59144 Christmas Lad.......... - L R

59145 Chris Weldon............. - R R

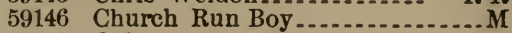

59147 Cid...................... P S

59148 C. J. Spillman.............

59150 Cleon

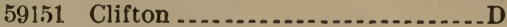

59152 Clifton Dan

59153 Clifton Duke $3 \mathrm{~d} . \ldots . . . . .2 \mathrm{R}$

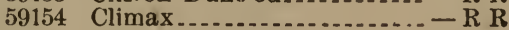

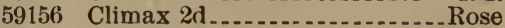

59157 Clinton Duke 2d...M

59159 Cloud

59160 Cloverdale Duke.........

59161 Clover Hill Prince.......... Cox

59162 Cock of the Walk........... P S

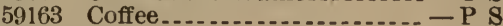

59164 Cold Wave............. P S

59165 Colonel

59167 Colonel Joe........ R R

59168 Col. Hatch .................. I

59169 Col. Judy $2 \mathrm{~d}$

59171 Col. Peet......

59172 Col. Redbank.....

59173 Col. Rosethorn

59175 Col. Waldberg ......... R R

59176 Col. Wheeler........ KV I

59177 Colorado _............ P S

59178 Combination ........ D

59179 Comet

59180 Comet ............ Cox

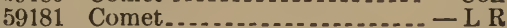

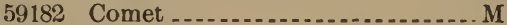

59183 Comet

59184 Comet .................. Ky I

59185 Commander $2 \mathrm{~d} \ldots \ldots . . . . .$.

59187 Commodore .............. R R

59189 Commodore................

59190 Compton 4th M., C. B

5919 I Concord ................... R R

59192 Confederation......... Cox

59193 Constance Duke............ - T

59197 Coonskin.................... Ky I

59198 Coral's Oxford........... D

59199 Cora's Oxford

59200 Coriolanus........ R R

59201 Corning ................. R

59202 Cornwallis ................ R

59203 Cortland Airdrie 2d.......... L R

59204 Cottonwood Louanjo 25th........M

59205 Cottonwood Louanjo 26th....... M

59206 Count Berry ...............

$542072 d$ Count of Oneida....... $\mathrm{R} R$

$5920 \Omega$ 4th Count of Oneida....... $-R R$

59211 Craggs of Ridgevale....... L R

59212 Crescent

59213 Cresco

59214 Crittenden

59217 Crossland

59221 Crown Prince............ Rolla

59:222 Crown Prince................. R R

59223 Cruickshank .............. Cox

59225 Crystal Prince Knightley .... - L R

592:8 Cuyahoga Plumwood.......... R R

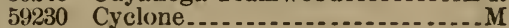

59231 Cyclone........................ Cox

59232 Cyclone

59233 Cypress Duke.............. R R

59234 Cypro $\ldots \ldots \ldots \ldots$ Ky I 
59235

59236

59:238

59240

59243

59244

$59: 245$

$59: 46$

59247

59249

59250

59251

59252

59253

59255

$29 \div 56$

59:259

59260

59261

59262

59263

$59: 264$

59265

59266

59267

59:268

59:270

59271

59272

59273

59274

59275

59276

59:277

69278

59:279

59281

$5928 \%$

59283

59284

59286

59288

$59 \cdot 290$

59291

$59: 242$

59:294

$59: 96^{\circ}$

59297

59298

59301

59302

59303

59305

59306

59307

59308

$59: 311$

59312

59313

59314

59315

59316

59318

59320

59321

59322

59324

59326

59328

59329
Cyrus ................... - P S

Cyrus

Dairy Boy

Daisy Challenger...... $\mathrm{R}$

Daisy's Duke

Daisy's Duke.........

Daisy's Duke ............... Cox

Daisy's Duke.................. - D

Daisy's Duke of Cleveland.... Ky I

Daisy Prince............... $\mathrm{R} R$

Dakota Duke...........

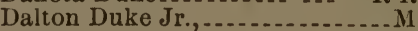

Dandy ........

Dandy ... Cox

Dandy

Dandy

Daniel Kirkwood...... P S

Daniel Reeds.................. I

Dan Price.......................

Dan Webster................ Cox

Dartmouth ............. - Rolla

Dave......... P S

David........

Davy Crockett.............

Deacon Zinn

Deacon Lee...........

Defiance ............

Detiance $2 \mathrm{~d}$ of Oakland...... - M

Dentatus..................

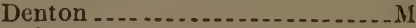

DeSegur

Dexippus ..................

Dexter ............................

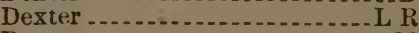

Dexter................

Dexter ...... P S

Dexter..................... Cox

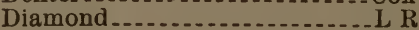

Diamond........ $\mathrm{R} R$

Dick …..................... Ky I

Dick Lesley ...............

Dick Raven.............. Ky I

Dick Swanson...................

Dick Taylor............ Rolla

Dictator...........

Dictator...

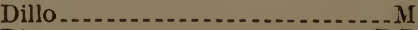

Director.........

Discount ......................

Doc Barkley ........ Milk Spring

Doctor Linn ................ $\mathrm{R} \mathrm{K}$

Doctor Moore 2d..................

Doctor Pratt......................

D'Olney ............

Dombey

Don Carlos...................

Don Labette.........................

Don Pedro

Don Porter..................... B

Don Roderick.........

Dora Lad............... $\mathrm{R}$

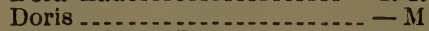

Double Bates Duke.....

Dover Duke............... Cox

Dover Lad................... $M$

Doxander ................ $-L R$

Drivewell

Dude

Duke

Duke
59330

59331

59333

59334

59335

59336

59339

59340

59341

59342

59343

59344

59345

59346

59347

59348

59349

59350

59351

59352

59353

59354

59355

59357

59358

59359

59360

59361

59362

59363

59364

59365

59368

59369

$593 \pi 0$

59373

59374

59375

59376

59377

59378

59329

59380

59381

54382

59384

59385

59386

59387

59388

59389

59390

59391

59392

59393

59394

59395

59397

59395

59401

59402

59403

59404

59408

59409

59410

59411

59412

59413

59414

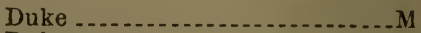

Duke .................... D

Duke

Duke........................ R

Duke...... L R

Duke $2 \mathrm{~d}$

Duke of Adeline...................

Duke Adriance..............

Duke of Airdrie....... Cox

Duke of Albany

53d Duke of Allendale..... - Ky I

10th Duke of Altaham....... M

Duke of Anita............ $R$

Duke of Armada........... $R$

17th I)uke of Armada...... - Cox

Duke of Ash Creek.............. D

2d Duke of Ashippon....... R $R$

16th Duke of Ashland........ - M

3d Duke of Athlone.......... - M

Duke of Athol............

5th Duke of Athol......... R R

14th Duke of Athol......... R R

Duke of Audrain.......... $-\mathrm{L} R$

Duke of Bainbridge............. S S

2d Duke of Barrington........ Ky I

3d Duke of Barrington..... - Ky I

4th Duke of Barrington........ Ky I

5th Duke of Barrington........ Ky I

6th Duke of Barrington........ Ky I

Duke of Beaver.........

Duke of Bendor....................

Duke of Benton......

2d Duke of Bloomfield

3d Duke of Bloomfield........... L R

5 th Duke of Bloomtield......... $\mathrm{L}$

Duke Bob.................... - II

Duke of Bowling........

Duke of Boynton.............. Ky I

15th Duke of Brownsville...- $\mathrm{K} R$

16th Duke of Brownsville... - R R

17th Duke of Brownsville...- $-\mathrm{R} \mathrm{R}$

6th Duke of Brownwood........M

Duke of Buena Vista...... - L R

Duke of Bushfield...........

Duke of Canton.........

Duke Champion........ R

Duke Charley ............ P S

Duke of Chautauqua...... - Cox

5 th Duke of Cherry Wood...- P S

6th Duke of Cherry Wood... - P S

Tth Duke of Cherry Wood...-P S

8th Duke of Cherry Wood...-P 8

Duke of Chester......... R R

Duke of Cisco............ Rolla

Duke of Clemon's Grove......... M

Duke of Clermont..........- $-\mathrm{R} \mathrm{R}$

Duke of Cleveland............. Ky I

Duke of Clinton.........

Duke of Clyde..............

Duke of Colebrook............ R

Duke of Como............. Cox

Duke of Cottonwood Farm $-R \quad R$

Duke of Creston............... P $\mathrm{S}$

Duke of Decatur.....................

Duke of Denver............. Cox

Duke of Deposit........... - Cox

Duke Dewdrop............ - Cox

th Duke of Dewitt............. Ky I

Duke Donald ................

Duke of Dorchester........ 
59416

59417

59418

59420

59421

59423

59424

59425

59428

59429

59430

59431

59433

59435

59436

59437

59439

59440

59441

59443

59444

59445

59446

59447

$594+8$

59450

59451

$5945 \%$

59453

59454

59455

59456

59458

59459

59460

59461

59462

59464

59465

59466

59467

59469

59470

59471

594 i2

59473

59474

59484

59485

59487

59488

59489

59490

59491

59492

59495

59496

59497

59498

59499

$5950 n$

59501

59502

59503

59504

59505

59506

59508

59510

59511
Duke Dunmore 2d........... - R R 7th Duke of Edgewood...... - P S Duke of Effingham........... T Duke of Esmond.......... Rolla Duke of Fairfield........... Duke of Fairview 2d Duke of Fairview......... D 3d Duke of Fairview .......... D Duke of Forest Hill.......... - P S Duke of Frankton......... $R$ Duke of Fulton .............. D Duke of Furnas.................. Duke of Geneva........... $R$ Duke of Gleeland.......... $-\mathrm{L}$ Duke of Glenerie................... 9th Duke of Glenwood......... D Duke of Goodness............ P S 54th Duke of Goodness..... - R R roth Duke of Goodness....... - R R Duke of Grant.............. Duke of Greenwood........- $R \mathrm{R}$ 2d Duke of Greenwood........ Duke Gwynne 2d......... - Rolla 2d Duke of Hadley........... Cox 3d Duke of Hadley ........... Cox Duke of Hancock............. - M Duke of Harmony ............. - M Duke of Harrison ...........- R R Duke of Harristown.............. M Duke of Hazel Dell.............. 2d Duke of Hazel Dell........ - D 3d Duke of Hazel Dell........ - D Duke of Hempfield............ - M 2d Duke of Hempfield......... - M Duke of Hesper.................... Duke of Highland.......... Duke of Highland.......... 11th Duke of Highland......... L R 14th Duke of Highland......... L R 16th Duke of Highland.......L R 17th Duke of Highland....... L R Duke of Holden.............. - M 7th Duke of Homestead....... Ky I 8th Duke of Homestead....... - D gth Duke of Homestead.....- Ky I Duke of Houston .......... - R R Duke of Houston $2 \mathrm{~d}$ Duke of Iroquois......... Duke of Jackson .... - Long Horn Duke of Jefferson............. - D 3d Duke of Jefferson............ R Duke John ................ Ky I Duke of Kearney Duke oi Keenton....... Rolla Duke of Kenney 25th Duke of Kenton.......- $R$ 26th Duke of Kenton........- R R 27th Duke of Kenton............. 28th Duke of Kenton........... R R 29th Duke of Kenton........... R Duke Kirklevington .................M Duke of Klinewood................. Duke of Knox................. Duke of Lake Pleasant......-L R Duke of Lancaster........... P S Duke Lea........... - Milk Spring 2d Duke of Lebanon............ L R Duke of Lewis Grove.......... Duke of Liberty Duke of Liberty Creek........ $-C$
59512

59513

59514

59515

59516

59517

59518

59519

59522

59523

59524

59525

59526

59527

59528

595:9

59530

59531

59532

59533

59534

59535

59540

59541

59542

59544

59545

59546

59548

59549

59550

59551

59552

59553

59554

59555

59556

59557

59560

59561

59562

59563

59564

59565

59566

59567

59569

59570

59571

59572

59573

59574

59575

59578

59579

59580

59581

59582

59583

59584

59585

59586

59588

59589

59590

59591

59592

59593

59596

59597
Duke of Locust Grove ...... $-\mathrm{R} R$

1st Duke of Locust Hill............M

Duke of Locust Lane.......... - D

Duke of Locust Lane 3d...... - D

Duke of Locust Lane 4th ....... - D

Duke of Locust Lane 5th ....... - D

Duke of Logan's Creek........... D

Duke of Long Branch............ B

4th Duke of Lowell............ Ky I Duke of Loyalhanna....... $\mathrm{R} R$ Duke Lyle Jr.,............... _ M 2d Duke of Madison...........L R 2d Duke of Mahoning...........P S Duke Major................... T Duke Major............. Duke of Makin ................. Ky I Duke of Malcolm .............. Ky I Duke of Manchester......... - R R Duke of Mandane.......... - L R Duke of Maple Grove....... - R R 2d Duke of Maplewood..... - R R 2d Duke of Marion..... Duke of Meadow Brook........R R 3d D. of Mecklenburg-Milk Spring 4th D. of Mecklenburg-Milk Spring Duke of Mitchell...........-L R Duke of Modena.......... Duke Mojeska................ Wrong. Duke of Monroe 7 th ............ Duke of Monroe 8th.......... M 9th Duke of Monroe Duke of Montgomery ..... Multiflora Duke of Morgan ............... Rose Duke of Morgan 2d....... Duke of Morgan 3d...........Rose Duke of Morgan 7th Duke of Morgan 4th ........... Ky I Duke of Morgan 8th .......... Daisy Duke of Mt. Vernon .......... S Duke of Murphy Hill......._- R R Duke of Netherby ................. Duke of Nucholls ........... Duke of Oaks............. Duke of Oak Grove......... - Rolla Duke of Oak Home 1st Duke of Oakland....... $R$ Duke of Oneida........... Duke of Orville........... Duke of Osborn ............ D Duke of Oxford Duke Ozi ................. R R Duke of Paris............ P S Duke of Parkman Duke of Pleasant Hill 2d Duke of Pleasant Hill........ Cox Duke 2d of Pleasant Hill .........L $R$ Duke of Raymond.............L R Duke of Reading............ Duke of Redden .................. Duke of Rich Hill....... Duke of Richland .......... - P S 5th Duke of Richland.......- Cox 15th Duke of Ridgeland ........... M 40th Duke of Ridgeland............ Duke of Rolling Fork ........ P S Dnke Roscoe................... P S Duke of Rose Hill Duke of Rosewood 3d......... - D 2d Duke of Rutland........ $\mathrm{R} R$ Duke of St. Elmo............ 
59598

59599

59600

59603

59604

59605

59606

59607

59608

59610

59611

59612

59613

59615

59616

59617

59619

59621

59623

59624

59625

59626

59627

59628

59629

59630

59631

596332

59634

59636

59637

59638

59639

59640

59641

59642

59643

59644

$596+5$

$596+6$

59647

59649

59651

59654

59655

59657

59659

59660

59661

59662

59663

59664

59665

59666

59670

59675

59681

59682

59683

59684

59336

59587

59688

59639

59691

59693

596.44

59696

54697

59698
Duke of Salem.............. Ky I

Duke of Selma................

Duke of Sharon............

Duke of Sharon

Duke of Sharon.................. Daisy

Duke of Sharon.... - Milk Spring

Duke of Sharon............... R R

2d Duke of Sharon........ Daisy

Duke of Sharon $2 d . . . . . . . .-B$

Duke of Sharon Geneva....- $R$

7th Duke of Shawnee Springs..... M

13th Duke of Smith's Grove. - R R

Duke of South Fork ........ M

4th Duke of Spring Hill ......... Cox

Duke of Spring Mills............ L R

Duke of Stockland.........- Cox

Duke of Sumner............... Ky I

Duke of Sylvan Ridge..........

Duke of Tall inadge......... $\mathrm{L} \mathrm{R}$

Duke of the Blue.........

Duke of the Border................

Duke of the Plains.......

Duke of the Ridge........ $R$

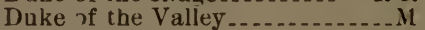

Duke Thomas..............

Duke of Thorndale........

Duke of Thornton............

12th Duke of Trout Run.....L R

Duke of Tyner..............-P S

Duke of Villanova............. R

Duke of Walnut Grove.....- R R

Duke of Walnut Hill ............. P S

Duke of Walnut Lodge $2 \mathrm{~d}_{\ldots} . . \mathrm{Kv}$ I

Duke of Walnut Park.......-P S

2d Duke of Walnut Park.....

Duke of Washington............. Cox

Duke of Waterford

Duke of Wellington.........

Duke of Wellington...............

Duke of Wellington.......

Duke of IVetona ............. M

Duke of Willow Brook $3 \mathrm{~d}_{-.-}-\mathrm{R} R$

15 th Duke of Windingvale..- $R$

Duke of Woodlawn ...........

Duke of Worthington

Duke of Yates $30 \mathrm{th} . . . . . . .-$ Cox

Duke of York 2d..........-Rolla

Dunlap 2d ............... Cox

Dunmore Lad................

Duplex .............................

Duplicate...

Durand ................... M

Durand

Durant Iad

Earl of Belmont.......... $R$

Earl of Colfax ...........

Earl's Duke............. P S

Earl of Edgewood .......

Earl of Elmgrove............. D

Earl of Fox Run ............- L R

1st Earl of Green Meadow .... - C

2d Earl of Green Meadow ....-- M

3d Earl of Green Meadow... - Cox

4th Ear! of Green Meadow.. - Cox

Earl of IIillside ............ - Rolla

Earl of Lamoille $3 \mathrm{~d}$

Earl of Lamoille $4 \mathrm{th} . . . . . . . . . .$.

Ear\} of Montgomery ............ M

Earl of Osborn ................

Earl of Oxford ...............
59700

59701

59702

59703

59704

59705

59707

59710

59711

59713

59714

59715

59717

59718

59719

59720

59721

59722

59723

59724

$597: 26$

$597: 30$

59731

59732

59733

59736

69737

59738

59739

59740

59742

59744

59745

$59 \tau+6$

$597+9$

.59750

59753

59754

59757

59759

59760

59761

59762

59763

59764

59765

59766

59767

$597 \% 2$

54744

59776

5978

59779

59780

59781

59782

59783

59784

59785

59788

59790

59791

59792

59793

59795

59796

59797

59798

59799

59800
Earl Prince................. R R

Earl Profit.....................

Earl of Ridott..............

Earl of Roseberry

Earl of Runnymede........ L R

Earl Sancho................

Earl of Springdale.......... Ky I

Earl of Tarkio .............

2d Earl of Tarkio........

Earl of Townsend ........... $R$

1st Earl of Trout Farm....

Earl of Waco ............... P S

Earl of Wellington......... Rose

Earl of Wellington $2 \mathrm{~d}$

7th Earl Wheatland........ $R$

Earl of Wilderness .......... - T

Earl of Woodvale.........- Rolla

Ed Funston................ M

Edman

Edward................

Elgin Duke .............. M

El Medico

Emerson . ................... Ky I

Emma's Airdrie ...........

Emma's Duke ............ - P S

Emporia Duke............... S

Endymion ....... R R

Engineer - ............... Rolla

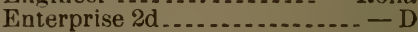

Enterprise 3d....................

Esquire McCune.................. M

Eva's Lad.....................

Excelsior

Expressman

Fairfax Duke.............. L

Fairy's Richmond.....

Fancy -.................. P S

Fanny's Duke........... Daisy

Fantachini.............. $\mathrm{L}$

Farmer Boy ............

Fascinator ........... T

Faula's Muller .......... $\mathrm{R}$

Favorite .............

Favorite............. R

Favorite $7 \mathrm{th}^{-\ldots . . .}$

Favorite 8th

Favorite 9th .............. - P S

Favorite Prince ......... Rose

Fayette City ..............

Felix Duke

Filibuster..................

First Word

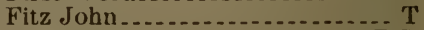

Fitz Lee.........

Five Mile Duke 2d...... P S

Fliberack ...............

Flora's Napier.............

Flora's Oxford................ L R

Florence Duke............... L R

Forester Lad............... L R

Fort Wayne Commander....... $T$

Foster ..................... R

Fox Hunter...... $R$

Frank James................

Franklin Lad............ P S

Frank Morris............... R

Frank of Sonora........... $R$

Frank V.................... $\mathrm{R}$

Frantic Duke............ Rolla

Frederick ............... P S 
59801

$598^{\circ} 02$

59803

59805

$598(16$

59807

59808

59809

59811

59812

59813

59814

59818

59819

59820

$598: 21$

$598: 32$

59823

598:4

59825

59826

59827

$598: 28$

59829

59830

59831

59832

59833

5983.

59836

59337

59838

59839

59840

59841

59842

59843

59844

59845

59846

59847

59848

59849

59850

59851

59852

59853

5985.5

59856

59857

59858

59360

59863

59864

58865

59866

59867

59868

59870

59871

59872

59876

59877

59878

59884

59885

59886

59887

59888

59889
Frederick

Frederiek Lowell $2 \mathrm{~d}$

Fred W. Plumwood........... Ky I

French Duke ............ P S

Freya Duke................ - R R

Frisco Duke........................

Fritz

Frontier............

Frosty's Goodness........ S

Fruitland King ............ - R R

Fruitland King $2 \mathrm{~d}$

Fur Coat ............... - R R

Garfield ........

Garfield .................... Ky l

Garfield _....................... B

Garfield

Gartield _.......... P S

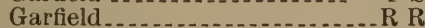

Garfield ......................... D

Garfield of Bell Creek...... Ky I

Garibaldi ................

Gault _....................... Cox

Gem's Duke............. R R

Gem's Duke.............. $\mathrm{R}$

3d Gem's Duke............ - Cux

4t $h$ Gem's Duke........ - Cox

Gem's Duke of Anoka..........L R

Gem's Duke of Grand Meadow_L R

Gemina.................. L R

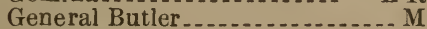

Gen. Clay ........... R

Gen. Custer........... L R

Gen. Custer.......... P S

Gen. Dix _.......... P S

Gen. Ewing .............

Gen. Garfield .................. T

Gen. Garfield .........

Gen. Garfield. .................. I

Gen. Garfield ........ R R

Gen. Gordon ........ P S

Gen. Grant

Gen. Grant................. I

Gen. Gute

Gen. Hancock

Gen. Harrison

Gen. Hurlbut .............. - Cox

Gen. Jerry

Gen. Lafayette..............

Gen. Lyon ........

Gen. Logan .............. $-\mathrm{R}$ R

Gen. McGrant Jr., ........ R R

Gen. Murgan. .................. T

Gen. Prentice ............

Gen. Sheridan

Gen. Sherman ................. B

Gen. Sherman

Gen. Sherman

Gen. Singleton

Gen. Taylor................. $R$

Gen. Washington ......... Cox

Gen. Weaver............... L R

Geneva Craggs............. - L R

Geneva Hopewell...........- R R

Geneva Rose's Duke ........... M

George .................

George ..........

Geurge

George $2 \mathrm{~d}$

George A............... $-R$ R

George Brown................. P S
59891 George Jr.

59892 George Villard ........... - P S

59893 George Washington....... - Ky I

59894 George Washington ....... - Cox

59895 Geraint_................... $-\mathrm{L}$ R

59897 Gerald

59898 Gernot 2d.........................

59899 Gertrude's Duke ............. D

59900 Gilham's Noxubee........ D

59901 Gilmore...................... R

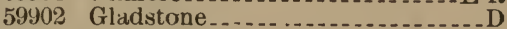

59903 Gladstone...............

59905 Glen Athol ...... P S

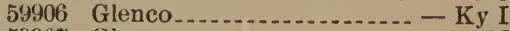

59907 Glenco ....................................

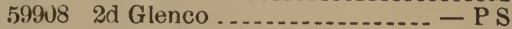

59909 Glen Mary's Sharon....... - R R

59910 Glenmore ............... P S

59912 Gloster of Orleans

59913 Gold Digger....... - Milk Spring

59914 Gold-dust .................. - PS

59918 Golden Chief $2 \mathrm{~d} \ldots \ldots$

59918 Golden Crown $2 \mathrm{~d} \ldots . . . \ldots \ldots$

59920 Gold Eagle................... R

59921 Goldtinder $10 \mathrm{th} . . . \ldots \ldots \ldots$ Disy

59922 Goliah................ R

$59(923$ Gonello

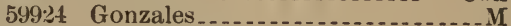

599:5 Goodenouugh

599:36 Goodness Duke........... P S

59927 Governor .......... R

59928 Gov. Begole

599:29 Gov. Begole

59930 Gov. Gliek .

59931 Gov, Glick........

59933 Gov. Martin ............... P S

59934 Gov. Tilden

59935 Gov. Wright ............

59937 Grace's Prince ......... Ro

59938 Grahain................... T

59939 Granada ...................... R

59940 Grand Army

59942 Grand Duke............... R R

59947 Grand Master $\ldots \ldots \ldots$

59950 Grand River Aribur..... - Daisy

59951 Grand Si.ky Duke......... - D

59952 Granger .......... D

59953 Granite Rock ....... Cox

59954 Granite Star....... Long Horn

59955 Grant ..................... R R

59957 Graves................... Daisy

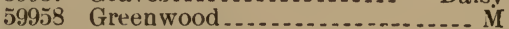

59960 Grove Duke................... T

59961 Grundy King $2 \mathrm{~d}$

59962 Guadalupe....................... M

59963 Guilford Duke Imperial .... $\mathrm{R} R$

59964 Guilford Sir Bertram ........ - D

59965 Gustave Dore ............. R R

59967 Gwynne's Gooduess........ - Cux

59968 Gwynne Louanjo...........

59969 Hal

59970 Hamilton

$5997 \%$ Hampton ................ R R

59973 H

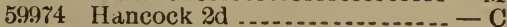

59475 Hancock of Newark ............ Ky I

59976 Harbinger.................... Cox

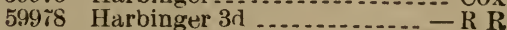

59980 Harman's Duke ............ - Cox

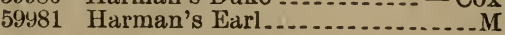


59982

59983

59984

59985

59986

59987

59988

59990

59991

59992

59994

59995

59996

59997

59998

60000

60001

60002

60003

60004

60005

60006

60007

60008

60009

60010

60011

60012

60013

60014

60015

60016

60017

60018

60020

60025

60026

60027

60028

60032

60037

60038

60039

60040

60042

60043

60044

60045

60046

60047

60049

60050

60051

60055

60058

60059

60060

60061

60062

60063

60064

60065

60066

60068

60069

60070

60071

60072

60074

60075
Harold

Harrison

Harrison Duke........ $\mathrm{R} R$

Harry Greenwood....... L R

Harry S. ................... L R Hartington ............ Rolla Haulpa................ Cux Hawkeye............ L R Hawkeye.......... PS Hawkeye................

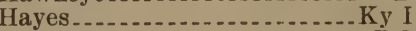
Hayes 2d.................. Hayward Duke........... L R Head Light............... $\mathrm{L}$ R Hector Henry 1st.............. Cox Henry 2d....... Cox

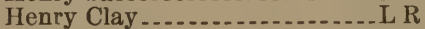
Henry Clay ..................... Ky I

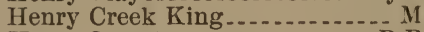
Henry Sinoot ............ R R Henry Ward Beecher..... $\mathrm{T}$ Henry Wilson ............ R R Hercules............. Hercules.................. Ky I Hercules.......... R R Hero Hesper Tom ..................... M Highland Duke 1st............... M Highland Duke 2d.......... - M Highland Duke 2d......... Highland Duke 3d....... Ky I Highland Duke 4th......... M Highland Duke 6th ....... Highland Knight............. Hilda's Muscatoon........ M Hillhurst Bettie.......... R R Hillhurst Fashion $6 \mathrm{tb} \ldots \ldots . . \mathrm{R} R$ Hillhurst Fashion 7th.....-R R Hoffman's Bull............. $-\mathrm{R} R$ Honest John........... Ky I Hookstown Duke.......... $-\mathrm{M}$ Hoosier Boy ................ R R Horace Greeley ............ H. Spurgeon............ Cox Hugo Hulda's Duke........... Cox Hull's Victory ............ $-\mathrm{L} \mathrm{R}$ Hunter Airdrie.................. Ida's Proud Duke...... Ky I Ike..... $\mathrm{R}$ Ike D.,. Ilderton Chief............. Cox Imperial Plumwood.... R Independence........... R Independence ..... Independence ........... $\mathrm{L}$ R Indian Chief.................. Inspiration.............. Daisy Iowa Iowa Chief........... Irene Duke......... - Milk Spring Irene Duke................... Ireneus $4 \mathrm{th}$ Iroquois ........ Irwin's Sharon Duke.........L R Ivanhoe ......................... Ivanhoe ....... Ixion ......... Jack Dillard.
$600 \% 6$

60078

60081

60082

60083

60084

60085

60087

60088

60089

60090

60091

60092

60093

60094

60095

60096

60097

60099

60100

60102

60104

60107

60108

60111

60112

60113

60114

60115

60116

60118

60119

60120

60121

60122

60124

60125

60127

60128

60129

60131

60132

60133

60134

60135

60136

60137

60138

60139

60141

60143

60144

60145

60150

60151

60152

60153

60155

60156

60157

60159

60160

60161

60162

60163

60165

60167

60168

60169

60170
Jack Finter........- - Milk Spring

Jack Holcomb ................. Cox

James G. Blaine ...................

James G. Blaine.......................

James G. Blaine

Jamie Smith ................ Daisy

Jay Bell..................

Jay L.,.............

Jeff Penix

Jenny's Duke.............. - R R

Jenny's Kirklevington......... Ky I

Jerry ...................... - Ky I

Jerry Murphy ................ M

Jesse _...................... L R

Jesse James.............. R

J. Hampton....... P S

Jim.........................

Jim

Jim Blaine.................... Ky I

Jim Coulehan.............. - R

Jim Lind................ - Cox

Joanua's Duke............ T

Jo Blackburn ........... R R

Joe Blackburn ............... - P S

Jo Dun.............................

Joe Gibson ................... Ky I

Joe Hall_................ Rolla

Joe Hedges ............. R

Joe Johnson 5th ................... Cox

Joe Morey ........................

Joe Willis................ $\mathrm{R} R$

John

John ............................

John ....................... I

John ................... M

John Bright................... R

John Fitzhugh........... R

John Kasson................... T

John Logan..........

John Martin .................... Cox

John O' Gaunt.............. - Cox

John Opp ............ - Long Horn

Johnny Bull ................... M

John P. St. John..... P S

Johnson ......................... S

John Stoller Jr.,

John T. Crisp....................

John W.,................. R

Jnker

Joseph E.,

Josephine's Duke of Sharon...$-T$

Josephus........................ L R

Josephus..................... S

Judge Ellington.....................

Judge Patch........... P S

Judge Paul ........................

Julius De Long.............. - C

Juubo............................. R

Jumbo........................... Cox

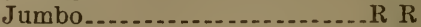

Jumbo................... $M$

Jumbo.................. $\mathrm{R}$

Jumbo................... M

Jumbo.................... - Cox

Jumbo

Juno ........................ Cox

Kansas .................................

Kansas Duke..........

Kansas Duke.................... M

Kansas Duke of Woodson..- Ky I 
60171 Kansas Oakland Duke........ - B

60172 Kansas Red..................... P S

60174 Katie's Orange Boy .......... D

60175 Keene ........................... R

60176 Keene..................... D

60177 Kellogg

60179 Kenneth .................. R

60181 Kentucky Prince.............

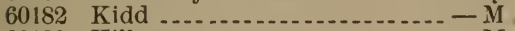

60183 Kilbourne..........................

60184 Kimball ...........

60185 King

60186 King Bates...............

60188 King George ............... Ky I

60189 King Lear.... R

60190 King's Loudon Duke 13th.. - R R

60191 King Milne............. R R

60193 King Philip

60195 King Richard................... I

60196 King Richard $2 \mathrm{~d}$

60197 King Splendor.................. R

60198 Kingston Maynard

60199 King of the Hills

60200 King of the Roses........ Rolla

60201 King of West Grove...... - L R

60202 King William ............. R R

60203 King of Harristown.......

60:204 Kirk Kirklevington........ D

60:205 Kirk Lee..............................

60214 Kirklevington Gwynne.... - Rolla

60215 Kirklevington of Prairie Hall_ _ M

60217 Kittie's Boy................. - M

60219 Knight of Pleasant Ridge..... - D

60221 Lacy's Duke............ - R R

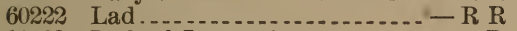

60223 Lad of Lamartine........ D

60224 Lady Maud's Oxford........... R R

60225 Lady's Mazurka............... D D

60226 Lafayette Lad................ M

60227 Lafe Athlestane.......... - R R

60228 Lancaster ................. R R

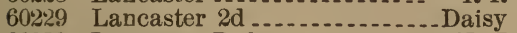

60230 Lancaster Duke................ I

60231 Lansing Bull.................

60233 Lavender...................... B

60235 Leander

60236 Lee Albert................... I

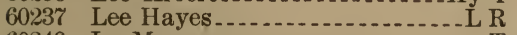

60240 Le Moyne....... T

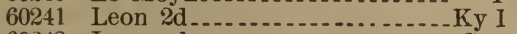

60242 Leonard ...................

60243 Leonard.................... I

60244 Leonard..................

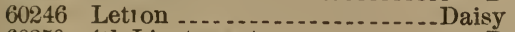

60250 4th Lieutenant............ D

602515 th Lieutenant...........

60253 Linda's Duke............... M

60254 Little Giant............ R

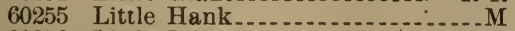

60256 I.ittle John

$60 \% 57$ Little Phil..................... R

60258 Littleton _........ Milk Spring

60259 Llewellyn's Sanborn........ - R R

60260 Locomotive.....................

60261 Locomotive.................... R

60262 Locum of Oakland ......... M

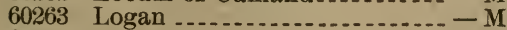

60264 Logan $\ldots \ldots \ldots$

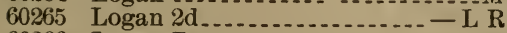

60266
60267

60269

60269

60270

$602 \% 1$

60272

60273

60274

60275

$6027 \%$

60280

60284

60286

60287

60288

60289

60290

60291

60293

60296

$6029 \%$

60298

60299

60300

60301

60302

60303

60304

60305

60307

60308

60311

60312

60313

60314

60315

60316

60317

60318

60319

60321

60324

60325

60326

60327

60328

60329

60330

60333

60334

60335

60336

60337

60338

60339

60340

60341

60342

60343

60344

60345

60346

60347

60348

60349

60352

60354

60356

60359

60361
Logan Chief................. R R

Logan Duke.......................

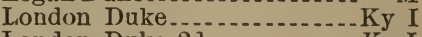

London Duke $2 \mathrm{~d} . . . . . . . . . . K v ~ I$

Lone Star............... P S

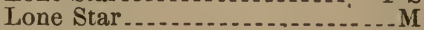

Longfellow................ R

Long John .................

Loran ........ Rolla

Lord Bacon ..... R

Lord Barrington of Huron.....Cox

Lord Beauty .................

Lord Bickerstaffe F........ D

Lord Bloom

Lord Bloom 2d...

Lord Bloom 3d.......... Cox

Lord Bloom 4th......... L R

Lord Bloom 5th...... Cox

Lord Cavendish.......... Daisy

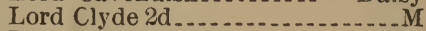

Lord of Concord

Lord Derby of Dromore...... - D

Lord Ducie............... Cox

Lord Falmouth................ Ky I

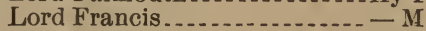

Lord Garrison Lord Gillham ..................... Lord Hilpa 2d........... Rolla Lord Lieutenant............ M Lord Manning .............. R Lord Mar........ R 4th Lord Marquis of Miami ....... M Lord Maxwell............. $\mathbf{P}$ S Lord Meredith........... Rose Lord Morgan............. Lord Nelson Lord Red Rose................ 6th Lord Red Rose........ Lord Reginald ...........

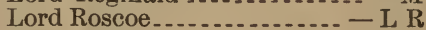
Lord of Sharon.......... P S Lord Shefflelder $2 \mathrm{~d} \ldots \ldots \ldots$ Lord of the Valley . Lord Valentine 2d........ Cox Lord Vernon............. R Lord Wellington....... P S Lord William ....... Lord Winchester 5 th Lord Winchester 8th........

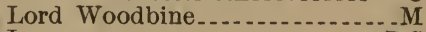
Lorne ................ Lost Boy 7th Louan of Crethmere's D. - L R Louan's Duke............. R R Louan Duke.............. Louan's Duke............ Louan Duke............. Louan Duke 2d............. Ky I Louan Duke of Calumet.... $-R R$ Louan Genera of Bell Creek - R R Louan's Governor......... $R$ R Louanjo ............... R R Louanjo 2d.......... Louan's Mazurka....... $\mathrm{R} R$ Louan Tip ......... R R 3d Loudon Duke......... Loudon Duke 50th Loudon Duke of Cedar..... Ky I Loudon Duke of Weston... - R R Loudon Lad 2d............. 
60362

60364

60366

60367

60368

60369

$603 \% 0$

60371

60373

60374

60379

60382

60.383

60386

60387

60390

60.392

60393

60394

60395

60396

60397

60398

60399

60400

60401

60402

60403

60405

60406

$6040 \%$

60409

60410

60411

60412

60413

60416

60417

60418

$604: 0$

60421

60422

$604: 24$

$604: 36$

60427

60428

604:39

$60+30$

60431

$60+32$

60433

60434

60485

60437

$6043 \mathrm{~s}$

60439

60442

60413

60444

60448

60451

60454

60455

60456

$6045 \%$

$60+59$

60460

60462

60463

60464
Loudon Townley

Loyal Kansan..........

Lucina's Hero............. P S

Luck........................

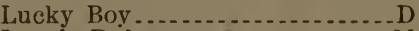

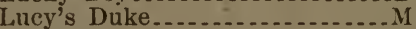

Lualow

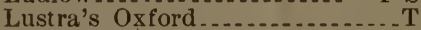

Lynden Duke

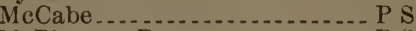

MePherson Ranger ......... P S

Mad River Duke.......... M

Magenta Cherub .......... R R

Maggie's Oxford ......... $\mathrm{R}$

Magistrate 13th ............- L R

Maid's Duke 2d...............

Majesty ...

Major

Major .

Major

Major

Major Burnett.......... L R

Major Butterfly

Major Climax........... $\mathrm{R} R$

Major Conger.............. $\mathrm{R}$

Major Conger.............

Major Cundiff................ M

Major Everly ......... M

Major Gwynne... L R

Major Hill of Durham....... Ky I

Major Hum of Oakland........ P S

Major McConkey.......... $\mathrm{R}$ R

Major Oxford.................... R

Major Redman............

Majur Reno.............. L R

Major Vanmeter .......... $\mathrm{R} R$

Major Wilkin

Major William ..................

Malaka Lad.................. M

Muquoketa Duke......

Mareus ..... R R

Margrave....... P S

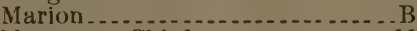

Marquette Chief ................. M

Marquis.

Marquis

2d Marquis of Bourbon Place - P S

Marquis De Kirk.......... - Rolla

3d Marquis of Floyd ....... $-\mathrm{L} R$

9 th Marquis of Floyd...... $-\mathrm{L} R$

Marquis of Liberty ......... $-\mathrm{L}$ R

Marquis of Linkville........ $-\mathrm{T}$

Marquis of Lorne.......... Cox

Marquis of Walnut Hill ...... - M

Martina's Duke.............. - C

Mary's Airdrie...............

Mary's Duke................. R

Mary's Duke.............. R R

Mary's Duke 2d .......... $\mathrm{R} R$

Mason Duke 4th.............. Ky I

Master.................... R

Master Dick............... Ky I

Master Hopkins............

Master Mazurka............... M

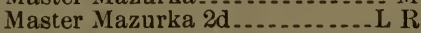

Masterpiece............... L R

Master Price $2 \mathrm{~d} . . . . . . . .6-R$

Master Ruby .............. P S

Master Taylor 6 th........ P S

Master Wallace............ $\mathrm{R} R$
60466

60467

60469

60170

$604 \pi 1$

$604 \pi 3$

60473

60474

60475

60476

$604 \pi 7$

60479

60480

60481

60482

60483

60484

60485

60486

$6018 \%$

60488

60490

60491

60492

60493

60494

60495

$60+96$

60497

60498

60499

60500

60501

60502

60503

60504

60506

60507

60508

60510

60511

60512

60513

60515

60516

60517

60518

60519

605220

$605: 1$

60522

605:24

60525

605:26

$6052 \%$

605:28

60.5:29

60530

60531

60532

$605: 33$

60534

60535

60536

60537

60538

60540

60541

$605+2$

$605 \pm 3$
Mat Hill 2d................. Daisy

Matilda Duke......... R R

Månd S's Baron........ P S

Masimilian .................

May Duke.................. $R$

May Duke .............. Ky I

Ma! fl'wr of Fennimore 2-L'g Horn

Mayflower 3d of Fennimore... - C

Mayflower Duke $31 . . . . . . .-$ Cox

Mayflower Duke 4 th........... R R

Mazurka's Bell Duke.... . - Rolla

Mazurka Duke............... - M

Mazurka D. of Sp'ng Vulley - Cox

Mazurka Oxford ............... S

Mazurka Oxford $2 d . . . . . . . . . . .4$

Mazurka Oxford 3d............ $\mathrm{R}$

Mazurka Oxford 4th......... R

Mazurka Oxford 5th

Mazurka's Oxford 34th............ M

Mazurka Prince............ Daisy

2d Mazurka's Treble Duke.. - R R

Melbourn Duke ............ - R R

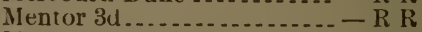

Mercury .............. R

Mermaid's Valentine......... D

Miami Cbief ......................

Middleton .................. Rolla

Miller's Duke.................... Dai

Mime Duke................. Cox

Minerva Duke...................

Minna's Barrington ....... - Rolla

Minnie's Duke............... M

Minnie Renick's D. of Sharon. - T

Minstrel Duke 7th............. L R

Minstrel Prince........... - Rolla

Minstrel Prince $2 \mathrm{~d}$

Missies Richmond.......... - R R

Miss Wiley's Jubilee $2 \mathrm{~d}$....... M

Mithridates ............... $-\mathrm{L} \mathrm{R}$

Modest Count $2 \mathrm{~d}$

Modoc........................

Morloc Chief ....................

Mollie's Duke.............. $\mathrm{R}$

Monarch ..................

Monareh

Mouareh.......... R

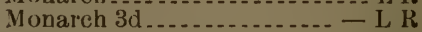

Monarch of Scotland

Monitor............... R

Monitor

Montana Ranger .......... - Ky I

Mont Calm Bedaconsfield....- - L R

Monte Cristo .............. $-D$

Monterey................... R R

Montgomery Chief $2 \mathrm{~d}$

Montreal....................

Montrose...................

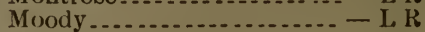

Morgan

Morning Glory

Morning Star.............. Ky I

Morte D'Arthur............ - L R

Moseow .................

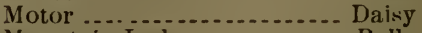

Mountain Lad ............ - Rolla

M. Rudolph ........... Daisy

Murray Duke ..........

Muscatine...........

Na-au-say ............

Nail Airdrie................ 
60544

60545

60546

60547

60549

60550

60551

60553

60554

60555

60557

60558

60559

60560

60561

60563

60568

60569

60571

60572

60573

60574

$605 \%$

$605 \%$

60578

60579

60580

60581

6058 ?

605ร3

60584

60585

60586

60587

60588

60590

60594

60595

60596

60597

60598

60599

6060:

60603

60604

60605

60606

60607

60608

60014

60615

b0616

60618

60619

606:20

60621

606:22

$606: 26$

60627

606:28

60629

60630

60631

60632

60633

60634

606:35

60636

60637

60638
Napoleon .............. Cox

Nasby ............................

Nat Leonard......................

Neander......................... S

Ned Duke of Lyndale....... $R$

Nelson ...................... L R

Nero ................... $L$ L R

New England ............ Daisy

New Year's Day ................. Cox

New Year's Night............ - M

Nimbus....

Nimrod...................... L R

Ninnescah Boy........... - L R

Nip...................... $-M$

Nippersink Bull........... L R

Nobleman ................... - D

North Elm Duke 1st............ Ky I

Northumberland............ - P S

Oakland ...................... - C

Oakland Duke $3 \mathrm{~d}$

Oakland Lad............... L R

Oakland Lad................... P S

Oakley's Pride............. R R

Oak Ridge Lad............

Oakwood Duke ........... - R R

Oberlin Duke............ $-\mathrm{R} R$

O'Brien .................. L R

Occidental............. L R

Ohio Frank ........

Old Jamie ............ Cox

()e Bull...................

Oliver.

Oliver Twist....................

Olymphus Duke

Omega ............ M

Orange Boy $2 \mathrm{~d}$

Orphan ...............

Orphan....................

Osage ............. Ky I

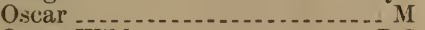

Oscar Wilde..............

Oscar Wilde................. $R$

Oswego Duke............ Ky I

Otley ...................... L

Ottawa

Owsley

Oxford

Oxford Äirdrie............ - Cox

Oxford of Appanoose ....... - R R

Oxford of Canton ........ - Ky I

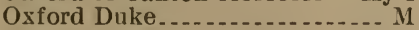

Oxford Duke

Oxford's 8th Grand Duke .. - R R

Oxford's 9th Grand Duke... _ R R

Oxford Gwynne.......... - Daisy

Oxford Junior ............... M

Oxford Knightly $3 \mathrm{~d}$

Oxford of Preble............. L R

Oxford Prince.........

Oxford Prince $2 \mathrm{~d}$

Oxford Prince $3 \mathrm{~d}$

Oxford's 2d Royal Duke.... - R R

Oxford's 3d Royal Duke.... - R R

2d Oxford Royal Prince..... - $R$ R

16 th Oxford of Vermillion ...... - T

Pageant of Orange...... Ky I

Palingenesis .................. T

Palos Boy ..........

Pannell.-
60639

60640

60642

60643

60644

60645

60646

60647

60648

60649

60650

60652

60654

60656

60657

60658

60659

60660

60661

60662

60663

60664

60665

60656

60669

60670

60671

60672

$6065 \% 3$

60674

$606 \% 5$

$606 \% 6$

60677

60678

60680

60681

60682

60683

60684

60685

60687

60688

60690

60691

60692

60693

60694

60695

60696

60697

60699

60700

60701

60702

60703

60705

60706

$60 \%(\pi)$

60708

60709

60710

60711

60712

60713

60714

60716

60717

60724

60728

60731
Parr........................ M

Pat

Peabody of Oakland ........... M

Peacock 3d.............. Cox

Pearl Duke ...........

Pearl's Duke............. L R

Pearl's Loudon Duke 7th......- - D

Pedro ............. P S

Peerless ................

Pekin Duke...... Rolla

Pension ............. D

Perfection......... Rolla

Peri's Duke............... - Rolla

Peri's Emperor ..... ......... - Cox

Peri's Prince............... C

Peri's Wellington .......... - Rose

Pete of Pleasant Ridge.......... M

Peter ......................... M

Peter Johnson ........... Cox

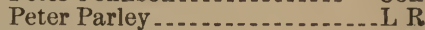

Phallas ..................... $M$

Pharaoh.............

Philip .................... R

Philip Sheridan.......

Phyllis Lad .............. $R$

Pickwick ............... - Rolla

Pilgrim ........... L R

Pilot_............................

Pilot....... Cox

Pilot ................... Daisy

Pilot Grove .............

Pine Hill Chief

Pine Hill Favorite ......... L R

Pioneer.......... Long Horn

Pioneer Allen .......... R R

Pizarro

Pleasant Hill Duke......... - D

Pleasant Lad............... - R R Plumwood Boy ........... L R Plumwood B. U............. R R Pogio Marquis .............. D

Pontefract $2 d$. ............... Ky I

Portage Lad . ............... Ky I

Porter $2 d$.....................

Posey Duke.................. L R Post Boy ............. R R

Prairie Duke................... S S

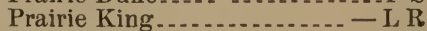

Premier.................

President_........................

Pretty Boy ................. D

Pride of Barrington ........- - L R

Pride of Madison............ $-\mathrm{D}$

Pride of Windsor............. P S

Prince

Prince

Prince................... Ky I

Prince............

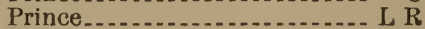

Prince............... L R

Prince ........... Daisy

Prince ......... R R

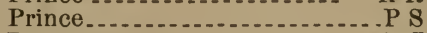

Prince $2 \mathrm{~d} . .$.

Prince of Adeline.............

Prince Albert .................. R R

Prince Albert................ $\mathrm{R} R$

Prince Azurine............. $-R R$

Prince Bismarck............ R R

Prince of Delavan......... 
60732 Prince of Drum Creek............ M

60733 Prince Eclipse................ M

60735 Prince of Elk County .......... R

60736 Prince Eugene............... M

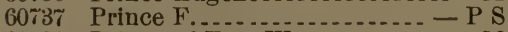

60738 Prince of Fort Wayne....... - M

60 i40 Prince Frederick.......... Cox

60741 Prince Geneva................ M

60742 Prince George.............. L R

60\%44 Prince Goodness........... R R

60745 Prince Greenwood.............. R R

60746 Prince of Hanover 10th.........

60747 Prince Imperial ........... R R

60749 Prince Jerome

60752 Prince Kirklevington........

60753 Prince Lad of Bell Creek ..... - M

60754 Prince Leo.......... - Long Horn

60756 Prince Leslie ............... P S

$60 \% 57$ Prince of Lima

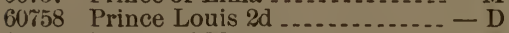

60759 Prince of Macon

60760 Prince of Marion .... Long Horn

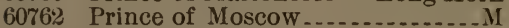

60763 Prince Napier 2d.........

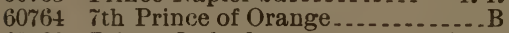

60766 Prince Oxford ................

60767 Prince Oxford Tite

60768 Prince Rialto.................. Ky I

60770 Prince of Seneca......... P S

$60 \approx 71$ Prince of Springdale..... - Rolla

60775 Princeton .................

60,76 Princeton

$607 \%$ Princeton M.

60778 Prince Victor..............

60\%79 Prince of Villanova......... R

60781 Prince of Walnut Grove.... - Cox

60782 Prince of Waterloo........ $\mathrm{R} \mathrm{R}$

60783 Prince William.............. M

60784 Prince of Wolviston .........

60785 23d Prince of Wyaconda...... - T

60786 34th Prince of Wyaconda........ B

60787 35th Prince of Wyaconda..........

60788 Prince Uxbridge............... T

60790 Prince Zeno............... Cox

60791 Prohibition ..............

60792 Prospect....................... Ky I

60793 Prospero...................

60794 Protection

60795) Proud Duke 5th.......... R R

60797 Proud Duke of Illinois......- Ky I

60798 Punch .................. R R

60799 Putnam

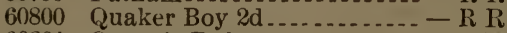

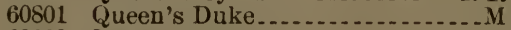

60802 Rackensacker ........ R R

60803 Rail Road Duke........ $\mathrm{P}$ S

60804 Rainey.................

60805 Raleigh .........

$60800^{\circ}$ Ralph..................

60807 Ralph Emmerson.

60808

60809

60811

60814

60815

60816

60817

60818

60819

60820
60821

60822

60823

60824

60825

60827

60829

60830

60831

60832

60833

60834

60836

60837

60839

60840

60841

$608+2$

60843

60844

60845

60846

60847

$608+5$

60850

60851

60552

60853

$60 \$ 56$

$6085 \%$

60858

60859

60861

60863

60864

$60866^{\circ}$

60867

60869

$608 \% 1$

$608 \pi 3$

60874

$608 \approx 5$

$608 \pi 6$

608กั

60878

60879

60880

60583

60384

60885

60886

60887

60888

60889

60890

60891

60892

60893

60894

60895

60896

60897

60898

60599

60902

60906

60907

60908

60909

60910

60911
Red Champion ............. - L R

Red Chief ..................

Red Cloud

Red Cloud 6th............ $R$

Red Cloud Jr............... Ky I

Red Comet................... Ky I

Red Dick ..................... L R

Red Duke.........

Red Duke.............

Red Duke............... Ky I

Red Duke................

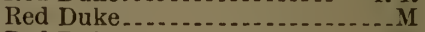

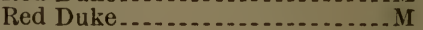

Red Duke..............

Red Duke............... $\mathrm{L}$

Red Duke........................

Red Duke...................

Red Duke........ Ky I

Red Duke................................

Red Duke......... R

Red Duke............... $\mathrm{R}$

Red Duke.......... - Milk Spring

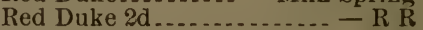

5 th Red Duke of Vine Hill.... - M

Red Forester...................

Red Frost.......................

Red Gen, of Sunnyside ...- - Ky I

Red Hillhurst.............. Daisy

Red Jacket ............

Red Jacket................ Rnlla

Red June ............ R R

Red Knight....... Cox

Red Leaf.................... Ky I

Red Major........ Ky I

Red Marquis .

Red Mazurka $2 \mathrm{~d}$

Red Prince............. Cox

Red Prince............

2d Red Prince............. R R

Red Romeo ................ Ky I

Red Rose Duke of Cleveland..Ky I

Red Rover.................. T

Red Rover..............

Red Rover.............................

Red Rover.................... M

Red Rover 2d

Red Ruby's Duke $2 \mathrm{~d} . \ldots . . . . . .4$

Red Soldier................

Red Stanhope.................

Red Star................... S S

Red Turk

Red Wing

Reformer ............ R

Reformer.................

Regent..................

Renick's Duke....

Renick's Duke of Glendale...- $R$ R

Reno

Reserve

Reserve Duke.......

Reuben Butler............

Reward .....

Rex.........................

Rhoderic Dhu ....... Cox

Richard Hanson............ - L R

Richmond Favorite........ - Ky I

Richwood's Chief............ R R

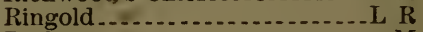

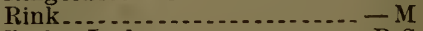

Ripley Lad.....................

Riverside Boy................. 
60912

60915

60916

60917

60918

60921

60922

609:24

60925

60927

60929

60932

6093

60934

60935

60936

60938

60939

60940

6094:

60943

$609 \pm 1$

60945

$609+7$

60948

60949

60950

60951

6095:

60953

60955

60956

60957

60958

60959

60961

60963

60964

6096 .

60966

60967

60969

60971

60972

60675

$609 \% 6$

60979

60980

60981

60982

60945̃

$6098 \pi$

60988

60989

60990

60992

60993

60994

60996

60997

60998

60999

61001

61002

61003

61004

61005

61009

61010

61011
Riverside Chieftain

Roan Boy ............... P S

Roan Boy ....... R

Roan Chief............... L R

Roan Dick......................

Roan Duke............ Rose

Roan Duke...................... R

Roan Duke.................... S

Roan Duke ................. Cor

Roan Duke of Fairview ..... - D

Roan Duke of Suez........

Roan Jack ................ P S

Roan Jim ..................... T

Roan John ............ Multiflora

Roan Lad............. R R

Roan Lad .................. $-\mathrm{L}$ R

Roan Prince.........

Roan Sharon.

Roan Underedge........... Rose

Rob................. C

Robert......... P S

Robert............. $\mathrm{L}$

Robert Bruce.............

Robertson's Roan........... M

Robin Adair........................

Robinson.................... - D

Rob Roy $2 \mathrm{~d} \ldots \ldots \ldots$

Rock.................... P S

Rockbridge Prince........ R R

Rock Run Chief........... L R

R derick Dhu .......... Rolla

Rodney Thompson........ - M

Roebling ..............

Roger's Bull ............. R R

Roland .................... R

Romeo Duke.............. P S

Romulus.................... R

Romulus.................

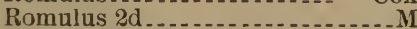

Roscoe Barrington ........ Rolla

Rosebud Duke............

Rose Duke ............... L R

Rose's Duke.............. Rolla

Rose's Duke.................... L R

Rose Duke ................. R

Rose Duke $2 \mathrm{~d}$

Rose Duke 5th................. Ky I

Rose Duke of Elmwood..... - Cox

Rosamoud's Bates..........

Rosamond Duke ............... M

Rose's Prince........ Multiflora

Rosewood Lad.....................

Rosewood Lad ..............

Rosicrucian ............... L R

Ross Boynton.................... Ky I

Rothschild .................

Rough and Ready....... R R

Rough and Ready........ L R

Roxabulus .............. R R

Roy .................... R

Royal Bates............ R R

Royal Commander........ - L R

Royal Duke.............. Rolla

Ruyal Duke..............

Royal Duke $2 \mathrm{~d} . . . . . . . . . . .$.

Royal Duke 2d..............

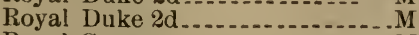

Royal George ...................

Royalist 5 th

Royal Jack.

...
61013 Royal Prince................... Ky I

61015 Royal Renick W.,........... S

61016 Royal Sedgwick ............ D

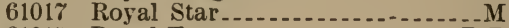

61019 Royal Tom.................. R

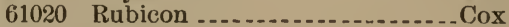

$6 \mathrm{I022}$ Rubv Prince............. R R

61023 Rucawa Duke............ L R

61024 Rufus....................... R R

61025 Rufus $2 \mathrm{~d}$

610:6 Rufus 2d................. Cox

61027 Rugby ....................... R

61028 Sailor Boy

61030 St. Julian

61031 St. Patrick..... P S

61032 St. Paul...................

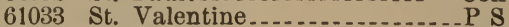

61034 Sallie's Lad ............... Rolla

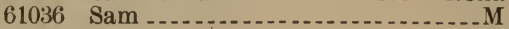

61037 Sam ............................

61038 Sam Brady 2d............... Ky I

61039 Sam Hazzard............ L R

61040 Sam Hazzard.............. Ky I

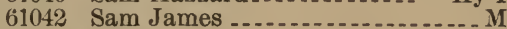

61043 Samson

61044 Sampson....................

$610+5$ Samson ....................... Daisy

61046 Sam Stedman.................

61047 Sam Taylor ................ R R

61048 Sam'l J. Tilden.................M

61049 Sam'l J. Tilden..............

61050 Sanborn Duke.............

61051 Sanborn Healy...............

61052 Sandusky Duke.......... R R

61050 Sandwich Duke.........

61054 Sangamon ..................

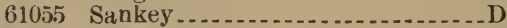

61057 Santa Claus ............... R

61058 Santa Fe................ L R

61059 Santee's Duke......................

61060 Sassamon .................... Daisy

61061 Satisfaction .................. I I

61062 Scottish Chief of Kirkland....Ruse

61063

$6106 \pm$

61065

61066

$6106 \%$

61068

61069

610\%2

61073

61075

61077

$6108 \%$

61084

61085

61086

61087

61092

61093

61095

61097

61098

61099

61100

61101

61102

61104

61105
Senator Senator Booth $3 \mathrm{~d}$............

Senator Booth 4th.............. S S

Senator Booth 6th ...............

Senator Conkling -.............. R R

Senator Vest ............... - M Sensation .................... R Seymour $2 d$.............. $R$ Sharon................... $-M$ Sharon Boy $3 \mathrm{~d} . . .2 \mathrm{R}$ Sharon Duke............ - Rolla Sharonius $3 \mathrm{~d}$ Sharon's Oxford............ Sharon's Oxford............... R Sharon Severs............... - L R Sharon of Sunnyside.......... - D Shep ....... Cox Sheridan ................... - D Shield's Orphan Boy Silver Crown Silvio ....... D Simon.......... Sir Athol................ I

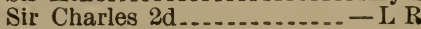
Sir Charles Clarke......... P S Sir Edward ................ D Sir Henry .............. Cox 
61106 Sir Henry Havelock ........ - P S

61108 Sir Hugh Parker............. D

61109 Sir James................. R

61110 Sir John................... Ky I

61111 Sir John

61112 Sir John..................

61114 Sir Richard......

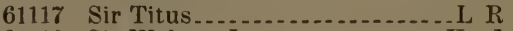

61118 Sir Walter, Jr.,................. I

61119 Sir Wilfrid.............. R R

61120 Sizer Duke.................

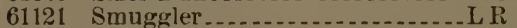

61122 Snider Boy .............

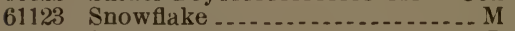

61124 Snow's Geneva...........

61125 Snowstorm.............. R R

61129 Sooy $2 \mathrm{~d} \ldots \ldots \ldots$

61130 Sooy $3 \mathrm{~d} \ldots \ldots \ldots \ldots \ldots \ldots-\mathrm{L}$ R

61131 Sorrento Duke of Elmwood_- $-\mathrm{L} R$

61132 South Elm Duke................ I I

61135 Spartan

61136 Speckle John............. Ky I

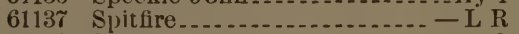

61138 Spot.........

61139 Spotted Duke............... Ky I

61141 Spotted Tail ............... P S

61142 Springdale Bright Eyes......... Ky 1

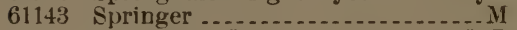

61144 Springfield Lad

61145 Springwood's Duke........ Cox

61146 Squire Buckner........... $\mathrm{R}$ R

61147 Squire Chaplin........

61150 Star Duke................. R R

61151 Star Duke.................. Ky I

61152 Star Duke of Maine Valley.....Ky I

61153 Stai Duke of Maple Hill.... $\mathrm{R} R$

61154 Star of Granger 2d....... $-\mathrm{L} R$

61155 Starlight.............. P S

61156 Star of Nelson.

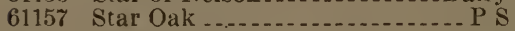

61158 Star of Ohio .

61159 Star Renick............. R R

61160 Star of Springfield........

61161 Star of the West $2 \mathrm{~d} \ldots \ldots \ldots-\mathrm{T}$

61162 Stately Lad .....................

61164 Statesman Junior ........... - M

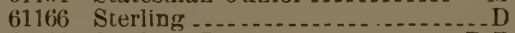

61168 Sterling Duke....... R R

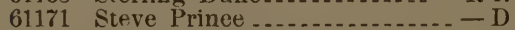

61174 Stonewall Jackson.......

61175 Stonewall Jackson......... D

61178 Summit Chief...............

61179 Summit Lad..................

61180 Surprise.......................... R

61181 Sut Luvingood............

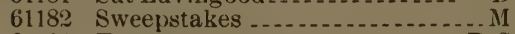

61184 Tacoma...............

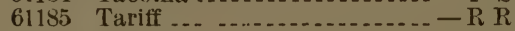

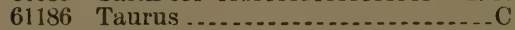

61187 Taylor Duke........... R

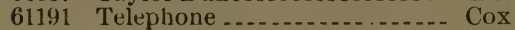

61193 The Commodore......... R R

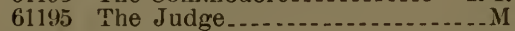

61196 Theodore

61197 Theodore Bates........... R R

61199 The Squire............. R R

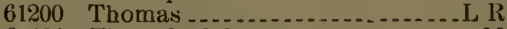

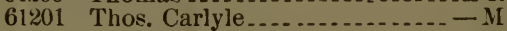

6120:2 Thorndale Duke .................

61203
61204

61205

61207

61208

61209

61210

91211

61212

61213

61214

61215

61218

61219

61220

61221

61222

61223

61224

612:5

61226

612:28

61229

61231

61232

61233

61234

61236

61237

61240

61241

61242

61245

61246

61247

61250

61251

61252

61253

61254

61255

61256

61257

$61 \% 58$

61259

61261

61263

61264

61266

$6126 \%$

61268

61269

61271

61272

$612 \% 3$

$612 \% 4$

$612 \pi 5$

$612 \% 7$

61278

61281

61254

61286

$6125 \pi$

61288

61289

61290

61292

61293

61294

61295

61296
Thorndale Duke 2d. $-\mathbf{R} \mathbf{R}$

Thorndale Duke 4th............

Thurlow...............

Tim Bates...................

Timber...........

Tim Finegan .......................

Titus 2d.......

Tom Barns 3d..... P S

Tom Brown

Tom Corwin ................. Ky I

Tom Grabam ..................

Tom Sawyer............. S

Topsman..................

Traveler...................

Treble Sharon............ D

Triumph of Sunnyside.......

Tuberose of Endicott........ T

Tuekahoe ................... R

Tulip's Oxford.......... Beauty

Twister .................

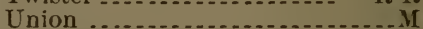

Union Bill

Valentine ....................... R

Valentine .................

Valentine ...............

Valentine's Loudon....... Cox

Valleyhurst 2d......- Milk Spring

Vanderbilt .............. T

Vanquisher ............... R

Van Wert Duke............ $\mathrm{P} B$

Van Wert Prince............

Vermillion Duke............. $\mathrm{D}$

Victor ...........................

Victor Duke................. $\mathrm{P}$

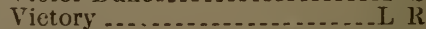

Vietory

Vienna Lad................

Violet's Prince......... R R

Virginius ...........

Virginius ...........

Virginius ................. Rose

Viscount Windsor......... L R

Volga Valley Duke...............M

Volunteer .................. L R

Wabash Lad.................

Wade Hampton.............. - M

Wade Hampton........... R R

Wakelee's Bull............ $\mathrm{R} R$

Wapsie Valley Milk Boy... - Ky I

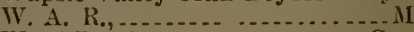
Ward Beeeher............

Warren B.,....

Warrior ......... Daisy

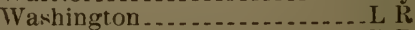

Washington Fairfax....... $\mathrm{R} R$

Washington Star............. R R

Waterloo .................. R

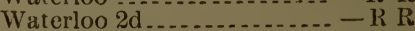

Waterloo Earl............ R R

Wauldron.................

Waveland Prinee......... L R

Wellington................ $-\mathrm{L} R$

Wenona's Duke of Orleans _ $-\mathrm{R} R$

Western Duke............. . R R

Western Lad.............. L R

What Care I?

Whitecloud Duke............ L R

IVhite Dúke of Sharon ....... T

White Jacket.............

White Jacket .................. 
61297 61298

61299

61300

61301

61302

61305

61306

61307

61308

61309

61312

61313

61314

61315

61317

61318

61319

61320

61321

61324

61325

61326

61327

61328

61330

61331

61332

61333

61334

61335

61336

61337

61338

61341

61342

61343

61345

61346

61347

61348

61349

61351

6135 ;

61354

61355

61424

614:6

61431

61433

61436

61439

6 1440

61441

61412

61443

61445

61446

61448

61452

61453

61456

61457

61460

61462

61466

61468

61469

$614 \% 0$

61471
White Jacket ................. Cox White Socks of Bell Creek _ - R R White Stockings ............. - L R Whitefields Brampton........ Cox Whiting Boy ............... Cox Wickliffe_................... - C

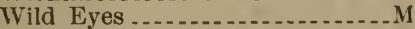
Wild Eyes Duke................. T Wild Eyes Hilpa......... Wild Star............... Ky I Wiley's Duke............. P S Wilkes _......... William H., William H.,........................ Wm. of Mulberryvale......... P S Willie Martin............... $-M$ Winner Wisbey's D. of Goodness .. - Rose Wolviston A.,............ P S Woodbine's Airdrie.......... - C Woodfurd ........... Woodford .......... Wnodstock ............ $\mathrm{L}$ Wotton Duke......... Wyandot..................... Young Airdrie 2d.......... D

Young America................M

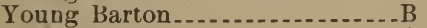
Young Byram ..... P S Young Derby ............... Young Duke.............. R R Young Duke............. L R Young Hamlet............. Cox

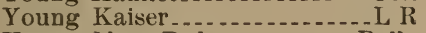
Young Mary Duke....... Rolla Young Milo................ Ky I Young Oliver 2d............ Young Prince... M Young Prince ............. Young Prince Albert.......- L R Young Ranger Prince 2d........M Young Robson .............. Young Starlight............ Zach Chandler ....................

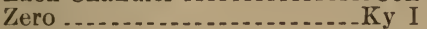
Zion Abe's Buckeye...... R Accident .......... P S Acorn Ad......... Adelaide Oxiord...... L R Adophus..... Adonis ..... T Agnes 7th's Lad......... L R Ainsworth ................. Airdrie.................. $\mathrm{R}$ Airdrie .......... Airdrie 2d .............. Airdrie Bates............ R R Airdrie Duke.......... P S Airdrie's Duke..... Milk Spring Airdrie Duke 2d........... $\mathrm{P}$ S Airdrie Duke 3d............ Daisy Airdrie Duke of Sharon...... - II Airdrie of Kentucky ....... $-\mathrm{R} R$

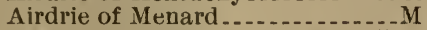
Airdrie of Pleasant Hill 5 th_ $-\mathrm{R} R$ Airdrie of Pleasant Hill 6th $-\mathrm{R} R$ Airdrie of Pleasant Hill 7th. $-\mathrm{L} R$ Airdrie of Pleasant Hill 8th. $-\mathrm{R} R$
61473

61475

$614 \pi 6$

61477

61478

61479

61480

61481

61482

61484

61486

61488

61489

61490

61496

61497

61498

61499

61500

61501

61502

61503

61501

61505

61506

61507

61508

61509

61510

61511

61512

61514

61515

61517

61518

61519

61520

61521

61522

615:3

61524

61525

61526

61527

61528

61529

61530

61531

61533

615:35

61536

61537

61539

61540

61541

61542

61543

61544

61545

61546

61547

61553

61554

61555

61556

615.57

61558

61559

61560

61561
Airdrie Sutherland $2 \mathrm{~d} \ldots \ldots . . .-\mathrm{R} R$ Airdrie of Wildwood $4 \mathrm{th} . . . . . . . M$ Ajax...................... P A kron .... Albert ............ Milk Spring Albert Ashton........... P S Alexander........................ I I

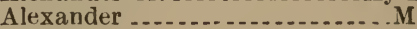

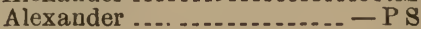
Alexis .................... Cox Alfred ............ Ali.................. $\mathrm{P} S$ Alice Maud's Cherub $2 \mathrm{~d} \ldots \ldots-\mathrm{R} R$ Alick Richie.............. Alioth....................... Ky I Allen

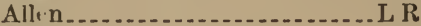
Allen...... Allendale Loudon Duke $2 \mathrm{~d}_{\ldots} . \mathrm{R} \mathrm{R}$ Allendale Loudon Duke 5th - - L R Allendale Loudon Duke $6 \mathrm{th}$ - $-\mathrm{L}$ R Allendale Lrudon Duke 16th - L R Allendale Loudon Duke 17th.. _ T Allendale Loudon Duke 18th .. - T Allendale Loudon Duke 20th.. - T Allendale I.oudon Duke 21st.. - M Allendale Loudon Duke 24th _. - T Allendale Lnudon Duke 25th.$--T$ Allen Duke 7th.......... $\mathrm{R} R$ Allen Hall....... R R Alma ............. Almer of $\mathrm{O}$ ak Hill ............... Alonzo..................... Ky I Alto Breastplate of Sp'ng Branch.M Ambassador 2d.................. T Amelia's Duke $2 \mathrm{~d} . . . . . . . . . . .-D$ America's Duke 2d............ America's Duke 3d......... Amherst Duke................. Ky I Amherst Duke 2d............ Ky I Amish Boy .................... $\mathrm{S}$ Amish Duke 3d...

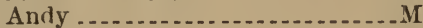
Andy

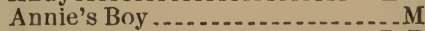
Anna's Duke......... R

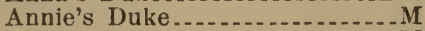
Annie's Lad......................... Anthony Archie........ Aretic Marquis .......... Cox Ardra Duke................ - Cox Argyll.................... L R Arijgton ................. Arlington Duke......... $\mathrm{R} R$

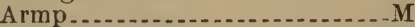
Arrow Arthur Arthur ...... P S Arthur Higgins ........... S Ashby ..................... Athol's Red Jacket........ - Cox Athlone's Soldier......... L R Augustus ...................... Ky I Aureus.................... R

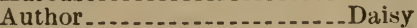
Ayers.......... Baden Duke 18th .............. Ky I Bad Luck................. D

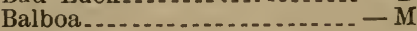


61562

61563

61564

61565

61566

61567

61570

61572

61573

$615 \%$

$615 \% 8$

61580

61583

61588

61594

61595

61596

61597

61598

61599

61601

61605

61606

61607

61609

61610

61613

61614

61620

61621

6162:

61625

61631

61633

61636

61637

61638

61641

61645

61646

61647

61649

61650

61651

61652

61653

61654

61655

61656

61657

61659

61660

61661

61662

61663

61665

61666

61667

61668

61669

61670

61671

$616 \pi^{2}$

61673

61675

$616 \pi \%$

61678

$616 \% 9$

61680

61681
Balco

Balco ....

Bannister ................. B

Barmpton's Jubilee ....... $\mathrm{R} \mathrm{K}$

Barmpton Lad ........... $-\mathrm{R} R$

Barmpton Lad $2 \mathrm{~d} . . . . . . .-\mathrm{R} R$

Baron Acomb 9th........ - Rolla

Baron B ,.................... Cox

Baron Bates........................

Baron of Carroll......... R R

Baron Claxton................. P S

Baron of Farmington.......-Cox

Baron Gwynne 4th........ - Rolla

Baron La Grange............ - Ky I

Baron Linwood................

Baron Mansfield $2 \mathrm{~d}$

Baron of Oakwood ........ Cox

Baron Pierce......

Baron Pixey.............. Daisy

Baron Prince.....................

Baron Richmond......... Ky I

Baron Siddington $3 \mathrm{~d} . . . . .-$ Daisy

Baron Sidney ................ R

Baron Steuben ...............

Baron Trimble $2 \mathrm{~d} . . . . . . .-C$

4th Baron Wupsie............. Daisy

Baron Wellington

Baron William 3d............

Barrington of Arcadia......

Barrington Bell Duke...... $\mathrm{R} R$

Barriugton Boy -.......... Rolla

Barrington Duke.......... R R

Barringtonia's Grand Duke - Ky I

Barrington Lad..........

Barrington Ward......... P S

Barrington of Zanesville... $-\mathrm{R} R$

Bar Wild Eyes..............

Bassett

Bates' Red Kirk.......... Cox

Bates' Roan Duke............ $-\mathrm{C}$

Beau Brummel........... $\mathrm{R} R$

Beauty's Don B.,

Beauty's Duke...............

Beauty's Duke.....................

Beauty's Lad ................ - Cox

Beauty's Mosske...................

Beauty's Oxford..................

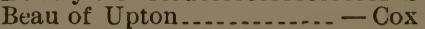

Beaver Prince.........................

Beck's Airdrie.............

Beecher

Beecher ........ L R

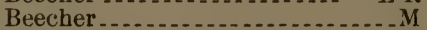

Beecher

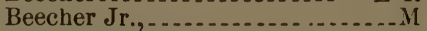

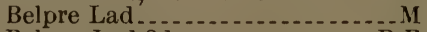

Belpre Lad $3 \mathrm{~d} \ldots \ldots$

Belpre Lad 5th............. L R

Belpre Lad 6th ..................

Belpre Lad 7th............. M

Belpre Lad 8th............ R R

Belpre Lad 10th............ $-\mathrm{L}$ R

Belpre Lad 13th........... - Rolla

Belina Peri.......

Bell Boy ...........

Bell Duke .......... R R

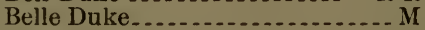

Belle's Duke................

Belle's Dnke ................... L P

Belle's Duke..................
61682

61683

61684

61687

61691

61692

61693

61696

61697

61698

61699

61701

61702

61704

61705

$61 \% 06$

61707

61 ช 08

$61 \% 09$

61710

61711

61713

61714

61 \%16

$61 \% 17$

61718

$6 \mathrm{i} 719$

61720

61721

61723

61724

61726

61727

$6172 \mathrm{~s}$

61729

61730

61731

61732

61734

61735

617336

61737

61738

61739

61741

61742

61743

61744

61745

61746

61747

61748

61749

61750

61751

61752

61753

61754

61755

61756

$6175 \%$

61758

61759

61760

6r761

61762

61763

61764

61771

$61 \% 72$
Belle's Duke 2d.................. R

Bell Duke 4th of Mapledale .- $-\mathrm{P}$ S

Bell Duke of Sonore........-Rolla

Bell Muscatoon .................

Bellview ...................

Belleview Duke...........

Belle's Wild Eyes...............

Bemus Point .................... R

Ben . Point -

Ben

Ben

Ben Bear.....................

Ben Bolt................ Ky I

Ben Butler .................. M

Ben Butler................... L R

Ben Butler

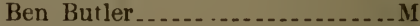

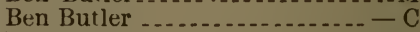

Ben Butler ........... Ky I

Ben Butler............. Cox

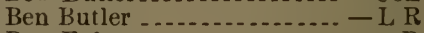

Ben Enloe.......................

Ben Favorite ............. Ky I

Benny Bidlake ........... $\mathrm{R} R$

Ben Richmond $2 \mathrm{~d}$

Ben T. Frederick..... R R

Benton Boy ................... D

Ben of Walnut Hill ...................

Beppo 2d.................. Batsey's Duke of Pichmond _ $-R R$ Bettie's Prince............. $R$ R Bev Tucker .................

Big Sam . . . . . . Bill

Bill Ferguson.... $R$

Billie

Bill Miller.....................

Bill Morgan

Billy

Billy

Billy $3 \mathrm{~d}$

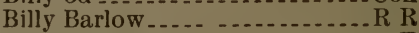

Billy Barney -...................

Billy Boy ................ Multiflora

Billy Button ............ $R$

Billy Green...................

Billy Harrison.......... $R$

Birt.................... $\mathrm{R}$

Bishop ...... Cox

Bishop Hughes........

Bismarck

Bismarck .................. Cox

Bismarck

Bismarck..........

Blaiue ..............................

Blaine .................. M

Blaine

Blaine

Blaine..................

Blaine ..............

Blaine ........... $\mathrm{R}$

Blaine.............

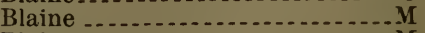

Blaine

Blaine of Sonora.........

Blair ...................... $-L$

Bletso Prince................

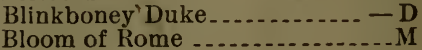

Bloom's Earl ............... R 
61774 Blossom Duke.............. - L R

61776 Blucher .................. Cox

617 Blucher ....................... D

61779 Blue Grass Duke........... . R R

61780 Blue Jacket .................. M

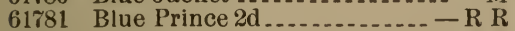

61782 Blythedale Alice.............. - M

61784 Bob Grant....................... S

61785 Bob Ingersoll ................

61786 Bob Lyon.................

61787 Bob of P. B.................. K

61788 Bob Ridley ................. P S

61789 Bob Sharon .................

61790 Bob Stratton .............. Ky I

61791 Bobtail ........................ Ky I

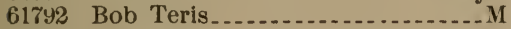

61793 Bob Wild

61794 Bub Wiley

61795 Bodego....................

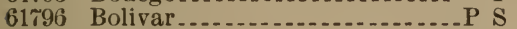

61797 Bonanza

61799 Bonaparte......................

61802 Bonus ................ P S

61803 Boone..................... Cox

61807 Boss Luca

61808 Boston 2d

61809 Boston Boy

61810 Boston Duke...............

61911 Botetourt.............

61812 Bouncer............................

61813 Bourbon ................ R R

61811 Bourbon Duke..........

61818 Bourbon Star.............. T

61819 Bourbon Star ............... S

618:1 Bradshaw ........................

61822 Brandywine ............... D

61823 Breastplate

61824 Breastplate........................ R

61825 Bridgeport Chief....... Daisy

618:26 Bridgeport King ............... R

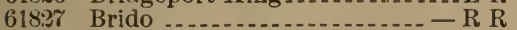

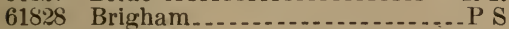

61832 British Prince of Sonora .... - R R

61834 Brokenstraw .............. Cox

61835 Brome Duke of Bedford .... - L R

61836 Bromley ................. R R

61837

61838

61839

61840

61841

61842

61843

61845

61846

61848

61849

61850

61851

$6185 \%$

61853

61854

61856

61858

61861

61862

61863

61864

61866

61867
$618 \pi 0$

61872

61874

61877

61878

61879

61880

61884

61886

61888

61889

61890

61893

61894

61895

61903

61904

61905

61906

61907

61908

61912

61913

61914

61915

61917

61918

61919

61920

61921

61922

619:23

61924

61925

61926

61827

61928

619:29

61930

61931

61932

61933

61934

61938

61910

$619+1$

61942

61943

61944

61945

61946

61950

61951

61952

61953

61954

61955

61956

61960

61961

61963

61966

61967

61970

61973

61974

61976

61977

61978

61979
Butterfly Duke ........... - L R

Byron -................. - Cox

Cabot Boy . . . .

Cadmus................... Cox

Cadmus of Melleray ........ - R R

Cage Barmpton............. $R$

Cain Boy - .................. - D

Calla's Duke...........

Callie's Duke............... $-R$ R

Calvin ............... $R$

Calvin ......................

Cambria's Duke.............. - P S

Cambria Rose of Sharon...-Kv I

Cambridge .................-R R

Cambridge Duke ........ R R

Canon Hill Exeter ............ - D

3d Capital Prince .......

Captain ................

Captain .............. $\mathrm{R}$ Captain Balco..........

Captain Barnes ...........-Rolla Captain George Leigh $2 \mathrm{~d} . \ldots . .-\mathrm{R} R$ Captain George Leigh 3d........ Rose Captain Hawk ............. - R R Captain Hill.................... $R$ Captain Jim ................. Ky I Captain Jinks........... S Captain Mono............. L R Captain Napier .......... Captain Pike .................... Captain Sharon Captain Walker............. Car Boy - ............. Carl Carlisle............... Daisy Carlisle ........... R R

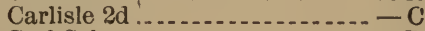
Carl Schurz ....... Carroll ................ Beauty Carroll Duke ...................P S Carroll's Oxford....... $R$ Cash Boy ....................... Cox Cashier ......................... Cox

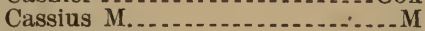
Castana Duke............... - M Castana Duke $2 \mathrm{~d}$ Castana Duke 3d .............. Ky I Castor .................... Cato Cato of Canton............ Cox Caucashian ................... Cox Cawdelle ................ R R Cayuga Chief $2 \mathrm{~d} . . . . . . .$. Cecil_.................... R R Cedric ............. Beauty Cedric . Centennial ................. Ky I Centennial Duke .......... Cox Challenger Bates ........... - R R Challenger of Clear Creek Camp Clark .................... Champion -................ Champion ................... L R Champion's Lesley ........... - T Charlie................. Charlie_..................... $-\mathrm{D}$ Charley Ford................. I Charlie Jones............-Beauty Charles Martell............... $-\mathrm{R}$ R Charlotte's Duke............-Daisy 
61980

61953

61985

61986

61987

61988

61989

61991

61992

61993

61994

61996

61998

61999

6:2000

62001

62002

62004

62005

62006

62007

62010

62011

62012

62013

62014

62016

$6301 \mathrm{~s}$

62019

62020

62021

6202:

62023

62024

62025

62026

62027

62ง28

$6: 20 \div 9$

62030

6:031

62033

$6: 2036$

62038

62040

62041

62042

62043

62044

62045

62046

62047

62050

62052

62053

62054

62055

62056

62057

62058

62059

62060

62061

62062

62064

62065

62066

6:068

62070

62071
Chase

Cherry Duke

Cherry's Lad

Chief Joseph

Chief Justice

Chief Ouray

Chieftain

Chieftain. ................ $R$

Chieftain of Dunleith...... - L R

Chillicothe ................

Christmas King

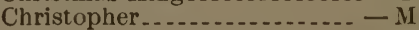

Chunk ........ $R$

Cicero of Southview........ $\mathrm{R}$

Clancy ................. M

Clarence ...................... Ky I

Claude

Claudius $2 \mathrm{~d}$

Clay .................

Clayton....... $R$

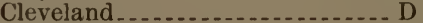

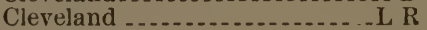

Cleveland..................

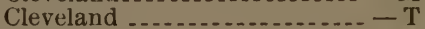

Cleveland...................

Cleveland ...........

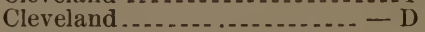

Cleveland ................... - D

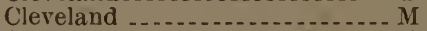

Cleveland .........................

Cleveland ........................

Cleveland - .......................

Cleveland

Cleveland ..........................

Cleveland .......... Milk Spring

Cleveland ..............................

Cleveland................ - Cox

Cleveland .................. R

Cleveland ........................

Cleveland...................... Ky I

Cleveland Boy

Clifton.................... - D

Climax

Clinton ................. Ky I

Clinton Duke............. R R

Clinton Duke...... Rolla

Clytus ...............

Coachman .......

Coffman's Duke............... Ky I

Cohasset Boy .........................

Colfax Grant ........ P S

Collin's Boy 3d............ Rose

Coleman Younger........ Ky I

Colonel .................... L R

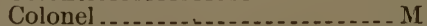

Colonel Briggs ............... R R

Colonel Burr . ....................

Col. Cooper................

Col. Gorman ............... R R

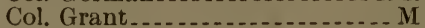

Col. Hepburn -......... - K R

Col. Hutton

Col. Kitson.................. $\mathrm{R}$

Col. Moore.............. Cox

Col. Mowbray............ Ky I

Col. Muir........... P S

Col. Panton........

Col. Redman

Col. Sharon
$620 \% 2$

62076

6:2077

62078

62079

62080

62081

62082

62084

62086

62087

6:2088

62089

62090

62091

62092

62093

62095

62104

6ะ105

6210 ז

62110

6:112

62113

62114

62118

6:119

62121

62122

62125

62127

6:129

62130

62131

62133

6:2135

62137

62142

62143

62144

62145

62146

62147

62148

62149

62151

62152

62153

62154

62155

62156

62157

62159

62160

$6: 161$

62163

62165

62166

62167

62169

62170

62171

62172

62173

62174

62175

62176

62177

62178

62179
Col. Sink .............

Columbia Duke........... - L R

Columbus ..................... M

Comet.................. Rolla

Comet................ Ky I

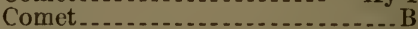

Comet....... R R

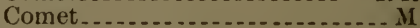

Commander Bly $2 \mathrm{~d} . .$.

Commodore............... $\mathrm{M}$

Commodore................... Ky I

Commodore .............. Cox

Commodore Harley ........ - Cox

Complete ...............

Compton $2 \mathrm{~d}$ M. ...............

Compton 5th M...........................

Concord's Laird ............... - D

Conkling

Coolbrook Prince........... Cox

Cooley .................. Beauty

Cora's Duke.........

Cosgrove..........

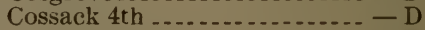

Costigan's Red ..........................

Cotton Allen .

Count Noble

3:d Count of Pickaway..... $R$ R

Country Gentleman ........ - L R

Country Gentleman -........ M

Cowboy .................. Ky I

Craig ................

Crethmere Duke.........

Crimson Duke............... R

Crimson Duke $2 \mathrm{~d}$

Crofty -............... $R$

Croton

Crown Prince...... P S

Crusoe .....................

Crystal Clayton

Crystal Clifford .......... - M

Crystal Cloud...............

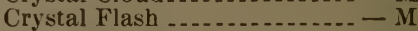

Crystal Prince ............... - M

Crystal Prince Albert.......... - M

Crystal Victor..............

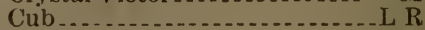

Cullen Burlington............... M

Cullen Duke..................

Cultus ............................

Cumberland Boy .............. - II

Custer Lewis..............

Cypress Duke $2 \mathrm{~d}$

Cypress Lad.............. - Rolla

Cryus. . . .

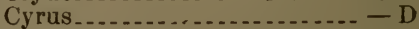

Dainty Davey .........

Daisy Boy ............ R

Daisy Duke........

Daisy Duke........... Daisy

Daisy's Jubilee.............. L R

Daisy Lad.................

Daisy Lad................ R

Daisy Lad of Macon....... $R$ R

Dakota Lad.................... P S

Dakota Lad.................

Dakota Prince.............. P S

Dakota of Yankton

Dakota of Yankton 2d............C

Dal Murphy............

Dalton............... Rose 
62180

62181

62182

62183

62184

62185

62187

62188

62189

62190

62192

62193

62194

62196

62198

6:199

$62: 200$

6:2201

$6: 2203$

$6: 2204$

62:205

6:206

6:2007

6:2:208

6:22:209

6:2210

$6: 2211$

6:2:12

6:2213

$62: 14$

6:2:15

6:2:16

6:2:218

6.2219

6:2:2:20

6:22:21

62:2:2

6\%:223

6:2:24

$62: 2: 5$

$6 * 22: 27$

62:zy

62:333

6:2:34

6:2235

62:236

6:2:237

62:238

622239

$6: 2240$

62:24:2

62:24:3

623344

6ะ2 45

62: 46

$6: 2249$

$6: 2250$

6:2:25:

6:2:53

6:2:254

62:25

62:556

6:258

62:259

622260

63:2:261

$62266^{\circ} 2$

6:22:3 3

6:2564

6:2:265
Dandy

Dandy

Dandy ............. R

Dandy ................. Rose

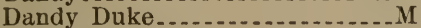

Dandy Jim ........................

Danforth . . . . .

Daniel :........ Cox

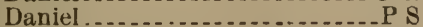

Daniel Boone.........

Danny ..............

Dan's Choice...................... I

Darby ................ R

Darby Duke 24 th

Darlington Duke 5th..... - Rolla

Darlington Duke 6th..... - Rolla

Dalwin ................. L R

Dave

Dave on the Creek.......... P S

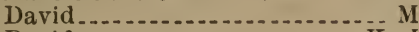

David............. Ky I

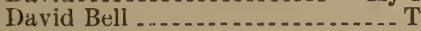

Davy Crockett.........

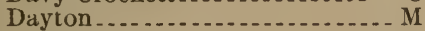

Deanville................

Deer Lick Lad................ R R

Delight's Frank................. S S

Delhi Prince..................... S S

Demosthenes....... D

Denton ................ Rolı

Depot..........

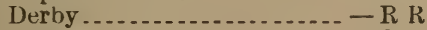

Dexter...................... Cox

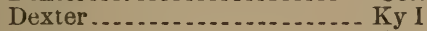

Dexter.................... Cox

Diamond

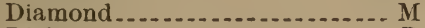

Dick.......... D

Dick

Dick Darrell.....................

Dick Mellvaine ........ $\mathrm{L}$

Dick Taylor ........................

Dick Turpin

Dick Wild ....... S S

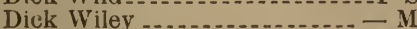

Dilworth................ P S

Dimple.................... $\mathrm{R}$ R

Diogenes...........

Dixun _................ D

Dixon Boy

Dr. Berbank ....... Beauty

Dr. Jesse

Dr. Jo 2d.........

Dr. McKee............. P S

Dr. Redmon

Dr. Stubs................... L R

Dr. West

Dolphin ........

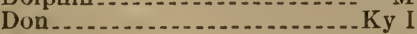

Don

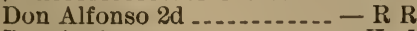

Don Carlos........................ Ky I

Don Clayton............... M

Don Pedro.................. R

Don Pedro...... Ky I

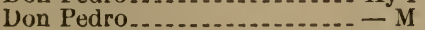

Don Pedro $2 \mathrm{~d}$

Don Waldberg........... Rolla

Dora's Lad 2d............. - R R

Duuble Monarch ........... - L R
62266

$62: 67$

62268

62269

62270

62271

62272

62273

62274

$6: 275$

62277

$6: 3 \% 8$

$6: 3279$

62280

62281

62:282

62286

62287

6:2288

622289

62290

622291

62:292

6:2294

62:295

62296

62:297

6:2998

62300

6:2302

62:306

62307

62308

62310

623311

62312

62313

6:2315

62316

62:317

62318

62319

6:3:20

623:21

6:32:2

6:323

62324

6:33:5

623326

62:327

62328

6:33:29

623331

623332

62333

62334

6:2336

623337

62339

62340

62341

62343

622344

$623+5$

62348

6:2351

62353

62354

6:3355

62357
Douglas ................. - Cox Douglas .............

Douglas.......................

Douglas of Oak Hill..............

Druggist .................... Rose

Duchess Duke ................. D

Duchess Lad................

Dude.......................

Uude...................... S

Dujac_.................

Duke_................... $-\mathrm{R} R$

Duke...................... R

Duke _.................. R K

Duke...............

Duke.....................................

Duke

63d Duke of Allendale

Duke of Almer ............. - L R

14th Duke of Aitaham...... - R R

15th Duke of Altaham ....... - M

16th Duke of Altaham..... $R$ R

Duke of Arcadia .......... - M

Duke of Argyll ........... - Cox

Duke of Ashland ........... R

3d Duke of Ashland ......... - M

4th Duke of Ashland.......... - M

7th Duke of Ashland.......- R R

4th Duke of Athlone ........ M

Duke Auvergne.......... $R$

Duke Barmpton ................ D

Duke of Bath 15th ...... Cox

Duke of Bath 16th ......... - L R

Duke of Bath 17th

Duke of Beaver 2d.......... - Cox

4th Duke of Beloit................ M

5th Duke of Beloit............. R R

6th Duke of Beloit................

Duke of Benton......................

Duke of Berwick................

Duke of Bethel .................. Ky I

Duke of Bizer Place........... M

Duke of Blacklick........... - L R

Duke Bonaparte........... - R R

Duke of Bow Park 2d...... - L R

Duke of Bradford............ $-M$

Duke of Bradford ............. Ky I

2d Duke of Brant ........ R R

Duke of Brockhurst 5th...... - M

19th Duke of Brownsville.... - R R

3d Duke of Buffalo....... Multiflora

Duke of Campbrook ........... Ky I

2d Duke of Campbrook...... Ky I

3d Duke of Cannondale ... - Rose

Duke of Carroll .............. - R R

Duke of Champion......... - L R

Duke Charles.............. - P S

Duke of Chester ......... P S

Duke of Chilicothe .........- L R

Duke of Clinton ............ $\mathrm{L} R$

Duke of Clinton........... $\mathrm{R} R$

2d Duke of Clinton ........

Duke of Coonewar..- Milk Spring 2d Duke of Coonewar-Milk Spring

Duke of Corners ............. M

Duke of Darby Plains ........ - M

Duke of Darwin 3d ........ - Cox

Duke Deets................ Cox

4th Duke of Dewitt ...... - Cox

5th Duke of Dewitt ........ - Cox

2d Duke of Dover................ Daisy 
62358 Duke of East Lynn .......... - M 62359 Duke of Edinburg .......... R 62360 Duke of Elmendaro ........ D 62361 2d Duke of Elm Glen..... - Ky I 62363 Duke of Elmhurst 2d.............. 623675 th Duke of Elmwood $\ldots \ldots \ldots-$ D 62368 6th Duke of Elmwood..... R R 62369 8th Duke of Elmwood ...... - D 62370 Duke of Enon ............... Ky I 62372 11th Duke of Evergreen Hill - R R 62373 Duke of Fabius............... Ky I 62374 Duke of Fairview 623ri Duke of Fayette........... - C 62382 Duke of Forest Shade.... $\mathrm{R} R$ 62353 Duke of Forrester 4th....... - D 62384 3d Duke of Fremont........ R 62385 Duke of Fryer Creek....... P S 62356 Duke of Ganbier......... R R 6:387 Duke of Genesee Valley ........... 62:389 Duke of Gentle Slope....... - M 62391 18th Duke of Glenflora..... - R R 62392 Duke of Glenwood........ - L R 6:393 42d Duke of Goodness...... - R R 62394 61st Duke of Goodness.....-R R 62395 75th Duke of Goodness..... - R R 6:3397 86th Duke of Goodness..... - R R 62399 Duke of Grandview....... - L R 62100 Duke of Grant............ P S 62401 Uuke of Greenwood....... R R 6:402 Duke of Guelph........... R R 62404 2d Duke of Hamilton........ D 6:2405 3d Duke of Hamiltou........ - D 62406 Duke of Harlan................ S 62407 Duke of Harrison 62408 Duke of Hastings.......... P S 62409 4th Duke of Hazel Dell...... - D 62410 5th Duke of Hazel Dell.......... D 62412 Duke of Helendale......... T 62414 4th Duke of Highland........ R 62415 5th Uuke of Highland...... M 62417 Duke of Hillhurst......... - L R 62418 Duke Imperial.............. 62420 5th Duke of Isahella....... - L R $6: 24: 21$ 6th Duke of Isabella........ - L R 62422 Duke of Jackson......... 6:24:3 Duke of Jamestown $\ldots \ldots \ldots \ldots$ P S 62424 Duke of Jefferson 62425 Duke of Jefferson.......... Cox $6242 \%$ 62436 62437 62438

62439

62440

62442

62443

62444

62445

62446

62447

62448

$62+49$

62450

62451

62452

$62+53$

62454

62455

$62+56$

62457
62459 62461 6.246 62464 $624 \div 0$ $62+71$ $62+\% 2$ 62473 62474 $62+75$ $624 \div 6$ $624 \pi$ $6: 278$ $624 \pi 9$ 62480 $62+81$ $62+8 \%$ $62+83$ 62485 6:4S6 62487 $6: 488$ 6:2489 62490 624192 6:2194 62496 62497 6:2499 6:501 6:5502 62503 62504

6:)505 6:506 62507 62509 6:513 6:514 6ะ515 6.516 62517 $6: 2518$ 625:30 6:5:21

6.25:4 6:5:25 625:28 6:25:29 62530 62531 62532 6:533 6:5334 62535 6ะ5:36 6:538 62539 6ะวั40 6:5ั1 $6: 542$ 62545 $6: 546$ 62548 62549 62550 6ำรั1 62553 62555 6:5556 2d Duke of Madison....... $\mathrm{R} R$ 2d D. of Maple Lawn Herd.. - R R Duke of Maple Valley ...... - Cox Duke of Marseilles......... $-\mathrm{D}$ Duke Merlin................ R Duke of Milan Duke of Milford.......... Duke of Mill Creek....... P S 2d Duke of Mill Creek...... - P S Duke of Morgan ............. M Duke of Mound Farm ..... R R 6th Duke of Moundview.... - Cox 7th Duke of Moundview.... - P S 9 th Duke of Moundview ..... - P S 10th Duke of Moundview... _- C 11th Duke of Moundriew....- -C 13th Duke of Moundview ... - Cox 15th Duke of Moundview .... -C Duke of Newton............. II Duke Nimrod.............. R Duke of North Plains ...... Ky I 4th Duke of Northumberland. $-\mathrm{D}$ Duke of Novi......... $\mathrm{R} R$ 2d Duke of Oak Grove.... $\mathrm{R} R$ Duke of Oakland.......... - Cox Sth Iuke of Oakwood.... - Daisy 10th Duke of Oakwood...... - T Duke of Ontario......... Cox 20th Duke of Orleans...... $\mathrm{R} \mathrm{R}$ 12th Duke of Osage....... - Cox 13th Duke of Osage......... - Cox 14th Duke of Osage........ Duke of Oxford ........ Woods Duke of Oxford........... Cox Duke of Palmer......... Cox Duke of Palmyra...... Duke Philip.......... Duke of Pine Valley......... Ky I Duke of Pleasant Hill......... L R 2d Duke of Pleasant Hill....... L R Duke of Pleasant Ridge....... Ky I Duke of Porterville...... 4th Duke of Preston...... D Duke Rena........... R R Duke of Richland $2 \mathrm{~d} . \ldots . .-\mathrm{R} R$ D. of Rose Lawn _. Sire not Recorded Duke of Roseneath........ L R Duke of Rothesay .......... L R Duke of Rush.................... Duke of Russel ....... R Duke of Salem 12th Duke of Salix...... D Duke of Sandusky Duke of Shackleford Duke of Sharon............ Cox Duke of shonkin......... D Duke of Sonora......... Duke of Sparta..... 3d Duke of Spring Arbor... - Cox 4th Duke of spring Arbor.- - Cox 5 th Duke of Spring Arbor.-- Cox Duke of State Line......... Duke of Stockdale 20th Duke of Stock Place.... Daisy 21st Duke of Stock Place.... $-R$ R Duke of Sugar Grove...... - Cox Duke of Sunbeam of the Glen Duke of Tindall...... Daisy Duke of Traer........... Rolla 
62557

62558

62559

62561

62563

62564

$6: 2565$

62566

62567

62568

62569

62571

62572

$625 \% 3$

62575

$625 \% 6$

$625 \% 7$

62581

6.258

62585

62586

62587

62588

62589

62590

62591

62593

62595

62596

62597

62598

62599

62600

62602

62603

62604

62605

6:2606

62607

62608

62610

6:611

62612

62613

62616

62617

62618

62619

62620

626:1

62622

62623

62625

62626

62628

62630

62633

62636

62637

62638

62641

$626+2$

62643

62645

62646

62647

62648

62650

62651

62652 21st Duke of Trout Run........R R

Duke of Troy ................ - L R

Duke of Trufant............. - L R

3d Duke of Tyne........... - L R

Duke of Villanova........... - Cox

Duke of Walnut........... - PS

Duke of Walnut $2 \mathrm{~d} . \ldots . . . . . . . M$

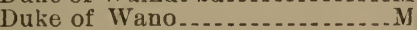

Duke of Wano $2 \mathrm{~d} . . . . . . . . . . . . \mathrm{M}$

Duke of Warren .............

Duke of Wayne...............

Duke Weston ................. - B

3d Duke of West View

4th Duke of West View .........L R

10th Duke of Wildwood......- D

Duke of Willow Grove.......... R R

Duke of Wilton.............. Ky I

Duke of Woodlawn $2 \mathrm{~d} . . . . . . . . \mathrm{P} S$

Duke of York .............. - Cox

Duke of Zanesville........... - P S

Duke of Zanesville $2 \mathrm{~d} . . . . . . .-\mathrm{P} \mathrm{S}$

Duke of Zanesville $3 \mathrm{~d} \ldots$

Dunkirk .................. _ D

Dunmore 8th ............. - Rolla

Dunmore Duke of Enon.. _ - L R

Dunstan ................. _- Cox

Dwight

Earl of Au-Claire....... Cox

Earl of Baden 2d......... Rolla

Earl of Bond ............

Earl of Brockhurst.......... - Cox

6th Earl of Brookdale.....- Rolla

2d Earl of Cato............ - Cox

Earl Chief ..................... Cox

Earl of Dane........... $R$

Earl Derby ...................... Rose

Earl of Eagledale 2d.......... - D

Earl of Eden ................. Ky I

Earl of Edgewood......... D

Earl of Fairchance........... - D

9th Earl of Hillsdale.......

Earl of Huron ................ Cox

Earl King -....................... Cox

Earl of Lakeview......

Earl Lorain .................. Cox

Earl of Mentz..............

Earl of Nettiewyyunt.......... Ky I

Earl of Oak Creek ......... - Rolla

Earl of Oakland .......... Cox

Earl of Omaha.............. $\mathrm{R} R$

Earl of Oregon..........

2d Earl of Raymond........... R

16th Earl of Ruralusia...... - P S

Earl of Saline.............. C

Earl of Sharon

Earl of Spring Brook...... - P S

Earl of Sunnyside......... D

Easter Lad.................

Ebenezer ................... R R

Ebenezer.................................

Ed Challenger.......

Edgar ................... - Woods

Edgwood Garfield ............ P S

Ed Mitchell ................... - M

Edmond

Edmond 3d........... Beanty

Edmond 7th ........... - Beauty

Einch - .

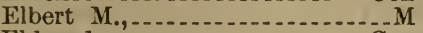

Eldorado..............
62653

62654

6:2656

62657

62659

62660

62661

626662

62663

62664

62667

62668

62669

62670

$626 \% 1$

626\% 2

6:2673

62674

62675

636\%6

62677

$626 \pi 8$

62679

62681

6:2682

62683

62684

62685

62687

62688

6:689

62690

62691

62693

62694

62695

62697

62698

62699

62700

62701

62702

62703

$62 \% 05$

62709

62711

62712

62713

$6: 2714$

62715

62716

62718

62719

$62 \pi 22$

62723

$627 \% 2$

62726

$62 \pi 28$

62729

62730

62731

62732

62733

62734

62735

62736

62737

62738

62739

62740
Eli _ _.................... $-\mathrm{R} R$

Ella's Breastplate......... - R R

Ella's Duke of Goodness ..... - R R

Ellen 11th's Duke.............. Daisy

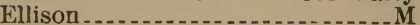

Elmer................

Elmore ................... $\mathrm{M}$

Embarrass Bell

Emerald Lad................... D

Emerson ....................... R

Emperor................. R R

Enchanter .................

Enemy

Eng -................. $R$

Enterprise................ R R

Enterprise -............. - Cox

Euvoy ...........................

Erichson

Esquire Walburg......... $\mathrm{R} R$

Ethelred ....................

Eugene......................... Ky I

Eureka

Eureka................... - R R

Excelsior ................... D

Excelsior .............. P S

Excelsior 2d............... D

Fairchance Oxford $2 \mathrm{~d}$

Fairfield 2d .............. $\mathrm{R} R$

Fair Play ................. L

Fairy's Prince............. - Rolla

Falstaff _.................. Cox

Fanatic ............. Rolla

Fancy Duke............. P S

Fancy Homely.....................

Fancy Lad of Sonora

Fancy Richmond.......... $\mathrm{P}$ S

Farmer......................

Farmer Boy ................ - M

Farmer's Friend.......... $\mathrm{L}$

Fashion's Earl............... - D

Fashion's Prince.......... $L$ L R

Favorite ................ $-\mathrm{L} R$

Favorite 6th ................ - M

Favorite of Lady Jane 17th_- R R

Fern Lad.................... - R R

Fidget's Duke of Glendive... $-\mathrm{R} R$

3d Fidg't's Oxf'd of Sunny Hill-R R

Filigree Gem 10th.......... - R R

Finance.................. $-\mathrm{L} R$

Finance $3 \mathrm{~d}$

Fitz Lewis............... Rolla

Fleka's Duke.............. Cox

Flink ........................... R

Florid D. of Mt. Zion $2 \mathrm{~d}$

Foot's Duke............. P

Forest ............................... S

Forest Duke of Spring Branch_Ky I

7 th Forest King................

8th Forest King........

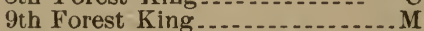

Fort Cribbs D. of Mt. Zion....-M

Fortune Teller.................... Ky I

Foy

Francis Murphy........... $M$

Frank ......... $R$

Frank

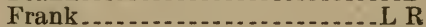

Frank

Frank ........................ Cox

Frank Bryant............... R R 
62741 Frank Church....................

62742 Frank James............. L R

6:74t Franklin ...................... R

62745 Franklin Duke 6th.......... Ky I

62 146 Frank Pierre.............. R R

62747 Frank of Waterford........ D

62749 Frederick .................. Ky I

62751 Fred G.,...................

62752 Frederick of Sonora....... $\mathrm{R} \mathrm{R}$

6:753 Frederick of Sunora $2 \mathrm{~d} \ldots . . .-\mathrm{R} R$

62754 Fritz................... R

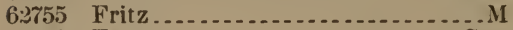

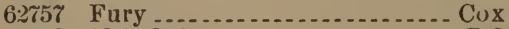

62758 18th Gaban.............. P S

6:\%61 Gambreno................. L R

$62 \tau 63$ Garfield ....................... Ky I

6:76t Garfield .................... KV I

6:765 Garfield ...................... Ky I

62766 Gartield.

6: 167 Garland.....................

6:769 Garland D. 3d of Calumet .. _ - R R

62\%70 Garnet's Duke...... - Milk Spring

6:тช2 Gaudy ................... L R

6:\%74 Gem ...................... Cox

627テ6 Gem's 2त Duke............. R R

62777 Gem Duke of Highland ...... $-\mathrm{M}$

6:380 Gem's Prince............... R R

6:781 Gem Prince .............. R R

6:782 Gena....................... D

62783 General .................. P S

62784 General

62786 Gen. Clark.............

6:787 Gen. Custer........ R

62788 Gen. Desha .............. R R

62759 Gen. Flash of Elm Grove ... - R R

62790 Gen. Gartield.............. L R

6: 792 Gen. Garfield..............

$62 \pi 93$ Gen. Gold ................ T

6:794 Gen. Girrdon.................. T

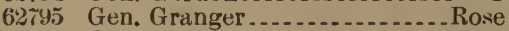

6*796 Gen. Grant................... R

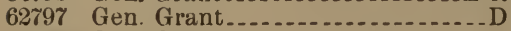

6:798 Gen. Grant

6280:2 Gen. Gwynne $2 \mathrm{~d} . . . . . . . . . .-$ Cox

6:2803 Gen. Hancock............... S S

62804 Gen. Hancock................ Cux

6:805 Gen. Han'k of Native Grove F'm-T

62806 Gen. Hill ................ R R

62807 Gen. Huwe

62808 Gen. IIighn................. T

6:2409 Gen. Jackson........... R R

62811 Gen. Jackson ...............

62812 Gen. Jackson ............... R

62813 Gen. Lee.................. R

62814 Gen. Lee ....................

$62 \times 15$ (ien. Lee....................... Ky I

62816 Gen. Logan ................ R

6:817 Gen. Lingan.....................

6:2818 Gen. Logan .............. P S

$62 \$ 19$ Gen. Loyan..................... Ky I

62820 Gen. Logan..............

6*2*:1 Gen. Logan.................. S S

628:2 Gen. Logan .................. R

62823 Gen. Logan ..................

6:882t Gen. Lugan .................... M

6\% $\diamond^{\circ} 25$ Gen. Mcluok

6\%8:66 Gien. Manderson........... C

6:28:2 Gen. Marmaduke ........... Ky I

628.28 Gen. Marmaduke........... - $\mathrm{P}$ S

$6: 8 * 29$
62830 Gen. Miles.................

628:31 Gen. Price ......... R R

628:32 Gen. Rice..................

62834 Gen. Sheridan ............ P S

6:8336 Gen. Sheridan............. - Cox

6:838 Gen. Slocum ............. R P

62839 Gen. Taylor............

6:28+1 Gen. Ward.............. R R

6284: Gen. Warren............ Daisy

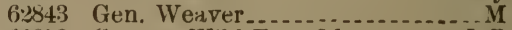

6:8.53 Geneva Wild Eyes 2d.... L R

628.58 Gentle Prince.............

62859 George.....

62860 George.

62861 George

6*862 George $2 \mathrm{~d} \ldots \ldots \ldots \ldots$

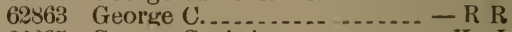

62365 George Guthrie.................

6:866 Geo. G. Vest............ Ky I

6:8667 Geu. Hammond.............

$6: 868$ Geo. Hloadly ........... R R

62869 Geo. Lee.............. L $\mathrm{L}$

62870 Geo. Sherwood.......... R R

62572 Georgetown Duke ........ - L R

62873 Geo. Washington ..........

62874 Geo. Washington ........ Ky I

62875 Geo. Zimmerman........ R R

6:876 Gift's 2d Duke............. Daisy

6:3878 Gilford Buy ............... - Cox

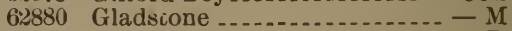

62882 G. L. Chase... D

6288t Gilenlock........... D

62886 Glenwood

6:888 Glenwood 5th.......... - Beauty

62889 Glenwood Lad ............ $\mathrm{R}$ R

6:391 Gloster ............. M

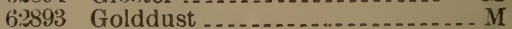

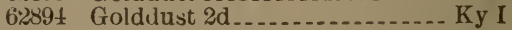

6:2895 Gulden Age 4th ......... - Cux

62897 Gulden Hare............. - Cox

62898 Golden King $2 \mathrm{~d} \ldots \ldots . . . . .$. Cox

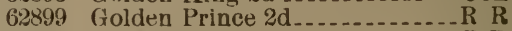

6:3900 Gold Finch .............. R R

6:2y01 Goldtinder............. R R

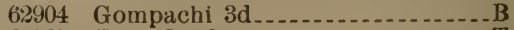

6:2905 Good Luck ........

6:3906 Good Luck

6:3907 Gurdon..............

6:908 Gushen...... Cox

622909 Governor ................ D

$6: 2910$ Governor

62911 Governor................. R

62912 Guvernor

6:913 Governor ........ Daisy

62914 Governor......

62916 Gov. Cleveland

62917 Gov. Cleveland................ R

6:2918 Gov. Cleveland ...........

6:2919 Gut. Furnas ........ R

629:1 G()v. Martin..............

6:29:2 Gov. Mondy

6:29:24 Guv. St. Juhn.............. R R

629:5 Guv. St. John.......... L R

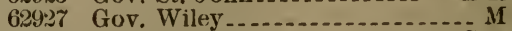

6:29:28 2d Grand Baron .......... Cox

6:29:29 Grand Blanc........ - Long Horn

6:2931 Grand Duke ............

62935 Grand Duke of Cumberland - Ky I

6:936 Grand Duke of Hightower.. $-\mathrm{L} R$ 
62937 Grand Duke of Lee......... - T

62942 Grand Renick ......... R

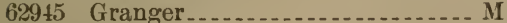

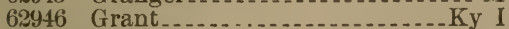

62949 Greeley .......................

62950 Greenwood

62951 Greenwood ............... L R

62952 Greenwood of Sonora

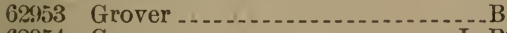

62954 Grover

62955 Grover C.

62956 Grover Cleveland

62957 Grover Cleveland ........

62958 Grover Cleveland ....... L R

62960 Grover Cleveland ........... M

62961 Grover Cleveland

62962 Grover Cleveland.........

62963 Grover Cleveland

62964 G. T. Eli......... B

62965 Guardsman

6:2967 Guardsman of Neosho..... - L R

62969 Guttenburg............. R R

62970 Gwinett

62972 Gwynne Duke ......... Cox

62974 Gwynne Paxton ............

62975 Gwynne Underedge......... - P S

62976 Hamilton

62977 Hamilton's Duke........ R R

62978 Hamlet ................... L R

62981. Hamlet of Melleray ...... - R R

63982 Hamp Watts .....

62983 Hancock ......... M

62984 Hancock

62985 Hancock

62986 Hancock ................ P S

62987 Hancock

62958 Hannibal

62989 Hannibal ..... Cox

62990 Hannibal of Melleray ..... - R R

62991 Hanson County Chief.......... P S

62992 Hardin Grant.................. P S

62993 Harold

62995 Harriet's Oxford $3 \mathrm{~d} \ldots \ldots \ldots-\mathrm{R} R$

62996 Harris Challenger............. $-\mathrm{C}$

62997 Harry

62998 Hawkeye Lad............ Cox

62999 Hays..................... D

63001 Heck's Grant.................. S

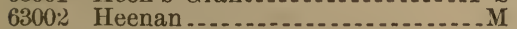

63004 Helianthus $2 \mathrm{~d} . . .6 . . .60 \mathrm{Cox}$

63005 Hendricks....................

63006 Hendricks...................

63007 Hendricks.........

63008 Hendricks................ R

63009 Henry

63011 Henry

63012 Henry Clay............... R R

63013 Henry Lewis

63014 Henry Ward Beecher....... - Cox

63015 Hercules ................ Ky I

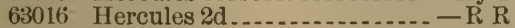

63018 Hero

63019 Hero........

630:0 Hero.............

6302: Hero............... R R

63025 Hick Grant............ S

630:26 Hickory ................... R

63027 Hickory Boy

63029 Highlander............. P S

63030 Highland Duke............. R R
63031 Highland Duke 5th......... - $\mathbf{M}$

63032 Highland Gem 2d..........

63035 Hindoo.................... R

63036 His Honor

63037 Honest Abe.................. R

63038 Hopeful _.................. P S

63039 Hope's Baron..... Cox

63040 Hopewell................

63042 Hopkins ................. Cox

63043 Horace Drake ......... R R

63044 Horace Miller, Jr.,......... R R

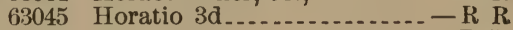

63046 Horatio 4 th.............. $\mathrm{R}$

63047 Housatonic Major 2d...... - L R

63048 Howard Breastplate........

63049 Hubert........... R R

63051 Hulda's Chief.......................

63052 Hunt's Duke................ M

63054 Hurley Porter ....................

63055 Hurrah ..................... Rose

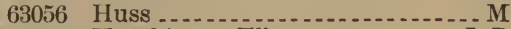

63057 Hutchinson Ellaot............ R R

63060 Idalia's Lad.................

63061 Ida's Duke................... M

63062 Ida's Proud Duke........ Ky I

63063 Idol ........................ Ky I

63066 Independence

63067 Independence ...............

63068 Independence .......

63069 Inigo

63070 Iowa Chief.............

63071 2d Iowa Lad .............. R R

63073 Ironsides ...........

63074 Irvine

63075 Isabella Chief ......... R

63076 Ivanhoe ......................... Cox

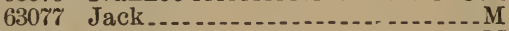

63079 Jack

63080 Jack Logan ................... I

63081 Jack Robinson............. - P S

63082 Jackson ............ M

63083 Jackson

63081 Jacob B.,

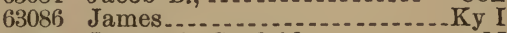

$6: 3087$ James A. Garfield.......... M

63088 James A. Garfield............. R

63089 James G.,.............. P S

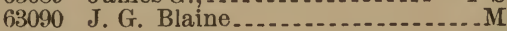

63091 J. G. Blaine

63092 James G. Blaine.... R

63093 James G. Blaine........ R R

63091 James Jeffreys ............. - M

$6: 3095$ Jane's Champion......... R R

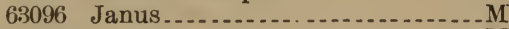

63097 Jasper

63099 Jayhawker .................

63103 Jeff Witch .........

63104 Jennie's Bob.... P S

63105 Jerry ............... R

63106 Jerry

63107 Jerry .......... R R

63108 Jerry Duncan ........... R R

63109 Jerry Morcon............ L R

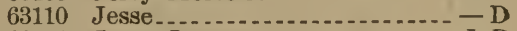

63111 Jesse James.............. L R

63112 Jesse James ................. - C

63113 Jesse Vineyard ............ P S

63114 Jessica's Duke ........... - Cox

63115 Jessie's Grant.........

63116 Jim 


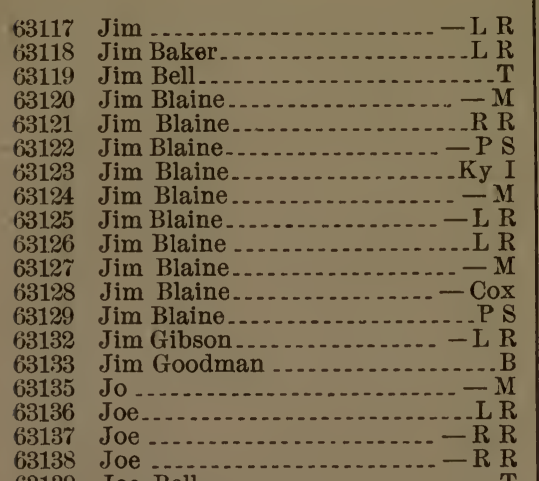

63139 Joe Bell

63141 Joe Dandy

63142 Joe Darby

63143 Joe Darling

63144 Joe Estes................... R R

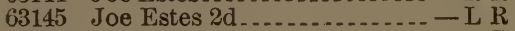

63147 Joe Hooker

63148 Joe Jefferson

63149 Joe Johnson

63150 Joe Johnson 6th.................. Cox

63151 Jo Luckey ...................... Ky I

63152 Joe Mitchell .................. - M

63153 Joe Smith....................

63154 Joe Taylor................... - C

63156 John A. Logan.................. Cox

63157 John A. Logan............. - Cox

63158 John A. Logan ................... S

63159 John A. Logan of Hill's Grove ...T

63161 John Baldwin ................... P S

63162 John Burgess ................ C

63163 John Clark 2d............... Ky I

63164 John Cleveland

63166 John Crockett..........

63167 John Gillette .........................

63168 John Gwynne............. - Cox

63169 John Hancock, Jr.,..... - L R

63170 John Jones .......

63171 John Killgore.................. S

63172 John Lee

63173 Johnnie Yost............... M

63174 John Perryman.............

63175 John Scott..................

63176 John Sherman............ - T

63177 John Sherman................. S S

63180 John Tetzel ..................... B

63181 John Ulmer................... - D

63182 John W., ............... Cox

63184 Jonathan .................

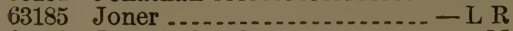

63186 Joseph Combs

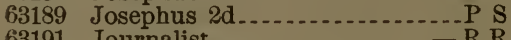

63191 Journalist_.................. R R

63194 Judge Buckner.............. Ky I

63195 Judge Davis..................

63197 Judge Elijah Robinson......... Ky I

63199 Judge Phelps............... - R R

63201 Judge Terrill ....................

63202 Julia Clay's Duke............ - D

63203 Jumbo...................... Rolla

63204
63206

63208

63209

63210

63211

63213

63214

63215

63216

63218

63219

63220

63221

63222

63223

63228

63229

63230

63232

63233

63234

63236

63237

63238

63239

63241

63242

63244

63245

63246

63247

63248

63249

63252

63253

63254

63256

63258

63259

63260

63261

63262

63263

63264

63270

63271

63272

63273

63274

63275

63276

63278

63279

63282

63284

63286

63289

63290

63291

63292

63293

63294

63295

63296

63297

63298

63299

63300

63301

63302

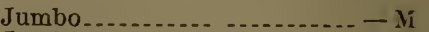

Jumbo

Jumbo..................... $R$ R

Jumbo -................. $-\mathrm{L} R$

Jumbo

Jumbo -....................

Jumbo $2 \mathrm{~d}$..................... Cox

Jumbo of Bell Creek....... Ky I

June Boy ................ - R R

Jupiter

Justice Lad.................. M

Kansas......................

Kansas ..............................

Kansas Boy ................

Kendall ..................... $\mathrm{R} R$

Keota Lad................ M

Kernan

Khedive .................... Ky I

King Airdrie.............. $R$

King Airdrie..................

King Bolivar..................

King Cyrus ........................

King Fairy

King George -....................

King John

King Lewis...................

King Midas..................

King Red Rose $3 \mathrm{~d} . . . . . . .-\mathrm{R} R$

King Richard .... ................ Cox

King Rose ......................

King of Saline.................

Kingscote

King of the Meadows........

King Tom .....................

King William ...................T

Kinellar Kirklevington ....-- $\mathrm{R} R$

Kirk ….........................

2d Kirklev'gton of Forest Home_L R 3d Kirklev'gton of Forest Home _...II 4th Kirklev'ngton of Forest Home M 5th Kirklev'gton of For'st H'me - C 7th Kirklev'gton of Forest Home_M 9th Kirklev'gton of Forest Home.MI 10th Kirkl'v'gt'n of For'st H'me L R Kirk Walters .................- MI Kirkwood ................. $R$ Kirkwood ….......... Kirkwood of Evergreen....- P S Kirkwood Lad.............-R R Kit Low .................... Knight of Barton............... Knightly ...................-Rolla Knightly Bates $2 \mathrm{~d} . . . . . .$. Knight of Iowa............- Rolla Knight of Redwood....... - R R Kossuth..................... D Lad $2 \mathrm{~d}$ Lad of Clear Creek........-R R Lad Duke 2d..............- $R$ Lady Watson's Cherub..... Lafayette ................ $R R$ Laird 3d.............. Lancaster ...................... Lancaster-............... - Cox Lancaster .................... Lassie's Gloster................ Cox Laudable................. Laura Ascot's Duke............ 
63304 Lawn Lad .................... .

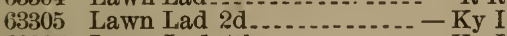
63307 Lawn Lad 4th................. Ky I 63308 Lawn Lad 5th .................. L R 63309 Lawn Lad 6th............ R R 63310 Lawn Lad 7th ............ - R R 63311 Lawn Lad 8th............. - R R 63312 Lawn Lad 9th............ - R R 63313 Lawn Lad 10th............ - R R 63314 Lawn Lad 11th.............. - R R 63315 Lawn Lad 12th............. - R R 63316 Lawn Lad 13th........... - R R 63317 Lawn Lad 14th............. - R R 63318 Lawn Lad 15th................L R 63319 Lawn Lad 16th........... - R R 63320 Lawn Lad 17th............ - R R 63322 Lawn Lad 19th............ - R R 63323 Lawn Lad 20th............. - R R 63324 Lawn Lad 21st............. - R R 63325 Lawn Lad 22d............. - R R 63326 Lawrence ..................... R R 63327 Lawson...............$-P S$ 63328 Leader $2 \mathrm{~d} . \ldots . . . . . . . . . . . .-D$

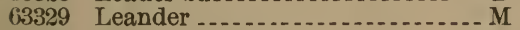
63330 Leap Year Duke........... 63334 Leno Duke 2d................... 63335 Lentus Airdrie $2 \mathrm{~d} . . . . . . . . . . . . .-\mathrm{T}$ 63336 Lentus Airdrie 3d.............. - T 63337 Lentus Airdrie 4th ............. T 63338 Lentus Duke 5th ............... - T 63339 Leo .........................

63340 Leo Boy ............ 63341 Leo of Ceres............. T 63342 Leonard ........................ 63343 Leonard ................... - L R

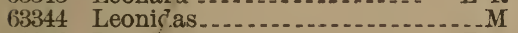
63346 Leoni las ................ R R 63347 Lero 63348 Ler y

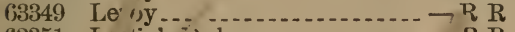
63351 L ilie's Juke............. R R $63353 \mathrm{~J}$ : wis $\ldots 3354 \ldots$ $63355 \mathrm{Le}^{*}$ is $\mathrm{D}$ ke $2 \mathrm{~d} . . . . . . . . . .2 \mathrm{P}$ 63353 J vbie' Duke $3 . . . . . . . . . . . . . . .1 \%$ 633 i 7 ibell', Duke............. - L R 633: $5 \backslash$ Libe cy Duke ................ $\mathrm{M}$ 63319 Lie tenant Barker......... $-\mathrm{L} R$ 633 0 Li utenant Cobb.................M 6331 I reutenant Greeley.............. Ky I 63 घ 2 lilac's Oxford ............. - Cox 63 5 5 Lincoln Duke............. 63507 Listless Duke............. Beauty 63368 Little Phil .......................

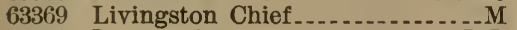
63370 Locomotive .............. $633 \pi 1$ Locum .................. 63373 Logan .................... - L R 63374 Logan ..................... 63375 Logan......................

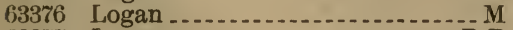
63377 Logan ................... R R 63378 Logan..................... R 63379 Logan 63381 Logan ................................... 63382 Logan ......................... P S 63384 Logan................................ 63385 Logan .............................. 63387 Logan Prince............ 63388 Logan of Sonora........... - D
63389 Longfellow ................ - R R 63390 Longfellow................ $\mathrm{D}$ 63391 Longfellow ........ 63396 Lord Bright Eyes............. - - M $6339 \%$ Lord Byron.............. 63399 Lord Byron 63400 Lord Carnhurst $2 \mathrm{~d}$ 63402 Lord Centerdale $2 \mathrm{~d}$ 63403 Lord Centerdale 4th ......... - M 63404 Lord Chatham ......................... 63405 Lord Chatham 63406 Lord of Cliffland...... Rolla 63407 Lord Clyde............... _ C C 63408 Lord Crook 63409 Lord Darnley 63410 Lord Duke 2d..... 63415 Lord Harry 63416 Lord Henry ................. Ky I 63419 Lord Hubback 4th. ....... - R 63420 Lord Hubback 6th.......... - P S 63421 Lord Hubback 7th........... R R 63422 Lord Hubback 8th ............ R R 63423 Lord Keifer ................. - D 63424 Lord Kenyon 63425 Lord Lassie.................. - D 63427 Lord Leslie................ 63428 Lord Livingston..... - Milk Spring 63433 Lord Matchless 3d........... D 63434 Lord Milnor................... 63443 Lord Renville.......- - Long Horn 63444 Lord Rio..................... Ky I 63445 Lord Robins .............. - Cox 63446 Lord of Rosedale.......... 63448 Lord Rowley 3d.............. Ky I 63449 Lord Rowley 4th............- - M 63450 Lord Rowley 5th............ Ky I 63452 Lord Sale 3d............. R R 63453 Lord Sale of Huron 63454 Lord Shaftoe 63456 Lord of the Dale.......... 63458 Tord Valiant.............. $\mathrm{R} R$ voxu, Lorena's Logan............ - Rolla 63463 Lost Nation................ - R 63465 Louan Chief ............. - R R 63466 Louan's Duke................. 63467 Louan's Duke 2d........ - D 63468 Louan's Duke 2d.......... $\mathrm{R} R$ 63469 Louan Duke 3d of Calumet. - R R 63470 Louanjo ................... P S 63471 Louanjo 2Sth of Courtland.....L R 63472 Louan Muscatoon 3d........ - D 63473 Louan Prince 4th............- R R 63474 Loudon's Duke 2d......... - R R 63475 Loudon Duke 2d.......... D 63478 Loudon Fairfax......... Rolla 63479 Louis Hautboy........... 63480 Lou-Lou's Duke........... - L R 63483 Lucullus Jr.,............ - R R $6348 \tilde{~ L u l a ' s ~ L a i r d ~ . . . . . . . . . . . . . . ~}$ 63486 Luzerne Duke............... - D 63487 Lynden Duke............. Cox 63488 Lysander....................... Ky I 63490 McClellan Goodness Duke ... - P S 63491 McConnell's Major .........-Daisy 63492 McConnell's Major 2d......Daisy 63493 McCormick's Duke.......... - D

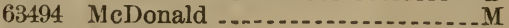
63495 McDonald 63496 McElwain's Cubin 63497 McElwain's Monster......... M 
63498

63499

63500

63501

63502

63.503

63504

63506

63511

63513

63514

63515

63516

63517

63518

63519

63520

63521

63522

63524

63525

63527

63529

63529

63531

63532

63534

63535

63536

63539

63540

63541

63354

63544

63545

63548

63549

63551

63552

63553

63555

63556

63557

63558

63559

63560

63561

63562

63563

63566

$6356 \%$

63568

63569

635\% 0

63571

63572

$635 \% 3$

63574

63575

63576

63577

63580

63582

63584

63585

$6: 3586$

63590

63592

63594

63595
McGregor ................. $-L R$

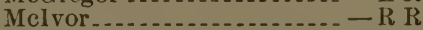
Mack ................ Daisy Mack .................... MeKinzie ............... $\mathrm{L}$ Maclagan .................... R Mc Millen's Flag......... Rolla Mad Cap......... R

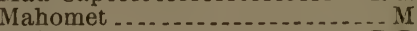
Major........ Major Major ............... Cox Major Major...................... Major Major Maj. Alleu ............. R Maj. Antrim Maj. Benham Maj. Booth.......... Maj. Brown Maj. Cloyd ..... P S Major Dee Maj. Dobbin ........... Daisy Maj. Edwards..... Maj. Gracy............... - R R Maj. Hiper.......... Maj. Holmes ....... Maj. Keith ................... Ky 1 Maj. MeConkey -....... Maj. Manors... Maj. Martin Maj. Randolph Maj. Richmond $2 \mathrm{~d} . . . . . . . . \mathrm{P} S$ Mallory ................... Mandane's Duke........... M Mandane Lad $2 d$........... Maple Creek Duke............. - D Maple Grove Lad............ D Marine Duke................ L R Mark Twain ...................... Marlboro Lad ............. Marmaduke ................... Ky I Marmaduke...................... Marmaduke ....................... Marmaduke................. Marmaduke....................... R Marmaduke $2 \mathrm{~d}$ Marmion ........................ Marquis of Silver Ridge.....- R R Marshall .................. Ky I Marshall Chief-....... Ky I Marshall Duke............ R R Marshall MeAnulty.......... R Mary Ann's Duke........ M Mary's Boy .......... Mary's C. K.,......... R R Mary Clay's Duke 4th Mary Clay's Duke 5th Mary Clay's Duke 6ch...... - T Mary Duke................ Mary Duke of Sharon.... $R$ Mary's King ............... Mary's Sharon Duke......- $-\mathrm{R} \mathrm{R}$ Mlason of Sonora ........... $\mathrm{R} K$ Masontown Duke................ Master Airdrie 8th......... - Cox Master Buttercup .............. M Master Craggs............ P S

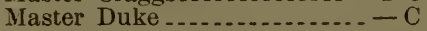

63596 63597 63598 63599

63600 63603 63605 63606 63607

63608

63610

63611

63614

63615

63616

63617

63619

63620

63622

$636: 33$

$636 \% 4$

$636 \div 26$

63627

63628

63633

63634

63636

6:3639

63640

63641

63612

6:3643

$6: 3644$

63645

633646

$6364 \tau$

63648

63649

63650

63651

63653

63654

63655

63658

63660

63661

63664

63666

$6366 \%$

633668

63669

$6: 36 \pi 0$

63671

$6367 \%$

63663

633674

63675

$6: 3676$

636577

63678

$636 \tilde{7} 9$

63650

63681

$6368: 2$

6:3683

63684

63650

63686

63687

63689
Master Ellis ............... - Rolla

Master Hillhurst ........... $-\mathrm{D}$

Master Illustrious......... $-\mathrm{L} R$

Master John .............. $-\mathrm{L}$ R

Master John ............... $-\mathrm{C}$

Masterman $2 \mathrm{~d} \ldots \ldots \ldots \ldots$

Masterpiece of Spring Branch _ - T

Masterplate of Spring Branch _ - T

Master Prince........... $\mathrm{R} \mathrm{R}$

Master Richmond ........ R R

Master Stark............. Ky I

Master Wellington .......... $-\mathrm{D}$

Master Zilla

Mat Hill 3d ................... Daisy

Matilda's 2d Duke ......... - R R

Matilda's Grand Duke...... $\mathrm{R}$ R

Max............. Long Horn Maximus................ P S

May Boy

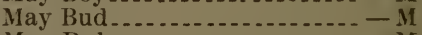

May Duke...

May Duke................. - Cox

Mazurka Bell Duke............ R P

Mazurka Boy ................. R

Mazurka Oxford 6 th.......... P S

Mazurka Oxford 7 th........... $\mathrm{L}$ R

Mazurka Roau Duke............. M

Medalist of Fairview ......... D

Melton Duke............

Melville.............

Meritorious.......... $\mathrm{R}$

Messina's Earl............... Ky I

Midday ................... P S

Midnight............. Daisy

Miguel................... Ky I

Mikado ....................

Mike

Mike of Hillside.......... $\mathrm{L}$ R

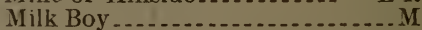

Milo

Milo ....................

Milo P. Smith.............. R

Milton .........

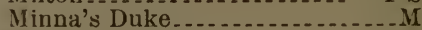

Minna's Prince........... R R

Minnie's Duke $2 \mathrm{~d} . . . . . . . .-$ Daisy

Minor......

Minor.........

Missouri Duke...............

Missouri Duke........

Miss Pugsley's Duke........ D

Miss Wiley's Jubilee........

Mudoc.......... R R

Mudoc.................. R

Hodoc of Brokenstraw ..... Cux

Monareh ................... Cox

Monarch..................

Monareh..................

Monareh..............

Monarch............. R

Mouarch Jr.,.......... D

Monarque............ $\mathrm{R}$

Money Maker............ $\mathrm{R}$

Monitor................. R R

Monitor.......... Ky I

Monitor.............

Monitur...................... II

Munitor Duke............. P S

Montezuma............... R R

Moody ........... 


\begin{tabular}{|c|c|}
\hline 63690 & Moody. \\
\hline 63691 & $\ldots \ldots+P_{S}$ \\
\hline 63693 & Mosske's Duke 4th \\
\hline 63695 & Mountain Home Duke....... - D \\
\hline 63696 & Mountain Meadow Duke....- R R \\
\hline 63697 & 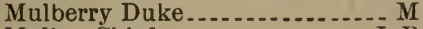 \\
\hline 63698 & Muline Chief ............ L R \\
\hline 63699 & Muline Earl \\
\hline 63700 & Mungo $\ldots \ldots \ldots-\mathrm{R} R$ \\
\hline 63701 & Murkey \\
\hline $6: 3702$ & Muscatoon 2d............ \\
\hline 63703 & Mussel Shell ............... Cox \\
\hline 63704 & Nain \\
\hline 63705 & Nanetta's Roan Duke....... - L R \\
\hline 63706 & Napier Climax......... L R \\
\hline 63707 & Napoleon . . . \\
\hline 63708 & Napoleon \\
\hline 63709 & Napoleon Breastplate........ \\
\hline 63710 & Napuleon Duke............. \\
\hline 63711 & Napoleon of Fairview \\
\hline 63713 & Nat Robinson $\ldots \ldots \ldots-D$ \\
\hline 63716 & Nell's Duke 2d. \\
\hline 63717 & Nelson ..... \\
\hline 63718 & Nelson ... \\
\hline 63719 & Neptune. \\
\hline 63722 & 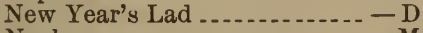 \\
\hline 63724 & Noah ......... \\
\hline 63727 & Nobleman \\
\hline 63728 & Norman J. Colman.......... $\mathrm{L}$ \\
\hline 63729 & Northrup ............. D \\
\hline 63733 & Noxubee Royal................ Ky 1 \\
\hline 63734 & Number One......... $\mathrm{R}$ \\
\hline 63736 & Oakland ..... B \\
\hline 63737 & - Daisy \\
\hline 63738 & Oakwood Prince....... \\
\hline $63 \sim 39$ & Oetavius \\
\hline 63740 & October Morning \\
\hline 63741 & Oelwein Bull .............. Cox \\
\hline 63742 & Ogden Prince . . . . . . Rose \\
\hline 63743 & Ohio Boy .... \\
\hline 63744 & Okoboje... \\
\hline 63745 & Oldham ... \\
\hline 63746 & Ole.... \\
\hline 63747 & - $-1-2-1-2$ \\
\hline $63 \pi 48$ & - \\
\hline 63749 & Oliver . \\
\hline $63 \% 51$ & Oliver Butterfly ..................... Daisy \\
\hline 63753 & Oliver Perry \\
\hline 63754 & Oliver Twist _.... D \\
\hline 63755 & 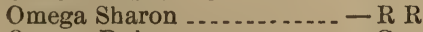 \\
\hline 63759 & Orange Duke .............. Cox \\
\hline 63768 & 12th Orange Duke $\ldots \ldots \ldots-\mathrm{R} R$ \\
\hline 63769 & Orange George $\ldots . . . . .$. \\
\hline 63770 & Orin ........... \\
\hline 63771 & Orontes Jr., . . \\
\hline 63773 & Orphan Beauty's Oxford $\ldots \ldots-R R$ \\
\hline 63774 & Orphan Boy ........ P S \\
\hline 63775 & Orphan Boy \\
\hline $63 \%$ & Orphan Boy ........... R R \\
\hline 63777 & 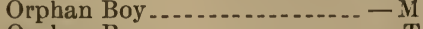 \\
\hline 63778 & Orphan Boy ................ $\mathrm{T}$ \\
\hline 63779 & Orphan Boy \\
\hline 63780 & Orphan Boy Wild Eyes......... Ky I \\
\hline 63781 & Orphan Duke........... \\
\hline 63782 & Osear \\
\hline 63784 & P S \\
\hline 63785 & 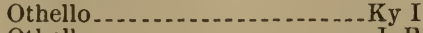 \\
\hline 63786 & Othello......... $\mathrm{R}$ \\
\hline 63787 & Otto \\
\hline & \\
\hline
\end{tabular}

63789 Oxblood...................

63790 Oxford 63791 Oxford Airdrie........... - Cox 63792 Oxford of Airdrie.......... - Cox 63794 Oxford Airdrie 2d............ - M 63795 Oxford Airdrie 3d........... 63796 Oxford Airdrie 4th..........- M 63797 Oxford Airdrie 5th......... 63798 Oxford Airdrie 6th............. Ky I 63801 Oxford Boy .............. C 63802 Oxford Bright Eyes 63805 Oxford Duke................. - M 63807 Oxford Duke of La Harpe .... - M 63808 Oxford Earl_................ - D 63809 Oxford Geneva.......... 63810 Oxford Junior ............ P S 63811 Oxford Jumbo.......... - Rolla 63812 Oxford Lad .................

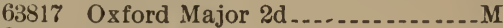
63818 Oxford Monarch.......... 63819 Oxford Nashua............ Ky I 63820 Oxford Prince............... D 638:2 Oxford's 4th Royal Duke... - R R 638:24 Oxford Taylor........... - Cox 63826 Oxford Wild 63829 Packenham ....................... S 6:3830 Paddy Malone ........ Rolla 63831 Paragon .............. R 63832 Paragon Taylor $3 \mathrm{~d}$ 63833 Parepa's Duke............. 63834 Paris Lad .................. R 63835 Parlor Gem 63836 Partch's Favorite................ 63837 Pataskala.................... 63838 Patrick ................... L R 63839 Patrick Parnell 63810 Patrick Wells.............. 63842 Paul Clifford ............. S 63843 Paul Jones ........................ 63814 Pawnee Chief ... Rolla 63845 Pawnee Prince.......... Ky I 63846 Paxton ......... 63847 Pearl 63848 Pearl of Decatur 63849 Pearl Duke..................

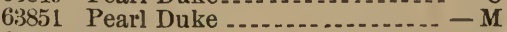

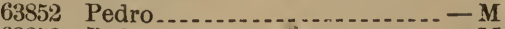
63853 Pedro 63854 Pedro of Evergreen 63856 Pegram .......................... 63857 Pelops 63858 Perdix 63859 Perfection 63860 Perfection.................. 63861 Perfection King 63864 Peri Duke 2d ........... R R 63867 Perryton Lad ....

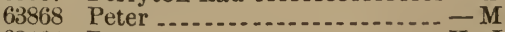
63869 Peter A. 63870 Peter Boyd................. R 638\%2 Peter Gray ............... P S 63873 Peter McNeill ............. L R 63875 Pet of Wildwood........ D 63878 Phil Thompson 63882 Phyllis Prince......... Cox 63884 Pike............... P S 63885 Pilot 63886 Pilot_.............. 63887 Pilot Bernhardt........... Ky I

63889 Pine Hill Lad I. .......... D 
63890

63891

63892

63893

63894

63895

63896

63897

63898

63899

63900

63901

63907

63908

63909

63910

63911

63912

63913

63914

63915

63916

63917

63919

63920

63921

63922

63923

63924

63926

63927

63928

63929

63930

(63931

(j3932

63933

63934

63935

63936

63937

63938

63939

63942

63943

63944

63945

63946

63947

63948

63950

63951

63953

63954

63955

43956

63958

63960

63961

63964

63967

$6396 \mathrm{~S}$

63969

63970

6.39\%?

63973

63974

63975

๒3976

$6397 \%$
Pioneer -

Plantagenet 10th

Plato........

Pleasant................... $\mathrm{R}$

Plumed Knight..............

Plum Run Duke...........

Plumwood Lyon ............ R R

Plymouth Duke........

Pollux................ Cox

Pomona's Booth....... R R

Pomona Boy .............. - Rolla

Pope Boy .......

Post Boy ........

Potawatomie Duke......... P $\mathrm{S}$

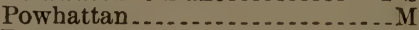

Prairie Boy .......... R

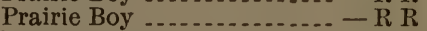

Prairie Duke ............. $\mathrm{D}$

Prairie Duke............. Rolla

Prairie Lad ........... R R

President................... R

President ................... Daisy

President............ COE

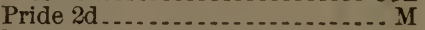

Pride of Brookdale....... $\mathrm{R} R$

Pride of Lone Jack........ $\mathrm{R} R$

Pride of Nebraska......... $\mathrm{R}$ R

Pride of Prairieville..........

Pride of Thurston........ Cox

Prince.................

Prince ....... R R

Prince ................... Ky I

Prince

Prince .........

Prince............ Ky I

Prince....... P S

Prince..........

Prince.

Prince . . Daisy

Prince ...............

Prince 2d ................. Cox

Prince 2d.................. Rose

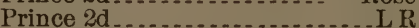

Prince Albert............ L R

Prince Albert............. R R

Prince Albert ...........

Prince Albert ............. R R

Prince Albert...............

Prince Albert ...... Cox

Prince Albert................

Prince Albert............. P S

Prince Albert..................... M

Prince of Anoka 5th.... Multiflora

Prince Arabella........... - L R

Prince Arthur................. Ky I

Prince Arihur............. - M

Prince Arthur ........ L R

Prince of Baldwin ............

Prince Bismarck .......

Prince Carmel 2d........

Prince of Clark.......... $R$

Prince of Corwin ............ Ky I

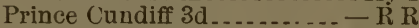

Prince Edward............ $\mathrm{R} R$

Prince of Elmwood...... R R

Prince of Fish Creek...... $-\mathrm{L}$ R

Prince Frederick 5th....... - P S

Prince Frederick 6th...... Kolla

Prince of Geneva.......... - R R

Prince Gwynne 5th..........
63978

63979

63980

63981

63983

63989

63990

63991

63993

63994

63995

$63995^{1}$

63996

63997

63998

63999

64000

64001

64003

64005

64006

64007

64008

64009

64010

64011

64012

64013

64016

64017

64090

64022

64023

64025

$6402 \pi$

$6 \pm 0 \% 28$

64029

64030

64031

64033

64034

64036

$6403 \tilde{r}$

64038

64039

64040

64041

64042

64043

64044

64045

64046

64048

61049

64050

64053

64054

64055

64056

$6405 \%$

$6405 \mathrm{~S}$

64059

64060

64061

64062

64063

64064

64066

64067

$640 \% 0$
Prince Gwynne 6th ............. T

Prince of Hesper................

$2 d$ Prince of Highland........

Prince Imperial........... Cox

Prince of Jackson $3 \mathrm{~d} . . . . . . .-\mathrm{P} \mathrm{S}$

Prince Lebanon ........... $\mathrm{L} R$

Prince of Lenawee..............

Prince Leopold ............ - Cox

Prince Loudon $3 \mathrm{~d} . . . . . . . . . .-\mathrm{M}$

Prince of Lovette.............. L R

Prince Malone 8th......... Prince Malone 9th.........

Prince of Maple Grove......... B

Prince Mazurka..............

Prince Napier............... R R

Prince Napier 11th........ $\mathrm{R}$ R

Prince Napier 19th....... - Ky I

Prince of Oaklawn $2 \mathrm{~d} . . . . .-\mathrm{P} R$

Prince of Palmer.........

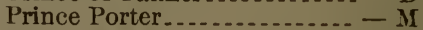

Prince Powers..............

Prince of Putnam

Prince Red Rose.............. D

Prince of Ripley.............. $-\mathrm{D}$

6th Prince of Riverdale.... $\mathrm{R} R$

Prince of Rome ........... $-\mathrm{L} R$

Prince Royal............... $-\mathrm{D}$

Prince Royal............... PS

Prince of Sharon 12th ...... Cox

Prince of Smyrna :..........- Cox

Prince of the Oaks........

Prince of the Roses........ Ky I

Prince of the Valley........

Princeton 2d.............

Prince of Wales........... Cox

Prince of Wales............... $-\mathrm{M}$

Prince of Warren.......... L R

Professor ............... Daisy

Prohibition ...............

Proud Duke of Cass County - Ky I

Proud Duke of Persia........ $-\bar{C}$

Proud Wells ............... $\mathrm{R}$ R

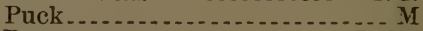

Pug . Daisy

Pulaski's Red Duke........... Ky I

Pulsation ................

Punch.............. R

Punch......... Rolla

Quaker............................

Quarryman .................. Rolla

Queen's Airdrie................ R

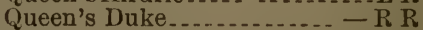

Queen's Oxford............... R

Queen's Richmond ..........

Quirk's Run Prince......... L R

Ralph ..........................

Ralph

Ranger....................

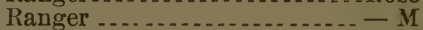

Ranger $2 \mathrm{~d} . . . . . . . .2 R$

Raquet $\ldots \ldots \ldots$

Rathbone ................

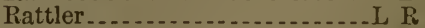

Ray's Earl...................

Raymond........................

R. D. Logan ......................

Reconstruction

Red Arthur .................

Red Baron .................... Ky

Red Billy ........... 
$640 \pi 1$

64072

t54074

64075

64076

$6407 \%$

64078

(64081

64082

64083

64086

64087

64088

64090

64091

64092

64093

64094

64095

64096

64098

64099

64101

64102

64103

64104

64105

64106

64108

64109

64110

64112

64113

64115

64116

64119

64120

64122

64123

64124

64125

64126

64127

64128

64129

64130

64131

64132

64133

64134

64135

64138

64139

64140

64141

64142

64143

64145

64146

64147

64149

64151

64152

64153

64154

64159

64160

64161

64162

64163
Red Boy

Red Boy

Red Boy of Calumet.......... Ky I

Red Buck ...................... M

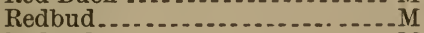

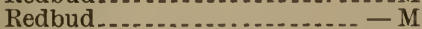

Redbud........................

Red Cherry Duke.....

Red Chief...................

Red Chief............... Ky I

Red Cloud

Red Cloud ...................... D

Red Cloud .................. D

Red Dalmahay

Red Dick.....................

Red Dick.......

Red Duke.....................

Red Duke.......... $R$

Red Duke..........

Red Duke............ $R$

Red Duke........

Red Duke........... Daisy

Red Duke............................

Red Duke....... P S

Red Duke........

Red Duke..................

Red Duke................

Red Duke .............

Red Duke of Bear Creek....... - M

Red Duke of Fairview...... $\mathrm{L}$ R

Red Duke of Glendive............M

Red Duke Rose 5th............ L R

Red Duke of Sharon - Milk Spring

Red Eagle ................... - M

Red Elm............................

Red Fiash

Red Fox .....................

Red Geneva................. - C

Red Jack ............ D

Red Jacket.............. P S

Red Jacket............... - R R

Red Jacket. .........

Red Jacket ................ - Daisy

Red Jacket ..... .

Red Jacket.................... R

Red Jacket ........... Cox

Red Jacket............ P S

Red Jacket ............... - Cox

Red Jim.................... Ky I

Red Joe.................. C

Red John ........... T

Red Lad ............

Red Lad ................... KyI

Red Lad $2 d$................ R

Red Lark ..................... - T

Red Lightning............. - P S

Red Logan ................ - R R

Red Lord of Salt Creek ............

Red Maid's Duke........... - P S

Redman ................ R R

Red Manchester 2d......... P S

Red Monarch .............. - L R

Redmond Duke.............. $-\mathrm{T}$

Red Montcalm ................. L R

Red Oak 2d................ R R

Red Prince....................

Red Prince .................. M

Red Prince...................... C

Red Richmond............

Red Roan........................
64165 Red Rose's Duke............ - P \&

64166 Red Rose Gloster................L R

64168 Red Rover of Colmar........... P S

64169 Red Royal..................

64170 Red Royal............... R

64171 Red Royal Duke.......... D

64172 Red Ruby

64174 Red Waterloo................ M

64175 Red Wing

64176 Red Winston.............. - R R

64178 Reformer....................... I

64179 Reformer .............

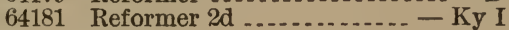

64182 Regent....................... Ky I

64186 Remington.............. D

64187 Remus......................... R

64188 Renick Duke ..............

64190 Reno 5th

64191 Representative............ D

64193 Reuben.................... M

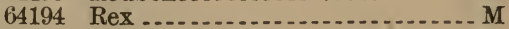

64195 Rhoderick Dhu ........... D

64196 Richland Duke..... Milk Spring

64197 Ridgeland Grover............... D

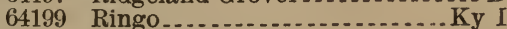

64200 Riverview Lad.............. - L R

64201 Riverview Lad $2 \mathrm{~d} . . . . . . .-\mathrm{R} R$

64202 Riverview Lad 3d........... - Cox

64203 Riverview Lad 4th .......... - Cox

64204 Riverside Lad 5th.......... - R R

64206 Roan Bob.................

64207 Roań Boy

64208 Roan Boy

64209. Roan Boy ................... I

64210 Roan Boy.................

64213 Roan Duke ................ M

64214 Roan Duke.....................

64215 Roan Duke................... Ky I

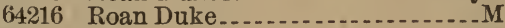

64217 Roan Duke.....

64218 Roan Duke

64219 Rean Duke............... D

64220 Roan Duke 2d............. Cox

64221 Roan Duke 2d.............. - L R

64224 Roan Duke of Sharon........- L R

64226 Roan Earl ................ - Cox

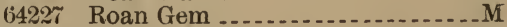

64228 Roan Hero

64229 Roan Jupiter............ R R

64230 Roan's Kirklevington........ - P S

64231 Roan Lad ....................

64232 Roan Lad

64235 Roan Lord.................... R

64237 Roan Oak.................. R

64239 Roanoke ........................ M

64240 Roan Prince....................... Cox

64242 Roan Prince of Vine Hill ...... - D

64243 Roan Robin ............. R R

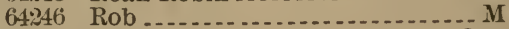

64247 Robber ..............................

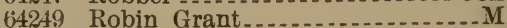

64250 Robin Hood ............... S S

64251 Robin Hood..................

64252 Robin Hood

64253 Robin Redbreast ...............

64254 Rob Roy ......................

64255 Rob Roy .................

64256 Rob Roy

64257 Rob Roy ................. L R

64258 Rob Roy 
64260 Rockefellers Lad........... - L R 64261

64262

64263

64264

64265

64266

64269

$642 \% 0$

64271

64272

64273

64274

64275

64276

64277

64278

64279

64280

64281

64282

64285

64286

64287

64288

64289

64290

64:291

64292

64294

64:295

64296

64297

64298

64302

64303

64307

64308

64310

64311

64312

64313

$6431 \%$

64318

64319

64320

64323

64325

64326

64328

64329

64330

64332

64333

64334

64335

64336

64337

64339

64341

64342

64343

64344

64345

64346

64348

64350

64352

64354

64355

Rock Rose's Duke-...... - Daisy

Rock Rose 4th's Duke.... - Daisy

Rock Rose 5th's Duke...... - Daisy

Roderick ................ Ky I

Rodger

Rolla Kossuth

Rome's Duke............ Cox

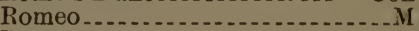

Romeo

Romeo

Romeo

Romeo

Romulus.............

Romulus .............

Rosamond's Bates $2 \mathrm{~d} . . . . .-\mathrm{R} R$

Rosamond's Bates 3d........ M

Rosco

Roscoe...........

Roscoe

Rose Boy .......... - Long Horn

Rosebud...............

Rosebud...................

Rosebud 2d........ L R

Rosebud Duke............... D

Rosedale Duke.............

Rose Duke..................

Rose Duke........... Ky I

Rose's Duke .................

Rose Duke 2d..... P S

Rose Duke 3d..........

Rose Duke 4th.........

Rose Duke 5th............. - P S

Rosemary Boy .............. - L R

Rosemary Lad..............

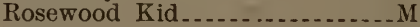

Rothwell . . .

Rover .............. P S

Rover........ R

Rover....................

Rover...............

Roy

Royal -..................... L R

Royal

Royal Airdrie 4th .......... - D

Royal Chief................. B

Royal Commander........ D

Royal Commander............. S S

Royal Cottonwood

Royal Crown ............ P S

Royal Dalton ............

Royal Dane $2 \mathrm{~d}$

Royal Duke .......

Royal Duke...........

Royal Duke ................... Ky I

Royal Duke ................ R R

Royal Duke 2d.............

Royal Duke of Fort Cribbo _- $R R$

Royal Duke of Rochester......Ky I

Royal George.............. - L R

Royal George ............. - Cox

Royal Goodness....- Milk Spring

Royal Hope..............

Royal Jupiter........

Royal Lad ....................

Royal Leslie $2 \mathrm{~d} . . . . . . .2 R$

Royal Oak.................

Royal Prince

Royal Prince 5th.........
64356

64357

64358

64360

64361

64362

64366

64367

64:369

64370

64371

64372

64373

64374

64376

$6437 \%$

64378

64379

64381

64382

64384

64385

64386

64387

64388

64389

64390

64392

64393

64394

64395

64396

64397

64398

64399

64400

64401

$6+402$

64403

64404

64405

64407

$6440 \mathrm{~s}$

64409

64410

64411

64412

$6+417$

64419

64421

64422

64424

64425

$6+426$

64427

64428

$6+4: 29$

64430

64431

64433

64437

64439

$6+414$

64445

64446

$6444 \pi$

64451

64452

64453

64456
Royal Ranchman.......... - L R

Royal Red Duke...........

Royal Red Rose.......... R

Royal Syracuse............. - Cox

Royalton Hero.......... Cox

2d Royalton Hero......... Cox

Ruby's Duke............ R R

Ruby Duke................ - R R

Rufus.................

Rufus $2 \mathrm{~d}$.

Rufus B.,

Ruler

Rullus -....

Rural

Sacramento .........

Safford .................. S

Sailor $3 \mathrm{~d}$

Saint Elmo .............. R R

Saint Elmo.................

St. John ................ R

St. John........ D

St. John

St. John

St. John

St. John ............... D

St. John of Carnahan......... L R

St. John Sharon................

St. Patrick .........

St. Valentine.........

St. Valentine.......... $-\mathrm{L} \mathrm{R}$

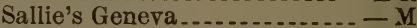

Sam .... Cox

Sam

Sam Bell.

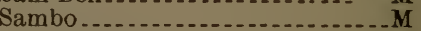

Sam Ennis........ R R

Sam Huxley ................

Sam Patch...............................

Sampson . .

Samson

Sam Tilden........ D

Samuel.................

Sancho ....... Cox

Sankey -...

Sankey

Santa Claus....... R

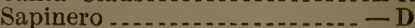

Scotsman's Duke $2 \mathrm{~d}$

Scott......................... Ky I

Seaplate..............

Seaplate $3 d . \ldots \ldots$

Seigmond .................. R

Senator -............. C

Senator

Senator ..............................

Senator .............. Ky I

Senator ............................

Senator ................ R

Senator Blaine............. L

Senator Wonn ..........

Shaker Duke............. Cox

Sharon 2d................ P S

Sharon Butterfly ......... Daisy

Sharon Duke ........ P S

Sharon Duke............. - B

Sharon Duke..............

Sharon Duke of Carroll

Sharon D. of Silver Ridge - - Rolla

Sharon D. of Forest Hill .... - R R

Sharon Favorite............ $M$ 
64460

64461

B4462

64464

64465

64466

64468

64469

64470

64472

64473

64474

64475

64476

64477

64479

64480

64481

64482

64484

64485

64486

64487

64488

64489

64490

64492

64493

64494

64495

64496

64500

64502

64503

64504

64505

64506

64508

64509

64511

64512

64513

64514

64516

64517

64518

64519

64520

64521

64522

64524

64525

64527

64529

64530

64532

64533

64534

64535

64536

64537

64538

64539

64540

64542

64543

64544

64545

64546

64547
Sharon Lad $2 d$.

Sharon Pride............... $\mathrm{R} R$

Sharon Prince..........

Sharon Venice.............. R R

Sharon Wiley $2 \mathrm{~d} . . . . . . . . .2 S$

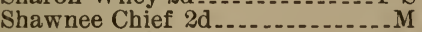

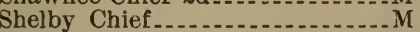

Shenandoah...............

Sheridan of Sonora....... R R

Sherman ...

Sherman's Delight.............

Shiloh _..................

Short Legs

Sidney Dunmore....... D

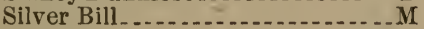

Silverside

Simroe................. L R

Sir Anthony.............

Sir Clay 2d................. I

Sir Corbin. ................

Sir Garnet.........

Sir George.

Sir George............................ R

Sir Hakes...... P S

Sir Hawthorn .......... R R

Sir Henry ...........

Sirius

Sir John ........ R R

Sir John $2 \mathrm{~d}$

Sir John of Pleasant Ridge....Ky I

Sir Joseph...............

Sir Marmaduke ...... R R

Sir Oxford.......... P S

Sir Profitable.............

Sir Richard.............. R

Sir Rupert..............

Sir Thomas ....... R R

Sir William

Sir William .

Sitting Bull

S. J. Nichols ............. Cox

Skipper ........ L R

Slater _..............

Snag Foot........ P S

Snow Cloud........

Soko .

Soldier Creek Lad.......... L R

Soldier Creek Lad 1st...........R R

Soldier Creek Lad $2 \mathrm{~d} . . . . . .-\mathrm{L} R$

Soldier Creek Lad 3d...... - R R

Soldier Creek Lad 5th...... - L R

Soldier Creek Lad 6th...... - R R

Solon of Edgewood......... - P S

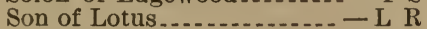

Soothsayer ...........

Spillman Derby ... D

Splendor ........ R

Sportsman

Spotted Boy

Spotted Duke........ R R

Spotted Duke .

Spotted Lad of Bell Creek .. - Ky I

Spotted Prince.............-Cox

Spotted Tail ....... R R

Sprightly _..........

Springdale ................ R

Springdale Airdrie 4th .... $-\mathrm{R} R$ Spring Hill Duke......... $-\mathrm{R} R$

Spring Hill Duke ..............

Spruce Duke.... L R
64548

64549

64550

64551

64552

64554

64555

64556

64558

64560

64561

64562

64563

64564

64565

64566

64567

64570

64573

$645 \% 4$

$645 \% 6$

$6457 \%$

64578

64579

64580

$6+581$

64583

64584

64585

64586

64587

64588

64590

64591

64592

64593

64594

64595

64596

64597

$6 \pm 600$

64601

64602

64603

64606

64607

64610

64612

64613

61616

64617

64619

64620

64621

6462:

64623

64625

64626

$6+6: 7$

64628

64629

64630

6+1631

64632

64633

64634

64635

64636

64637

64638
Spupendycke ............. R R

Squire Acomb...................

Stafford . . .

Staley's Favorite............. $\mathrm{K}$

Standard................

Stanley ................. R R

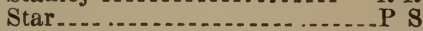

Star

Star Duke..........................

Starlight .....................

Starlight . .

Starlight .........

Starlight..................

Starlight Major

Star Richmond................

Star of Udolpho...............

Stephen's Duke............... Ky I

Stillwater .................... R

Stonewall _.............

Stonewall ............... R R

Straight Line......................

Seventh Strawberry Duke......P S

Success ........... R R

Sultan .............

Summit Duke ..............

Summit Duke $2 \mathrm{~d} . \ldots . . . . . . . .-\mathrm{C}$

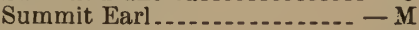

Sunnyside Duke..........

Sunshine .................

Sunshine's Duke.......... P S

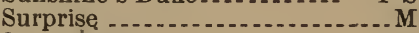

Sweepstakes ...............

Sylvander ............

Sylvester....................

Tallula Duke............. P S

Tammany ......... R R

Tam O'Shanter..........

Tanner

Taurus . . . .

Taurus

Tecumseh ..................

Telemachus of Melleray.... $R$ R

The Baron ................. $-\mathrm{L}$ R

The Doctor ..............

Themistocles............

Theobold .................

The Squire....... R

Thomas Duke............. $\mathrm{L}$ R

Thomlinson's Duke......

Thorudale $2 \mathrm{~d} \ldots . . . . . . . .2 \mathrm{R}$

Thorndale Lad................ $-\mathrm{C}$

Thornton

Threlkeld's Duke........... Cox

Thurman ...................... I

Thurman .................

Tilden

Tipton .

Tobe ........... P S

Tobe Cooke.............

Tom .......... Daisy

Tom ...

Tom Bell

Tom Bell

Tom Brown

Tom Embry .

Tom Hall ...............

Tom Hendricks........ P S

Tom Moore ........

Tommy Smith........

Tom Paine ........... P S 
64639

64640

64642

64643

64644

64646

64648

64649

64650

64651

64652

64653

64654

64655

64656

64657

64658

64659

64660

64661

64662

64663

64664

64665

64666

64667

64668

64670

64671

64672

64673

64674

64675

64676

$6467 \%$

64680

64682

64690

64691

64692

64696

64697

64699

64700

64701

64702

64703

64704

64705

64706

64707

$64 \% 10$

64711

64713

64 \%16

64718

64719

64722

64723

64726

64728

64730

64731

64732

64734

64736

64737

64739

$6 \pm 740$

64741
Tom Pansy

$-\mathrm{C}$

Tom Rose-............

Tom Smith $3 \mathrm{~d}$

Tom Wappett.................... S

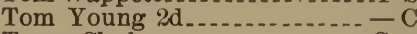

Town Clerk......................

Traveler

Trenton Duke

Trenton Duke 2d................. Cox

Trenton Lad .....................

Trenton Prince....................

Trojan ................... D

Trojan

Trouble -...

True Blood.................

True's Minister.......

Trustee ...............

Turley Read...........

Turney's Prince............ R R

Tuscola Chief................... R

Twin Baron ............... - R R

Twin Baron $2 \mathrm{~d} . . . . . . . . .$.

Twinkle's Barmpton........ - R R

Tyee ..................... - L R

Ulysses of Melleray........ $-R R$

Uncas.......................

Underedge Prince......

Union Duke...................

Unknown .................

Uxbridge Lad $2 \mathrm{~d} . . . .$.

Vain Lad of Ada............ $\mathrm{M}$

Valentine ................... Ky I

Valentine's Day .......... Cox

Valley Duke.............. Ky I

Valley's Duke......... P S

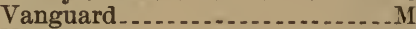

Vanity

Verdict................. Daisy

Vest ...............

Vest $\ldots . . . . . . . . .18$

Victor ..........................

Victor

Victor

Victor

Victor ..................

Victor

Victor

Victor...................... $\mathrm{R}$

Victor $2 \mathrm{~d}$

Victor Hugo ................... M

Victor Hugo ........................

Victoria D. of Mayville 5th

Vigo _............. $\mathrm{R}$ Villard....................... $-\mathrm{L}$

Vinnie's Duke 4th ......... - R R

Viscount Peterkin ............. M

6th Viscount of Glen Leven... - C

Wabash ............

Waco ................

Walter Davis.......

Walter Scott..................

Wamba ................ Ky I

Wane Prince.....................

War Dance................. - L R

Warrior........................

Washburn -.......... $\mathrm{R}$

Washington

Washington Bates......... $\mathrm{L}$ R

Wash Jones.............. - Cox

Wat
64742

64743

64749

64751

64752

64753

64754

64755

64756

$6475 \%$

64758

64759

$64 \div 60$

64761

64762

64763

64764

64765

64766

64777

64778

6479

64780

64781

64782

64783

64784

64785

64786

$6478 \tilde{1}$

64789

64791

64794

64795

64798

64799

64800

64801

64802

64803

64804

64805

64806

64807

64809

64810

64811

64812

64815

64816

64518

64819

64820

64821

64822

64823

64824

64825

64826

644:2

64829

64830

64832

64834

64837

64840

64841

64842

64846

64848
Waterloo _....................

Waterloo Baronet $2 \mathrm{~d}$

Waterloo Prince 2d........ $\mathrm{R} R$

Wave Duke of Evergreen.......P S

Wea Commander.............. M

Wea Dude........................ S S

Webster.................... T

Weehawken 8th's Duke..... - M

Welcome............ D

Weller ................... Ky I

Wellington ........................

Wellington Duke.......... $\mathrm{R} R$

Wellington $2 d$ of Sonora.... $-R$

Wesley - .......................

Western

Western Comet.......

Weston......................

Wheatland's Pride......... $\mathrm{P}$ S

Wheeler ................... M

W. H. English............ L R

White Belt................... $\mathrm{B}$

White Cloud..............

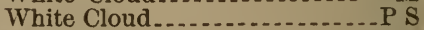

White Comet............. - Ky I

White Earl .......................

White Foot...................... S

White Prince...........

White Rose Duke......... $\mathrm{R}$

White Stockings.............. - M

Whitewater Duke Boy ........ - M

Wild Eyes................. - Ky I

Wild Eves Duke................ $\mathrm{R}$

Wild Eyes Lad................. M

Wild Eyes of Limestone..- - Daisy

Wiley of Ogden.................

Wiley Oxford......................

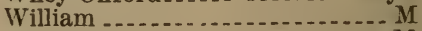

William Rufus..................

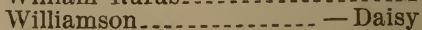

William Tell _..............

William Walker...............

Willow Duke 4th.......... $\mathrm{R} R$

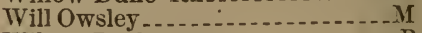

Wilson Darlington

Winfield of Sonora...... $R$

Winnebago Chief $2 \mathrm{~d}$

Wonder ........

Woodbine..............

Yankee Boy ................ R

Yankton Dick

Yellow Bill 2d...........

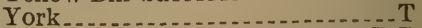

Young Airdrie........

Young Airdrie

Young Albert............. R R

Young Aleck .........

Young Billy

Young Breastplate.............. M

Young Breastplate ....... M

Young Cato................ Rose

Young Champion ........

Young Charley Davis....... - L R

Young Duke............. $\mathrm{R}$ R

Young Duke $2 \mathrm{~d}$

Young Emperor.......... R R

Young Goshen............

Young Hero .............

Young Kickapoo.........

Young Mary's Dukę..........

Young Napoleon ........... 
64849 Young Oliver of Oakhill...... - D 64852 Young Tameron..................... 64853 Young Washington ....... R R 64854 Young Wingard........... L R 64855 Zenaida's Earl................. D

64856 Zenaida's Pearl............... D 64857 Zero......

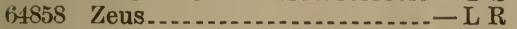
64877 Bushbury Count of Oxford - Rolla 64948 Aaron ............ L R

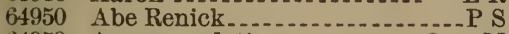
64952 Accommodation 64956 64957 64958 64959 64960 64961 64962 64963 64964 64965 64966 64967 64968 64969 64970

$649 \% 1$

64973

64975

$649 \% 7$

64980

64981

64982

64983

64984

64985

64986

64987

64988

64989

64993

64996

64998

65002

65003

65007

65008

65009

65011

65012

65013

65014

65016

65017

65019

65020

65025

65026

65027

$6502 \mathrm{~S}$

65029

6.5030

65031

65032

65033

65035

65036

65038

6.5039

Acme

Acme Barmpton 6th

Acme Barmpton 7th..... - Daisy

Acomb Boy ............ Rolla

Acomb Prince ........... Cox

Acorn's Duke 6th........ - Rolla

Adam 5th... L R

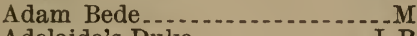

Adelaide's Duke...... L R

Adolpus............ P S

A. D. Temple

Agnes Duke 4th

Agnes Duke 5th ........ Daisy

Agnes Duke 6th ......... Daisy

Ahava Duke ...........

Airdrie

Airdrie 15th............. Rose

Airdrie Bates............ L R

Airdrie Chief............. $\mathrm{R}$

Airdrie Duke 2d.........

Airdrie Duke 3d.........

6th Airdrie D. of Constance-Rolla

Airdrie Duke of Forest Home. - M

7th Airdrie Duke of Sandusky - D

8th Airdrie Duke of Sandusky - M

9th Airdrie D. of Sandusky - $-\mathrm{R} R$

10th Airdrie Duke of Sandusky - D

11th Airdrie Duke of Sandusky - D

27th Airdrie Duke of Sciota......D

Airdrie Goodness 2d.......... - M

Airdrie Gwynne......... Cox

Airdrie Knight.......... R R

Airdrie Napier............ R R

Airdrie Prince........... $\mathrm{R}$

Albert. . .

Albert....... R

Albert......

Albert 5th

Albert Wood...... D

Albin

Alexander Hope........ R R

Alexis Duke............ Rolla

Alexis Prince ...............

Alfonso Duke 2d.........

Alleen Duke............... Ky I

Alonzo......

Alphonso ........ R R

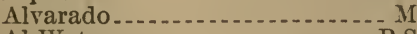

Al Watson............... P S

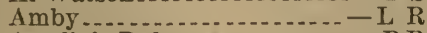

Amelia's Duke........... R R

Ancestor................... R

Andrew Samuel 2d........ $-\mathrm{L}$ R

Andy . . .

Annie's Duke............

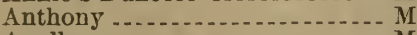

Apollo.

April Day .........................
65040

65041

65042

65043

65045

65046

65049

65051

65052

65053

65054

65056

65058

65060

65064

65065

65068

65071

65073

65074

65075

$650 \% 6$

65077

65078

65079

65080

65082

65084

65085

$6508 \%$

65089

65090

65092

65093

65094

65095

65096

65097

65100

65105

65111

65113

65114

65115

65116

65119

65126

65128

65129

65130

65132

65133

$6513 \%$

65140

65143

65144

65146

65147

65148

65149

65160

65161

65162

65163

65166

6.5167

65168

65170

$651 \% 1$

65172
April Fool...................... T

April Fool......

Araby Bey................

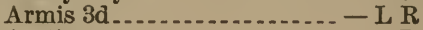

Arctic...................

Aristos.............

Arlington

Artesia Dick............. R R

Artesia Joe................. R R

Arthur............. D

Arthur................... COX

Aruba's Duke $2 \mathrm{~d} . . . . . . . . . . \mathrm{L}$ R

Ascot's Prince.............. $R$

Ashley ...................

Athelstane 4th....... D

Athelstane 4th

Athelstane 7 th

Athelete

Atlanta Monarch..........

Atlas Duke......................

Autocrat............. $\mathrm{R}$

Autocrat.....................

Autumn Duke.... P S

Avanee .............

A. W. Lamb...

Ayer's Duke............... $R$

Aylesford.................. $\mathrm{R}$

Badger Boy

Bailey Red..................

Balco J. ...............

Bandit ........... D

Bangormar

Barmpton Aylesby Duke .... - P S

Barmpton Duke of E!m Grove-P S

Barmpton Lad 2d...........- - R R

Barmpton Prince .......... P S

Barney

Baron Äirdrie ............ Daisy

Baron Bates of Maple Nook _ - P S

Baron Booth .............. D

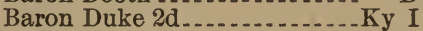

Baronet.................... Ky I

Baronet Dunmore..... $R$

Baronet Williams........ Ky I

Baronet Winfield......... D

Baron George........ $R$

Baron Knoyle 4th ......... - Cox

Baron of Lakeside.......... - L R

Baron Leslie of Orleans...........

Baron L. G. C. ...................

Baron of Maple Grove....... - M

Baron May .........................

Baron of Oxford

Baron Pixy .......... P

Baron Rose Duke ....... M

Baron of Sunnyside... Cox

Baron Tuberose.........- Beauty

2d Baron Tuberose......- - Beauty

B'n Tuberose of Sp'ngboro-Beauty 2d B. Tuberose of Sp'ngboro-Bea'y

Barton.......... P S

Barzy -.....

Basil.................... Ky I

Basil Duke.................

Bates' Oxford 4th ........ $\mathrm{R}$

Bayard......................

Bay's Red Rose Duke..... - Ky I

Beauty's Duke............... D

Beauty of Elysian......... L R

Beauty's Prince,................ 


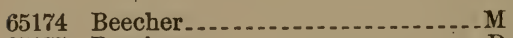

65175 Beecher....

65176 Beecher....

65177 Beecher.............................

65178 Begole...................... I

65181 Bell Boy

65183 Bell Duke.......................

65186 Bell Duke ......... D

65187 Bell Duke........ D

65189 Bell Duke................... P S

65190 Bell Duke 2d..... R R

65191 Bell 3d's Duke................ M

65192 Bell Duke 3d......... P

65193 Bell Duke of Greendale .... $-\mathrm{R} R$

65194 Belle's Duke of Maine Valley _L R

65195 Bell Duke of Marengo....... - D

65197 Belmont 2d................. D

65200 Belvedere 2d

65201 Ben

6502 Ben............

65203 Ben

65206 Ben Butler................... Ky I

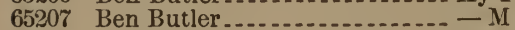

65208 Ben Butler..................

65210 Ben Butler.......... P S

65211 Ben Butler.................... S

65212 Ben Butler ................ D

65:13 Ben Butler........... Ky I

65214 Ben Butler..........................

65216 Ben Franklin 65217 Ben Fredericks

65218 Ben Funk ................ D

65219 Ben Hill

65220 Ben Oliver..................... R

65221 Ben Thompson

65223 Ben Wade................. $\mathrm{L}$

65225 Berlin

65227 Bernard

65230 Bethel Duke..... R R

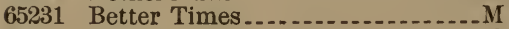

65232 Beverly

65233 Beverly Bright Eyes.......... P S

65235 Beverly Star Duke............... D

65237 Big John

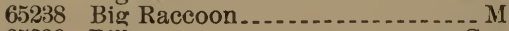

65239 Bill

65240 Bill Fox. Daisy

65241

65242

65243

65244

65245

65246

65247

65248

65249

65250

65251

65252

$65 \% 53$

65254

65255

65256

65258

65259

65260

65261

65262

65\%63

65264
65265

65266

65267

65268

65269

65270

65271

65272

65273

$652 \% 4$

$65 \% 76$

65277

65278

$652 \% 9$

65290

65281

65284

65286

65287

65290

65291

65292

65297

65298

65300

65301

65302

65303

65304

65305

65307

65309

65311

65312

65315

65316

65317

65318

65320

65322

65323

650324

65325

65326

65327

65328

65329

65330

65332

65333

65334

65336

65338

65339

65340

65343

65344

65346

65347

65348

65349

65350

65351

65353

65354

65356

65357

65358

65359

65360
Blaine-...................... Cox

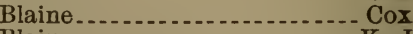

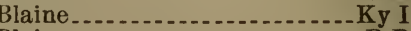

Blaine-....................---R R

Blaine -........................- - $M$

Blaine.............................. Ky I

Blaine -.......................- -

Blaine -............................ M

Blaize......................-.- $\mathrm{M}$

Blanche's Duke -.........-- - $-\mathrm{L} R$

Blanche Duke $3 d$.............- $-\mathrm{L} R$

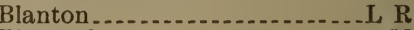

Blizzard -...................-..--

Blocky

Blocky 2d -................-M

Blocky 3d..................-- -

Bloom's Prince 2d...........-- - M

Blue Grass Boy ..............-- $\mathrm{R} R$

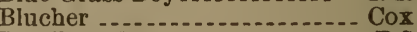

Bob Lincoln -...-..-......--- - P S

Bob Miller -...-...-.........-- $-\mathrm{R} R$

Bob Munson -................- - M

Bolivar......................- -

Bonanza Lad................- $-\mathrm{R} R$

Bonhomie Ben-.....................T

Bonito-..........-...-...-...-... Cox

Bonny-......................-T

Bonnie Knight..............-- $\mathbf{R} R$

Boss Renick................-.-C

Boston

Bostwick-.................-L $-\mathrm{L} R$

Bouncer-.................-Cox

Bourbon Lad 2d............--Ky I

Bourbon of Ninigret..........--D

Bourbon Star..................-- - D

Bowman

Bracelet Boy -..................- $-M$

Bracelet Duke-.............

Bracelet's Satellite.............- - D

Brady -........................P S

Brainard-...................-- $\mathrm{R} R$

Bravo

Breadalbane-...-...-.....---Daisy

Breastplate.............

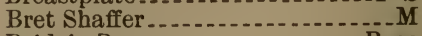

Bride's Baron.......... Rose

Bride's Favorite................. Rose

Bridegroom............ Rose

Brigham .............

Brigham-.... R R

Bright Boy $2 \mathrm{~d}$

Bright Eyes Jr.,............ Ky I

Bright Night...........

Brilliant - ................ M

Britannia Duke $2 \mathrm{~d}$

Briton Airdrie ............ $D$

Britt Star..............

Brooks................

Bruce.....................

Brutone..........

Brutus................

Brutus...........

Buck .................

Bud Junior.

Buffalo Bill .............. Cox

Buffalo Duke 4th

Buffalo Duke 5th............ Ky I

Bugler ........................

Bugler..........

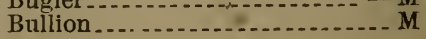




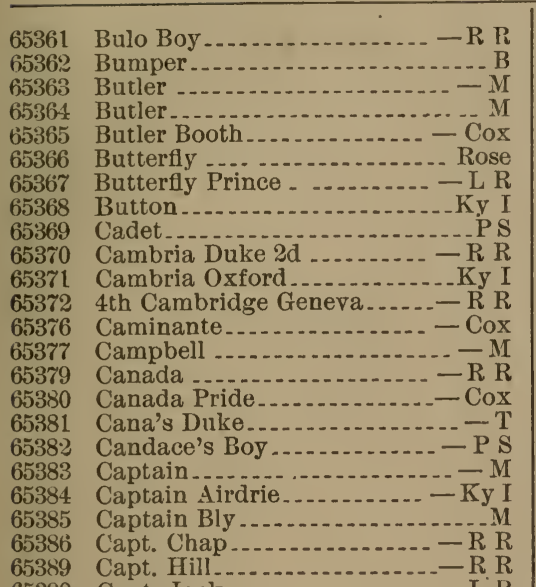

65390 Capt. Jack..................... L R

65391 Capt. Jack

65393 Capt. Lawson

65394 Capt. Thomas........... - Ky I

65395 Capt. Sharon.............. R R

65396 Capt. Wallace ............... - M

65398 Capt. W. H. Flint.......... - L R

65399 Carleton .................. - R

65400 Carlisle .................... R

65401 Carlisle

65402 Carlos....................... P S

65403 Carlotta's Duke $3 \mathrm{~d} . . . . . . . . .-\mathrm{T}$

65405 Carter Harrison ............. P S

65406 Casabianca............ Daisy

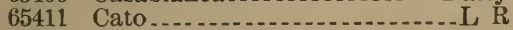

65413 Cedar Duke........ R R

65414 Challenger .................. R R

65415 Challenger Lad 9 th......... $\mathrm{R} R$

65416 Champaign Duke............... Ky I

65417 Champion.................. D

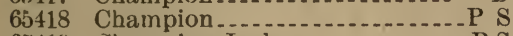

65419 Champion Jack............-P S

65420 Champion Roan.............. Ky I

65421 Charles D'Orleans............ - P S

65422 Charles Harman............. - M

65423 Charles Pink............... - L R

$65+24$ Charles Ross

65425 Charles Sweatham ........... - M

$654: 6$ Charles Swetmon ............ - L R

65427 Charley .......................... M

65428 Charley

$65+29$ Charley Lad.............. Cox

65430 Charlie....................... - T

65431 Chautauqua Duke............. R

65432 Cherry Duke.............. - K R

65433 Cherry Duke of Maine Valley .... L R

65435 Cherry Orchard Chief ........- M

65433 Cherrywood Athol .......... R R

65443 Chief.

$65+44$ Chief of Lanark...... P S

65445 Chieftain

65149 Cicero

65450 Clara's Duke $2 \mathrm{~d} . . . . . . . . . .-\mathrm{P}$ S

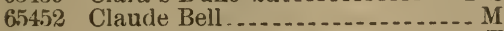

65454 Clay Duke $2 \mathrm{~d}$

65455 Clay Duke $3 \mathrm{~d}$

65456 Clay Duke 4th.........
65458

65461

65463

65464

65466

655467

$65 \tilde{4} 468$

65469

65470

6 อ̃471

65472

65473

65474

65475

$65+76$

$6547 \%$

65478

65479

65480

65481

65482

65484

65485

650487

65488

65489

65540

65492

65493

65495

65496

65497

65498

65500

65501

65502

65503

65504

65505

65506

65507

65508

65510

65511

65513

65514

65515

65516

65517

65518

6 ธร็น19

655123

65521

$655 \% 5$

655203

655:77

65528

65529

65530

65531

65533

65535

65536

6.55538

655 วิ39

$6.55+1$

65543

65544

65545

65546
Clayton Dick ................ - D

Cleveland ................ $L$

Cleveland................. - L R

Cleveland......................

Cleveland................. Rose

Cleveland .................

Cleveland .............. Rose

Cleveland .............

Cleveland............ $\mathrm{R}$

Cleveland.................. $\mathrm{M}$

Cleveland

Cleveland .............

Cleveland

Cleveland..................

Cleveland ................

Cleveland

Cleveland

Cleveland ........

Cleveland ................

Cleveland......................

Cleveland........................

Cleveland .......................

Cleveland Duke............ Ky I

Cleveland Duke........ P S

Clifton .......................... R

Ciifton Duke............. $\mathrm{L}$

Clifton Duke............. - R R

Climax Barrington....... - R R

Coburg - . . . .

Coin of Nineveh

Collingwood ................ - P S

Columbus Lad $2 \mathrm{~d}$

Colonel......................... S S

Colonel ............... $\mathrm{R}$

Colonel

Colonel ........... Daisy

Colonel .......... D

Col. Allen............. Cox

Col. Barmpton $3 \mathrm{~d}$

Col. Benton .................. L R

Col. Breckenridge..........

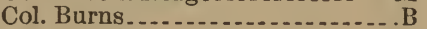

Col. Joe Meek ............... - D

Col. J. W. Judy

Col. Lucas ........... R R

Col. Lynn................ Cox

Col. Page.................. Cox

Col. Richland....................

Col. Rose ........

Col. Scott_....... R R

Col. Shaw ........... Rolla

Col. Vest................ R

Col. Vest...........................

Comet ...........

Comet........

Comet.............................

Comet P.

Comet R.

Commander.............. Cox

Commander................. $-\mathrm{M}$

Commodore.................... Ky I

Commodore..........................

Commodore Nutt ....... Cox

Como .................... C

Compton .............. $\mathrm{R} R$

Conewango Lad........... - L R

Conqueror ................... M

Conqueror

Conqueror..................

Conrad $2 \mathrm{~d} \ldots \ldots \ldots$ I 
65547 Conspirator................. - R R 65550 Constance Duke............. Rose 65552 Constance Duke of Athens_- Rolla 65556 Conway ....................... B

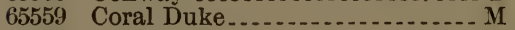

65560 Cora's Duke ............... M

65561 Cornwallis......................

65562 Corazon

65563 Cora's 2d Airdrie......... - Daisy

65565 Corsair .....................

65566 Cottage Duke 14th........ - Rolla

65567 Count ................................

65568 Count Brickerstaffe 2d.... - Rolla

65569 Country Lad................... Ky I

65570 Craymor....................

65571 Crinoline's Duke....... Rolla

65573 Crombo................ R

65574 Crownover.......

65576 Crown Prince.......................

65578 Cunningham 26th .......

65579 Curley Duke

65580 Curly Logan

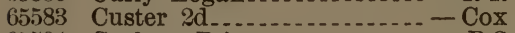

65584 Cyclone Prince

65587 Cypress Prince............. - M

65589 Dahlia's Duke........ Beauty

65590 Dairyman.........................

65591 Daisy Boy

65595 Daisy Duke 2d...........

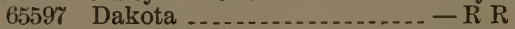

65599 Dandelion................ $\mathrm{L}$

65600 Dandy

65601 Dandy

65602 Dandy $2 \mathrm{~d}$

65604 Dandy Duke.........

65606 Dangerous Duke

65607 Daniel .................... R R

65608 Daniel Boone............. P S

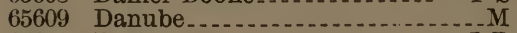

65610 Darby

65611 Darby Duke

65612 Darius........................ Rolla

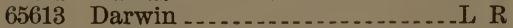

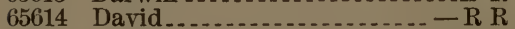

65615 David ......................

65617 David Copperfield........... R R

65618 Day's Thorndale...........

65619 Dayton

65620 Deacon

65623 December Duke.............

65624 Decoration Duke............. R

65625 De Long ............

65626 Delta's Duke $2 \mathrm{~d}$

65627 Dennis ...............

65632 Diamante....

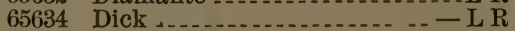

65635 Dick Hill 4th........ $\mathrm{R}$

65636 Dick Hill 5th................ $\mathrm{R} R$

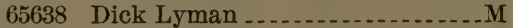

65640 Dick Oglesby

65641 Dick Reed........

65642 Dick Reid 65643 Dicksonburg

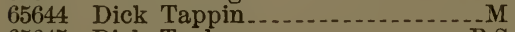

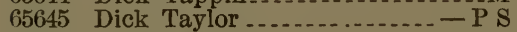

65646 Dick Taylor

65647 Dick Turpin

66649 Dick Turpin

65649
65650

65651

65652

65654

65655

65656

65659

65660

65661

65662

655663

65664

65665

65666

65667

65668

65669

$656 \% 0$

65672

65673

65674

65675

65676

65677

65678

65679

65680

650681

65682

65684

65685

65686

65687

65689

65691

65092

65693

65691

65695

65696

65697

65698

65699

$65 \% 01$

65702

$65 \% 03$

65704

65705

65707

65709

65710

65712

65713

65714

65715

65716

65717

65719

65720

65721

65721

65725

65726

65727

65728

65729

$65 \% 30$

655731

65733

65734
Dictator........... $\mathrm{R} R$

Director........ $\mathrm{R}$

Dirigo

Dixie Duke 4th

Doctor Dadd................... R

Doctor Golden Block......... Ky I

Dolly's Duke.....................

Dom Pedro $3 \mathrm{~d} \ldots \ldots . . .2 \mathrm{R}$

Don

Don

Donald Duke 18th.................

Donald Duke 19th

Donald Duke 20th

Donald Duke 21st............ R

Donald Duke 22d

Donald Duke 23d.......... R R

Donald Duke 24th

Donald Duke 25th............Ky I

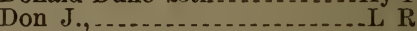

Don Pedro $2 \mathrm{~d}$

Dora's Duke of Richmond...- - D

Dora's Lad................... M

Dorsey

Douglas

Driver ..............................

Duane's Duke $23 \mathrm{~d}$

Duke ............. R R

Duke.......

Duke........ $D$

Duke.......... $\mathrm{R}$

Duke

Duke............. Ky I

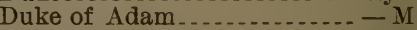

Duke of Adena..........

Duke of Alum Creek

Duke of Apple Grove

3d Duke of Argentine.......... $R$

Duke of Arlington......... $R$

18th Duke of Ashland..- - Woods 2d Duke of Avon ............ L R

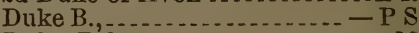
Duke Balco Duke of Balinger.

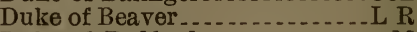

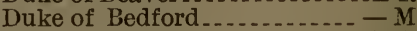
Duke of Benson Duke of Bentley Creek.... - Cox Duke of Benton. Duke of Berlin ................. Duke of Black Creek..... Duke of Black Hand....... Ky I Duke of Bourbon.......... $\mathrm{R}$ Duke of Brady Duke of Buccleuch Duke of Buccleuch........... Cox Duke of Buffalo............ - Rolla 3d Duke of Bunker Hill..... - R R Duke of Burnsville........... Ky I Duke of Busti.................. 3d Duke of Butler Duke of Canon Hill Duke of Cedar Valley ......- Rolla Duke of Chautauqua...... - Cox Duke of Chemung -........ - L R Duke of Clair ............... Duke of Clarence...... Rolla 9th Duke of Clinchdale-Milk Spn'g Duke of Cloverdale ........... - Cox 5th Duke of Clover Meadows - L R Duke of Clyde............. 
65735

65736

65737

$65 \% 40$

65741

65742

65743

65745

$65 \% 48$

$65 \% 5 \%$

65753

65754

65755

65757

65758

$65 \% 60$

$65 \% 61$

55762

65763

65766

65767

$65 \% 68$

$65 \% 69$

$65 \% 70$

65771

65772

65773

$657 \% 4$

65775

65777

65778

$65 \% \% 9$

$65 \% 80$

65781

65784

$65 \% 85$

65789

65792

65793

65794

65795

65796

65800

65807

65808

65809

65811

65812

65813

65815

65817

65818

65819

65820

65822

65823

65824

65825

65826

65827

65829

65830

65832

65833

65834

65835

65836

65837

65838 2d Duke oí Colebrook......... Cox

Duke of Colorado ...............

Duke of Columbus........... L R

Duke of Corning

Duke of Cornwall.......... Cox

Duke of Crawford .......... R

Duke of Crescent Vale..... - R R

Duke of Crow Lake..........R R

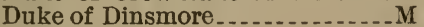

Duke of Elk.............. L R

Duke Ellis......

Duke of Elmvale.......... Cox

2d Duke of Elmwood....... R R

Duke of English............ M

2d Duke of Erin........... R R

5 th Duke of Fairview

6th Duke of Fairview........ - D

Duke of Fenton...M

Duke of Fife.......

Duke of Florence..........

2d Duke of Florence.............. M

7th Duke of Forest Home..... - T

8th Duke of Forest Home.... - T

9th Duke of Forest Home ... $-\mathrm{P} \mathrm{S}$

10th Duke of Forest Home... -

11th Duke of Forest Home... - T

12th Duke of Forest Home.... $\mathrm{T}$

13th Duke of Forest Home... - T

Duke Fosco..................

Duke of Genesee........ Cox

Duke George.......... R R

Duke of Glens Creek........ $-\mathbf{M}$

Duke of Glendale.......

2d Duke of Glen Rock........P S

2d Duke of Goodness

69th Duke of Goodness.....- R R

2d Duke of Green Valley...... $-\mathrm{D}$

Duke of Hale..................... D

Duke of Hale $2 \mathrm{~d}$

Duke of Hallard

2d Duke of Hallard.........

Duke of Haywood

Duke of Hemdoka 2d....... - P S

Duke of Highland........ R R

Duke of Hill's Grove......... D

Duke of Hillside....... R R

Duke of Hilpa.......... Rolla

Duke of Jasper.............

7th Duke of Jefferson.

8th Duke of Jefferson....... - M

Duke Joe......................

Duke John .........................

Duke Jumbo...................

Duke of Kensington.... Fradulent

Duke of Kent................ D

Duke of Lafayette....... D

6th Duke of Lapwing ....... - M

3d Duke of Leaf River........ L R

5th Duke of Leaf River...... L R

6 th Duke of Leaf River...... R

Duke of Lebanon ............ Cox

Duke of Liberty

Duke of Licking $4 \mathrm{th} \ldots \ldots$

7th Duke of Locust Grove.... - M

Duke of Lodi................

Duke of London

Duke of Lost Creek...... P S

Duke of Lynnville........ Cox

Duke of Lynnville $2 \mathrm{~d} . \ldots . . . . . M$

Duke of Marble........ P S
65839

2d Duke of Manchester.... - L R

65842 Duke of Manor............... C

65843 Duke of Maple Grove

65844 2d Duke of Maple Grove..... - P S

65845 Duke of Maple Hill........

65846 Duke of Mapleton............ M

65847 Duke of Maplewood

65848 Duke of Maplewood.

65849 Duke of Marengo.............. Cox

65851 3d Duke of Mazomanie....... S

65852 Duke of Meadow Lake........ - C

65853 2d Duke of Meadow Springs _ P S

65854 Duke of Mecklenburg ...Fradulent

65855 Duke of Medina............. S

65856 Duke of Merwin ....... R

65857 Duke of Miami............... I I

65859 2d Duke of Miffin

65860 Duke of Milwaukee 2d........ R

65861 Duke Monmouth ..............

65862 Duke of Monroe

65863 Duke of Montcalm........ - Cox

65865 Duke of New Salem

65866 Duke Newtonian........ Rolla

65868 Duke of Oakdale........

658695 th Duke of Oakdale......... - T

658715 th Duke of Oakland

65872 2d Duke of Oak Park...... - P S

65873 3d Duke of Oak Park ........ - D

65874 4th Duke of Oak Park ...... -D

65875 Duke of Oakwood $2 \mathrm{~d} \ldots \ldots \ldots-\mathrm{D}$

6587\% Duke of Otto

65880 Duke of the Nemeha ..... - Ky I

65881 Duke of Paddy's Run ...... R R

65884 Duke of Perry

65887 Duke of Pleasant Ridge....... Ky I

65888 Duke of Pleasant View.... - L R

65889 20th Duke of Pleasant View - Cox

65890 22d Duke of Pleasant View_ _ Cox

65894 2d Duke of Poverty Hill_._-Daisy

65895 3d Duke of Poverty Hill..... _ P S

65896 13th Duke of Preston........

65897 Duke of Red Cherry ......... - M

65898 Duke 5th of Richland..... P S

65899 Duke 6th of Richland...... - P S

65900 Duke 8th of Richland ....... P S

65901 Duke 9th of Richland

65902 17th Duke of Ridgevale....... Cox

65903 20th Duke of Ridgevale.... - L R

65904 Duke of Riverside ........ - Rolla

65906 Duke of Rock Creek ........ Ky I

65907 Duke of Rockford......... P S

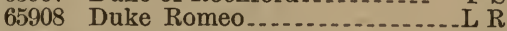

65909 Duke of Rose $3 \mathrm{~d}$

65911 2d Duke of Rosedale.......... R R

65912 3d Duke of Rose Lawn ........ D

65913 4th Duke of Rose Lawn ...... - B

65914 4th Duke of Rosewood ... - Daisy

65915 Duke of Ross............. R R

65916 Duke of Rostraver...

65917 Duke of Royal Spring.....

65919 Duke of the Ranch.......... M

65920 Duke of Seaham

65921 2d Duke of Severn's Run ..... - C

65922 Duke of Sharon

65923 Duke of Sharon .......... D

65924 Duke of Sharon.......

65925 Duke of Sharon ........ Daisy

65927 2d Duke of Shiloh......... $\mathrm{R}$ R

65928 11th Duke of Skaneatles.... - Cox 
65929 Duke of Smithfield ........ - L R 65931 Duke of Spring Branch.... - R R 65933 Duke of Spring Brook...... - P S 65934 Duke of Spring Creek ........ - D 659)35 Duke of Stanhope Jr.,.... - Ky I 659:36 Duke of Star Mills .............. M 65938 2: d Duke of Stock Place..... - D 65939 Duke of Sutton .................. M 65910 2d Duke of Sycamore 65944 Duke of Tellico..... - Milk Spring 65945 Duke of The Dominion.... - L R 65946 Duke of The Louans...... $\mathrm{R} R$ 65947 Duke of The Loup 65948 Duke of The Mounds...... L R 65949 Duke of The River ......... - Cox

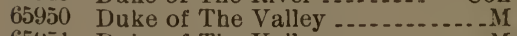
65951 Duke of The Valley 65953 Duke of Tiger........... L R 65954 2d Duke of Toulon......... P S 659.6 Duke of Troy 65957 Duke of Turkey......... Ky I

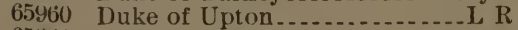

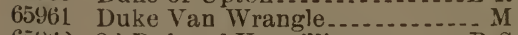
6596:) 3d Duke of Vermillion........ $\mathrm{P}$ 6590 i3 Duke of WValnut Grove..... $\mathrm{R} \mathrm{K}$ 65965 Duke of Wardsboro ....... R R 65966 Duke of Warlaby 65967 Duke of Warrick 65968 Duke of Warwick.... R 65969 I 65973 Duke of Watson 65974 Duke of Wayne...... M 65975 Duke of Wayne...... D 65976 2d Duke of Wayne....... M $659 \pi$ Duke of Wellington 65978 Duke of Wellington...... Cox 65979 Duke of Westburg........ - P S 65980 Duke of West Penn................. $659 \mathrm{~s} 2$ Duke of Wild Grass $\ldots \ldots \ldots \ldots$ 65983 Duke of Wodeneath.......... P S 65984 2d Duke of Woodbine 65986 3d Duke of Woodland ..... - P S 659 \% 3d Duke of Woodland..... - Cox 65988 4th Duke of Woodlawn .... - R R 65990 Duke Worcester.......... - C

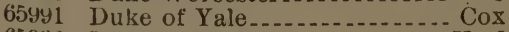
6599.2 Duke of York................... I 65993 3d Duke of York

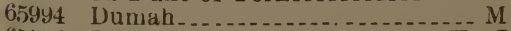
65996 Duncian of Farmington...... Ky I $659 y 7$ Dun D. 65998 Dunkle's Republic $65 y 99$ Dunlap $2 \mathrm{~d} \ldots \ldots \ldots$ 66000 Durbin WVard..... M 6́6uul Dutehman........ Long Horn 66002 Eidgle Point Duke........... Ky I

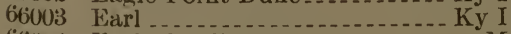
66004 Earl of Arlington 660 Earl of Cummington 66010 Earl of Darlington 66013 Earl of Dexter ................ Ruse 66015 Earl of Elın Grove 66018 Eirl of Lima............. 66019 Earl of Loudon......... Cox $660: 0$ Earl of Merwin 66023 Earl of Morton..... P S 66027 Earl of Oak Ranch $2 \mathrm{~d} \ldots \ldots \ldots . \mathrm{L}$ R 66029 2d Earl of Oakshade........ D 66031 4th Earl of Richmond ........ D 66033
66034 66035 66036 66037 66038 66039 66040 66042 66043 66044

66045

66046

66047

66050

66051

66052

66053

66054

66056

66057

66058

66059

66061

66062

66064

66065

66067

66069

66070

66071

66072

66073

66074

66075

66077

66078

66079

66080

66081

66082

66083

66084

66086

66087

660089

66090

66091

66093

66094

66095

66097

66099

66100

66101

6610:2

(66103

66104

66106

66108

66109

66111

66112

66113

66114

66115

6́6117

66119

66120

66122

66123
Earl of Roseneath ............. $\mathrm{M}$

Earl Royal _............. Cox

Earl $2 d$ of Saline.........

Earl of Tillamook........ $\mathrm{D}$

Farl of Upton................ $\mathrm{R}$

Earl Vane

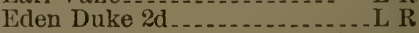

Edmond 6 th ........... Beauty

Erl Mosley .......... T

Edmond Chief

Edmond Chief $2 \mathrm{~d}$

Ed Riley ......................

Edward

Elfinfoot Jr................. $\mathrm{R}$

Eli............................

Elmo Airdrie ... M

Elster..........

Emory

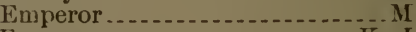

Emperor

Emperor 2d

Enchanter $2 \mathrm{~d}_{0 .}$

Ephsiham ........ Daisy

Esau 2d ..............................

Esquire Waldberg

Eugenie's Challenger .......

Excelsior .............

Excelsior -........

Excelsior

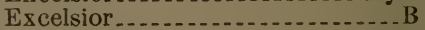

Exchange............... Ky I

Exivera's Duke............. Ky I

Expectation ......... R

Fairview ....................

Fairview Duke 4th......... R R

Fairview Duke 5th......... R R

Fairy Boy ................

Fairy's Duke '-.... P S

Fairy's Duke $2 \mathrm{~d}$

Fairy's Duke 4tb ....... - Daisy

Falconer ...............

Faney .......... Cox

Fancy Boy ....... P S

Fancy Boy ........ R

Fancy Duke............ $R$

Fancy Duke..........

Fancy Major 4th.......... Ky I

Fannie's Champion ....... - D

Fannie's Duke........... $\mathrm{R} R$

Fashion's Duke...........

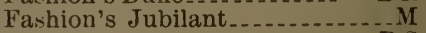

Favorite.................. S

Favorite ............... $R$

Favorite of Edmund....... Cox

Fayette Duke........... $-\mathrm{L}$

Fay's Prince...................... I

Fearnaught ............

Ferdinand ...................

Fidella's Jubilee........... R R

Finney

Firefly .............. Ky I

Fitz-Duke ......... Cox

Fitz-Duke $2 \mathrm{~d} . .$.

Fitzgerald ...............

Flattery's Duke.......... $R$

Floghiu _............. R

Flora's Kirk

Florian's Duke.............. $\mathrm{R}$

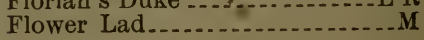




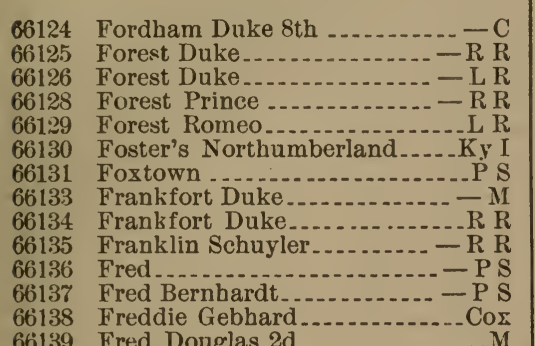

66139 Fred Douglas 2d..................

66140 Frederick

66141 Frederick of Clover Dale........ R

66142 Frederick Valentine......... D

66143 Fred Grant...................... R

66144 Fred Moppin.... P S

66145 Fremont........... R

66146 Fritz ........... D

66147 Frontier O.,................

66148 Frontier P.,........... R

66149 Frontier R.,....... R

66150 Frontier S., $\ldots \ldots \ldots \ldots$

66151 Frontier T.,..... R

66152 Frontier U.,............ R

66154 Fulton Duke....... - Milk Spring

66155 Fulton Jumbo

66156 Fulton Lad .................. Cox

66157 12th Gaban of Eastside.... - P S

66158 Gancho ................... R

66159 Garden City Duke...... R R

66161 Garfield

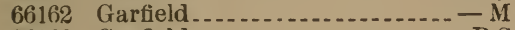

66163 Garfield

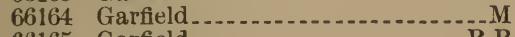

66165 Garfield

66166 Garfield ........

66167 Garfield.......... D

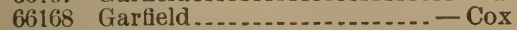

66169 Garfield King

66170 Garnet's Airdrie.

661 1 Gath............... R

66172 Gaylord

66175 General

661 \%7 General

66178 General B.,.................

66179 Gen. Blucher...M

66180 Gen. Cass ........ Ky I

66181 Gen. Clyde........ R

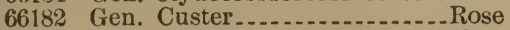

66183 Gen. Garfield ......... Cox

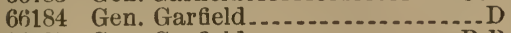

66185 Gen. Garfield

66186 Gen. Garfield $2 \mathrm{~d} \ldots \ldots \ldots \ldots$ P

66187 Gen. Garfield 2d

66188 Gen. Geo. B. MeClellan...... - D

66189 Gen. Gordon ............. R R

66190 Gen. Grant................ Ky I

66191 Gen. Grant.................... Ky I

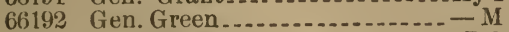

66194 Gen. Hancock....... P S

66196 Gen. Hill

66197 Gen. Hood

66198 Gen. Jackson......... Cox

66199 Gen. Jackson....... M

66:00 Gen. Logan .............. D

66:201 Gen. Logan

$6620 \%$ Gen. Logan...... R R
66203

66204

66305

$66: 206$

66207

66208

66209

66210

66211

66212

66213

66214

66215

66216

66217

66219

66220

66221

66222

66223

66224

66225

66226

66230

66232

66234

66236

66:41

66242

66243

66244

66245

66246

66247

66248

66:49

66250

66251

66252

66253

66255

66256

66257

66258

66259

66260

66261

66263

66265

66266

66267

$66 \% 68$

66269

66:2\%

$662 \pi 1$

$662 \div 2$

66273

66274

66277

66284

66285

66288

66289

66290

66291

$66 \div 922$

$66: 293$

66294

66295

66297
Gen. Logan ............. R R

Gen. Logan......

Gen. Logan .......

Gen. Logan ......................

Gen. Logan ...................

Gen. Logan ....................

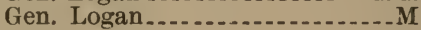

Gen. Logan

Gen. Logan .............. $-\mathrm{P}$ S

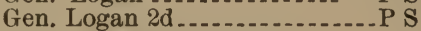

Gen. Logan of Beallsville....- - D

Gen. Moreton ............... - C

Gen. Otoe............................

Gen. Potts .........

Gen. Rosecrans .........

Gen. Sheridan............. - Ky I

Gen. Sherman

Gen. Sherman of Beallsville.....M

Gen. Singleton............. L R

Gen. Smith........

Gen. Taylor.............

Gen. Washington..... R

Gen. Weaver................. R

Genera Duke .............. Cox

Geneva Lad................. - L R

Geneva Star................ $-R$ R

Gent Barmpton ...............

George........................ $R$

George ..........................

George

George Ballentine........ $-L ~ R$

George Brown ............

George C., .........

George Foot ................. - L R

George $\mathrm{G}, \ldots$

George H,

Geo. Stephenson ........ M

Georgetown Duke ........

Geo. Washington.........

Gill..............

Gipsy Boy

Gladiator ............. - Beauty

Gladstone.......... Cox

Gladstone ................. - T

Gladstone..........

Gladstone .............

Glaucus.................. R R

Glendower...........

Glenso .............

Glick ..........................

Glick

Glitter ...............

Glory ..............

Gloster 4th ........ Cox

Gloster's Boy ............... - M

Gloster's Chief.................. R

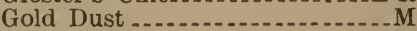

Gold Dust...........

Golden Duke................

Goldsmith ............. D

Goldsmith

Gordomin

Goshen Chief.................. R R

Governor..................

Guvernor .................... D

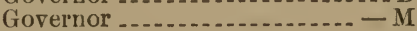

Governor....

Governor.........

Governor......................

Gov. Cleveland.............. - D 
66298 Gov. Cleveland................ M

66299 Gov. Cleveland.................

66300 Gov. Glick ...................

66301 Gov. Gwynne...........

66302 Gov. Hamilton............ Cox

66304 Gov. Lucas......................

66305 Gov. Palmer ...............

66306 Gov. Phelps................ Woods

66307 Gov. Sherman ................ M

66308 Gov. Sherman

66310 Gov. Thayer $2 \mathrm{~d} . \ldots \ldots \ldots \ldots \ldots$

66311 Gov. Tilden 2d.....................

66313 Graceland Duke ..........- - Rolla

66317 Grand Duke of Harding ...... - M

66318 Grand D. of Pleasant View - - Cox

66325 Grand Prince .............. - Cox

66326 Grand Turk

66328 Granger.................. R

66329 Granger 2d............... $-\mathrm{R} R$

66330 Grant..........................

66331 Grant...................................

66332 Grant $\ldots$

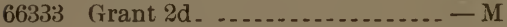

66334 Grant Richmond.......... L R

66336 Greeley .................

66337 Greenback

66338 Greenwood Boy ...............

66339 Greenwood Boy $2 \mathrm{~d} . . . . . . . . . .$. Cox

66340 Greenwood Champion....... - D

66342 Greenwood's Duke ......... - Ky I

663.3 Grenola....................

66344 Grover........................

66346 Grover...........

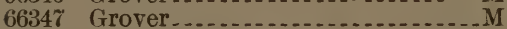

66349 Grover Booth............... - Ky I

66350 Grover C., .................

66351 Grover Cleveland............ Daisy

66352 Grover Cleveland ...............

66354 Grover Cleveland...........

66355 Grover Cleveland ............. $\mathrm{D}$

66356 Grover Cleveland........... $\mathrm{L} \mathrm{R}$

66357 Grover Duke............... - L R

66358 Gwynne Bull of Maple Grove - Cox

66360 Gwynne's Hillhurst......... - Cox

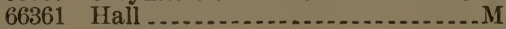

66364 Hancock

66365 Hancock

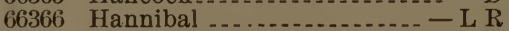

66367 Happy Medium...............- $\mathrm{B}$

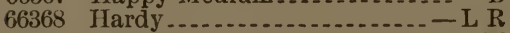

66369 Harold

66370 Harpado..................

66372 Harry ..................... - P S

66373 Harry Cleveland........... - P S

66375 Harry of Maine Valley...... $\mathrm{R} R$

66376 Harrington ................ - Rolla

66377 Harvester................... Rolla

66378 Harvey's Duke.....................

66379 Hawkeye............................

66380 Hawley ............................... S

66381 Hawthorne Duke.......... - R R

66382 Hayes............................

66383 Hayes...........................

66384 Hector

66385 Hector

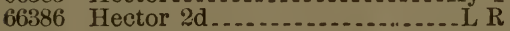

66387 Hedger

66388 Heir of Bates............. - Ky I

66389 Heir Duke....................

66390
66391

66392

66393

66394

66395

66396

66397

66398

66399

66400

66401

66402

66404

66405

66406

$6640 \%$

66409

66410

$66+11$

66413

66414

66415

66416

66417

66418

66419

66422

66424

$664: 5$

$66+26$

66427

66428

66429

66430

66431

66432

66433

66434

66437

66438

66439

66440

66441

66442

66443

$66+44$

66445

66446

66447

66448

66449

66450

66451

66452

66453

66454

66455

66456

66458

66459

66460

66461

66162

66466

66468

66469

66473

66474

66476

66477
Hendricks..................

Hendricks..............

Hendricks.

Hendricks ................

Hendricks

Hendricks................

Henry ...................... Rose

Henry

Henry

Henry Clay ....................

Henry Magistrate............... Ky I

Hercules ......................... Ky I

Hercules .................

Hero .......................

Hero....................... R

Hero of Marshall-.........

Heyday ....................

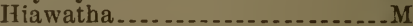

Highland Boy ............ - C

Highland Duke -.............-M

Highland Duke..............- $\mathrm{R} R$

Highland Duke

Highland Duke.............. - M

Highland Duke.............- $\mathrm{L}$ R

Highland Duke.................. Ky I

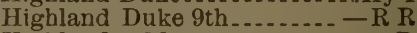

Highlander $2 \mathrm{~d}$....................

Highland Napier $2 \mathrm{~d} . . . .$.

Highland Napier 4th......- - Ky I

Highland Prince 5th........- $-R$

Highland Prince 6th.......- - R R

Hillhurst.

Hillhurst Moore........... - R R

Hillsboro Lad .................

Hilpa's Good Boy............-D

Hilpa's King Duke..........- - P S

Hoadley ...............................

Holy Lad................ Cox

Homer........................

Honest Abe............. - Daisy

Honest Cleveland.............- - C

Honest John ................- L R

Hoosier Bob -.................-M

Hopeful

Hopeful of Farmington....- - Cox

Hope's Airdrie ............. - C

Hope's Hilpa .............-Rolla

Hope's Wild Eyes $2 \mathrm{~d} . . . . . . . .-M$

Howell Jr., .............. R R

Horace

Hough's Granger-..........- $R R$

Howard White

Hopewell Duke $2 \mathrm{~d} . . . . . . .-$ Ky I

7th Hubback ..............-R R

Hubback of Edmund.....-- Cox

Humboldt....................... T

Ianthe's Pride.............. $\mathrm{R} R$

Ida's Duke..................

Ida's Duke...............

Ida's Star of the West...........M

Illinois .....................

Illinois Chief-................ Ky I

Independence Lad............ Ky I

Industry ..................

Ingalls

Irving Boy

Isaac ......................... Ky I

Ivanhoe..................................

Ivanhoe..................... Ky I 


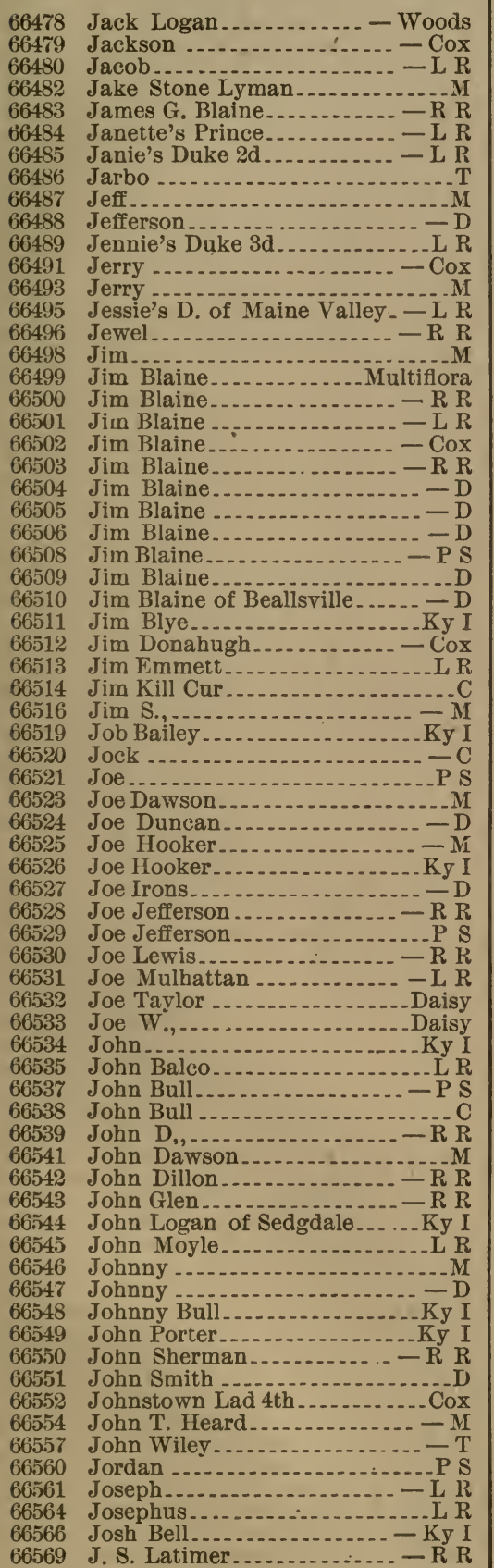

66570

66571

66572

66573

66574

66575

$6657 \%$

$665 \% 8$

66579

66580

66581

$665 \mathrm{~S} 2$

66583

66585

66586

66587

66588

66589

66590

66591

66592

66593

66594

66595

66596

66597

66598

66599

66602

66603

66604

66605

66607

66608

66609

66610

66612

66613

66614

66618

66621

66622

66623

66625

66626

66627

66628

66629

66630

66631

66632

66634

66635

66636

66639

66640

66641

66642

66643

66644

66645

66646

66647

66648

66649

66650

66651

66653

66654

66659
Juba of Oak Grove............ - M

Juba $2 d$ of Oak Grove........ -

Juba $3 d$ of Oak Grove......... - M

Jube .......................... Woods

Jubilee Duke $2 \mathrm{~d} . . . . . . .$.

Jubilee Prince $\% \mathrm{~d} . . . . . . .-$ Rolla

Judge Bowling ............... - C

Judge Clark

Judge Hoadly ................... Cox

Judge Lauby .................. Ky I

Judge Vance ............... S

Julia's Lad................... _ L R

Julius Cæsar ..........................

Jumbo ................... Cox

Jumbo . . . . . . . . . . . . Ky I

Jumbo .......................

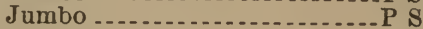

Jumbo ................ R

Jumbo ..................

Jumbo ..................

Jumbo..................

Jumbo.......... R R

Jumbo $2 d$. . . .

Jumbo 3d................... Cox

Jumbo Burdick..............

Jumbo of Sedgdale............ Ky I

Junction Duke............ R R

Juno 2d ...........................

Jupiter................. R

Jupiter..........................

Jupiter..........................

Kæsar............. Ky I

Kansas Duke...................

Kansas Lad ........ - Long Horn

Kaster ….............. $\mathrm{R}$ R

Kate's Baron..

Kennedy ................. $R$

Kentuck .......................

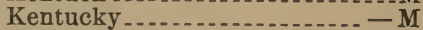

Kentucky Chief......... L R

Kete ....................... Daisy

Killbuck Ranger......... $-\mathrm{P}$ S

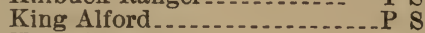

King Aylesby ......................

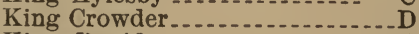

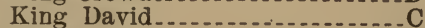

King Henry ............

King John ......................

King of Kansas..........

King of Maplewood......... D

King Mazurka 2d.......... $\mathrm{R} R$

King Splendor ................. R

King of the Meadow........... Ky I

King of the Park ............ - Cox

King of the Valley..........

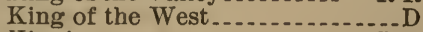

Kinnie ....................

Kirklevington of Conneaut... _-M

4th Kirklevington King.......-M

Kirklevington Kirk ........... - P S

Kirklevington Lad 3d.....- R R

Kirklevington Lad 4th......- Ky I

Kirklevington Lad 6th.....- - R R Kirklevington Oxford 4th - - Rolla Kirklevington Prince........- $R$ R $K^{\prime} k l^{\prime} v^{\prime} g$ 'n of t'e G'vePra'ie H'd Ky I Kirklevington Waverly.....-Rolla

Kirksville -............... T

Kirkwood...................

Knox ..................... 


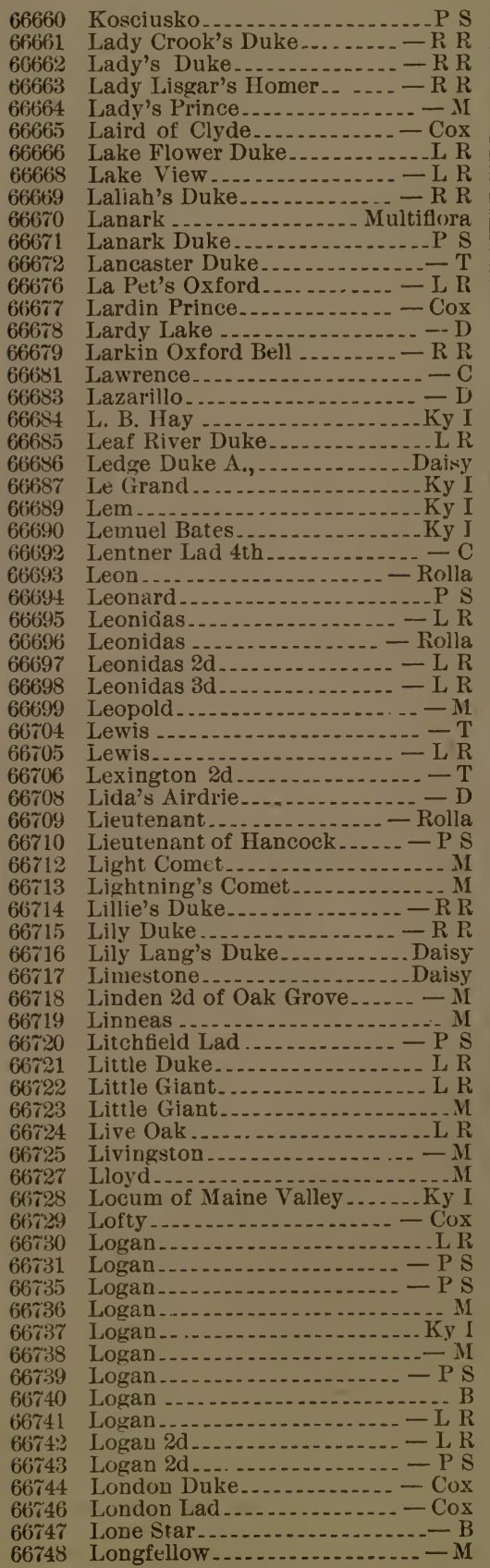

66749

66750

$66 \% 51$

66754

66755

66756

$66 \% 58$

66761

66762

$66 \% 63$

66 \%64

$66 \pi 6 \%$

66768

66774

$667 \% 6$

66777

$667 \% 8$

66779

66783

$6.57 \times 5$

66788

$66 \pi 91$

66 \%96

66797

66798

$66 \% 99$

66800

66801

66805

66507

66808

66810

66811

66812

66814

66815

66816

66817

66818

$66 \$ 19$

668:20

66821

66822

66823

66826

$668: 29$

66830

66831

66832

66833

66834

668:36

66838

66541

66842

fitist3

66846

66547

$66 \diamond 48$

66849

66850

66853

66854

66855

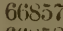

66858

66859

66860

66861

66862
Longfellow .................... P S

Longfellow 4 th

Longfellow A.....................

Lord Ayleshy ....................

Lord Barmpton.......... R R

L. Barmpton of Maine Valley - R R

Lord Barrington .............- - L R

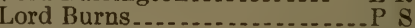

Lord Byron .........

Lord Byron .......... Ky I

Lord Byron.....................

Lord Chesterfield ............... P S

2d Lord Clancharlie.......... II

Lord Goodness ......- Milk Spring

Lord Ioyful..............

Lord Jumbo........... P S

Lord of Kirklevington .....- - Rolla

Lord of Kirklevington $2 \mathrm{~d} . .-$ Rolla

Lord Llewellyn.................- D

Lord Lytton

Lord Oxford .............. - Rolla Lord Princess Barrington - Beauty Lord Russell ............... - P S

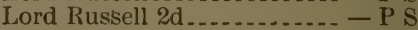
Lord Russell 3d............. - P S Lord Russell 4th........... - P S Lord Russell 5th........... - P S 4th Lord Sale ................ - M Lord of The Manor $3 \mathrm{~d} . . . . .$. Lord Turncroft Oxford 4th - Rolla Lord U'pton................... L R Lord Wiley $2 \mathrm{~d}$ Lord Wylia.......... T Lorne.................... Louan Duke....... R R Louan's Dnke........... R R Louan Duke $2 \mathrm{~d}$ Louan's Duke of Belmont..... - M Louan's Duke of Fort Cribbs_ - M Louan's 2d D. of Walnut Grove-R R Louan's Kirklevington.........- - D Louan's Mazurka_........ $R$ Louan's Muscatoon ......... - M Louan Prince...............-R R Loudon D. of Liberty Green_-R R Lowell ............... S S Loyalist................. $\mathrm{R} R$ Lucas........ M Lucian . Lucretius.................... Ky I Lucy Bly's Gem....... R R Luke................ L. Wildcat.............. $\mathrm{R}$ Lyndon ................ Rose Mabel Duke................. MeAdow ......... D MeDonald ................. Mack ............... M

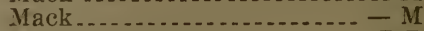
Mack of Hopewell......... $R$ McLain's Duke............. Madison.................... Kolla Magician Jr., ............. Ky I

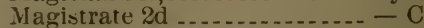
Magyar Oxford............. P Majur .............. $R$ Major Major Major 
66863

66864

668605

66866

66867

66868

66570

668 ชิ

$668 \% 2$

66873

$668 \% 4$

66875

66877

66878

66879

66880

66582

66883

66884

66588

66890

$668 \pm 1$

66893

66894

66895

66897

66899

66900

66903

66904

66905

66906

66908

66910

66911

66912

66913

66914

66915

66916

66917

66919

66921

669:22

669:23

66924

66925

66931

66432

66933

66935

66936

66938

66939

66941

$669+2$

66943

66945

$6694 \pi$

66948

66949

66950

66951

66952

66953

66954

669.56

66957

66958

66959
Major 2d

- R R

Major Brenda........ Rolla

Major Brenda $2 \mathrm{~d} . . . . . . .-$ Rolla

Major Clay.................... R

Major Cloyd.................... S

Major Courson............. $\mathrm{R} R$

Majur's Duke ............. - Cox

Major Grant ............ - M

Major Harman........ - M

Major Jack . . . . . . .

Major Lake ..............

Major Lee................ $\mathrm{L}$ Major of Maple Grove 3r... - Cox Major Mazurka ............... - M Major Moore................ Major Morris Major Oxford............. Rose Major Renick ......... D Major Robertson........ Major Warner .............. T Malta........................... Manfred.................... Mankato Duke......... - Cox Manilus _................. $-L R$ Mapleton Lad.............. - L R Marion.................................. Mark Twain ................... Mark Twain Marmaduke............... Ky I Marmaduke................... P S Marmaduke................................. Marmaduke............. D Marquis..................... Cox Marquis of Buckeye Farm ...... L R 2d Marquis of Buckeye Farm ... Ky I Marquis Duke..............._-M Marquis of Fairland Marquis of Lapwing ......... M Marquis of Lorne.............. Marquis of Maplewood....... Ky I Marquis of Solway Marshall Neil................ D Mary's Albert............. - L R Mary's Cambridge Duke_..._.__ M Mary Duke.............. KyI Mary's Duke.............. R Mary Duke.................... Master Airdrie................. D Master Bates.................. - P S Master Buttercup 6th......... - M Master Fitz ................ Master Gwynne .......... Master Medora ............ R R Masterpiece................. R R Master Richmond 4th.....Multiflora Master Richmond 5th ......... - M Ması er Richmond 6th .......... M Master Richmond 8th ..... - Rolla Master Watson 2d......... $-\mathrm{R} R$ Master Zilla .............. Matchless................... - D Mathias Lee .......... - Beauty Mat's Tom.................. Matthias.................... Matt Trimble.......... $\mathrm{R}$ Miaud's Aylesby ........... - Cox Maxwell Davison............. L R May Bell's Hillhurst.......... - D Mayberry ................... - M Maybird's $2 \mathrm{~d}$ Duke........ Cox
66960

66962 May Duke............. R R

66963 Mayflower Duke 2d...........R R

66965 Mayfrost 2d................... R

66966 Mayhugh........................... R

66967 Mayor........................ S S

66970 Meadow's Barrington Duke _ - R R

66971 11th Meadow Duke........... - D

66972 2d Meadow D. of Mazomanie - D

66973 Meadow's Furd............. - L R

66975 Medallist $3 \mathrm{~d} . . . \ldots \ldots \ldots \ldots \ldots \ldots$

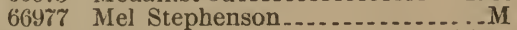

66979 Mercer.........................

66981 Michigan Lad................... C

66983 Midnight .................. R R

66985 Mike ....................... - D

66986 Mike Bell Duke ........ P S

66987 Mike Muldoon.................. Ky I

66989 Milford Lad............ - Cox

66990 Miller....................... D

66992 Milton Duke...................

66994 Minna's Duke............... - M

66995 Minna's Duke 4th.............. D

6 6000 Miranda's Duke............. - R R

6r001 Missouri Magic................ - B

67002 Miss Pickett's Duke....... - L R

67004 Misteltoe Boy .............. - R R

67005 Mistress Hanna's Duke...........M

$6 r 006$ Model.....................

67007 Model...................... $\mathrm{D}$

67008 Modoc........

$6 r 009$ Modoc Chief........

67012 Monadnock Boy ............ - L R

67013 Monarch....................... Ky I

67014 Monarch................... PS

67015 Monarch......... R

67016 Monarch................. - Cox

67017 Monarch......

67018 Monarch $2 \mathrm{~d} . . . . . . . . . . . .-$ Cox

67019 Monarch of Kansas...........-M

6r020 Monarch of Merwin ........... - M

67022 Monday...................... R

67023 Monitor.......................

67024 Monitor....................... P

67025 Monitor....... D

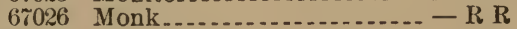

67027 Monkey Jim.....

6r0:38 Monroe Lad..............

6ru29 Monroe Rover

67031 Montgomery Chief............ Daisy

$6 \% 033$ Moonlight Lad................ $-\mathbf{C}$

6r034 Moorehouse............. R R

67037 Morton.......................

67038 Moscow................ Ky I

67039 Moses.................... R

67040 Mossie Bryan.............. - L R

6r044 Moundale Prince............. - D

$6 \pi 045$ Mountain Boy............. - L R

$6 \pi 047$ Muchacho .................... L R

$670+9$ Muller.....................................

67050 Murray Lad.............. - Rolla

67052 Myres...................... R

67054 Niada's Boy ................ - M

67058 Nantura's Duke ............ B

$6 \tilde{6} 059$ Napier.................. R R

$6 \pi 061$ Napoleon ............... $\mathrm{L} R$

6r06: Napoleon................ - Ky I

67063 Napoleon................ - L R

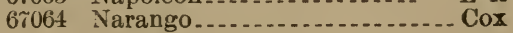




\begin{tabular}{|c|c|c|c|}
\hline 67066 & Nectar. & 67168 & Paris Boy \\
\hline 67067 & Ned... & 67169 & Parson 2d \\
\hline 67069 & Nellie's Duke. & 67170 & Partnership \\
\hline 67071 & Nero.......... & 67171 & Patrhoulia's D \\
\hline 67072 & Nero.. & 67173 & Petterson Duk \\
\hline 67073 & Newport Boy & 67175 & Pawnee Chief \\
\hline 67074 & Newsboy & 67176 & Paymaster.. \\
\hline 67077 & Newton C., & $671 \% 7$ & Pearce..... \\
\hline 67078 & New Year's Du & 67178 & Pearl. \\
\hline 7030 & Nick Worthingtor & 67179 & Pearl Diver \\
\hline 7081 & Nimrod $=. .$. & 67180 & Pearlette's Du \\
\hline 37082 & Nina's Muscatoo & 67181 & Pearlette's Du \\
\hline 67084 & Nolond ......... & $6 \% 182$ & Pearlette's Kir \\
\hline $6 \% 085$ & Nonsuch.. & 67183 & Pedro........ \\
\hline 67087 & Nora's Prince & 67184 & Pedro.. \\
\hline 67089 & Norfolk ....... & 67188 & Peter \\
\hline 091 & Normandy & 67189 & Peter B. \\
\hline 67092 & Norte..... & 67190 & Peter Cool \\
\hline 67094 & Northwest. & 67191 & Peter Co \\
\hline 67097 & November Duk & 67193 & Petrie. \\
\hline 098 & Noxall . & 67194 & Philip. \\
\hline 67105 & Oakland $3 \mathrm{~d}$ & 67203 & Phyllis Du \\
\hline 106 & Oakland 5th. & 67204 & Phyllis Duke \\
\hline 67107 & Oakland Boy & 67208 & Pickway Oxfo \\
\hline 108 & Oakland Duke & 67209 & Pickwick \\
\hline 67109 & Oakland Duke. & 67210 & Pidemont. \\
\hline 67110 & Oaklawn Duke & 67212 & Pilot. \\
\hline 111 & Oglesby........ & 67213 & Pilot \\
\hline 112 & Oglesby & $6 \tilde{2} 214$ & Pilot of Banc \\
\hline 113 & Olive Branch ] & $6 \% 215$ & Pilot Grove \\
\hline 67114 & Oliver of Maple G & 67216 & Pilot Leor \\
\hline 67116 & Omer & 67217 & Pioneer \\
\hline 67118 & Onondaga $2 \mathrm{~d}$ & 67218 & Plant \\
\hline 67119 & Onondaga $3 \mathrm{~d}$. & 67219 & Plato \\
\hline 120 & aga 4 th & $672: 30$ & Plato $2 \mathrm{~d}$ \\
\hline 67121 & Orange Duke. & 67221 & Pluto.. \\
\hline 67123 & Orangeman... & 67223 & Plymouth \\
\hline 67124 & Oriental Princ & $6 \pi 223$ & Pompey - \\
\hline 67125 & Orlando $2 \mathrm{~d}$.. & 67225 & Pony Richr \\
\hline 67126 & Orpha & 67226 & Popcorn Harve \\
\hline 67128 & Orpheus .. & $672: 27$ & Popcorn's Prid \\
\hline 67129 & $-\mathrm{K} R$ & 67230 & $-\mathrm{M}$ \\
\hline 67130 & Osceola C & $67: 231$ & Pottawattami \\
\hline 67131 & Othello. & 67232 & Pounce. \\
\hline 67132 & Ottumwa Lad. & 67233 & Powers . \\
\hline 67133 & Ouachia ... & 34 & Prairie Duke. \\
\hline 67134 & Oxford $3 \mathrm{~d}$ & 6 б23ว & Prairie Duke. \\
\hline 67135 & lof Arca & 36 & Ky I \\
\hline 67138 & Oxford Bly $2 \mathrm{~d}$ & 672338 & Presley's Bull 2d \\
\hline 67139 & Uxfora Bull Erne & 67241 & Pride of Avon 4th \\
\hline 67141 & Oxford Duke.. & $672+2$ & Pride of Burton \\
\hline 67142 & Oxford Duke. & 43 & Pride of Gle \\
\hline 67143 & Oxford Duke. & 6245 & Pride of Humph \\
\hline 67145 & Oxford Duke. & 67246 & Pride of Upton \\
\hline 67147 & Oxford Duke $2 \mathrm{~d}$ & 67248 & Prince. \\
\hline 67149 & Oxford of Eagle D & 67249 & Prince. \\
\hline 67150 & Oxford Earl.. & & Prince... \\
\hline $6715 \%$ & Oxford Hillhurst.. & $6 \pi 251$ & Prince.. \\
\hline 67153 & Oxford Hilpa's Son & 67252 & Prince \\
\hline 67154 & Oxford Knightly & $6 \tilde{2} 254$ & e $3 d$ \\
\hline 67155 & Oxford Prince... & 67255 & Prince Agatha \\
\hline 67156 & Oxtord Prince.. & 67256 & Prince Airdrie $2 d$ \\
\hline 67157 & Oxford Prince $3 d$ & 67257 & Prince Albert - - \\
\hline 67158 & Oxford Prince 4th & 67258 & Prince Albert - \\
\hline 67159 & Oxford Red.. & 67259 & Prince Albert \\
\hline 67163 & Ozark . - & 67260 & Prince Albert \\
\hline 67164 & Palmyra Phyllis. & 67261 & Prince Albert - \\
\hline & 2d Palmyra Phylli & 67262 & Prince Albert \\
\hline 67166 & 3d Palmyra Phyllis & $6 \tau 263$ & Prince Albert \\
\hline 67167 & 4th Palmyra Phyllis..... & 67264 & Prince Albert. \\
\hline
\end{tabular}


67265

67266

67267

67268

$6 \approx 271$

67274

6 ชัน5

$6 \% 2 \%$

67279

$6 \% 282$

67283

67284

67285

67287

67288

67289

67290

6\%291

67292

67295

6ชั296

67297

67298

67299

67300

67301

67302

67303

67304

67305

67306

67308

67310

67311

67313

67316

67317

67318

67321

67325

67327

$673 \%$

67332

67333

67336

67337

$6 \% 340$

67341

67342

67343

67341

67348

67349

67350

67351

67352

67354

67355

67356

67357

67359

67360

67361

67362

67363

67365

67366

67368

67369

67370
Prince Albert.

Prince Albert.....................

Prince Albert of Hancock ...... Ky I

Prince of Anoka 4th ..... Multiflora

Prince Arthur.................. Cox

Prince of Bluffdale..........-P S

2d Prince of Bourbon....... - D

Prince of Buffalo ..........- $-R R$

Prince Dan ................ II

Prince Edward ............- Cox

Prince Edwin ............... - II

Prince Frederick

Prince George...........- $\mathrm{R} R$

Prince Gwynne........... D

3d Prince of Hancock...... P P

12th Prince of Hanover............ M

13th Prince of Hanover.............

14th Prince of Hanover............ M

Prince Hilpa ....................... II

Prince Joe ........................ B

Prince John ....................

Prince Kirklevington Duke_-_ - P S

Prince L., ...............- - Cox

Prince Lapode.................- II

2d Prince of Lapwing .........- M

Prince Leopard .....................

Prince of Lincoln .................

Prince of Lingwick

Prince Ly ford of Roscoe - - Beauty Prince of Maine Valley........ L R Prince of Maple Grove......- - L R Prince of Meadow Brook ........... Prince of Mt. Hope............. C Prince Napier 7th ...........- $\mathrm{R} R$ 11th Prince of Orange ......- - - L R 15th Prince of Orange ............ B Prince Phyllis............... $\mathrm{k}$ Prince of Portland....... Prince Ray ................... Prince Royal............... - is Prince Royal 2d.............. Ky I Princess Lad................-Cox Prince of The Valley........... L $\mathrm{R}$ Prince of The West..........-- $-\mathrm{L} R$ Prince of Unadilla........... - Cox Prince of Upton .............- - L R 3d Prince of Virginia....- - Beauty Prince of Wales............ - L R Prince of, Warlaby.......... - $\mathrm{L} R$ Tth Prince of Woodlawn....- $\mathrm{R} R$ Prince of Wyoming............ - M Produce Prohibition-................. - T Prohibition -............ Prohibitionist............ Prospect.................. Proud Duke 7 th..........- Ky I Proud Duke of Cedar....... Proud Prince...............- $-R$ Punch ................... P S Raleigh $2 \mathrm{~d}$ Ralph ............... Rambler ......... Cox Rambler ........................ C Rambo ...................... Randall Randolph.................. Ranger Ranger .................. Ranger of Maple Grove.....-Cox
67372

67373

67374

67375

67376

67377

67378

67379

67380

67381

67382

67383

67384

67385

67387

67388

67389

67390

67391

67392

67393

67395

67397

67401

67402

67403

67404

67405

$6 \approx 406$

67407

6 ส708

$6 r 409$

67410

$6 r 412$

67413

67414

67415

67417

$6 \% 418$

$6 \widetilde{719}$

$6 \approx 421$

$6 \pi 422$

67423

$674: 24$

67425

$6 \% 426$

67427

67428

67429

67430

$6 \% 431$

67432

67434

67435

67436

67438

67440

67442

$6 \pi 443$

$6 \pi 444$

67445

$6 \approx 4+6$

$6 \pi 447$

67448

6 ช 449

$6 \% 450$

67451

6r452

$6 \widetilde{454}$

67455
Red Airdrie.................

Red Bat...................

Red Bill...........................

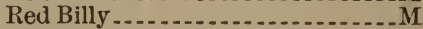

Red Billy

Red Bird ............ S

Red Bird.................

Red Boy

Red Boy of Tollon Prairie.. - Cox

Red Buck................- $\mathrm{R} R$

Redbud..................... -PS

Redbud..................... - P S

Redbud Knight............. Ky I

Red Butterfly..............- $\mathrm{R} R$

Red Champion................R R

Red Chief

Red Chief.................-L $R$

Red Chief of Rose $2 \mathrm{~d} . . . . . . . . \mathrm{L}$ R

Red Cloud .......................C

Red Cloud.................- - R R

Red Cloud ............................

Red Cloud ................ - Cox

Red Cross................ Rolla

Red Duke..............................

Red Duke...............

Red Duke........... Milk Spring

Red Duke.................. - L R

Red Duke...................

Red Duke................. P S

Red Duke..........................

Red Duke........

Red Duke ....................

Red Duke

Red Duke ..................

Red Duke............... Rolla

Red Duke...........

Redfield ...............

Red House........... R

Red Jacket..........

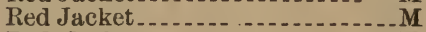

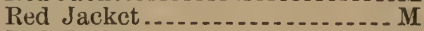

Red Jacket..............

Red Jacket........................

Red Jacket...................

Red Jacket .....................

Red Jacket.............. R R

Red Jim ................... R

Reà Joe..............

Red Lad....................

Red Lark 2d......

Red Light ............

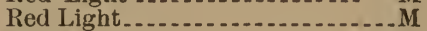

Red Oak................ R

Red Plumed Knight....... $-\mathrm{D}$

Red Prince.............. R R

Red Prince....

Red Prince....... Milk Spring

Red Prize of Aylesby ..........P S

Red Ranger .................

Red Rattler.

Red Rex............ Rolla

Red Roan.....

Red Robin .....

Red Rose Delight........

Red Rover............... - Cox

Red Rover..................... R

Red Rover.............. R

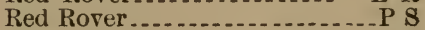

Red Rover ................. D

Red Rover of Columbus...-Cox 
67456

$67+58$

67459

67460

67461

67462

67463

$67+65$

67466

$6746 \%$

67468

67472

$67+73$

$674 \pi 4$

67475

67477

67478

67479

67480

67481

67482

67483

67484

67485

67487

67488

67489

67490

67491

67492

67493

6 6 494

67495

67496

67497

67498

67499

67500

67502

67503

67504

67505

67506

67507

67508

67509

67510

67511

67512

67513

67514

$6 \% 15$

67516

67517

67519

67520

675:1

675:2

67523

67524

67525

67526

67527

67528

$675 \div 9$

67531

6753 ?

67533

67534

67535
Red Rowdy ............ R Red Star Red Star ............... Red Tiger Duke....... R d Tom . Red Tom Red IVillow Prince

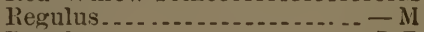
Regulns........ Reliable .......... Cox Renick

Reno

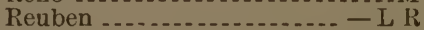
Rex ........................ Richmond............. Richmond.................. P S Richmond $2 \mathrm{~d}$................. $\mathrm{L}$

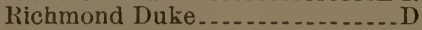
Ridgeland Joe Ridgeland Porter .........

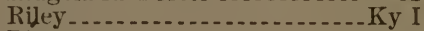

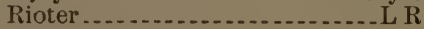
Ristori's Duke....................... Riverdale Jewel Roan Architect...................... Roan Boy ........... Roan Boy Roan Bull Roan Charley ............. - D Roan Chief.................. Cox Roan Duke................... Ky I Roan Duke...................... Roan Duke....... D Roan Duke Roan Duke..........

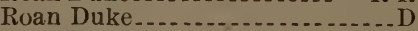
Roan Duke............ Roan Duke........ Roan Duke.................. - C Roan Duke of Maine Valley - - L R Roan Kirklevington..........- PS Roan Lad.................... P S Roanoke...................... R Roan Prince........ Rolla Roan Prince.................. Ky I Roan Prince of Dumma...... Cox Roan Richard ............ Daisy Roan Rover........... Roarer ................... Robert Burns ..... R R Robert Mack ............................ Robert Williams..... $\mathrm{R}$ Robin ................. Rob Roy Rob Roy Rob Roy ..... Rob Roy ........... $R$ Rochelle ............. Rocket ............. Woods Rocket 4th............. R Rock Highland ........ Rock River Lad 90th .......... Rock River Lad 91st ....... - Cox Roderic Dhu.............. Rodman ................ Rolla of Rose Hill Rolling Chief............... Ky I Romeo...................... $R$ Romeo

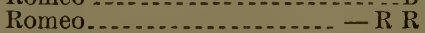

67536

$6 \pi 537$

67538

$6 \% 540$

67541

67542

67543

$6 \% 547$

67549

67550

67552

$6 \% 553$

67554

67555

67556

67557

67558

6ัวัริ9

65560

67561

67562

67563

67564

67565

67566

67568

67570

67571

67573

$675 \% 6$

67577

67578

67581

67582

67583

67585

67586

67557

67588

67589

67591

67594

67596

6 бั597

67598

67600

67602

67604

67605

6ribus

67609

67610

67611

67612

67614

67615

$6 \% 616$

67617

67619

67620

67621

67622

$6 \% 6 \div 3$

67625

$676: 26$

67627

67628

$676: 29$

676332

6 6ั633
Romney

Romulus..........

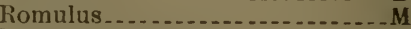

Rosebud Duke.......... Ky I

Rosebud Duke 2d......... - Ky I

Rosebud Sharon $2 d . . . . . .-L R$

Rosedale ...................... R R

Rose's Duke.......................

Rose Duke........................

Rose Duke of Walnut Itill ......P S

Rosemary Bly ............. $\mathrm{R} R$

Rosemond Duke 3d......... - B

Rosemonger ................ - D

Roscoe

Rosy Barrington......

Rosy Duke .............

Rosy Lad $2 \mathrm{~d} . \ldots \ldots \ldots$

Rossi..........................

Rosso ................ C

Rover

Rover....................

Rover .................... Ky J

Rover $2 \mathrm{~d}$ of Lake Park...... $\mathrm{C}$

Rowdy Belmont ............ P S

Roxana's Lord Chancellor 2d $\mathrm{d}_{-}-\mathrm{M}$

Royal Airdrie............... - R R

Royal Booth's Duke.......

Royal Booth Jr.,........ - Cox

Royal Bright Eyes.......... - Cox

Royal Crown .......... Cox

Royal Duke.................. R R

Royal Duke.................. Ky I

Royal Duke 2d............... Kv I

Royal Duke of Maine Valley - R R

Royal D. of Wheatland..- - Beauty

Royal George................. - D

Royal George............

Royal George................ Ky I

Royal Grandissime........ - Rolla

Royalist ........................ M

Royal Knight..................

Royal Oak..................

Royal Prince............

Royal Prince................

Royal Prince...............-R R

Royal Prince of Woodlawn

Royal Reserve.............. R R

Royal Roan...................... R

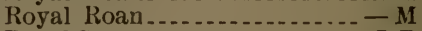

Royal Star

Royalton Duke 2d...... R

Royalty .................... $\mathrm{R} R$

Royal Viewland.............

Roy Roxie..........

Rufus.......... $R$

Rufus......

Rupert............

Rural Duke... Cox

St. Clair-................. - D

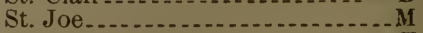

St. Joe

St. John

St. John .............. Ky I

St. John ............ P S

St. John

St. Nicholas ............. - C

St. Patrick ............

Salamonie Duke ....... M

Sam Gardner.....

Sam Patch....... 
67634 Samson .......... Daisy 67635 67636 67638 67639 67640 67641

67642

67643

$6 \pi 644$

$6 \% 6+5$

67646

67647

67648

67649

$6 \% 6 \overline{1} 0$

67651

67652

67653

67655

67656

67657

67658

$6 \div 663$

67664

67665

67666

676677

$6 \% 668$

67669

67670

67672

67673

67675

67682

67633

67684

67685

$6 \% 686$

6 6758

67688

67689

67690

67691

67692

67693

$6 \% 694$

67697

67698

67699

67700

67701

67703

67704

67706

6 6rios

6รช09

$67 \% 11$

67712

67714

67715

6 พร16

67717

67718

67719

$67 \% 20$

$67 \% 21$

$67 \% 22$

67723

67724

Sam Tilden

Sam Tilden

Cox

Samuel ................... R R

Sandy ........

Sankey ............... Cox

Sankey .............

Sankey

Santa Claus.................. R

Santa Claus ................ - L R

Santa Claus 2d................ S

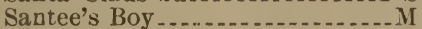

Sappho's Lord .............

Sappho's Prince............. T

Saxton's Glick.......... R

Sciota Airdrie Lad............ L R

Scotland's Hero............. Cox

Scottish Chief................ Cox

Searsport ...

Seigle

Senator .......................

Senator Heard.............. S

Senator Pierce...................

Seventy Six............................

Shakespeare ................ P S

Shakespeare................. R R

Shakespeare................

Shamrock 2d............. Ky I

Sharon.................. D

Sharon Atkinville......... - Daisy

Sharon Baronet........... $\mathrm{R} R$

Sharon Duke.............. $\mathrm{R} R$

Sharon Duke............

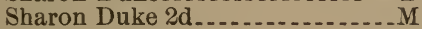

Sharon Lad...................

Sharon's Oxford ...........

Shawnee Chief............... M

Shelbrook Boy.................. Ky 1

Shelby ....................

Shelby .

Shelby's.Hillhurst................

Shellenberger ............. Multiflora

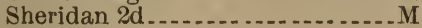

Sherman....................

Sherman ...................

Sherman ..........

Sherman 2d........ R

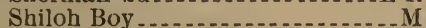

Short-legged Joe 2d........ - Cox

Sid Baron...................

Siggle...........

Signal........... P S

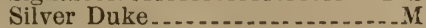

Silver Duke $2 \mathrm{~d}$

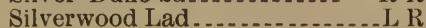

Sir Alfred............... $\mathrm{R} R$

Sir Arthur..........

Sir Brutus.....

Sir George..............

Sir Guy of Greenland..... Rolla

Sir Henry .............. P S

Sir John ............. P S

Sir Knight ............... R R

Sir Rubert ........

Sir Robert Bruce.......... P S

Sir Rufus...................

Sir Rupert 2d......... R

Sir Tom

Sir Walter

Sir Walter Hamilton ........... Cox
67726

$6 \div 7 \cdot 27$

$67 \% 29$

$6 \% 730$

67732

67733

67734

$67 \% 35$

6\%737

$67 \% 35$

67739

67740

67741

$67 \%$

$6 \% 74$

67746

67748

$67 \% 49$

$67 \% 50$

$67 \% 51$

$67 \pi 52$

67753

67754

67755

67756

$6775 \%$

$67 \% 58$

6 \% 60

$6 \pi \% 6 \%$

$67 \% 67$

$677 r 0$

67771

67772

6 7778

67775

67776

67778

6 ช779

$6 \% 781$

67783

6 \% 84

67786

67787

67788

$6 \% 789$

$6 \% 790$

$6 \pi 791$

$67 \% 92$

$6 \div 793$

67795

$67 \% 96$

6ัศ97

$67 \% 99$

67800

6 \%801

67802

6 \%03

67804

67805

67806

67807

67809

67810

67811

67812

67813

67814

67815

67816

67818
Sir Whitsitt.................

Sir Wiley ............... Ky I

Skeen ....................

Skowhegan ............ - Beauty

Sly Dude...................... L R

Smuggler................ $\mathrm{R} R$

Smuggler ............................

Snowball ...................

Soldier.................... R

Solomon ...............

Solomon _.................

Somerset_............. Beauty

Son of Airdrie Duke............ L R

Sonora Chief.................. - M

Sonsie Duke............... P S

Sovereign .................... S S

Spectator - .

Speedwell................ - L R

Spink County Duke....... $-\mathrm{R} R$

Spot .........

Spot Cash.............. $\mathrm{R}$

Spotted Duke..................

Spotted Lyman .................M

Springvale Duke $3 \mathrm{~d} . . . . . . . . . . \mathrm{Ky} I$

Springvale Duke 4th........... Ky I

Springvale Duke 5th.......... Ky I

Springvale Duke 6th........... Ky I

Stafford ..................... Ky I

Standoff ............

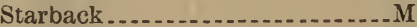

Star Duke......................

Star Duke of Maine Valley ..... Ky I

Star of Hope 2d........... - Cox

Stark ..................... Ky I

Starlight................... Cox

Star of the West................ Cox

Sterling's Duke of Woodlawn.Ky I

Stillwater Duke........... -C

Stoneman's Duke............ - Cox

Stonewall ...................

Stonewall of Beallsville....... $\mathrm{L}$

Storm Duke .................. Daisy

Storm King .............

Strawberry Duke.................M

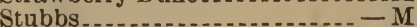

Stunner ................ P S

Sturgeon Duke........... P S

Sultan ............. R

Sunbeam ....... R R

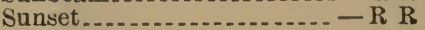

Surprise................. R R

Surprise .............

Sycamore Duke........... $\mathrm{R}$ R

Tagnarook..................

Tallook ...................

Tama Jim.................... R R

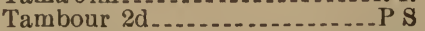

Taopi Lad............... - Rolla

Tasso ......................... R

Tasso .................. $\mathrm{R}$

Taurus.................. Cox

Ted

Tulluria Duke 6th......... - Rolla

Tulluria Duke 7th........ - Rolla

Tulluria Duke 8th......... _ Rolla.

Thaddeus of Warsaw...... - Cox

Thad Hickman............

The Kid ........ Cox

The Laird...... T

Theodore ....................... 
67819 Theodore ..................... T

67820 Therino

6r821 The Wing Bull............... R

67822 Thomas.................. D

67824 Thos. A. Hendricks ........

67825 Thos. A. Hendricks......... P S

67826 Thunder................... - Rolla

67827 Thurman ........................

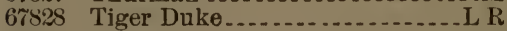

67830 Tilford....................

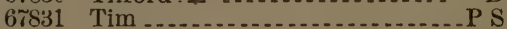

67832 Timothy

6r833 Tinnie's Duke.............

67834 Toby ..................

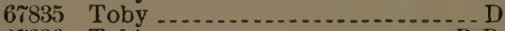

67836 Tokio................

67837 Tom...................

67838 Tom

67839 Tom..............

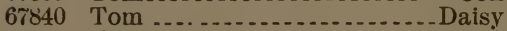

67841 Tom Bangs.....................

67842 Tom Benton..................

67843 Tom Brown.............. R R

$678+4$ Tom Brown................ $\mathrm{D}$

67845 2d Tom Dutt.....................

67846 Tom Granger

$678+7$ Tom H.,...................... S

67848 Tom Hendricks............

67849 Tom Hendricks............. $\mathrm{R}$ R

67850 Tom Hendricks..............

67851 Tom Hendricks........... - Rolla

67852 Tom McGee...........

67853 Tom Maloney

6785̃4 Tommy .........................

67855 Tom Paine

67856 Tom Porter..............

67857 Tom Rodman

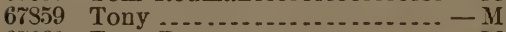

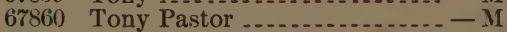

67861 Topsy's Prince...........

67863 Tremont......................

67864 Triumph...........................

67865 Trubadour................. - N

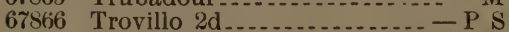

67867 True Duncan ................. R R

67868 Trumbull .......................

67869 Trusty Boy ................. Ky I

67870 Tuberose Prince ........ - Beauty

67871 Turk .............................

67872 Twin Boy .................. P s

67873 Twin's Duke ....................

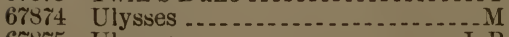

67875 Ulisses...........

67876 Ulysses .....................

67877 Uncle John

67879 Union Duke 3d............. Ky 1

67880 . Upright ....................

67881 Vaile Prophet............. Daisy

6 rss3 Valley Duke.............

$6788 t$ Vampire..................

67886 Vandalia.................. $\mathrm{R}$

67887 Vanderbilt $2 \mathrm{~d} . . . . . . . . . . . . .-\mathrm{P} \mathrm{S}$

67889 Venor..........................

6тธ92 Viceroy

67893 Vici..............................

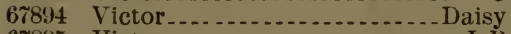

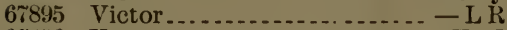

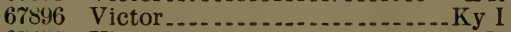

67897

67898
67900

67902

67903

67906

67907

67908

67914

67916

67917

67919

67920

67921

67922

6ั9:3

67924

67925

67928

67930

67932

67933

67934

67942

67943

67944

67945

67947

67952

67954

67957

6รงอั8

67959

67960

67961

6796:

67963

67965

$6 \% 967$

67968

$6 \% 969$

$6 \% 970$

$6 \% 971$

6\%972

67973

$6 \% 974$

$6 \% 976$

6ส97\%.

67978

67979

67980

67983

67.984

67985

67986

67987

67989

67995

67996

6ับ999

68000

68001

68003

68004

68006

68007

68008

68009

68010

68011

68012

68013
Victor H.,

Victoria Duke..............

Victoria's Duke

Victor of Maple Shade....... - Cox

Victor of Raytown

Vidi...........................

Virginia's Gloster ........ Cox

Voltaire............... T

Waco

Wakefield ................ Rose

Walker ..........

Wallace...............

Wallace...........................

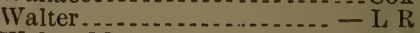

Walter $2 \mathrm{~d}$

Warlaby ................

Warren

Washington...............

Washoe Prince......... Daisy

Wataga..................

Vaterloo..........................

11th Waterloo of Raccoon V'y Ky I

12th Waterloo of Raccoon V' $y-R$ R

13th Waterloo of Raccoon V' $y$ Ky I

Waterloo of Upton............. L R

Watson Lad................. L R

Weehawken Prince.........

Welcome .....................

Wellington..................

Wellington

Wenona's Duke of Boody ....- M

IVest.......................

West Burlington Boy

West Burlington Bud ......... - M

West Burlington Lad ....... Cox

Western Lad...............

Whalebone..............

Wharfdale Prince $2 \mathrm{~d}$

White Balco .................. L R

Whitebreast Ben.......... P S

Whitebreast Fly

Whitebreast Garfield......... - P S

Whitebreast Logan........... $\mathrm{T}$

Whitebreast Luther..........

White Flint.................. R R

White Foot

White Legs .......................

Widower .................

Wiggins... .............. Daisy

9th Wild Eyes of Armada....... K R

10th Wild Eyes of.Armada.....L R

11th Wild Eyes of Armada....... L R

12th Wild Eyes of Armada..... $L$ R

Wild Eyes of Avon.............. $\mathrm{L}$

Wild Eyes Duke..................

Wiley

Wiley

Wiley of Peoples..........

William F.,..................

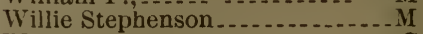

Wilson ...........................

Windsor of Maine Valley...... L R

Wisdom................ $R$

IV. J. Lucas.......... D

Woodland Prince............

W. T. Sherman............... L R

William T. Sherman.......

Xenophone.................. $-\mathrm{L}$

Yanker.....................

York 
68014 Young Airdrie 68015 Young Arthur ................. Cox 68016 Young Brick Pomeroy ............. T 68017 Young Bridegroom ............... C 68018 Young Chancellor.............. D 68019 Young Clement..............- Cox 68020 Young Cypress.................... R R 68022 Young Dom................ - Cox 68023 Young Duke............... Cox 68024 Young Erasmus................ T $680: 25$ Young George................... M 68026 Young Grant of Cedar Creek - P S 68027 Young Gunter................. - T 68028 Young Hopewell 68029 Young King ..................... M 68030 Young Liverpool .............. - D 68031 Young Livingston ........... - C 68032 Young Lord................. - M 68033 Young MlcGregor........ Cox 68034 Young Manfred ............. - L R 68038 Young Oliver................ 68039 Young Peri ..... . .............. Rose 68040 Young Richard 5th......... - R R 68041 Young Royal Oxford.......... L K 68042 Young Ruby .................. M 68043 Young Tempest................ L R 68044 Young Vicior............... - Cox 68046 Zeilenople Duke.............. - T 68047 Zeno...............................

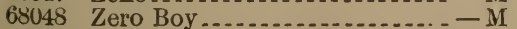

68049 Ziwankee.....................

68051 Aanthna...................... Ky I

68052 Abdallah................... Rolla

68057 A. C. Briant........

68058 Accident...........................

68059 Acern $3 \mathrm{~d} . . . . . .2 \mathrm{R}$

68060 Acklam's Duke.................. R

68061 Acomb Duke $3 \mathrm{~d} . . . . . . . .-\mathrm{R} R$

68065 Ada's Duke................. Kolla

68066 Addison

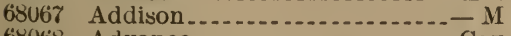

68068 Advance.......................... Cox

68073 Airdrie Constance .......... - Daisy

68 78 Airdrie's Duke.............. - L K

68080 Airdrie Duke 4th ............. Daisy

68 us1 Airdrie Duke of Elmwood 3d _ - B

68082 Airdrie of Etna................ R

68483 Airdrie Goodness ............. - M

68086 Airdrie of Guy Park........ $-\mathrm{R} R$

68087 Airdrie Imperial............ - R K

68088 Airdrie J.,................... $\mathrm{K}$

68089 Airdrie Lee..................

68090 4th Airdrie Prince..............

68091 Airdrie Prince 4th.......... Cox

68093 11th Airdrie of Rosewood. - Daisy

6 sug4 14th Airdrie of Rosewood. - Daisy

68096 Airdrie Sharon $2 \mathrm{~d} . . . . . . . . . . . .-$ Rose

68097 Airdrie Sharon 3d............. Kose

$680 y 8$ Airdrie Sharon 4th............. Rose

68099 Airdrie Sharon 5th ........... - M

68100 Airdrie Sharon 6th ............. Rose

68101 Airdrie Sharou 7 th .............. Rose

68102 Airdrie of Spring Hill....... K K

68104 Airdrie Woodhill ............ L R

68107 Ajax........................ R

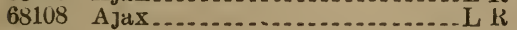

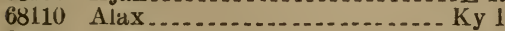

68111 Albert............................... 1

68113 Alberta's Oxford...........

68114
68115

68116

68117

68119

68120

68123

68125

68126

68127

68128

68129

68130

68131

68132

68133

68134

68136

68137

68138

68139

68143

68144

68145

68147

68148

68149

68150

68151

68152

68153

68154

68155

68156

68157

$6 \$ 158$

68159

68160

68161

$6816 \%$

68163

68164

68165

68171

$6817 \%$

68174

68176

68177

68178

68179

68180

tisir1

68182

68184

68185

$6 s 186$

68187

68188

68189

68190

68191

$6 \triangleleft 192$

68193

63194

$6 \$ 195$

$68: 200$

68201

68202

68203

68204

68:205
Albion 3d.................. - L R

Albion 3d................

Albion Beaconsfield ........ - L R

Aleck MeKenzie............. $-R R$

Aleck McKenzie $2 \mathrm{~d} . . . . . . . .-\mathrm{R} R$

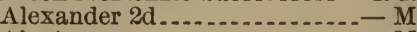

Alexis..........................

Algoner................... $-\mathrm{L} R$

Alias Duke of Wyandot....... - D

Allendale Loudon Duke 19th..- M Allendale Loudon Duke $23 \mathrm{~d} . .-$ M Aliendale Loudon Duke 26th. Daisy Allendale Loudon Duke 27th-Ky I Allendale Loudon Duke 29th .. - T Allendale Loudon Duke 30th- Ky I Allendale Loudon Duke 31st.. - $\mathrm{T}$ Alpha ......................... C Alphens $2 \mathrm{~d}$ Alphouso Amar................ Anna's Quaker ............. P S Anna's Duke................ $M$ Antony .................... $\mathrm{R}$ April Lad................ Archduke Deland......... Archer.................. $\rightarrow$ Ardmore................ R Ares Duke............. Aristos 3d................. Arnol Lad............................ Arr Aytch Arthur..............................

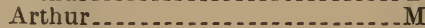
Arthur ..............

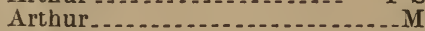
Arthur.............. Arthur................ - Cox Arthur 2d................. Arthur Watts............. Arth Wild ....................... Ashland Duke ............. Daisy Ashton .....................

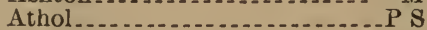
Athol Duke .......... R R Atwood ......................... August Belmat........ Cox August $\mathrm{K}, \mathrm{n}, \ldots$ Aurora .................... Aurora's Oxford Australia's Lad ......... Cox Avalanche.................. A von Prince............ $\mathrm{R}$ Aylesby Baronet........... R R Babraham's Royal D. of Salem_L R Baby Duke.................... T Badyer Boy .................. Badger Jim.......................

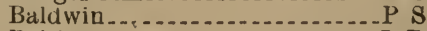

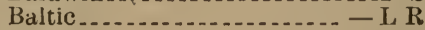
Banker...................... Banker..........................

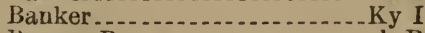
Banner Boy ............ Bannister........................ S Barinpton's Admiral ........ - Cox Barmpton's Canbria ....... - Ky I Barmpton's Chief........... - K R Barmpton's Lad ............. - M Barnaby Rudge.................. Barnet.................... $\mathrm{R}$ 
68206

$68: 07$

68209

68:16

68:17

68218

68:21

65223

68:26

$68: 28$

$68: 32$

68233

68:2:34

68235

65:2:36

$68: 37$

68:38

$68: 239$

$68 \% 4 \%$

$68: 43$

68248

68252

63:5ั3

68:5̃5

68:256

68:257

68258

$6820{ }^{\circ} 3$

68:264

68:65.

68:267

68269

68:70

68272

68274

68275

68: 6

68:51

68285

68290

68292

68:243

68294

68297

68:98

$68: 299$

68300

68304

68305

68306

68307

68308

68309

68310

68311

68312

68313

68314

68316

68317

68318

68319

68321

683325

$683: 26$

683228

6ธ3:29

68330

68331

68332
Barney Baron Acomb of Monmouth .... P S Baron Bly 5th .............. - Cox Baron Booth of Brook Farm - R R Baron Bright Eyes 5th ......- - Rolla Baron Clyde.................. L R Baron of Cresabrook Baron Duke 3d .............. Ky I Baron of Eatun $2 \mathrm{~d}$ Baron Gwynne 6th ........ Cox Baron Gwynne 7th ..........-Cox Baron Gwynne 21 of Oakland - R R Bıron G wynne of Sugar Creek-Cox 2d B. Gwynne of Sugar Creek - Cox Baron Harriet Baron Hopewell.......... Baron Hopewell.......... $-R$ Baron Judy ................ $-R$ R Baron of kewanee ......... - L R Baron Lewis $3 d$.................. Baron Mansfield $3 \mathrm{~d}$ 3d Baron of Maple Hill...... D Baron Oxford ............ Rolla Baron Oxford $2 \mathrm{~d}$.......... $-R R$ Baron Oxford 14th ....... - Rolla Baron Peri ............... Rolla Baron Prince $2 \mathrm{~d}$ Baron Red Rose............ Baron Red Rose $2 \mathrm{~d} . . . . . .$. Baron sharon 4th .......... - L R 3d Baron of The Valley..... - L R B ron Varna ............... - M Baron of Wahoo Valley .... - Cox Barrington 7th.............. - M Barrington 8th.......... Barrington 9th 13th Barriugton $\mathrm{U}$. of Bluff $\mathrm{Cr}^{3} \mathrm{k} L \mathrm{~L}$ Barrington D. of Silver Creek $-T$ Barryscourt Duke.......... - R R Bartja........................ $R$ Bartlet ............... Rulla Basil ..... . . Bates Darby Duke......... Bates Duke .................. - M Bates Duke 3d............... M Bates Emperor............ - L R Bayard ...................... $\mathrm{R} R$

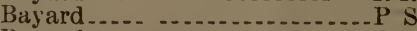
Bayard....................... Bayard of Edbrun........... L Beaconsfield............... P S B zallville................ Beaumont

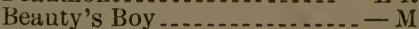
Beauty's Duke........ Daisy Beauty Prince............ Beaver Breastplate....... P S Beecher................ Beecher.......... Beecher .............. Beechy Mire Lad.......... II Beldale .................... Bell Chief Belle's Dandy..................... Belle's Duke ................ R R Bell Duke...................... D Bell Duke........ Bell Duke...... Bell Duke 4th ............
68334

683335

68:337

68:338

63340

68:341

6834:2

$6 \$ 343$

$68: 344$

$6 \$ 345$

68346

65352

68353

65354

$6 \$ 356$

68:357

68358

68360

68361

$6536 \%$

68363

68364

68355

68366

68367

68368

68369

68370

65372

68373

65374

68375

68376

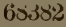

68383

6\$3ऽ5

65387

685388

68390

68391

68394

$6 ธ 345$

68396

65397

68398

68349

$6 \$ 400$

bstu3

68404

68405

$6 s+47$

68408

68409

68410

$6 \checkmark \$ 11$

$6 \$ 412$

$68+13$

$68+15$

$6 s+16$

$6 s+17$

$6>418$

$6 s+19$

$65 \pm 21$

$6 s+2: 2$

68123

$68+24$

$68+25$

$68+26$

68427

$68+28$
Bell Duke of Beloit..........- R R

5th Bell Duke of Lyndale... - R R

4th Bell Duke of Lyndale... - R R

Bell Duke of Stonington.... $-R R$

Bell Duke of Wyandot......... - D

Bellfounder............... Cox

Bell Sharun Duke........... - D

Bell Victor...................... P S

Bellville Duke................... T

Bellville Golddust ............. Ky I

Belmont $2 d$..................

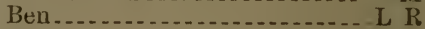

Ben ............. Ky I

Ben B., .............

Ben Butler............. Daisy

Ben Butler................. - M

Ben Butler............... PS

Ben Butler....... D

Ben Butler................... R

Ben Butler...........................

Ben Butler ...................

Ben Butler.............

Ben Butler................

Ben Butler............

Ben Butler.................. P S

Ben Butler ............

Benedict................ P

Ben Garwood ............

Ben Logan .........................

Ben Swindler

Benton......... $\mathrm{R}$

Benton

Benton Duke...................

Bernard ....................

Bernardino Wiley 7 th ......-Cox

Bernardino Wiley 24th........_- M

Bernardino Wiley 2ith.........Rose

Bernardino Wiley $28 \mathrm{th} . . . .-$ Cox

Bernardino Wiley 30th ............ M

Bernardino Wiley 31st.........-M

Bernardo.................. $R$

12th Berthold ................

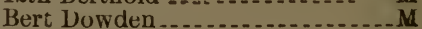

Bertram

Bertr.m 9th

Bertram 10th.......

Bertram 11th............. R R

Beulah Duke 10th

B. F. Butler .............. - Rolla

Big Ben.....................

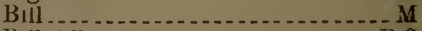

Bill Allen..........

Bill Baldwin...........

Bill Bates ...............

Bill Bulluck...............

Bill Dow ................

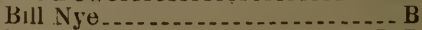

Bill Rawlings

Bill Kiner of Evergreen Hill... . Cox

Bill Tate .............

Billy $\ldots . . \ldots, \ldots$

Billy

Billy

Billy

Billy $2 \mathrm{~d} . \ldots . . .6$.

Billy Barstow ..... L R

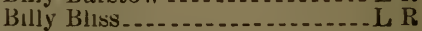

Billy Buy ..................

Billy Breckenridge

Billy Cambridge......... 
68429

68430

68431

68+3:

$684: 33$

68434

68435

68436

68437

68438

68439

$68+40$

68441

68442

$68+43$

$6: 3444$

68445

68446

68447

68448

68449

68450

68451

$6 \triangleleft 452$

$6 \triangleleft 453$

68454

68456

63457

68458

68459

$68+61$

68462

$68+63$

$6 \times 464$

65465

68467

$684 \pi 0$

68471

$6847 \%$

68473

$6 \triangleleft 474$

$684 i 6$

$68 \pm 77$

$6 \diamond 480$

$6 \triangleleft 4>1$

68483

68484

68485

$6 \varangle \pm \varangle 6$

68487

68488

$68+59$

$6 \triangle 440$

68491

$6849: 2$

$68+43$

68494

$68+45$

65496

$6 \triangleleft 497$

$68+9 y$

6ธวับ0

6sout2

6ธ5บ3

6S:Sut

68: 46

6rour

68.308

68509

68510
Billy Dixon ................. $\mathrm{M}$

Billy Edgewood ............... L R

Billy Gray ....................... Ky I

Billy Marble.................. R

Billy Patterson................L R

Billy Ray ............... - L R

Billy Smith $2 \mathrm{~d} . . . . . . . . . . . . . . \mathrm{L}, \mathrm{K}$

Billy Smith 3d................. K

Billy The Kid...............

Birdie Woodson's Duke...... Ky I

Birnie Oxford ........... Ky I

Bismarck .................. - cox

Bismarck ................... Ky I

Bismarck.................... $-\mathrm{D}$

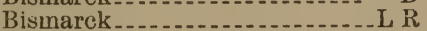

Bisniarek....................... M

Bismarck ..................

Bismarck................... D

Bismarek................... - M

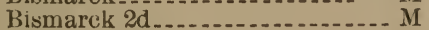

Bismarck 2d ..................... P S

Bismarck Duke..................

Blaine............................

Blaine ................ Cox

Blaine..................................

Blaine............................

Blaine................ D

Blaine.................. $\mathrm{R} R$

Blaine...................... R

Blaine........................ L R

Blanche's Edmond........... - $\mathrm{K} \mathrm{K}$

Blanche's Oxford.............. R

Blanco ................... $-L R$

Blanton-................... $R$

Blazer -..... R

Bluckey ...................

Bloomer..........................

Blossom Boy ............

Blossom's Duke........... - L R

Blossom's Mariner .......... - P S

Blucher........................... T

Blue Island Duke.........

Bluemont Wiley 5th............

Biythedale Champion 15th.. - L R Blythedale Champion 16th.. - L R Blythedale Earnesty 3d......... R R

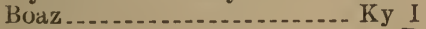

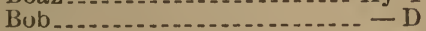

Bub........... M

Bub_.................... D

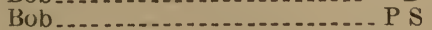

Bob .................... Rolla

Bub Burdette ........... Cux

Bub Cortland ........ Cox

Bob Ingersoll....................

Bob Ingersoll $2 \mathrm{~d}$

Bob Jacks....................... M

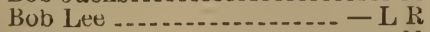

Bub U Link................. - M

Bub Smith.........................

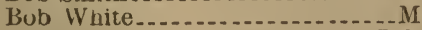

Bohemian.........

Buling ....................

Bolivar .................. Cox

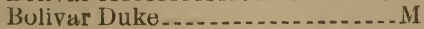

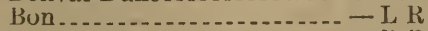

Bunanza...............

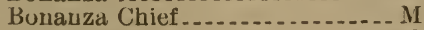

Bunaparte............ P S

Bonito................... Rollá
68511 Bonny Boy ...............

68512 Bonny Lad....................

68513 Bonnie's Pet................ Ky I

68515 Boomer ......................

$6 \times 516$ Booth ..............

68517 Boss of Sugar River ............. P S

68518 Boston Boy ..................

68519 Boston Duke..........

68520 Boston Duke 2d ............ - T

68521 Bothwell ......................... Ky I

68522 Boulder Boy ............... - L R

68523 Bonncer................... $\mathrm{R} R$

68524 Bourbon

68525 Bourbon Duke............ - D

68529 Bourbon Duke of Trout Farm - D

68531 Bourbon Spot.............. - R R

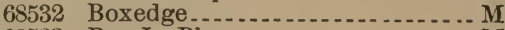

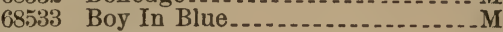

68534 Bozeman

68536 Bracelet's Lad............. R R

68537 Bradford ..................

68539 Branch Hall ............... - R R

68541 Bravo ..................

68542 Breastplate .................. - M

68543 Breastplate of Clay's Grove... -

68544 Bret Harte ..................

68545 Brett Champion...........

68546 Brick Pomeroy ............... - C

68547 Brick Pomeroy ............

68548 Bride's Duke of Lancaster.... Rose

$685+9$ Bridegroom of Riverdale.......Rose

68550 Brigham ..................... R

68551 Bright Boy ................ Cox

68552 Bright Eye

68554 Brighton ....................

68556 Brink ........... Cox

68557 British King........................

68559 Broady ..........

68560 Brokenhorn

68561 Brokenleg................- Cox

68562 Brooklyn

68563 Brownell ........................

68565 Bruce........................

68566 Bruce Charmer........

65567 Bruno ............................. Cox

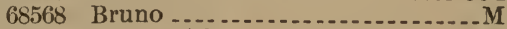

68569 Brutus 4th ............... R

68570 Brutus .............

68571 Buchanan

68572 Buckeje Boy .....................

685\%3 Buckeye Duke................. R

685 it Budd Doble.............. R R

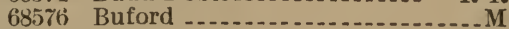

68578 Bulver .................... R

$6 \$ 579$ Bully Boy ........................

68580 Bunker's Ben...............

68581 Bunty ............................

68582 Burglar.......................

68583 Burks ............ R

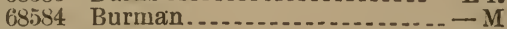

68585 Burt .................... Daisy

68586 Burt Mack .................. - I I

65587 Burton............................

68588 Butler......................................

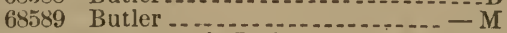

68590 Buttercup's Lad............. Daisy

65591 Buttertiy .............. - Rolla

68.593 Butterfly ................... - D

68593 Butterfly's Duke............ - M

68594 Butterfly Prince.......... Rolla 


\begin{tabular}{|c|c|}
\hline $\begin{array}{l}68595 \\
68599\end{array}$ & $\begin{array}{l}\text { Byas Beasiy } \\
\text { Cadiz Comer }\end{array}$ \\
\hline 8600 & Cadiz Dexter \\
\hline 601 & Cadiz Major \\
\hline 602 & Cadiz Porter \\
\hline 3608 & Cambria Duk \\
\hline 609 & Cambria Duk \\
\hline 610 & Cambrian .. \\
\hline 311 & Cambridge \\
\hline 612 & Cambridge D \\
\hline 622 & Cambridge $R$ \\
\hline 623 & Canefield Johy \\
\hline 624 & Canefield Prin \\
\hline 625 & Cannon \\
\hline 3628 & Cantor ....... \\
\hline & Captain.. \\
\hline 631 & Captain \\
\hline & Capt. Bill \\
\hline 33 & Capt. Booth \\
\hline 634 & Capt. Clay - - \\
\hline 335 & Capt. Clyde. \\
\hline 636 & $\begin{array}{l}\text { Capt. Cook -. } \\
\text { Capt. Couch. }\end{array}$ \\
\hline 638 & Capt. Cressb \\
\hline & Capt. Deupre \\
\hline 641 & Capt. Fisher \\
\hline 643 & Capt. Gram - \\
\hline 644 & $\begin{array}{l}\text { Capt. Jinks. } \\
\text { Capt. Joe... }\end{array}$ \\
\hline 645 & Capt. Kidd. \\
\hline 40 & Capt. Kidd- \\
\hline 647 & Capt. Orgain \\
\hline 48 & Capt. Oriole. \\
\hline 352 & Capt. Sawyer \\
\hline 353 & Capt. Shaf toe \\
\hline 655 & $\begin{array}{l}\text { Capt. Thorne. } \\
\text { Capulet }\end{array}$ \\
\hline 57 & $\begin{array}{l}\text { Capulet } \\
\text { Cardinal } 2 \mathrm{~d} . .\end{array}$ \\
\hline 58 & Carleton ..... \\
\hline 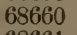 & Carlyle.... \\
\hline 61 & Caroline's Prir \\
\hline 3 & Carroll \\
\hline 65 & $\begin{array}{l}\text { Carter Harriso } \\
\text { Cash Boy } . . . . .\end{array}$ \\
\hline 367 & $\begin{array}{l}\text { Cash Boy }-. . . . . \\
\text { Cash Boy } . . . . . .\end{array}$ \\
\hline 368 & Cash Boy -- \\
\hline 69 & Cashier \\
\hline 0 & Casquet's Oxf \\
\hline & Catalpa-Duke \\
\hline 34 & Cato....... \\
\hline & Cavour \\
\hline 7 & Cayuga Lad 2 \\
\hline & $\begin{array}{l}\text { Cayuga Prince } \\
\text { C. B. Palmer's }\end{array}$ \\
\hline & $\begin{array}{l}\text { C. B. Palmer's } \\
\text { Cedar Prince. }\end{array}$ \\
\hline 82 & Cedar Prince $2 \mathrm{~d}$ \\
\hline & Cleocia's Duke. \\
\hline & Chaco ..... \\
\hline & Challenger \\
\hline & $\begin{array}{l}\text { Challenger of Ore } \\
\text { Champlon }\end{array}$ \\
\hline & $\begin{array}{l}\text { Champlon.- } \\
\text { Champion.. }\end{array}$ \\
\hline & $\begin{array}{l}\text { Champlon.- } \\
\text { Champion.- }\end{array}$ \\
\hline & Champion.- \\
\hline & Champion.- \\
\hline & $\begin{array}{l}\text { Chancellor } \\
\text { Charles Ha }\end{array}$ \\
\hline & Charlie Daley \\
\hline
\end{tabular}

68701

69702

$68 \% 03$

68704

68708

68709

68710

$68 \pi 12$

68713

68715

68716

68717

68715

68719

68720

68721

$68 \% 22$

68723

68724

68725

$6872 \%$

$68 \pi 28$

68729

68730

68732

68733

68734

68735

68736

$6873 \pi$

68738

65742

68744

68746

68747

68748

68750

68751

68752

68753

65754

68755

68756

68757

68758

68759

68760

68761

68762

68763

68764

68765

68766

68767

68768

68769

68770

68771

68772

68773

68774

$687 \% 5$

$687 \% 6$

$687 \% 8$

63779

68780

68781

68782

68784

68785
Charlie Humphrey ............. Ky I

Charlie Ray ................. D

Charley Vaughn

Charlotte's 2d Prince.........

Chautauqua .............. Cox

Chautauqua Duke........ $\mathrm{L} R$

Cheeny -..............

Cherry's Moro........

Cherry Oxford...... $R$

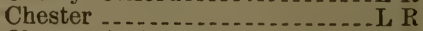

Chester A. Arthur.

Chestnut Ridge Duke.......... L R

Chet ..........

Chetopa 2d .

Chief

Chief Joseph .......

Chief Joseph...

Chief K. C. B.,

Chieftain .......... Cox

Chieftain

Chief of Woodlawn

Chilton's Best Bull........ Rolla

Chipillo ................... R

Christmas Day

Chromo ........ P S

Chub .

Chubb

Chumley $2 \mathrm{~d}$

Cibola

Cicero

Cicero

Clarence

Clarence Duke...........................

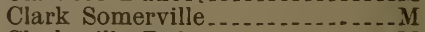

Clarksville Duke....................

Clarno Oxford .............. Cox

Claydean

Claysville Lad........ $\mathrm{R}$

Clear Water 4th

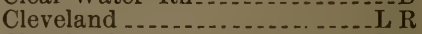

Cleveland

Cleveland

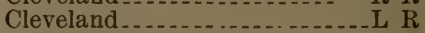

Cleveland ...... P S

Cleveland

Cleveland ......... P S

Cleveland

Cleveland ....... R

Cleveland

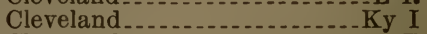

Cleveland

Cleveland .........

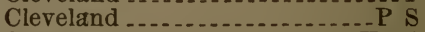

Cleveland...........

Cleveland..................

Cleveland...........

Cleveland ......

Cleveland

Cleveland .......

Cleveland

Cleveland

Cleveland

Cleveland

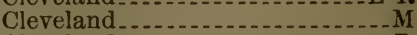

Cleveland

Cleveland

Cleveland ........ 


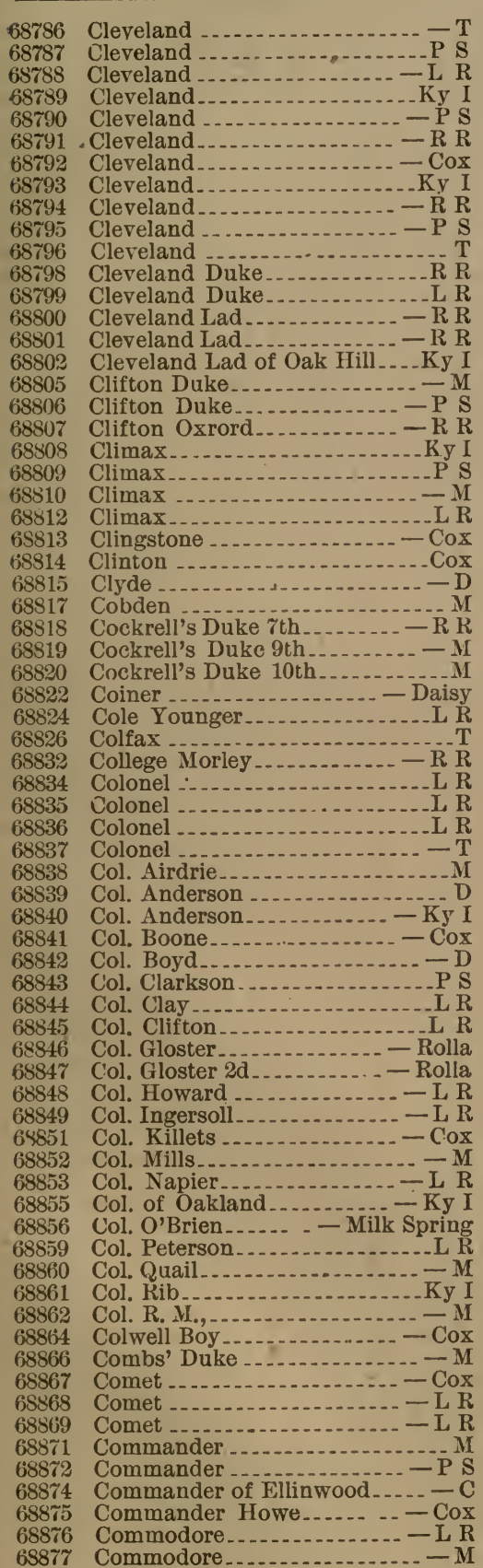

68880 Conewango Lad 9th .......... - L R

68882 Conqueror $2 \mathrm{~d} \ldots . . . . \ldots . . . . . . .$.

68886 Constance Lad

68888 Constitution

68890 Cordelia Duke ...... Cox

65893 Cornwallis.................... Cox

68894 Corporal ................

68895 Cortez ...........................

68896 Cossack ........................... M

68897 Count Bassang.......... Cox

68900 Count of Minglewood....... - D

68901 Count of Rousillon..........

68902 Count Teillen .............

68903 Cowan Duke............. - R R

68905 Crescent...................

68906 Creston Duke....... - Long Horn

68907 Crimson Bell........... R R

68909 Crispin ..................... R

68911 Cruickshank Sharon ........ - L R

68913 Curley ................... Ky I

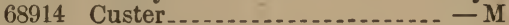

68915 Cypress Baron.................

68916 Cypress Duke of Anoka ...... - M

68919 Dacon

68920 Daily's Oxford ............. M

68922 Daisia Jim .............. R

689:4 Daisy Boy

68925 Daisy Bull ................ Cox

6892 i Daisy Duke............

68928 Daisy Duke ................. Daisy

68930 Daisy Duke............... L R

68931 Daisy Duke.................

68932 Daisy Duke..................

68934 Daisy's Duke 2d.......... L R

68935 Daisy's Duke of Sharon....... - T

68936 Daisy Lad............... P S

68937 Daisy Lad......................

68938 Daisy Thornton's 2d Duke.........

68940 Dakota Duke ............... L R

68941 Dakota Duke.................... Ky I

68942 Dakota Prince............. R R

68943 Dandy

68944 Dandy Duke .......... $\mathrm{R}$

68945 Dandy Jim....................

69946 Dandy Jim ............... Cox

68947 Dandy of Woodlawn............. Ky I

68948 Dane

68949 Dan Hopper..............

68950 Dan Lee

68951 Dan Manning ................. Ky I

68952 Dan Manning .......... Daisy

68953 Dan Rice ............... L R

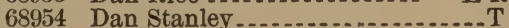

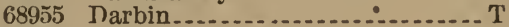

68956 D'Arcy

68957 Darius......................... R

68958 Darlington ................... Ky 1

68959 Darlington ...................

68960 Dartmouth $2 \mathrm{~d}$

68961 Dash ...................... Cox

68962 Dassel ..................... R R

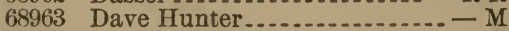

68964 David Crocket ............. M

68965 David Dows

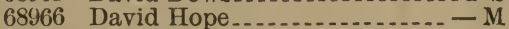

68967 Davis Climax...B

68968 Daybreak......................

68969 Deacon ..................... Cox

68970 Deacon Jones............

68971 Deacon Pogram.......... Daisy 
$689 \pi 2$

68973

68974

68975

68976

$6897 \%$

68978

68979

68980

68981

68982

68983

68986

68957

68988

68989

68990

68991

68992

68993

68996

68949

69000

69001

69002

69003

69004

69005

69006

$6900 \pi$

69008

69010

69012

69013

69014

69015

69016

69017

69019

69020

6902\%

69024

69025

69026

69027

69028

69029

69030

69031

69033

$6903 \overline{5}$

69037

69038

69039

63040

$690+1$

69042

69043

69044

69045

$690+6$

69047

$690 \mathrm{ts}$

69049

69050

69051

69053

69055

69056

69057
Deadwood _......... - Long Horn

Decatur Chief.............

Decoration........ Milk Spring

Ded Duke.......... P

Deer Creek Lad

Defiance..........................

Delaware Chief

Delegate

Delmar........

Denmark ....................... M

Dn's Saunders of Wahoo V'y-Cox

Darinda Duke................... M

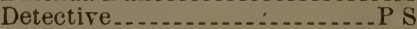

Dexter...............

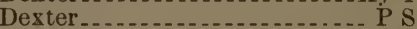

Diamond....................

Diamond Duke ...............

Diamond Joe.......................

Dick........... D

Dick

Dick Hardy

Dick Leslie................... S

Dick of Russell

Dick Shelby

Dick Taylor ...........

Dick Taylor ........... $\mathrm{R} R$

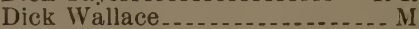

Dictator ................. II

Dictator

Dilke..................

Dimple's Duke.................... S

Director

Dock

Dock

Doc Kenick.........

Doctor Caperton

Doctor Emerson

Doctor Favorite

Doctor Leslie.............

Dr. of Maine Valley

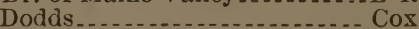

Dolly's Duke..... Woods

D Oney $3 \mathrm{~d}$

Don Pedro.......... Ky I

DonPedro ........... $\mathrm{R}$

Don ald .....................

Don ald Duke of Marble Cliff _. - D

Donald Duke of Marble Cliff 2d-D

Donald Duke of Marble Cliff $3 d-D$

Don Carlos ................. $\mathrm{R} R$

Don John................. R R

Don Juan $2 \mathrm{~d}$

Don Pedro............ R R

Don Pedro................ Ky I

Double Airdrie.............

Druble Duke.............. - P S

Double Grand Airdrie........ M

Dougal Thomson...................

Douglas ................ P S

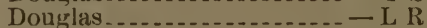

Downey Lad............... D

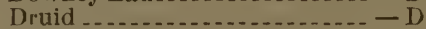

D. S. C., Dares

Duchess Duke.......

Uuchess Loudon Duke..... - Cox

Dude.......................... M

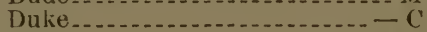

Duke-........

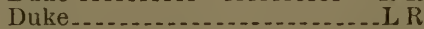

Duke.

T
69058

69059

69060

$6906 \dot{1}$

$6906 \pm$

69065

69066

$6906 \pi$

$6906 \mathrm{~s}$

69069

69070

69071

69072

69073

69074

69075

69076

69077

69078

69079

69080

69081

69083

69054

69085

69086

69089

69090

6\%991

69092

69093

69094

69096

69098

69100

69101

69102

69103

69104

69105

69106

69112

69115

69116

69117

69119

69120

69121

69122

69123

69124

69125

69127

69128

69130

69131

69132

69133

69134

69135

69136

69138

69139

69140

69141

69142

69143

69144

69145

69146
Duke

7th Duke....................

Duke Acomb.... L R

Duke of Airdrie

Duke Albert of Mud Creek .. _- L R

Duke of Albion.......... $\mathrm{R} \mathrm{R}$

Duke of Alby

6th Duke of Alderly........

7 th Duke of Alderly .......- $-R$

8th Duke of Alderly

10th Duke of Alderly......- $-R$

11 th Duke of Alderly .......- $\mathrm{R} \mathrm{R}$

Duke of Alma .............. $R$

3d Duke of Almont......... $\mathrm{R}$

Duke of Arab................

Duke of Argyll...........

Duke of Arlington ....................

Duke of Arndahl .............. Cox

3d Duke of Astoria ........ - R R

5 th Duke of Astoria....... $R$

6th Duke of Astoria........

Sth Duke of Astoria........ $\mathrm{P} R$

Duke of Avoin ............ $-\mathrm{P} S$

Duke of Avondale ......... P S

$2 \mathrm{~d}$ Duke of Avondale ........

3i Duke of Avondale ........ - PS

Iuke of Belle Vernon........ - M

Duke of Benton............... Ky I

Duke of Rerlin ............

Duke of Bertram

Duke of Big Reed...........

Duke of Blooming Grove....- R R

Duke of Bandon..........

2d Duke of Blue springs......

Duke of Brenot_................ L R

$3 d$ Duke of Bridgewater ....- $L R$

Duke of Brookside........ - $\mathrm{Ky}$ I

Duke of Bunker Hill........... Ky I

Duke of Burr Oak............ P S

Duke of Bushnell ........ Cox

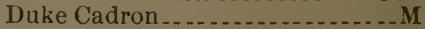

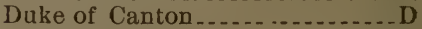

Duke of Caro ................

Duke of Carroll.............. $\mathrm{R}$

Duke of Carroll .....................

Duke of Cedar Home........................

2d Duke of Cedarview ....- - Daisy

Duke of Champion...........

2d Duke of Chartiers...... $\mathrm{L} R$

Duke Cbief ..............

Dukr of Clark.........

Duke of Clements............. T

Duke Cleveland ...................

Duke of Clifton........................

6th Duke of Clinton.......- Cox

2d Duke of Clorer Meadow - L R

Duke of Clyde........... - L R

Duke of Clyde............ Cox

2d Duke of Clyde......... - L R

3d Duke of Clyde...............................

Duke of Climer.............. R

Duke of Compton........ - L R

Duke of Conewango

Duke Constant 5ch ........ - L R

3d Duke of Coonewan-Milk Spring

Duke of Cornwall $2 d$...... - Rolla

Duke of Corsondale.......... . D

Duke of Cowley ........... $R$

Duke of Crawford.................

2d Duke of Crawford..... R R 
69147

69150

69154

69155

69156

6915 i

69158

69159

69160

69164

69165

69166

69167

69170

69172

69174

69175

69176

69177

69178

69179

69181

69182

69183

69184

69185

69186

69189

69191

69192

69193

69194

69195

69196

69197

69198

69199

69200

69201

6920:2

69203

69204

69205

69206

69208

69209

69210

69212

69213

69216

69217

69218

$69: 219$

69220

69221

$692: 32$

69223

69224

69225

$69: 226$

69227

69228

692:29

69230

69231

69233

69234

69235

69236

69237
Duke Cymodice Duke of Darby Duke of Deland............ P S Duke of Denver $2 \mathrm{~d} \ldots \ldots . . . .-\mathrm{Cox}$ Duke De Sac ............. - Cox Duke Desdemonia....... Daisy Duke of Dodge........... Duke Donald .............. Daisy Duke of Doylestown Duke of Durham......... - Ky I Duke of Eagle Point........ -R R Duke of East Branch........ 3d Duke of Eastwood........ Duke of Edgerton 3d Duke Elkhorn.......... - Rolla Duke of Enterprise........ R Duke of Enterprise 2d...... $-\mathrm{C}$

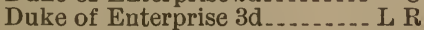
Duke of Enterprise 4th....... Ky I Duke of Evans Farm....... $\mathrm{R}$ R Duke of Fairfield............. Ky I 2d Duke of Fairview.......... R R 3d Duke of Fairview ......... R R Duke of Farmington........ Duke Favorite ................ D Duke of Fawn River............ 12th Duke of Fayette........ 15 th Duke of Fayette ........ 17th Duke of Fayette ........ - T 2d Duke of Fern Leaf ...... - L R Duke of Fletcher....... Rolla Duke of Florence........ Ky I 2d Duke of Florence....... 3d Duke of Florence.......... Ky I Duke of Forest Hill 17th Duke of Forest Hill...... - M 18th Duke of Forest Hill..... - M 2d Duke of Franklin........ - D 3d Duke of Franklin ......... - D

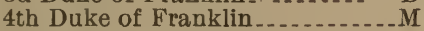
5th Duke of Franklin........ D Duke of French Creek...... L R Duke of Ganges................ Ky I 20th Duke of Glen Flora.... $\mathrm{R} R$ Duke of Goodness.............. Ky I Duke of Goodness $2 \mathrm{~d} . . . . . .-\mathrm{R} R$ 82d Duke of Goodness...... R R 12th Duke of Granville....... - P S 21st Duke of Granville..... $\mathrm{R} R$ 2d Duke of Greenwood.....-R R 3d Duke of Greenwood..... R R Duke of Grove Farm 7th Duke of Gun Plains...... Cox Duke of Hamilton .......... P S 3d Duke of Hamilton ......... Ky I 4th Duke of. Hamilton.......... Ky I 5 th Duke of Hamilton 6th Duke of Hamilton ....... Ky I Duke of Hanover 2d............ Ky I Duke of Harper............. - M Duke of Hartsville................. Duke of Hastings ........ Cox 6th Duke of Hazel Dell........ D 7th Duke of Hazel Dell ... - Daisy 3d Duke of Hazel Grove.......... D 3d Duke of Hempfield......... C 4th Juke of Hempfield.....5 th Duke of Hempfield. 6 th Duke of Hempfield Duke of Hershaville....... R R
69238

69239

69240

69241

69242

69243

69245

69246

69247

69248

69249

69250

69251

69252

69253

69254

69256

69257

69258

69259

69261

69262

69263

69264

69265

69266

6926.9

69269

69270

$692 \% 1$

$692 \% 2$

69273

69274

69275

69278

69279

69280

69281

69282

69283

69284

69285

69:386

69287

69288

69289

69291

69292

69293

69294

69295

69296

69297

69298

69299

69300

69302

69304

69305

6930 F

60507

69308

69309

69310

69311

69314

69315

69316

69317

69318
Duke of Heyworth...........-D

Duke of Hickory

Duke of Hickory Grove..... - Cox

Duke of Hickory Grove......

20th Duke of Highland.... - R R Duke of Highland Park ..... $-\mathrm{L}$ R 2d Duke of Hillside......... - Cox 3d Duke of Hillside ........ $-\mathrm{R} R$ Duke of Hinckley ....... $\mathrm{L}$ R 14th Duke of Homestead..... - D 15th Duke of Homestead...... D Duke of Honey Creek........ - D 6th Duke of Hoosick........... Cox Duke of Hope............... Cox Duke of Howard............. - D Duke of Huron ............... Cox Duke of Ionia $3 \mathrm{~d}$ Duke of Ionia $4 \mathrm{th}$ Duke of Ionia $5 \mathrm{th}$ Duke of Iowa................ 2d Duke of Jenevive........ Duke Jesse................... Cox Duke John of Durham.... - Ky I Duke of Keno Valley .............M Duke of Kent ............. L R Duke of Kent............ Ky I 3d Duke of Kent.... - Long Horn 4th Duke of Kent.... - Long Horn 5th Duke of Kent..... - Long Horn 6th Duke of Kent....- Long Horn 7th Duke of Kent.... - Long Horn 8th Duke of Kent.... - Long Horn 9th Duke of Kent.... - Long Horn Duke of Kiowa Duke of La Belle ......... D Duke of Labrador...............Cox 3d Duke of La Crosse 5th Duke of La Crosse....... - PS 6th Duke of La Crosse........ - D Duke of La Crosse Valley.... $\mathrm{PS}$ Duke of Lakeside........... - Cox 3d Duke of Lapeer............. R 4th Duke of Lapeer............ R 5th Duke of Lapeer........... R Duke of Laramie $2 \mathrm{~d} . . . . .-$ Woods Duke of Lauderdale.......... - M Duke of Lawrence......... Duke of Lawrence........ R R Duke of Leatherwood.... - L R 2d Duke of Leatherwood..... - D 3d Duke of Leatherwood .... - D 4th Duke of Leatherwood...-L R 3d Duke of Lebanon....... - Ky I 5 th Duke of Leesburg .... - L R 6th Duke of Leesburg ....... - M 8th Duke of Leesburg I) 9th Duke of Liberty .......... 10th Duke of Liberty 11th Duke of Liberty 5 th Duke of Lima 3d Duke of Lincoln........... - M 4th Duke of Lincoln .......... 5th Duke of Lincoln........... - D Duke of Lindsey ........... $\mathrm{R} R$ Duke of Linwood 4 th....... $-\mathrm{R} R$ Duke of Locust Lane Sth..... - D Duke of Locust Lane 9th..... - D Duke of Locust Lane 10th....... M Duke of Locust Lane 11th ...... D 
69319 Duke of Locnst Lane 12th ..... - D

69320 Duke of Locust Point ........ - M 69321 2d Duke of Long Branch...- Ky I 69322 Duke of Longview........... - P S 69323 Duke of Lost Creek.......... - D 69324 Duke of Lyons............... Cox 69326 Duke of Macon.................. R 69328 4th Duke of Madison ........ - R R 693295 th Duke of Madison ......... - R R 69330 Duke of Madisonville...... - - Cox 69332 Duke of Maple Home....... M 69333 Duke of Maple Shade ...... - L R 69335 2d Duke of Maplewood........ T 69336 4th Duke of Maplewood....... R R 69337 Sth Duke of Maplewood ........R R 69338 4th Duke of Marble Cliff ......-D 69339 Duke of Marengo ............ $-\mathrm{D}$ 69340 Duke of Marion

69341 Duke of Marion.............. Beauty 69342 69343

69344

69346

69347

69355

69356

69357

69358

69359

69360

69361

69362

69363

69364

69366

69369

$693 \% 0$

693\%2

69373

69379

69381

69382

69383

69384

69385

69389

69390

69391

69392

69393

69394

69395

69396

69398

69400

69401

69402

69403

69404

69405

69406

69407

69408

69409

69411

69412

69413

69415

69416

69417
Duke of Marlborough

Duke of Marsh Head....... II

Duke of Maumee.......... $\mathrm{R} R$

3d Duke of Mayslick....... - L R

Duke of Mentor 2d..................

Duke of Mentar 3d......... M

Duke of Meredith

Duke of Sill Creek......... $\mathrm{R} \mathrm{R}$

Duke Modoc...............

Duke of Montgomery $2 \mathrm{~d} . . . . . . . . \mathrm{C}$

Duke of Morton ................

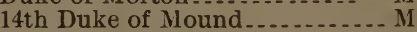

Duke of Mt. Sterling .

Duke of Neenah

Duke of Nora........... Ky I 2d Duke of North Plains...- Ky I

Duke of Oak Dale

3d Duke of Oak Dale........... - D

Duke of Olmsted ................. P S

3d Duke of Orchard Hill ........ Cox

4th Duke of Orehard Hill .... - P S

Duke of Osage .............. M

Duke of Ottawa...........

Duke of Outlet Farm........... B

Duke of Paw Paw .............. Cox

Duke of Peach Hill

2d Duke of Peach Hill.......... P S

Duke of Pearl ................. Cox

Duke of Penn Line............ Ky I

4th Duke of Perry

Duke of Pine Valley

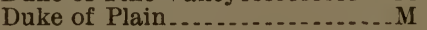

2d Duke of Platte.........

3d Duke of Platte...........

4th Duke of Platte......... P S

Duke of Plumwood............

Duke of Plumwood $2 \mathrm{~d} . . . . . . .-\mathrm{D}$

Duke of Plumwood $3 \mathrm{~d} . . . . . .-\mathrm{C}$

Duke of Poplar Grove....... $R$

Duke of Portland ............ PS

Duke of Prairie Heart......- $-L R$

Duke of Prospect.........-Daisy

Duke of Reading $2 \mathrm{~d} . . . . .-\mathrm{Cox}$

Duke of Redbank .......... L R

$2 d$ Duke of Redbank........ - L R

Duke Renick 2d............ $R$

Duke of Republic........... $\mathrm{L} R$

Duke of Rice................ $\mathrm{R} R$
Duke Mitchell ............. - Rolla

Duke of Perry ................ L R
69418

69419

69420

69421

69422

69423

69424

69425

69426

$6942 \tau$

69429

69430

69431

$69+32$

69433

69434

69435

69436

69437

69438

69439

69440

69441

69442

69443

69444

$6944 \tilde{~}$

69446

69417

69448

69449

69450

69451

69452

69453

69456

69457

69458

69460

69461

69462

69466

69467

69468

69469

69470

69471

69478

69480

69481

69483

69484

69485

69486

69487

69488

69491

69492

69493

69494

69495

69497

69498

69499

69500

69501

69503

69504

69505

69506
Duke of Richfield ..........- $-\mathrm{R} \mathrm{H}$

Duke of Richland...........-

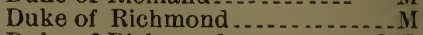

Duke of Richmond

16th Duke of Ridgevale.....- $L R$

18th Duke of Ridgevale..... - L R

$22 d$ Duke of Ridgevale.....- $\mathrm{L} R$

23d Duke of Ridgevale.......... Cox

Duke of Riley .............. R R

14th Duke of Rio.............

Duke of Roscoe..................

Duke of Rosedale............

Duke of Running Creek ...... - P S

Duke of Rush

Duke of St. Croix................

$2 d$ Duke of St. Croix.......... L R

3d Duke of St. Croix ........ R 4th Duke of St. Croix ........ R Duke of St. Paul............. Duke of Salem............... $R$ Duke of Salem

Duke of Sarpy

Duke of Scio................... Ky I

$3 d$ Duke of Scipio

Duke of Seacome............

Duke of Seward..................

Duke of Sharon ............... M

Duke of Sharon $2 \mathrm{~d}$

4th Duke of Sharon....... Daisy

Duke of Shelby .......... R R

Duke of Sinelby

5th Duke of Sheldon

6th Duke of Sheldon...........

ith Duke of Sheldon.........

Duke of Shenango ......... M

12th Duke of Skaneatles.... - Cox

Duke of Sparta..................

Duke of Sparta............. Cox

th Duke of Spring Branch - Daisy 8th Duke of Spring Branch - Daisy 9th Duke of Spring Branch - Daisy Duke of Spring Grove........... D Duke of Spring Hill......... Daisy Duke of Spring Hill......... - D 2d Duke of Spring Hill....... Cos Duke of Spring Valley....... - R R Duke of Springwood ........... L K Duke of Stark............. 28th Duke of Stock Place... - L R 34th Duke of Stock Place - Beauty Duke of Sugar Creek ........ - Cox 2d Duke of Sugar Creek.... - Cox Duke of Sumner............ P S Duke of Sunbeam.............. Ky I Duke of Sunny Side.......... Duke of Susquehanna.......... $R$ Duke of Sutherland $2 \mathrm{~d}$ Duke of Sutherland 5th.....- $\mathrm{R} R$ Duke of Sylvester.............. Duke of The Heights.......- Rolla Duke of The Highlands...... $-M$ Duke of The Valley $2 \mathrm{~d} . . . . . . . . . \mathrm{P} \mathrm{S}$ Duke of Thornapple........ - Cox Duke of Thorndale ........... Ky I Duke of Tingle Star......... Cox Duke of Tipton.................. $R$ 2d Duke of Trenton. ............. Ky I Duke of Trojan Farm ........ Cox 18th Duke of Trout Run....... II Duke of Twolick........... $\mathrm{R} R$ 
69508

69509

69510

69511

69512

69513

69514

69515

69519

69520

69522

69523

69525

69526

69527

69528

69529

69530

69532

69533

69534

$6953 \tilde{5}$

69536

69537

69540

69541

69542

69543

69544

69545

69546

69547

69548

69549

69551

69552

69553

69554

69555

69556

69557

69558

69559

69560

69561

69563

69563

69564

69565

69566

69567

69568

69569

69571

69576

69577

69580

69583

69585

69586

69587

69588

69589

69591

69592

69595

69597

69598

69599

69601 5th Duke of Underley

Rose

Duke of Vermillion.......... - Cox

6th Duke of Vermillion .... - Ky I

Duke of Vernon ............. - M

Duke of Verona..........

2d Duke of Victor........ $\mathrm{R} R$

3d Duke of Victor........... - R R

29th Duke of Victor.......... Daisy

Duke of Wabash Avenue.......- D

Duke of Walnut Bend.......... R R

$2 d$ Duke of Walnut Shade......R R

Duke of Walnut Wood... - Daisy

Duke of Wano 3d ................

Duke of Warrendale.........

Duke of Warwick............. Ky I

Duke of Wayne ...........-Daisy

Duke of Wellington........ - L R

Duke of Wellington........- $R$

D. of Westmorel'd County Home-C

$2 \mathrm{~d}$ Duke of Weston..............

5th Duke of West View ......... R

Duke of Wetona $2 d$.............. Cox

Duke of Wheatland.......... Ky I

2d Duke of Whitecloud......- R R

6th Duke of Woodhill ....... - L R

Duke of Woodlawn......... $-M$

Duke Worth ............. Cox

Duke of Wuirdenberg....... - P S

9th Duke of Wyandot.......- R R

Duke of York ................. Ky I

Duke of York .............. - Cox

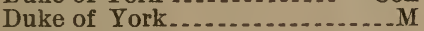

Duke of York .............. R

Duke of York

11th Duke of Yule Grove.... - Cox

Dumont.................. R R

Dundas............

Dunglen Lad

Dunglenna's Plumwood....- $\mathrm{R} R$

Dunmore Duke 2d................

Dunmorix.................... M

Dunreath ...................... S

Du Page Chief......... P S

Duquesne ....................

Durachen Duke...........

Durachen Duke $2 \mathrm{~d} . . . . . . . . .$.

Durham Duke................ - D

Duroc..................... $R$

Duroc ............ P S

Duroc of Oak Hill ..................

Dynamite............................ S

Eagle................. R

Eagle Dale Booth.......

Earl ..................... Cox

Earl Boyd.................... R R

Earl of Carroll $2 \mathrm{~d} . . . . . . . . . . . . . T$

2d E. Chesterf'd of Sun'y Hill-Rolla

Earl of Concord............... M

Earl of Ellisburg........... Cox

6th Earl of Endwood........ D

10th Earl of Endwood........ $\mathrm{L} R$

3d Earl of Erie.............. - P S

Earl of Fairfield.............. R R

Earl of Geauga ............. - R R

Earl of Glen Flora.......... $R$ R

Earl of Gloster............. - Cox

47th Earl of Greenbush......- L R

48th Earl of Greenbush.....- - L R

49th Earl of Greenbush.....- - L R

6th Earl Hanover..........-Beanty
69606

69608

69609

69611

69612

69613

69614

69616

69618

69619

69621

69622

69623

69624

69625

69626

69627

69628

69629

69632

69633

$6963 \tilde{5}$

69636

69637

69638

69640

69643

69644

69645

69647

69649

69651

69653

69655

69656

69657

69659

69660

69661

69662

69663

69664

69665

69666

69667

69668

69669

69670

69671

69672

69674

69675

69677

69679

69680

69681

69682

69683

69684

69686

69687

69688

69689

69690

69691

69692

69693

69694

69695

69696
Earl of Kiowa................. S

Earl of Lenawee.............. Ky I

Earl of Linwood ............ L R

2d Earl of Macglen.........

3d Earl of Macglen....... D

4th Earl of Macglen ............ D

Earl Manlinder ................... M

Earl of Paris ............. R R

3d Earl of Red Oak........ Cox

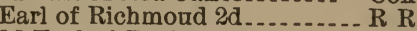

2d Earl of Rochester.............

4th Earl of Rochester.......... M

Earl Rosamond 2d.........-R R

Earl Rosamond 3d.......... - R R

Earl Rosamond 4th.......- $-\mathrm{R} R$

Earl of Rosedale ............ - D

Earl of Sharon ...............

Earl of Springbank........

Earl of Springdale $2 \mathrm{~d} . . . . . .-\mathrm{L} R$

2d Earl of Spring Valley.... - R R

2d Earl of Sussex ................ Ky I

3d Earl of The West......... - C

Earl Thornapple 8th.........- Cox

Earl of Warlaby ............ - L R

Earl of Willow Park ......... D

Early's Sharon .............. - P S

Easter Lad.......................

Easter Lad.....................

Eckert............... Ky I

Eclipse ............... _ _ Long Horn

Edbrun's Butterfly ........... - B

Edgewood's Prince.............. P S

Edwin Drood ................

Egmont of Circleville........ - C

Eighth of January........... R R

Elbert..........................

Eldon.............

Elihu................. Ky I

Eli Perkins ..................

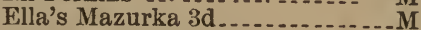

Ella's Roan Duke.......... - Ky I

Ellick ........................ D

Ellick ..................... D

Ellsworth Duke.......... R R

El Mahdi...................... $\mathrm{P}$

El Mahdi...................... I

El Mahdi............... R

El Mahdi......................

El Mahdi

Elmwood Prince....................

Emerald..........................

Emerson 2d...............

Emperor............. R R

Emperor 2d of Silverleaf.... - $\mathrm{R} R$

Emperor of Willow Park...... - D

Enchanter 2d............. - Rolla

English Duke................ - C

Enterprise 4th ............. Ky I

Ephraim .................

Erastus .........................

Escot ........................

Esquire Stinson.............

Essex Chief................. $\mathrm{L}$

Ethan Allen ................. - D

Eudora's Duke........... R R

Excelsior ........................

Excelsior.........................

Expansion Duke $2 \mathrm{~d}$

Exquisite Kirklevington........ - C

Faber...................... 
69698 Fable Duke..............

69703 Fairy Gift..................

69704 Fairy Prince $2 \mathrm{~d}$

69705 Falconer.......................

69706 Falstaff........................ Ky I

69707 Famous Phil.....................

69708 Fancy Boy ............... R

69710 Fancy's Duke.......... P S

69711 Fandango................... R

69713 Farmer Boy...................

69714 Farmer Boy........ Rolla

69715 Faro

69716 Fashion's Barrington

69717 Fashion's Barrington $2 \mathrm{~d} . . . .-\mathrm{R} R$

69718 Faustina's Duke............. - C

69720 Favorite's Wild Eyes........ - P S

69721 Fawsley Duke 2d........-Rolla

69722 Fayette Duke ........... D

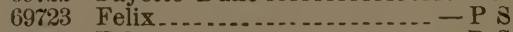

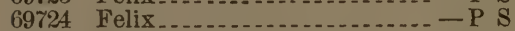

69726 Fellow Craft................. I

69727 7th Fennel D. of Lancaster..... Cox

69728 8th Fennel D. of Lancaster - - R R

69729 9th Fennel D. of Lancaster ....R R

69732 Festus ....................................

69733 Fidella's Chief................

69734 Fidella's Gem.......... R

69735 Fidget's Hillhur'st $3 \mathrm{~d} . . . . . .-$ Cox

69737 4th Fid't's Oxf'd of Sunny H'l-R R

69738 Filligree Gem 11th............. M

69740

69742

69743

69745

69746

69747

69748

$69 \pi 49$

69750

69751

69752

69754

69755

69756

69758

69759

69760

69761

69762

69763

69764

69767

69768

69769

697\%0

69771

69\%72

69774

$697 \% 6$

69777

69778

69779

69780

69782

69783

69784

69785

69786

69792

69793
69794

69797

69798

69799

69800

69801

69802

69804

69805

69806

69809

69810

69813

69814

69815

69816

69815

69819

69820

69821

69823

69825

69827

69828

69829

69830

69531

69832

69833

69834

69835

69836

69837

69838

69839

69840

69841

69342

69843

69844

69848

69849

69850

69851

69852

59853

6985

69555

69856

69857

69858

69859

69860

69861

69862

$6986 t$

69865

69866

69867

69369

69870

69871

69876

$698 \% 7$

69878

69879

69880

69881

69882

69883
Free Trade

Fresco

Gabriel......................

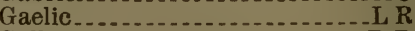

Gallant .......

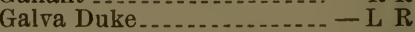

Garfield ............

Garfield..............

Garfield

Garibaldi...................... R

Garland's Duke..... Rolla

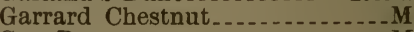

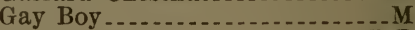

Gay Duke....... R R

Gay's Kingscote.......

Geauga Boy ....................... P S

Gem's Duke of Anoka 2d......L R

Gem Duke of Clinton..... R R

General ..................... R

General Bolivar....... R R

Gen. Bright....... D

Gen. Clay

Gen. Custer....... $R$

Gen. Flash

Gen. Fremont..............

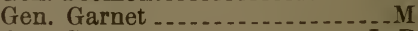

Gen. Gordon

Gen. Gordon,

Gen. Gordon

Gen. Gordon

Gen. Gordon .....

Gen. Grant ............. Ky I

Gen. Grant..... Ky I

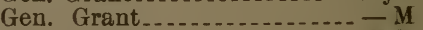

Gen. Grant........ R R

Gen. Grant.....

Gen. Grant

Gen. Grant .......

Gen, Groves

Gen. Huncock

Gen. Lee................

Gen. Lee $2 \mathrm{~d}$

Gen. Leibold .

Gen Logan ........ R R

Gen. Logan............... Cox

Gen. Logan.....................

Gen. Louan

Gen. Logan....... R R

Gen. Montcalm

Gen. Montgomery....................

Gen. Moscow ........

Gen. of Oakland..... R

Gen. Oglesby . . .

Gen. Palmer...

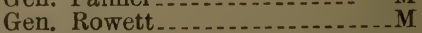

Gen. Sheridan....................

Gen. Sherman ................ Daisy

Gen. Sherman ................ S

Gen. Sherman

Gen. Weaver

Gen. Weaver

Gen. Weaver........

Geneva Prince

Geneva of Syracuse.......

Geneva Wellington........ Rose

Geneva Wild Eyes......... L R

Genera Wild Eyes........... D

Geneva Wild Eyes $3 \mathrm{~d} . . . . .-$ M

Gentry 


\begin{tabular}{|c|c|}
\hline 884 & George \\
\hline 9885 & George. \\
\hline 8886 & George \\
\hline 887 & George Evan \\
\hline 8888 & Georgiana D \\
\hline 890 & Gessler.. \\
\hline 891 & Gibralter. \\
\hline 893 & Ginger - - \\
\hline 9894 & Gipsy Barring \\
\hline 3895 & Give-Or-Take \\
\hline 896 & Gladiator .. \\
\hline & Gladstone. \\
\hline 899 & Gladstone. \\
\hline 900 & Gladstone. \\
\hline 905 & Glencoe Duke. \\
\hline 906 & Glossy Duke.. \\
\hline 907 & Gloster $2 \mathrm{~d}$.. \\
\hline 908 & Gloster Boy $\mathrm{J}$ \\
\hline 909 & Gloster's Duke \\
\hline 910 & Glyndon \\
\hline 9912 & Godolphin . \\
\hline 69913 & Golddrop ... \\
\hline 914 & Golddust.... \\
\hline 915 & Golddust. \\
\hline 320 & Golden King ? \\
\hline 921 & Goldfinder... \\
\hline 22 & Goldfinder... \\
\hline 923 & Goldfinder .. \\
\hline & Good Friday \\
\hline 926 & Good Luck.- \\
\hline עזt & Governor.. \\
\hline 928 & Governor... \\
\hline 929 & Governor .. \\
\hline 930 & Governor $2 d$. \\
\hline 31 & Governor 2d. \\
\hline 32 & Gov. Cleveland \\
\hline 933 & Gov. Cleveland \\
\hline 934 & Gov. Glick .. \\
\hline 35 & Gov. John M \\
\hline 36 & Gov. Marmaduke. \\
\hline 37 & Gov. Richard. \\
\hline 88 & Gov. St. John. \\
\hline 39 & Gov. Sherman. \\
\hline 40 & Gov. Sherman \\
\hline 41 & Gov. Sheron... \\
\hline 942 & Gov. Todd... \\
\hline 943 & Gov. Thaver $3 \mathrm{~d}$. \\
\hline & Gracie's $2 \mathrm{~d}$ Duke of Eln \\
\hline 46 & Grace's 2d Grand Duke. \\
\hline & Gracy Lad ... \\
\hline 49 & Gr'd Airdrie of Pl'sant \\
\hline 0 & Grand Duke... \\
\hline 53 & Grand Duke of Athens $2 \mathrm{~d}$ \\
\hline & 3d Grand D \\
\hline 55 & 4th Grand Duke of Boardman..P S \\
\hline & 2d Grand Duke of Gloster. \\
\hline 59 & 3d Grand Duke of Gioster. \\
\hline (0) & 4th Grand Duke of Gloster. \\
\hline & Grand Duke of Hazelwood.. _- PS \\
\hline 3 & 2d Grand Duke of Oxford \\
\hline & Grand Duke of Rosedale. \\
\hline & 2d Grand Duke of Rosedale... _- M \\
\hline & 3d Grand Loudon Duke \\
\hline & 4th Grand Loudon Duke. \\
\hline & Grand Prince............. $R$ \\
\hline 977 & Granger.. \\
\hline & Granger \\
\hline & $-\mathrm{R} \mathrm{R}$ \\
\hline & Grant of B \\
\hline & $\ldots \mathrm{Ky}$ \\
\hline
\end{tabular}

69983 Greeley

69984 Greeley Cone.................

69985 Green Lawn Duke.............

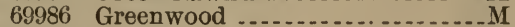

69987 Greenwood Boy ............. Ky I

69989 Greenwood Duke.............

69990 Greenwood Duke...................

69991 Greenwood Duke $2 \mathrm{~d}$

69992 Greenwood Prince............... Cox

69993 Gresham's Prince ............

69994 Greystone

69995 Grove City Duke............ - Cox

69996 Grover

69997 Grover

69998 Grover

\%0000 Grover

70001 Grover .................. R R

70002 Grover.....

70003 Grover C., ...................

70004 Grover Chambers........

70005 Grover Cleveland............ D

70006 Grover Cleveland................ I I

70007 Grover Cleveland... - Long Horn

70008 Grover Cleveland ...................

70012 Grover Cleveland

r0013 Grover Cleveland............ - Cox

r0014 Grover Cleveland ......... R R

70015 Grover Cleveland.............. R R

70017 Grover Cleveland ............ Ky I

70018 Grover Cleveland............ D

70020 Grover Cleveland ............ - D

70021 Grover Cleveland............

70022 Grover Cfeveland......... Ky I

70023 Grover Cleveland...............

700:24 Grover Cleveland ......... Cox

70026 Grover Cleveland ............... T

70027 Grover Cleveland........... L R

70028 Grover Cleveland............ - D

r0029 Grover's Crop ............. R R

70031 Guberly Moultrie ............... Ky I

70032 Gwynne Tompkins........... C

70034 Halberdier .................. R

r0036 Halleck ................. R

70037 Hallet Sheetz................ R

70038 Halpin Cleveland............... Ky I

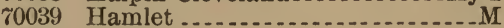

70040 Hamlet .................... R

70041 Hamlet

70043 Hampton's Duke........... Ky I

70045 Hancock..................

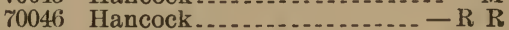

70049 Handybuilt .................... Ky I

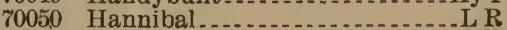

70051 Hannibal.................... R

70052 Hannibal .........................

70053 Hannibal..................

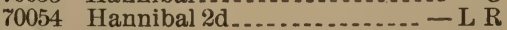

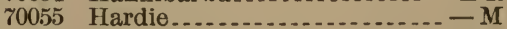

70056 Harlan Duke John......... L R

70057 Harlequin 2d................

70058 Harmean...................

70063 Harper...................... R

70065 Harrison Bates............. D

70066 Harry Bassett................ I

70067 Harry Dollinger...... Multiflora

70068 Harry Lee...................

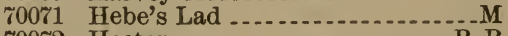

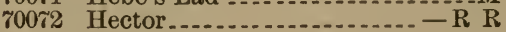

70073 Hector 
$70075=$ Heir of Hillhurst............. - P S 70076 Helen's Kirklevington..... - Ky I 70077 Hendricks ................. - Cox $700 \%$ Hendricks..... 70079 Hendricks.............. 70080 Hendricks................... R

70081 Hendricks ........................

70082 Hendricks

70083 Hendricks................. Cox

70085 Hendricks.................... M

70087 Henry ............

70088 Henry 2d................. R

70090 Henry B.,...................... T

70091 Henry Clay .....................

70092 Henry Clay

70093 Henry of Syracuse..............

70094 Henry Ward Beecher............ M

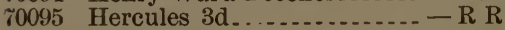

70097 Herman .................... R R

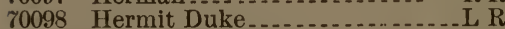

70099 Hermit Harold............... R R

70100 Hermit Prince.................... R

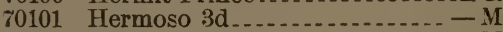

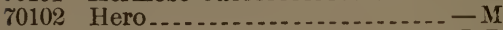

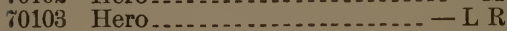

70104 4th Hero..... R

70105 Herod

70106 Hero of Elkhart.............

70107 Hero of Hillsboro

70108 Hero of Upton................

70109 Hidalgo....................

70110 Hidalgo............................ R

70111 Highland Airdrie........... $\mathrm{R}$ R

70112 Highland Chief............ - Daisy

70113 Highland Chief........... - Cox

70114 Highland Duke ........... - M

70115 Highland Duke.............. L R

\%0116 Highland Duke

70117 Highland Duke $2 \mathrm{~d}$

70119 Highland Lad............. - Cox

70121 Highland Lad $2 \mathrm{~d} . .$.

70122 Highland Napier $3 \mathrm{~d} . . . . . .-\mathrm{L} R$

70126 Hillhurst .................

テ0129 Hilpa Duke............... Rolla

70130 Hilpa's Mazurka Duke........ - C

70133 Hold The Fort ............. R R

70134 Holton of N'tive Gr've F'm _. Multitl'a

70135 Homer ...................... R

70136 Homer..........................

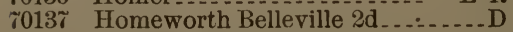

70138 Homeworth Gamester.......... Cox

70139 Honest Abe ................ - Cox

70140 Honest Abe..................... R

70141 Honest John

70143 Hoosier ....................

70144 Hoosier Boy

70145 Hoosier Duke........... R R

70146 Hopeful

70149 Horatio........

70150 Horatio 6th...............

\%0151 Horatio 7th................

70152 Hospodar................ - L R

70153 Hotspur..........................

70154 Hotspur .....................

70155 Howard

r0156 Howell Duke of Sharon..... - Cox

70157 Hubback ...................... Ky I

70158 Hubback $3 \mathrm{~d} . \ldots \ldots \ldots \ldots$

70159 Hubback Jr.,................... R

70160
70161

70162

70166

$\% 0168$

70170

$\% 0172$

70173

\%0174

70175

\%01\%6

$7017 \%$

70181

70182

70183

\%0184

r01S5

70186

$7018 \gamma$

т0188

\%0189

70190

\%0191

70194

70195

70196

\%0197

\%0198

70199

70200

70201

70202

70203

70204

70205

70206

70207

70208

70209

70210

70211

70213

70214

70215

70216

70217

70218

70219

r0220

テ0221

70222

r0223

ๆ0224

70225

70226

70227

70228

r0229

70230

\%0231

70232

70233

\%0235

70237

\%0235

70239

70240

70241

70242

70243

70245
Hudson................... $\mathrm{L}$

Hudson .................... $\mathrm{C}$

Humphrey's Duke........ R

Hunter ................ P S

Hurlburt...................

Huron Chief.........

Hutton ..........

Hylas

I. A. Renick ...........

Ida's Pride............ R R

I'm Coming

Imperial Grover............... L R

Imperial Prince.................

Independence............... Cox

Independence ..........

Independence ...... R

Independence $2 \mathrm{~d}$

Index...........................

Indiana...... Ky I

Innuce

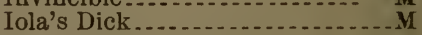

Irene's Hillhurst Duke .....- R R

Isaac ................... $R$

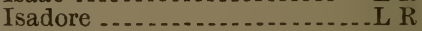

Ishmael

Ivanhoe $2 \mathrm{~d}$

Jack

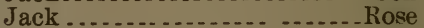

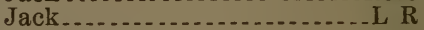

Jack Falstaff -......... $\mathrm{R} R$

Jack Logan...............

Jack Monroe.............. Ky I

Jackson ........................

Jackson

Jackson Prince...........

Jack On The Green.......

Jack Vickory ................. - T

Jacob

Jacoby

James..........................

James A. Garfield......

James Clay .................... $\mathrm{R}$

James G. Blaine......

James G. Blaine.............. Ky I

James G. Blaine.............

James G. Blaine.............. R

James G. Blaine...................

James G. Blaine........... R

James G. Blaine............. Ky I

James G. Blaine................. S

James G. Blaine... Cox

James G. Blaine............. M

James G. Blaine........... $R$ R

Janesville Star............ - L R

January Airdrie......... Daisy

Jasper 2d................ R

Jay Bickerstaffe

Jay Gould ..........................

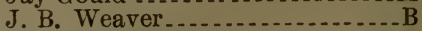

J. C. Jenkins .............

Jeff Davis.................

Jefferson............

Jefferson

Jefferson

Jefferson

Jefferson

Jefferson ..................

Jefferson Lad...............-D 
70246

70247

70248

70249

70250

70252

70253

70254

70255

70256

70257

70258

千0259

70260

70261

70262

70263

70264

千0265

\% $026 \%$

$\tau 0269$

$702 \% 0$

70271

\%0272

70273

\%0274

70275

$702 \% 6$

r027

\%0278

70283

70284

\%0285

70286

70287

\%0:288

70289

70290

70291

70292

70293

70294

т0ะ95

70296

$7029 \pi$

70298

70300

r0301

70302

\%0303

70304

70305

80306

r0307

70308

70309

$\checkmark 0310$

70311

70312

70313

70314

70315

70316

\%0317

70318

テ0319

70320

70321

70322

г0323
Jehu

Jemmie

Jennings' Red Duke.........

Jennie's Oxford $3 d \ldots . . . . . .-R$ R

Jeroboam .................

Jerry _..........

Jerry

Jerry .

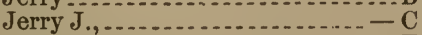

Jerry Teters ................

Jersey's Choice ................... M

Jesse James ...................... R

Jesse Woodford........... $-\mathrm{L}$ R

Jeweller................... $R$ R

Jim ....................

Jim

$\operatorname{Jim} 2 \mathrm{~d} \ldots \ldots \ldots$

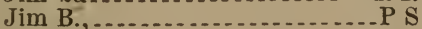

Jim Blaine .................... D

Jim Blaine.............. L R

Jim Blaine................ R

Jim Blaine .................. D

Jim Blaine.................... P S

Jim Blaine .................. Ky I

Jim Blaine...................

Jim Blaine..................

Jim Blaine............... Ky I

Jim Brown ............ P S

Jim Crow

Jim Way .................

Joe

Joe . . . . . . . . .

Joe

Joe

Joe Arnold ...................... Ky I

Joe Ashby ............... D

Joe Bell ...................... D

Joe Blackburn

Joe Blackburn.............. - P S

Joe Blackburn

Joe Combs................. M

Joe Dale.........................

Joe Gargery

Joe Hooker..................

Joe Hooker................. R

Joe Ingman $2 \mathrm{~d} . . . . . . . . . .-\mathrm{P} S$

John A. Logan............ D

John A. Logan.......... D

John A. Logan................ D

John A. Logan ..............

John A. Logan ..................

John Anderson ...............

John Bishop................. L R

John Bright Eyes.......... R R

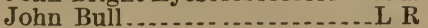

John Bull...................... Ky I

John Clay ...................... R

John Diamond 2d............ D

John Gilpin.........................

John Knox.......... P S

John L.,...................... Ky I

John Mac............... $\mathrm{R}$

John M. Barker........... - L R

Johnny ....................

Johnny Bull.................. D

Johnnie Gleason............ Ky I

Johnny Lad of Evergreen Hill_Cox

Johnnie Vaughn ............. - D

Johno .................. R

John Sharon .................... T
70324 John S. Marmaduke........ - P S

70325 Johnston ................. Cox

70327 Jonathan ..................

70328 Jonathan .................. Cox

70329 Joseph.................... M

70330 Joseph ...................... S S

70331 Joseph 4th.................... R

70332 Josephus................ - R R

70333 Joshua.................. L R

70334 Joshua.......................... R R

70335 Josiah Allen .................. R R

70336 Jubelo ............................

70338 Jubilee Duke 9th ........... - R R

70340 Jude....................... Beauty

70341 Judge Clark ................. - L R

70342 Judge Dunmore........... $\mathrm{R} R$

70343 Judge Hawkins............ - L .R

70344 Judge Leslie ................ - P S

70345 Judge Lincoln.............. $-\mathrm{R} R$

70346 Judge Welty ............... - L R

70347 Judy ....................... Daisy

70349 Julia's Duke............... - Ky I

70351 Julius ..................... - Rolla

70353 Julius of Tany Creek ........

70354 Jumbo....................

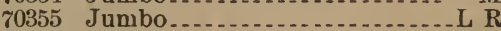

70356 Jumbo......................... Cox

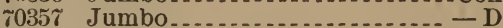

70358 Jumbo............

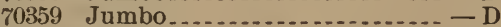

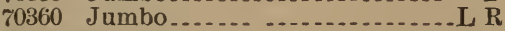

70363 Jupiter.................... R

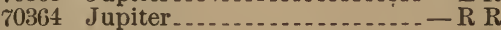

70365 Jupiter .............. M

70366 J. W. Eller.........

70367 Kaiser .................

70369 Kansas Rose Lad ..................

70370 Katie's Duke of Elmwood.... Ky I

70371 Kenelm Chillingly _...............M

70372 Kenesaw....................

70374 Kent W., Ken

70375 Keystone

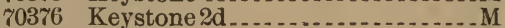

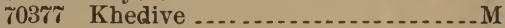

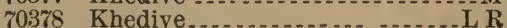

70379 Kilpatrick 2d............. Woods

70380 Kindel Star................ R

70381 King 3d................... Cox

70382 King of Atha............. R

70383 King Bell ........................ I

70384 King David...... S S

70386 King George...................

70387 King Henry .................... Ky I

70388 King Lear 2d............... P S

70390 King of May.................... I

70391 King Philip................. R

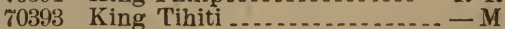

70394 King Waif .................. Ky I

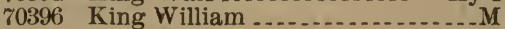

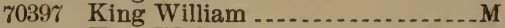

70398 King William ...................

70400 Kirkl'v'gt'n Airdrie of Prairie H'l.T

70403 Kirklevington Duke 5th....... - B

r0405 K'klev'gton D. of Bluff View - R R

\%0409 Kirklevington Lad................ Ky I

70410 Kirklevington Lad 2d.......... - D

70412 Kirklevington of Monmouth...... D

70414 Kirklevington of Oakland 7 th.... P S

70415 Kirklevington Prince....... - Rolla

70417 Kirk Peri ................. - Rolla 
70418 Kirk Phyllis................. - L R

70419 Kirk Walters $2 \mathrm{~d} \ldots \ldots \ldots$

70420 Kit Carson ..................... S S

70423 Knightly Duke.................. R

70426 Knightly Prince

70427 Knightly Prince $3 \mathrm{~d} . . . . . . .-$ Rolla

r0428 Knightley Sharon......... - Rolla

70432

\%0133

\%0436

70437

70438

r0439

70440

70441

70442

70443

70445

70446

r0447

70448

\%0449

70451

ช045?

\%0453

70454

70455

70456

70457

70458

\%0459

70460

r0462

r0464

r0166

i0467

\%0469

70470

$704 \pi 1$

\%0472

ช04\%

\%0475

70476

r04ir

70480

70483

\%0484

70485

r0487

70489

70490

70492

70494

70496

70497

70498

70499

70500

70501

r0504

70505

\%0506

70507

\%0509

70510

\%0511

70512

70513

70514

70515

Lacka wannock ............. - D

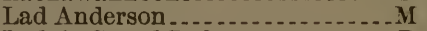

Lady's Grand Duke........... - D

Lafayette ......................

Laird Airdrie...............

Lamoille Boy . ..........

L'Argent.................

Lary O'Day .........

Late Hubback

Lathrop

Laurel Airdrie ........................

2d Laurel Airdrie..............

Lauriston .........................

Lawn Lad $23 \mathrm{~d}$

Lawn Lad 24th............ $\mathrm{R} R$

Lawn Lad 26th.............. - R R

Lawn Lad 27 th.......... - R

Lawn Lad 28th...........- R R

Lawrence ............... Cox

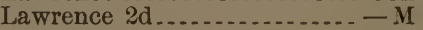

Lawson Hatch........... Cox

Leander ..................... Ky I

Leander Chief................ Ky I

Lee

Lee Wolf

Lehr's Grant................ S

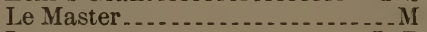

Leo ........

Leo ............... R

Leo of Grassfield..........

Leon

Leon

Leonard .......

Leonidas...............

Leopold ........

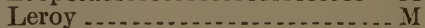

Leslie Duke................... S

Levi Breastplate........... - R R

Lewis Duke 5th ..........

Lexington ........... P S

Lieutenant

Lighteliffe $2 \mathrm{~d} . . . . . . . . . . . .-\mathrm{C}$

Light Don ................... Cox

Lily's Buck of Gardner........ Ky I

Lily's Duke................. - L R

Limbus .................. R

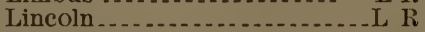

Linn Duke......................

Linwood Duke...............

Lionheart of Elm Grove....... Ky I

Little Beaver.................. Ky I

Little Bobby .................... R

Little Duke.................... R

Little Giant.............. R R

Little Goldie...........................

Little Jo........................ R

Little Mack ................. Ky I

Live Oak ..............................

Liverpool ..................... $-\mathrm{C}$

Lizzie's Duke................ $\mathrm{R} R$

Llewellyn Prince $2 \mathrm{~d} . . . . .$.

Loan's Duke..................
70516

\%0517

70518

70519

70520

70521

70522

ז0523

70524

ז0525

70526

70527

70528

ז0530

70531

70533

70535

70536

70537

70538

r0539

テ0540

70541

r0543

70544

70545

\%0546

70547

70548

70549

70550

70553

70554

ๆ0555

70557

70558

70560

\%0561

70567

70568

70571

ז05\%

70573

70574

$\tau 05 \% 6$

70580

70582

70583

70584

70585

\%0590

70591

70593

70594

70595

70598

70599

70603

\%0604

70605

70610

70612

70613

r0614

70616

70617

\%0618

70619

70620

70621
Locomotive ..................... Ky I

Locomotive........................

Lodi Romeo............

Logan ..........

Logan ...........

Logan...................

Logan .......... $R$

Logan............

Logan

Logan ................

Logan

Logan.............. R

Logan ........

Logan .......... Ky I

Logan ..................

Logan

Logan ................. T

Logan

Logan ...........

Logan

Logan -............. - Long Horn

Logan Boy ............... R R

Lone Duke........... Ky I

Lone Star.......................

Long Branch Lad......-Daisy

Longfellow .................... Ky I

Longfellow ..................... Cox

Longfellow $2 \mathrm{~d}$

Long Point Star..... Cox

Lonsdale................. $\mathrm{R} R$

Lord Althorp..................

Lord Anson...............

Lord Athelstane ....... P S

Lord Bacon

Lord Baron...........

Lord Barrington $3 \mathrm{~d} . . . . . . .-\mathrm{Cox}$

Lord Barrington 4th...........M

Lord Barrington of Huron $2 \mathrm{~d}-\mathrm{M}$

Lord Barrington of Windsor 2d L L R

Lord Beecher.............. - P S

Lord Belleville $3 \mathrm{~d} . . . . . . . . .$. Rose

Lord Bernhardt........... P S

Lord Byron................... Ky I

Lord Byron.......... R

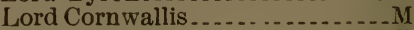

Lord Cundiff..........

Lord Derby .............

Lord Dunmore................. Ky I

Lord Dunmore............ - Ky I

Lord Geneva 3d........... - Cox

Lord Graham 8th.......... R R

Lord Harry ................

Lord Henry

Lord Hylas .......... Rolla

Lord Loftus...........

Lord Lorne.................. L R

Lord Lytton

Lord Mlayor -........ Milk Spring

Lord Mayor 4th ......... R R

Lord Nithsdale........... R R

Lord Ogan 2d..........

Lord Oram ..................

Lord of Orleans .................

Lord Raglan.......................

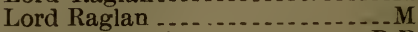

Lord Randolph........... R R

Lord Roscoe.............

Lord Roscoe ................ - Cox

Lord Roseberry........... R R 
70622

70623

70624

70625

$7062 \%$

70628

70629

70630

70631

70632

70633

70638

70639

70640

70641

70643

70645

70646

70647

70648

70649

70650

70651

70652

70653

70654

70655

70656

70657

70658

70659

70660

70665

70667

70669

70670

70671

70673

70674

70675

70676

70677

70678

70679

70680

70681

70683

70684

70685

70686

7068

70688

70689

70690

70692

70693

70694

70695

70696

70698

70699

70702

70703

70704

70705

70707

70708

70709

70711

70712
Lord Ross

Lord Ruby

Lord Sharon $3 \mathrm{~d}$

Lord Shelburn........... - Rolla

Lord of Springhill $2 \mathrm{~d}$

Lord Stanhope............

Lord of The Valley...... - Cox

tord of The Valley ............ M

Lord Turncroft Ox ford 4th - Rolla

Lord Vincent............. $R$ R

Lord Wild Eyes........... - Cox

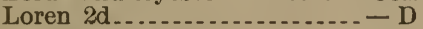

Lorena's Darling ..........

Lotta's Thorndale............

Louan Duke .................

Louan's Duke 4th.......... $R$

Louan's Duke 5th........... $-R$

Louan's Duke 6th..........-R R

Louan's Duke 7th.......... - R R

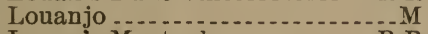

Louan's Montauk ....... R R

Louan's Montauk $2 \mathrm{~d} . \ldots . . .-R \mathrm{R}$

Louan's Montauk 3d...... - R R

Louan Prince..............

Loudon's Best.................... D

Loudon Duke ........

Loudon Duke............ Ky I

Loudon Duke 3d......... D

2d Loudon Duke of Forest_._ $R R$

Loudon Duke of Bath $2 \mathrm{~d} . . . . .-\mathrm{D}$

Loudon D. of Macacheek 2d-R R

Loudon D. of Macacheek 3d_._- M

Loudon Duke of Randolph... - P S

Lucknow -................

Lucy's ThorndaIe

Luke ............................

Lula's Duke $2 \mathrm{~d}$

Luther ..........................

Lutrelle...................... R

Lyndon Duke................. I

Macbeth ......................

Macbeth

Macbeth ....................

MeClellan .......................

McDuff .............................

McKay's Duke ................ S S

Madriver Valley Chief $3 \mathrm{~d} . .-\mathrm{Ky}$ I

Magellan ..................... M

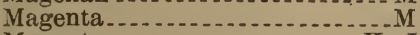

Magnet ..............

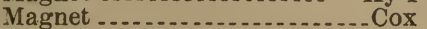

Maid's Airdrie.............. $R$

Major ..........................

Major

Major

Major

Major.............. Daisy

Major

Major

Major.................. $-\mathrm{L}$

Major

Major Bates $2 \mathrm{~d}$

Major Bell

Major Bly $3 \mathrm{~d}$

Major Booth............ - M

Major Breckenridge..............

Major Brown

Major Bull of Manor Oak_.. _ L R

Major Cressbrook........... $-\mathrm{R} R$

Major Dixie......................
70713 Major Don Anderson.............M

70714 Major Doxey............... D

70715 Major Duke............... L R

70716 Major G.,................. Cox

70717 Major Gault.............. - R R

70719 Major Hill 2d of Durham..... - D

70720 Major Hopeful ................ - T

70721 Major Hopewell..........

$7072 \%$ Major Io.....

70724 Major Lee...................

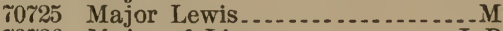

70726 Major of Lima..........

70727 Major Marmaton ......... - L R

70728 Major Pierson...............

70729

70730

70731

70733

70734

70735

70737

70738

\%0739

70740

70741

70742

70744

70745

70746

70747

70748

70749

70751

70752

70753

70754

70757

70763

70767

70768

70769

70770

70771

70772

70774

70775

70776

ซ0777

70778

70779

70780

70781

70782

70783

70784

70785

70786

70787

70789

70792

70798

70800

70801

70803

70804

70805

70806

70807

70813

r0814

Major Thomas ............... Ky I

Major Vanmeter 2d........

Major Whittaker.........

Malcolm .......

Mallardean

Malvern Chief........ $R$

Mammoth Duke.........

Mammoth Roan........ D

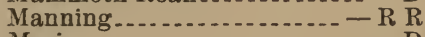

Mario....

Marion ........

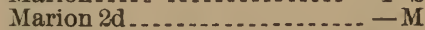

Marius ....... Rolla

Mark Twain ............ Daisy

Marmaduke............... Ky I

Marmaduke................ $-\mathrm{D}$

Marmaduke............. R R

Marmaduke ..............

Marmion

Marquis....................

Marquis .... C

Marquis............ R

Marquis of Boone.... $R$

Marquis Duke .........

Marquis of Lorne.....

Marquis of Lorne........

Marquis of Maple Shade...... L R

Marquis Pierce.......... R R

Marquis of Rushford....

Marquis of Tipton .......... $R$

Mars ...................... $\mathrm{P}$

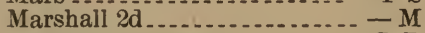

Marshall Dickerson........ $\mathrm{L}$ R

Marshall Duke................ Ky I

Marshall Duke 2d...........

Marshal Morgan .......... L R

Martin ............. $R$

Martin Daffodil ....... L R

Marvelle........... Rolla

Mary's Airdrie .......... - Rolla

Mary Airdrie's Duke...... $-R R$

Mary Airdrie's Duke 2d.... - R R

Mary Bell's Duke............ $-\mathrm{M}$

Mary's Cypress Duke.......-C

Mary Duke.................. Ky I

Mary's King ............. S

Master Baron.......... R R

Master Elizabeth.......... R R

Master Gem.............. R R

Master Mason.............

Master Phyllis...... P S

Master Price $3 \mathrm{~d}$

Master Royal ....................

Master Sharon $2 \mathrm{~d} . . . . . . . . . .-M$

Matilda's Airdrie........... $\mathrm{R}$ R

Matilda's Pride........ $R$ 


\begin{tabular}{|c|c|}
\hline 70815 & Maxwell Chief. \\
\hline 70816 & May Boy... \\
\hline 817 & May Duke \\
\hline 70818 & May Duke 2d \\
\hline 0819 & May's 2d Duke of Elmwoo \\
\hline 70820 & Mayflower Prince......... \\
\hline 70821 & 7th Mayflower Prin \\
\hline t0823 & May's Monarch -- \\
\hline $\begin{array}{l}70824 \\
70825\end{array}$ & $\begin{array}{l}\text { Mayor of Oakland } \\
\text { Mav's Sycamore }\end{array}$ \\
\hline 70826 & Mazor............ \\
\hline & $\mathrm{B}$ \\
\hline
\end{tabular}

70833 Mazurka Judge 2d .............. P S

70837 Mazurka's Oxford 38th.........Ky I

70838 Mazurka Prince.............. - L R

70840 Meadow King................. R

70841 Melbourn ....................... R

70842 Melita's Duke.............. - L R

70843 Melton Duke................ S S

70846 Merry Lad.......................

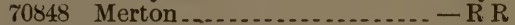

70849 Michigan

70851 Midshipman..............$-M$

70852 Mike......................... L

70854 Milan Duke.............

70856 Millard Fillmore............... R

7085 Millard's Knight.............................

70858 Milo .................... R

70859 Mingo

70860 Minister.................. R R

70861 Minnesota Chief...............

70862 Minnesota Duke............. M

70863 Minnesota Lad............ Cox

70865 Minna's Duke.........

70866 Minnie's Duke $2 \mathrm{~d}$

70867 Miramonte................. L R

70870 Miss Pugsley's Duke.......... - D

70873 Model 2d ...........................

70874 Model's Lad

70875 Modesty's Belle Bates...... - L R

70876 Modoc ..........................

70877 Mohawk................... B

70878 Mohawk

70879 Mohawk

70880 Mollie's Airdrie ............. $\mathrm{R}$ R

70881 Mollie Eight's Oxford of Risk _ D

70882 Mollie Eight's Oxf'd of Risk 2d-D

70883 Monarch

70884 Monarch ................ D

70885 Monarch......................

70887 Monroe Duke.............. R R

70888 Montana Boy

70890 Montgomery

r0891 Mora's Duke $2 \mathrm{~d} . . . . . . . . .-$ Rolla

70892 Mordica....................

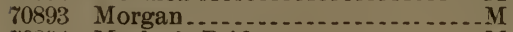

70894 Morley's Pride

70895 Mormon ...................... R

70896 Morning Star.............. R R

70897 Morris Treble Gloster $2 \mathrm{~d} . . . . . . . \mathrm{L} \mathrm{R}$

70898 Mosboro Chief .............. - Cox

70899 Moses ............................ B

70900 Mossback ..................

70901 Moss Cherub..................

70902 Moss Duke................

70903 Moss Duke 2d................

70904 Moss Prince.................

70905 Moss Red Duke ............. D

70906 2d Moss Rose's Duke....... - Rose

70907
70908 Mountaineer................

70909 Mount Joy ....................

70910 Mugwump..........................

70911 Mulachey

70912 Muldoon

70913 Muldoon....................

70914 Murdock...................... R R

70915 Muscatoon 6th........... P S

70916 Muskingum Chief $2 \mathrm{~d} . . . . . . . . . . .4$

70918 Myrtle Prince $3 d$...........

70920 Nabob.....................

70921 Nodaway Duke............ Ky I

7092: Nannetta's Lad............... - L R

70924 Napoleon -............... Ky I

70925 Napoleon of Shirley .........

70926 Narcissus.................. $-\mathrm{L} R$

70927 Narcissus of Beallville...... $-\mathrm{L} R$

70928 Nasby

70929 Nat Bronaugh........... - Rolla

70931 Navajoe.................

70932 Neal Dow

70933 Nebraska Duke.............. - P S

0934 Nebraska Duke............... M

r0935 Ned......................... P\&

70936 Ned Bates......

70937 Nellie's Duke.................... S

70938 Nellie's Oxford.............

70939 Nelson

70940 Nelson Boy 8th................. R

r0941 Nelson Boy 9th ............... R R

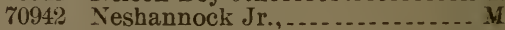

70943 Newham Duke $2 \mathrm{~d}$

r0944 Newman ................... L R

70945 New Model...................

70946 New Rumbley Duke....... - Cox

70947 New West...................

70948 New-Years...................... I

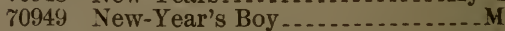

70951 Nicator of Melleray .......

70952 Nick B.,....................... P S

r0953 Nickle Plate............

70954 Nimbus...................... Ky I

70955 Ninescah Duke............ Ky I

70956 Nip...........

70958 Norfolk

70959 Normal Duke ..........

70960 Norman .............. Ky I

70962 Northman...............

70963 North Star...................

70964 North Star.................... M

70965 North Star................ Ky I

70966 North Street Wild Eyes...... - P S

70967 North West............... - L R

70968 Norwegian Boy ......................... M

i0969 Norwood.................... D

70974 Nustid Abby of Beallsville.. - L R

70976 Oak Island Chief.................

70978 Oakland Dhief................. - R R

70979 Oakland Duke.............. P S

70980 Oakland Duke $2 \mathrm{~d} . . . . . . . . .-\mathrm{L}$ R

70981 Oakland Lad 28th..........

70982 Oakland Pride ................... M

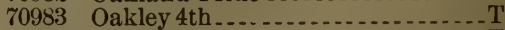

$7098 \tilde{5}$

70986

70987

70988

70989

70990
Oglesby

Ohio Boy

Ohio Boy

Oklahoma..................

Ole Bull ........................ 


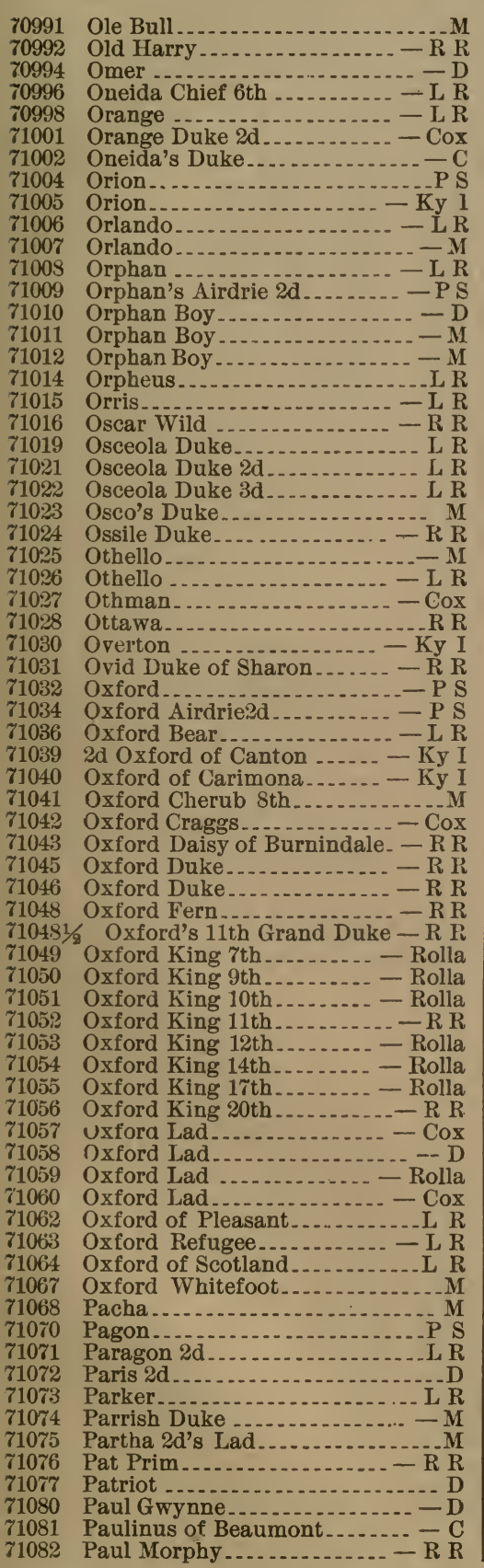

71083 Paul Pry.................. P S 71084 Paul Vane ............... R 71085 Pawnee Chief $2 \mathrm{~d} . . . . . . . . . . . .8$ P S 71086 Pawnee Prince................. Cox 71087 Paxton ....................... M 71089 Pearl's Duke ............. R R 71090 Pearl Duke 2d.......... P S 71091 Pearl's Wild Eyes.............. - P S 71092 Pedro 71093 Pedro................ 71094 Pedro Moultrie................ Ky I 71095 Peek-A-Boo 71096 Peerless Duke........ R R 71098 Peko.............................

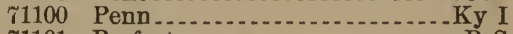

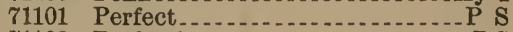
71102 Perfection ....... 71103 Peri Duke................. - M 71107 Peri Lad 6th............. R 71108 Peri Trump.............. R R 71109 Peter.......... 71110 Peter Cooper Jr............. - Cox 71111 Peter Cooper of Woodlawn ... Ky I 71112 Peter The Great. ............. - M 71113 Peter Wakam ............... D 71114 Pewanee Chief.............. L R 71115 P. Grover ........

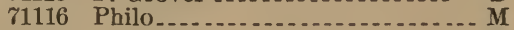
71117 Philosopher................ R 71118 Phil Sheridan.................. L R 71119 Phœnix Duke.......... Cox 71120 Phyllis .................... R R 71121 Phyllis Boy ................. R R 71128 Phyllis Duke 4th............ R R 71129 Phyllis Duke of Hamburg 2d-L R 71130 Phyllis Duke of Hazelwood - Rolla 71133 Pickwick.................. - Cox 71134 Pilgrim.............. 71135 Pilot......................... I 71136 Pilot...............................

71137 Pineapple Duke........... -

71138 Pine Creek Hero ...............

71140 Pioneer .................

71141 Pizarro ................... D

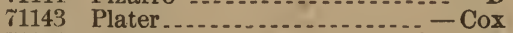

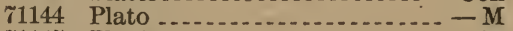

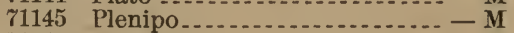

71147 Plow Boy .....................

71149 Plumber

71150 Plumed Knight................ R

71152 Plum Lad.................. - Cox

71153 Plumwood Lad............... Ky I

71155 Plutarch.................... Cox

71156 Pluto.............................

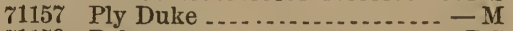

71158 Poke ............... P S

71159 Poland's Lad...................... R

71160 Polo............................ Cox

71161 Pomona Duke..............

71165 Porsena $2 \mathrm{~d} . . . . . . . . . . . . .2 \mathrm{R}$

71166 Porter.......... L R

71167 Porter

71168 Portersville Duke ......... - L R

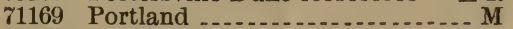

71170 Prairie Duke............. - Daisy

71172 Prairie Duke $3 \mathrm{~d} . . . . . .6$

71174 President ........................

71175 President...........

71176 President ....................

71180 President Grant........... - L R 
71181 Price Connaught........... L R

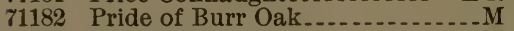

71183 Pride of Cold Run............ M

71184 Pride of Linesville............ Cox

71185 Primrose Duke.......... D

71186 Prince..................

71187 Prince.........

71189 Prince............... M

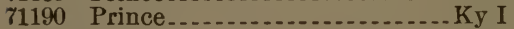

71191 Prince.......

71192 Prince............... P S

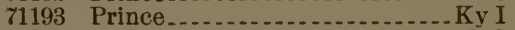

71194 Prince............. P S

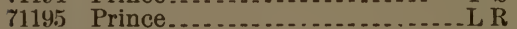

71196 Prince.................. R R

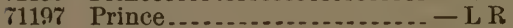

71198 Prince $2 \mathrm{~d}$

71199 Prince 2d...................... I I

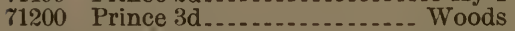

71202 Prince Airdrie.......... - Beauty

71203 Prince Airdrie......... Beauty

71204 Prince Albert ......... D

71205

71206

71207

71208

ช1209

71210

ช1211

71212

71215

71216

$7121 \%$

ช121S

71219

テ1220

$\gamma 1221$

\%122\%

71223

712:4

71225

71226

$712: 27$

ॠ1229

\%1230

ช1231

71232

71233

71234

71235

71237

71238

71239

71240

ช1241

71243

71244

71245

71246

71247

71250

71251

71252

71254

71255

71256

71257

71258

71261

71262

Prince Albert.................. MI

Prince Albert ................. $R$

Prince Albert -............. - L R

Prince Albert................ Ky I

Prince Albert.............. - Cox

Prince Albert................ Cox

Prince Albert................ $\mathrm{R} R$

Prince Albert $2 \mathrm{~d} . . . . . . . . .-\mathrm{L} R$

Prince Alfred ............... M

Prince Arthur . .............. - M

Prince Arthur

Prince Arthur

Prince of Avondale.........

Prince B.,................. $-\mathrm{D}$

Prince Bismarck.............. Ky I

Prince Bismarck..............- KyI

Prince Bismarck ............. - L R

Prince Charles ................... MI

Prince Charles ............... M

Prince Charlie.............. Cox

Prince of Cherry Creek.....- - Cox

Prince of Dexter............ - L R

Prince Earl

Prince Ecton

Prince Edward

Prince Edward..............

Prince Edward ................- $\mathrm{L} R$

Prince of Fair Plains........... L R

Prince of Fairview .............- M

Prince of Four-Miles.......... M

Prince George............... - L R

Prince George

6th Prince of Grass Hill. - Beauty

Prince Hal ............... - L R

Prince of High Prairie....

Prince Henry -............. - Cox

Prince Hood ................ - P S

Prince Hubert.............- - Cox

Prince of Jefferson........- $R$

Prince John -................ - D

Prince John ..................

Prince Jubilee.............- Ky I

Prince Kansas..............- - L R R

Prince Kirby ................... R

Prince Leo -.................. - Rose

Prince Logan ..............-Rolla

14th P. of Lombardy P'k-Multif'ra
71263

71264

$\tau 1265$

71266

71267

71269

71270

71271

71272

71273

71274

71275

71276

71277

71279

71282

71284

71285

71286

71287

71289

71290

71291

71292

71293

71294

71295

శ1296

71297

71301

テ1302

71304

71305

71306

$7130 \tau$

ז1308

71309

71310

71311

テ1313

71314

71315

$7131 \tau$

71318

71320

71323

71324

71325

z1326

71327

71330

71332

71333

71337

71339

71340

71341

71342

71344

71346

71347

71348

71349

ซ1350

71351

71352

71353

71354

71355

71356
Prince of Lost Creek...... - Rolla Prince Loudon ............ - L R Prince of Madison Prince Malone 10th ........- Cox Prince Malone 11th ........... - P S Prince of Mapleshade....... L R Prince of Maplewood ........ Ky I Prince Marquis .......... Cox Prince of Miami..... Prince Moore................. Ky I Prince Napier 9th Prince Napier 12th....... R R Prince Napoleon ............ L R Prince of Oakland $2 \mathrm{~d} . . . . . . .-\mathrm{D}$ Prince of Orange........... $-\mathrm{L} R$ Prince Owen ........... D Prince of Platte $2 \mathrm{~d} . . . . . . .-$ Daisy Prince of Platte $3 \mathrm{~d} . . . . . . .-$ Daisy 2d Prince of Pleasant Point ... - C Prince of Prairie Farm ......- $R$ Prince of Riverdale ........... $\mathrm{L}$ Prince of Riverdale $7 \mathrm{th}_{\mathrm{n}} . . . .-\mathrm{R} R$ Prince of Romulus ........ $R$ Prince of Root River...... P S Prince Royal .............. - L R Prince of Sarcoxie Prince of Short Creek....... - P S Prince of Sorrento .........- L R 2d Prince of Springdale....- Rolla Princess Lad............... - R R Princess Sultan ............. $-\mathrm{D}$ Prince Teck Prince of Ten-Miles......... M Prince of The Grove......... Ky I Princeton .................. Princeton $2 \mathrm{~d}$ Princeton Duchess Duke...- $\mathrm{R} R$ Princeton Duke of Clay Pool .._- M Prince Torning .............. M Prince of Vinewood Prince of Wales......... Cox Prince of Wales............. $\mathrm{R} R$ Prince William .............. Ky I Prince of Willow Park $2 \mathrm{~d}$ Prince of Woodland ........... D Printice................. Prior .................. Proctor Knott..... P Profit........... Prolific ..... Cox Prospector Prospero $2 \mathrm{~d}$ Proud Duke.................... R Proud Duke 6th ........... - Cox Proud Duke of Springwood. $-\mathrm{R} R$ Proud Peter.................... R Proud Prince.......... $R$ R Pugsley Quinto Raindown ....... Rajah Roll's Duke of Perry Ralph Ralph Rambler ....... Daisy Rambler.... R R Rambler ............. M Ramsey Duke.... Daisy

Ranchman 


\begin{tabular}{|c|c|}
\hline & \\
\hline & Randolph $\mathrm{R}$ \\
\hline & -............. \\
\hline & Ranger... \\
\hline & Rattler.. \\
\hline & 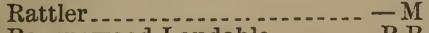 \\
\hline & audable..... $-\mathrm{R}$ \\
\hline & $\ldots+\ldots$ \\
\hline & - \\
\hline & $\ldots \mathrm{Ky}$ \\
\hline & $-\mathbf{R}$ \\
\hline & L $\mathrm{H}$ \\
\hline & - R \\
\hline & R R \\
\hline & Ky $]$ \\
\hline & 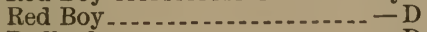 \\
\hline & - D \\
\hline & ar Creek....... \\
\hline & - n...... \\
\hline & 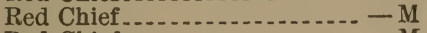 \\
\hline & (n) \\
\hline & - \\
\hline & $\cdots-1$ \\
\hline & $\ldots \ldots-K y$ \\
\hline & $\mathrm{Ky}_{\mathrm{y}}$ \\
\hline & 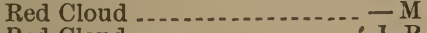 \\
\hline & $-\mathbf{L}$ \\
\hline & $-R_{1}$ \\
\hline & - Co \\
\hline & 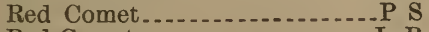 \\
\hline & - \\
\hline & Red Comet $2 d$ \\
\hline & 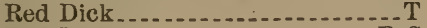 \\
\hline & P \\
\hline & Roll \\
\hline & (n) \\
\hline & - \\
\hline & 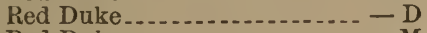 \\
\hline & -1 \\
\hline & 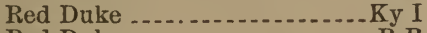 \\
\hline & - R \\
\hline & 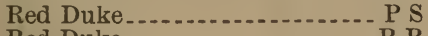 \\
\hline & -........- $-\mathrm{R}$ \\
\hline & . L \\
\hline & 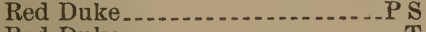 \\
\hline & 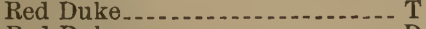 \\
\hline & 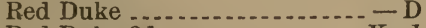 \\
\hline & $\ldots \ldots \ldots$ \\
\hline & $\ldots-\mathrm{L}_{\mathrm{T}}$ \\
\hline & $\ldots$ L \\
\hline & $3 d \ldots$ \\
\hline & f Glasgow ............. \\
\hline & ke of Vine Hill.... \\
\hline & $2 \mathrm{~d} \ldots-\mathrm{P}$ \\
\hline & Co \\
\hline & non \\
\hline & $2 \mathrm{~d} \ldots \ldots \ldots$ \\
\hline & et $2 \mathrm{~d}$ \\
\hline & $-R$ \\
\hline & . Long Ho \\
\hline & . \\
\hline & . \\
\hline & 然- \\
\hline & Ky \\
\hline & $-\mathrm{L}$ \\
\hline & \\
\hline & \\
\hline & \\
\hline & \\
\hline & \\
\hline
\end{tabular}

71439 Red Lad $-\frac{T}{}$

71440 Red Lad...... Cox

71441 Red Lad......................

71442 Red Leopard $2 \mathrm{~d}$

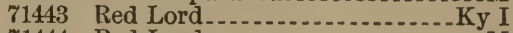

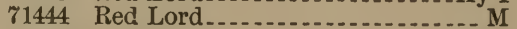

71445 Red Major.............. Ky I

71446 Red Man........................ Daisy

71447 2d Redman of Trout Farm .... - D

71448 3d Redman of Trout Farm.... - M

71450 Red Montauk ............. D

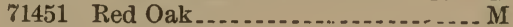

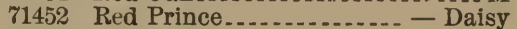

71453 Red Prince ............... P S

71454 Red Prince....................

71455 Red Prince............. Rolla

71456 Red Prince............. Cox

71457 Red Prince................ D

71458 Red Prince.................. D

$\gamma 1459$ Red Ranger

71460 Red Robin ....... D

71461 Red Rose's Duke......... L R

71463 Red Rose Prince 5th..... - Rolla

71465 Red Rover................. Daisy

71466 Red Rover......... Ky I

71467 Red Russell ................. T

71468 Red Sam. Rolla

71470 Red Tom...................... R

71471 Red Tom

71472 Red Tom

71473 Red Twin............... R

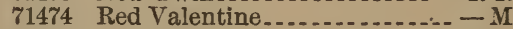

71476 Red Wolf ................. R

71477 Reformer of Oak Lawn - Milk Sp'g

71478 Renick's Dollinger ..............

71479 Renick Duke......

71480 Reno.............................

71481 Republican Postmaster... - R R

71483 Reuben.... Rolla

71484 Reuben

71485 Reuben E. Fenton... R R

71486 Reuleux

71487 Revere

71488 Rex........ Cox

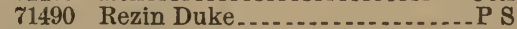

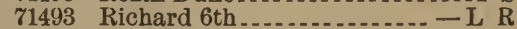

71494 Richard 7th ......... D

71495 Richard Booth............ R R

71496 Richard of Orange........ R R

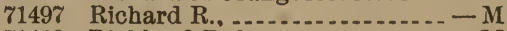

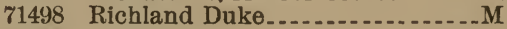

71499 Richmond............. R

71500 Richmond

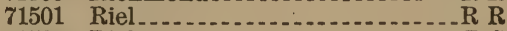

71502 Riel

71503 Rigdon

71505 Rinaldo ................... P S

71507 Ringtail ............

71508 Rip Van Winkle....... Cox

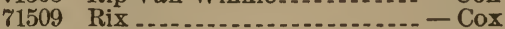

71512 Roan Bly

71513 Roan Boy

71514 Roan Boy .................. P

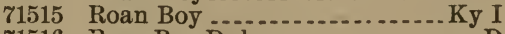

71516 Roan Boy Duke........

71518 Roan Dan............... R R

71519 Roan Duke.........

71520 Roan Duke............... P S

71522 Roan Duke.................. Rolla

71523 Roan Duke................. R R 
71524

71525

71526

71527

71528

71529

71532

71537

71538

71539

71540

71541

ז1542

71543

71544

71546

71547

71548

71549

71550

71551

71552

71553

71554

71555

71556

$7155 \%$

71558

71559

71560

71561

71562

71563

71564

71565

71568

71569

71570

71571

$715 \% 2$

71573

71574

71575

71576

71578

71579

71580

71581

71582

71583

71584

71585

71586

71587

71588

71589

71590

71592

71593

71594

71596

71597

71599

71601

71603

71604

71605

71607

71608

71610
Roan Duke

Roan Duke

Roan Duke.............

Roan Duke.................. R R

Roan Duke 2d............ $\mathrm{R} R$

Roan Duke 4th .............-R $R$

Roan Duke of Eastwood....-R R

Roan Duke of Upton..........L R

Roan George................ P S

Roan Jupiter $2 \mathrm{~d} . . . . . . . . .-$ Cox

Roan Jupiter $3 \mathrm{~d} . . . . . . . . . .-$ Cot

Roan Lad ................. D

Roan Lewis..............

Roan Oakland.............. $\mathrm{R} R$

Roanoke .................

Roan Prince.................... Ky I

Roan Zelia's Jumbo of Risk ... - D

Rob .................... $\mathrm{L} P$

Robert

Robert Bruce................. L R

Robert Bruce......

Robert Burns................... M

Robert Lincoln............

Robert Lincoln ............. D

Robert Richard........... Ky I

Robin .......................

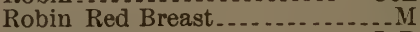

Rob Ridley ........

Rob Roy .........................

Rob Roy ............................

Rob Roy ................... Rose

Rob Roy ......................

Rob Roy

Rob Roy ........... S

Rock ....................

Rockville Lad........ Rolla

Rockwell ..............

Rocky Mountain

Rocky Mountain Boy ........ - D

Rocky Mountain Dnke...... $\mathrm{R} R$

Roebuck ........... - Milk Spring

Roger .................... Ky I

Roger

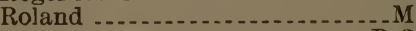

Rollo

Romelo......

Romeo.........

Romeo....... R

Romeo....................... Ky I

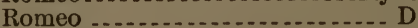

Romeo

Romeo $2 \mathrm{~d} . . . .60 x$

Romeo $3 \mathrm{~d}$

Romulus...............

Romulus........

Romulus_................ $R$

Romulus........... Rolla

Ronald _............... - Rolla

Ronaldo........ R

Roscoe.........................

Roscoe..................

Roscoe

Rose's Barrington .............. L R

Rosy Buck of Gardner......... Ky 1

Rosy's Bedford.............. - Cox

Rnsy's Boy................ Daisy

2d Rosedale's Duke........ - Cox

Rosy Duke................. $\mathrm{R}$

Rosy Duke $2 \mathrm{~d}$

Rose Duke $3 d$................. R
71615 R'e of E'gle Dale's Pr'e Gwy'e 2d-D

71617 Rosemary Duke............. - M

71618 Rosamond's Bates $2 \mathrm{~d} . . . . . . . . .-\mathrm{M}$

71621 2d Rosamond D. of Norwood-Rolla

71623 Rosamond Favorite............... M

71624 Rosamond's Oxford $2 \mathrm{~d} . . . . . . .-\mathrm{M}$

71627 Rosewood .....................

i1628 Rosewood's Duke......

71639 Roubadoux.............

71631 Rover ......

71632 Rover ..........................

71633 - Rowdy

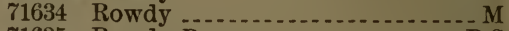

71635 Rowdy Boy

71636 Rowdy Boy ...

71637 Rowland.......

71638 Roy

71639 Royal ...........

71640 Royal Bates.............. Rolla

71642 Royal Booth of Riverdale... - R R

71643 Royal Butterfly 2d ........... D

71644 Royal Bull of Manor Oak...-L $\mathrm{R}$

71645 Royal Champion............. $-\mathrm{L} R$

71648 Royal Duke....................

71649 Royal Duke 2d........... R

71650 Royal Duke 3d.................. P S

71651 Royal Duke 4th.........- - Woods

శ1654 Royal George $2 \mathrm{~d} . \ldots . . . . . . .-\mathrm{L}$ R

71655 Royalist 6th

71656 Royal Joe..................

71658 Royal Mayor......................

$\tau 1660$ Royal Oxford ........... - L R

71664 Royal Prince.............. R R

71666 Royal Purple............ D

71667 Royal Rheam 2d......... Daisy

71668 Royal Richard............... II

71669 Royal Waterloo................. Daisy

71671 Royal Wiley 3d............. - R R

71673 Royal Wiley 5th...........- - R R

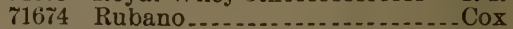

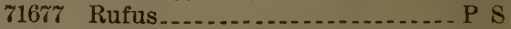

71678 Rugby

71679 Rupert...........

71680 Rush Duke...

71681 Ruskin..........................

71682 Rustler........................ I

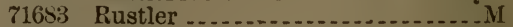

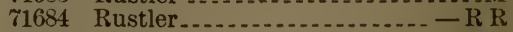

71685 Sailor...................

71686 Saint

71687 Saint Elmo_.............

71688 St. Elmo 4th....................

71690 St. Jacob............ - R

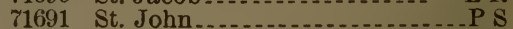

71693 St. John........

71694 St. John

71695 St. John....................

71696 St. John .................. R R

71697 St. John

71698 St. Jonn..................... R

71699 St. Lawrence Duke........... Ky I

71700 St. Nicholas of South View -..L R

71702 St. Patrick ................. D

71703 Sam Loury

71704 Sam Pratt...........

71705 Sam Snook................

71706

71707

71708

71709

Samson

Samson...........................

Samson

Samson.............. 
71710 Sam Taylor...................... Ky I

71711 Sam Tilden............... P S

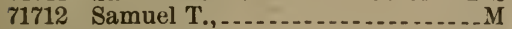

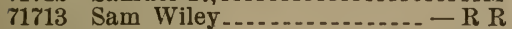

71714 Sam Wiley............... $\mathrm{R} R$

71715 Sancho

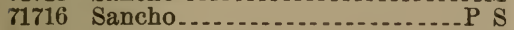

71717 Sancho Panza..... M

71718 Sandoval................. R

71719 Sandy ..........................

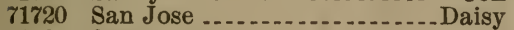

71721 Santague 2d....................

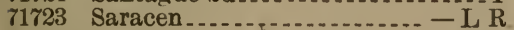

71727 Saxon..............................

71728 Sciota

71729 Scio Victor $3 \mathrm{~d}$

71730 Scotchman's Lad........... Daisy

71731 Scott....................... D

71733 Scranton

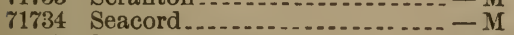

71735 Sebastian Cardec........ Cox

71736 Secrecy's Chief........... R P

r1738 Senator........................ S

71739 Senator

71740 Senator Logan........ P S

71742 Senator Sharon

71743 Senator Vest ............ Daisy

71744 Seneca of Melleray........ - L R

71745 Senor Cherry ............. Cox

71746 Senor Clay

71748 Sentinel..................... R

71749 Seward Boy

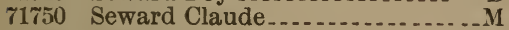

71751 Seward Duke............

71753 Shamrock...................

71754 Sharon

71755 Sharon...........

71758 Sharon Boy

71759 Sharon of Cedar Hill

71760 2d Sharon of Cedar Hill.... - R R

71761 Sharon Duke................ Ky I

71762 Sharon Duke............. R

71766 Sharon Duke........ P S

71767 Sharon Duke............... P S

71768 Sharon Duke............. Rolla

71769 Sharon Duke............ Rolla

71770 Sharon Duke ................ I I

71771 Sharon's Duke .......... Cox

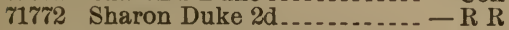

71776 Sharon Geneva Duke ......... D

71777 Sharon Geneva's Duke $2 \mathrm{~d} . . . .-\mathrm{D}$

71778 Sharon Geneva Duke 2d.........D

71779 Sharon Geneva Duke 3d........ D

71780 Sharon Lad................ R R

71781 Sharon Lad 2d......... R R

71782 Sharon-Netherby Duke..... - Cox

71783 Sharon Oxford of Glenwood-Daisy

71787

71788

71789

71790

71791

71792

71794

71795

71796

71797

71798

71799

71800
71801

71802

71803

71804

71805

71806

71808

71809

71810

71811

71812

71813

71815

71816

71817

71818

71819

71821

71822

71823

71824

71825

71828

71529

71833

71834

71835

71836

71837

71838

71840

71841

71842

71844

71845

71846

71847

71849

71851

71852

71854

71857

71858

71859

71860

71861

71862

71863

71864

71865

71866

71867

71868

71869

71870

71871

71872

71873

71874

71875

71876

71877

71878

71879

71882

71884

71885

71886

71887

71888
Simon Rogers ................. - D

Sir Alexander................

Sir Archie................

Sir Arthur...................

Sir Charles.................. S S

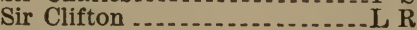

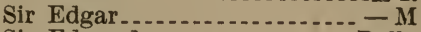

Sir Edward ............. Rolla

Sir Ellwood .................. R

Sir Garnett Wolsey ......... - R R

Sir George................. D

Sir Hancock ..... Cox

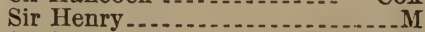

Sir Henry.............. R R

Sir Henry

Sir Henry........... Ky I

Sir Isham .................

Sir Jasper .................

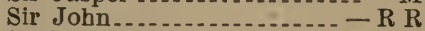

Sir Knight .....

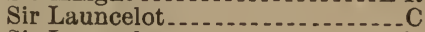

Sir Launcelot....................

Sir Oxford............ Rolla

Sir Phillip........... Rolla

Sir Walter..........

Sir Walter Scott $2 \mathrm{~d} . . . . .$.

Sir Westcott..........

Sir William.....................

Sir Woodcock........ P S

Sittiag Bull.....................

Sitting Bull............

Siva

Smuggler .........

Soco

Soldam Bru .............................

Solomon of Wanoo Valley .. - Cox

Solon

Somonauk $2 \mathrm{~d}$

Sonoma Prince.........

Son of Pearletta......... Rolla

Sonsie Boy $2 \mathrm{~d}$.......... - Beauty

South Carolina Duke.......

Southern Prince......... - Rolla

Sovereign ................. $\mathrm{L} R$

Spangled Banner............. L R

Spangler ........................

Specie............

Speedwell ....... Spencer......................

Spencer Duke Spot'd Chief of Lodi St'k F'm - P S Spotted Wells................ $\mathrm{C}$ Sprightly Duke...... S Springbrook Lad Springbrook Lad $2 \mathrm{~d}$ Springdale Beaconsfield....- L R Spring Grove Chief .........- Cox Stafford .................... Rolla Stanhope Duke ....... L R Stanley .................. R Stanley ............. P S Stanley

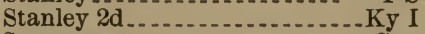
Star.................. Star Duke ......... L R Star Hopewell.......... R R

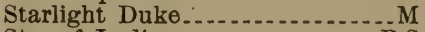
Star of Lodi............ P S Starry Duke .......... D Star of St. Oloff ............... 
71889 2d Star of St. Oloff

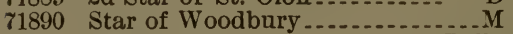

71891 Stately Kirk............. Rolla

71893 Stillwater......................... R

71894 Stillwater Duke 2d...... Cox

71895 Stillwater Lad............. Ky I

71896 Stockbridge Chief............ $-M$

71897 Stockens Ravenswood ..... - R R

71898 Stockings ........... - Long Horn

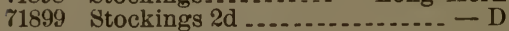

71900 Stone City Prince........ L R

71901 Strawberry Duke 3d....... - Cox

71902 Success .................. R R

71903 Success ................. R

71904 Sucker Boy

71905 Sullivan $\ldots . . . \ldots \ldots \ldots \ldots$ R R

71906 Sullivan $\ldots$

71907 Sullivan

71909 Summit Duke........... R

71910 Sunflower of Forest Hill ...... - D

71911 Sunset................... P S

71912 Sunshine.................. R R

71913 Sunshine.......... T

71914 Snperior

71916 Swartz's Bright Eyes Duke - Daisy

71918 Sweet William ............ Ky I

71919 Swope

71920 Sycamore........................

71921 Sydney Mac

71923 Sylvester..................

71924 Symmetry ..........

71925 Symmetry

71926 Symmetry .........

71927 Talbot 2d.................... R

71928 Tally Ho............... R

71929 Tally Ho...............

71930 Tallyrand....................

71931 Tam O'Shanter............. D

71933 Taopi Boy

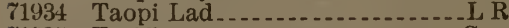

71935 Tariff

71936 Tascar........

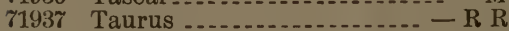

71938 Taurus

71943 Tecumseh..... M

71944 Tecnmseh............................

71945 Tecumseh Duke............ - Cox

71946 Teddy Bell .................. Ky I

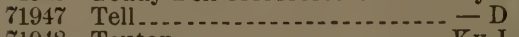

71948 Teuton

71949 Thayer

71951 The Earl

71952 The Favorite....................... R

71953 Theos

71954 The Scot

71955 Theseus $3 \mathrm{~d}$

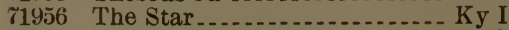

71957 Thomas A. Hendricks ...... - P S

71958 Thomas A. Hendricks....... - R R

71959 Thomas Jefferson......... - L R

71960 Thompson ........ Cox

71961 Thompson

71962 Thorndale Boy

71963 Thorndale Duke.......... R R

71964

71965

71966

71967

71968

71969
71970

71971

71972

71974

71975

ซ1976

71977

71979

71981

71982

\%1983

71984

71985

71987

71988

71989

71990

71991

71993

71994

71995

71997

71998

71999

72001

72002

72003

72006

$7200 \%$

72008

72010

\%2011

72012

72015

\%2016

72017

72018

72019

72020

72021

7*2022

72023

72024

ช2025

ช20\%6

72027

72028

72029

72030

72031

т2032

72033

72034

72037

72039

72040

72041

72042

72043

72049

\%2050

72051

72052

\% 2053

72054

72056

72058

72059

72060

72061
Tiffin Duke ............... - L R

Tillie 4th's Duke..........

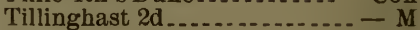

Tintype ...............

Tioge.............. Ky I

Tip............ Ky I

Titus.................-Milk Spring

Tom

Tom

Tom Benton..............

Tom Carl......... Rolla

Tom Don ......

Tom Hardy .

Tom Hendricks

Tom Hendricks........ P S

Tom Hendricks ............ Ky I

Tom Hendricks ......... Ky I

Tom Hendricks ......

Tom Marline

Tommie G., .... P S

Tom Moore......... $\mathrm{R}$

Tommy H., ..... L R

Tom Nelson ...........

Tom Paine....... D

Tom Smith . - .

Tom Thompson ............ - M

Tony Charmer ....... D

Trade Mark ............ $\mathrm{R}$

Tramp.........................

Trenton Star ................ Cox

Tristram Shandy $2 \mathrm{~d}$

Triumph ............... Ky I

Trojan

Troubadour

Trumpeter................... - M

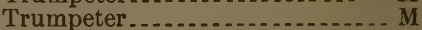

Tuberose Duke $2 \mathrm{~d}$

Tuberose of Willow Park.... - D

Tuck

Tug Wilson.......

Turk ................

Turley Duke $2 \mathrm{~d}$

Turuer....................

Twin Duke........

Twin Dake $2 \mathrm{~d}$

Tycoon .......

Uncle Mell

Uncle Sam

Uniou Duke.......

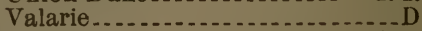

Valentine...........

Valentine

Valentine's Oxford......... - Cox

Valley Duke......... - Milk Spring

Valorous....................... R

Van Adelaide ............. P S

Van Daffodil ................

Vandal........................

Van Flora

Vennor

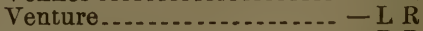

Vermillion

Verona Loudon -........... - L R

Veto........................

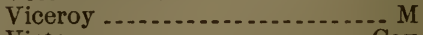

Victor

Vietor

Victor

Victor

Victor $2 \mathrm{~d}$ 
72062

72063

72064

72066

\%2067

$720 \pi 1$

7207\%

72073

72074

72075

72079

72081

72082

72083

72084

72086

72087

72088

72089

72090

72091

72092

72093

\%2094

ז2096

72097

72098

72099

72100

โ2102

72104

72105

72106

72107

72108

72109

72110

72111

72112

72113

72114

72116

72117

72118

72119

72121

72122

72123

72124

$721 \% 5$

72126

72134

72135

72137

72138

72139

72140

72141

72142

72143

72145

72146

72147

72148

72149

72150

72151

72152

72153

72154
Victor of Bartondale....... - Cox

Victor D.,.................. - Cox

Victor Emanuel............... $\mathrm{L} \quad \mathrm{R}$

Victor Hugo...................

Victoria Duke $3 \mathrm{~d} . . . . . . . . . .-\mathrm{L} R$

Victoria Prince 5th ........ - Rolla

Victoria Prince 6th......... - Rolla

Victor $3 \mathrm{~d}$ of Springdale........ - M

Victor of Snnnyside................ T

Vienna Prince..............

Villa Nova Chief .......... - Cox

Vinna's Pride................ Ky I

Viola's Thorndale............ Ky I

Violet's Breastplate_............ $\mathrm{R}$

Violet's Pride.................... R R

Viscount's Jewel......... - Daisy

Wa-As ................. - Rolla

Wabash Duke.............. T

Wabash Duke $2 \mathrm{~d}$

Wabaunsee Lad........... R R

Vade Hampton............ R R

Wade Hampton $2 \mathrm{~d} . . . . . . . . . .$. L R

Wagner..................... Cox

Wakefield 4th.............

Waldemar.................

Walker........................ $\mathrm{R}$

Wallace

Wallace.........................

Walnut.................

Walter................... Rolla

Wamba $2 d$................ - L R

Wapato

Wapsie Prince............ $R$

Wapsie River Prince........ - L R

Wardell ....................

Warfield ............... Rolla

Warren Duke ...............

Warrick Star ......................

Warrior Johnson

Warwick ................. Daisy

Washington_................... T

Washington Duke $2 \mathrm{~d}$

Watchman ................. - L R

Waterford Prince.................. M

Waterloo............ $R$

Waterloo........... R R

Waterloo $2 \mathrm{~d}$

Waterloo 2d.................. P S

Waterloo 5th....................... R

Waterloo 6th............. - R R

Waterloo 7th............ $\mathrm{R} R$

Watonwan .................

Waveland Duke.........

Waverly

Wayne Duke............

Webster 2d....................... Ky I

Webster 3d.........................

Weehawken's Duke...............

Welcome Duke $2 \mathrm{~d}$

Welcome Duke 3d......... - M

West Burlington Eclipse.... - Cox

West Burlington Jim....... - Cox

West Burlington Joe............. M

Western Chief.............. - L R

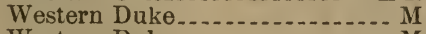

Western Duke...........................

Western Duke............. $\mathrm{R}$

Western Lad.................. D

Western Prince ....... Cox

Westfield
72155

72156

72157

72158

72159

72160

72163

72165

$\tau 2166$

72167

72168

72169

ซ2171

7.172

72173

72174

72176

โ2179

72180

$\tau 2181$

$7: 2182$

$7 \% 184$

72185

72188

$7: 2189$

72190

72191

ๆ2193

72195

72196

$\gamma 2197$

72198

72199

72:201

72:203

72:204

72:205

72206

72210

72:211

72:13

72216

72219

$7 \% 220$

7:22:1

7\%2:23

72:224

722225

722:26

72:227

722228

7\%2:29

72:230

722332

72233

72:234

72237

72:238

72240

72241

$7 \cdot 2243$

72244

72245

72246

72247

72248

72249 .

72250

$72: 54$

72:255
Weston Duke ............. - $\mathrm{R} \mathrm{R}$

Whistler.......................

White Rover

White Royal.................... $\mathrm{R}$

White Stocking -..................

White Stocking......... Rolla

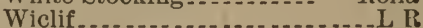

Wildame Duke............. T

Wildare_.................... R R

Wild Bill ...................

Wild Cat Prince.............. Cox

Wild Clair......................

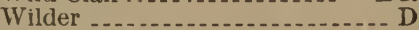

13th Wild Eyes of Armada..... L R

14th Wild Eyes of Armada.....L $R$

15th Wild Eyes of Armada...... L R

Wild Eyes of Columbus.........L R

Wild Eyes Louan.............. M

Wild Eyes Prince .......... Cox

Wild Eyes Prince............ - L R

Wildwood

Wiley Duke......

Wiley Duke........... R

Wilfred............. $\mathrm{R}$

Wilks_................ Rolla

Will..............................

William .............. B

William Dorritt........ $\mathrm{R}$

William Tell................ $\mathbf{R}$

Williamton

Willia's Prince........... - Cox

Willie

Wilmington Duke.......... Rose

Wilton Boy $2 \mathrm{~d}$.................. M

Windsor

Winfield Duke............ D

Winnebago Duke.............

Winneshiek Prince........... Cox

Wisconsin

Wolseley

Woodbine's Admiral.......... - C

Woodland Prince ............. - M

Yellowstone.................. Ky I

Yoeman Jr.,

Young Airdrie

Young Archibald ............ M

Young Bertram .............

Young Bismarck............. Ky I

Young Briton ......... Ky I

Young Captain ................ M

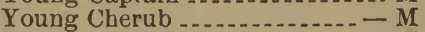

Young Cherub $2 \mathrm{~d} . . . . . . .$.

Young Claremont...........

Young David..................... S

Young Duke................. T

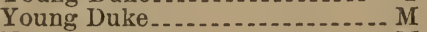

Young General

Young Giant .........

Young Hancock.............. R

Young Harry ............... $-M$

Young Irishman........... $-\mathrm{L}$ R

Young Kirklevington......- $\mathrm{R} R$

Young Knight of Maple Mo'nd-R R

Young Lexington_..........- L R

Young Mac ............... B

3d Young Mary's D, of Bath - R R

Young Mazurka's Duke.... - $R$ R

Young Optimus.......... Ky I

Young Pembroke.........

Young Prince............... 
72256

72257

72258

72:259

72260

72261

$7226 \%$

72263

72364

72265

$72: 266$

72267

72268

$722 \% 1$

72272

$722 \pi 3$

72274

72275

72276

72218

72279

72280

72281

$72: 282$

72353

72236

72291

$72: 242$

ช2:293

72294

72295

72296

$72: 297$

$72: 498$

72299

72300

$7 \cdot 2301$

7.302

72303

7:2304

72305

72308

72310

$7 \cdot 2311$

7.2313

72314

72315

72316

7.31\%

ร2:318

72319

$7 \cdot 2320$

72322

723:33

$723: 4$

72325

72328

72329

$7+2330$

72337

72338

72340

$723+1$

72342

72343

\%2344

72345

72346

72352

72354
Young Prince

Young Riggs............... $\mathrm{R} R$

Young Robarts......................

Young Rockingham.... - Beauty Young Rufus _................. P Young Scott Winfield.......... R Young Thorndale ......... R R Young Wolsley........... L R Ziadee's Prince.................. Zama.......... MI

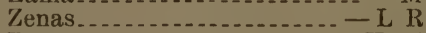
Zeno Zenobia's Duke ............ Zuni.:................ R R Aaron Aaron A. B. Carver. $\mathrm{R} R$ Abe Abe Johnson..... $\mathrm{R}$ Abe Renick Abe Renick........... P S

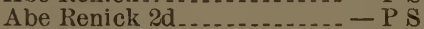
Abigail Prince............ Abradatus............... $\mathrm{R} R$ Abraham ....... P S Achilles................. Daisy Acme Barmpton 8th..... - Daisy Acme Barmpton 9th...... - Daisy Acme Barmpton 10th..... - Daisy Acmon ....................... R Adam Adam Adam 6th.......... R Adam Bede........... C Adam Dufferin Addie's Duke............... - M Adelaide Duke..... Long Horn Adelaide's Oxford......... Adelaide Prince........... R Adelaide Prince....... P S Adeline's Baron....... R R Admiral Farragut........ $\mathrm{R} R$ Admiral Nelson.......... R R Adolph $\mathrm{S} ., \ldots \ldots \ldots$ Adonis Adonis Adonis $2 \mathrm{~d}$ Adrian.... L R Advance.....................

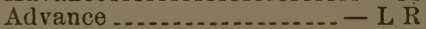
Ad Walker....................... Aneas ............ P S Agamemnon Agassiz . . Agatha's Duke... L R Agtator......... L R Agricola......... Cox Airdrie......... L R Airdrie Airdrie Duke... R R Airdrie Duke............. $C$ Airdrie Duke $3 \mathrm{~d}$ Airdrie Duke 4th.......... P S Airdrie Duke 5th............. - D Airdrie Duke 6th.......... P S Airdrie Duke 7th........ P S Airdrie Duke Sth.......... P S Airdrie Duke 9th .......... Airdrie Joe 2d........... L R

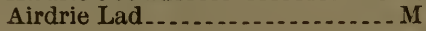

72355

\%2357

พ 2358

72359

72361

$72: 362$

ז236)3

ซ2:364

7236.5

72366

$\tau 2368$

72376

ส23\%

$723 \% 9$

72380

־2381

72382

72383

72384

\% 2385

T2386

7:3388

־2390

\% 2391

ז2392

\% 2394

72395

7ะ3แก

\%2398

\%:399

72100

72401

72402

ก.2403

$7: 2404$

72107

$\pi 2408$

72409

$\tau 2411$

$\tau 2412$

$\tau 2414$

72415

72418

72120

$\tau 2421$

72422

ช2423

\% 2424

72425

72426

72427

$\tau 2430$

พะ) 431

\% 2432

72433

72434

$\tau 2435$

72436

$72+37$

$\tau 2438$

т.239

ז2440

$72+41$

72442

72443

72444

72445

$\tau 2446$

$72+47$

72449
Airdrie Lad $2 \mathrm{~d} \ldots \ldots . . . . . . . .$.

Airdrie of Mar-h Head........ D

Airdrie 2d of Marsh Springs..... D

Airdrie Nectar................ S

Airdrie of Pleasant Hill 14th - R R

Airdrie of Pleasant IIill 15th $-R R$ Airdrie of Pleasant Ifill 16th $-\mathrm{R} R$ Airdrie of Pleasant Hill 17th - L R Airdrie of Pleasant Hill 18th $-\mathrm{P} R$ Airdrie Plumwood........ Multiflora Airdrie Prince............ Airdrie Sharou 5th .......... - M Airdrie Sharon 9th ............ M Airdrie Sharon 10th........... Ky I Airdrie Sharon 11th ........- - Rose Airdrie Sharon 12th ....... - Rose Airdrie Sharon 13th ........-Rose Airdrie Sharon 14th ........- Rose Aircirie Sharon 16th ........... - M Airdrie Sharon 17th ......... Daisy Airdrie Sharon 18th ........... - M Airdrie Th'ndale of Calumet-Daisy Airdrie of Walnut Grove.... - P S Airdrie of Waveland........- $\mathrm{R}$ Ajax..................... Aladdin

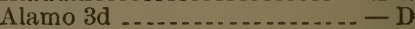
Albert.................. P S Albert................ Albert Lea............... $\mathrm{R} R$ Albert Lee ..................... T Albert Lee......... $R$ Albia Duke......... Albia Lad ............... T Albina's Duke.............. D Alex........................ Alex

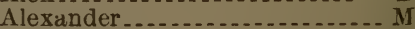
Alexander Alexander Alexander Garfield............ Alexander Meigs.......... Alexis.... . . Alexis of $\mathrm{Oak}$ Hill ............... Alfonso Alfonso Duke $3 \mathrm{~d}$

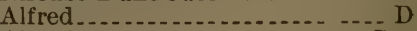

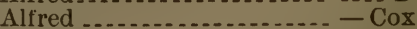
Alfred........... Ky I Alfred Tennyson........... D Alice's Royal.............. $R$ Allen's Bull ............ Allendale Loudon Duke $32 \mathrm{~d}-\mathrm{Cox}$ Allendale Loudon Duke 33d ... - T Allendale Loudon Duke 34th - Cox Allendale Loudon Duke 36th .....-C Allendale Loudon Duke 37th - Cox Allendale Loudon Duke 39th - Cox Allendale Loudon Duke 40th - Cox Allendale Loudon Duke 41st - Cox Allendale Loudon Duke 42d - - Cox Allendale Loudon Duke 43d... Daisy Allendale Loudon Duke 44th - Cox Allendale Loudon Duke 45th - Cox Allendale Loudon Duke 46th Allen Duke............. R R All Right................... All Right_.................... $T$ Alma........................ $\mathrm{R}$ Alonzo Grandiflor.......... Daisy 
72450

72451

72452

72453

72455

72456

72457

72458

72459

72460

72461

72462

$72+63$

72465

72466

72468

72470

$7 \cdot 2471$

72472

72473

72474

72475

72476

72477

$72+78$

72479

$72+80$

T2481

72484

72485

72486

72487

72488

โ2490

T2491

72492

72495

72196

72497

72498

72499

72500

72504

72505

72506

72507

72508

72509

72510

72511

72512

72513

72516

72517

72518

72519

725:20

$725 \div 21$

725\%2

72524

$7 \div 2525$

72527

72528

72529

$725 \div 30$

72531

72532

72533

72534

72535
Alpha ........................

Ambassador 4th.......... Cox

Amelia's Duke..............

Amelia's Lad ..............

Americus...................

Ammon ........

Amrah Duke......... - Beauty

Anak........... R R

Anderson ................ R R

Anderson ................... Ky I

Andrew Jackson ............ - Cox

Andrew Jackson ........... R

Andrew Mead. .......... P S

Angelo .............. P S

Annapulis................ $-L$

Antiquary of Oakland.... - Daisy

Apoll . ..................... - M

Apollo 4th........

Appleton .................. - Cox

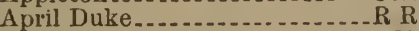

A pril F.,.................. $-M$

Aprillus.................. I

Apriolus ........... Cox

Arab..................... $\mathrm{R}$

Arabella Comet ...........

Arabella Comet $2 \mathrm{~d}$.......... - Cox

Arabella Comet $3 d$......... - Cox

Arabella Comet 4th.......... - L R

Arbutus .....................

Archbald Duke.......

Archbald Duke 2d............ - M

Arch Duke..................

Arch Duke of Deland $2 \mathrm{~d}$

Aretas of Bealsville............ L R

Argus ........................

Argus.................. Cox

Ariosto ......... Cox

Arlington ................... - M

Artemas Ward ..........

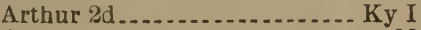

Ar ie........................

Artless.............. P S

Athelstane 9th ............ $\mathrm{R} R$

Athelstane 10th............. $R$

Athelstane 13th ............. - R R

Athelstane 13th................... Ky I

Atlantic....................

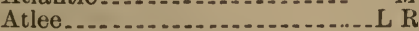

A. T. Stewart........

Auctioneer................ $\mathrm{R} R$

Audi:or 3d..................... R R

Auditor Brown........... Ky I

Augdon Airdrie................... Ky I

Augusta Prince ............ $R$

Angust Duke............... — P S

August Duke.................. R

Augustus........................ $\mathrm{L} R$

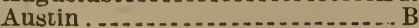

Auten of Oak Hill

Axis

Aylesby Lad............. $\mathrm{R}$

Badger ................... - Cox

Badger Boy ............ P s

Badger Boy .................... Rose

Badger Boy.....................

3d Badger Boy ...............

Balco...............................

Baldwin

Baldy $2 d$........... 1

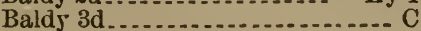

72536

72537

72538

72539

72540

$\tau 2542$

72543

72544

$725+5$

72546

$7: 2547$

72548

72549

72550

72551

โ2552

72553

72554

725.5

72556

$7255 \pi$

72559

72560

72561

72562

72563

72566

72567

72568

72569

72570

72571

ז 2573

$725 \% 5$

72576

72577

72578

72580

\% 2581

72586

\%2587

$7: 2593$

72594

72595

72596

72597

72598

72600

723601

72602

$7 \cdot 2605$

72606

72607

72609

72614

72615

72617

72618

7.2623

$7 \% 26: 55$

72631

72635

72637

72638

\% 2639

\% 2640

72641

72642

72646

72647
Baltimore .................... M

Banco .......................... R

Bancroft................. $-L R$

Banger...................

Banker-................. - C

Banker......................... S

Banner.................... $M$

Bannock.................... L R

Barharossa Chevalier.......... Rose

Barbecue Jr.,............... P S

Barlow ..................... $-M$

Barmpton -........... R R

Barmpton Äirdrie .......... $\mathrm{R} R$

Barmpton Boy .............. Cox

Barmpton Duke.............. - M

Barmpton Lad............... - T

Barmpton's Sidon.......... $R$ R

Barnaby ...................

Barnes.......................... P S

Barnett.................. Ky I

Barney ................... - C

Barney McCoy...........

Barnis Bravo .............. $-\mathrm{L} R$

Bar None.............. $R$

Barnum ...................... Ky I

Barnwall ................... - L R

Baron Barmpton............

Baron Barrington .......... - Rolla

Baron Bates................ - R R

Baron Bates of Cedar Springs - I, R

$2 d$ Baron Bates of Cedar Sp'gs-L R

Baron Blarney ............. - Cox

B 1 ron Bly ............... - R R

Baron Booth......

Baron Booth ................. R R

Baron Booth of Maplewood. - R R

Baron Bright Eyes .........- - Rolla

2d Baron of Carroll......... - R R

Baron Clarence............. $R \mathrm{R}$

Baronet................... - Cox

Baronet.................. Cox

Baron Frantic 5th .......... - Rolla

Baron Geneva of Melrose... - R R

Baron of Glen 2d.......... - L R

Baron Goodness............ $\mathrm{R} R$

Baron Goodness ..........................

Baron Grant................... L R

Baron Gwynne 6th........ - Rolla

Baron Gwynne 7th......... - Rolla

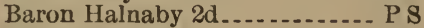

2d Baron Jupiter.......... - Cox

Baron of Kedron............. R R

Baron King .............. P S

Baron of Lakeside......... Cox

Baron Liud 3d................... M

Baron Lind 4 th of Kink River... P S

Baron Mazurka............. - P S

Baron Mazurka $2 \mathrm{~d} . . . . . . . . .-\mathrm{P} S$

Baron Oxford ................ - D

Baron Raby .................... Cox

Baron of Smyrna............. P S

Baron Taylor 2d............. $L R$

Baron Trench............... $-R$

Baron Tuberose......... - Beauty

Baron Tuberose $3 \mathrm{~d} . . . . . .-$ - Beauty

$3 \mathrm{~d} B$. Tuberose of Springboro-L $\mathbf{R}$

4th B.Tunerose of Sp'gboro-Beauty

5 th B.Tuberose of Sp'gboro-Beauty

Baron Watson ............. - Rolla

Baron of Westchester ...........M 


\section{8}

72650

72651

72654

72655

72656

72664

72665

72668

$7: 2669$

72670

72672

726\%3

72674

$7: 2675$

72686

72689

72690

72691

72694

72695

72696

72699

72700

72702

72703

$72 \% 04$

72706

72707

$72 \% 09$

72712

72713

72714

72715

72716

72ส17

72719

$727: 20$

72721

72723

72725

72726

72727

7 2 T28

$72 \% 29$

72\% 30

72731

72733

72734

72735

ซ2\%37

$72 \% 38$

72 739

72740

72741

72742

72743

ส2744

$72 \pi 45$

72 $74 \pi$

72748

ซ2น49

72750

72751

72752

72755

72757

72758

72760

72761
Baron Whiteman........... - PS

Barrington ............... Daisy

Barrington......... Cox

Barrington 12th..........

Barrington 13th

Barrington 14th ..............

Barrington Duke............ Daisy

Barrington Duke $2 \mathrm{~d} . . . . . . . . . . \mathrm{Ky}$ I

Barrington Duke of Banta.... - T 6th Barrington D. of Bluff Creek-T 18th Barrington $\mathrm{D}$. of Cope $\ldots$ - $-\mathrm{T}$ Barrington D. of Whiteland.- - T $2 d$ Barrington of Elmwood....Cox Barrington Genera............ - D Barrett Gwynne 2d.............. Ky I Bart Anderson.............. - L R Bascom ........................ Daisy Bascom 2d ..... Basil .................... Cox $2 d$ Bates of Chestnut Grove ... - D 3d Bates of Chestnut Grove...- - D Bates Cruickshank.......... - Cox Bates Duke ................ - Cox Bates Duke of Goodness ..... - R R Bates Redman of Trout Farm - R R Battie Duke . .............. P S Bayard .................... Ky I Bean Shooter.............. $R$ Beauchamp ........................ Beau of Oxford $4 \mathrm{th}$.......-- Rolla Beauty's Aird'e D. of Silver C'k Cox B'ty's Barmpton of Maine V'y - R R Beauty's Baron ................. Beauty's Boy ......... R Beauty's Chief .................... Beauty's Duke.............. Beanty's Geueva............. Ky I Beaver Prince.............. Berk ..................... P $\mathrm{S}$ Bedford .................... Bedford Forest........ Beecher ................... Beecher-......... $R$ Beecher.............. M Beecher .. . . . Beecher ....... Daisy Beecher Belding Boy... Belina Duke ................ - M Belle Airdrie's Walnut Hill ... - i Bell Bates Waterloo......... - R R Bell Crescent................... P S Belle's Duke............. P S Belle's Duke ...........................

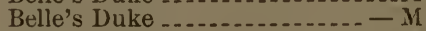
Belle's Duke.................. - M Belle's Duke.............................. Belle Duke................ 2d Belle Duke............ - L R 3d Belle Duke............. L R Bell Duke of Cottonwood...- - L R Bell Duke of.Livingston..... $-R R$ 6th Bell Duke of Lyndale... $-R R$ 5 th Bell Duke of Milford.... - $R R$ 6th Bell Duke of Milford....- $R$ Bell Duke of Ripley ............. M Bell Duke of Walnut...... $R$ Bellfield of Eagle Dale........ - D Bell Phonso ...................... S S Bell Prince.....................
72762

$7: 2764$

72766

72767

72768

72769

$727 \% 0$

72771

$7: 272$

72773

72776

72777

72778

7:2779

$7 \% 2780$

72781

72752

$7: 2784$

$7: 2785$

72786

72757

$7: 2758$

72789

72790

7.2791

72792

72793

72794

$7: 2795$

72796

72797

72798

72799

72800

72801

$7280: 2$

72804

72805

72806

72507

72508

72809

72810

72811

72812

$72 \$ 13$

72814

$7: 2815$

72818

\% 2819

$725: 20$

$728: 21$

$7: 28: 22$

$728: 4$

$728: 5$

$728: 26$

72825

72829

$7: 2830$

72831

728:33

72834

$728: 36$

72837

72838

$7: 2839$

72841

72842

72843

72844
Bell Renick

Bellevue Ned...............

Belpre Lad 9th........... - Rolla

Belpre Lad 12th.......... - Rolla

Belpre Lad 14th............... M

Belpre Lad 15th.............. - D

Belpre Lad 16th............ - Rolla

Belshazzar................ L R

Belvedere Duke......... $\mathrm{R}$

Ben ......................... - M

Ben $2 \mathrm{~d}_{0}$

Ben Boggs......... Rolla

Ben Butler............ P

Ben Butler........

Ben Butler .................

Ben Butler ............. Cox

Ben Butler................. R

Ben Butler ......... Multifiora

Ben Butler ....................... R

Ben Butler .................. $D$

Ben Butler................. $\mathrm{R}$ R

Ben Butler......................

Ben Butler of Chestnut Grove_-D

Ben Clyde ........... A

Ben Davis .............. - Cox

Ben Davis............. R

Bendigo -.................. $M$

Benedict

Ben Foraker ...........

Ben Franklin................ $R$

Ben Franklin............ $R$

Ben Franklin ................

Ben Goff. - .

Ben Hamilton ....................

Ben Hord..... .... Milk Spring

Ben IIur...............

Benjamin

Benjamin B.,....... Daisy

Benjamin Sonsie.......... P S

Benny ...........

Benny $2 \mathrm{~d}$

Bennie B., -...

Ben Myers

Ben Pasha .............

Benson .................... D

Ben Swanson .....................

Benton Oxford......................

Ben Wade.............

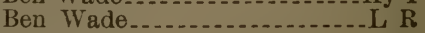

Ben West...........................

Ben Winn ......................

Bergin Lad........ Ky I

Berlin Duke.........

Bernard_........

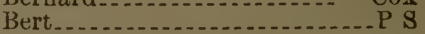

Bert Byron

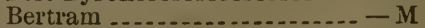

Bertram ........ D

Bertram Lad........

Bethlehem Boy.............

Big Dick Duke...

Bijah Joy -................ Ky I

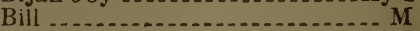

Bill Bass ......

Bill Boy ............................

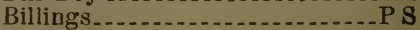

Bill Miartin 
72845

72846

72848

72850

$7: 3851$

72855

72856

72857

72858

72859

72860

72862

72863

72864

72865

72868

72869

72870

$728 \% 1$

72872

72874

72875

72876

72877

72878

72879

72880

72881

72882

72883

72884

7.2885

72886

72887

72858

72889

72890

72891

72892

72893

72894

72896

72899

72900

72902

72903

72906

72907

72908

72909

72910

72911

72912

72913

72914

72915

72916

72917

72918

72921

72922

729:33

$729: 4$

72925

729:36

72927

72929

72930

72931

72932
Bill Morrison

Bill Nye

Billy

Billy .................. $\mathrm{R} R$

Billy

Billy Boy ...................... I

Billy Boylen......... R

Billy Button..........

Billy of Castlerock......... D

Billy Morgan...............

Billy Noble.................L R Billy Pitt of Elm Grove

Billy Richmond .............. D

Billy Shortt...................... Cox

Bimetalist................ - C

Bismarck ..........................

Bismarck -......................

Bismarck -.......... $\mathrm{Ky} 1$

Bismarck .......... R

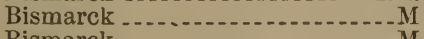

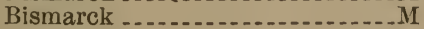

Bismarck ... Cox

Bismarck ................... R

Bismarck ............... Ky I

Bismarck .......................

Black Hawk

Black Hills

Black Jack............... R R

Blaine........... P S

Blaine ............... $R$

Blaine ............... Ky I

Blaine .................... - M

Blaine............................

Blaine -...

Blaine -...

Blaine -.........

Blaine ......................... D

Blaine .................... R R

Blaine...................... D

Blaine of Beaver....

Blaine Lad.................... L R

Blaize -................. - Cox

Blanche's Duke...... C

Bland .............

Bloom D. of Grand Prairie_.- - L R

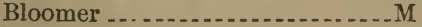

Bloomington

Bloomington King...........

Bloomy's Duke.................. S

Bloomy's Jasper.............. P S

Blossom Duke............ - R R

Blossom's Son of Oxford .... - R R

Blucher.................. R R

Blucher ...................... R

Blucher .................. $R$

Blucher ................... - L R

Bluebell's Lad.............. - P s

Bluegrass Dick:............... S

Bluehill Boy .................... R R

Blythdale Champion 17th...-L R

Blythedale Champion 18th.- - L R

Blythedale Champion 19th.- - L R

Blythedale Champion 20th.. - L R

Blythedale Duke ................ Cox

Blythedale Duke of Elmwood - L R

Blythedale Ernesty 4th.........R R

Board of Trade............. Cox

Bob Duke................. - M

Bob Ingersoll.............. $R$

Bob Ingersoll ................ $-M$
72934

72935

72936

72937

72938

72939

72942

72943

72944

72946

72947

72948

72949

72950

72951

72952

72954

72955

72957

72959

72960

72961

72963

72964

72966

72969

72970

72971

72973

72974

ว2975

โ2976

72979

72980

72981

72982

72983

72984

72985

72986

72987

72988

72989

72990

72991

72992

72993

72994

7:2996

7\%999

73001

73002

73003

73004

73005

73006

73007

73008

73009

73010

73011

73012

73013

73014

73015

73018

73019

73020

73021
72933 Bob Ingersoll 2d...........- R R

Bob Lad................... $\mathrm{R}$ R

Bob Lee............. R R

Bob Lincoln ..... . .............. Rose

Bob Lincoln.....................

Bob Lincoln................ - Cox

Bob Lincoln ..

Bob Wellington........ D

Bob of Wetherby

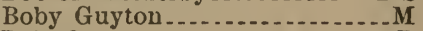

Boiardo.................... - D

Bolivar .............

Bolivar............. R R

Bolivar................... R

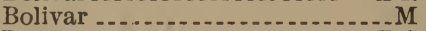

Bon .............

Bonanza ...........

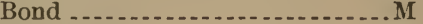

Bon Homme.........

Bonner...........

Bounicastle ............... - Cox

Bonnie's Climax $2 \mathrm{~d}$

Bonnie Duke ............... $\mathrm{R} R$

Bonnie Oaks................. R

Bonnie Oxford.... .............M

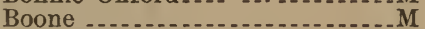

Booth of Sonora...... R R

Bordeaux ............... R R

Boreas of Osborn ............ - D

Bosam......................

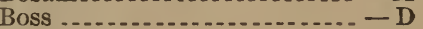

Boss Taylor ............... - Cox

Boston Boy ...............

Bourbon Prince.......... R R

Bower Boy 2d .............. D

Bowery Boy 2d.............. R

Bowery Boy 3d................L R

Bowling.................... R

Boxer .....................

Boxer................... Cox

Bradford........ R R

Bradley -............ $M$

Bradley ...................... M

Brampton Hero 2d............ Cox

Brandon .................. L R

Brandywine ....................

Brantford Lad ..............

Breadlebane 25th............. Ky I

Breadlebane 26th................ R R

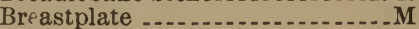

Brian .............

Bridgeport ......... R

Brigadier.................... $-D$

Brigadier................. $\mathrm{R} \mathrm{R}$

Brigand ................ $R$

Brigham _.................. $R$

Brigham .................... Ky I

Brigham Young

Brigham Young............ Daisy

Bright Boy ..................... R

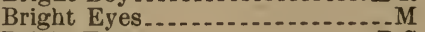

Bright Eyes.................

Bright Eyes Bates......... - Rolla

Bright Eyes Duke............ - M

Bright $\mathrm{E}$ es Duke......... - Cox

Bright Eyes Duke $3 \mathrm{~d}$

Bright Eyes D. of Champlin-Ky I

Bright Eyes King 2d......- - Rolla

Bright Eyes of Troy............... Cox

Bright Hope.................. 
73022

73023

73024

73025

73028

73029

73030

$7: 3031$

73032

73033

73035

73036

73037

73038

$7303 ! 1$

73040

73041

73043

73044

73045

73046

73047

73048

73049

73050

73051

73052

73053

73054

73055

73056

73057

73058

73059

73061

73062

73063

73064

73065

73066

73067

73069

73070

73071

73072

73073

73074

$730 \% 5$

$730 \% 6$

73077

73078

73079

73080

73086

73087

73089

73090

73091

73092

73094

73095

73096

73097

73098

73099

73100

73102

73103

73104

73105
Bright Noble

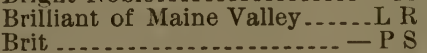

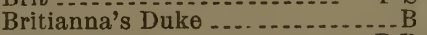
Briton...................... Brock Duke Broderick Junior.......... $\mathrm{R} R$ Broker …................ Broken Horn................ Ky I Brokenstraw Prince............ Brookside..................... L R Brookside Favorite $2 \mathrm{~d}$ Brother Jonathan ..........- Cox Brother Murphy .......... Bruce..............

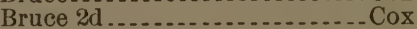
Bruce of Riverside......... I R Bruno...................... Bruno............................ I Bruno Brunswick -.............. $R$ Brutus..................... - Cox Bryan Boy ........ R R

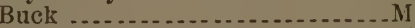

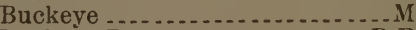
Buckeye Boy .......... Buckeye Boy ................... I Buckeye Brigadier.............. R Bhckeye Lad................ Daisy Buckeye Lad ................. Buckner .....................

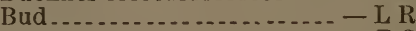
Budwell Buffalo Bill Buffalo Bill........ Buford ............ Bullion ......................... Cox Bull Jim........................ Cox Bully Boy ........... Bulmer ................... Daisy

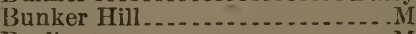
Burlington

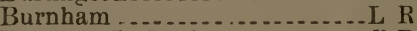
Burnie's Lad 4th.......... R R Burnie's Lad 5th....... R Burr Oak ................. $\mathrm{C}$

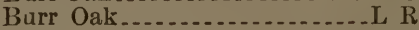
Burr Oak Prince..........- Cox Burton Beecher........... Ky I Bushy Head................ Busti Rajah

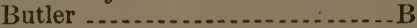
Butler Duke.......... Byron Byron

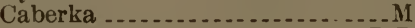
Cabery Lad .......... $\mathrm{R}$ Cable $2 d$. Cactus........... Cadmus................. $-\mathrm{D}$ Caesar................... P S Caesar Calapoia................ Caledonian Prince........... L R Calico Charley .................. $\rightarrow \mathrm{D}$ Califf Calm Lad Cambria Duke $2 \mathrm{~d}$ Cambria Winfield......... Cambridge ................. $\mathrm{R} \mathrm{R}$
73106 Cambridge Duke............ - R R 73107 Cambridge D. of Hill's Grove $-\mathrm{T}$ 73114 Capital Airdrie............ - L R 73115 Captain ..................... 73116 Captain 73117 Captain ............ 73118 Captain 73119 Capt. Allen.......... $\tau 3120$ Capt. Barber......... 73121 Capt. Bates ......... 73122 Capt. Boy 73123 Capt. Cuttle.................. 73124 Capt. Dandy ................... Ky I 73125 Capt. Duke 73126 Capt,"Duke 73127 Capt. ¿Dumars................. 73130 Capt, (ray $21 . . . . . . . . . .2 \mathrm{R}$ 73131 Capt. General............ 73133 Capt. Jack............... R R 73134 Capt. Jack........ 73135 Capt. Jack............... D 73136 Capt. Jack......... Cox 73137 Capt. Jack 73138 Capt. Jack.................. ז3140 Capt. Jack $2 \mathrm{~d}$ 73141 C pt, Jinks 73142 Capt. Johnson ................... R 73143 Capt, Jones 73145 Capt. Latimer...................... 73146 Capt. Leon..... R $\tau 3147$ Capt. May \%3148 Capt. of Oakland........ - Kv I 73149 Capt. Oglevee............ - R R 73150 Capt. Rink................... R R 73151 Capt. Shields............ R R 73152 Capt. Thomas............. Cox 73153 Capt. Tom 73155 Capt. Winkler......... R R 73156 Capt. Wolviston............. T 73157 Cardinal ........................... 73159 Carleton Duke................. D 73161 Carlisle ................................. 73163 Carlisle 73164 Carlos.......... 73165 Carlos $2 \mathrm{~d}$ 73166 Carpenter's Loudon Duke 6th - R R 73167 Carrie's Chief................ - B 73168 Carrie's Duke.............. R R 73169 Carroll Duke............ Cox 73170 Carron Prince.......... 73171 Carsondale .............. Daisy $731 \tau_{2}$ Carter Harrison......... Ky I 73173 Carter Harrison........... P S 73174 Cash Boy................... R 73175 Cashier.................... Ky I 73177 Cashus .........................

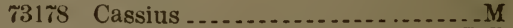

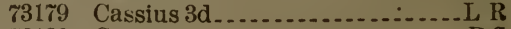
73180 . Castor...................................

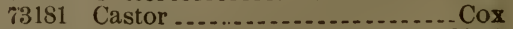
73153 Cavalier 73185 Cedar Boy ............... R

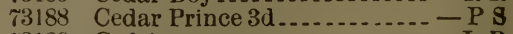
73189 Cedric .................... 73190 Cepheus ................... Cox 73191 Challenge........................ 73192 Challenge B............. 73193 Challenger 73194 Challenger $\ldots . . . . . . . . . . . . . .-M$ 


\begin{tabular}{|c|c|}
\hline & 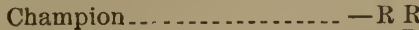 \\
\hline & Champion \\
\hline 02 & R \\
\hline 203 & 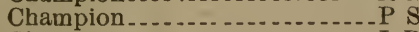 \\
\hline 204 & Champion .... \\
\hline 05 & Champion... \\
\hline 006 & Champion... \\
\hline 207 & Champion... \\
\hline 10 & Champion Boy \\
\hline 11 & Champion Comet. \\
\hline 12 & Champion Duke.......... \\
\hline 13 & Chancellor ........... \\
\hline 215 & Charley ...... L \\
\hline 216 & Charley ..................... \\
\hline 217 & Charley ...... \\
\hline 219 & Charles . . \\
\hline 20 & 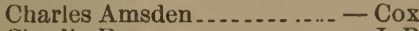 \\
\hline 21 & Charlie B., . \\
\hline 22 & 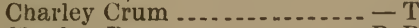 \\
\hline 23 & Charley D., \\
\hline 35 & Charles Felix ................... \\
\hline 26 & 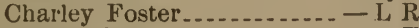 \\
\hline 7 & 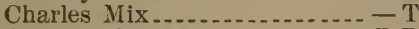 \\
\hline 228 & Charles Napier........... R R \\
\hline 9 & Charley Steel ............ P S \\
\hline 230 & Charles The Kid......... $\mathrm{R} R$ \\
\hline & Charleston . \\
\hline & Charmer ........... Rolla \\
\hline & Chautauqua... \\
\hline & Chautauqua Boy......... \\
\hline & Chatauqua Duke. \\
\hline & Chenault \\
\hline & Cherokee Bill \\
\hline & Cherokee Chief... \\
\hline & Cherry's Duke .............. \\
\hline & Cherry Duke \\
\hline
\end{tabular}

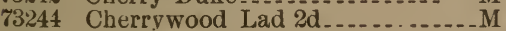

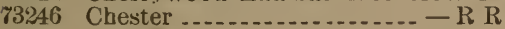

73247 Chestnut Boy

73:48 Chevalier............ Long Horn

73219 Cheyenne Chief.......... R

73:250 Chicago.................... T

73251 Chief

73253 Chief........ P S

73:251 Chief ..................... Spring

73:355 Chief of Jasper $2 \mathrm{~d}$

73257 Chiefrain...................... R

73:558 Chieftain

73:59 Chieftain.................. L

73:360 Chieftain

73261 Chilton............... I

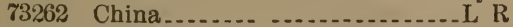

73264 Christmas King

73:267 Chrittenden................... M

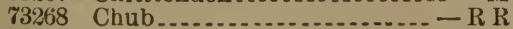

73269 Chub

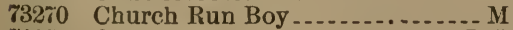

73:71 Chuzzlewit......... R

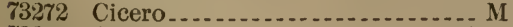

73274 Cicero......

732\% Cincinnatus............ R R

73276 Clara's Duke...............

73:7\% Claremont...................... R

73278 Clarence................ Rolla

73279 Clarence 2d............ $\mathrm{R} \mathrm{R}$

73280 Clarence Duke............ Ky I

73282 Clark Duke...............

73284 Claud of Fairview

73285 Clay Chief .............. $\mathrm{R}$

73286 Clear Creek Embassador........ S S
73287

Clem - ...

73288 Cleo............................. Cox

73289 Cleopatra's Kirklevington .. - R R

73291 Cleveland ................... R

7329:2 Cleveland ................. L R

73293 Cleveland

73294 Cleveland .............. R

73295 Cleveland ............ R

73296 Cleveland ................. L R

73297 Cleveland

7329S Cleveland.................. $\mathrm{P}$ S

73299 Cleveland..........................

73300 Cleveland

¡3301 Cleveland.......... - Long Horn

73302 Cleveland..................... Ky I

73303 Cleveland ..................

73304 Cleveland

73305 Cleveland..................

73306 Cleveland................ Cox

73307 Cleveland.......... R R

73308 Cleveland............... Rolla

73309 Cleveland....... L R

73310 Cleveland.................. S

73311 Cleveland ................... Daisy

73312 Cleveland

73313 Cleveland...................

73314 Cleveland...................... Ky I

73315 Cleveland................. R R

73316 Cleveland

73317 Cleveland

73318 Cleveland.................... S

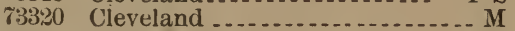

73321 Uleveland.................. R

73:3:2 Cleveland

73323 Cleveland................ $\mathrm{R} R$

73324 Cleveland....... $\mathrm{R} R$

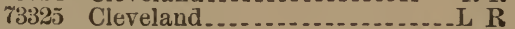

73326 Cleveland ................ L R

733:27 Cleveland

73328 Cleveland..................

73329 Cleveland

73330 Cleveland

73331 Cleveland

$73: 332$ Cleveland

73333 Cleveland................. P S

7:3334 Cleveland 2d................... Ky I

73335 Cleveland 2d........ Long Horn

73336 Cleveland $2 \mathrm{~d} . . . . . . . . .28$

7,3338 Cleveland Boy ......... Woods

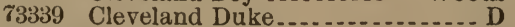

73340 Cleveland Duke ........... R R

73343 Cleveland Lad................. $\mathrm{D}$

73344 Cleveland Lad.............. L R

$\lceil 3345$ Cleveland Oregon............

73346 Cleveland X., ............. T

73347 Clifford

73348 Clifford........

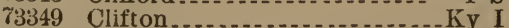

73350 Clifton 5 th

73351 Clifton Glenwood

73352 Climax ......................

73353 Climax

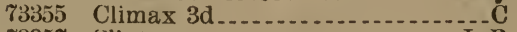

73357 Clinton

73358 Clinton

73361 Clinton Duke ............... - D

\%3362 Clinton Duke $2 \mathrm{~d} \ldots . . . . . . . .$.

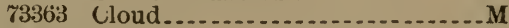

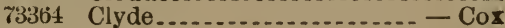

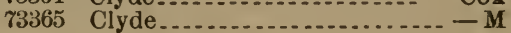


73365 Clyde Airdrie............... - Ky I

73367 Cockrell ................ Daisy

73368 Cockrell Duke.........

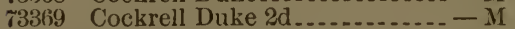

73371 Cordus of Melleray........... - M

73372 Coin Lad ...............

73373 Coin Prince.................. L R

73374 Coke............................

$733 \pi 5$ Colby

73378 Collins Boy 5th........... - Rose

73379 Colman..................... Ky I

73380 Colonel

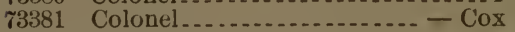

73382 Colonel....................... - M

73384 Col. Acomb 3d d............... Ky I

73385 Col. Airdrie.................

73386 Col. Bennett............. Cox

73388 Col. Chambers ............... R R

73390 Col. Cressbrook ............... D

73391 Col. Davis....................

73392 ' Col. Dick .................. M

73393 Col. Ellsworth.............

73394 Col. Ellsworth ............... P S

73397 Col. Fry ..................... R R

73398 10th Col. Gloster.......... Cox

73399 Col. Grizzy ................ - R R

73400 Col. Hale........................ S S

73401 Col. Hepburn 2d............. Daisy

73402 Col. Hickman ............. - Daisy

73403 Col. Huntington............... Ky I

73404 Col. Hutton

73405 Col. Johnson

73406 Col. Jubilee $2 \mathrm{~d}$

73407 Col. Judy

73408 Col. Keeler.................

73409 Col. King.....

73410 Col. Kregger ........... B

73411 Col. Muir

73412 Col. Muir .................

73414 Col. Rhinerson ............. - R R

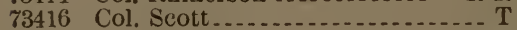

73417 Col. Scott $2 \mathrm{~d}$

73418 Col. Sheldon...................

73419 Col. Stone

73420 Col. Stone........................

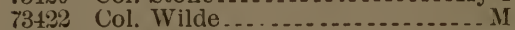

73423 Comanch $\mathrm{Boy}$

$73+24$ Comanche Boy $2 \mathrm{~d} . . . . . . . .-\mathrm{Ky} I$

73425 Comanche Boy 3d.............. I I

$734: 6$ Comanche Boy 4th............. M

73427 Comanche Boy 5th....... R R

73429 Comanche Duke............... II

73130 Comanche Duke 2d........ - Rolla

$73+31$ Comanche Duke 3d ............. . M

73432 Comanche Duke 4th....... - Rolla

73434 Comanche Duke 6th........ - L R

73435 Combination.............. - R R

73436 Combination.................. P S

734:38 Comet..................... R

73439 Comet.............................

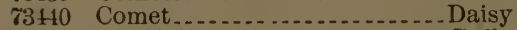

73445 Comfort

7 +47 Commander .............. S

73448 Commander............... $\mathrm{R} R$

73450 Commander-In-Chief.......- L R

73452 Commodore.................. Daisy

73453 Commodore...................... Ky I

73454 Commodore

73455 Commodore................

73456
$7345 \tilde{r}$

73459

73460

73461

$7346 \%$

\%3463

ז3465

73466

73467

73468

73469

73471

$734 \pi 2$

73473

73474

73475

$734 \% 6$

73478

$73+79$

73455

73488

73489

73490

73491

73493

73494

\%3495

73496

73497

\%3498

73499

73500

73501

73502

ז3503

73504

73508

73509

73510

73514

73516

73521

73523

73524

$735: 25$

735:26

7352\%

$735 \% 28$

73529

73530

73531

$735 \overline{3} 3$

73536

735337

\%3538

73540

$735+2$

73543

ז3544

73545

73516

73547

73548

73549

73550

73551

73552

73553

73554

73555
Commodore

Commodore Barney

Commodore Blaine......... - Cox

Commodore Foote........... - R R

Commodore Nut... ............. M

Commodore Perry ............. P S

Compeer ....................... B

Conpeer Duke........

Compromise............. P S

Comus ................... Cox

Comus................ Rolla

Concord Rose............. - M

Conductor...............

Conewango Lad $4 \mathrm{th}$

Confidence.........................

Congressman

Conqueror.......................

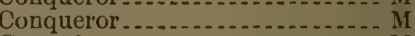

Conrad......................... M

Constance Duke of Ridgeland - M

Constitution.................. Ky I

Conundrum .....................

Convivial .........

Copley

Corn Cracker................. R R

Corporal ........................ Ky I

Corporal's Belle's Duke..... - L R

Corporal's Kate's Duke ........ Ky I

Corporal Whitaker...........

Corsak.................

Cortland Duke....... L

Cosmos...........

Cossack

Cottonwood Louanjo 14th.... Ky I

Counsellor ................ $\mathrm{R}$

Count Butterfly $2 \mathrm{~d} . . . . . . .-$ Rolla

10th Count of Oneida..... $R$

Count Reniek..............

Count Sharon ............. P S

Count Wetherby

Cozine......................

3d Crescent Hill Duke..........

Cricketer......................

Cricketer $2 \mathrm{~d}$

Crimson Lad............. Cox

Cromart...................

Crook Tail........

Crop Ear............... $\mathrm{L}$

Crown Prince

Crown Prince........

Crnickshank............. P S

Cub ............. D

Cudjo.........

Cullom Duke 3a

Cupid ................ Cox

Curdella's Duke........... - L R

Curley .................. $D$

Custer .................. - Cox

Cutler

Cuyahoga Prince.............. Ky I

Cayahoga Rover $2 \mathrm{~d} . . . . . . . . . . . \mathrm{L}$ R

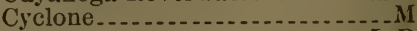

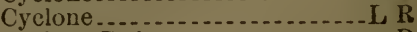

Cyclone Duke........ D

Cypress Duke.............. $\mathrm{R} R$

Cypress Duke...........

Cypress Prince

Cypress Valentine............ C

Cyrus................................ 
73556

73557

73558

73559

73560

73561

73562

73563

73564

73565

73568

$735 \%$

$735 \% 1$

$7357 \%$

73573

73574

73575

735\% 6

73577

ช $35 \% 8$

$735 \% 9$

73580

73581

73582

73583

73584

73585

ร 3586

73587

73588

73589

73590

73591

73592

735.93

73594

73595

73596

73597

73593

73600

73601

73603

73605

73606

\%3607

73608

73809

73610

73611

73612

73613

73615

73616

$7361 \%$

73618

73619

73620

73621

73622

73623

73624

73625

7362 6

$736: 7$

73628

73629

$736: 30$

736ื31

Ү3632
Cyrus......................... Ky I

Cyrus.................... $\mathrm{R} R$

Czar.................

Czar -..................... R R

Dagon .......... Woods

Dainty Davy $2 \mathrm{~d} . . . . . . . .0-\mathrm{D}$

Dairy Boy -................ - L R

Dairy Boy_............... $R$

Dairy Clerk -

Dairyman...................

Daisy's Duke.................... I

Daisy Duke ................ Daisy

Daisy Duke .................. - M

Daisy Duke .......... L R

Daisy's Duke............ - Daisy

2d Daisy Duke............... Daisy

Daisy Duke 4th........... S

Daisy Ecton............... - L R

Daisy Lad.................... Daisy

Daisy's Lad.............. $R$

Daisy Lad $2 d$........... - Rolla

Daisy's Mazurka............. D

Daisy Red Boy ............. $R$

Dakota

Dakota....................... Daisy

Dakota ...................... M

Dakota $2 \mathrm{~d}$

Dakota Boy -............. - Rolla

Dakota Chief......................

Dakota Chief............ - Cox

Dakota Chief 9............ - R R

Dakota Duke...............

Dakota Duke................ - D

Dakota Duke.......................

Dallas ......................... B

Dallas Duke.......... Cox

Dalton Duke ................ M

Dambach.................... D

Damon ........ Cox

Dan

Dan Du ...........................

Dandy

Dandy

Dandy

Dandy ........

Dandy 3d................................

Dandy Dick

Dandy Dude.......

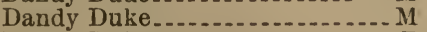

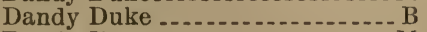

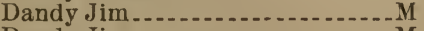

Dandy Jim ..........................

Dandy Jim of Walnut Grove.... M

Dandy King................

Daniel Boone..................... S

Daniel Boone........................

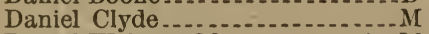

Daniel Webster $2 \mathrm{~d}$

Dan Mace ......... P S

Dan Oxford............ C

Dan Rice...........................

Dan Sharon $2 \mathrm{~d}$

Danton ...................

Dan Warrior

Darlington of Catalpa.......... S S

Darlington of Elmot........ - P S

Darnley ................. - $M$

Darwin

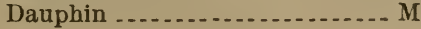

73633

73634

73635

73636

73637

\%3638

73639

73640

736๋41

73642

$736+3$

\% 3644

\%3645

73646

73647

$736+8$

73649

73650

73651

73652

73653

73654

73656

73657

73658

73660

73661

$7366^{\circ} 2$

73663

73664

73665

73666

73668

73669

$736 \%$

73671

73672

$736 \% 3$

73676

73677

73678

73679

73680

73681

73682

73683

73684

73685

73686

73687

73688

73689

73690

73691

73692

73693

73694

ז3695

73696

73697

73698

73701

73702

73703

73704

$73 \% 06$

73707

73708

73709

73711
Daveo.................. L R

David......... $R$

David................ T

David................... - L R

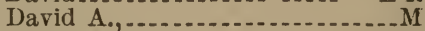

David A., .......... Ky I

David Christy................. L R

David Copperfield

David H., ............ - Long Horn David Noble $3 \mathrm{~d}$

David Ross...................... M

Davie...................... $R$

Davy Crockett............. - Ky I

Davy Crockett................. Ky I

Davy Duke................... P S

Davy Wilson............... - L R

Deacon ........................

Deacon Phelps................ R R

Dean .........................

Dean of Linwood........... P S

Dean of Linwood $2 \mathrm{~d} . . . . . . . . . . . \mathrm{P} S \mathrm{~S}$

Jean of Linwood 3d........... P S

Deceiver ........................ P S

December King......... P S

Decoration .............. Ky I

Deerfield Duke............... R R

Delevan.................... R R

Delaware...................... $\mathrm{L} \mathrm{R}$

Dell Files ................. R R

Delos........................... R

Dennis....................

Dennis Fleet.......

Denverado........ $R$

Derby ..................

Derby ................. $\mathrm{P} S$

Derby ................ P S

Derby $2 \mathrm{~d}$

Deronda.................. Ky I

Dexter................... Beauty

Dexter................. Ky I

Dexter............. P S

Dexter................ P S

Dexter ..........................

Dexter...........................

Dexter................ Cox

Dexter .............................

Dexter Duke................ - P S

Dexter of Hopewell..........Ky I

Diamond ..................... M

Diamond -

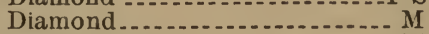

Diamond $2 \mathrm{~d}$

Diamond Duke...........

Dick.................... R

Diek

Dick.....................................

Dick..... Daisy

Dick........ - Cox

Dick ..... P.S

Dick.............. Ky I

Dick .................... $\mathrm{R}$

Dick Bright Eyes........ - Cox

Diek Byron Jr., .................. S S

Dick Ellis............. Cox

Dick Hopper......... C

Diek of Melrose ....... - Daisy

Dick of Oakland.............

Dick Oglesby ............. - Cox

Dick of Sunnyside..............

Dick Taylor Jr.,............. 
73713

$73 \% 14$

73715

73717

73718

73719

73720

ส3\%ะ1

$7372 ?$

73\%23

73725

73726

ช3\%28

73729

73730

$73 \% 31$

73732

$\tau 3733$

73734

73735

73736

73738

73739

73741

73 it3

$737+4$

73745

73746

73747

73748

73749

73750

73751

$73 \% 52$

73753

73754

73755

73756

73757

73758

73759

73760

73761

73762

73763

73764

$73 \pi 66$

73767

73768

$73 \% 69$

73\%ซ 0

73771

73772

73773

73774

73775

737\%6

73777

73ั78

73779

73780

73781

$73 \% 83$

73784

ส378.

73786

73787

73788

73789

73791
Dick Turpin ..................... M Dick Turpin ....................... M Dick Wellington............... Dictator....................... Ky I Dictator ..................

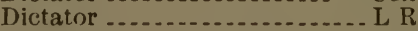

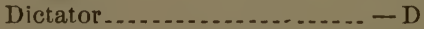
Dictator................... Rolla Dictator...................... Ky I

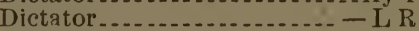
Dictator Dictator $2 d \ldots$ Dingenes.................... Ky I

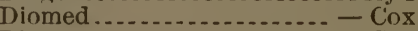
Dinn ..................... Cox Dobbin's Duke.......... M Doc ..... D Doc Black $2 \mathrm{~d}$ Doc Logan.................. - M Doctor -................... I) Doctor Davison ............. R Doctor Dunn Doctor Leonard ................ C Doctor Russell............. Ky I Dod . . Dolah................ $\mathrm{P}$ S Dolphus................. $-\mathrm{L}$ R Dombey Dombey ............... Daisy Dom Pedro.......... Dom Pedro Don Don Donald......... $R$ Donald Donald Duke 26th............ L R Donald Duke 27 th................ M Donald Duke 28th............. R Donald Duke 29th ............. R R Donald Duke 30th ................. Donald Duke 31st................ Donald Kay ..................... R Don Cameron................ R Don Carleton ............ Cox Don Carlos................... R R Don Cleophas............ P S Donham..................... Don Juan ............ L R

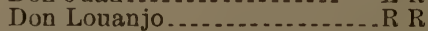
Don Pedro Don Pedro...... P S Don Pedro .............. Don Pedro Don Pedro.................... D Don Pedro................ Don Reddale............... Don Roderick ................. Ky I Dora's Duke............... P S Dora's Duke................. L R Dora's Duke............... P S Doster Prince................. Douglas...................... Douglas........................... Dover Duke.................... Doxey Draco of Melleray Duchess Duke................. Duchess Lad................ Dudau .............................
73792 Dude $\ldots \ldots \ldots \ldots \ldots$ 73793 Duenna Duke.......... 73794 Duenna Duke 4th......... 73795 Duke................

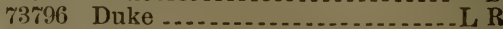
73797 Duke................. 73799 Duke

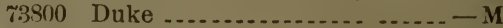
73801 Duke

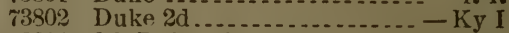
73804 6th Duke of Acklam........ $\mathrm{D}$ 73805 Duke of Addison............. L R 73806 Duke of Airdie.............. - R 73810 Duke of Alderside.......... - L R 73811 Duke Alexander ............ - Rolla

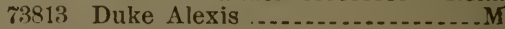
73814 2d Duke Alexis 73815 3d Duke Alexis................. 73816 Duke Alexis 5th 7?319 Duke Allen ............... 73822 Duke of Alloa.......... D 73823 Duke of Alpine............ 73९24 Duke of Alpine 3d.......... R 73525 3d Duke of Alpine......... D 73826 4th Duke of Alpine....... L R $738: 7$ 5th Duke of Alpine........... 73828 6th Duke of Alpine......... R 73830 Duke of Applewood............ M 73831 Duke of Arendahl ............ Daisy 73832 Duke of Argyll ................ Cox 73833 Duke of Arqpll .............. R R 73835 18th Duke of Armada.......- Cox 73836 19th Duke of Armada..... — Cox 73837 20th Duke of Armada...... - Cox 73838 21st Duke of Armada...... - Rnlla 73339 22d Duke of Armada........ - Cox 73840 23d Duke of Armada...... - Cox $738+1$ 2d D. of Armstrong Prairie_ - R R 73843 3d D. of Armstrong Prairie - $-R$ R 73843 4th D. of Armstrong Prairie - R R 73814 5th D. of Armstrong Prairie-R R 73845 6th D. of Armstrong Prairie - R R 73846 7th D. of Armstrong Prairie - Cox 73847 8th D. of Armstrong Prairie-R R 73848 Duke of Ashbrook $2 \mathrm{~d}$.......- $-\mathrm{R} \mathbf{R}$ 73849 Duke of Asher................ M 7385. Duke of Ashgrove ........ - K J I 73851 2d Duke of Ash Grove........ Ky I 7385̃2 9th Duke of Astoria........-R R \%385\% 3 12th Duke of Astoria.......- $\mathbf{R} \mathbf{R}$ 73854 14th Duke of Astoria....... - R R 73855 Duke of Aurora........... 73856 Duke of Ballville.......... 73860 Duke of Bath 18th.......... - Cox 73861 Duke of Bath 19th ......... - Cox 73563 Duke of Bedford.... ....... - M 73864 Duke of Beech Fork .......... - C 73865 Duke of Belknap.......... D 73869 Duke of Belmont................. $7: 3870$ Duke of Bennington........... 73871 Duke of Big Prairie_._ - Long Horn 73872 Duke of Black Hawk ....... - P S 73873 2d Duke of Black Hawk .... - Cox 73874 3d Duke of Black Hawk .... - Cox $738 \% 6$ Duke of Blue Springs 6th..... - C $738 \% 7$ Duke of Blue Valley .......... 73879 2d Duke of Boardman........ S 73880 Duke of Bow Park 2d...... - L R 73881 4th Duke of Brandon.......... Ky I 73882 Duke of Bridgeport.............. R 
73883 2d Duke of Bridgeport.......... L R 73884 3d Duke of Bridgeport.... Daisy 73885 Duke of Broad Creek........ - L R 73886 6th Duke of Broadlawn..... - P S 73887 13th Duke of Broadlawn.... - P S 73888 Duke of Brooklyndale...... - L R 73989 Duke of Brookside............ D D 73890 Duke of Brookville............. I I 73892 Duke of Buccleuch.......... D 73893 5th Duke of Buffalo ........ Daisy 73895 Duke of Buins ........... - R R 73896 Duke of Burr Oak....... - Cox 73899 Duke of Carpenter's Creek .. _ P S 73900 Duke of Carroll.............. R 73901 Duke of Carroll................. 73904 Duke of Cattaraugus........... L R 73905 Duke of Cedar View ...... Daisy 73906 Duke $2 \mathrm{~d}$ of Cherry Grove... $-\mathrm{R} \dot{\mathrm{R}}$ $73 \subseteq 07$ 4th Duke of Cherry Grove.. - R R 73908 Duke of Cherry Tree....... - Cox 73909 Duke of Chili.............. - Cox 73910 Duke of China........... L R 73911 Duke of Clare.................... 73912 Duke of Clarence.................. 73915 Duke of Clay County ......... D

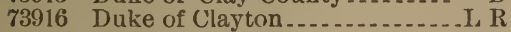
73918 Duke of Cleveland........... R 7:3919 Duke of Cleveland........... D

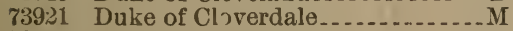
7392: Duke of Clyde............ P S 73923 2d Duke of Clyde............... 73921 4th Duke of Clyde................ 73925 5th Duke of Clyde............... 73926 2d Duke of Cnlon............ Ky I 73927 5th Duke of Columbia....... P S 73928 3d Duke of Columbus........ R 73929 Duke of Commerce......... L R 73930 Duke of Cowley .............. - D 73931 Duke of Crab Grove........ P S 73933 8th Duke of Crawford .............. S 73934 Duke of Crescent Grove...... - D 73935 73936 73937 73938 73939 73940 73941 73942 73943

Duke of Dakota.................... Duke of Danby.......... Ky I Duke of Darwin 7th....... - Cox Duke of Darbyville........ $-\mathrm{R} R$ Duke of Darlington ....... $\mathrm{R} R$ 3d Duke of Dawson ........R R 4th Duke of Dawson....... $\mathrm{R} \mathrm{R}$ Duke of Decatur............... Cox Duke of Decorah................ Duke of Deer Creek.......... D Duke DeGarmo............ Cox Duke of Deland $2 \mathrm{~d} . . . . . .-\mathrm{R} R$ Duke of Dellona................ Duke of Denver............ $\mathrm{PS}$ Duke of Diamond Springs.... - T Duke of Donelson ................ D Duke Donna Maria........ $\mathrm{R} R$ 8th Duke of Dover........ - Cox Duke Draketown ...................... Duke of Duchess $3 \mathrm{~d} \ldots \ldots . . \mathrm{R} R$ 2d Duke of Duchess 3d..... $-\mathrm{P}$ S Duke of Durand............ $\mathbf{M}$ 3d Duke of Earl............. Cox 1Ith Duke of Eastside....... - P S Duke of Edinboro ........... R Duke of Edinburgh Duke of Edinburgh ........ Rolla Duke of Edmund......... Duke of Egypt.................
$739 \% 0$. 73971

73972

73973

73974

73975

73977

73983



73987

73988

\%3989

73990

73991

73992

73993

73991

\%3995

73996

73997

73998

73994

74000

74001

74002

74003

74004

74005

74006

74007

74008

74009

74010

74011

74012

$\lceil 4013$

74014

74015

74016

74017

74018

74023

74027

74028

74029

ז 4031

74032

ז4033

74034

74035

74036

千4039

\%4040

$7 \pm 043$

74044

$740 \pm 5$

74050

$7 \pm 0.51$

74052

74053

74054

74055

74056

74057

740.58

74059

74060

74061

74062

74061
Duke of Elkhart ................. B 2d Duke of Elk Run 3d Duke of Elk Run ............ Ky I Duke of Ellington $2 \mathrm{~d} . . . . . . .-\mathrm{M}$ Duke of Ellinwood....... I. R 3d Duke of Ellison.......... R R Duke of Elmhurst 3d .............. 7th Duke of Elmwood....... 9th Duke of Elmwood......... - D 10th Duke of Elmwood........ - D 11th Duke of Elmwood....... - C 12th Duke of Elmwood........ - D Duke of Emerson................M Duke of Ervin.............. Duke of Ervin 2d........... Duke of Essex Centre....... L R 2d Duke of Essex Centre.... - L R 12th Duke of Evergreen Hill - R R Duke of Exeter........... Multiflora Duke of Fairhury .......... - Cox 2d Duke of Fairbury....... - Cox 3d Duke of Fairbury ......... - Cox 4th Duke of Fairbury........ - Cox 9th Duke of Fairfield....... $-\mathrm{R} R$ Duke of Fairview ............. R R Duke of Fairview 2d Duke of Fairview......... $-\mathrm{D}$ 2d Duke of Fairview....... $\mathrm{R} R$ 2d Duke of Fairview......... - Cox 2d Duke of Fairview....... - Ky I Duke of Fairview 3d............ Ky I 7th Duke of Fairview....... Ky I 8th Duke of Fairview...... $-\mathrm{Ky} \mathrm{I}$ 9th Duke of Fairview...... - Ky $\mathbf{~}$ Duke of Farm Creek........ Duke of Farmington....... $-\mathrm{R} R$ Duke of Farragut........... 5th Duke of Ferndale........... L R 6th Duke of Ferndale........... L R 7 th Duke of Ferndale.......... L R Duke of Florence............. 5th Duke of Fort Scott..... - Cox Duke of Franklin .................M Duke of Franklin.............. Duke of Franklin $2 \mathrm{~d}$. Duke Frederick 2d....... $\mathrm{L} \mathbf{R}$

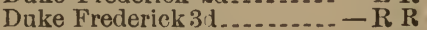
Duke of French Grove............ 18th Duke of Gabilan........ R R Duke of Gales............. R Duke of Galva.......... $-\mathrm{L}$ R 2d Duke of Gentry.............. Duke George...................... Duke of Gladwin ............. Cox Duke of Glasford................ Duke of Glenwood......... R R Duke of Goodness......... - Rolla Duke of Goodness.......... - L R 46th Duke of Goodness..... $\mathrm{R} R$ Duke of Goshen............. Duke of Granby ............ Duke of Grand View........ Ky I Duke of Grant County. ...... - $\mathbf{M}$ Duke of Gray's Flat............. Duke of Greenwood........... D 2d Duke of Greenwood....... Ky I 3d Duke of Green wood ........ R R 4th Duke of Greenwood..... P P Duke of Griswold............. Duke of Hamilton ................M 
74066

74067

74069

74070

74071

74072

74073

74076

74077

74078

74079

74080

74081

74082

74087

74089

74090

74092

74093

74094

74095

74096

74097

74098

74099

74100

74103

74107

74108

74109

74112

74113

74114

74116

$7+117$

74119

74120

74121

74122

74123

74124

74125

74126

74127

74130

74132

74133

74134

74135

74136

74140

74141

74142

74144

74146

74147

74149

74150

74152

74153

74154

74155

74156

74157

74158

74159

74160

74161

74162

74163
Duke of Hampshire..........- Cox

Duke of Hancock ............. - D

21 Duke of Hanover........... P S

Duke of Hardilee............ - R R

Duke of Hastings 5th........ - P S

Duke of Hayfleld .............. L R

Duke of Hayfield............. - D

8th Duke of Hazeldell...... - D

9th Duke of Hazeldell............. D

Duke of Hazelwood ............ P S

2d Duke of Heathwood..... - Cox

Duke of Hickoryhurst........ - M

Duke of Highland .......... - D

Duke Highland ..................

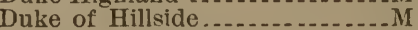

5th Duke of Hillside............ T

8th Duke of Hillside..............

Duke of Holt ...................... M

Duke of Holton

Duke of Homerville......... - L R

Duke of Hopewell...............M

Duke of Hudson..............

Duke of Hume............... S

Duke of Huntsville........... R R

Duke of Illinois .............R R

2d Duke of Ingham .............. R

5 th Duke of Iverness........... L R

Duke of lowa............. - L R

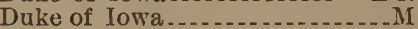

Duke of lowa.............. D

Duke of Jefferson............. $\mathrm{R}$

Duke John ................ L R

Duke J. W., ................. Ky I

Duke of Kansas........................

Duke of Kansas.......................

Duke of Kedron ................... R

2d Duke of Kedron........... L

Duke of Kenderhook...... - Rose

Duke Kent................ R R

Duke of Kent.................... Cox

Duke of Kent.............

Duke of Kent................... R

Duke of Kent.................

Duke of Kingsbury

9th Duke of Kirklevington..... Cox

Duke of Knoxville..........-L R

Duke of Lake Crystal ....... - - R R

Duke of Lake Farm...........

Duke of Lake Pleasant......... L R

Duke of Lakeside.............. -

Duke of Larch Lawn

Duke of Larned .............. S S

Duke of Levander.......... P S

3d Duke of Lebanon........ S

Duke Lester.................... R

3d Duke of Lester............ - C

Duke Lewis 2d............ $\mathrm{R} R$

Duke L'Homedien ........... - Cox

2d Duke of Liberty ......... - R R

3d Duke of Liberty .......... R R

3d Duke of Liberty ................

4th Duke of Liberty...... D

5th Duke of Liberty

12th Duke of Liberty .......... - M

13th Duke of Liberty.......... - M

14th Duke of Liberty......... - M

Duke Linden............... $\mathrm{L}$ R

2d Duke of Linn .............

Duke of Linwood............. - C

4th Duke of Linwood....... $R$ R
74165

74167

74169

74170

$7+171$

74172

74173

74174

74175

74176

74177

$74178^{\circ}$

$7+180$

74181

74183

74184

74186

7418

r4188

74190

74191

74192

74193

74195

74196

74198

74201

74202

74203

74204

74206

74207

$7420 \mathrm{~s}$

74209

74210

74211

ז4212

$74: 14$

74216

74217

74219

74221

74222

\%4223

74224

74225

74226

74227

$742: 29$

74230

74231

74232

74233

74234

74235

74236

74237

74238

74239

74240

74241

74242

74243

74245

74:247

74248

74250

74251

74252

$74: 253$
Duke of Locust Grove.............

Duke of Logansport............ M

4th Duke of Lone Oak....... Ky I

Duke of Longton.............

6th Duke of Lone Oak......... Ky I

2d Duke of Lost Creek........ - C

Duke of Lyon ............... - D

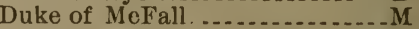

14th Duke of McLean Park - Rolla

Duke of Madison........... - Rolla

3d Duke of Madison......... R

6th Duke of Madison.......- R R

7th Duke of Madison...... $R$ R

8th Duke of Madison......- $\mathrm{K} \mathrm{R}$

Duke Mandane............... $-R$ R

Duke of Mankato..................

Duke of Mansfield...........

Duke of Maple Grove.............

Duke 2d of Maple Grove..........M

Duke of Maple Lawn............. Cox

Duke of Mapleton........ - Woods

Duke of Marengo......... $-\mathrm{Cox}$

Duke of Marengo 2d........ - Cox

2d Duke of Marion............-M

Duke of Marlboro............ $\mathrm{R} R$

2d Duke of Marsh Head.....- $-M$

Duke of Midday............ $\mathrm{L}$

Duke of Middlebury...........P S

Duke of Middlesex .........

Duke of Middletown.......-D

Duke of Millbrook ...........

Duke of Milton........... Ky I

Duke Mizpah.................... Ky I

Duke of Mohican.............. Ky 1

Duke of Molly 4th............. L R

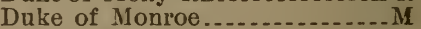

Duke of Montgomery ....... - Cox

Duke of Montrose......... R R

Duke of Munson......... $R$

Duke of Mutual......... $R$

Duke Nectar...............

Duke of Nicholas..............

Duke of Nicollet.................

Duke of Nine Spring

Duke Noble................ $\mathrm{L}$

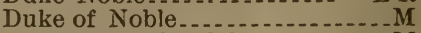

5th Duke of Oakdale............. M

Duke of Oakficld........... P S

Duke of Oakland............. M

Duke of Oakland

2d Duke of Oakland....... - L R

5 th Duke of Oak Park......-Cox

6th Duke of Oak Park.........-M

7th Duke of Oak Park............ M

8th Duke of Oak Park...... - Cox

9th Duke of Oak Park........ - M

10th Duke of Oak Park.......- - M

11th Duke of Oak Park......- - M

12th Duke of Oak Park........ - M

Duke of Oak Ridge......... - Cox.

Duke of Oberlin ............. S

Duke of Oceola................ Ky I

Duke of Ohio ................. Ky I

Duke of Orchard Hill ....... P

Duke of Orleans..................

Duke of Orleans........... Daisy

15th Duke of Osage......... P S

16th Duke of Osage........-Cox

17th Duke of Osage......... P S

18th Duke of Osage.......... - Cox 
74254 Duke of Osceola..... - Long Horn 7425.5 2d Duke of Osceola.. _ Long Horn 74256 Duke of Oshawa......... 74257 Duke of Ottawa......... R R 74258 Duke of Ottawa

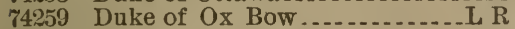

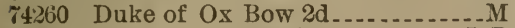

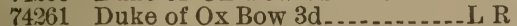
74262 Duke of Oxford ............ 74263 Duke of Oxford 74264 Duke of Oxford 74265 Duke of Oxford ......... R R 74266 Duke of Oxford $2 \mathrm{~d} \ldots \ldots \ldots-\mathrm{R} R$ 74273 Duke of Pawnee........... - D $742 \tau 4$ Duke of Pawnee.......... 7427.5 Duke of Pawnee $2 \mathrm{~d}$ 74276 2d Duke of Pearl Lawn ..... $\mathrm{R} R$ ₹427\% Duke of Penfield................ S S 74278 2d Duke of Perry .......... P S 74279 3d Duke of Perry 74280 4th Duke of Perry 74:281 5th Duke of Perry 74:82 6th Duke of Perry ......... 74:383 7ith Duke of Perry ........ L R 74284 Duke of Petersburg............. Ky I 74287 74288 $7+289$

74290 $74: 991$ 74292 742193 74296 74297 74298 74299 74300 74302 74303 74305 74307 74308 74309 74310 74311 74312 74313 74314 \%4315 テ4318 74319 74320 74321 74322 \%4323 743 ?4
Duke of Pike 4th Duke of Pittsfield........ S 5 th Duke of Pittsfield .......... S 3d Duke of Pleasant Hill ..... $-\mathrm{T}$ 4th Duke of Pleasant Hill ....- R R Duke of Pleasant Lane $2 \mathrm{~d}_{-.}-\mathrm{R} R$ $3 d$ Duke of Pleasant Run..... - D Duke of Pleasant Vale.... - Daisy Duke of Pleasant V ew...... - Cox Duke of Plum Grove R.,.....-C Sth Duke of Portage.......... - D 5th Duke of Poverty Hill.... - P S 6th Duke of Poverty Hill.. - Daisy 2d Duke of Prairie VieW........ T Duke of Princeton Duke of Purple Cane....... $\mathrm{R}$ R Duke of Ranchito.......... Duke of Red Rose.......... D Duke of Redstone....... $R$ Duke of Rich Hill...... $\mathrm{R} R$ Duke of Richland ............ Duke 13th of Richland.... M Duke of Richmond........ $\mathrm{L} \mathrm{R}$ 4th Duke of Ridgevale....- Ky I Duke of Riley County ......-L R Duke of Riverside.......... - Cox Duke Rosamond........ R R 3d Duke of Rosedale............ 6th Duke of Rosedale............

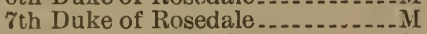
5 th Duke of Rose Lawn......D Duke of Roseville.............. Duke Royal 3d Duke of Runnymede... Rolla 2d Duke of Rush............ - C Duke of Sabina......... $R$ Duke of St. Valentine.... $-\mathrm{R} R$ 2d Dnke of Salem............. Duke of Saline....... R

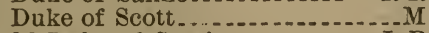
2d Duke of Service............ R Duke of Sharon Duke of Sharon Duke of Sharon...........
74341

74312

74344

74345

74346

$\div 4347$

$743+8$

$7+349$

74351

74352

74353

74354

74356

74357

74358

74359

74360

74362

74363

74364

74365

T4366

74867

74368

₹4369

$7 \pm 370$

74371

ז4372

74373

74374

74375

$743 \% 6$

74377

$7+378$

74379

$74: 382$

74383

74384

74385

74386

74388

74389

74390

74392

74393

74394

74395

74396

74397

74398

74399

74400

74401

74403

74405

74406

74407

74408

74409

74410

74412

74413

74414

74415

74416

74417

74418

74419

74421

74422 3d Duke of Sharon........ - Daisy

6th Duke of Sharon....... - L R 8th Duke of Sharon Duke of Sharon Knight.....- $R \mathrm{R}$ Dute of Sharonville......... - R R Duke of Shelby .......... Duke of Shenango........ - Cox Duke of Sheridan ................. 13th Duke of Skara Glen ..... - R R 14th Duke ef Skara Glen....- - R R 15th Duke of Skara Glen.... - R R 16th Duke of Skara Glen..... - R R Duke of Soldier Creek.........R R Duke of Soldier Creek........R R 2d Duke of Scldier Creek .......... 3d Duke of Soldier Creek... - L R 4th Duke of Soldier Creek - - Rolla 6th Duke of Soldier Creek._ - L R 2d Duke of South Fork .... - Rolla Duke of Southington ............. Duke of Springboro Duke of Springdale........... Daisy Duke of Springdale........ - Ky I Duke of Springfield Duke of Spring Grove Farm .. - B Duke of Springhill ............ - D 2d Duke of Springhill.........-D Duke of Spring Valley 4th Duke of Stafford........ Daisy Duke of Stanton ........... $R$ Duke Sterling 7 th Duke of Stockbridge....... Cox 35̃th Duke of Stock Place.... - R R Duke of Sugar Grove 10th.:- - M Duke of Summit ................... Duke of Sutherland........ - Cox Duke of Sutherland $3 \mathrm{~d} . . . .-\mathrm{R} R$ Duke of Sutherland $4 \mathrm{th}_{\text {...... }} \mathrm{M}$ Duke of Sycamore......... P S Duke of Tama Duke of The Hills......... 22d Duke of The Meadows .. - Cox Duke of The Pines...........- R R Duke of The Valley .............. Cox Duke of The West............Ky I Duke of The West.............. B Duke of The Woods.....- - Daisy Duke of Trego...................... 6th Duke of Trout Farm 7th Duke of Trout Farm........ Duke of Trowbridge:.......... R Duke of Troy -................ $R$ Duke of Underly Duke Van Airdrie 2d Duke of Venedocia...... - P S 3d Duke of Venedocia....... - R R 4th Duke of Venedocia...... - R R 5 th Duke of Venedocia....... - P S Duke of Vernon Place......- R R 36th Duke of Victor........ - R R Duke of Vinewood.......... D Duke of Wabash................ T Duke of Wadsworth Duke of Walnut............... Ky I Duke of Walnut Grove.....- R R Duke of Walnut Hills......- - Ky I 5th Duke of Walnut Park........ D Duke of Wappengers........ - Cox 4th Duke of Watson.......... Ky I Duke of Wayne.............. 8 
74423 14th Duke of Wayne ..............

74424 Duke of Wellington

74425 D. Wellington of Walnut G'e-L R

74426

$7442 \%$

744:28

74139

74431

74433

74435

\% 4439

$74+43$

74444

74445

74446

ז4449

Ү4450

74452

74453

74454

$7445 \overline{5}$

$7445 \%$

74458

74459

74460

74462

74465

74468

\%4469

テ44\%0

74472

ז44\%3

74474

$\div 4475$

74476

74477

74480

74481

$7448 \%$

74483

74184

74485

74487

74489

r4490

74491

74492

74493

74494

r4496

74498

74500

74502

74503

74504

74505

74506

74507

74509

74512

74514

74515

ז4516

74518

74519

74520

745:21

$7+524$

$745 \% 5$

74526

Duke of Wellman........... - M Duke of West Beaver....... $\mathrm{R} R$ Duke of Westblue.......... $-\mathrm{L} R$ Duke of West Grove Duke of Wetona 3d......... - Cox Duke of Whitingham........ $-\mathrm{M}$ Duke Wild Eyes 2d..............M 8th Duke of Willow Creek _. - R R 2d Duke of Winchester..... - Cox 13th Duke of IVindingvale.. $-\mathrm{R} \mathrm{R}$ Duke of Winsome ........ - Rolla Duke of Woodeock........... Duke of Woodland Hall.... $-\mathrm{L}$ R Duke of Woodside.......... $-\mathrm{Ky} \mathrm{I}$ Duke of Woody Glen......- Rolla Duke of Worthington.......... S 2d Duke of Wyandot....... $\mathrm{R} R$ Duke of Wythe............ L R Duke of Yankton.......... M Duke of York.................. Ky I Duke of York.............. Duke of York $2 \mathrm{~d}$ 5 th Duke of York Duke of Yutan........... R R

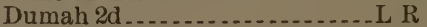
Dumah 3d ...................

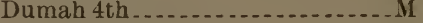

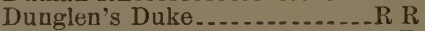
Dunlap 2d................ Duplain Jack............. L R Duplicate ............. Durand ............. R R Durango Duroc........................ Ky I Dusk ........... Dutch....... Duteh Airdrie............ D Dutch Boy .......................... Daisy Dutchman .......................... Eagle Boy ................... Earl of Alamo 4th........... $10 \mathrm{th}$ Earl of Allen......... Cox 11th Earl of Allen.......... Cox 12th Earl of Allen.......... - Cox 13th Earl of Allen.......... $\mathrm{R} R$ 14th Earl of Allen......... Cox Earl Antrim ............. Earl Barrington........... Rolla Earl of Beach Grove........ $\mathrm{R} R$ Earl of Blanchard........... $D$ Earl of Brooklyndale...... $-\mathrm{L} R$ Earl of Carimona........... $D$ Earl of Catalpa............... $\mathrm{P}$ Earl of Cedar Home......... D Earl of Chesterfield....... Rolla Earl of Chetopa ........... 7th Earl of Darlington..... $-\mathrm{L} R$ 8th Earl of Darlington...... $-\mathrm{L} R$ Earl's Derby .................. Ky I Earl Dufferin .............. L R Earl of Elkhorn.......... Earl of Evansburgh....... - Ky I 2d Earl of Fairview......... P S Earl of Fontenelle........... Daisy Earl Goodness 2d...- Milk Spring Earl of Grass Hill........ - Beauty 51 st Earl of Greenbush ..... - L R
74527 74528 74529 74530 $\tau 4531$ 74532 74533 $\tau 4534$ 74585 74536 74540 74541

74542

$\tau 4543$

$\pi 45+4$

74545

74547

ז4548

$\lceil 4549$

74550

74552

$745 \overline{4} 4$

\%4556

74557

74558

74559

74561

ז4563

74564

74565

74566

$\pi 4567$

74568

74569

$745 \% 0$

$745 \pi 4$

$745 \% 6$

$745 \% 8$

74579

74580

74581

74582

74583

74554

74586

74587

74590

74591

74592

74593

74594

74595

74596

74597

т4. 98

74600

74601

74602

74603

74604

74605

74606

74607

74608

74609

74610

74611

74612

74615

74616
52 Earl of Greenbush ...... - L R $53 \mathrm{~d}$ Earl of Greenbush..... $-\mathrm{L} R$ 54th Earl of Greenbush...... $-\mathrm{L}$ P Earl of Greenwood........ $-\mathrm{R} R$ $2 \mathrm{~d}$ Earl of Greenwood..... $\mathrm{R} R$ 3d Earl of Greenwood...... - R R Earl of Hawthorn ............. P S Earl of Highland ........ D Earl of Hillside........... Cox Earl of Hainesville.............. M Earl of Jasper............. Cox Earl of Kansas........... 2d Earl of Knox.............

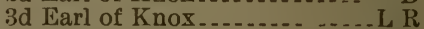
Earl of Lakeside................. Earl of Lakeview............ Cox Earl of Linday....... D Earl of Linn

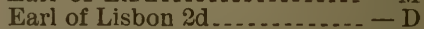
2d Earl of Locust Grove..... - D Earl of Mar Glen.......... Cox Earl of Mohawk.......... Cox Earl of Mosenw Earl of Mounds......... R R Earl of Nemaha........... Ky I

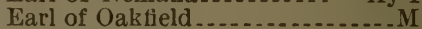
Earl of Oxford............. D Earl of Paw Paw........... $\mathrm{L}$ Earl of Peabody Earl of Prairie Grove...... $\mathrm{R} R$ Earl of Ridgeview............. Ky I Earl of Rome .............. Cox Earl of Sarpy Earl of Shady Side.......... 5 th Earl of Sharon......... Earl of Somers............. Rolla Earl of Springbrook........... Cox Earl of Springdale......... R 2d Earl of Springdale....... $\mathrm{L}$ Earl of Springwood......... R Earl of Suffield .......... Beauty Earl of Syracuse Earl of The Glen....... Daisy Earl of Two Pines............... 4th Earl of Townsend..... R R Earl Wadsworth............. Ky I Earl of Westchester........ Earl of Worcester........ Rolla Earnest.................

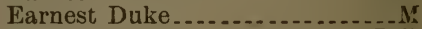
Eaton Farmer......

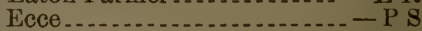
Echo.....................

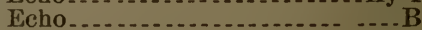
Echo................ Eclipse................

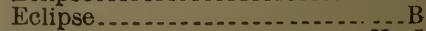
Eclipse...............

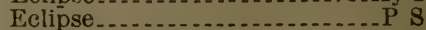

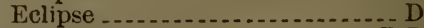
Ecllpse............ Eclipse. . . . Eclipse .................. Eddie E., .................. Eddie Hulse's Lad........ Eden Duke 3d.............. Eden Duke 4th...... D Edgar ........................ Edgehill Prince 2d...-Long Horn Edinburg Duke 5th........... - D 
74617 Edinburg Duke 7th ......... - L R 74618 Ed Moll............... R 74619 Edna's Duke............... 74620 Edward .........................

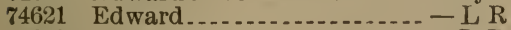
74622 Eglintoun Lad ............ L R 74624 Elba's Best................ P S 74625 Elba Boy............................ 74627 Elector 74628 Electric Light........... R R 74630 Eli

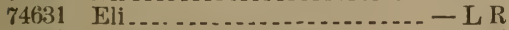
74633 Elizabeth's Geneva.......... - M 74634 Elkhorn Boy .......... - Multiflora 74635 Elkhorn Lad......... - Multiflora 74636 Elk Run Duke...............R R 74637 Elk Run Lad................... R R 74638 Ellah's Duke 2d.......... - L R 74640 Ellah's Underly............ R R 74641 Ellen's Duke............... 74642 Ellwood 74644 El Mahdi.................. $D$ 74645 El Mahdi...................... 74646 El Mahdi

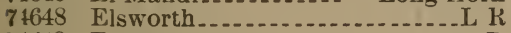
74649 Ember 74650 Emma's Duke 74651 Emma Kendall's Airdrie...... - M 74652 Emory .................... 74653 Emperor ........ 74654 Emperor 74655 Emperor................... R 74656 Empernr................... 746557 74658 74660 74661 746602 74663 $7 \pm 664$ 74665 74666 74667 74668 74669 746670 74672 74673 74674 74675 74676 74677 74678 74679 74680 74682 74685 74686

Emperor Englemar ......... Equinox.............. Erick ............... Eric .......... Eric ........... Erwin Esau Esau $2 \mathrm{~d}$. Esperanza's Duke ......... Esther's Sidon ...................

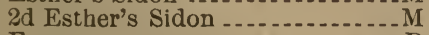
Eugene ................................

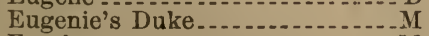
Eureka .................... Eureka Eureka's Lad............... Eureka's Star.............. Europa ................ L R Eurymachus ............ - Daisy Eva's Daisy Duke......... - P S Eva's Duke of Walnut Grove - P S Evergreen Sharon............-M Evergreen Sharon $2 \mathrm{~d} . . . . . . .-\mathrm{Cox}$ Evergreen Sharon 5th........-M Excelsior ............. Excelsior...................... Expansion Duke ............. Ezra's Duke Fairest Eclipse............ -1 R Fairfax Fairholm Baron $2 \mathrm{~d}$. Fairholm Duke............... Fair Manager
Fair Weather............... D Fairy's Duke 3d............... Ky I Fanchon's Duke..........-Daisy Fancy Boy ................. D Fancy Boy Fancy Boy Fancy Boy 2d of Whiteland .. - - T Fancy's Duke................. - M Fancy Major 5th ................. Ky I Fancy Major Booth............. I Fancy Prince.................. M Fanny's Duke................. Fanny 3d's Duke........... L R Fannie's Kirklevington Duke - R R Farmer Boy ................... L R Farmer's Friend.......... $-\mathrm{L}$ Farmer's Pride.............. $-\mathrm{T}$ Farragut ............... Fashion's Captain Fashion's Earl 2d ........... D Fashion's Pride ........... L R Fashion's Sidon_.......... - L R 2d Fashion's Sidon........ - L R Fatty ................... Multiflora Favorite............ Favorite....... Favorite $2 \mathrm{~d}$ Favorite Boy .................... Favorite Briton of Tyron FarmsL $R$ Favorite of Glenwood.........- D Favorite $2 \mathrm{~d}$ of Sunnyside....... T Fawn's Duke................... Ky I Fayette Duke................ R R Fearless

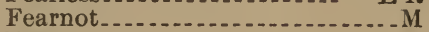
Felix ......... $\mathrm{R}$

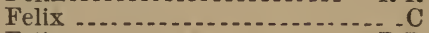
Felix Felix.................. $R$ Fenton Ferney Fern Lad.............

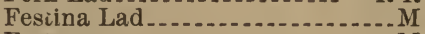

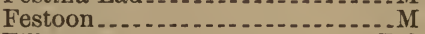
Fillmore............. Fingermark First Chance............ Ky I Fitz Adrian .............. - Rolla 5th Fitzhugh of Maple Grove_ _ M Fitz Walter .............. - L R Five Spot................ $R$ Flash Flora's Duke............ L R Flora's Lad............ Flora's Pride............. Ky I Flora's Prince............ R Florence Duke.............. $T$ Florence Duke.......... Florence Duke............... M Florence Lad................. R Florentia's Duke $2 \mathrm{~d}$. Florenz Duke ........... Floyd Foggathrope of Mellary......_- M Fondy Duke................. Foraker.................. $\mathrm{R}$ Foraker...... Forester................... Forest Inlow.................... 
74803

74805

74807

74808

74809

74810

74811

74812

74813

74814

74816

74817

74818

74819

74820

74821

748:2:2

74323

74825

74827

$748: 28$

$748: 29$

74830

74831

74832

74833

74834

74835

74836

74837

74838

74839

74841

74843

74814

74845

74846

74847

74848

74849

74851

74852

74853

74854

74855

748506

74857

74858

74859

74860

74361

74862

74863

74864

74865

$7486 i$

74868

74869

$7487 \%$

74873

74874

74876

74878

74879

74881

74882

74883

74884

74885

74886
Forest. King -

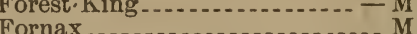

Fortune................... P S

Fortune.............. P S

Fort Wayne Chief...........

Fosters Airdrie................ P S

Fountain Gus .............. D

Four Mile Chief ........... $R$

Fourth of July Duke.......... M

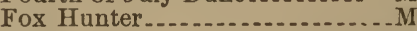

Frank .......... Long Horn

Frank...................... R R

Frank Critzie................_P S

Frank De Garmo...............

Franklin ..................

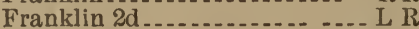

Franklin's Bull .......... - Daisy

Franklin J.,.................. L R

Franklin Pierce................. L R

Fred _..................... D

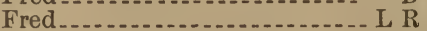

Fred

Fred Bell

Fred Booker....................

Frederick .................. $-P$ S

Frederick William 4th........ Ky I

Frederick William Jr.,......- - L R

Fred Grant..................... - M

Fred Webster ..............

Fred Zerenberg ........... Ky I

Freedom ................. L R

Freedom Lad................... Ky I

Freezeout..................... Ky I

Fremont ........................

Fremont of Kedron...........

2d Fremont of Kedron..........L R

Frigid ..............

Frisco ..................... II

Friskine ................ $R$

Fritz

Frost Duke................ - T

Frosted ............................

8th Gab $\rightarrow n$ of Eastside ...... - P S

15th Gaban of Eastside....... _ P S

2sth Gaban of Eastside....... - P S

Gabba..................

Gaffer Green

Gallant Duke 2d............ - D

Gallant Lad ................... Ky I

Gallant Wiley ............

Game Bird................... I

Gamester ..........................

Garfield ..................... C

Garfield

Gartield................

Garland......................... 1

Garland D. of Soldier Creek - Rolla

Gaston

Gazelle's Oxford........ Rolla

Gem's Chief................. - R R

Gem Duke....................

Gem's Duke........ Cox

Gem Duke $2 \mathrm{~d} . . . . . . . . .$.

Gem's 3d Duke.............. $\mathrm{R}$ K

Gem's D. of Grand Meadow 2d_L R

Gem's D. of Grand Meadow 3d_L R

Gem Maxurka's Oxford ...... - R R

General .................... D

General ...........................

General..................... Ky I
74887

74888

74890

74893

74894

74895

74896

74899

74900

74901

74902

74903

74904

74906

74907

74908

74910

74911

74912

$\tau 4913$

๘4914

74915

74917

T4918

74919

74920

74921

74922

$749: 23$

74924

74925

74926

74927

74928

74929

74930

74931

749:32

74933

74934

74935

74936

74937

74938

74939

74940

74941

74942

ז4943

74944

74945

74946

74947

74948

74949

74952

74953

74954

74955

74956

74959

74960

74961

74962

74963

74964

74965

74966

74967

74968
General .................. - Cox

Gen. Black

Gen. Bly of Riverdale $3 \mathrm{~d}$

Gen. Cleveland.............. P S

Gen. Custer..................... Cox

Gen. Day

Gen. Dick .....................

Gen. Fremont.............

Gen. Gates..........

Gen. Gordon................

Gen. Gordon...............

Gen. Gordon..............

Gen. Gordon ................. L R

Gen. Gordon .............

Gen. Grant ................ P S

Gen. Grant......................

Gen. Grant .............

Gen. Grant ........

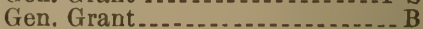

Gen. Grant .............. - Rolla

Gen. Grant........

Gen. Grant -............ - Cox

Gen. Hancock .

Gen. Hancock ............. - R R

Gen. Hancock $2 \mathrm{~d}$

Gen. Hooker ...........

Gen. Hunter.............. $-\mathrm{R} R$

Gen. Jackson .........

Gen. Jones ................ P S

Gen. Kitson ...........

Gen. Lee..............

Gen, Leonidas................ M

Gen. Lewis.................

Gen. Logan ....................

Gen. Logan............ Cox

Gen. Logan .... Daisy

Gen. Logan..... R R

Gen. Logan .......... M

Gen. Logan ................. Ky I

Gen. Logan .......... Ky I

Gen. Logan............... - L R

Gen. Logan ...........

Gen. Logan ... ............ - M

Gen. Long...........

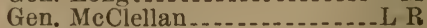

Gen. Marmaduke.............

Gen. Matt Ransom.

Gen. Moore .............

Gen. Napier..................... Ky I

Gen. Nay ................... $\mathbf{S}$

Gen. Ned................

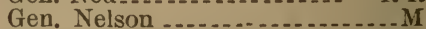

Gen. Norton .................... Ky I

Gen. Porter

Gen. Ray .

Gen. Sale...........

Gen. Sale $2 \mathrm{~d}$.

Gen. Scott.............

Gen. Scott $2 \mathrm{~d}$

Gen. Sedgwick ............. Daisy

Gen. Sheridan....... C

Gen. Sherman

Gen. Sherman ....................

Gen. Shields of Beallsville

Gen. Sigel .................... Cox

Gen. Vespasian of Beallsville_.L R

Gen. Warren .................... $\mathrm{L}$ R

Gen. Washington .............

Gen. Washington......... $\mathrm{R} R$

Gen Weaver................ Ky I 
74969 Gen. Weaver........... R

74970 Gen. Wright ...........

74971 Genesee.................... L R

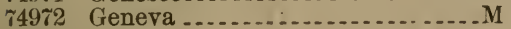

74976 Geneva's Duke................ D

¡4977 Genera Duke................... I I

74978 Geneva Duke.............. - R R

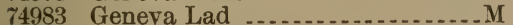

74985 Geneva's Oxford.......... - Rolla

74988 Geneva's Star....................... M

¿4990 Genoa Chief ................ Cox

¡4991 Gentleman George............. L R

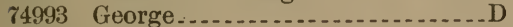

74994 George

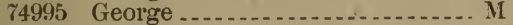

74996 George

74997 George .............................

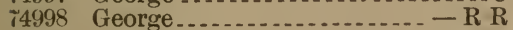

74999 George A.,........... R R

75001 George B.,............... Cox

75002 George Ford............

75003 George Gem...............

75004 George Long ............ R R

7500 5 George May ................ Cox

75006 George Oglan ............... R R

70007 George of Swede Home............. M

5008 George T.,...................

75009 Geo. Thorndale Jr.,....... - Cox

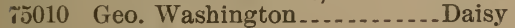

75011 Gerald .................... Daisy

75016 Gideon

i5017 Giftie's Bell Duke....... R R

75018 Gilboa Duke................

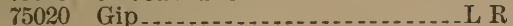

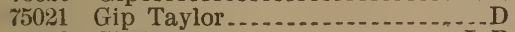

75023 Gladiator ................... R

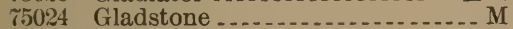

75035 Gladstone.....................

75026 Gladstone

75027 Gladstone.......... R R

¡502S Gladstone $2 \mathrm{~d}$

i5029 Gladstone of Albion..............

5030 Gladstone Jr., ............. - L R

5031 Glancer .......................

5032 Glancer.................... M

75033 Glaucus.......................

75034 Glazier's Molly's Oliver..... - L R

千5035 Glen Avin .................... S

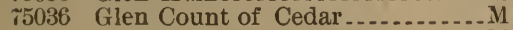

¿5037 2d Glen Count of Cedar..........M

75038 Glen Duke.................... R

\%5039 Glenroy............... Cox

75040 Glenwood Lad 2d.......... - L R

75041 Glenwood Lad 3d........ L R

¡5042 Glenwood Lad 4th.......... - L R

75043 Glenwood Phyllis............ - L R

75044 Glenwood Richmond....... - Cox

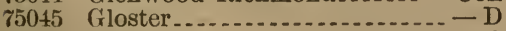

75046 Gloster................

75047 Gloster of Burr Oak............ Cox

75048 Gloster's Duke............ Cox

75050 Gloster of Easton......... - Cox

75051 3d Gloster of Ionia........ Cox

75052 Gloster's Oxford................ R

75053 Gloster of Sharon............. Cox

75054 Gold Coin.................. L R

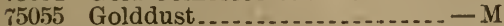

75056 Golddust.........................

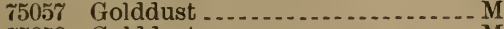

75059
75060

75064

75065

75070

75072

75073

75076

75078

75079

75081

75082

75083

\%5084

75085

75086

75087

75088

75089

75090

$\lceil 5091$

76094

75095

ร5096

75097

75098

75100

75101

$\% 102$

75103

75104

75105

75107

75108

75109

75113

\%5114

75115

75116

75117

75126

75128

75130

$75 ๊ 138$

75140

75146

75148

75150

75151

75153

75154

75155

75156

75157

75158

75159

75160

75161

75162

75163

75164

75166

75167

75168

75169

75170

75171

75173

75174

75175

$751 \% 6$
Golddust 2d.............. R R

Golden Duke................ - M

Golden Duke.................

Golden Prince $\mathrm{ith}$

Goldfinder ...............

Goldfinder

Goldsmith ................ R R

Good Boy .....................

Gooddie Boy

Governor..........................

Governor.........

Gov. Alfred Scales............ - C

Gov. Cleveland ............

Gov. Crittendon......... $\mathrm{R} R$

Gov. Dawes...................

Gov. Glick ...........................

Gov. Grover ................ D

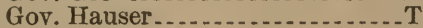

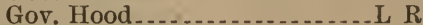

Gov. of Kedron..... R

Gov. MeCreary ..... D

Gov. Martin

Gov. Martin........... Daisy

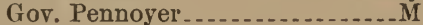

Gov. Rusk................. Daisy

Gov. Zeb Vance..............

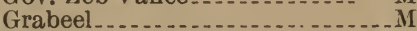

Gracchus ............... - Rolla

Grace's D. of Kirkwood... - Rolla

Grace's Duke of Salem..........

Grace's Hillhurst.......... - L R

Graduate......................

Grafton Lad................. Ky I

Grand Admiral 2d... R R

Grand Duke................. Daisy

Grand Duke............ R R

Grand Duke.................. R

Grand Duke.......................

Grand Dủke $2 \mathrm{~d}$

כth Grand Duke of Broadman ...P S

6th Grand Duke of Gloster - Daisy

Sth Grand Duke of Gloster.... - - T

Grand Duke of Goodness ... _ $R$ R

Grand Duke of Oakhill $2 \mathrm{~d} . . . . .-\mathrm{D}$

Grand Duke of Wells............. D

Grandiose ................... C

Grand Leyburn.......... R R

5th Grand Loudon Duke.... - R R

Grandview Duke........... L R

Granger .................... $-M$

Granger............... Ky I

Granger.................. - P S

Granger....................... $\mathrm{R}$

Granger................... $\mathrm{R} R$

Granger................ $R$

Grant_................... $-D$

Grant................... R R

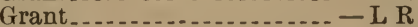

Grant .................

Grant

Grant .............

Grant........................ $R$

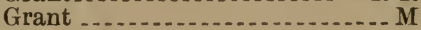

Grant..................... D

Grant

Grant's Duke.................. T

Grayson

Greeley $2 \mathrm{~d}$

Greeley $3 \mathrm{~d}$............... $\mathrm{T}$

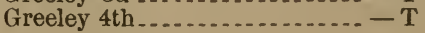


75177 Green Mountain Boy....... - L R

75178 Greenwood Boy................ Cox

75179 Greenwood Duke $2 \mathrm{~d} \ldots . . . . .$.

75180 Greenwood Duke 3d........ M

75181 Greenwood Duke 5th......... L R

75182 Grey Bull .................... R

75183 Grissim's Pride............ - Rose

75184 Gritney Green ............. - R R

75185 Grove City Prince........... Daisy

75187 Grover ....

75188 Grover...$\ldots$

75189 Grover.....

75190 Grover $\ldots \ldots \ldots$

75191 Grover ....................... II

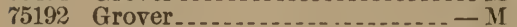

75193 Grover.....................

75195 Grover-..............

75196 Grover..... Cox

75198 Grover........................ Ky I

75199

75201

75202

75204

75205

75206

75207

75208

75209

75210

75211

75212

75214

75216

75217

75218

75219

75220

75221

75222

75223

$752: 24$

75225

75226

75227

75228

75229

75230

75231

75232

75233

75234

ז5235

75237

75238

75239

75240

75242

75243

75244

75245

75246

75248

75249

75250

75251

75252

75253

75254
75255

75256

75257

75258

75259

75260

75261

75262

75264

7526 .

75266

75268

75269

752\% 0

75271

75272

75273

75274

75275

752ส6

75277

75279

75280

75281

75282

75283

75284

75285

75286

75288

75290

75291

75092

75293

75294

75295

75296

75297

750298

75299

75301

75302

75305

75306

75307

75308

75309

75310

75312

75313

75314

75315

75316

75317

75318

75319

75320

75321

75322

75323

75324

75325

75326

5328

75329

75330

75332

75333

75334

75335
Happy Prince............. $-\mathrm{L} \mathrm{R}$

Harden - . . . . . . . . .

Hardin

Harold ...............

Harold ....... $\mathrm{T}$

Harold $3 \mathrm{~d}$

Harry ............... Daisy

Harry

Harry Clay ....

Harry Clay

Harry Cook

Harry Granfield

Harry Hazel...............

Harry L.,

Harry L.,

Harry Napier ................. M

Harry Sargent............. P S

Hartford Duke.......... $\mathrm{R}$

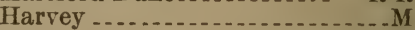

Harvey Sheldon.... Cox

Haus . . . . .

Hawkeye.................

Hawkeye ...................... M

Hawkeye................... R

Hawley .................. M

Hawthorne ..........................

Hazel Duke............ P S

Hazeltine ..................... B

Hazel Bell

Headsland Duke.............. - D

Heber.......................

Hector

Hector

Hector ...........................

Hector....................... $-M$

Hector

Hector .................. $-R$ R

Hector ............

Hedgerow

Heenan .................. $-R \mathrm{R}$

Heir of Broad Creek ....... - R R

Heir of Englishman ..........- M

2d Helen's Compton M.,........ B

Helen's Duke............... - L R

Hemlock Prince................ S

Hendricks................. - L R

Hendricks.............

Hendricks ............

Hendricks................... Ky I

Hendricks.................... S

Hendricks................. $\mathrm{R} \mathrm{R}$

Hendricks ..................

Hendricks..................... L R

Hendricks..................... M

Hendricks................

Hendricks ...................... S

Hendricks .................. Cox

Hendricks........... P S

Hendricks ..................

Hendricks............ $\mathrm{R}$

Hendricks................. - Cox

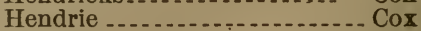

Hengis............. R R

Henry

Henry Clay

Henry Cole............

Henry L., ............... $\mathrm{R}$

Henry R.,

Henry Ward Beecher......... - B

Henry Ward Beecher....... Daisy 
75336 Herculaneum .................-M $7533 r$

75310

75341

75342

75343

г5344

75345

75346

75347

т5318

75349

75350

75351

75353

ธรือบ

75355

75359

75362

75363

75365

75366

\%5368

$753 \% 9$

75373

$75 \div 374$

ซ53\%6

75.3น's

75380

75382

75383

75384

75386

75387

$\tau 5389$

พ5ั390

75391

75392

75393

75394

พริ395

75396

75397

5398

75399

75400

75401

75402

$75+03$

75405

75406

75408

พ5409

75411

75413

75414

75415

75416

$75+17$

75418

75419

75420

$7542 \%$

75424

75426

\%5427

75428

75429

75431

75432

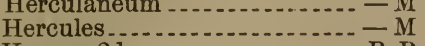

Hermes 2d............. R R

Hermes $3 \mathrm{~d}$

Hermes 4th

Hermes 5th.................. R

Hermit Duke 2d........ R

Hero.........

Hero

Hero of Shiloh ...........

Herod ........ Cox

Herr Hans..........

Hiawatha

Hibernia Duke......... L R

Highland Chief ............

Highland Chief ............. Ky I

Highland Chief ........... - Rolla

Highland Duke 13th........ - R R

Highland Duke 16th........

Highland Duke 17th....... Rose

Highland Duke 21st......... $-\mathrm{C}$

Highland Duke 22d........ R R

Highland Duke 26th........-C

Highland Napier 7th.......- L R

Highland Prince...........

Highland Prince...........

Hill Dom

Hillhurst Duke........... - Rolla

Hillside Joe.............. - Daisy

Hilpa's Oxford.......... Rolla

Hilpa's Pixley Duke........ - P S

Hinkle ......... R

Hippias..... L R

Hippo .........

H. C. Carter .......

Hoadley ......

Hoarfrost ............ P S

Hobson

Hogarth $2 \mathrm{~d} \ldots . . .6 \mathrm{Ky} I$

Holt............. P S

Homer

Homer...... R

Homer...............

Homestead ....... R R

Homesty ............. R R

Honesi Tom................ Cox

Hood .................... R R

Hoosier Boy

Hope

Hopeful

Hopetul Duke $2 \mathrm{~d}$

Horatio ........

Horton

Howard

Howell's Duke............ P S

Howland Duke............

Hoyle..........

Hubback 4 th ................. R

Hubback Jr.,............. P S

Hudson Dennis $2 \mathrm{~d}$

Hudson's Duke 2d............. L R

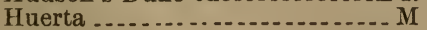

Hulse Geneva 3d......... $\mathrm{R} R$

Huntingdale Chief........ $\mathrm{R}$ R

Ianthe Duke.........

Ianthe Duke............

Idalia Duke 2d........ L R

Ike Harris ................. $\mathrm{L}$

Imperial Clav.

Imperial Duke...... R
75434 Independence............ R R

75435 Indepeudence.......... R R

75436 Independence ............................

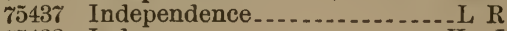

75438 Index.....................

75439 Indian Chief.................

75440 Indian Kentuck............ R R

75442 Instanter.............. Cox

75444 Invincible.................. R

75445 Ingo Chief ................

75146 Ingo Prince............... Daisy

$754+7$ 3d Iowa Lad ............ $\mathrm{R} R$

75448 4th Iowa Lad............. Ky I

75449 5th Iowa Lad............... Ky I

75450 Irak ................ R

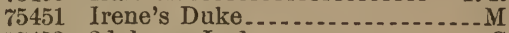

75452 2d lrene Lad...................

75453 3d Irene Lad................

75454 Iron Ore...... R

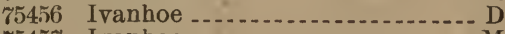

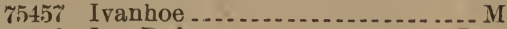

75458 Ivy Duke...... R R

75459 Jacho ..................

75460 Jack Bell $\ldots \ldots \ldots$

75461 Jack Johnson.............

75462 Jack of Maine Valley ...... $-\mathrm{L} R$

75463 Jack of Negro Town...... $-\mathrm{R} R$

75465 Jack Russell ..................

75466 Jack Shepard.............. R

75467 Jackson..................... R

75468 Jackson.......... R

75470 Jack Wright......... R

75471 Jacob.................. R

75472 Jacquemont......... $\mathrm{R}$

75473 Jake Springer................. T

75474 J. A. Logan................ R

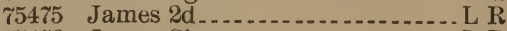

75476 James Clay ............... R

75477 James G.,............... Rolla

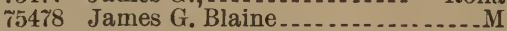

75481 Jamie................... R R

75482 Janolus................... Cox

75484 Jasper Airdrie...... Long Horn

54485 Jasper Chief................... Ky I

75486 Jasper Prince..... Long Horn

75487 Jasper Prince $2 \mathrm{~d} \ldots . . .-$ Long Horn

75489 Jay Gould ................. - Cox

75490 Jay Gould

75491 Jay Gould.................. Daisy

75492 Jay Gould

75493 Jay Gould ........... R

75494 Jeanette's John ......-Milk Spring

75495

75496

75497

75498

75499

75500

75501

75502

75503

75504

75506

75507

75508

75510

75511

75512

75513

75514
Jean's Roan Duke.................

Jeb Stewart....................

Jeff

Jeff Davis............................

Jeff Davis..................

Jefferson ...................... $\mathrm{R}$

Jefferson ....................

Jefferson $2 \mathrm{~d}$

Jefferson $3 \mathrm{~d}$..................... $\mathrm{R}$

Jefferson Duke........

Jericho ..................... C

Jerome.................

Jerome.................... D

Jerome B., ................. Daisy

Jerome Red...........

Jerry

Jerry

Jerry .......................... 
$\pi 5515$

$\tau 5516$

$7551 \%$

75518

75519

75520

75521

ז5523

75524

75525

75526

75529

75531

75532

75533

75534

75535

75536

75537

75538

$\tau 5539$

75540

75541

75543

75544

75545

75546

75547

75549

75550

75552

75553

75555

ๆ5556

7555

75558

75559

75560

75561

75562

755์63

$\tau 5564$

ז5565

755666

755667

ז5569

75570

75571

$755 \pi 2$

75574

75575

75576

$7557 \%$

75579

75580

75581

75583

75584

75585

75587

75.588

75589

75591

75592

75593

75594
Jerry Jerry

Jerry Tyson...........

Jesse......................... - D

Jesse ......................

Jesse.....................

Jesse...........................

Jesse James.....................

Jesse James $2 \mathrm{~d}$

Jeweler .................. - D

Jewel's Oxford............. D

J. G. Blaine..................... Cox

Jim........................ Ky I

Jim.............................

Jim Blaine............

Jim Blaine............... L R.

Jim Blaine..............

Jim Blaine............. Ky I

Jim Blaine............... R R

Jim Blaine ...............

Jim Blaine.......................... Rose

Jim Blaine....................... R

Jim Blaine....................... R

Jim Blaine............. R R

Jim Blaine................ - M

Jim Blaine................

Jim Blaine............. R R

Jim Blaine...................... R

Jim Blaine............ T

Jim Blaine 3d............. D

Jim Burner ........ Daisy

Jim Charlie................ Ky I

Jim Crack............ P S

Jim Davis............ - D

Jim Dixon:

Jim Duke.................. - M

Jim Ecton ................... T

Jim Fenton.......... - Rolla

Jim Fisk .........................

Jim Granite.......................

Jimmy Blaine.......... P S

Jimmy Murphy ........... Ky I

JimmV R.,.................. M

Jim Salzlor............ Daisy

Jingo ......................

Jingo ..........

Joab $2 \mathrm{~d}$

Joab's Duke of Athol...... R R

Joaquin .......................

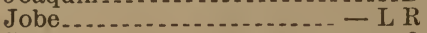

Joe.........................

Joe

Joe Aillen.................... Ky I

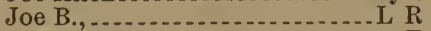

Joe Biggar .........

Joe Favorite .....................

Joe Granger $2 \mathrm{~d}$

Joe Hooker.............. P S

Joe Jefferson ................... Ky I

Joe Johnson ................

Joe Johnson 7 th

Joe Rea.........................

Joe Scott......... $\mathrm{R}$

Joe Scott...............

Joe Turner ................... P S

John ....................... M

John .

John $\tau 5595$

75597

75598

75599

75600

75601

75602

\%ั603

75604

75605

75606

\%560\%

ז5็08

75609

$\tau 5610$

75611

75613

75614

75615

75617

75619

$756: 0$

$\tau 5621$

กัต 622

ธ5623

75624

75625

75627

75628

75629

75630

75631

ๆ5632

75635

75636

75637

75638

75639

75640

75641

$\tau 5643$

75644

75645

75646

75647

75648

75649

75650

75654

75657

75658

75659

75660

75661

75662

75664

ז5665

75666

75667

75668

75669

ז56\%0

75671

75672

75673

75675

75676

75677

75678

75679

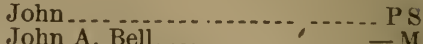

John A. Bell ................. M

John A. Logan........ P S

John A. Logan

John B., .............. R R

John Bell.......................

John Belmont...............

John Brown...................

John Brown........... $R$

John Bull .............. L R

John Eckes........................

John G. Brown .................. S

John Gillette.............. P S

John Gilpin ................... D

John Hollingsworth.......- $\mathrm{R} R$

John Logan ................ - L R

John L. Sullivan ............ T

John Machin ............... R

Johnnie Logan ................. D

Johnny Bull .....................

Johnny Rich.................. B

Johnuy Wick................ M

John Osborn .............. Ky I

John Riley .................

John $S_{.,}, \ldots$

John Sherman...... D

John Sherwood ............... - I

John Wesley .................. Ky I

Jolly ..............................

Jonathan ......................... M

Jonathan . . . . . . . . . . Cox

Jonathan......................... S S

Joseph ...........................

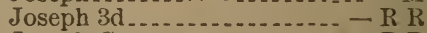

Joseph Geneva............ R R

Joseph Geneva 2d.......... - R R

Josephine Duke ............. B

Josephine Duke $2 \mathrm{~d}$

Josephine Duke 4th...............

Josephine's Oxford......... $\mathrm{L}$ R

Josiah Allen................. - P S

Josie's Noble ................ R R

Josie's Prince ................. . Cox

Jotapata of Beallsville........... M

Joy Duke 2d............... - R R

J. S. Morton...........................

Jubalo ...................... D

Jubilee Duke of Airdrie..... - $R$

Judge .....................

Judge ................... $-\mathrm{P}$ S

Judge......................... R

Judge....................... $B$

Judge Ambler.................. S

Judge Bass ................. $\mathrm{K}$

Judge Buckner ............. $R$

Judge Greene $2 d . . . . . . . . P S$

Judge Hoover............... - D

Judge Jewett ................ Rolla

Judge Matson ............. - Cox

Judith's Duke.............. D

Jules Victor..................... S

Juliet 3d's Duke.......................

Julius_._.

Julius

Julius Cæesar......... Cox

Julius Vetter...........

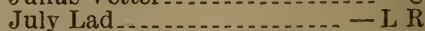

Jumbo..................... $R$

Jumbo......................... 
75681

75682

75683

75684

75685

ช5686

75687

75688

75689

75690

75691

75692

75693

ช5695

75696

75697

75698

$75 \% 01$

7570:

75703

$75 \% 04$

$75 \% ั 05$

$75 \% 06$

รธิช0ิ

รธิง

ช5709

รักร10

$75 ช 11$

75712

สิธน13

75714

7ริน1ร

ชัน 16

พรัช 19

$75 \% 20$

$75 \% 2 \%$

$75 \% 23$

$757 \% 5$

75726

$75 \% 27$

75728

$75 \% 29$

75730

75733

75735

$75 \% 36$

$75 \% 37$

75738

75740

75742

5743

ช5744

$75 \% 45$

75746

75749

75751

$75 \% 5 \%$

$75 \pi 54$

7575๊

75756

$75 \tilde{ธ} \%$

75758

รัพ59

75760

$75 \% 61$

75763

75764

75766

75769

75770
Jumbo

Jumbo.

Jumbo

Jumbo

Jumbo -

Jumbo

Jumbo

Jumbo

Jumbo

Jumbo

Jumbo

Jumbo.

Jumbo

Jumbo .

Jumbo..

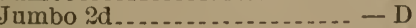

Jumbo 2d...........

Juneau ................

June Boy ............. Mi

Junebug ...................... D

June Duke of Warren...... P S

Junior Duke................ - P S

Junius................. R

Juno ..........

Juno

Juno -

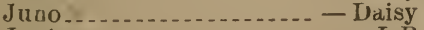

Jupiter...........

Jupiter ............. R R

Jupiter .......... R

Jupiter .....................

Jupiter Jr.,.............. - Ky I

Jupiter Lad...............

Justice.............. R R

Justice Baronet.

Kansas Boy.......................

Kansas Duke.........

Karl Retzer........... Multiflora

Katie's Boy 2d.............. D

Katie's Duke................. B

Kate Kendall's Airdrie....... P S

Katy's Prince............ $\mathrm{R} R$

Kenosha................. L R

Kenton Duke.......... M

Kentuck 2d................. M

Kentuck $3 \mathrm{~d} \ldots \ldots \ldots$

Kentucky Duke ........... - L R

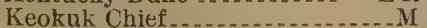

Kilpatrick

Kilpatrick........

Kincade ......

King . . .

King

King Antiquary ........ R R

King B.,....

King Briton .

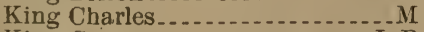

King Comet............ R

Kingeraft.................. $\mathrm{L} R$

King David.......................

King George.............. R R

King Granite....................... I

King Hall .....................

King Harman. ................ B

King Harry ............ Daisy

King of Kansas.......... $\mathrm{R} R$

King of Ohio................ P S

King Phonso................ Cox

King Pineapple.
75771

75773

75774

75776

$75 \% 77$

$757 \% 9$

75780

75781

75782

$75 \% 83$

75784

75785

75786

75787

$75 \% 89$

75791

75792

75793

75798

ก580\%

75803

75804

75806

75807

75809

75811

75812

75814

75815

75816

$7581 \%$

75818

75819

75820

$758: 23$

75824

75825

75830

75834

กั5839

75841

75842

75844

75845

75846

75847

75848

75849

75550

75852

75853

75855

75858

75859

75860

75861

т5862

75863

75864

$\widetilde{75865}$

75866

75867

75870

75871

75873

75874

75875

75880

75882

75886
King Richard............... - Ky I

King Sharon .................

King of Stafford ...............

King of the Prairie....... $R$

King Tom ................ $-\mathrm{M}$

King William

King William.......................

King William 4tb.........

King of York

Kinloc ......................... M

Kinnie's Cypress Duke...... - C

Kinzua.................. $\mathrm{L}$

Kirk .........

Kirk Don Pierola........ - R R

Kirklevington A., ............. M

Kirklevington of Andover .. - Cox

Kirklevington Bell Duke......... M

Kirklevington Boy ................ M

Kirklevington Duke of Elba..._- C Kirklerington Duke of Madison_._B Kirklevington D. of Mayville $3 d-M$ Kirklev'gton D. of M'yville 4th-P S Kirklevington D. of Mt. Zion - R R Kirklev'gton D. of Mt. Zion 2d-P S Kirklevington $\mathrm{D}$. of York ... - R R Kirklevington Knight....... - P S Kirklevington Lad.............- M Kirklevington Lad 10th......- - C Kirklevington of Oakland 8 th-R R Kirklevington of Oakland 9th-Cox Kirklev'gton of Oakland 10th - Cox Kirklevington of Oakland 11th-R R Kirklevington Oxford .......... - C Kirklevington Prince $2 \mathrm{~d}$ Kirkwood................... R Kirkwood Royal........... - R R Kitturah's Duke of Sharon. - Ky I Knight of Gwynne........ - L R Knightly Duke ............... C Knight of The Plains......... - M 4th Knight of The Ridge...... - M Knight of The Rose..... - Beauty Laclede . . . . . . . . . . . . . . . . - L R Lactucarius.................... B Lad ............................. $R$ Lad Duke......................... Lad of Meadow Lawn 2d.....-M Lad of Meadow Lawn 3d..... - M La Due ................. $R$ Lady's Duke................. R R 2d Lady's Duke ................ R R Lady Kirk's Lad........... D Lady Moselles Duke........ - R R Lady's Prince.............. - R R Lady's Thorndale .............. M Lalla's Kirklevington Duke_ _ L R Lalla's Sprightly Duke ....... - L R Lalla's Duke............. - Rolla Lambert.................... Cox Lancaster ................. $\mathrm{R} R$ Lancaster Prince............. Ky I Lancaster Prince........... P S Lark's Booth ................ Ky I Larrabee...................... Last Chance.................. Last Chance's Hero......... L R Laura Ann's Oxford.......... - T Lawn Lancaster............ L R Lawrence Airdrie ... - L Long Horn Leander..................... $\mathrm{P}$ S 
75887

75888

75889

75890

75891

75892

75893

75894

75895

75896

75897

75898

75899

75900

75901

75902

75903

75904

75905

75906

75907

75908

75909

75910

75911

75912

75913

75914

75915

$759^{1} 6$

75917

75918

75919

75920

75922

75924

75927

75928

75929

75930

75931

75932

75933

75934

75935

75936

75937

75938

ซ5939

75940

75941

75943

75944

75945

75947

75948

ז5949

75950

75951

75956

75957

ז5958

75959

75960

75961

75962

75964

75965

75966

75967
Ledan......................

Lee............ $R$

Lee

Lee 5th

3d Lee Duke.............. R

Lee Joseph.............. - L R

Lee Porter ........................

Legal Tender.....................

Lelia's Monarch ........... - R R

Lemuel Bouth................ Ky I

Lentus Duke 6th............

Lentus Duke 8th.......

Lentus Duke 10th..........

Lentus Duke 11th............ - T

Lentus Duke 12th.............

Leo ........................ M

Leo-.................. $R$

Leo

Leo

Leo $2 \mathrm{~d}$

Leo of Kedron................ L R

2d Leo of Kedron...................

Leo Lurat................... Ky I

Leonard......................

Leonard .........................

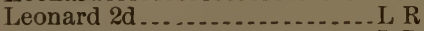

Leonidas................

Leopard .................. - Cox

Leopold

Leroy ............. Cox

Leroy

Leroy Albion..........

Leroy Briton................. L R

Leslie

Leslie Chief...................

Leslie Duke........ Ky I

Lester......................

Lester............... Ky I

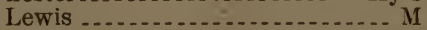

Lewis Airdrie...... $\mathrm{R}$

Lewis Croxton............ Daisy

L'Homedien .................. $\mathrm{L}$

Lieutenant Greeley.......

Lillian Duke............. $R$

Lily's Duke .................. - T

Lily's Duke ....................... B

Limestone............... R R

Linda's Duke........ Multiflora

Lingrove Prince........

Link 5th . . . .

Lion.......... Ky I

Lisbon Boy

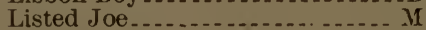

Little Dan.......... S

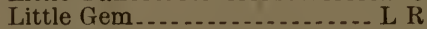

Little Man....... L R

Little Pete ............ $\mathrm{R}$ R

Lively Dean ...... Rolla

Llewellyn Prince 4th ...... D

Logan ............... K I

Logan ......

Logan ...................... Cox

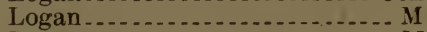

Logan

Logan

Logan -........................ D

Logan ........................ M

Logan ........ L R

Logan .................... Ky I

Logan..............................
75968

75969

75970

75972

75973

75974

75975

75976

75978

75979

75980

75982

75984

75985

โ5986

75987

75988

75989

75990

75992

75994

75999

\%6001

76002

76004

76005

76006

$\pi 6007$

76008

76011

76013

\% 6014

76015

76016

76020

$\tau 6021$

76022

760:23

76024

76025

76031

76033

$\tau 6034$

76035

\%6037

76038

76039

76040

76045

$7604 \pi$

76049

76051

76053

76054

760อ๊ร

76059

76061

76062

76063

76064

76065

76066

76067

$\pi 6068$

76069

76070

76073

76074

76075

ร6076
Logan .................... R R

Logan $3 \mathrm{~d}$

Logan sth Elm Grove .... - Rolla

Logan's Renick ................ M

Logan of Willow Grove Farm ....T

Lohrville Prince............ - L $\mathrm{R}$

Lord Wenlock .............. S S

Lone Star................ - Cox

Lone Star .......... R R

Lone Star - .

Longinus ...................

Longton Chief....

Lord Adrocate . . . . . . . . . . . M

Lord Agnes......... Rolla

Lord Airslie.............. - R R

Lord Alvin $2 \mathrm{~d}$

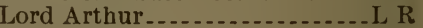

Lord Athol............. R

Lord Baltimore............

Lord Barrington ............-Cox

Lord Barrington of Elmwood _. - C

Lord of Beech Grove.........- $\mathrm{R} \mathrm{R}$

Lord Bellville 2d............... Rose

2d Lord Bickerstaffe...............

Lord Blanch............... R R

Lord Byron..........

Lord Byron ............... - R R

Lord Byron ................

Lord Castleton ........ D

Lord Chalham .............. $\mathrm{L} R$

Lord Chatham 5th........... - $\mathrm{T}$

Lord Chesterfield.............

Lord Cheswick............. Cox

Lord Compton .............. - R R

Lord Compton $2 \mathrm{~d} . . . . . . . . . .-\mathrm{C}$

Lord Compton $3 \mathrm{~d} . . . . . . . . . .-\mathrm{C}$

Lord Cowell .................... M

Lord Cromwell............ $\mathrm{R} \mathrm{R}$

Lord Derby 3d............... S S

5th Lord of Forest Grove....- $R$

Lord Foster............

Lord Fox.............. Ky I

Lord Galloway

Lord Garrison 3d.......... - R R

Lord Garrison 4th......... $\mathrm{R} R$

Lord Goodness.............. R R

Lord Goodness $2 \mathrm{~d} . . . . . . . . .-\mathrm{R} R$

Lord Leslie.................

Lord Lind ..............................

Lord Lonsdale................. Rose

Lord Lynn ..................... S

Lord Marmion

Lord Mordaunt ............. M

Lord Morrington.......... R R

Lord Nithsdale $3 \mathrm{~d}$

Lord Oxford 6th......... $R$

Lord Peri.............. Rolla

Lord Perry ................ M

Lord Reno

Lord Richmond ......... $\mathrm{R} R$

Lord Roscoe................. Ky I

Lord Rose Foote............. R

Lord Rose of Clark............ Ky I

2d Lord Rose of Clark.....-L R

3d Lord Rose of Clark....... $R$

Lord Sharon............. Ky I

2d Lord Sharon........... R

Lord Sharon of Clark........-M

2d Lord Sharon of Clark...... - M 
$7607 \%$

76078

\%6079

76083

76085

76088

76089

76090

76093

76094

76095

76096

76097

76098

76099

76100

76101

76102

76104

76106

76107

76108

76109

76110

76111

ช6115

76116

76118

76119

76120

76123

76124

76126

76127

76128

76129

76131

76132

76134

76135

76137

76138

76141

76143

76145

76146

76147

76148

76150

76151

76152

76153

76154

76155

76156

76157

76158

76159

76160

76161

76162

76163

76164

76165

76166

76167

76163

76170

76171
Lord Spencer - -

Lord of Springwood

Lord Stanley of Merrill..... - Cox

Lord Thornapple 3d........ - L R

Lord Ward

Lord Wild Eyes .............

Lorena's Duke................. S

Lorena's Prince..... ........... L R

Lorraine.............................

Lota's Duke............ R

Louan Airdrie's Rocket...... - R R

Louan Breastplate.......... - R R

Louan's Gloster ............ $\mathrm{R} R$

Louan's Hillhurst............ $R$

Louan Jumbo.............. - R R

Louan's Dake ............. Ky I

Louan Duke $2 \mathrm{~d}$

Louan Duke 3d............. $-R$

Louan Duke 4th of Calumet_ - R R

Louan's Duke of Silver Creek - R R

Loudon Boy .................. $-\mathrm{M}$

Loudon's Climax........ $\mathrm{R}$

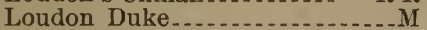

Loudon Duke..............................

Loudon Duke 15th

Loudon Duke 64th........ - R R

Loudon D. of Woolworth $2 \mathrm{~d}_{-}-\mathrm{R} R$

Loudon D. of Woolworth 3d - R R

Loudon Fairfax 2d......... - Rolla

Loudon's Gunter........... L R

Loudon Lad................ M

Loudon Prince ........... $L$

Loudonville Jim........... Ky I

Louis 11th................. - R R

Louis Riel................... $-\mathrm{P}$ S

Louis Sherley ............. $-\mathrm{L}$

Louisville..................... $-\mathrm{L}$ R

Lowell.............................

Lowell Duke.......................

Lucas .................... $R$

Lucius ..................

Lucknow .............

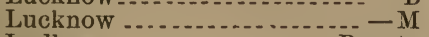

Ludlow ............. - Beauty

Luke.........................

Lurt .......... Ky I

Lustro

Luther Nonpariel .........

Lutzen Duke...............

Lycurgus ..........

Lyndon .................... Cox

Lyndon Chief................... Ky I

Lynn Lawn Lad............. - R R

Mac.........................

Macawber .......................

McBeth .......

McCann

McClellan's Duke 3d....... - L R

McCullough........... - Multiflora

Macduff........................ $\mathrm{R}$

McElwain's Alexander........ - M

McElwain's Best............. - M

McElwain's Boston.........

McElwain's Burnside..........

McElwain's Duke ............. M

McElwain's D. of Darling ... - L R

McElwain's Pilot................. T

McElwain's Red Cloud.....- L R

Macgregor ....... Milk Spring
76172

76173

76174

76177

76178

76179

76180

76181

76186

76187

76188

76189

76190

76191

76192

76193

76194

76195

76196

76197

76198

76199

76200

76201

76202

76203

76204

76205

76206

76207

76208

76209

76210

76212

76213

76214

76215

76216

76217

76218

76219

76221

76223

76224

76:226

76228

76229

76230

76231

76235

76236

76237

76240

76243

76244

76245

76246

76247

76250

76251

7625?

76253

76254

76255

76256

76257

76259

76260

76261

76262
Mack .................... $\mathrm{R} \mathrm{R}$

MeLaren .

McManus .................

Madelia..........

Madison ..............

Madison

Maggie's Duke

Maggie 2d's Duke...........

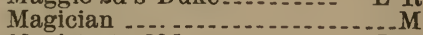

Magistrate $32 \mathrm{~d}^{2}$

Magnus ..................... Cox

Magoun ...................

Mahogany Duke........... P S

Mahomet …............ Cox

Maid's Duke ............................

Majesty .....................

Majesty

Major

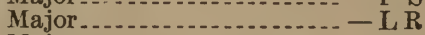

Major

Major ............................

Major

Major

Major

Major .............

Major

Major Allen -........

Maj. Arthur..............

Maj. Ascot............... Ky I

Major B.,...............

Maj. Bagstock ....................

Maj. Belden

Maj. Bloom

Maj. Booth

Maj. Brown

Maj. Bruno

Maj. Burke........ $R$

Maj. Clark .......... $R$

Maj. Dean ... Rolla

Maj. Doc....................

Maj. Dunn ......... Ky I

Maj. Frantic...........

Maj. General.............. $-R$ R

2d Maj. General....... $R$ R

Maj. Gould ...............

Maj. Gwynne $2 \mathrm{~d}$

Maj. Gwynne of Hector...._- L R

Maj. Hill.................. $\mathrm{R} R$

Maj. Kent............................

Maj. of Maine Valley

Maj. O'Doud .............. Multitlora

Maj. Perfection .................. R

Maj. Rose ........................

Maj. Sharon ........................

Maj. Strait

Maj. Vanderbiltt.......... $L R$

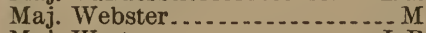

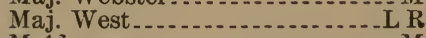

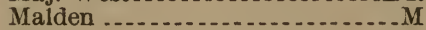

Malry

Malvolio of New Castle...... - B

Maylneaux .................. $\mathrm{L}$

Manchester

Mandon................

Manhatten Prince.......... $-M$

Manzat ......................... M

Mareh ............ P S

March Boy............

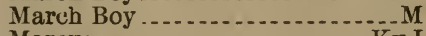

Mareus....... 
76263

76265

76266

76267

76268

76271

76272

76273

76274

76275

76276

76277

76278

76279

76280

76281

76282

76283

76284

76285

76286

76287

76289

76290

76291

76293

76294

76296

\%6297

76298

76299

76300

76301

76302

76303

76304

76306

76308

76310

76312

76313

76314

76315

76316

76318

76319

\%6320

76321

76323

76324

76325

76328

76331

76332

76333

76335

\%6336

76337

76338

76339

76341

76342

76343

76344

76345

76349

76350

76351

76352

76353
Marcus

Marenus

Margrave..................

Margrave 2d......

Margrave $3 \mathrm{~d} . . . . . . . . . .1 \mathrm{~T}$

Marion Chief.................

Mark .................... $\mathrm{R}$

Mark Anthony............ S

Mark Anthony .............

Mark of Mapleshade.......... L R

Mark Smith................. R

Mark Twain ..................... R

Mark Twain...............

Marmaduke................. M

Marmaduke...............

Marmaduke.................

Marmaduke.............. $\mathrm{R} R$

Marmaduke............... L R

Marmaduke.............. $-\mathrm{L}$ R

Marmaduke............. $\mathrm{L}$ R

Marmaduke ............... Daisy

Marmaduke of Maple Herd _ R R

Marmion................. $-M$

Marmion......... R R

Marmontel................

Marquis ................... $\mathrm{P}$ S

Marquis.................. R R

Marquis of Barrington.....- $\mathrm{R} R$

3d Marquis of Bourbon Park $-\mathrm{P} \mathrm{S}$

Marquis of Harper.......... $-\mathbf{P} \mathrm{S}$

Marquis 2d of Kengrove..... $-\mathrm{D}$

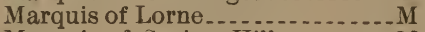

Marquis of Spring Hill..........

Mars ... R R

Marseilles Chief.................

Marshall ................ Cox

Marshall Henderson........ - P S

Marshall Ney................ $-\mathrm{T}$

Marshland Prince........ Daisy

Martin .................. Ky I

Martina's Pride......... R R

Mary's Breastplate....... R R

Mary's Climax.......... R R

Mary Duke...............

Mary Duke .............. - Cox

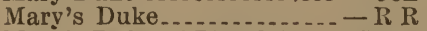

Mary's Duke of Bluffdale.. - Ky I

Mary's D. of Bluffdale 2d.. - Rolla

Mary D. of Spring Arbor .... - Cox

Mary D. 2d of Spring Arbor + Cox

Mary's Grant

Mary Sharon Duke........... T

Master Booth

Master Butler.......... Rolla

Master Clover ...................

Master Dick.............. Ky I

Master Frank.

Master Glitter......... R R

Master Mason 2d......... $-\mathrm{C}$

Master Morgan .............. T

Master Moss ... ............ D

Master Oxford ............... Daisy

Master Pricie.............. R R

Master Richmond of Melrose _. Rose

Master Spirit..............

Matchem _............ R R

Matchem 2d............ Daisy

Matchem 3d.............. L $\mathrm{R}$

Matchem 4th............. L R

Matchless Bridegroom....
76354

\%6:35.

76356

76357

76359

$\% 6360$

テ6362

r6:363

r6364

76365

$\tau 6366$

ช6368

$763 \% 2$

ช6373

76374

76376

76377

\%6378

76379

\%6380

\%6381

\%638\%

76385

76386

\%6389

76394

\%6396

\%6397

г6398

\%6399

$\gamma 6400$

\%6401

76405

$\tau 6406$

76407

r6408

\%6409

76410

₹6411.

76412

$\% 6413$

$\% 6414$

\%6415

76417

76420

\% 6421

76422

$76+23$

$765: 4$

$76+25$

\%61:66

\%64:27

76428

\%6429

\%6430

$76+31$

76433

ช64:35

\%6436

76437

$\tau 6438$

76439

76440

$\tau 6441$

76443

76444

76445

76446

76447

76448
Matilda's Airdrie........... - K y I

Matilda 11th Airdrie........ - R R

Matilda Duke ................

Matilda's Prince............... $R$

Maximilian ................... L R

Maximilian .............. $\mathrm{R}$

Maxwell _..................

Maxwell $\ldots \ldots \ldots$

May Belle's Gem Duke..... D

May Boy 2d................ R

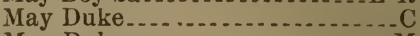

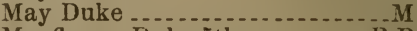

Mayflower Duke 5̃th......... R P

Mayflower 2d of Oakland... - R R

Mayflower's Oxford..............M

Mayflower's Red Rase...... P S

Maynard ..................

Mayolus ....... Cox

Mayor of Mapleshade..... - L R

Mayor of Reedsburg....... $\mathrm{R} R$

May's Twin Duke........... $\mathrm{B}$

Mazurka.................. R R

Mazurka Boy

Mazurka Chief.............

Mazurka Duke $2 \mathrm{~d} . . . . . . . . .-C$

Mazurka Duke of Lima........ Ky I

Mazurka's Oxford 39th........ Ky I

Mazurka's Oxford 40th........ C

Mazurka's Oxford 41st....... Ky I

Mazurka's Oxford 42d......... Ky I

Mazurka's Premium .........-R R

Mazurka Prince 2d............ R R

Meadow Ridge Duke....... $\mathrm{R} R$

Mecaskey's Duke.......... - L R

Mecca 4th................ Daisy

Medera................

Medora Duke.......... Cox

Melrose Boy ................. $-\mathrm{D}$

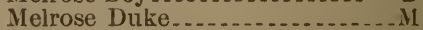

Mel's Challenger........... $\mathrm{R} R$

Melvin .................... R R

Merchant Prince......... $\mathrm{R} R$

Meritorious 2d............. R

Merrill Duke 2d.............. $\mathrm{T}$

Merry Snowball ............. L R

Meteor

I. Felix Tolemais......... D

Viami's Lad..................... Ky I

Miami's Prince.............

Mickey Free................ $\mathrm{R}$

Mico

Mikado ....................

Mikado .... Rolla

Mikado .................. Daisy

Mildred's Duke

Mill Creek Lad............... M

Milo Goodness............ R R

Milton's Pride

Milton Randolph............ D

Milwaukee

Mina Rose.................... Ky I

Minerva's Duke............ $\mathrm{R}$

Minerva Duke....................

Minerva's Lord

Minnie's Duke........ Daisy

Minnie's Duke of Ashland .. - $R$ R

Minnie Renick's D. of Sharon $2 d-T$

Mississippi Charley ......... - P S

Mitchell.......................

Mitchell................. 


\begin{tabular}{|c|c|c|c|}
\hline $76+49$ & Mlodel.. & 76527 & 5th Napier \\
\hline 76451 & Modoc. & 76528 & -Ky I \\
\hline 76452 & Modoc & 76539 & Fth Napier \\
\hline 76453 & Modoc Chif & 76530 & Napoleon. \\
\hline 76454 & Modoc Chief 2 & 76531 & Napoleon \\
\hline 76456 & Mogul _.......... & 76532 & Napoleon \\
\hline $76+57$ & Mohawk 20 & 76533 & Nasby \\
\hline 4.58 & Mohawk Ai & 765334 & Nasby.. \\
\hline 459 & Mohawk Prir & 76535 & Nate \\
\hline 460 & Mollie's Con & 76536 & Nate Putn \\
\hline 462 & Monarch . & 76539 & Nebraska I \\
\hline 463 & Monarch. & 76540 & Nebraska 1 \\
\hline 464 & Monarch & 76541 & Nebraska 1 \\
\hline 465 & Monarch . & 76542 & Nebraska I \\
\hline 466 & Monarch & 76543 & Nectar's Gl \\
\hline 468 & Monarch & 76544 & ... Ky I \\
\hline $4681 / 2$ & 2. Monarch' & 76545 & Neil Dow \\
\hline 170 & Monarch N & 76547 & Nell's Duk \\
\hline 76471 & Monarch N & 76549 & Nellie's Ju \\
\hline 76473 & Monro .... & 76550 & Nellie's Prir \\
\hline 76474 & Monroe .. & 76551 & Nell's Tilder \\
\hline 40 & Monroe... & 76552 & Nelson.... \\
\hline 76476 & Monroe Ch & 76553 & Nelson .. \\
\hline$t 7 i$ & Monroe Ru & 76554 & Nelson of I \\
\hline 76478 & Montebello & 76555 & Neptune.... \\
\hline 479 & Monte Cris & 76557 & Neptune .. \\
\hline 76480 & Montgome & 76560 & Nero..... \\
\hline 481 & Montmor & 76561 & Cox \\
\hline $76+52$ & Moody -.. & 76564 & Nestor \\
\hline & $--\mathrm{B}$ & 76565 & Nettie's Bo \\
\hline 185 & Moore_.... & 76566 & New Haven \\
\hline $48 \pi$ & Morgan Dn & $\tilde{i} 6567$ & Newt Duk \\
\hline 488 & Morley .... & 76.568 & New Year Bc \\
\hline 489 & Morning Di & 76569 & $-\mathrm{R} R$ \\
\hline 491 & $-\operatorname{Cox}$ & 76570 & $-\operatorname{Cox}$ \\
\hline 492 & Morning Sta & 76571 & Nicholas B \\
\hline 93 & $\ldots-M I$ & 76573 & - R R \\
\hline 194 & Morning Sta & 76575 & - Cox \\
\hline & Morning Sta & 76577 & $-\mathrm{R} R$ \\
\hline 196 & Morning Sta & 76580 & Noah Smith \\
\hline 197 & - Rolla & 76581 & Noah Stark \\
\hline 498 & Morris Duke & 76582 & Noble.. \\
\hline 199 & Morton's Prid & 76583 & Noble 2 \\
\hline 500 & Moscow ... & 76586 & Noble Ced \\
\hline 01 & Moscow & 76587 & Noble Duke. \\
\hline 76502 & Moselle's Dr & 76588 & Noble Du \\
\hline 765003 & Mose of Mia & 76589 & Noble Du \\
\hline 76504 & Moses..... & 76590 & Noble Duke \\
\hline 6505 & Moses Dixo & 76591 & $-\mathrm{L} \mathrm{R}$ \\
\hline & Moss Rose Ch & 76594 & Daisy \\
\hline 507 & Moss Rose I & 76596 & $-\bar{M}$ \\
\hline & Motteen ... & 76601 & North Newport \\
\hline 9 & Mountain B & 2 & \\
\hline & ain Boy & 4 & Nor \\
\hline 76511 & Mountain Chief. & 5 & $R R$ \\
\hline 76512 & Mountain Cloud. & 76606 & R \\
\hline 76513 & Mozark .- & 76607 & Ni \\
\hline & $\begin{array}{l}\text { Mozark } \\
\text { Mulford 2d }\end{array}$ & & $-\mathrm{R} \mathrm{R}$ \\
\hline 76515 & $\begin{array}{l}\text { R...M } \\
\text { Rolla }\end{array}$ & 1 & $\ldots B$ \\
\hline & Rolla & & Oak H \\
\hline 17 & $\ldots M$ & 76613 & Oakla \\
\hline 518 & $-\mathrm{L}$ & & Oakland Geneva \\
\hline 519 & $\begin{array}{ll}L & R \\
P & S\end{array}$ & 76616 & $\begin{array}{l}\text { Oak Wreath Lad } \\
\text { Occident......... }\end{array}$ \\
\hline 20 & $\begin{array}{l}\text { Music } \\
\text { Muskingum Duke. }\end{array}$ & 76617 & $\begin{array}{l}\text { Occident........ } \\
\text { Octo }\end{array}$ \\
\hline 521 & $\begin{array}{l}\text { Muskingum Duke } \\
\text { Nabob.... }\end{array}$ & 76618 & \\
\hline 76523 & Nabob.... & $\begin{array}{l}76620 \\
76621\end{array}$ & Ohio Boy \\
\hline 76524 & Natura's Duke 2d & 78622 & Ohio Boy \\
\hline & 2d Napier................ & & Ohio Boy \\
\hline & 3d Napier. & 76624 & Ohio Duke \\
\hline
\end{tabular}


76625

76626

76627

76628

76629

76630

76631

76632

76633

76634

76635

76636

76637

76638

76639

76643

76644

76645

76646

76648

76652

76653

76657

76658

76659

76660

76661

76662

76663

76664

76665

76666

76667

76669

76670

76671

76672

76673

76674

76676

76677

76679

76680

76681

76682

76683

76684

76685

76686

76688

76689

76690

76691

76692

76693

76694

76695

76696

76698

76\%00

76701

76702

$76 \% 08$

76710

76714

76718

76722

76723

76724

76725
Ohio Duke.................

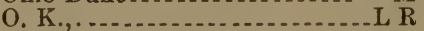

Oklahoma Chief..............

Oklahoma Duke............

Olaf ................ P S

Olathe ................. P S

old Gold

Olena Duke............... - L R

Ole Stoughton........

Olio Ben.................... Ky I

Olio Dick..........

Olive's Duke.......

Oliver........................ M

Omega ......... D

One Eye

Oneida King............... - Cox

Ooita's Duke............

Opatunka ..................... M

Operator ........................ S

Orange Blossom of Durham ....... C

Orange Duke........ Cox

Orange Duke 2d......... Ky I

Orange Watson

Orator ....................... R

Orient .....................

Orient

Orin 7 th.....................

Orion

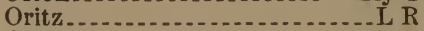

Orlando

Orlando Booth ....

Orontes Duke 3d........ R R

Orontes Duke4th

Orphan Boy ............... - D

Orphan Boy

Orphan Boy

Orphan Lad........... Ky I

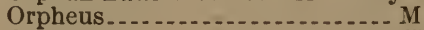

Oscar Boy ..........

Oshkosh .......... Rolla

Othello..................

Otis Airdrie........ $R$

Ottawa Duke............ R

Otto

Ottomarus.................. $R$

Overall ..................... $M$

Ovid Bell Duke.................. R

Ovid Bell Duke A.,........... R

Oxblood Jr., ................

Oxford ........

Oxford...... R

Oxford $23 \mathrm{~d}$

Oxford 35th ...................... M

4th Oxford of Aspen Grove - R R

$3 \mathrm{~d}$ Ox'rd Bar'ngton of Br'dlawn-C

Oxford Boy ............ $\mathrm{L} R$

Oxford Boy .............

Oxford Duke........... R R

Oxford Duke $2 \mathrm{~d} . \ldots . . . . .$.

Oxfora Duke of King Creek_- L R

2d Oxford Duke of King Creek- $\mathrm{L} R$

Oxford's Duke of Lawland.. - L R

Oxford 10th Grand Duke.... - - L R

Oxford Myrtle............... $T$

Oxford Prince................... R

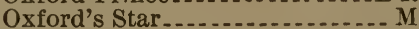

Ozark .

Ozark

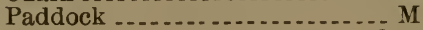

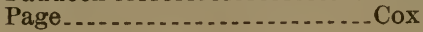

76726

76727

76728

76729

76730

76731

76732

76733

76734

76737

76r38

76739

76740

76741

$76 \pi 42$

76743

76744

76745

76746

$76 \% 4 \pi$

ז6749

\%6750

76752

76753

$76 \% 55$

76756

76757

76758

76759

\% 6761

76762

$76 \% 63$

76\%64

ช6\%65

$76 \% 66$

$76 \tilde{6} 6 \pi$

ช6r68

76.69

76ช70

76771

ช6ร72

76773

76774

76776

76777

76780

ז6781

76782

76783

76784

76785

76786

76787

76791

76792

76793

76796

76800

76804

76805

76810

76812

76813

76815

76816

76818

76819

76821

76822

76823 Palmyra Duke of Oxford..... Ky $\mathbf{I}$ Palo Alto.............. P S Pangus ........... Ky I Pansy Duke of Stanton .. - Beauty Pansy Duke of Stanton $2 d-$ Beauty Pansy Duke of Stanton 3d-Beauty Paragon -............ - Long Horn Paragon Cambrian........ Ky I Park .......................... Parkers Duke Park Prince............... - L R Parnell ...................... Parnell ................... S Parsee...................... Pat Bell.............. Ky I Pathfinder........................ Patrick Henry ................... Patriot of Waveland .......- $R$ R Pattie Duke................. - PS Paul G.,.................. Ky I Paul Jones ............ Ky I Paulsen's Duke............ Paxton Duke 2d.......... $R$ Pearcy

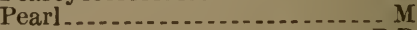
Pearl.................. Pearl..........

Pearl Laddie...... D

Pearl Richard......... Cox

Pearl Richard $2 \mathrm{~d} . . . .$.

Pearly Duke............... - L R

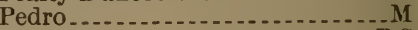

Pedro ................

Pedro $2 \mathrm{~d}$

Peerless

Peirce

Peko...............

Pendennis ................

Percy

Perfection ......... R

Peri's Emperor $2 \mathrm{~d}$........ Rolla

Peri Lad.................... D

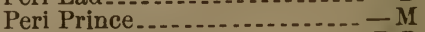

Peri of Springdale....... $R$

Pete McDole .......... $R$

Peter Cooper.......

Peter Parsel ..........................

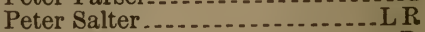

Peter Tyson $2 \mathrm{~d} . . . . . . . . . . .-D$

Peto..... Cox

Phil Cline

Philip ..................... M

Phœnix Duke 14th

Phœnix Duke 15th.........- Cox

Phœnix Duke 16th..........-Cox

Phyllis Belle's Duke....... - Rolla

Phyllis Duke =.......... T

Phyllis Duke 5th

Phyllis Duke 5th

Phyllis Duke of Maplewood - $R$ R

Phyllis Duke of Springwood__ - P S

Phyllis Prince ........... $\mathrm{R}$

Pierce Prince.................. $\mathrm{R}$

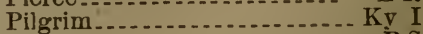

Pilot

Pilot.................

Pilot.........................

Pine Hill Duke............ Ky I

Pink Second's Duke....... - L R 
76824

76825

76826

76827

76828

76829

76330

76832

76833

\%6834

76835

76836

76837

76838

76810

76841

76842

76845

76847

76848

76849

ช 6850

\%68.51

76852

ז6853

\%6854

76855

\%6856

76857

76858

\%6859

76860

76862
76863

\%6865

76866

76867

76868

76869

\%6870

76871

$768 \% 2$

76874

76875

76877

76878

76879
76882

76883

76885

\%6886

76887

76888

\%6889

76890

\%6892

76893

76894

76895

76896

76897

76899

76900

76901

76902

76903

76904

76905

76906

76907
Pioneer

Pioneer

Piper 2d Granite........... L R

Pippin's Lad.............. R R

Pisgar's Chief .................... I

Pisistratus of Melleray .........

Plato...............

Plum Bob ........

Plum Tree Dick

Plumtree Major ............. R

Plumwood Jr., ............ $\mathrm{R} R$

Plumwood Jr. $2 \mathrm{~d} \ldots \ldots . . . .-\mathrm{R} R$

Pluto .................. R

Pluto......... P S

Poco.................. Ky I

Poinsett.........

Polka Dot................... R

Pollux........................ Cox

Pomona Prince...........

Pomp................... R R

Pompey .................. Ky I

Pompey ..................

Pompey ....................

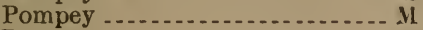

Pontiac .................... D

Ponto....... B

Portage Duke $3 \mathrm{~d}$

Porter ........................ R

Porter

Port Hudson ................

Powell's Duke.............. P S

Powhattan ............. Daisy

Prairie Boy

Prairie Boy .............. Rolla

Prairie Chief. ................ Rose

Prairie Duke..... I R

Prairie Duke................. - P S

Prairie Duke............... D

Prairie Duke............... Multiflora

Prairie Duke............... L R

Prairie Lad 2d............. R R

Premier Gladstone......

President.......... P S

President................. R R

President ...................

Preston Boy .................... I

Pretty Boy 2d ................. D

Pride

Pride of Avon ............. $\mathrm{R}$

Pride of Fairview............ Ky I

Pride of Howland............. L R

Pride of Marengo......................

Pride's Red Duke...............Rose

Pride of Utah.................. Ky I

Primrose Duke 2d........... D

Prince......................... M

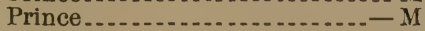

Prince.....................

Prince

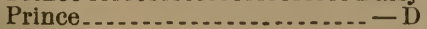

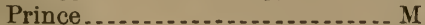

Prince.....................

Prince.......................... Ky I

Prince................

Prince.......... $R$

Prince....................... $\mathrm{R}$

Prince .....................

Prince........................ M

Prince ..................... Cox

Prince............................. S $\tau 6908$

76910

76911

76912

76913

76914

76915

ซ6916

76917

76918

ร6919

76920

76921

ร69:22

76923

\%6924

ร6925

76926

\%6927

76928

\%6969

76930

\%6931

\% 6932

76933

\%6934

\%6935

76936

76937

т6938

76940

76943

76945

$\tau 6946$

76947

76948

76949

76950

76951

76952

76953

76954

76955

76956

76957

76960

76961

76962

76963

76966

76969

76970

76971

76973

76974

76975

76977

76978

76979

76980

r6983

76984

76987

76988

76989

76990

76991

76992

76993

76994
Prince

Prince $2 \mathrm{~d}$

Prince 2d...................... Ky I

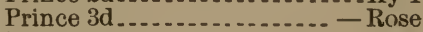

Prince 4th.............

Prince Acklam........... R R

Prince Acomb............ $\mathrm{L}$ R

Prince Adams............. $\mathrm{R} R$

Prince Adams 2d............. R R

Prince Adams 2d......... $-\mathrm{R} R$

Prince Agatha 2d.......... $\mathrm{C}$

Prince Airdrie............. R R

Prince Albert ....................

Prince Albert...................

Prince Albert.......

Prince Albert.

Prince Albert..............- Cox

Prince Albert...............

Prince Albert.......... $\mathrm{L}$

Prince Albert...................

Prince Albert.................... R

Prince Albert............. Daisy

Prince Albert. ...................

Prince Albert.

Prince Albert.......... Daisy

Prince Albert......... $\mathbf{R} \mathbf{R}$

Prince Albert.............. R

Prince Alkert................ Ky I

Prince Albert .............

Prince Albert 2d... R R

Prince Alexander......... $\mathrm{L} R$

Prince Arthur ............. P S

Prince Atha...........

Prince of Banner........ $\mathrm{L} R$

Prince Bismarck............ Ky I

Prince Bismarck $2 \mathrm{~d} . . . . . . . .-\mathrm{R} R$

Prince of Bourbon.......

Prince Boy ................

Prince of Burton...... R

Prince Butterfly .....................

Prince C., ............... B

Prince of Calumet...............

Prince of Cato.....

Prince of Cedar Ridge ........

Prince Charles.................. L R

Prince of Chautauqua...... - Cox

Prince of Colonus........... - Cox

Prince Constance............ - L R

Prince Cranor...................

Prince Deronda........... Ky I

Prince Duke............... $\mathrm{R} R$

Prince of Edmund $3 \mathrm{~d}$

Prince Edward............... - D

Prince Edward.....................

Prince Edward

Prince Edward ...................

Prince of Elk Park.......... L R

Prince of Elmwood....... $-\mathrm{L}$ R

Prince Euclid...................

Prince of Fair Oaks........ Rolla

Prince of Glenwood......... $-\mathrm{L} R$

Prince of Glenwood .........

Prince of Harmony ........ $-\mathrm{L} R$

Prince Hecla............... R R

Prince Herbert.........

Prince of Hill Grove.......... - C

Prince Hubback .............

Prince Imperial ................. - D

Prince Imperial .............. Cox

Prince Imperial .................. 
т6995

76996

ช6997

76948

76999

ชั000

$7 \% 001$

77002

7r003

77004

ชั005

$7 \% 008$

rro11

ช7015

77017

$7 \% 018$

ช 020

77022

\%7024

ชั025

$7 \% 0: 26$

77028

$770: 29$

ชั031

7\%032

77034

77037

$7 \% 038$

7ซ039

77040

77041

7r042

77043

$7 \% 044$

77045

ช 7047

т7049

$7 \% 050$

テ7051

77053

$7 \% 054$

77055

ร7057

$7 \% 059$

7テ060

ชั061

77064

$7 \% 066$

\%7067

77071

ซ7072

77073

\%7074

$7 \% 076$

77077

ชr078

77079

77080

77081

77082

77083

\%ros4

77085

77086

$7708 \pi$

77088

rros9

$7 \% 090$

77091

77092
Prince Jerry L R

Prince Jim ...........

Prince John ................... Ky I

Prince Leo ................ Cox

Prince Leopold. ....... Beauty Prince Leslie..................... C

Prince of Liberty ............ Prince of Livingston........ Cox Prince of Lost Creek.......... Prince of Loudoun....... Prince M.,................. L R Prince of Melrose...... Daisy Prince of Morrow.......... R Prince Ole........................ R 17th Prince of Orange...... L R 20th Prince of Orange...... - L R Prince of Otter Creek......... P S Prince Pearl................. $-\mathrm{D}$ Prince of Randolph....... $\mathrm{R} R$ Prince Realm ............ R R

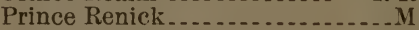
Prince of Riverdale Sth..... $\mathrm{R} R$ Prince Rollins............. $-\mathrm{L}$ R Prince Rossa................. Prince Royal............. Cox Prince Royal.............. R Prince Royal $3 \mathrm{~d}$ Prince Royal 4th............... I Prince Rudolph.............. Rose Prince Rupert................. Prince Sam Prince Scherbatoff.......... M Prince of Schuyler.......... Daisy Prince Sharon of Blufidale.... - D Prince of South Union...... - L R 3d Prince of Springdale.... - Rolla Princess Duke............. - R R Princess Duke of Mayville .. $-\mathrm{R} R$ Princess Lad 2d............ - Cox Prince of The Valley........ - L R Prince of The Valley .......... Prince of The Valley $3 \mathrm{~d} \ldots \ldots . . \mathrm{D}$ Princeton .................... Princeton Louan Duke 4 th Prince Varna............. $-R$ R Prince Victor............... $\mathrm{L}$ R Prince of Wales........... Prince of Wales......... Prince of Warren Prince of Wilderberg ........ Prince Wiley ................. Prince William.......... D Prince William................. Prince of Windsor....... Daisy Prince of Woodford ............. Ky I 26 th Prince of Woodlawn ... $-\mathrm{R} R$ 27th Prince of Woodlawn........ C 28th Prince of Woodlawn........ C 30th Prince of Woodlawn... $\mathrm{R} R$ 31st Prince of Woodlawn... $-\mathrm{R} R$ 34th Prince of Woodlawn... $-R R$ 36th Prince of Wyaconda......... B Prize Boy .................. Ky I Prize Winner......... Professor Powell ......... - Daisy Profitable Boy 2d.......... $-\mathrm{R} R$ Prolific's Lord Rose........... Ky I Prospect King . Prospect Lad................ L R Protection $\tau \% 093$

\%ซ096

77097

$\checkmark 7100$

77101

77102

$7 \gamma 104$

77106

$\gamma \gamma 107$

77108

77110

77111

$7 \% 112$

77114

77115

77116

77118

77119

$7 \gamma 120$

\%ซ121

77123

77124

$7 \% 125$

77126

77127

$7 \pi 128$

พ7129

รั130

$7 \% 131$

ชั132

ชิ 133

\% 134

77135

77136

$7713 \%$

\%138

ช7139

\%7140

ส7141

\% 142

\%7144

\%1147

$7 \% 148$

77152

สช153

$\tau 7154$

ช 1155

77156

$7715 \%$

7715s

ชช159

$7 \% 160$

$7 \% 161$

\%ั163

ชช164

$7 \gamma 166$

$7 \% 167$

77168

\%7169

$7 \% 1 \% 0$

\%ั171

ช71\%:

7r173

77175

77176

$7 \% 17 \%$

สr17s

$771 \% 9$

ชั180

\%7181
Proud Duke................ - Cox

Proud Duke......... R

Proud Duke.............. $\mathrm{R} R$

Proud Duke 10th............... B

Proud Duke 11th.......... - Ky I

Proud Duke 12th............ - R R

P. T. Barnum ......... M

Puck.................... - R R

Punch

Punch

Quaker.............

Queen's Booth........... D

Queen's Boy .................... Ky I

Queen's Duke.........................

Queen's Duke.................. S

Quilp 2d................... Rolla

Rainbow . .

Rain in the Face.......

Ralph.......

Rambler..............

Randel.........

Randolph ................ $\mathrm{R}$

Range......

Ranger........ R R

Ranger.......... R R

Ranger...

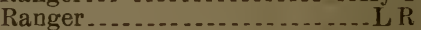

Rarus .

Rattler.............

Rattler.......

Rattler ....... R R

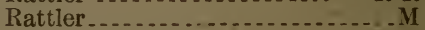

Ravenswood $3 \mathrm{~d} \ldots \ldots . . . . .2 \mathrm{R}$

Ravenswood Boy......... R R

Raymond................. Ky I

Raymond.................. - M

Realm Prince......... P S

Rebecca's Duke......... C

Rector................ R R

Red

Red Airdrie .............

Red Airdrie of Pittsfield........ P S

Red Bank .................... Rose

Red Beauty's Duke........ R R

Redbird ................ P S

Red Boy ............... I

Red Boy ............

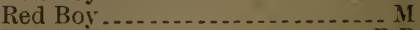

Red Boy

Red Boy

Red Boy

Red Boy

Red Boy

Red Cambridge Duke......

Red Chatham

Red Chief ..................

Red Chief .................. $M$

Red Cloud................ Ky I

Red Cloud.................

Red Cloud .....................

Red Cloud

Red Cloud............... R

Red Cloud

Red Cloud.............

Red Clover.........

Red Coat.................

Red Comet.............

Red Comet...............

Red Daisy 4th's Duke.......... M

Red Derby ................ R 
77182

77183

77184

77185

77186

77189

77190

77191

$7 r 192$

7ซ193

77194

77195

77196

$7 \% 197$

7ซ198

77199

77200

77201

77202

77203

77204

$7 \% 205$

77206

77207

77208

77 210

77211

77212

77213

ส7 214

$7 \% 215$

$7 \% 216$

$7 \% 21 \%$

$7 \% 218$

$7 \% 213$

77220

7า223

772:5

รสะ26

$772: 27$

77228

$772: 39$

77230

77231

77232

77233

ร7234

77235

77236

รส $23 \%$

77238

77239

$7 \% 240$

77241

77242

77243

7ช244

77245

77247

77248

77249

77251

77252

77253

77254

77255

77256

$7725 \%$

77258

77259
Red Dick

Red Dick

Red Dixey

Red Duke

Red Duke

Red Duke

Red Duke.................. - D

Red Duke................. - P S

Red Duke........... R

Red Duke...................

Red Duke....................

Red Duke...................... Ky I

Red Duke.................... -M

Red Duke..............

Red Duke.................. - M

Red Duke........... J

Red Duke .................

Red Duke................... M

Red Duke.................

Red Duke .................... M

Red Duke.................. Rose

Red Duke..... ............ - D

Red Duke.........................

Red Duke.................... Ky I

Red Duke................ R

Red Duke...............

Red Duke............... - L R

Red Duke.............

Red Duke......

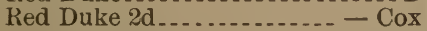

Red Duke $2 \mathrm{~d}$

Red Duke 2d..............

Red Duke 3d .............

Red Duke 4th

Red Duke of Lakin............

Red Eagle................. L R

Red George of Oak Hill....... - D

Red Giant ...................... M

Red Governor.............. Daisy

Red Harry 4th ............. - Ky I

Red Hawk................. L R

Red Ike....................

Red Jack....

Red Jack............. R

Red Jacket................... - B

Red Jacket.......................

Red Jacket ...................... P S

Red Jacket ...................... C

Red Jacket.........................

Red Jacket.................

Red Jacket............. Cox

Rer Jim ...........................

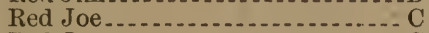

Red Joe............ C

Red Jupiter.......... Cox

Red King - . . .

Red Knight.................. Rose

Red Knight ............ Daisy

Red Knight ................. D

Red Lad........................

Red Lad..................... C

Red Lad ............... Daisy

Red Lad................... R R

Red Lad......................... R

Red Lad of Longton....... - R R

Red Lark $3 d$..................... C

Red Louan Duke.........

Redman..........................
77260

รั262

7т263

77264

77265

77266

77267

77268

$7 \% 269$

$\tau 72 \% 1$

77272

テ7273

77274

77275

77276

77277

77278

77279

77281

77282

7ั283

77285

77286

77287

77288

テ7289

ร7292

$7 \% 293$

77294

$7 \% 295$

ซร296

$7 \% 297$

ส7298

ช7299

77300

77301

77302

77303

77304

77305

77306

77307

77308

$7 \% 309$

77312

77314

ช7315

$7 \% 316$

$7 \% 31 \%$

77318

77319

$7 \% 321$

77324

77325

77326

77327

77330

7ซ331

77334

77336

77337

77338

77339

77340

77341

ז7342

77343

77344

77345

\%ซ347
Redman 2d...............-Rose 4th Redman of Trout F'm Multiflora 5th Redman of Trout Farm_- Ky I 6th Redman of Trout Farm ...- D ith Redman of Trout Farm.- $R$ R Red March.................... Ky I Red Mary Duke.............. - M Reã Mendelssohn............. - D Red Monarch 2d............ - L R Red Pompey ............. R R Red Prince...............- - Beauty Red Prince.................. D 3d Red Prince............. Red Prince.................. Red Prince.................. Red Prince............... D Red Prince................. Red Prince .................. - C Red Prince................ Red Prince................... - M Red Prince................. - L R Red Robin .................. Rose Red Robin............ R R Red Rock......... Cox Red Rocket................... M Red Rock of Richmond...... - M Red Rover................. $-R$ R Red Rover............... $\mathrm{R}$ R Red Rover.................. Rose Red Rover.................... C Red Rover............... Ky I Red Rover.................. Red Rover................. Ky 1 Red Rover.................... S Red Star......................... M Red Taylor............... Red Tom ................. $\mathrm{R} R$ Red Twin's Duke.......... - Daisy Red Victor................. - L R Red Victor Red Warrior............... R R Red Wiley ................ $\mathrm{M}$ Red Wilks...................... $\mathrm{R}$ Red Wine................... Reformer ................. Ky I Regent's Belle's Duke........... Ky I Regulator .................. - C Riegulus..................... P S Reives Geneva...............Ky I Reliable................... Ky I Reliance Renick of Elm Grove......... Renick of Orleans ........... D Renick Payne................. L R Renick's Ribbon Robber..... - D Renick's Sharon.............. T Resolute ................... Cox Reuben ....................... Revilee........................... Rex Rex Rex $2 d$.......... Ky I Rex of Hart...................... Rezin Duke 2d............... P S Rich ....................... Ky I Richard Richard ................. $\mathrm{R} R$ Richard....................... B Richard Richard of Blue River............ 
77348 Richard Whittaker.......... - M

77349 Richland Chief................... M

77350 Richland Duke of Airdrie.... - M

77351 Richland Favorite.............. R

77355 Richmoud.................... R

7\%356 Richmond 2d............ R R

77357 Richmond 4th.......... S

77359 Richmond Duke.......... R R

77361 Ridgeville Boy ............. R R

77362 Ridot Chief ...................

77363 Rienzi ......... R R

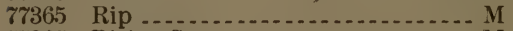

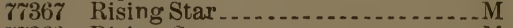

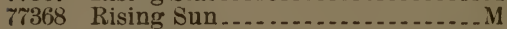

テ7369 Rising Sun $2 \mathrm{~d}$

$773 \pi 1$ Riverdale.................. P S

773\%2 Riverdale Dick........... R R

77374 Riverdale Tom................ M

77376 Roan Beauty .............. D

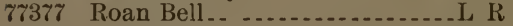

r73rs Roan Bill........................

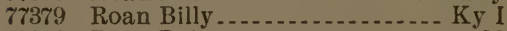

77380 Roan Bob................... M

77381 Roan Boy......................... Ky I

77382 Roan Boy ...................... Ky I

77383 Roan Boy ..................... Ky I

77384 Roan Boy ......................... R

77385 Roan Boy .........................

$7 \% 386$ Roan Boy.................. R

77387 Roan Boy ........... M

7 7388 Roan Boy ...........................

77389 Roan Boy

77391 Roan Boy

77392 Roan Boy .........................

77393 Roan Boy

$7 \% 394$ Roan Boy

77395 Roan Boy $2 \mathrm{~d}$

77396 Roan Boy of Maple Herd... $-R$

77398 Roan Champion............... I I

77399 Roan Chief...............................

77400 Roan Cyrus...................... R

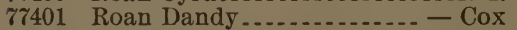

77104 Roan Duke............... R R

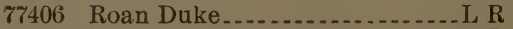

77408 Roan Duke..................... R

77409 Roan Duke................

77410 Roan Duke.......................... S

77411 Roan Duke..................

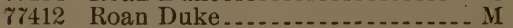

77414 Roan Duke................... R

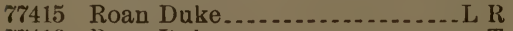

77416

$7741 \%$

77418

774:20

77421

77422

77423

77421

77425

77426

$7 \% 427$

77428

77430

$7 \% 432$

77433

$7 \% 434$

77435

77436

77437
77439

77440

77441

77442

$\tau 7443$

77444

77445

77447

77449

77450

77452

77453

77454

77455

77456

77457

77458

77459

7\%461

77462

77464

77465

77466

77467

77468

77470

77471

$7 \% 4 \% 2$

77473

7ั7 74

77475

77476

$7 \% 478$

77479

77480

$\approx 7481$

$\tau \pi+82$

77484

77485

77486

77457

77485

77489

77490

$\tau$ r 491

7ช 492

7 7493

77494

77495

77496

สั749

77498

77500

77501

77502

77503

77504

77505

77506

77507

77508

77509

77510

77512

77513

77514

77515

$7 \% 516$

77517

77518
Roan Prince............... .

Roan Prince......

Roan Prince........

Roan Prince........

Roan Puritan.....

Roan Tom .......

Roan Twin .

Robare.................. P S

Robbie............

Robert

Robert E. Lee............. P S

Robert Falconwood.......... Cox

Robert Ingersoll...............

Robert James................

Robie .........................

Robin Adair $2 \mathrm{~d} . . . . . . . . . .4 \mathrm{~L}$

Robin Hood .........

Robin Hood $2 \mathrm{~d} \ldots \ldots \ldots \ldots$

Rob Roy ....................

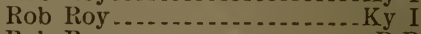

Rob Roy

Rob Roy -..................

Rob Roy

Rob Roy

Rob Roy........ M

Rochester ....... D

Rock Brook Prince....... P S

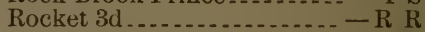

Rockville Monarch........ M

Rocky Ford................. R

Rodeo

Roderick.

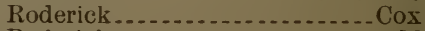

Roderick

Roderick Dhu............ R R

Roderick of Union..... k

Rody ......... R R

Roland....

Roland ......... R R

Roland........ R R

Rolla...... R

Romeo

Romeo

Romeo...... Ky I

Romeo.........

Romeo

Romeo

Romeo................ $\mathrm{R}$

Romeo

Romeo...... $R$

Romeo

Romeo .

Romeo - .

Romeo 4 th

Romeo 5th..........

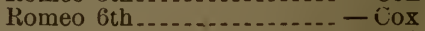

Romeo

Romulus .... Daisy

Romulus ........

Romulus ....... P S

Romulus.........

Romulus....... R

Romulus $2 \mathrm{~d}$

Rondeau .

Rosary Bead 2d.......

Roscoe ${ }_{\text {Roscoen }}$

Ruscoe ........

Roscoe.

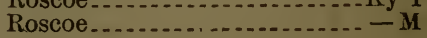


77519

77520

77521

77526

77527

77528

77529

77530

77532

ชัธ533

77534

77536

77537

77538

77540

77541

77545

$7 \% 546$

77547

77550

77551

$7 \% 552$

77553

77554

77555

7755์ 6

77557

77558

77559

77562

77563

77564

77565

ชัต56

77568

77569

77570

$775 \% 1$

77573

77574

$7 \% 576$

$7 \% 581$

77582

77583

77584

77585

ช7589

77590

77591

77594

77595

77596

77598

77601

77602

77603

77604

77605

77607

77609

77617

77618

77620

77621

77622

77623

77624

$7 \div 626$

77627

77628
Roscoe

Roscoe Conkling

Rosa Ayers 2d's Ruby Duke....L R

Rosebud Duke............. - R R

Rosebud Duke .....................

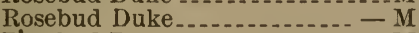

Rosebud Pince

Rose's Duke.................. R R

Rose Duke............... - Cox

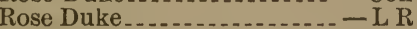

Rose Duke............

Rose's Kirklevington........ - D

Rosalie's Duke...............

Rosemary Duke......... $R$

Rosamond Duke.... $R$

2d Rosamond Duke...... $R$ R

Ross ........................

Ross $2 d, \ldots . . . . . M$

Rossey Duke

Rouser

Rouser $2 \mathrm{~d}$

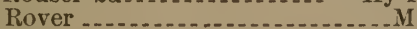

Rover...........................

Rover.......

Rover -

Rover

Rover of Maple Herd.....

Rowdy _..............

Rowdy Boy

Rowland............. Rolla

Roxey ................ Daisy

Roxey Duke.............. Ky I

Roy

Roy

Roy

Royal

Royal

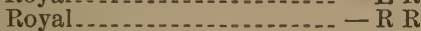

Royal $2 d . . . . . . . .2 R$

Royal Airdrie............

Royal America........

Royal Bourbon..............Woods

Royal Briton.................... PS

Royal Broad Tread

Royal Cass.................. $\mathrm{R} R$

Royal Chief.........................

Royal Constantine......... $\mathrm{R} R$

Royal Daisy .................... Daisy

Royal Derby

Royal Duke..............

Royal Duke............

Royal Duke .................. M

Royal Duke.............

Royal Duke.......................

Royal Duke................ D

Royal Duke.............

Royal Duke $2 \mathrm{~d}$

Royal Duke 3d................. M

Royal Duke of French Grove Royal Duke of Maine Valley.-.Ky I

Royal George................. D

Royal George................... Ky I

Royal George............... - P S

Royal George ............

Royal George............... - Cox

Royal Goodness............... Ky I

Royal Grundy ...........................

2d Royal Huntingdale.......- $R$ R

3d Royal Huntingdale.......- $-\mathrm{R} R$

Royalist 5th ................. - Cox
77629 Royal Knight.............. - R R

77631 Royal Major................... S

77633 Royal Mason............... - Rolla

$7 \% 634$ Royal Mazurka ............. - R R

77635 Royal Motte...................... M

77637 Royal Oak.....................

77638 Royal Oak $2 \mathrm{~d}$

77639 Royal Oakland...............

77640 Royal Oakland............ R

77641 Royal Oxford ......................

77642 Royal Oxford .............

77643 Royal Oxford.......

77646 Royal Pierce................

77647 Royal Prince ...................

77648 Royal Prince................ R

77649 Royal Prince................ L R

77650 Royal Prince $3 \mathrm{~d}$

77652 Royal Red Knight......... - L R

77653 Royal Rob........................ R

77657 Royal Sharon......................

77658 Royal Sharon ............. - Rolla

77660 3d Royalton Hero........ - Cox

77665 Roy Leslie........................

77666 Rubert........

77667 Rube Walton

77668 Ruby's Duke...........

77669 Ruby Duke............... $\mathrm{R}$

77672 Ruby Duke 4th.................. R

77673 Ruby Jewel .............. R R

77674 Ruby's Prince................... B

$776 \% 6$ Ruby's Prince ................ B

77677 Ruby's Princeton .................. B

77678 Ruby's Sidon ................. B

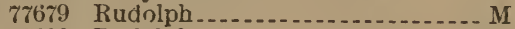

77680 Rudolph.....................

77681 Russel Duke................. Ky I

77682 Rustler

77684 Ruthford......

77686 Ryon

77687 Sabran................. $-\mathrm{R} R$

77690 Sagamore........ L R

77691 Saide's Prince._._._._._.....P S

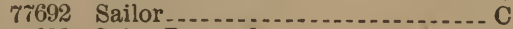

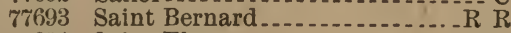

$7 \% 694$ Saint Elmo

77695 Saint Endo

77696 Saint John _................. Ky I

77697 Saint John

77698 Saint John ................ D

77699 Saint John

77700 Saint John................. Daisy

77701 Saint John of Beaver........ - M

77702 Saint Joseph.................. Ky I

77703 Saint Lawrence Joe........ - Cox

77705 Saint Louis................... Daisy

7\%707 Saint Paul ................ R R

rro8 Saint Patrick ............ T

7\%709 Saint Patrick.........

77710 Saint Paul................... Cox

77712 Saint Valentine........... R R

77713

7\%714

777.15

77716

77717

7ซ718

77719

77720

77721

77722

Saladin

Sallie's John....................

Salvini.................... T

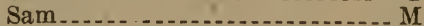

Sambo......

Sambo.......... Cox

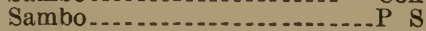

Samboke..................... R

Sam Hauser....................

Sam Hilderbrand.......... $\mathrm{R} R$ 
7ร723

77724

พั7เ25

77726

$77 \% 27$

777\%9

ซ7ซ30

77731

7ช732

รั7333

77734

77735

77736

77739

ส774\%

77743

77745

77746

77747

77748

77749

77750

$77 \% 1$

77752

77753

$7 \% 754$

77758

ช7759

$77 \% 63$

77766

$7 \% 767$

ร7769

ชชชั0

ร7ช71

$77 \% 73$

77774

77775

77776

สช7ส7

777\%8

77782

77783

77784

77785

77786

77787

77788

77789

77791

77792

77793

77794

$7 \% 796$

7ร798

77800

77802

77803

77804

77809

7811

77812

77814

77817

77818

77821

77822

77823

77824

77825

77826
Sam Jones.

Sam Jones

Sam Jones

Sam Jones ............... - Ky I

Sam Lawrence............... Rose

Sam Patch................. R

Sampson.............. $\mathrm{P}$

Sampson.................... R

Sampsom

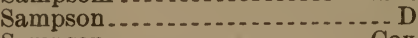

Sampson

Samuel Bluebell ............... M

Samuel Harvey .................

Sancho........................

Sandstone.............. R R

Sandy Jim................. R

Sangaree......................... T

San Joan

Sanspareil Duke......... - R R

Saracen.................

Sargeant...................

Satisfaction ................

Saturn ........................... R R

Savannah Duke......... Daisy

Scandamore ..................... T

Schiller

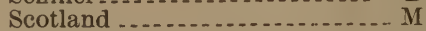

Scotland Duke......... L R

Scott Wike.................. M

Seaham Lad......

Secretary ...................

Sears Duke...............

Sedgewick............. Daisy

Sedgewick $2 \mathrm{~d}$.

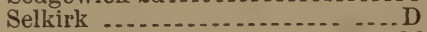

Seneca Duke............

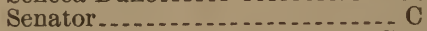

Senator................... Cox

Senator Beck............

Senator Logan ....................

Seneca Oxford............. Cox

Sero _.......................

Set-Em-Up.......

Seth....................... R

Settler......................

Severinus ................ Ky I

Seymour ................... M

Seymour Renick............. Ky I

Sharon Boy ............. - Cox

Sharon Boy

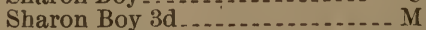

Sharon Dent ........... Daisy

Sharon Duke........... R R

Sharon Duke............ R R

Sharon's Duke................-M

Sharon Duke of Ashland......- - M

Sharon Duke of Carroll 2d.... - M

Sharon Duke of Ferndale... - R R

Sharon Earl..................... R

Sharon Fletcher.......... - Rolla

Sharon Gem............... R R

Sharon of Kengrove...... $R$

Sharon of Mound View ....... - B

Sharon Prince $2 \mathrm{~d} . . . . . . . . . .-\mathrm{D}$

Sharon Venice.............. $R$

Sharon Wellington .......-Rose

Sheared ....................

Shelburne....... Cox

Shelby ........................ M

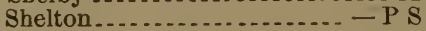

77827

77828

77829

\%ซ830

77831

77832

$7 \% 833$

77834

7\%835

77836

77837

77838

$7 \% 839$

$7 \% 840$

77841

77842

$\pi 7843$

77849

77851

77852

77853

77854

$7 \% 855$

778556

77558

ธ7859

ז7860

$7 \% 861$

77863

$7 \% 864$

$7 \% 866$

77867

77868

7\%869

$778 \% 0$

รั78 1

77873

7\%8\%4

77876

77877

77878

77879

77580

7\%81

7ั883

77884

$7 \% 885$

77888

77889

77890

77891

77892

77893

77894

77896

77897

77899

77900

77901

77902

77903

77904

77905

77906

77907

77908

77909

77910

77911

77912
Sheridan ............ - Long Horn

Sheridan..................

Sheridan................... I

Sheriff .............................

Sheriff Foster....................

Sherman ...............

Sherman................ $\mathrm{R}$

Sherman

Sherman Rnssell..........

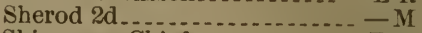

Shiawasse Chief............. $\mathrm{R} \mathrm{R}$

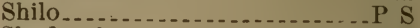

Shodsuch

Short Horn

Short Legged Duke

Short of Maple Herd......

Short Tail_....................

Sidney Grip.............................

Silkie Dan ..................

Silver Duke.................. Ky I

Silver Tail_................

Silverthron Duke........

Silverton .......................

Silver Tongue..........

Simon

Sinnemahoning

Sioux Chief............... Daisy

Sip........................... Ky I

Sir Atha of New Castle ....... - M

Sir Best ........................

Sir Charles Dilke.........

Sir Claypool.......................

Sir Cleveland

Sir Cochrane

Sir Duncan.................. $L$

Sir Dwight of New Castle..- $-R R$

Sir George................ $R$

Sir George .. ................... S

Sir George $2 \mathrm{~d}$

Sir Harry ........... Cox

Sir Harry

Sir Henry

Sir Henry $2 \mathrm{~d} . . . .$.

Sir Isaac.................

Sir James ................. - M

Sir John

Sir John Lyndale........... R

Sir Leonard ............ R R

Sir Loudon............. R

Sir Mack................. $\mathrm{R} R$

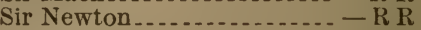

Sir Oxford

Sir Oxford Lee........... R

Sir Ramsey ................ R R

Sir Richard Ostler.......... R R

Sir Richard Benton...... Ky I

Sir Sumner................ R R

Sir Thomas....................

Sir Walter-..........

Sir Walter

Sir Walter $2 \mathrm{~d}$

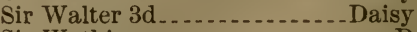

Sir Watkin

Sir White Rose........... R R

Sitting Bull...............

Siva_.................. Ky I

Slidell

Smoky

Smutty Bear...............

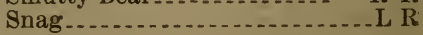


77914 Snowball ....................... M

77915 Snowbank................. Ky I

7 7916 Snowflake................... D

77917 Snowflake.................. - B

77918 Snug ...................... Cox

77919 Solid Silver

77920 Solon of Baileyville............ P S

77921 Solon's Dude................... M

77922 Solon of Melleray ........... $-\mathrm{P}$ S

77923 Son of Florence............. Ky I

77924 Songster.................. - Rolla

77925 Son of Hubback ........... - Cox

$7 \tau 92 \pi$ Soon............................. R

77928 Sostratus................. $-\mathrm{L} R$

79930 Sparswood............... R R

77931 Sparswood Duke 2d........ - R R

7 7933 Splentor 2d.................... M

77934 Spoke.......................... I

$7 \% 935$

7\%936

7ั93\%

77938

77939

77940

77941

77942

7ั943

ร7944

77945

77946

77948

$7 \% 949$

7740

7\%953

77954

ชัตริ5

77956

$7795 \%$

ส7958

77960

77961

77962

77964

77965

7\%966

7796 ช

77968

77969

77970

$7 \% 971$

77972

ชช9\%

$7 \% 979$

7\%980

77981

77982

$7 \% 983$

77984

สั985

77986

77988

77989

$7 \% 990$

77991

7799?

$7 \% 993$

77995

77996

77997

77998
Spotted Boy $2 d$.

$\mathrm{Ky}$ I

Spotted Chief

Spotted Duke............ - R R

Spotted Duke .................. U

Spotted Duke John......... L R Spotted Lad.............. - Ky I Spotted Montauk............. D Spotted Sheridan............. - M Spotted Tail ................. Spriggs _................. - Rose Springbr'k K'kl'v'gt'n-Long Horn Springer .................... - M Spurgeon Jr., ........... - Cox Spy Stanley .......................... B Stapleton Duke......... Star ....................... D

Star Bates......................... Star of Clinton Star Commander............ L R Star Duke................. - R R Star Duke.................. Star Duke..................... S

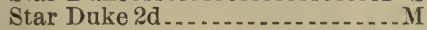
Siar of Kentucky...- Long Horn Starlight ................. Starlight................. R R Starlight_............ $R$ Starlight ........................ R Starlight of Dublin ......... - R R Star of Maine Valley........ - L R Star of Nelson.............. T Star Tumbler................... S S Stem Winder................. Ky I Sterling Duke 2d.......... - R R Sterling Kirklevington.....- Rolla Steven .................... Stiles.......................... Stillwater................. - Cox Stockman ................... - D Stockton Lemonweir........ - M Stonewall................... - P S Stonewall ................... M Stonewall Storm Stormado ................. - Cox Strait's Lake Duke.......... - L R Strawberry of Hickory Grove - T Strike..................... $-R \mathrm{R}$ Stubborn .................. Stube................. $R$ Springwood
77999

78000

78001

78002

78003

78004

78005

78006

78007

78008

78009

78011

78012

78013

78014

78016

78017

78018

78019

78020

78021

78022

78023

78024

78025

78026

78027

78028

78031

78032

78033

78034

78035

78036

78037

78038

¿8039.

78040

78041

78042

78043

78047

77048

78049

78050

78051

78053

78054

78055

78056

78057

78058

78059

78060

78061

78062

78063

78064

78065

78066

78067

78068

780681

78069

78070

78072

78074

78075

78076

78077
Sturgeon ................. - D

Success ....................... R

Success ....................

Success ................. - Daisy

Suceess $2 \mathrm{~d}$............... R R

Sucker Boy ......................

Sugar Creek Duke....... R R

Sugar Creek Lad.......... - Ky I

Sullivan.................... D

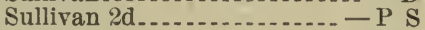

Sultan ....................... Ky I

Summer Lad................ - D

Summit Duke of Goodness.....R R Summit Earl 2d.............. - D

Summit Roan Duke............

Sunflower_............... - R R

Sunlight.............. P S

Superior .............. R

Superior.............. $R$

Superior ................. Ky I

Superior Briton 10th..........L R

Surprise ..................

Sussex ...................

Sutherland Duke 3d...... - R R

Sutherland Duke 4th......... - R R

Sweet William ............

Swift.................... $R$

Sycamore Duke............ - R R

Sylvester.................. R R

Sylvester.................... D

Sylvester................

Symmetry..................

Syrian ...................... Cox

Tacitus of Beallsville...... - D

Talmage _................ Cox

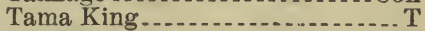

Tancred ...................

Taurus............. Milk Spring

Taurus.................. - Ky I

Taylor Richmond........ - R R

Taylor Wellington.......... $R$

Tebo Lawn's Chief......... - R R

Tecumseh Duke............. Daisy

Te Duke........................

Tempest .............

Templeton ........................

Teno ............................ R

Tevis Harwood .............

Thanksgiving.................

Thanksgiving.............. $\mathrm{M}$

The Clerk ............... R R

The Creeper................. R

The Czar................... Cox

The Dandy Duke............ - P S

The Doctor ................ Daisy

The Dude................ $\mathrm{R}$ R

The Judge..............

The Kaiser............... Cox

Theodore

Theodore Richmond............ M

Theodore Tilton .............

Thomas......................

Thomas A. Hendricks....... M

Thomas Hendricks ........... Ky I

Thomas J.,................... D

Thorn Benton ................. Daisy

Thorndale Duke 3d........ - R R

3d Thornda?e Duke of Melleray --C

Thorndale Prince......... $\mathrm{R} R$

Thornton

Daisy 
78078

78079

78080

78081

78082

78083

78084

78085

78046

78087

78089

78090

78091

78092

78093

76094

78096

78097

78098

78099

78101

78102

78103

78104

78105

78106

78107

78108

78109

78110

78111

78112

78114

78115

78116

78117

78118

78119

78120

78122

78123

78124

78125

78126

78127

78128

78129

78130

78133

78135

78136

78137

78138

78139

78140

78141

78144

78145

78146

78147

78149

78150

78153

78154

78156

78157

78158

78159

78160
Thornton

Thornwell Chief

Thurley Forest . ...............

Tiger

Tiger

Tilden

Tilden

Timothy Jones........ M

Tim Tuff.................. L R

Tippecanoe .....................

Tip Top

T. J. Jackson.................

Tobin

Toliff

Tom ................. R

Tomah

Tom Bell

Tom Benton.........

Tom Benton ............... R

Tom Benton.............. P S

Tom Corwin

Tom Heddleston

Tom Hendricks........

Tom Hendricks.......

Tom Hendricks.......... $-R$

Tom Hendricks ...................

Tom Hendricks.......

Tom Hendricks ............. $\mathrm{L} R$

Tom Hendrick's Granite..........M

Tom Moore ................. Cox

Tommy ................

Tommy - .

Tommy

Tommy Lincoln............. Ky I

Tommy Taft.....................

Tom P.,

Tom Paine............

Tom Sayer

Tom Scott.................

Tom Taylor...............

Tom Thumb.......

Toney ..............

Tonney

Top

Top Gallant..............

Torchlight ..................

Torpedo . ............ Rolla

Torris................. Ky I

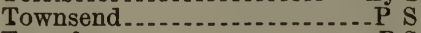

Transfer...........

Traveler.................. Rolla

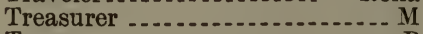

Trego

Trick

Trig

Trim

Triumph $2 \mathrm{~d}$

Trojan $2 \mathrm{~d} . . . . .$.

Trowbridge

Turk of Whitebreast........ $-\mathrm{PS}$

Turner's Duke of Sharon...-R R

Turner's Duke of Sharon $2 \mathrm{~d}_{-}-\mathrm{R} R$ Turtle Boy ................... R R

Turtle Lad............

Twenty-Two Below ........... S S

Twin Duke................ P S

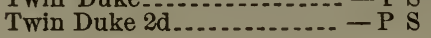

78161 Twin Napier ............. - R R

78162 Twin Oaks Brocton.......

78163 Twin Oaks Lad........... - R R

78164 Twin Oaks Lad $2 \mathrm{~d} . . . . . . .-\mathrm{R} R$

78165 Twin Oaks Lad $3 \mathrm{~d} . . . . .$.

78166 Tyler ....................

$7816 \%$ Tyler of Melrose...........

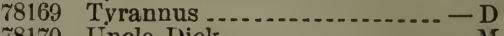

78170 Uncle Dick

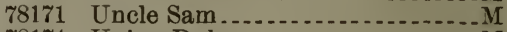

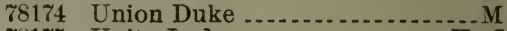

78175 Unity Lad...............

78176 Unk's Sam................. R

78177 Unquomonk Chief....... $\mathrm{R} R$

78179 Updike....................... P S

78180 Uranius .......................

78181 Urbana's Son ........

78184 U. S. Grant ................

78185 Ux̣bridge Duke...........

78186 Valentine.............. $\mathrm{R} R$

78188 Valentine of Forreston......... P S

78189 Valentine Perfection...... - Rolla

78191 Valiant .................. R R

78194 Valley Boy

78195 Valley Chief............... $-\mathrm{R} R$

78196 Valley Chief..............

78197 Valley Duke.................. Ky I

7819 Vall'y Rose's D. 11th - Milk Spring

78199 Val Sharon................ D

78200 Van Buren Duke...........

78201 Vanderbilt ..........

$78: 202$ Vanderbilt ................... Daisy

78203 Vanderbilt....................... S

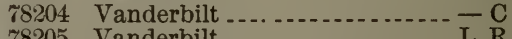

78205 Vanderbilt.........................

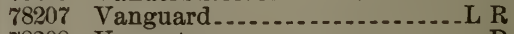

78208 Vanmeter .................... D

78209 Van Tromp's Airdrie $2 \mathrm{~d} . . . . . . \mathrm{L}$ R

78210 Varna's Chetopa............. - P S

78211 Varna's Duke............... Cox

78212 Varna's Perfection ........... - D

78213 Varna's Sampson........... $\mathrm{L}$ R

78214 Vaughey ..................... M

75215 Vedder

78219 Venus

78220 Venus Sidon

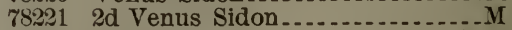

78222 Vermillion ................

78223 Vermillion

78224 Vermillion Barney....... R R

78226 Verndale....................... R

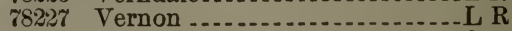

78228 Vernon Lad.................. Cox

78230 Vestris......................... R

78231 Veto ............................. S

78232 Vice President .............

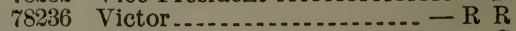

78237

78238

78239

78240

78241

78242

78244

78245

78246

78247

78249

78250

78251
Victor

Victor

Victor

Victor...............

Victor

Victor

Victor..... $\mathrm{L}$

Victor

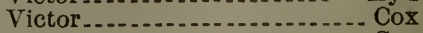

Victor....... Cox

Victor........

Victor................

Victor 
78252

78253

78254

78255

78257

78258

78259

78260

78261

78262

78263

78264

78265

78267

78268

78269

78270

78272

78276

$7827 \%$

78278

78280

78281

78282

78283

78284

78285

78289

78290

78291

78292

78293

78394

78295

78296

78297

78301

78302

78303

78307

78308

78309

78310

78311

78312

78313

78314

78315

78316

78317

78318

78326

78327

78328

78329

78331

78332

78333

78334

78335

78336

78337

78338

78339

78340

78341

78342

78344

78345

78354
Victor.

Victor of Cottage

Victor Emanuel

Victor Fairfax ...........

Victor Huerkamp.......... - R R

Victor Hugo ............ $-\mathrm{R} R$

Victor Hugo.....

Victor Hugo................ - P S

Victor Hugo .......... Rose

Victor Hugo

Victor Hugo................ Cox

Victor Hugo ......... Cox

Victoria's Dexter............. - M

Victoria's Duke

Victoria Duke 4th ........ - Cox

Victoria Lad ............... - L R

Victoria Prince 7th ........ - Rolla

Victorine's Duke ......... - Cox

Vilas................ P S

Village Boy ............... Ky I

Village Challenger............ Ky I

Village Lord ..................... K I

Villager.....................-M

Villas.................... $\mathrm{P}$

Villenovia John......... P S

Vinnie's Duke.............. $\mathrm{R}$

Vinnie's Red Duke........._- R R Virgil 2d........................ R Virginia's Lad................ - D Virginius ..................... Cox Viscount.................... Ky I

$2 d$ Viscount Geneva............Cox

Voltarie............................

Volunteer

Von

Von Ärrdrie........... Ky I

Vulcan ..................... R

Waagen.................. $\mathrm{R}$

Wabash.................... - L R

Walker Duke............... - L R

Wallace................... - L R

Wallace................... $R$

Walnut Duke of Sharon ..- - Daisy

Walter

Walter

Walter

Walter $3 \mathrm{~d}$

Walter 4th

Walter Airdrie............... -

Walter Poe 2d................ -

Waltham Lad $2 \mathrm{~d}$

Warren Duke.............. - M

Warrior ...........

Warwick.......... $R$

Washington .............. - Cox

Washington Airdrie........... Cox

Washington's Day............ - C

Washington Duke........... - M

Washoe Chief 2d......... Daisy

Washoe Chief $3 \mathrm{~d}$

Washoe Chief 4th....... - Daisy

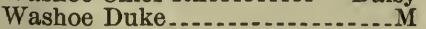

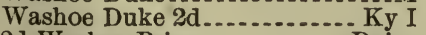

2d Washoe Prince ......... - Daisy

Washtenaw Duke............. Ky I

Waterloo.............

Waterloo......... Cox

Waterloo.............

Waterloo $2 \mathrm{~d}$

Waterloo Ned............. Ky I
78355

78360

78361

78362

78363

78365

78366

78367

78368

78370

78375

78376

78378

78379

78380

78382

78383

78384

78385

78386

78387

78388

78389

78390

78391

78392

78393

78394

78395

78396

78397

78398

78399

78400

78401

78402

78405

78406

78407

78408

78411

78418

78419

78420

78421

78422

78423

78424

78425

78426

78428

78430

78431

78435

78437

78438

78439

78440

78441

78443

78444

78446

78448

78449

78450

78452

78454

78455

78456

78457 17th Waterloo of Raccoon Va'y Ky I Waterloo Twin Duke 2d ....- R R

Watson ........................ Ky I

Waverly Lad.............. - Ky I

W. Dixon................. P S

Webb Genera........... R R

Webster...........................

Webster...... P S

Weimer.............. P S

Welcome............ Rolla

Wellington.................... Ky I

Wellington.............. - Beauty

Wellington............. - Cox

Wellington............ $R$

Wellington ............. - R R

Westbrook Barrington.......... M

Western Duke................ I

Western Duke 2d...............

Western Lad.............

Western Lad.................. R R

Western Star................. M

Westport Comet

Westview Duke 2d...... - KJ I

Westview Duke 3d....... - Ky I

Westriew Duke 4th ........ - Ky I

Wewoka ..................

W. H. Gibson.............

Which

White Cap..................... M

White Chief.............. - P S

Whitefoot....................... P S

White Stocking .............

White Stockings

White Stockings

White Stockings .........

Whitetail:...................

Wide Horns......................

Wilcox's Duke................

Wildame Duke.......... $\mathrm{L} R$

Wild Duke................ L R

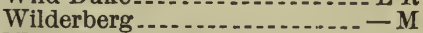

Wild Eyes Oxford........ - Cox

Wild Eyes Phyllis........... - R R

Wild Eyes Russell ............ - T

Wild Ranger.......................

Wildwood Agate.......... Ky I

Wildwood Favorite .............. Ky I

Wildwood Hermit............... Ky I

Wiley

Wiley's Duke................... R.

Wiley Duke of Crooked Lake - L R

Wilkes ...................... $\mathbf{M}$

Wilkins......................... $R$

William ..................

William................... R

William H.,.............. - P S

William Tell............

William Toll ............... P S

Willie of Center Point............M

Will Rogers................. Daisy

Will U. Tell ..................

Win

Winchester Chief

Winchester's Pride ......... D

Winchester Prince......... D

Winfred..................................

Winfield Jr.,............. P S

Winfield Lad................. L R

Winnebago Duke $2 \mathrm{~d} . . . . . . . .-\mathrm{M}$

Winnebago Duke $3 \mathrm{~d} . . . . . . . . . .-\mathrm{M}$ 


\begin{tabular}{|c|c|}
\hline 8 & Winnesheik Ch \\
\hline 459 & Winnimuck... \\
\hline 461 & Wisconsin. \\
\hline 463 & Witham... \\
\hline 78464 & Wonder Boy. \\
\hline $3+68$ & Worth. \\
\hline 78469 & Wotton Duke \\
\hline $78+70$ & Wright.... \\
\hline 78471 & Winyard. \\
\hline 78473 & Yankee.- \\
\hline 78474 & Yankton Boy \\
\hline 446 & Yeaman.. \\
\hline 477 & You Bet.. \\
\hline 3478 & Youghiogheny Du \\
\hline 480 & Young Acklam.. \\
\hline 481 & Young Acklam. \\
\hline 3482 & Young Acklan \&d \\
\hline 78484 & Young Airdrie Duk \\
\hline 78485 & Young Albert. \\
\hline 78487 & Young Apollo Bel \\
\hline 78499 & Young Banks. \\
\hline 78490 & Young Barringt \\
\hline 78491 & Young Ben. \\
\hline 78492 & Young Butterfly \\
\hline 78494 & Young Con \\
\hline 496 & Young Cou \\
\hline 497 & Young Dar \\
\hline 498 & Young Dimple.. \\
\hline $7 \$ 499$ & Young Duke.. \\
\hline 78502 & Young. Golden Cro \\
\hline 78503 & Young Governc \\
\hline 78504 & Young $\mathrm{Har}$ \\
\hline 506 & Young Kirklevington Lad \\
\hline
\end{tabular}

78507 Young Kirk of Shiloh.......- - L R ¿8508 Young Kirklev'ton of Shiloh-Rolla 78509 Young Kirkwood........... - Daisy 78511 Young Doudon............ $-\mathrm{R} R$ 785̃12 Young Mary Duke of Owen _._ M 78513 Young Mary's Lad..........- R R 78514 Young Mott................... Daisy 78515 Young Oak Hill.............. L R 78516 Young Oakland........... - Rolla 78517 Young Oliver.............. - P S 78518 Young Oneida.................. L R 78519 Young Oneida $2 \mathrm{~d} \ldots \ldots \ldots$ 78520 Young Oneida 3d............. M 78521 Young Oxford of Shiloh.... - R R 78522 Young Phantassie 2d......- - R R 78524 Y'g Prince Ed'd of Hic'ry Grove L R 785.25 Young Rocket 4th ............ R R 78526 Y'g Ry'l Booth's D. of H. Gr've-LR 78527 Young Rufus................... R 78528 Young Sampson............. M 78529) Young Sharon ..............

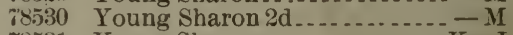
78531 Young Sherman............. Ky I 78532 Young Thunder.............. $-\mathrm{L} R$ 78533 Young Wilkes.............. $-\mathrm{L}$ R 78534 Zac............................ 7\$536 Zenaida's Duke ............. D ¿\$537 Zeniada's Oxford............ - C 785338 Zenas........................... Ky I 78539 Zero..................................... ¿8541 Zillah's Baronet.......... Rolla 78543 Zouroff ................. Multiflora 
